\title{
Initiation and Evolution of Matrix Cracking in Non-Symmetric Laminates under in-Plane and Flexural Loading
}

\author{
Adi Adumitroaie \\ West Virginia University
}

Follow this and additional works at: https://researchrepository.wvu.edu/etd

\section{Recommended Citation}

Adumitroaie, Adi, "Initiation and Evolution of Matrix Cracking in Non-Symmetric Laminates under in-Plane and Flexural Loading" (2012). Graduate Theses, Dissertations, and Problem Reports. 3595.

https://researchrepository.wvu.edu/etd/3595

This Dissertation is protected by copyright and/or related rights. It has been brought to you by the The Research Repository @ WVU with permission from the rights-holder(s). You are free to use this Dissertation in any way that is permitted by the copyright and related rights legislation that applies to your use. For other uses you must obtain permission from the rights-holder(s) directly, unless additional rights are indicated by a Creative Commons license in the record and/ or on the work itself. This Dissertation has been accepted for inclusion in WVU Graduate Theses, Dissertations, and Problem Reports collection by an authorized administrator of The Research Repository @ WVU.

For more information, please contact researchrepository@mail.wvu.edu. 


\title{
Initiation and Evolution of Matrix Cracking in Non-Symmetric Laminates under in-Plane and Flexural Loading
}

\author{
by \\ Adi Adumitroaie \\ Dissertation submitted to the \\ College of Engineering and Mineral Resources \\ at West Virginia University \\ in partial fulfillment of the requirements \\ for the degree of \\ Doctor of Philosophy \\ in \\ Aerospace Engineering
}

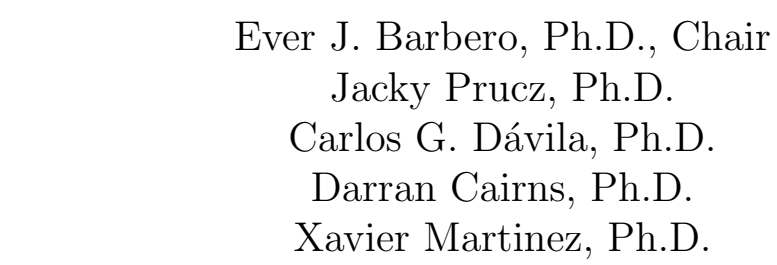

Department of Mechanical and Aerospace Engineering

Morgantown, West Virginia

2012

Keywords: progressive damage; matrix cracking; composite materials 


\begin{abstract}
Initiation and Evolution of Matrix Cracking in Non-Symmetric Laminates under in-Plane and Flexural Loading

by

Adi Adumitroaie

Doctor of Philosophy in Aerospace Engineering

West Virginia University

Ever J. Barbero, Ph.D., Chair
\end{abstract}

A constitutive model of progressive matrix cracking in fibers reinforced laminated composite is developed for the case of both membrane and flexural deformation. The progressive damage model makes use of the following key ingredients i) an appropriate material model for calculating the reduced thermo-elastic properties of the laminate containing individual plies affected by matrix cracking, ii) an energy based damage evolution criterion inspired by Fracture Mechanics, iii) an homogenization technique inspired by Continuum Damage Mechanics, iv) an iterative procedure in order to detect the conditions for damage growth in individual plies of the laminate, and to increase the damage level when the conditions are met, and v) the Classical Laminate Theory in order to describe the overall membrane and flexural deformation of the laminated composite. These elements are integrated into a new progressive damage model, where both the degraded mechanical properties of the laminate for given levels of matrix cracking in individual plies, and the matrix cracking process (both onset and progression) under applied loading are regarded.

Crack densities in individual plies of the laminates are the damage state variables of the model. This formulation is unlike the progressive damage models for laminated composites implemented in most of the FEA commercial packages, where softening laws are implemented in order to describe the stiffness reduction and the damage evolution. By using the ply crack densities as state variables the model is able to predict and to keep track of the crack density in individual plies during the loading history, which can be of interest in application where the permeability of the laminate is a limiting design factor. One example of this kind of application can be pressure vessels containing fluids or gases. Thermal residual stresses are taken into account in the present analytical model, which can extend the predictive capabilities of the model to applications in the range of cryogenic temperatures.

The process of matrix cracking under $I, I I$, or mixed $I-I I$ modes conditions are included in the present model. The loading case can be in-plane, flexural or combination of the two. There is no limitation on the configuration of the laminate or on the number of the cracking plies, as it is the case of the most models available in the literature, where only symmetric stacking sequences are addressed.

The analytical model is validated against available experimental data for the case of both membrane and flexural loading. 
I want to dedicate my work to my son Vlad, with hope and love. 


\section{Acknowledgments}

I want to thank all of my mentors and advisors that offered me guidance and support in order to accomplish my PhD work.

First of all, I want to express my gratitude and to bring my thanks to Dr. Ever J. Barbero, who besides being a great scientific advisor has also been a great friend, and whose elegance made my work under his guidance an always enriching experience. I want to thank all of my advising committee members for their input and feedback related to my research work, especially to Dr. Carlos G. Dávila, who offered me valuable support and opinions in order to improve the quality of my work. Special thanks to Dr. Jacky Prucz, whose support I could feel during the whole period of my time spent at West Virginia University. I would also like to acknowledge the contribution of my former professors in opening my interest for the world of composite materials: Dr. Elena Alamoreanu from Politehnica University of Bucharest and Dr. Ioan Fuiorea from the Military Technical Academy Bucharest, Romania.

My thanks to my friend and colleague Mr. Fritz Campo, a great mind and a great person as well, who has always offered me his opinions on any kind of problems, and who also provided a critical and constructive review of the present manuscript. 


\title{
Contents
}

\author{
Acknowledgments
}

List of Figures

1 Introduction $\quad 1$

1.1 Problem Statement . . . . . . . . . . . . . . . . . . . . 1

1.2 Main aspects of the problem . . . . . . . . . . . . . . . . 4

2 Problem Background. Literature Review $\quad 6$

2.1 Background of the reduced material properties aspect of the problem . . 6

2.1.1 Models based on $R U C$ method . . . . . . . . . . . . . . . . . . . 11

2.1.2 Models based on COD method . . . . . . . . . . . . . . . . . 18

2.1.3 Models based on CDM method . . . . . . . . . . . . . . 23

2.1.4 Models involving flexural deformation . . . . . . . . . . . 25

2.2 Background of the damage growth aspect of the problem . . . . . . . . . 27

2.2.1 Resistance-curve behavior of damage progression in laminated composites . . . . . . . . . . . . . . . . . 31

2.3 Concluding remarks on problem background . . . . . . . . . . . 33

3 Objectives $\quad 35$

4 Model Formulation $\quad 37$

4.1 Reduced Material Properties (Material Model) . . . . . . . . . . . . . . . 40

4.2 Ply homogenization technique . . . . . . . . . . . . . . . . . . . 41

4.3 Iterative procedure . . . . . . . . . . . . . . . . . . . . . . . . . . . . . . . .

4.4 Damage onset and damage evolution . . . . . . . . . . . . . . . . 43

4.4.1 Energy based damage growth criterion . . . . . . . . . . . . . 43

4.4.2 Modeling crack multiplication in laminated composites . . . . . . . . 46

4.4.3 Modeling the in-situ $\mathrm{R}$-curve behavior of damage evolution in laminated composites . . . . . . . . . . . . . . . . 50

4.5 Classical Laminate Theory . . . . . . . . . . . . . . . . . . . . . . 64

4.6 Analytical model formulation . . . . . . . . . . . . . . . . . . . 64

4.7 Additional comments on some modeling aspects . . . . . . . . . . . . . . 68

4.7.1 Displacement vs. Load control case . . . . . . . . . . . . . . 68

4.7 .2 Crack Closure model . . . . . . . . . . . . . . . . . 74 
5 Results and Discussion $\quad 77$

5.1 Analytical model output: analysis of expected trends . . . . . . . . . . . . 79

5.1.1 The influence of the laminate configuration . . . . . . . . . . . . . 80

5.1 .2 Extension vs. Bending loading . . . . . . . . . . . . . . . . 100

5.1 .3 Combined loading . . . . . . . . . . . . . . . . . 105

5.1 .4 In-situ R-curve behavior . . . . . . . . . . . . . . . . . . . 109

5.1.5 The influence of thermal residual stresses . . . . . . . . . . . . . . 114

5.2 Analytical model output: validation against experiments . . . . . . . . . . 118

5.2.1 Model validation, experimental data set $1 \ldots \ldots$. . . . . . . . . . . . . . . . . 118

5.2 .2 Model validation, experimental data set $2 \ldots \ldots . \ldots . . \ldots 125$

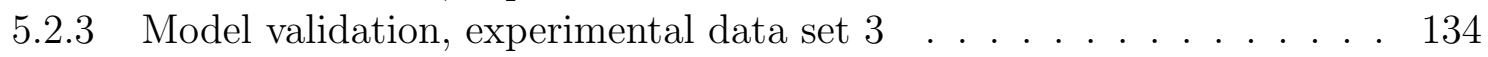

5.2 .4 Model validation, experimental data set $4 \ldots \ldots$. . . . . . . 155

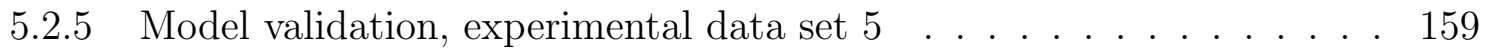

6 Conclusions $r$

A Classical Laminate Theory

$\begin{array}{lr}\text { considering the effect of thermal residual stresses } & 175\end{array}$

A.1 General constitutive formulation . . . . . . . . . . . . . . . . 175

A.1.1 The case of only mechanical loading . . . . . . . . . . . . . . . 177

A.1.2 The case of only thermal loading $\Delta T \ldots \ldots \ldots \ldots$. . . . . . . . . . . . . . . . . . 18

A.1.3 The case of both mechanical and thermal loading . . . . . . . . . . . 182

A.2 Laminate Strain Energy: effect of thermal residual stresses and damage mode separation . . . . . . . . . . . . . . . . . 183

B Analytical model for the reduced thermo-elastic properties of composite laminates with matrix cracks

B.1 Details of the modeling approach based on COD and energy balance: in-plane and bending deformation . . . . . . . . . . . . . . . .

B.2 Calculation of COD $\beta$ coefficients from analytical fracture mechanics and numerical methods . . . . . . . . . . . . . . 196

B.3 Calculation of membrane and flexural reduced material properties . . . . . . 200

B.4 Observations on the mathematical manipulation of the needed quantities of the material model . . . . . . . . . . . . . . . . . . . .

B.5 Details of the modeling approach based on COD and theory of elastic body with voids: only in-plane deformation . . . . . . . . . . . . 206

C Various mixed modes crack growth criteria 209

D Alternative approaches to crack multiplication in laminated composites 218

E Overview of progressive matrix cracking implementation in commercial FEA packages

E.1 The progressive damage model in Genoa . . . . . . . . . . . . . . . . 228

E.2 The progressive damage model in Abaqus . . . . . . . . . . . . . . . . . . 231

E.3 The progressive damage model in Helius . . . . . . . . . . . . . . . . . 238 
E.4 The progressive damage model in LS-Dyna . . . . . . . . . . . . . . . . . 243

References 


\section{List of Figures}

1.1 Ply Discount method vs. Progressive Damage Analysis. . . . . . . . . . . . . 4

2.1 Composite laminate featuring matrix cracking . . . . . . . . . . . . . 9

2.2 Modes of crack propagation . . . . . . . . . . . . . . . . . . . . . . . . 19

2.3 Matrix cracking in laminated composites and associated problems: row of cracks in an infinite transversely isotropic body, and row of cracks in an semiinfinite transversely isotropic body . . . . . . . . . . . . . . . . 22

2.4 Transverse ply strength as function of ply thickness . . . . . . . . . . . . . . 29

2.5 Back-calculated critical ERR based on analytical model and experimental crack multiplication data, Adolfsson and Gudmundson . . . . . . . . . . .

2.6 Back-calculated critical ERR based on analytical model and experimental crack multiplication data, Zhang and Soutis . . . . . . . . . . . . .

2.7 Back-calculated critical ERR based on analytical model and experimental crack multiplication data, Hahn . . . . . . . . . . . . . . . . . . . 32

2.8 Experimental measured critical ERR, Varna . . . . . . . . . . . . . . . . . . 32

2.9 The general evolution, with respect to the modeling capabilities, of analytical models for matrix cracking in FRP laminates. . . . . . . . . . . . . . . . . 34

4.1 CDM inspired ply homogenization technique. . . . . . . . . . . . . . . 41

4.2 Load vs. Displacement control crack multiplication . . . . . . . . . . . . . . 45

4.3 An equally spaced pattern of matrix cracks in one ply of a laminate. . . . . . 48

4.4 Mode I crack extension and critical ERR for homogeneous material . . . . . 56

4.5 Different possible directions of mode I matrix cracking in laminated composites 57

4.6 Mode I crack extension and critical ERR for UD fiber-reinforced composite: R-curve behavior . . . . . . . . . . . . . . . . . . . . 58

4.7 Mode I crack extension and critical ERR for laminated composite: $\mathrm{R}$-curve

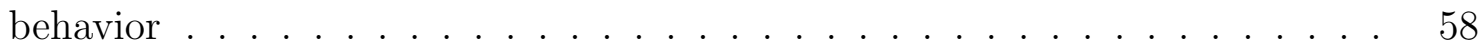

4.8 Mode I crack formation and critical ERR for cracking plies of different thickness: in-situ effect on the R-curve behavior . . . . . . . . . . . . . . 59

4.9 Typical matrix cracking multiplication data in laminated composites. . . . . 62

4.10 Model of R-curve behavior $G_{I c}=G_{I c}(\lambda)$ at $t=t_{\text {ref }} \ldots \ldots \ldots$. . . . . 62

4.11 Model of in-situ effect on the critical ERR for damage onset $\left(t \neq t_{\text {ref }}\right) \ldots$. . 63

4.12 Model of in-situ R-curve behavior . . . . . . . . . . . . . . . . 63 
4.13 The flow-chart of the membrane-flexural model for composited laminates subjected to damage in form of matrix cracking. . . . . . . . . . . . . . .

4.14 Elastic strain energy released in the case of Displacement control vs. Load control.

4.15 Loading cases and B.C. considered for numerical simulation

4.16 Crack closure effect . . . . . . . . . . . . . . . . . . . 74

4.17 The behavior of the analytical material model for different input LSS. . . . . 76

5.1 The influence of ply stiffness on the normalized extension stiffness $E_{x} / E_{x}^{0}$ of the laminate.

5.2 The influence of ply stiffness on the normalized CTE $\alpha_{x} / \alpha_{x}^{0}$ of the laminate. $\quad 81$

5.3 The influence of ply stiffness on the normalized Poisson $\nu_{x y} / \nu_{x y}^{0}$ of the laminate. 82

5.4 The influence of ply stiffness on the normalized shear modulus $G_{x y} / G_{x y}^{0}$ of the laminate. . . . . . . . . . . . . . . . . . 82

5.5 The influence of ply stiffness on the damage process, $\lambda=\lambda\left(\epsilon_{x}\right) \ldots \ldots 3$

5.6 The influence of ply stiffness on the damage process, $\lambda=\lambda\left(\sigma_{x}\right) \ldots \ldots$

5.7 The influence of ply stiffness on the stress-strain behavior of the laminate. . 84

5.8 The influence of the cracking ply thickness on the normalized extension stiffness $E_{x} / E_{x}^{0}$ of the laminate. . . . . . . . . . . . . . . .

5.9 The influence of the cracking ply thickness on the normalized Poisson $\nu_{x y} / \nu_{x y}^{0}$ of the laminate. . . . . . . . . . . . . . . . . . . . . .

5.10 The influence of the cracking ply thickness on the normalized shear modulus $G_{x y} / G_{x y}^{0}$ of the laminate. . . . . . . . . . . . . . .

5.11 The influence of the cracking ply thickness on the normalized CTE $\alpha_{x} / \alpha_{x}^{0}$ of the laminate.

5.12 The influence of the cracking ply thickness on the damage process, $\lambda=\lambda\left(\epsilon_{x}\right)$.

5.13 The influence of the cracking ply thickness on the damage process, $\lambda=\lambda\left(\sigma_{x}\right)$.

5.14 The influence of the cracking ply thickness on the stress-strain behavior of the laminate.

5.15 The influence of the cracking ply orientation on the normalized extension stiffness $E_{x} / E_{x}^{0}$ of the laminate.

5.16 The influence of the cracking ply orientation on the normalized Poisson $\nu_{x y} / \nu_{x y}^{0}$

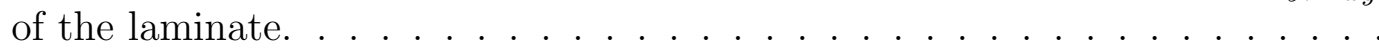

5.17 The influence of the cracking ply orientation on the normalized shear modulus $G_{x y} / G_{x y}^{0}$ of the laminate. . . . . . . . . . . . . . . .

5.18 The influence of the cracking ply orientation on the normalized CTE $\alpha_{x} / \alpha_{x}^{0}$ of the laminate. . . . . . . . . . . . . . . . . . . . . . 90

5.19 The influence of the cracking ply orientation on the damage process, $\lambda=\lambda\left(\epsilon_{x}\right)$. 91

5.20 The influence of the cracking ply orientation on the damage process, $\lambda=\lambda\left(\sigma_{x}\right)$. 91

5.21 The influence of the cracking ply orientation on the stress-strain behavior of the laminate.

5.22 Damage evolution in interior vs. surface cracking plies, $\left.\lambda=\lambda\left(\epsilon_{x}\right)\right) \ldots \ldots$. . . 92

5.23 Stress-strain behavior of the laminate for interior vs. surface cracking plies. .

5.24 The influence of the neighboring plies orientation on the normalized extension stiffness $E_{x} / E_{x}^{0}$ of the laminate. 
5.25 The influence of the neighboring plies orientation on the normalized Poisson $\nu_{x y} / \nu_{x y}^{0}$ of the laminate.

5.26 The influence of the neighboring plies orientation on the normalized shear modulus $G_{x y} / G_{x y}^{0}$ of the laminate. . . . . . . . . . . . . .

5.27 The influence of the neighboring plies orientation on the normalized CTE $\alpha_{x} / \alpha_{x}^{0}$ of the laminate.

5.28 The influence of the neighboring plies orientation on the damage process,

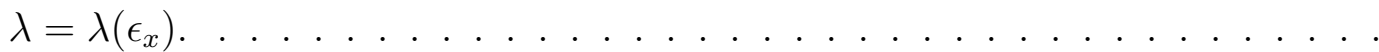

5.29 The influence of the neighboring plies orientation on the damage process, $\lambda=\lambda\left(\sigma_{x}\right)$.

5.30 The influence of the neighboring plies orientation on the stress-strain behavior of the laminate.

5.31 The influence of the neighboring plies thickness on the normalized extension stiffness $E_{x} / E_{x}^{0}$ of the laminate.

5.32 The influence of the neighboring plies thickness on the normalized Poisson $\nu_{x y} / \nu_{x y}^{0}$ of the laminate.

5.33 The influence of the neighboring plies thickness on the normalized shear modulus $G_{x y} / G_{x y}^{0}$ of the laminate.

5.34 The influence of the neighboring plies thickness on the normalized CTE $\alpha_{x} / \alpha_{x}^{0}$ of the laminate. . . . . . . . . . . . . . . . . . . .

5.35 The influence of the neighboring plies thickness on the damage process, $\lambda=$

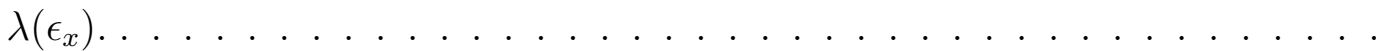

5.36 The influence of the neighboring plies thickness on the damage process, $\lambda=$

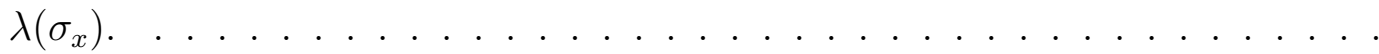

5.37 The influence of the neighboring plies thickness on the stress-strain behavior of the laminate.

5.38 Damage evolution under extension loading, $\lambda=\lambda\left(\epsilon_{x}\right)$

5.39 Damage evolution under bending loading, $\lambda=\lambda\left(\kappa_{x}\right)$.

5.40 Extension vs. bending loading: reduction of the normalized extension stiffness $E / E^{0}$ of the laminate.

5.41 Extension vs. bending loading: reduction of the normalized bending stiffness $B / B^{0}$ of the laminate.

5.42 Extension vs. bending loading: reduction of the normalized Poisson $\nu / \nu^{0}$ of

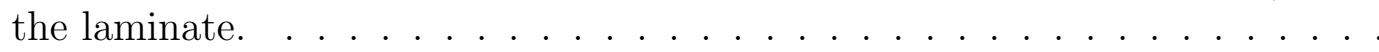

5.43 Extension vs. bending loading: evolution of the extension-bending coupling term, $E B_{x} / E B_{x}^{0}$, of the laminate. . . . . . . . . . . . . .

5.44 Extension vs. bending loading: reduction of the normalized shear modulus $G_{x y} / G_{x y}^{0}$ of the laminate.

5.45 Extension vs. bending loading: reduction of the normalized twisting modulus $T_{x y} / T_{x y}^{0}$ of the laminate.

5.46 Extension vs. bending loading: reduction of the normalized extension CTE,

5.46 Extension vs. bending loading: reduction of the normalized extension CTE,
$\alpha_{E} / \alpha_{E}^{0}$, of the laminate. . . . . . . . . . . . . . .

5.47 Extension vs. bending loading: evolution of the bending CTE, $\alpha_{B}$, of the laminate. . . . . . . . . . . . . . . . . . . 104

5.48 Damage process for the case of extension-bending combined loading, $\left(\epsilon_{x}-\kappa_{x}\right) .105$ 
5.49 Damage process for the case of biaxial extension loading, $\left(\epsilon_{x}-\epsilon_{y}\right),(0 / 90 / 0)$ LSS

5.50 Damage process for the case of biaxial extension loading, $\left(\epsilon_{x}-\epsilon_{y}\right),(0 / 90 / 0 / 90 / 0)$ LSS

5.51 The influence of $\beta_{\lambda}^{\text {ref }}$ parameter on the $\mathrm{R}$-curve behavior at the reference thickness $\left(t=t_{r e f}\right), G_{I C}(\lambda)$ coordinates.

5.52 The influence of $\beta_{\lambda}^{\text {ref }}$ parameter on the $\mathrm{R}$-curve behavior at the reference thickness $\left(t=t_{r e f}\right), G_{I C}\left(\epsilon_{x}\right)$ coordinates.

5.53 The influence of $\beta_{\lambda}^{\text {ref }}$ parameter on damage evolution at the reference thickness $\left(t=t_{r e f}\right)$.

5.54 The influence of $\beta_{\lambda}^{\text {ref }}$ parameter on the stress-strain behavior of the laminate at the reference thickness $\left(t=t_{r e f}\right) \ldots \ldots \ldots \ldots$

5.55 The influence of $\beta_{0}^{t}, \beta_{\lambda}^{t}$ parameters on the in-situ $\mathrm{R}$-curve behavior at $t \neq t_{\text {ref }}$, $G_{I C}(\lambda)$ coordinates. . . . . . . . . . . . . .

5.56 The influence of $\beta_{0}^{t}, \beta_{\lambda}^{t}$ parameters on the in-situ $\mathrm{R}$-curve behavior at $t \neq t_{\text {ref }}$, $G_{I C}\left(\epsilon_{x}\right)$ coordinates.

5.57 The influence of $\beta_{0}^{t}, \beta_{\lambda}^{t}$ parameters on damage evolution at $t \neq t_{\text {ref }}$.

5.58 The influence of $\beta_{0}^{t}, \beta_{\lambda}^{t}$ parameters on the stress-strain behavior of the lami-

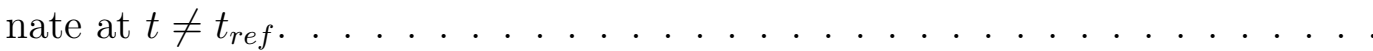

5.59 The influence of thermal residual stresses on damage process, Carbon-Epoxy material system. . . . . . . . . . . . . . . . . .

5.60 The influence of thermal residual stresses on damage process, Glass-Epoxy material system. . . . . . . . . . . . . . . . . . . .

5.61 Matrix cracking progression: analytical curve vs. experimental data, Wang. $\left( \pm 25 / 90_{2}\right)_{S}$ LSS.

5.62 Matrix cracking progression: analytical curve vs. experimental data, Wang.

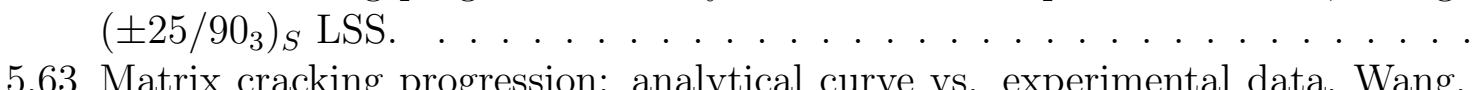
$\left( \pm 25 / 90_{4}\right)_{S}$ LSS.

5.64 Matrix cracking progression: analytical curve vs. experimental data, Wang. $\left( \pm 25 / 90_{6}\right)_{S}$ LSS.

5.65 Matrix cracking progression: analytical curves vs. experimental data, Wang. $\left( \pm 25 / 90_{n}\right)_{S}$ LSS.

5.66 Matrix cracking progression: analytical curves. $\left( \pm 25 / 90_{n}\right)_{S}$ LSS. . . . . . .

5.67 Matrix cracking progression: analytical curve vs. experimental data, Wang. $(0 / 90 / 0)$ and $\left(0 / 90_{2} / 0\right)$ LSS. . . . . . . . . . . . . . . .

5.68 Matrix cracking progression: analytical curve vs. experimental data, Wang. $\left(0 / 90_{3} / 0\right)$ LSS.

5.69 Matrix cracking progression: analytical curve vs. experimental data, Wang. $\left(0 / 90_{4} / 0\right)$ LSS.

5.70 Matrix cracking progression: analytical curve vs. experimental data, Wang. $\left(0_{2} / 90_{2}\right)_{S}$ and $\left(0_{2} / 90_{3}\right)_{S}$ LSS.

5.71 Matrix cracking progression: analytical curves vs. experimental data, Wang. $\left(0_{m} / 90_{n}\right)_{S}$ LSS. 
5.72 Matrix cracking progression: analytical curves. $\left(0_{m} / 90_{n}\right)_{S}$ LSS

5.73 Matrix cracking progression: analytical curve vs. experimental data, Nairn. $(0 / 90)_{S}$ and $\left(0 / 90_{2}\right)_{S}$ LSS, AS4/Hercules 3501-6 material system.

5.74 Matrix cracking progression: analytical curve vs. experimental data, Nairn. $\left(0 / 90_{4}\right)_{S}$ and $\left(0_{2} / 90_{2}\right)_{S}$ LSS, AS4/Hercules 3501-6 material system. . . . .

5.75 Matrix cracking progression: analytical curves vs. experimental data, Nairn. $\left(0_{m} / 90_{n}\right)_{S}$ LSS, AS4/Hercules 3501-6 material system. . . . . . . . . . .

5.76 Matrix cracking progression: analytical curves. $\left(0_{m} / 90_{n}\right)_{S}$ LSS, AS4/Hercules 3501-6 material system . . . . . . . . . . . . . . . . . .

5.77 Matrix cracking progression: analytical curve vs. experimental data, Nairn. $\left(0 / 90_{2}\right)_{S}$ and $\left(0 / 90_{3}\right)_{S}$ LSS, IM6/Avimid-K material system. . . . . . . . .

5.78 Matrix cracking progression: analytical curve vs. experimental data, Nairn. $\left(0_{2} / 90_{2}\right)_{S}$ and $\left(0_{2} / 90_{4}\right)_{S}$ LSS, IM6/Avimid-K material system. . . . . . . . .

5.79 Matrix cracking progression: analytical curves vs. experimental data, Nairn. $\left(0_{m} / 90_{n}\right)_{S}$ LSS, IM6/Avimid-K material system. . . . . . . . . . . . . . . .

5.80 Matrix cracking progression: analytical curves. $\left(0_{m} / 90_{n}\right)_{S}$ LSS, IM6/Avimid$K$ material system

5.81 Matrix cracking progression: analytical curve vs. experimental data, Nairn. $\left(90 / 0_{1 / 2}\right)_{S}$ and $(90 / 0)_{S}$ LSS, AS4/Hercules 3501-6 material system. . . . . .

5.82 Matrix cracking progression: analytical curve vs. experimental data, Nairn. $\left(90 / 0_{2}\right)_{S}$ and $\left(90 / 0_{4}\right)_{S}$ LSS, AS4/Hercules 3501-6 material system.

5.83 Matrix cracking progression: analytical curves vs. experimental data, Nairn. $\left(90 / 0_{m}\right)_{S}$ LSS, AS4/Hercules 3501-6 material system. . . . . . . . . . .

5.84 Matrix cracking progression: analytical curves. $\left[90 / 0_{4}\right]_{S} \mathrm{LSS}$, AS4/Hercules 3501-6 material system.

5.85 The $G_{I C}=G_{I C}(\lambda)$ in-situ R-curve evolution of the critical ERR for the AS4/Hercules 3501-6 material system, $\left(0_{m} / 90_{n}\right)_{S}$ LSS . . . . . . . . . .

5.86 The $G_{I C}=G_{I C}(\sigma)$ in-situ R-curve evolution of the critical ERR for the AS4/Hercules 3501-6 material system, $\left(0_{m} / 90_{n}\right)_{S}$ LSS

5.87 Reduced Young modulus $E_{x}(\lambda)$ : analytical curve vs. experimental data, Varna. $\left(0_{2} / 90_{4}\right)_{S}$ LSS, HyE 9082Af/Fiberite material system. . . . . . . . . .

5.88 Reduced Young modulus $E_{x}(\lambda)$ : analytical curve vs. experimental data, Varna. $\left( \pm 15 / 90_{4}\right)_{S}$ LSS, HyE 9082Af/Fiberite material system.

5.89 Reduced Young modulus $E_{x}(\lambda)$ : analytical curve vs. experimental data, Varna. $\left( \pm 30 / 90_{4}\right)_{S}$ LSS, HyE 9082Af/Fiberite material system.

5.90 Reduced Young modulus $E_{x}(\lambda)$ : analytical curve vs. experimental data, Varna. $\left( \pm 40 / 90_{4}\right)_{S}$ LSS, HyE 9082Af/Fiberite material system.

5.91 Reduced Poisson coefficient $\nu_{x y}(\lambda)$ : analytical curve vs. experimental data, Varna. $\left(0_{2} / 90_{4}\right)_{S}$ LSS, HyE 9082Af/Fiberite material system. . . . . . . . .

5.92 Reduced Poisson coefficient $\nu_{x y}(\lambda)$ : analytical curve vs. experimental data, Varna. $\left( \pm 15 / 90_{4}\right)_{S}$ LSS, HyE 9082Af/Fiberite material system.

5.93 Reduced Poisson coefficient $\nu_{x y}(\lambda)$ : analytical curve vs. experimental data, Varna. $\left( \pm 30 / 90_{4}\right)_{S}$ LSS, HyE 9082Af/Fiberite material system. . . . . . . .

5.94 Reduced Poisson coefficient $\nu_{x y}(\lambda)$ : analytical curve vs. experimental data, Varna. $\left( \pm 40 / 90_{4}\right)_{S}$ LSS, HyE 9082Af/Fiberite material system. 
5.95 Reduced Young modulus $E_{x}(\lambda)$ : analytical curves vs. experimental data, Varna. $\left( \pm \theta / 90_{4}\right)_{S}$ LSS, HyE 9082Af/Fiberite material system. . . . . . . . .

5.96 Reduced Poisson coefficient $\nu_{x y}(\lambda)$ : analytical curves vs. experimental data, Varna. $\left( \pm \theta / 90_{4}\right)_{S}$ LSS, HyE 9082Af/Fiberite material system. . . . . . . . .

5.97 Reduced Young modulus $E_{x}\left(\epsilon_{x}\right)$ : analytical curve vs. experimental data, Varna. $\left(0_{2} / 90_{4}\right)_{S}$ LSS, HyE 9082Af/Fiberite material system. . . . . . . . . .

5.98 Reduced Young modulus $E_{x}\left(\epsilon_{x}\right)$ : analytical curve vs. experimental data, Varna. $\left( \pm 15 / 90_{4}\right)_{S}$ LSS, HyE 9082Af/Fiberite material system. . . . . . . .

5.99 Reduced Young modulus $E_{x}\left(\epsilon_{x}\right)$ : analytical curve vs. experimental data, Varna. $\left( \pm 30 / 90_{4}\right)_{S}$ LSS, HyE 9082Af/Fiberite material system. . . . . . . .

5.100Reduced Young modulus $E_{x}\left(\epsilon_{x}\right)$ : analytical curve vs. experimental data, Varna. $\left( \pm 40 / 90_{4}\right)_{S}$ LSS, HyE 9082Af/Fiberite material system. . . . . . . .

5.101Reduced Poisson coefficient $\nu_{x y}\left(\epsilon_{x}\right)$ : analytical curve vs. experimental data, Varna. $\left(0_{2} / 90_{4}\right)_{S}$ LSS, HyE 9082Af/Fiberite material system. . . . . . . . . . 5.102Reduced Poisson coefficient $\nu_{x y}\left(\epsilon_{x}\right)$ : analytical curve vs. experimental data, Varna. $\left( \pm 15 / 90_{4}\right)_{S}$ LSS, HyE 9082Af/Fiberite material system. . . . . . . . 5.103Reduced Poisson coefficient $\nu_{x y}\left(\epsilon_{x}\right)$ : analytical curve vs. experimental data, Varna. $\left( \pm 30 / 90_{4}\right)_{S}$ LSS, HyE 9082Af/Fiberite material system. . . . . . . . 5.104Reduced Poisson coefficient $\nu_{x y}\left(\epsilon_{x}\right)$ : analytical curve vs. experimental data, Varna. $\left( \pm 40 / 90_{4}\right)_{S}$ LSS, HyE 9082Af/Fiberite material system. . . . . . . . 5.105Reduced Young modulus $E_{x}\left(\epsilon_{x}\right)$ : analytical curves vs. experimental data, Varna. $\left( \pm \theta / 90_{4}\right)_{S}$ LSS, HyE 9082Af/Fiberite material system. . . . . . . . . 5.106Reduced Poisson coefficient $\nu_{x y}\left(\epsilon_{x}\right)$ : analytical curves vs. experimental data, Varna. $\left( \pm \theta / 90_{4}\right)_{S}$ LSS, HyE 9082Af/Fiberite material system. . . . . . . . . 5.107Matrix cracking progression: analytical curve vs. experimental data, Varna. $\left(0_{2} / 90_{4}\right)_{S}$ LSS, HyE 9082Af/Fiberite material system. . . . . . . . . . . .

5.108Matrix cracking progression: analytical curve vs. experimental data, Varna. $\left( \pm 15 / 90_{4}\right)_{S}$ LSS, HyE 9082Af/Fiberite material system. . . . . . . . . . . . 5.109Matrix cracking progression: analytical curve vs. experimental data, Varna. $\left( \pm 30 / 90_{4}\right)_{S}$ LSS, HyE 9082Af/Fiberite material system. . . . . . . . . . . . .

5.110Matrix cracking progression: analytical curve vs. experimental data, Varna. $\left( \pm 40 / 90_{4}\right)_{S}$ LSS, HyE 9082Af/Fiberite material system. . . . . . . . . . . . .

5.111Matrix cracking progression: analytical curves vs. experimental data, Varna. $\left( \pm \theta / 90_{4}\right)_{S}$ LSS, HyE 9082Af/Fiberite material system. . . . . . . . . . . . .

5.112The $G_{I C}=G_{I C}(\lambda)$ in-situ R-curve evolution of the critical ERR for the HyE 9082Af/Fiberite material system, $\left( \pm \theta / 90_{4}\right)_{S}$ LSS . . . . . . . . . .

5.113The $G_{I C}=G_{I C}(\epsilon)$ in-situ R-curve evolution of the critical ERR for the HyE 9082Af/Fiberite material system, $\left( \pm \theta / 90_{4}\right)_{S}$ LSS . . . . . . . . . .

5.114Reduced Young modulus $E_{x}(\lambda)$ : analytical curve vs. experimental data, Varna. $\left(0 / 90_{8} / 0_{1 / 2}\right)_{S}$ LSS, HyE 9082Af/Fiberite material system. . . . . . .

5.115Reduced Young modulus $E_{x}(\lambda)$ : analytical curve vs. experimental data, Varna. $\left(0 / \pm 70_{4} / 0_{1 / 2}\right)_{S}$ LSS, HyE 9082Af/Fiberite material system. . . . . .

5.116Reduced Young modulus $E_{x}(\lambda)$ : analytical curve vs. experimental data, Varna. $\left(0 / \pm 55_{4} / 0_{1 / 2}\right)_{S}$ LSS, HyE 9082Af/Fiberite material system. . . . . . 
5.117Reduced Young modulus $E_{x}(\lambda)$ : analytical curves vs. experimental data, Varna. (0/ $\left.\pm \theta_{4} / 0_{1 / 2}\right)_{S}$ LSS, HyE 9082Af/Fiberite material system. . . . . . 5.118Reduced Poisson coefficient $\nu_{x y}(\lambda)$ : analytical curve vs. experimental data, Varna. $\left(0 / 90_{8} / 0_{1 / 2}\right)_{S}$ LSS, HyE 9082Af/Fiberite material system. . . . . . . 5.119Reduced Poisson coefficient $\nu_{x y}(\lambda)$ : analytical curve vs. experimental data, Varna. (0/ $\left.\pm 70_{4} / 0_{1 / 2}\right)_{S}$ LSS, HyE 9082Af/Fiberite material system. . . . . . 5.120Reduced Poisson coefficient $\nu_{x y}(\lambda)$ : analytical curve vs. experimental data, Varna. (0/ $\left.\pm 55_{4} / 0_{1 / 2}\right)_{S}$ LSS, HyE 9082Af/Fiberite material system. . . . . . 5.121Reduced Poisson coefficient $\nu_{x y}(\lambda)$ : analytical curve vs. experimental data, Varna. $\left(0 / \pm \theta_{4} / 0_{1 / 2}\right)_{S}$ LSS, HyE 9082Af/Fiberite material system. . . . . . 5.122Matrix cracking progression: analytical curve vs. experimental data, Varna. $\left(0 / 90_{8} / 0_{1 / 2}\right)_{S}$ LSS, HyE 9082Af/Fiberite material system. . . . . . . . . . . . 5.123Matrix cracking progression: analytical curve vs. experimental data, Varna. $\left(0 / \pm 70_{4} / 0_{1 / 2}\right)_{S}$ LSS, HyE 9082Af/Fiberite material system. . . . . . . . . . 5.124Matrix cracking progression: analytical curve vs. experimental data, Varna. $\left(0 / \pm 55_{4} / 0_{1 / 2}\right)_{S}$ LSS, HyE 9082Af/Fiberite material system. . . . . . . . . .

5.125Matrix cracking progression: analytical curves vs. experimental data, Varna. $\left(0 / \pm \theta_{4} / 0_{1 / 2}\right)_{S}$ LSS, HyE 9082Af/Fiberite material system. . . . . . . . . . . 5.126The $G_{I C}=G_{I C}(\lambda)$ in-situ R-curve evolution of the critical ERR for the HyE 9082Af/Fiberite material system, $\left(0 / \pm \theta_{4} / 0_{1 / 2}\right)_{S}$ LSS . . . . . . . . . . . 5.127The $G_{I C}=G_{I C}(\lambda)$ in-situ R-curve evolution of the critical ERR for the $H y E$ 9082Af/Fiberite material system, $\left(0 / \pm \theta_{4} / 0_{1 / 2}\right)_{S}$ LSS . . . . . . . . . .

5.128Reduced Young modulus $E_{x}(\lambda)$ : analytical curves vs. experimental data, Rubenis. $\left(0_{m} / 90_{n}\right)_{S}$ LSS, Vicotex 913/28\%/192/EC9756 material system.

5.129Reduced Poisson coefficient $\nu_{x y}(\lambda)$ : analytical curves vs. experimental data, Rubenis. $\left(0_{m} / 90_{n}\right)_{S}$ LSS, Vicotex 913/28\%/192/EC9756 material system. .

5.130Matrix cracking progression: analytical curve vs. experimental data, Varna. $\left(0 / 90_{2}\right)_{S}$ LSS, Vicotex 913/28\%/192/EC9756 material system. . . . . . . . 5.131Matrix cracking progression: analytical curve vs. experimental data, Varna. $\left(0_{2} / 90_{2}\right)_{S}$ LSS, Vicotex 913/28\%/192/EC9756 material system. . . . . . . . 5.132Matrix cracking progression: analytical curve vs. experimental data, Varna. $\left(0 / 90_{4}\right)_{S}$ LSS, Vicotex 913/28\%/192/EC9756 material system. . . . . . . . .

5.133Matrix cracking progression: analytical curves vs. experimental data, Varna. $\left(0_{m} / 90_{n}\right)_{S}$ LSS, Vicotex 913/28\%/192/EC9756 material system. . . . . . . .

5.134The $G_{I C}=G_{I C}(\lambda)$ in-situ R-curve evolution of the critical ERR for the Vicotex 913/28\%/192/EC9756 material system, $\left(0_{m} / 90_{n}\right)_{S}$ LSS . . . . . .

5.135The $G_{I C}=G_{I C}(\epsilon)$ in-situ R-curve evolution of the critical ERR for the Vicotex 913/28\%/192/EC9756 material system, $\left(0_{m} / 90_{n}\right)_{S}$ LSS . . . . . . . 5.136Reduced Young modulus: analytical curves vs. experimental data, Ogin. $(0 / 90)_{S}$ and $(0 / 90 /-45 /+45)_{S}$ LSS, $\epsilon_{x}$ loading. . . . . . . . . . . 5.137Reduced Poisson coefficient: analytical curves vs. experimental data, Ogin. $(0 / 90)_{S}$ and $(0 / 90 /-45 /+45)_{S}$ LSS, $\epsilon_{x}$ loading. . . . . . . . . . . 5.138Matrix cracking progression: analytical curve vs. experimental data, Ogin. $(0 / 90)_{S}$ LSS, $\epsilon_{x}$ loading. . . . . . . . . . . . . . . . 
5.139Matrix cracking progression: analytical curve vs. experimental data, Ogin. $(0 / 90)_{S}$ and $(0 / 90 /-45 /+45)_{S}$ LSS, $\epsilon_{x}$ loading. . . . . . . . . . 5.140Matrix cracking progression: analytical curve vs. experimental data, Ogin. $(0 / 90)_{2 S}$ LSS, $M_{x}$ loading. . . . . . . . . . . . . .

5.141Matrix cracking progression: analytical curve vs. experimental data, Ogin. $(0 / 90)_{2 S}$ LSS, $\kappa_{x}$ loading. . . . . . . . . . . . . . . .

5.142Reduced Young modulus: analytical curves vs. experimental data, Gudmundson. $\left(0 / 90_{n} / 0\right)$ LSS, $\epsilon_{x}$ loading. . . . . . . . . . . . . . . .

5.143Reduced Poisson coefficient: analytical curves vs. experimental data, Gudmundson. $\left(0 / 90_{n} / 0\right)$ LSS, $\epsilon_{x}$ loading. . . . . . . . . . . . . .

5.144Matrix cracking progression: analytical curve vs. experimental data, Gudmundson. (0/90/0) LSS, $\epsilon_{x}$ loading. . . . . . . . . . . . . . . .

5.145Matrix cracking progression: analytical curve vs. experimental data, Gudmundson. $\left(0 / 90_{2} / 0\right)$ LSS, $\epsilon_{x}$ loading. . . . . . . . . . . . . . .

5.146Matrix cracking progression: analytical curve vs. experimental data, Gudmundson. (0/904/0) LSS, $\epsilon_{x}$ loading. . . . . . . . . . . . .

5.147Matrix cracking progression: analytical curve vs. experimental data, Gudmundson. $\left(0 / 90_{8} / 0\right)$ LSS, $\epsilon_{x}$ loading. . . . . . . . . . . . . .

5.148Stress-strain evolution: analytical curve vs. experimental data, Gudmundson. $(0 / 90 / 0)$ LSS, $\epsilon_{x}$ loading. . . . . . . . . . . . . . . . . . .

5.149Stress-strain evolution: analytical curve vs. experimental data, Gudmundson. $\left(0 / 90_{2} / 0\right)$ LSS, $\epsilon_{x}$ loading. . . . . . . . . . . . . . .

5.150Stress-strain evolution: analytical curve vs. experimental data, Gudmundson. $\left(0 / 90_{4} / 0\right)$ LSS, $\epsilon_{x}$ loading. . . . . . . . . . . . . . .

5.151Stress-strain evolution: analytical curve vs. experimental data, Gudmundson. $\left(0 / 90_{8} / 0\right)$ LSS, $\epsilon_{x}$ loading.

5.152Matrix cracking progression: analytical curves vs. experimental data, Gudmundson. $\left(0 / 90_{n} / 0\right)$ LSS, $\epsilon_{x}$ loading. . . . . . . . . . . . .

5.153Stress-strain evolution: analytical curves vs. experimental data, Gudmundson. $\left(0 / 90_{n} / 0\right)$ LSS, $\epsilon_{x}$ loading. . . . . . . . . . . . . . . . .

5.154Matrix cracking progression: analytical curves vs. experimental data, Gudmundson. $\left(0_{n} / 90_{n} /+45_{n} /-45_{n}\right)_{S}$ LSS, $\epsilon_{x}$ loading. . . . . . . . . . .

5.155Matrix cracking progression: analytical curve. $\left[0_{n} / 90_{n} /+45_{n} /-45_{n}\right]_{S}$ LSS, $\epsilon_{x}$ loading. . . . . . . . . . . . . . . . . .

5.156Stress-strain evolution: analytical curve vs. experimental data, Gudmundson. $(0 / 90 /+45 /-45)_{S}$ LSS, $\epsilon_{x}$ loading.

5.157Stress-strain evolution: analytical curve vs. experimental data, Gudmundson. $\left(0_{2} / 90_{2} /+45_{2} /-45_{2}\right)_{S}$ LSS, $\epsilon_{x}$ loading. . . . . . . . . . .

5.158Stress-strain evolution: analytical curve vs. experimental data, Gudmundson. $(0 /+45 /-45)_{S}$ LSS, $\epsilon_{x}$ loading.

5.159Stress-strain evolution: analytical curve vs. experimental data, Gudmundson. $\left(0_{2} /+45_{2} /-45_{2}\right)_{S}$ LSS, $\epsilon_{x}$ loading. . . . . . . . . . . .

5.160Matrix cracking progression: analytical curve vs. experimental data, Gudmundson. $\left(90_{2} / 0_{2} /-45_{2} /+45_{2}\right)_{S}$ and $\left(90_{2} /-45_{2} /+45_{2}\right)_{S}$ LSS, $\kappa_{x}$ loading. 
5.161Moment-curvature evolution: analytical curves vs. experimental data, Gudmundson. $\left(90_{2} / 0_{2} /-45_{2} /+45_{2}\right)_{S}$ and $\left(90_{2} /-45_{2} /+45_{2}\right)_{S}$ LSS, $\kappa_{x}$ loading.

A.1 The influence of different CTE of different plies of the laminate.

B.1 Decomposition of ply stresses in uniform and linear components . . . . . . . 205

B.2 Rotation of surface tractions and COD . . . . . . . . . . . . . . 207

C.1 Mixed modes damage growth criteria: simple non-interacting; linear; power law $\left(G_{I}=f\left(G_{I I}\right) \ldots \ldots \ldots \ldots\right.$

C.2 Mixed modes damage growth criteria: simple non-interacting; linear; power

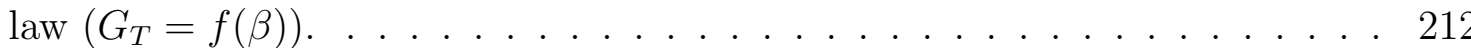

C.3 Exponential hackle mixed modes damage growth criterion $\left(G_{I}=f\left(G_{I I}\right)\right)$. . 213

C.4 Exponential hackle mixed modes damage growth criterion $\left(G_{T}=f(\beta)\right)$. . . 213

C.5 Exponential $K_{I} / K_{I I}$ mixed modes damage growth criterion $\left(G_{I}=f\left(G_{I I}\right)\right)$. . 214

C.6 Exponential $K_{I} / K_{I I}$ mixed modes damage growth criterion $\left(G_{T}=f(\beta)\right)$. . 214

C.7 Interaction mixed modes damage growth criterion $\left(G_{I}=f\left(G_{I I}\right)\right)$. . . . . 215

C.8 Interaction mixed modes damage growth criterion $\left(G_{T}=f(\beta)\right)$. . . . . . 215

C.9 $K_{I}$ mixed modes damage growth criterion $\left(G_{I}=f\left(G_{I I}\right)\right) \ldots \ldots \ldots \ldots$

C.10 $K_{I}$ mixed modes damage growth criterion $\left(G_{T}=f(\beta)\right) \ldots \ldots \ldots$

D.1 Damage evolution curves for different thicknesses of the cracking ply, $(\lambda-\sigma)$ coordinates. . . . . . . . . . . . . . . . . . . . . . . 219

D.2 Closely spaced damage evolution curves for different thicknesses of the cracking ply in $(\rho-\epsilon)$ coordinates. Selection of the $\rho(\epsilon)$ damage evolution criterion, Gudmundson. . . . . . . . . . . . . . . . . . . . .

D.3 Combination of $G_{C}(\lambda)$ and $\lambda(\epsilon)$ experimental data in order to obtain the

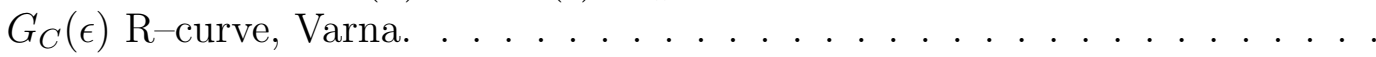

D.4 Required intersecting trends of the $\lambda(\epsilon)$ damage evolution curves for different thicknesses of the cracking ply. . . . . . . . . . . . .

E.1 Micromechanics RUC model developed for symmetric laminate featuring matrix cracks in the central ply, Soutis. . . . . . . . . . . . . . . 229

E.2 Model of stress transfer (cohesive zone) during crack propagation. . . . . . . 232

E.3 a) Linear softening $(\sigma-\delta)$ stress transfer model; b) The variation of the damage parameter in the $(\sigma-\delta)$ softening model. . . . . . . . . . . . . . 234

E.4 Mesh sensitivity in the $(\sigma-\delta)$ softening model . . . . . . . . . . . . . 237

E.5 Fiber-matrix averaging approach of MCT. . . . . . . . . . . . . . . . 239

E.6 Discrete (4 step) matrix stiffness degradation, Helius progressive damage model.242

E.7 Mesh sensitivity study for Helius progressive damage model. . . . . . . . . . . 244

E.8 Progressive damage model, $(\sigma-\epsilon)$ exponential softening law. . . . . . . . . . 245 


\section{Chapter 1}

\section{Introduction}

\subsection{Problem Statement}

The characterization of composite materials has been an intensive field of research since their emergence as a new class of high performance structural materials. On one hand, the advantages offered by composite materials are very tempting, opening a large pool of choices for both new applications and alternative solutions for traditional applications, e.g., lightweight structures. On the other hand, the use of composite materials requires the knowledge of limiting conditions under lifetime loading for each application demanding extensive amount of research. As a matter of fact, the same anisotropy and heterogeneity of the material properties that governs their unique behavior, raises the complexity in quantifying and understanding the behaviors and the properties. Thus, new experimental and analytical tools are constantly demanded to study composite materials.

In particular, damage and failure of laminated Fiber Reinforced Polymers (FRP) has been treated by many researcher under a variety of approaches. Damage is defined as any deterioration in the material integrity that triggers a change in mechanical properties and behavior. Usually, damage induces a reduction of the load carrying capacity of the structure. There is a moment of damage onset (damage initiation), followed by damage propagation under increasing loading. Failure is defined as the damage level when the material has totally lost his structural functionality due to damage. The failure of the structure, or of the structural component, is decided according with its structural role. For example, for a tensile loaded structural component, the failure can be considered the moment of breakage, when the structural component stops to carry tensile loading; there might be some forms of 
progressive damage up to the moment of failure. For a pressure vessel structure, the failure moment might be considered when the first crack appears in the material, and the structure stops meeting its functional requirements, i.e., containing the pressurized fluid or gas.

Damage mechanisms such as fiber breaking, fiber buckling, inter-ply delamination, fibermatrix debonding, and matrix deterioration, follow different laws particular of the material structure and loading case. These failure modes, also dependent on the material structure and loading case, can be catastrophic (i.e., structural failure) or not, but regardless of their primary structural impact, each one has to be very well understood and controlled. All of them cause a deterioration in the mechanical property and structural integrity, and in some cases they interact, as it is the case when a non catastrophic failure mode may work as a nucleation point of a catastrophic failure mode.

The traditional tools involved in damage analysis of isotropic homogeneous materials of course needed to be modified, adapted or totally replaced by new theories specially designed for the class of composite materials. The multiple modes of damage which can be encountered in composites under structural loading, and the interaction between these different and separate damage modes, complicate the problem even more. Traditionally, modified versions of failure theories (failure criteria) of isotropic homogeneous materials has been used. Recently, more advanced models have been proposed in order to describe the progressive process of damage propagation in composite materials up to failure. Damage tolerance of the composite structure (i.e., the capacity of the structure to withstand load after damage onset) has become a design criterion. However, since damage involves multiple damage mechanisms which can interact as the material is loaded in different ways, traditional approaches remain behind to predict this phenomenon.

The terminology dedicated to laminated composites has classified the various damage mechanisms that can be encountered in this materials in two main categories: i) interlaminar damage is the deterioration taking place between separate plies of the laminate, at the plies interface. The most common form of inter-laminar damage is delamination (debonding) between plies; ii) intra-laminar damage is the deterioration taking place inside of individual plies of the laminate. Forms of intra-laminar damage are transverse matrix cracking due to tensile/shear loading, matrix crushing in compression, fiber breaking in tension, fiber buckling or kinging in compression, fiber crushing.

This dissertation treats progressive damage through intra-laminar matrix cracking in laminated continuous fiber reinforced polymers under mechanical loading, including the ef- 
fect of residual thermal stresses. Even if this damage mode is not catastrophic in most of the structural applications, it has a negative impact on the structural performance. Thus, according to the laminate architecture, namely the mechanical properties of individual fiber and matrix constituents, ply orientation angles, plies thickness, the matrix cracking by itself has a degrading effect on mechanical properties, i.e., stiffness and thermal expansion coefficients of the material.

Moreover, experimental observations show that matrix cracking not only affects the ply locally, it also affects neighboring plies. Thus, due to stress concentrations, inter-ply delamination is initiated at the tip of matrix cracking, and especially at the intersection point between two cracks in two different adjacent layers. The same stress concentration effect at the tip of the matrix crack can develop fiber breakage. However, matrix cracking can be even regarded as a catastrophic model of damage in other applications, as it is the previous example of the pressure vessel, where matrix cracking can constitute a path way for gas or liquid penetration, leading to exterior leakage or more difficult to detect fibers corrosion. The latter may induce weakening of fibers and ultimately fibers breakage.

These are some of the reasons for spending a considerable amount of research in studying matrix cracking in composite materials. As a result, the theoretical models are expected to provide reliable tools to asses the matrix cracking effects on the overall structural level. According to this objective, the effort of the researchers has provided answers to some of the questions, but still other aspects remain to be investigated.

The problem of transverse matrix cracking in laminated composites has been extensively studied for the particular case of $\left[0_{m} / 90_{n}\right]_{S}$. Matrix cracking is found in the transverse $90^{\circ}$ plies. Successively extended to other laminate configurations such as $[0 / \pm \theta / 0]_{S}$ and $\left[0 / \theta_{1} / \theta_{2}\right]_{S}$, models featuring cracks in the off-axis $\theta$ plies have been developed. Acknowledging that the models are considering more general problems, the models are still limited to symmetric laminates under in-plane loading. The approaches considering a general laminate configuration and bending deformation are rare in the literature. The general case of a $\left[\theta_{1} / \theta_{2} / \ldots / \theta_{n}\right]$ laminate with matrix cracks in any ply under general membrane and flexural loading, is the subject of this work. 


\subsection{Main aspects of the problem}

The first basic approach to damage in laminated fiber reinforced composites is labeled in literature as the Ply Discount Method (PDM), where the entire ply is discarded (the ply stiffness is set to zero or is reduced to some arbitrary chosen value close to zero) along the damage direction as soon as damage is detected on that particular direction. While ply discount is a very expedient and easy to implement method, it has been proved to be inaccurate and rudimentary, because the affected ply obviously has stiffness and load carrying capacity after damage is initiated. The process of mechanical degradation is gradual requiring increase in the load. Thus, more refined and precise models have been deviced by researchers in order to characterize the progressive damage evolution in laminated composites. These refined models are referred to as Progressive Damage Analysis (PDA).

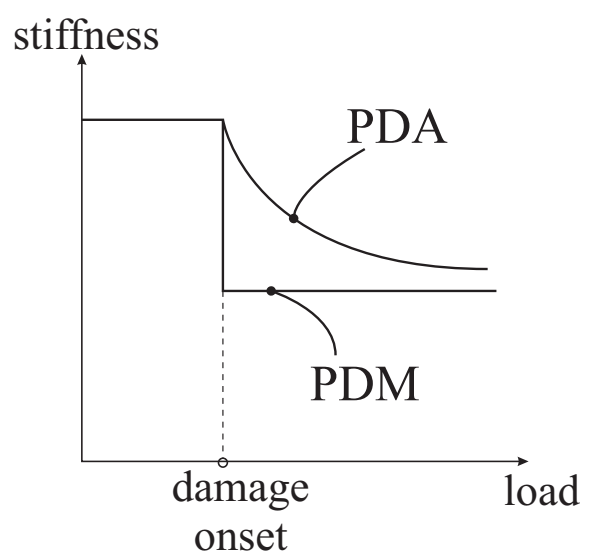

Figure 1.1: Ply Discount method vs. Progressive Damage Analysis.

Two main aspects regarding the material properties and behavior have to be considered in order to perform progressive damage analysis of laminated composites. First, the reduction in the material thermo-elastic constants at a given damage level has to be evaluated. This aspect will be regarded as the reduced material properties in the following, and can be described by the following relationship

$$
[C]=\left[C\left(\lambda^{(k)}\right)\right]
$$

where $[C]$ generically represents the thermo-elastic properties of the material, and $\lambda$ represents the state variable accounting for the damage level inside of the material. One example of damage variable for intra-laminar matrix cracking can be the crack density (i.e., number 
of cracks per unit length $\lambda=1 / d$, where $d$ is the distance between two consecutive cracks as presented in Fig. 2.1 (a)) inside of any ply $(k)$ of the laminate. Other damage variables can be defined as well.

The background of the problem of reduced material properties due to intra-laminar matrix cracking is presented in Section 2.1. A proper material model is selected from the literature and integrated in the present model of progressive damage analytical model, as described in Section 4.

The second aspect is the prediction of the damage onset and progression due external thermo-mechanical loads applied to the laminate. This aspect will be regarded as damage growth in the following, and it can be represented by the relationship

$$
\lambda^{(k)}=\lambda^{(k)}(\epsilon)
$$

where $\lambda^{(k)}$ is the damage variable representing the damage level in any ply $(k)$ of the laminate, and $\epsilon$ generically represents the external thermo-mechanical loads applied to the laminate. The background of the damage growth aspect is presented in Section 2.2, and the implementation and modeling specific to the goals of the present analytical model are presented in Section 4.4

Both aspects, generally labeled as damage mechanics, have been investigated through different approaches and research tools. The main goal of the present analytical model is to predict the progressive damage in the form of transverse matrix cracking for a general laminate case under a general membrane and flexural loading. 


\section{Chapter 2}

\section{Problem Background. Literature Review}

The main stages analyzing the problem of intra-laminar matrix cracking in laminated fiber reinforced polymers is presented in this section. The achievements and limitations of each model or class of models are briefly presented and commented, with two goals: i) to present the possible analytical tools used, modified, or developed in order to approach the problem formulated in Section 1.1, listing the assumptions and limitations of each tool, and ii) to create a frame in which the place and the achievements of the present work, with respect to other similar research studies, will be underlined.

\subsection{Background of the reduced material properties as- pect of the problem}

Various modeling approaches addressing the degradation in material properties due to matrix cracking can be found in the technical literature. These different modeling strategies are briefly listed and described in their core principles in the following.

Continuum damage mechanics (CDM) approach can be found in the work of Varna and Talreja [1, 2], Groves and Allen [3, 4], Li, Reid, and Soden [5, 6]. Continuum damage variables are used to express the reduction in mechanical properties of the material. The individual plies affected by damage, in this case in the form of transverse matrix cracking are considered as an homogenized medium having their properties expressed as a fraction of 
the undamaged (virgin) material properties through the use of continuum damage variables. Cracks are not regarded as separate entities. Usually, additional experimental parameters are involved in expressing the damage variables. Therefore, the method does not have a high level of generality, requiring independent tests for each of the different laminate configurations to obtain the desired experimental parameters.

However, the method by itself can be applied to the calculation of the reduced material properties for a given damage level. Since a detailed stress analysis due to presence of cracks is not possible due to the homogenization method, there might be difficulties in dealing with the second part of the problem, i.e., the damage growth. Even when the CDM method is used in damage growth evaluation, its dependence on experimental parameters makes the method difficult to apply in practical engineering design calculations.

Micromechanics of damage (MMD) approach can be found in the work of Nairn [7, 8, 9, 10, 11, 12, 13, 14, 15, 16], Hahn [17], Aboudi and Herakovich [18, 19], Crossman [20], Flaggs [21], Li [22, 23], Rebiere [24], Tan and Nuismer [25, 26], Yokozeki and Aoki $[27,28]$. This method is the most widely used modeling strategy when individual cracks are physically considered and stress-strain field due to presence of cracks is calculated using the Representative Unit Cell (RUC, Fig. 2.1(a)) technique. The RUC technique basically consists of selecting the space of material between two consecutive cracks. When applied, the symmetry of loading, geometry and material is exploited by reducing the analysis to only half or a quarter of the RUC. The solution can be reached by approximately solving the elasticity problem of the stress-strain field inside of RUC.

The term micro used in the name "micromechanics of damage" does not refer to individual fiber to matrix interaction, but instead to emphasize the detailed stress-strain analysis performed to the cracked ply, which is no longer considered as an homogenized one. The analysis is given a higher level of detail as compared to the meso scale level of the homogenized ply. The term micro found in the literature will be consequently used in the present manuscript.

Of course, the stress state and deformation in the cracked layered composite material, with cracks in some or in all of its plies, is a very difficult $3 D$ problem whose analytical solution is very difficult or impossible to calculate. Simplifications have been considered by various MMD models in the literature to express the stress-strain fields. The most common ones are plane stress, plane strain or generalized plane strain. The expense for using these assumptions is that only specific problems, or particular classes of problems satisfying the 
assumptions, can be analyzed. In this way, only some loading cases and some laminate configurations can be analyzed, but not general cases.

The advantage of MMD is the fact that, once the stress-strain field is calculated, analytical solutions of the reduced materials properties and the formulation of damage growth criteria are easy to implement, without a strong dependence on experimental parameters.

MMD and CDM have been improved or modified to overcome particular drawbacks and to extend their abilities to predict experimental measurements. For example, synergistic damage mechanics are methods that combine elements of CDM and MMD, as in Varna and Talreja [1], Li, Reid and Soden [5, 6], Barbero [29, 30]. Also, the statistical distribution of the material strength, which corresponds to a statistical distribution of material flows, has also been modeled and taken into account in Varna [1], Li [6], Wang and Chou [31], as an refinement of one of the base methods; usually, refinement of the method brings the need of incorporating additional experimental parameters.

An alternative approach to the problem of matrix cracking in laminated composites has been developed based on approximate solutions for crack opening displacement (COD, Fig. 2.1(c)), as in Gudmundson and Adolfsson [32, 33, 34, 35, 36, 37, 38], Varna and Lundmark [39]. The COD method is defined in the frame of Kachanov theory of elastic bodies with voids [40]. The COD parameter, which is defined as the relative displacement between two faces of the same crack lying along fiber direction of a ply (Fig. 2.1(c)), is difficult to calculate analytically for the case of matrix cracking in laminated composites. This happens because the laminate configuration influences the COD parameter, and consequently, the generalization of the analytical COD solution for any laminate configuration is not possible. Thus, the COD solution is approximated by either i) analytical laws suggested from extensive parametric FEA simulations [32, 35, 39], or ii) extension of the fracture mechanics solution developed for similar problems $[32,33,34,35]$. The latter approximation uses FEA analysis to asses the level of error and acceptance of the solution to the problem of interest, in this case, laminated composite containing cracked plies.

To be noted that, despite its name, the concept of COD is not limited only to mode $I$ crack opening relative displacement between opposite faces of the crack (as it is exemplified in Fig. 2.1 (c)), but in the general case it also includes mode $I I$ sliding displacement and mode III tearing displacement (see Fig. 2.2). The name COD is sometimes generically used in the literature for the crack displacements in all three modes.

The COD solution intends to analytically determine the constraining effect of the ply 
thickness undergoing matrix cracking, and to determine the constraining effect of adjacent neighboring plies which could have different thickness, orientation, and material properties.

Different limiting cases have been analyzed in the previous references cited which used extensive parametric FEA simulations, e.g., thick vs. thin cracking ply, dilute (when consecutive cracks are far away and do not influence each other) vs. high crack density (when cracks are close and influence each other), and internal (inside of the laminate) vs. external cracking ply (at the top or bottom of the laminate).

The derivation of analytical COD solution is made possible by extrapolating the FEA results or by generalizing the known solution of simpler fracture mechanics problems [32, 33, $34,35,36,37,38]$. The drawback of the method is the unclear level of generality, usability and error of the analytical COD solutions obtained by this extrapolation approach.

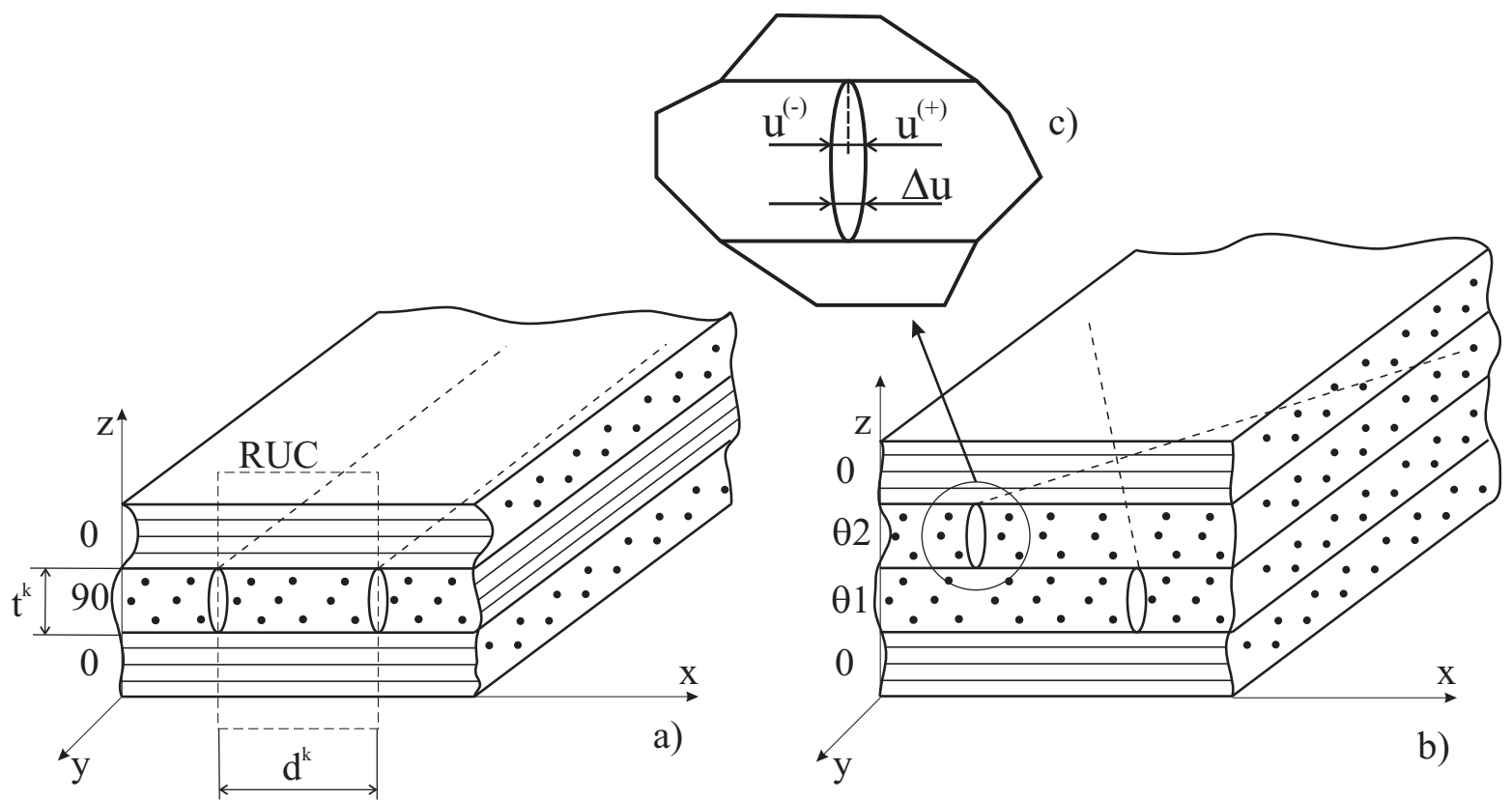

Figure 2.1: Laminate configurations featuring cracks a) only in plies transverse to loading direction (90 plies), and b) in plies with different off-axis orientations. c) $\Delta u$ crack opening displacement (COD).

Alternatively, numerical solutions, especially using the FEA method, allow the possibility of solving complex 3D stress-strain fields, as in Gudmundson and Adolfsson [35, 38], Aboudi [18], Yokozeki and Aoki [41, 42, 43]. Theoretically it is possible to solve the problem of the reduced material properties by FEA method, and detailed analyses can be performed, but usually these detailed analyses are particular of limited laminate configurations. A parametric study using FEA is difficult to implement, involving for example multiple meshing. With 
a skilled engineer, with no time or computational limitations, we have to remember that a real problem, of a real structure considering loading, material properties and boundary conditions as required by the FEA method, the problem becomes a double FEM problem when the level of damage (in form of matrix cracks in this case) is a variable of the problem. First of all, the whole structure is discretized and meshed to find the FEA solution for the considered loading case and boundary conditions. Subsequently, according to the structure geometry and the level of external loading, the resulting nonuniform stress field can locally reach the level of damage growth. At this moment, a second FEA problem has to be solved, to provide the damaged material properties at the structural (laminate) scale, for the corresponding extent of damage at the local (material point) level. The double FEM problem continues for each of the external loading increments. The double meshing process becomes very involved and hence, expensive computationally.

An easier way to solve the problem consists of having an analytical solution for the problem of reduced material properties at a certain damage level (material model), and for the problem of damage extent at a certain loading level (damage growth model). The analytical solution is then provided to the FEA problem of the real structure under loading and boundary conditions. Then, the material properties of the individual finite element is modified according to the analytical solution of the reduced material properties and damage growth problems.

The above considerations have imposed FEA method mostly as a verification and validation tool for analytical models of reduced material properties and damage multiplication (as it is the case in Gudmundson and Adolfsson [35, 38], Hahn [17], Aboudi [18]). Also, in the frame of a numerical approach, exhaustive computational Monte Carlo simulations can be used, where the probabilistic distribution of flaws through material is considered (Varna [1] , Wang and Chou [31]).

It has to be noted that each of the above modeling approaches have different levels of complexity imposed by the assumptions and simplifications used. For example, each problem can be taken from its $3 D$ complex nature (as every problem of matrix cracking in a laminate is) to a $2 D$ or even $1 D$ problem, imposing several assumptions such as plane stress (Nairn $[7,8,9,10,11,12,13,14,15,16]$, Talreja [44]), plane strain or generalized plane strain (Aboudi and Herakovich [18], Li, Reid and Soden [22, 23, 45], Tan and Nuismer [26]) and/or assuming a particular shape of the stress-displacement field generated by the perturbation effect due to the presence of cracks. If these assumptions might be wise and powerful tools 
in order to achieve a solution in within the limits of an acceptable precision, they also have a confining effect on the model that relies on them, because the model can not be applied to problems that do not fit the initial assumptions.

Examples of research and analytical modeling efforts selected from the technical literature, representative to the previously listed modeling strategies, are discussed in the following sections.

\subsubsection{Models based on $R U C$ method}

When the problem is approached in the frame of micromechanics of damage, the RUC method (Fig. 2.1 (a)) is developed in order to approximately solve for the stress-strain field inside of the laminate featuring matrix cracks.

It is widely accepted that the solution of a composite laminate without cracks under external loading is provided by Classical Laminate Theory (CLT), and the problem is solved under the plane stress assumption in the $(x-y)$ plane of the laminate. When cracks appear inside of the laminate, the uniform solution of CLT is superposed by perturbations of the stress-displacement field variables as described in eq. (2.1), this considerable increasing the complexity of the problem. Thus, local out-of-plane (along $z$ direction) stress-displacement components also appear, and the simplified $2 D$ plane stress frame (in the $(x-y)$ plane of the laminate) of CLT might not be any more adequate to represent the new problem of laminate with cracks.

The resultant (perturbed) stress-displacement field in the cracked laminate is regarded as a superposition between the uniform field of the uncracked laminate and a perturbation field generated by the presence of cracks,

$$
F^{c(k)}=F^{0(k)}+F^{p(k)}
$$

where $F^{0(k)}$ is the uniform field of the uncracked laminate (provided by CLT), $F^{p(k)}$ is the unknown perturbation field due to the presence of cracks, and $F^{c(k)}$ is the unknown resulting perturbed field. $F$ might be either stress, strain or displacement field, and $(k)$ represents a certain ply of the laminate.

Then, the field elasticity equations are considered in order to solve for the unknown strain and stress fields inside of the cracked laminate:

- strain-displacement equations: $\epsilon_{i j}=\frac{1}{2}\left(u_{i, j}+u_{j, i}\right)$; 
- stress equilibrium equations: $\sigma_{i j, j}=0$;

- constitutive equations : $\sigma_{i j}=Q_{i j k l}\left(\epsilon_{k l}-\epsilon_{k l}^{0}\right)$ (including the effect of initially residual strain $\epsilon_{k l}^{0}$, usually from thermal effect);

- stress free boundary conditions at the crack surface;

- stress and displacement continuity equations at the boundary of the RUC and at the interface between separate plies of the RUC;

- equilibrium equations between external applied loading and the stress resultant over the RVE.

The problem can be solved by imposing additional assumptions with regard to the distribution of the perturbation stress-displacement field $\left(F^{p(k)}\right.$ in $\left.(2.1)\right)$ which appears as a result of the presence of cracks, especially regarding through the thickness distribution (along $z$ direction, Fig. 2.1) of the sought stress-displacement field. The level of complexity and accuracy of the michromechanics model is given by these assumptions, among which the following can be remembered:

- out-of-plane stress/displacement components (along $z$ direction) are set to zero (neglected);

- stress/displacement components are considered independent of $z$;

- stress/displacement components are considered in an average sense along $z$ direction, and the above field elasticity equations are applied on these averaged quantities;

- a certain spatial variation law (polynomial in Han [17] and Aboudi [18, 19], Fourier expansion in Li [45], hyperbolic in Yokozeki [27, 28] and Barbero [29, 30]) is supposed for components of the perturbation stress-displacement field.

A way to improve the accuracy of the model is to use variational methods as in $\mathrm{Li}[23$, 45, 46], Nairn [10, 47, 48], Rebiere [24], Talreja [44] in order to find the unknown functions $F^{p(k)}$ of the perturbation field (either stress or displacement field), this method being more mathematically sound than simply assuming a certain law for the unknown field. Of course this method is preferred, but it can be applied only for simpler problems, as is the one of cross-ply symmetric laminates with cracks in the $90^{\circ}$ plies. 
After the stress-strain field $\left(\sigma_{i j}, \epsilon_{i j}\right)$ of the RUC of laminated composite containing cracked plies is calculated, the overall RUC strain $\tilde{\epsilon}_{i j}$ can be evaluated as

$$
\tilde{\epsilon}_{i j}=\frac{1}{V_{R U C}} \int_{V_{R U C}} \epsilon_{i j}
$$

and one way to evaluate the cracked material properties is by considering in turn cases of unit external loading; the compliance matrix of the cracked material results based on the previous determined strain field, as a function of the crack density, Yokozeki and Aoki [27], Barbero [29, 30], Li, Reid and Soden [45].

The first models of stress analysis, reduced material properties and cracks multiplication under mechanical and thermal loading use a classical $\left[0_{m} / 90_{n}\right]_{S}$ laminate configuration, under separate uniaxial or shear loading, with cracks developing in the 90 ply transverse to the loading direction (Fig. 2.1). This laminate stacking sequence and loading case allowed for expedient model simplifications, one of them being the plane stress assumption in the $(x-z)$ plane of the laminate, as in Nairn $[7,8,9,10,11,12,13,14,15,16]$. In this case all the field equations are expressed in terms of $(x, z)$ variables only according with the plane feature of the problem, and the unknown perturbation field $F^{p(k)}$ eq. in (2.1) is regarded as a stress field:

$$
\sigma_{i j}^{c(k)}=\sigma_{i j}^{0(k)}+\sigma_{i j}^{p(k)}(x, z), i, j=x, z
$$

The equation (2.3) is referred to as the stress transfer model in the cracked laminate, or shear lag model, due to the fact that the loss in stress transfer capacity of the $90^{\circ}$ cracked ply is transfered to the intact $0^{\circ}$ plies through the perturbation generated $\sigma_{x z}$ out-of-plane shear stresses. It has to be noted that the out-of-plane stress components $\sigma_{z z}, \sigma_{x z}$ do not appear in the undamaged laminate in the case of no out-of-plane loading. The undamaged stress field is an uniform field along $z$ direction (no variation in $z$ direction), and the plain stress assumption in the $(x-y)$ plane prevails for the for the undamaged laminate: $\sigma_{z z}^{0(k)}=$ $0, \sigma_{x z}^{0(k)}=0$. The out-of-plane stresses appear due to the perturbation stress components $\sigma_{z z}^{p(k)}(x, z)$ and $\sigma_{x z}^{p(k)}(x, z)$ generated by the presence of matrix cracks.

It is claimed in Nairn [7, 9, 16], that the $y$ components of stresses in the undamaged material are not considered $\left(\sigma_{y y}^{0(k)}=0\right)$ because they actually represent a minor Poisson effect, while the $y$ perturbation stress components in the cracked material do not appear due to the crack configuration (cracks along $y$ direction only, Fig.2.1 (a)) and the loading case considered (uniaxial loading along $x$ direction). By the use of the plane stress assumption, 
the in-plane axial (along $x$ direction in Fig. 2.1 (a)) elastic modulus of the cracked laminate is possible to be evaluated, but not the other elastic constants.

A a common characteristic of the plane stress based models is the fact that their applicability is limited to the mentioned $\left[0_{m} / 90_{n}\right]_{S}$ laminate configuration. This fact attracted some criticism toward those references who have been continuously dealing only with $\left[0_{m} / 90_{n}\right]_{S}$ laminates, even if the level of accuracy and refinement of their models have been continuously improved. The earlier named stacking sequence was labeled as an "academic" one, and it was pointed that the practical research interest requires studying other laminate configurations and loading cases, where the so helpful simplifications adopted for the $\left[0_{m} / 90_{n}\right]_{S}$ case are not valid any more. As a response, it was claimed that this problem is a good medium for developing reliable theoretical tools and for a good understanding of the governing laws for the matrix cracking in composite laminates, and only after well developing these tools and well understanding these governing laws, the subsequent more complicated problems can be successfully approached.

The easier solution of the $\left[0_{m} / 90_{n}\right]_{S}$ problem comes, on one hand, from the fact that there is only one ply (the term ply is applied here, and in the following, to the whole group of adjacent laminae having the same orientation, as it is the case of $90_{n}$ set of laminae) which undergoes matrix cracking. Then, the symmetric nature of the stacking sequence does not induce any in-plane/flexural coupling deformation, and the balanced nature of stacking sequence does not induce any in-plane extension/shear coupling deformation, this bringing a considerable contribution to the simplification of the analysis. Moreover, the uniaxial loading case considered allows for reducing the problem to its $(x-z)$ plane stress representation.

In the review in Nairn $[7,9]$ it is shown that even in the frame of $\left[0 m / 90_{n}\right]_{S}$ problem under plane stress assumption, different levels of accuracy of the analytical model have been reached by different authors, by making or not use of supplemental assumptions in order to solve for the resulting stress field in the cracked laminate. In this way, the elasticity problem of the resulting stress/strain field due to the presence of cracks can be reduced even to a $1 D$ problem (where the resulting stresses/strains field components are supposed constant along $z$ direction, and variable along $x$ direction only), or to a more complicated $2 D$ problem in the $(x-z)$ plane where the perturbation along $z$ direction is taken into account. It is shown in $[7,9]$ that the label $1 D / 2 D$ problem can be debatable, since sometimes a $2 D$ solution has been claimed by considering a simple Poisson effect along $y$ direction in Fig. 2.1 (a) , 
but still keeping the limiting assumption of constant field variables along $z$ direction, which strongly defines the problem as a $1 D$ one.

One first attempt to solve another laminate configuration than the traditional $\left[0_{m} / 90_{n}\right]_{S}$ is made in Tan and Nuismer [25, 26]. In this case supplemental assumptions are imposed on the resulting displacement field and a generalized plane strain model in the $(x-z)$ plane of the laminate is thus generated, Fig.2.1 (a). In this case, instead of writing the perturbed field (eq.(2.1)) in terms of stresses (as it was previously done in (2.3)), this is expressed in terms of displacement perturbations

$$
u_{i}^{c(k)}=u_{i}^{0}+u_{i}^{p(k)}(x, z), i=x, y, z
$$

It has to be noted that, similarly to the case of the perturbed stress field in (2.3), the out-ofplane displacement variations $u_{i}^{p(k)}(x, z)$ are exclusively the result of the perturbation field due to matrix cracking. The displacement field of the undamaged material $\left(u_{i}^{0}\right.$ in $\left.(2.4)\right)$ is uniform along $z$ direction, and it can be written as

$$
\begin{aligned}
& u_{x}^{0}=\epsilon_{x}^{0} \cdot x \\
& u_{y}^{0}=\epsilon_{y}^{0} \cdot y \\
& u_{z}^{0}=0
\end{aligned}
$$

in order to reproduce the constant strain field $\epsilon_{x}^{0}, \epsilon_{y}^{0}$ which is generated in the case of membrane deformation only. Average quantities ( $\alpha$ - either stress or displacement) over the ply thickness ( $z$ direction) are used in the field equation in order to simplify the problem:

$$
\bar{\alpha}^{k}=\frac{1}{h^{k}} \int_{h^{k}} \alpha^{k} d z
$$

A higher level of generalization is possible by the use of generalized plane strain assumption as compared against plane stress one, and the classical $\left[0_{m} / 90_{n}\right]_{S}$ laminate is extended to $\left[S / 90_{n}\right]_{S}$, where $S$ represents a balanced $\pm \theta_{m}$ sublaminate. The overall balance and symmetric feature of the laminate has to be kept in order to find a solution. The elasticity problem is solved in Tan and Nuismer $[25,26]$ for both tensile and shear in plane loading, since the resulting deformations due to the two loading cases are decoupled due to the balanced configuration of the considered laminate. The solution of the elasticity problem is an approximate one, not only through the assumptions made on the displacement field, but also through considering averaged quantities (stresses, displacements, see eq. (2.6)) over the ply 
thickness, and applying the general elasticity field equations (equilibrium, compatibility and constitutive equations) on these ply averaged quantities.

Finally, the reduction of all in-plane laminate elastic constants along loading direction for a given crack density is assessed in [26], and the damage growth is implemented in [25], based on strain energy release rate failure criterion, in both I (matrix cracking by tension normal stress) and II (matrix cracking by shear stress) failure modes. However, in order to maintain the plane feature of the problem, only the $90_{n}$ ply sequence is considered to undergo damage, while the $S$ sublaminate (which is constrained to a balanced $\pm \theta_{m}$ ply sequence) is considered at low ply angles, such that the $S$ sublaminate is not damaged under axial loading.

Under the same plane strain modeling approach, the model in Mayugo, Camanho and Dávila [49] considers the case of combined extension-shear in-plane loading. The application of the generalized plane strain method to the problem of matrix cracking in cross-ply laminates is well documented in $\mathrm{Li}[46]$.

The two above described approaches (plane stress/plane strain) to a solution of the stress/strain fields generated by the perturbing effect of the cracks have been selected as examples, these methods being also followed by other authors. For example, the generalized plane strain model, while making additional assumptions on the displacement field distribution in order to solve the problem of matrix cracking in $\left[S / 90_{n}\right]_{S}$ laminates is also adopted in Hahn [17], Aboudi and Herakovich [18].

As it can be noticed up to this point, the modeling efforts into the frame of micromechanics of damage has been confined to the $\left[S / 90_{n}\right]_{S}$ laminate configuration. The cause of this fact is identified in $\mathrm{Li}[23]$ as a lack of necessary boundary conditions and the way to overcome it is presented under the assumption of generalized plain strain. Here, the physical available boundary conditions that limited the level of generality of the previous models are completed with so called natural boundary conditions (mathematically sound boundary conditions from variational calculus), and the limitation with regard to the number of cracking plies into the laminate is removed, the model in [23] being able to approach a more general $[0 / 90 / 0 / 90 / \cdots / 90 / 0]$ type of laminate, with cracks in multiple 90 plies. However, the generalized plane strain (in the $(x-z)$ plane) assumption, together with other modeling assumptions, create limiting elements regarding the level of generality and applicability of the model in [23]. For example, the cracking plies have to have the same orientation and the same crack density (crack spacing), which could be considered strong limitations. Only 
stress distribution is treated in [23], as resulting from variational calculus.

A remarkable generalization level has been achieved in Yokozeki and Aoki [27, 28], where the case of $\left[S / \theta 1_{m} / \theta 2_{n}\right]_{S}$ under general in-plane loading, featuring cracks in both $\theta 1$ and $\theta 2$ plies, is treated (Fig.2.1 (b)). The solution is an original one, by adopting an oblique coordinate system along the $\theta 1, \theta 2$ cracking directions, and setting an equivalence between the constitutive behavior in the oblique coordinate system (given by the oblique $\theta_{1}, \theta_{2}$ off-axis directions) and the laminate orthogonal coordinate system. The method of solving the problem is applicable to any symmetric LSS, the limitation being that cracks has to be present in at most two different oriented plies. In this way, a more general $\left[S 1 / \theta 1_{m} / S 2 / \theta 2_{n} / S 3\right]_{S}$ laminate can be approached by further developing and generalizing the method in [27, 28]. However, the problem can not benefit by any of the previous plane stress - plane strain (in the $(x-z)$ plane) simplifications, but it is treated as a $2 D$ one in the $(x-y)$ plane of the laminate, by considering the averaged quantities of the in-plane stress-displacement components through the ply thickness (along $z$ direction) as in (2.6), and by neglecting the out-of-plane (along $z$ direction) normal stress and displacements. In this way, the perturbed displacement field in (2.4) is replaced by

$$
u_{i}^{c(k)}=u_{i}^{0}+u_{i}^{p(k)}(x, y), i=x, y
$$

and no variation of the displacement field unknowns along $z$ direction is treated. The solution makes use of the shear-lag method, where out-of-plane shear stresses are included. The elasticity field equations are applied in an averaged sense (along $z$ direction), and assumptions are made regarding the shape of the perturbing displacement field resulting as effect of the presence of cracks. Since the average quantities are considered over the ply thickness, the $z$ variable does not appear explicitly into the analysis, and the solution is generated as a function of $(x, y)$ variables only. The model is developed up to the level of calculating the whole set of membrane reduced material properties for given damage levels in the $\theta 1, \theta 2$ off-axis plies.

The model in [27, 28] if further developed in Barbero [29, 30], where general yet symmetric $[\theta 1 / \theta 2 / \cdots / \theta n]_{S}$ laminates under in-plane loading is possible are treated, from both reduced material properties and damage evolution point of view. By applying the homogenization technique together with an iterative procedure, one of the big limitations of the previous models is released, namely the number of plies that can be modeled as cracking plies. 


\subsubsection{Models based on $C O D$ method}

A different way to tackle the problem of laminate damage by matrix cracking is the one based on Kachanov theory of elastic bodies with voids [40]. Under this approach, the crack opening displacement (COD, Fig. 2.1 (c)) is the key in solving for the resulting strain of the laminate, and consequently for the reduced elastic constants of the damaged material, Gudmundson and Adolfsson [32, 33, 34, 35, 36, 37, 38] , Varna and Lundmark [39]. The COD parameter is defined as the relative displacement between the two faces of the same crack lying along fiber direction of a ply (Fig. 2.1(c)), and it is responsible for additional effective strain at the overall laminate level.

Because the material model in Gudmundson and Adolfsson [35] is implemented in the present progressive damage model, details of its formulation and background development $[32,33,34]$ are provided in the following.

The main approximation of the model in [35] (which is the extension to the flexural case of a previous model [34] dealing only with membrane deformation, yet based on the same type of approximation involved in finding a solution) is the fact that it uses a known solution for a related, yet different problem, i.e., the fracture mechanics solution to the problem of a row of cracks in an infinite plate, in order to analyze the problem of matrix cracking in internal layers of the laminate. Correspondingly, the solution to the problem of a row of cracks in a semi-infinite plate is used in analyzing the problem of matrix cracking in surface layers of the laminate. By involving the extrapolation of the solution to the problem of a row of cracks (as opposed to that one of a single crack, as in [32]), a former limitation of the "dilute crack density" (cracks far away from each other, so that they do not influence each other) is released, and the problem of intermediate and high crack densities can be approached.

Rather than selecting a RUC as the space in between consecutive cracks and running the analysis on this representative element, the COD method considers an arbitrary volume $V$ of the laminate containing cracks in each of its plies (see Fig. 4.1(a)) as being representative for the laminate.

The average crack displacement of cracks in ply $(k), \Delta u^{(k)}$, represents the relative displacement between individual faces of the crack, corresponding to either $I$-crack opening, 
$I I$-crack shearing or $I I I$-crack tearing modes (see Fig. 2.2), and it is defined as

$$
\Delta u_{i}^{(k)}=\frac{1}{t^{(k)}} \int_{0}^{t^{(k)}}\left(u_{i}^{(k)(+)}-u_{i}^{(k)(-)}\right) d t^{(k)}
$$

where the $(+),(-)$ superscripts indicate the displacements along the matrix crack over the thickness of the ply, on the two opposite faces of the same crack (Fig. 2.1(c)). $u_{i}^{(k)(+,-)}$ quantities are relative displacements of the crack faces, in ply material c.s. $\Delta u_{i}^{(k)}$ quantity is the average (over ply thickness) relative displacement between opposite faces of the crack.

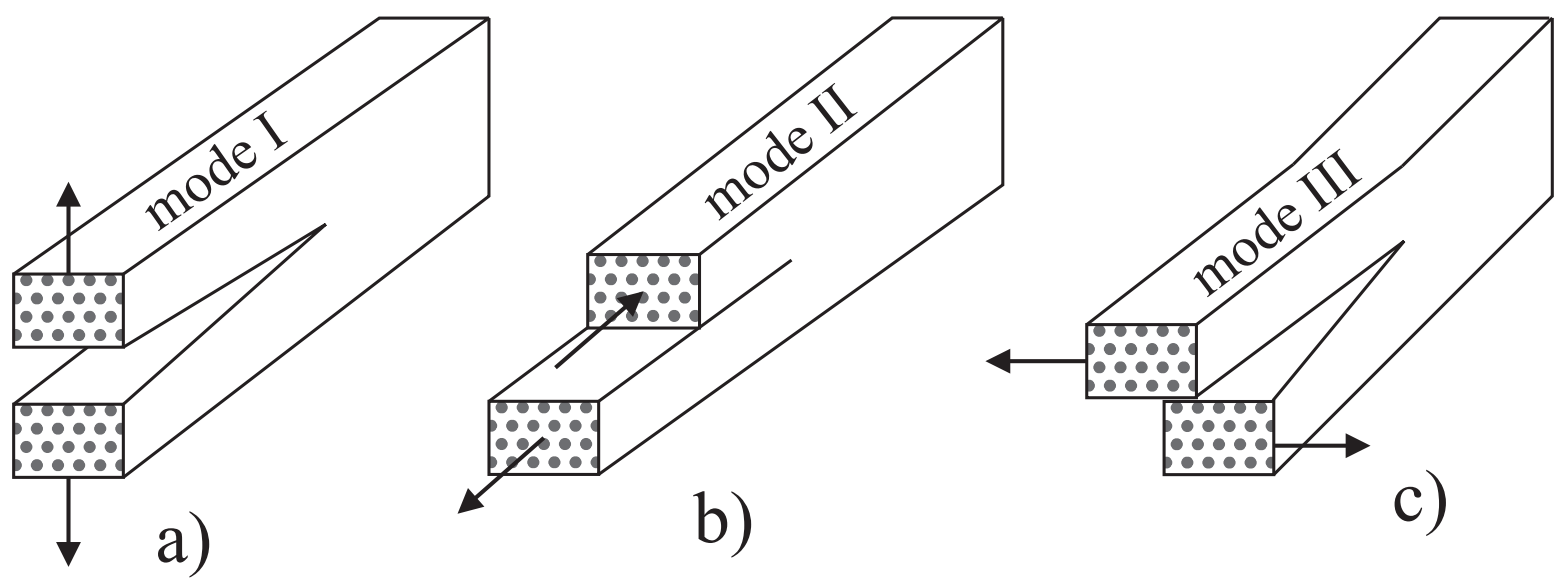

Figure 2.2: Modes of crack propagation: a) mode $I$, crack opening; b) mode $I I$, in-plane shear (sliding); c) mode $I I I$, out-plane shear (tearing).

The new unknown of the problem becomes the average crack displacement $\Delta u^{(k)}$. The problem of average crack displacement $\Delta u_{i}^{(k)}$ is regarded as the superposition of two separate elasticity problems: first the surface tractions on the prospective crack surface are calculated for the material regarded as uncracked; then the average crack displacement in ply $(k)$ is considered as the result of the contribution of surface tractions in all plies of the laminate $\tau^{l}(l=1 \ldots N)$, where $N$ is the number of plies in laminate. In this way, the average crack displacement in ply $(k)$ becomes

$$
\Delta u_{i}^{(k)}=t^{(k)} \sum_{l=1}^{N} \beta_{i j}^{(k l)} \cdot \tau_{j}^{(l)}, i, j=1 \ldots 3
$$

which is a linear combination of the surface tractions at the prospective crack location in all plies of the laminate, $\tau^{(l)}$. It can be observed that the linear combination is made through the coefficients $\beta^{(k l)}$, which quantify the effect of surface traction in ply $(l)$ on the COD in ply $(k)$. The coefficients $\beta_{i j}^{(k l)}$ which are function of LSS configuration, ply material properties and 
ply crack density (if cracks influence or not each other), become now the new unknowns of the problem. The physical meaning of the $\beta_{i j}^{(k l)}$ coefficients is not a direct intuitive one, they acting as COD compliances under the given surface tractions $\tau_{j}^{(l)}$, in the linear combination in $(2.9)$.

There is no direct analytical solution for the $C O D$ (or its equivalent $\beta_{i j}$ ) in laminated composites. At this point, the approximation of using $C O D$ solution to a closely related, yet different problem, i.e., the fracture mechanics problem of a row of cracks in an infinite plate, is proposed. This approximation is suggested by an earlier research [32], where the attempt to extrapolate a variation law of $C O D$ from extensive parametric FE simulations has shown that $C O D$ is a robust parameter and it can be approximated to a good precision from the fracture mechanics solution of one crack in an infinite orthotropic plate. In this way, the problem of determining the $\beta_{i j}$ parameters of $C O D$ is highly simplified, while introducing the aforementioned approximation. The level of error introduced by the proposed approximation is checked by comparing the output of the analytical model with experimental data and extensive FE simulations on a large variety of laminate types, and a good correlation is found between these data $[34,38]$.

The new introduced COD parameter is approximately evaluated, and analytical variation laws (see Appendix B.2) are extrapolated base on a) extensive FE simulations as in Gudmundson [32], Varna [39], or b) both generalization of FE simulations, and analytical fracture mechanics solutions of different, yet similar problems, as approached by Gudmundson et al. (e.g. the problem of one crack in an infinite orthotropic plate in [32], or the problem of a row of cracks in an infinite or semi-infinite orthotropic plate $[33,34])$. The effect of generalizing particular solutions (i.e., cracks in infinite/semi-infinite orthotropic plates) to more general problems (i.e., cracks in laminated composites) is analyzed in $[34,38]$ by multiple parametric FE simulations, in order to asses the level of error of this approach. The influence of different parameters (for example, the orientation of the neighboring plies with respect to the cracking ply) is analyzed, and the approximative analytical solution provided in this way is check for multiple laminates with different ply stacking sequences (thickness, fiber orientation) and ply thermoelastic properties.

The present method based on the concept of COD, where analytical laws of COD are obtained from extrapolating either parametric FE analysis or exact solutions of fracture mechanics for similar but simpler problems, provides a convenient closed form solution to the problem of reduction in material properties of the general $[\theta 1 / \theta 2 / \ldots / \theta n]_{S}$ laminate 
affected by matrix cracks in any of its plies, preserving in the same time good accuracy of the results. The main achievements of this approach is that the restriction on the number of cracking plies is released, and the whole set of thermo-elastic constants is possible to be evaluated by analytical closed form solutions.

While the COD model is initially applied in $[34,38]$ only to membrane deformation of thin laminates, it is further developed in [35] to out-of-plane flexural deformation. The validity of the flexural model output is checked against FE results in [35], and against experimental results in [36]. The model in [35] provides the whole set of membrane and flexural thermoelastic constants of the laminate, using as only input the uncracked plies properties and the plies crack density $\rho^{(k)}$. A more detailed presentation of the COD approach and the associated $\beta_{i j}$ parameters can be found in Appendix B.

Once the COD in each ply $(k)$ of the laminate is calculated based on $(2.9)$, it can be used in two different ways, under two analytical modeling approaches, in order to calculate the reduced material properties of the laminate:

1. based on COD and the theory of elastic body with voids [34]:

the effective (overall) strain of each cracked ply $(k)$ inside of the laminate comprised into the volume $V$ (see Fig. 2.3 (a)) can be expressed as

$$
\bar{\epsilon}_{c}^{(k)}=\epsilon_{c}^{a(k)}+\Delta \epsilon^{(k)}
$$

The overbar notation $\bar{\epsilon}$ denotes effective (overall, at macro level) quantity, and the subscript $c$ indicates the fact that the ply $(k)$ features matrix cracks. $\epsilon_{c}^{a(k)}$ is the average ply strain corresponding to individual material points inside of the cracked ply $(k)$, which is defined as

$$
\epsilon_{c}^{a(k)}=\frac{1}{2 V^{(k)}} \int_{V^{(k)}} \epsilon_{c}^{(k)} d V=\frac{1}{2 V^{(k)}} \int_{V^{(k)}}\left(u_{i, j}^{(k)}+u_{j, i}^{(k)}\right) d V, i, j=1 \ldots 3
$$

where $\epsilon_{c}^{(k)}$ is the elastic strain at individual locations, other than the crack location, inside of the material featuring cracks. These material locations other than the location of the matrix cracks are still affected by the presence of matrix cracks due to the induced perturbation in the stress-strain field. $\Delta \epsilon^{(k)}$ is the strain increment due to average crack displacement $\Delta u^{(k)}$ in ply $(k)$.

2. based on COD and an energy balance equation between two equivalent instances of the cracked laminate $[32,33,35,36]$ : 
First, the elastic energy $W_{c}$ stored in the composite material featuring matrix cracks is calculated as a linear elasticity superposition problem:

$$
W_{c}=W_{0}+\Delta W
$$

where $W_{0}$ is the elastic energy in the uncracked material, and $\Delta W$ is the change in the elastic energy due to advent of matrix cracks. The change in elastic energy $\Delta W$ is calculated as the mechanical work done by the surface tractions $\tau$ at the prospective crack location, through the COD displacement $\Delta u$ (see Fig. 2.3 (b)).

Second, the cracked laminate is considered in its effective (homogenized) instance, and the same $W_{c}$ is expressed in terms of effective (reduced) material properties $C_{c}, \alpha_{c}$ :

$$
W_{c}=W_{c}\left(C_{c}, \alpha_{c}\right)
$$

where $C_{(c)}$ and $\alpha_{(c)}$ are the reduced stiffness and the reduced CTE of the cracked laminate, respectively.

By identification of the two expressions (2.12), (2.13) of the stored energy $W_{(c)}$ in the damaged material, the reduced thermo-elastic material properties $C_{(c)}, \alpha_{(c)}$ can be calculated.

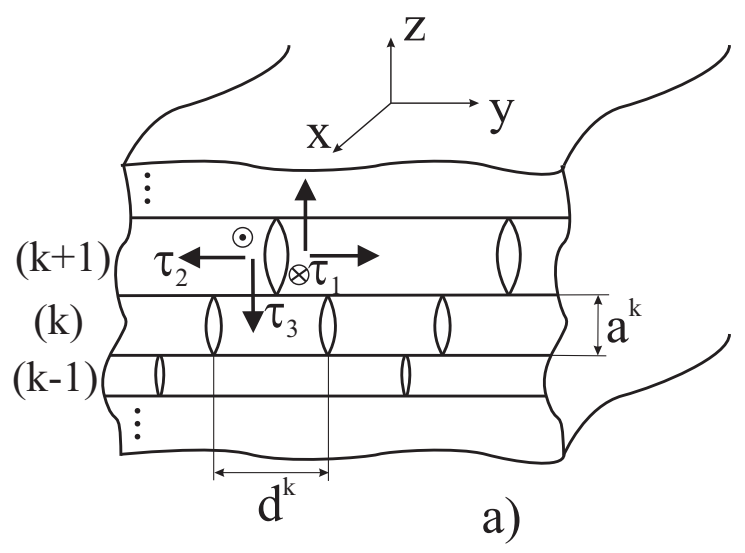

b)

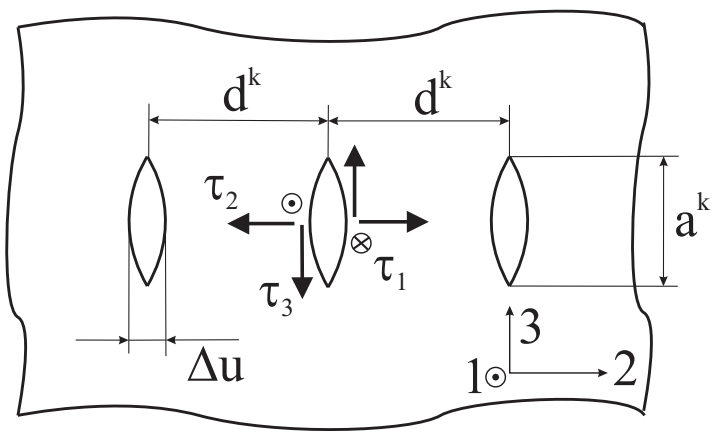

c)

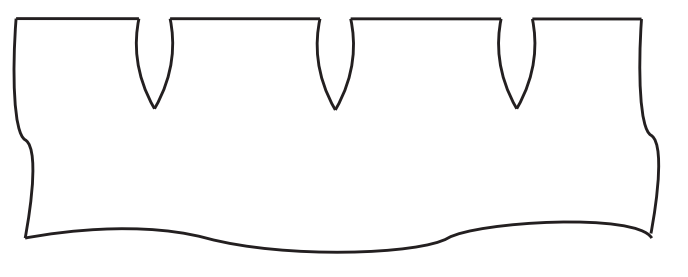

Figure 2.3: a) Intra-laminar matrix cracking in laminated composites; b) A row of cracks in an infinite transversely isotropic body; c) A row of cracks in an semi-infinite transversely isotropic body. 
Based on either of the two COD modeling approaches, close form analytical solutions for degradation in the laminate thermo-elastic properties for given levels of matrix cracking in individual plies can be derived:

$$
[C]_{6 \times 6}=\left[C\left(\rho^{(k)}\right)\right],\{\alpha\}_{6 \times 1}=\left\{\alpha\left(\rho^{(k)}\right)\right\}
$$

where $[C]$ is the reduced effective laminate stiffness matrix and $\{\alpha\}$ is the reduced effective laminate thermal expansion coefficients vector, and $\rho^{(k)}$ is the normalized crack density in each ply $(k)(k=1 \ldots N$, where $N$ is the number of plies in the laminate) of the laminate. The normalized crack density is defined as $\rho^{(k)}=t^{(k)} / d^{(k)}$, where $t^{(k)}$ and $d^{(k)}$ are the thickness and the crack spacing for the ply $(k)$, respectively (see Fig. 2.1 (a)). The normalized crack density is a measure of ply damage level equivalent to the crack density $\lambda$ in eq. (1.1). The $6 \times 6$ and $6 \times 1$ subscripts in (2.14) indicate that the reduced effective (laminate) material properties are for the case of combined membrane and flexural deformation.

Details of the two modeling approaches adopted in order to calculate the reduced material properties in (2.14) based on the concept of COD as expressed in eq. (2.8), (2.9) are presented in Appendix B.3. The modeling approach based on COD and theory of the elastic body with voids (Appendix B.5) is limited to predicting the reduced thermo-elastic properties of the laminate without considering the flexural properties. The modeling approach based on COD and energy balance (Appendix B.1 - B.4) is able to predict the whole set of membrane and flexural reduced material properties. The output of the COD model can be characterized as a "laminate based" one, since the reduced effective properties of the whole laminate are provided, without knowing the reduced effective properties of individual plies.

\subsubsection{Models based on $C D M$ method}

The $C D M$ has brought his own contribution to solving the problem of matrix cracking in laminated composites. Thus, a first step forward from the $\left[S / 90_{n}\right]_{S}$ laminate configuration is made in Varna [2], where both the reduction in material elastic properties as a function of matrix cracks density, as well as the damage evolution as a function of the applied external loading are treated for the case of $\left[0 / \pm \theta_{4} / 0_{1 / 2}\right]_{S}$ laminate, with cracks developing in the off-axis $\pm \theta$ plies. In this case, the problem is treated into the frame of synergistic damage mechanics, by combining elements from both continuum damage mechanics (where the crack opening displacement COD is considered as state variable) and micromechanics 
of damage. Moreover, the model has a stochastic nature by considering the statistical distribution of flaws through material. Additional experimental parameters are required by the model, and the computation cost and implementation difficulty are increased by the Monte-Carlo feature of the numerical simulation. The COD associated parameters requires experimental determination, and the model has an applicability only to the $\left[0 / \pm \theta_{4} / 0_{1 / 2}\right]_{S}$ stacking sequence.

Similarly, another LSS is treated in the frame CDM approach in Varna [1], where a solution is offered to $\left[ \pm \theta / 90_{4}\right]_{S}$ laminates based on the same concept of treating the COD as parameter of the constraining effect of the neighboring plies. This time the COD for the given laminate configuration is studied by parametric FEM analyses, and a FEA generated variation law for COD is used in the analytical model. There are also parameters requiring experiments, and the model seems to be applicable only to the given LSS.

The drawbacks of the CDM method, especially regarding their dependence on experimental parameters which in turn are dependent on the laminate type (material properties and LSS) are underlined in $\mathrm{Li}[6]$, where a new CDM model is meant to overcome these drawbacks. While the prior CDM models involved continuum damage variables in order to describe the behavior of the laminated composite material as a whole (this modeling strategy being regarded as "laminate-based damage reprezentation", and imposing the dependency of the model on the laminate configuration), the new CDM model proposed in [6] is built on the concept of "lamina-based damage representation". In this way, a release of the CDM model from experimental data is sought and expected. In this new "lamina-based damage representation" approach, continuum damage variables as a function of damage level in form of matrix cracking are defined for each ply of the laminate, and each cracked ply is considered as a fictitious homogeneous material characterized by the effective elastic properties of the cracked ply, this approach being an instance of the homogenization theory applied at ply level. The properties of the composite laminate are further calculated based on CLT, and due to this fact only in-plane ply stresses components are evaluated. Actually the model in [6] implements the new lamina-based damage reprezentation on an earlier CDM model (Talreja [50]), based on laminate damage representation. A michromechanics of damage model ( $\mathrm{Li}$ [45]) is coupled with the CDM model in [6], in order to provide the required individual ply damage variables, the model being conferred in this way a synergistic feature. The formulation of the model allows for treating laminates of general layup, under both membrane-bending deformation, though it has to be noted that the model in [45] which is 
called in [6] is based on the generalized plain strain assumption, which might have restrictions regarding the number and orientation of the cracked plies.

\subsubsection{Models involving flexural deformation}

The approach of bending loading-deformation represents a distinct stage of the more general problem of damage through matrix cracking in composite laminates. Contrary to the in-plane case (presented in Section 2.1.1 - 2.1.2), the flexural models are scarce in the literature.

In the category of $R U C$ based methods, a simplified model for the flexural deformation is offered in Smith and Ogin [51,52], where an earlier shear-lag stress transfer model is coupled to $1 D$ beam bending theory in order to derive the influence of matrix cracking on flexural modulus. The model has limited applicability to some particular 0/90 symmetric stacking sequences, supposing that matrix cracking takes place in only one transverse 90 ply. The matrix cracking onset is also evaluated by the critical energy release rate approach of fracture mechanics, considering only mode I mechanism of crack formation.

An extension of micromechanics of damage methods to bending case is done in Talreja [44], in the limiting frame of plane stress assumption (similar to Nairn [7, 9, 16] for the in-plane case), which allows the flexural analysis of only $\left[0_{m} / 90_{n}\right]_{S}$ laminates, with cracks in the transverse 90 ply. Only the approximate solution of the stress field is solved, serving as a foundation for further development to calculating reduced flexural elastic properties and damage evolution under external flexural loading.

Another approach based on RUC method to solving for the stress/displacement field under bending deformation is the semi-numerical solution "finite strip method" presented in Li, Reid and Soden [45], under the limiting generalized plane strain assumption. The general frame of the problem is the one presented before for the in-plane only deformation, see eq.(2.1), (2.4) and (2.5). In order to accommodate the bending deformation, the displacement perturbation field due to the cracks presence $\left(u_{i}^{p(k)}\right.$ in $\left.(2.4)\right)$ is superposed on an initial displacement field able to describe the flexural deformation of the laminate without cracks 
$\left(u_{i}^{0}\right.$ in $\left.(2.4)\right)$, and (2.5) needs to be accordingly modified as follows

$$
\begin{aligned}
u^{0} & =\epsilon_{x}^{0} \cdot x+\frac{1}{2} \gamma_{x y}^{0} \cdot y+\kappa_{x} \cdot x z+\frac{1}{2} \kappa_{x y} \cdot y z \\
v^{0} & =\epsilon_{y}^{0} \cdot y+\frac{1}{2} \gamma_{x y}^{0} \cdot x+\kappa_{y} \cdot y z+\frac{1}{2} \kappa_{x y} \cdot x z \\
w^{0} & =-\frac{1}{2} \kappa_{x} \cdot x^{2}-\frac{1}{2} \kappa_{y} \cdot y^{2}-\frac{1}{2} \kappa_{x y} \cdot x y
\end{aligned}
$$

where the additional $\kappa_{x}, \kappa_{y}, \kappa_{x y}$ terms describe the bending deformation of the laminate. It can be noticed that eq. (2.15) incorporates the kinematics of flexural deformation: $\{\epsilon\}=$ $\{\epsilon\}^{0}+z \cdot\{\kappa\}$, where the curvature $\kappa$ is given by $\kappa_{i j}=-\frac{\partial^{2} w^{0}}{\partial x_{i} \partial y_{j}}$.

The problem set up in this way becomes more complicated. The laminate can be under general membrane and flexural loading, and the model deals with cracks in multiple plies, but the orientation of the cracking plies has to be the same, in order to be consistent with the generalized plane strain assumption. Additional limiting assumptions are involved, since the crack spacing in different plies has to be the same, which is not real for bending loading. This limitation confines the model to only one cracking ply for bending deformation. The solution to the strain-stress field is sensitive to both the number of finite strip elements used to discretize the domain and the number of elements used in the Fourier series approximation of the displacement field. The crack multiplication (damage growth) is addressed in Li, Reid and Soden [5] into the frame of CDM, giving a synergistic feature to this modeling approach.

The $C D M$ homogenization method is involved in Li, Reid and Soden [6] for the case of general laminates under flexural loading, by shifting from the laminate formulation specific to former CDM models to lamina formulation (a damage variable is associated to each ply), eliminating in this way the necessity of experimental parameters for different stacking sequences. The material model has a synergistic feature, since the micromechanics model in [45] is used to calculate the damage variables of individual plies. It can be inferred in this way that the limitation in [45] are carried in [6] under the homogenization approach, and the user should be cautious and thoroughly validate the model against experimental data. Another similar approach of superposing a CDM inspired homogenization model on a micromechanics model $([27,29])$ was used in Barbero [30] and proved to provide good correlation to experimental data in the case of symmetric laminates. In the view of these facts, the model of general deformation of the laminate (flexural properties included) in [6] is a good option to be considered in modeling the behavior of general laminates affected by matrix cracking. However, the micromechanics of damage solution in [45] proves not only to 
be restricted by its built-in assumptions, but also to feature a double sensitivity: first, to the discretization procedure involved by "finite strip" method used in this model, and second to the number of terms used in the Fourier expansion series of the supposed displacement field. These facts make the procedure difficult to implement in its numerical aspects.

The approach of the $C O D$ for flexural deformation is applied in Gudmundson and Adolfsson [35]. The whole set of in-plane / flexural thermo-elastic constants of the laminate affected by cracks in individual plies are calculated, based on approximate solutions of the $C O D$ parameter for the general case of combined in-plane/flexural deformation. The model follows as a development of other previous models [32, 33, 34] (Section 2.1.2), where flexural deformation aspect of the problem was not considered (this limiting the applicability to only symmetric laminates).

\subsection{Background of the damage growth aspect of the prob- lem}

In literature, different approaches have been considered to solve the damage growth problem. As damage growth is understood the crack multiplication upon increasing loading conditions. Each approach has to define a damage activation criterion, and an evolution criterion commonly defined as a function of the internal stress-strain state of the cracked material, or the external loading level. Two principal categories of damage evolution criteria have been used: i) strength based, as in Li, Reid and Soden [5, 6], Dávila and Camanho [53], and ii) energy balance based, as in Nairn [7, 9, 16], Hahn [17, 54], Crossman [20], Flaggs [21], Li [22], Rebiere [24], Tan and Nuismer [25], Barbero [29, 30], Dávila and Camanho [53].

The strength based formulation considers that a new crack is formed when the stress or strain state, $x_{i j}$, inside the cracked material reaches a critical state defined as the material strength, $X_{i j}$. The strength based formulation is written as follows:

$$
F\left(x_{i j}, X_{i j}\right) \geq 1
$$

$F$ expresses the functional relationship between the internal stress/strain state of the material and the critical state of the material. In (2.16), the condition for crack formation is defined.

The strength approach to damage growth is based on traditional strength of materials failure criteria, e.g., the general maximum stress, maximum strain failure criteria, or the 
specific designed for fiber reinforced materials Tsai-Wu, Tsai-Hill quadratic failure criteria. The material strength parameters $X_{i j}$ are determined based on failure experiments on unidirectional (UD) fiber reinforced composite samples.

However, it has been shown through experimental observation that the material strength is not actually an objective material property, Groves [3], Crossman [20], Flaggs [21]. Instead, it is highly influenced by the laminate stacking sequence (LSS), ply thickness, and mechanical properties of the other plies in the laminate. This effect is known as the constraining effect by the neighboring plies (see Fig. 2.4). A strength based damage criterion is not able to reproduce the experimental trend presented in Fig. 2.4. For example, consider two laminate configurations $\left[0 / 90_{n 1} / 0\right]$ and $\left[0 / 90_{n 2} / 0\right]$, with $n 1>n 2$ under axial loading, and the maximum strain failure criterion, the same strain to damage onset, i.e., the level of the applied strain when the first matrix crack appears, is predicted for the two laminate configurations. This result is not observed experimentally as depicted in Fig. 2.4. The laminate configuration $\left[0 / 90_{n 1} / 0\right]$ with a thicker $90_{n 1}$ cracking ply should undergo matrix cracking at a lower strain compared to the laminate configuration $\left[0 / 90_{n 2} / 0\right]$ featuring a thiner $90_{n 2}$ cracking ply. The same limits of the predictive capabilities of strength based criteria stand true if the maximum stress or a quadratic stress criteria is used instead of maximum strain criteria. There is no differentiation between the thick/thin cracking ply once the critical stress condition defined in eq. (2.16) is satisfied.

The behavior in Fig. 2.4 shows that the strength of material is actually an in-situ material property (Flaggs [21], Dávila [53]) that depends on the laminate configuration. Experimental parameters which depend on the stacking sequence have to be used in conjunction with a strength based criteria, to successfully model the influence of the laminate configuration on matrix cracking. This disadvantage can be avoided by using an energy based criterion, as follows.

$$
F\left(G_{i}, G_{i}^{c}\right) \geq 1
$$

The energy based formulation in eq. (2.17) considers that the damage growth condition is reached when a critical amount of mechanical energy is available for the formation of a new unit crack surface. The energy released for the formation of an unit surface of crack is defined as energy release rate $(E R R) . G_{i}$ represents the ERR through mode I, II or III crack propagation, and $G_{i}^{c}$ represents the critical value of ERR, or the material resistance to I, II, III modes of crack propagation. The energy based formulation is based on Griffith energy 
balance criterion for crack growth from traditional fracture mechanics.

The laminate configuration influence, i.e., the thickness of the cracking ply and the constraining effect of the neighboring plies, is implied in the criterion. For this reason, most of the researchers have recently embraced energy balance based failure criteria for modeling the cracks growth in laminated fiber reinforced polymers.

Nonetheless, also based on experimental observations (Parvizi [55]), it has been shown that the in-situ thickness effect presented in Fig. 2.4 works only for a certain range of ply thicknesses, which is function of the material properties of the composite material system. Above a certain ply thickness, which is referred to as the thin-thick transition thickness in Dávila [53] and Barbero [56], the ply thickness does not influence any more the cracking onset and evolution of the laminate. Instead, energy based models predict a continuously reduction of the damage onset with increasing ply thickness. To account for the discrepancy, the method of using an energy based damage criterion for ply thickness less than the transition thickness, and a strength based criterion for ply thicknesses greater than the transition thickness, has been proposed. The problem of ply transition thickness is not addressed in the present model.

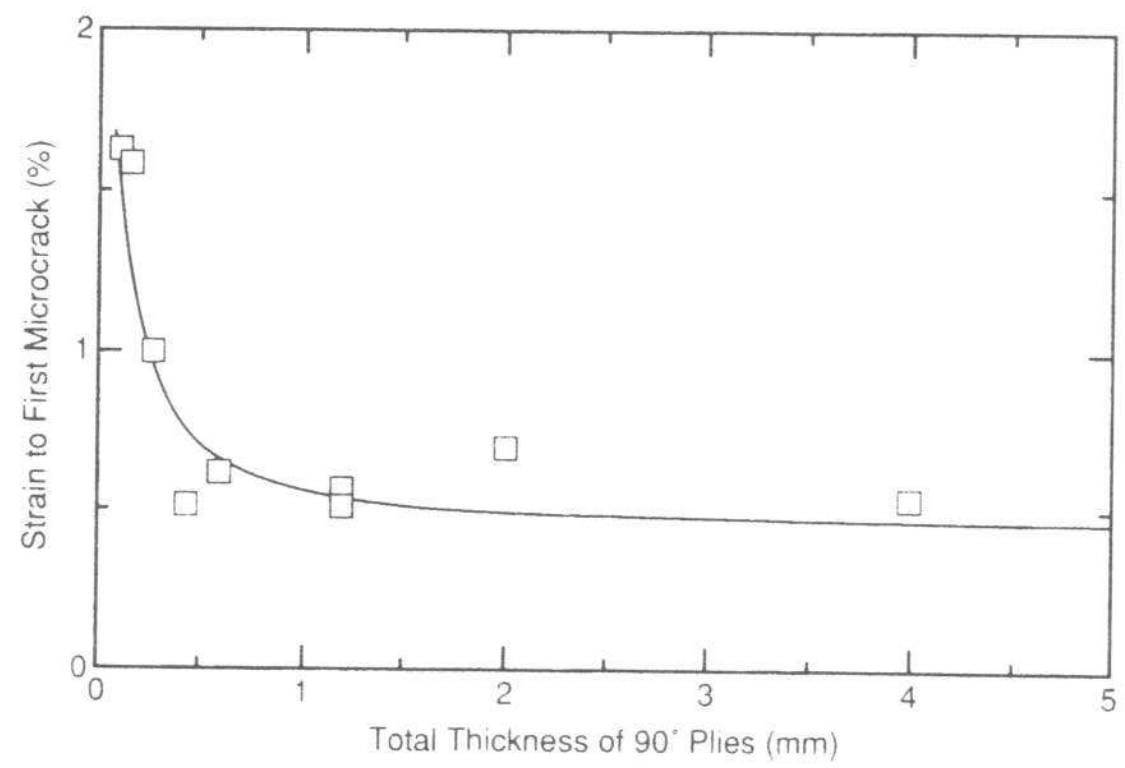

Figure 2.4: Transverse ply strength as function of ply thickness (from Nairn [7]).

The method of energy balance damage evolution criteria is an instance of traditional fracture mechanics, but applied in a particular manner, in order to reproduce the exper- 
imental observations and to allow for analytical solutions applicable to the more complex case of non-homogeneous composite materials. Thus, if in the case of traditional fracture mechanics the infinitesimal growth of crack is usually regarded, and specific theoretical tools have been developed in order to asses the conditions for this infinitesimal crack growth of one single crack at a time (stress state at crack tip, stress intensity factors, energy release rate), in micromechanics of damage for laminated composites usually the crack growth is regarded as a finite (discrete) event, where the difference between two consecutive damage states is a finite number of cracks which usually span the entire width of a sample, as it is the case of cracks developing in transverse $90^{\circ}$ plies with respect to the load direction. Even if there are experimental cases when the new formed crack does not span the entire width of the laminate sample (Nairn [7, 9], Yokozeki [41, 42], as it is the case of off-axis plies or even of thin transverse $90^{\circ}$ plies, the crack growth can still be considered as being finite (discrete), over a finite length, this being imposed by the brittle behavior of the toughened epoxy matrices used in most of the structural applications. Theoretical tools of fracture mechanics have been specifically adapted by different models in the literature, and used in micromechanics of damage for composite materials.

The finite fracture mechanics concept and energy based damage growth criterion are used in Barbero [29] for modeling damage progression. This model is further developed in [30], where the damage growth is also treated by ERR criteria, this time considering a CDM inspired ply homogenization technique and coupled with a plasticity inspired algorithm in order to establish the equilibrium state upon damage evolution.

Besides finite fracture mechanics approach, there have been other attempts to model the matrix cracking in laminated composites using methods of fracture mechanics, focusing on infinitesimal growth of an individual crack at a moment. In this case, the individual crack propagates through the ply thickness direction, through the ply width direction, or through the combination both.

Another method to define the damage evolution function is proposed in Gudmundson et al. [36, 37], Soutis [57], Hahn [17], Varna [58], Yalvac [59], Zhang [60] based on the resistance curve ( $R$-curve) of the laminated composite. The determination of the $\mathrm{R}$-curve requires experimental data and it is expected to have a laminate configuration dependency. Due to experimental evidence of the $\mathrm{R}$-curve behavior of the composite laminate undergoing progressive damage, this feature is considered and implemented in the present model. The background of the problem of $\mathrm{R}$-curve behavior in laminated composites is presented in the 
following section.

\subsubsection{Resistance-curve behavior of damage progression in lami- nated composites}

First observations regarding the possibility of the $R$-curve behavior of laminated composites are formulated in those models $[17,36,57,58,59,60]$ claiming that the critical material parameter $G_{c}$ is not a constant number, but it actually increases with increasing crack density $\lambda$. In this way the critical parameter for crack growth becomes $G_{c}=G_{c}(\lambda)$.

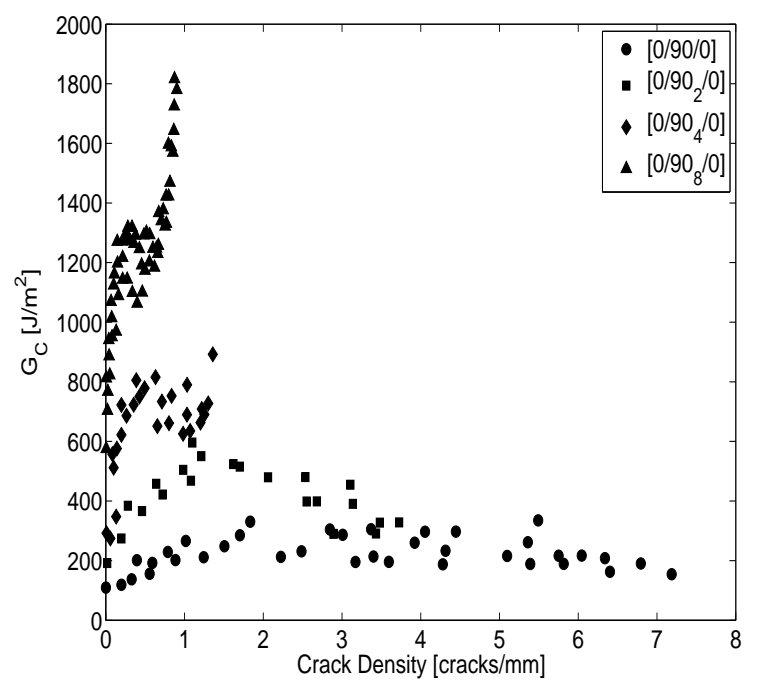

Figure 2.5: Back-calculated critical ERR based on analytical model and experimental crack multiplication data, Adolfsson and Gudmundson [36].

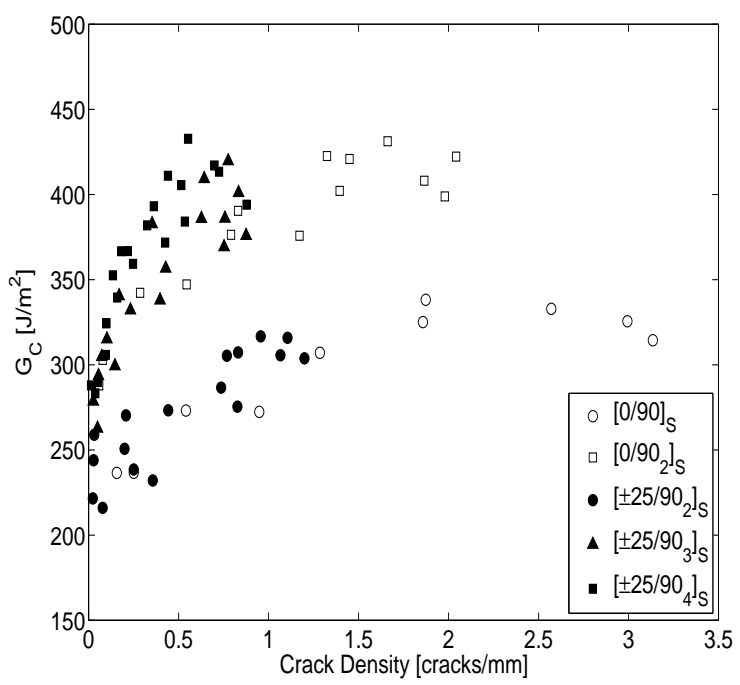

Figure 2.6: Back-calculated critical ERR based on analytical model and experimental crack multiplication data, Zhang and Soutis [57].

The phenomenon of increasing resistance of the material to crack growth with increasing damage level was labeled as the strengthening effect of the material in $\mathrm{Li}[5,6]$. A possible explanation of this effect can be formulated based on the influence of the material flaws on the material strength, which is referred as the size effect. According to this explanation, there is a statistical distribution of the inherent flaws in the material, which translates into a distribution of the material strength. The points with bigger flaws and lower strength will fail first, and the overall strength of the material will increase with increasing damage level due to the fact that the remaining flaws are less and less. This modeling approach can be applied to both strength of material for strength based criteria $\left(X_{i j}\right.$ in eq. $\left.(2.16)\right)[5,6]$ and 


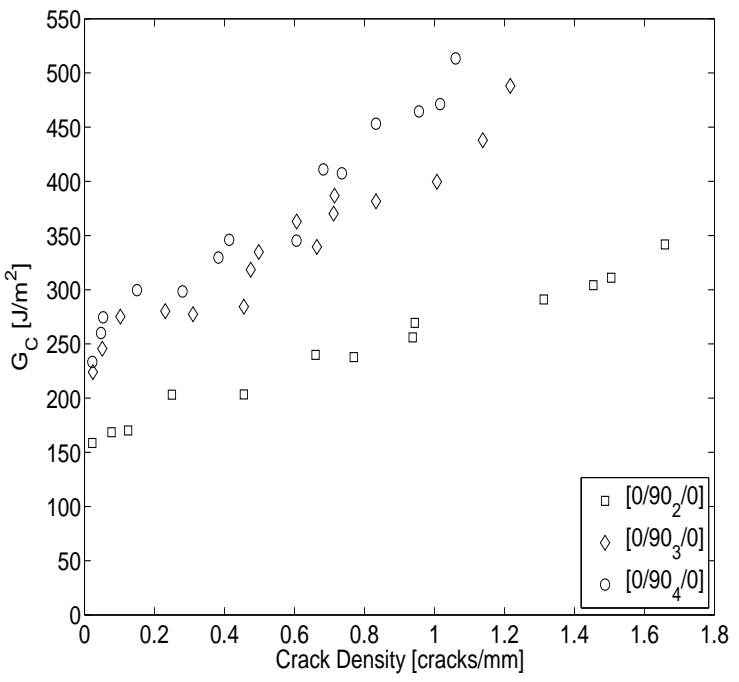

Figure 2.7: Back-calculated critical ERR based on analytical model and experimental crack multiplication data, Hahn [17].

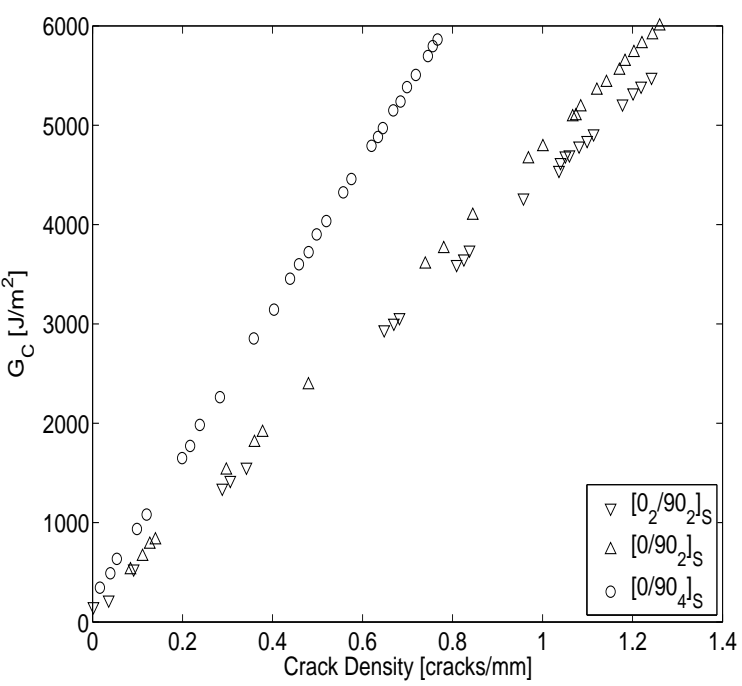

Figure 2.8: Experimental measured critical ERR, Varna [58].

to critical ERR for energy based criteria $\left(G_{i}^{c}\right.$ in eq. $\left.(2.17)\right)[31,61]$. However, the required parameters of the $X$ or $G_{c}$ distribution through the material are not readily available, and their determination requires additional experiments.

This possible $R$-curve behavior of laminated composites has been noticed in some energy based models $[17,36,57,59,60]$ not based on direct experimental determination, but based on the fact that the analytical model can not reproduce the experimental damage growth data using a constant value $G_{c}$. However, this brings a level of uncertainty on the assumption that $G_{c}=G_{c}(\lambda)$; it might be the analytical model itself that is not able to catch the important aspects of the physical behavior of the material. Based on the analytical equations of the models in $[17,36,57,59,60]$ and experimental data $\lambda=\lambda(\epsilon)$ crack multiplication points, the critical ERR in the moment of damage growth is back-calculated and it shows a clear $R$-curve behavior (see Fig. 2.5, 2.6, 2.7). Again, back-calculating the $G_{c}=G_{c}(\lambda)$ variation based on $\lambda=\lambda(\epsilon)$ experiments in conjunction with the analytical formulation of the model brings the analytical model as a filter between the $\lambda=\lambda(\epsilon)$ experiments and the backcalculated $G_{c}=G_{c}(\lambda)$ results. Any problematic assumption or formulation of the analytical model might translate in inaccurate $G_{c}=G_{c}(\lambda)$ results. However, these convergent opinions from different, even if scarce sources, has to be kept in mind and considered in the analytical formulation of the model, if it proves to be true and representative for the material behavior. 
Moreover, what can be observed in Fig. 2.5, 2.6, 2.7 is that not only the crack growth resistance parameter $G_{c}$ features an $G_{c}=G_{c}(\lambda) R$-curve behavior, but it also features a ply thickness dependence, which is labeled here as in-situ $R$-curve behavior: $G_{c}=G_{c}(\lambda, t)$. According to this observation, both the critical ERR at damage initiation $\left(G_{c}\right.$ at $\left.\lambda=0\right)$ and the subsequent slope of the $G_{c}=G_{c}(\lambda)$ variation seem to be function of the ply thickness $t$.

These observations of the possible in-situ $R$-curve damage behavior of the laminated composites tremendously complicate the problem of damage evolution for those models based on energy damage growth criteria, as compared with the formulation of Griffith principle from classical fracture mechanics which is based on constant $G_{c}$ value (see Section 4.4.1).

The only experimental determination of the $G_{c}=G_{c}(\lambda)$ for matrix cracking in laminated composites, at the best knowledge of the author of this manuscript, was done in Varna [58], using specific fracture mechanics experimental procedures. The result of this experiments is presented in Fig. 2.8, where the in-situ $R$-curve behavior $G_{c}=G_{c}(\lambda, t)$ can be clearly observed: there is an linear increase in $G_{c}$ with increasing crack density $\lambda$, and the slopes corresponding to different cracking ply thicknesses are different.

Upon noticing the possible in-situ $R$-curve behavior of the critical strength parameters of the material, some authors attempted to model this behavior, as it is the case in Soutis [57], Zhang [60]. In Nairn [8] a correction factor $f$ is applied to the model calculated $G_{c}$ at high crack densities, in order to improve the correlation with experiments. Different $f$ 's are needed for different material systems. Other authors, as Gudmundson and Adolfsson [36], Varna [58], simply considered the energy based methods for crack multiplication as inadequate for modeling damage growth in laminated composites, and alternative $\mathrm{R}$-curve formulation are proposed. The latter solution is briefly presented in Appendix D.

The possible in-situ $\mathrm{R}$-curve progressive damage behavior for laminated composites featuring damage in form of matrix cracking is considered and implemented in the present model, as it is presented in Section 4.4.3.

\subsection{Concluding remarks on problem background}

Considering the aforementioned review of the research approaches on the problem of matrix cracking in laminated FRP, an evolution of the models can be observed (Fig. 2.9), trying to release various limitations on maximum number of cracking plies possible to be solved, maximum number of different orientations of the cracking plies possible to be solved, 
and more recently trying to solve the case of general stacking sequence under both in-plane and flexural deformation.

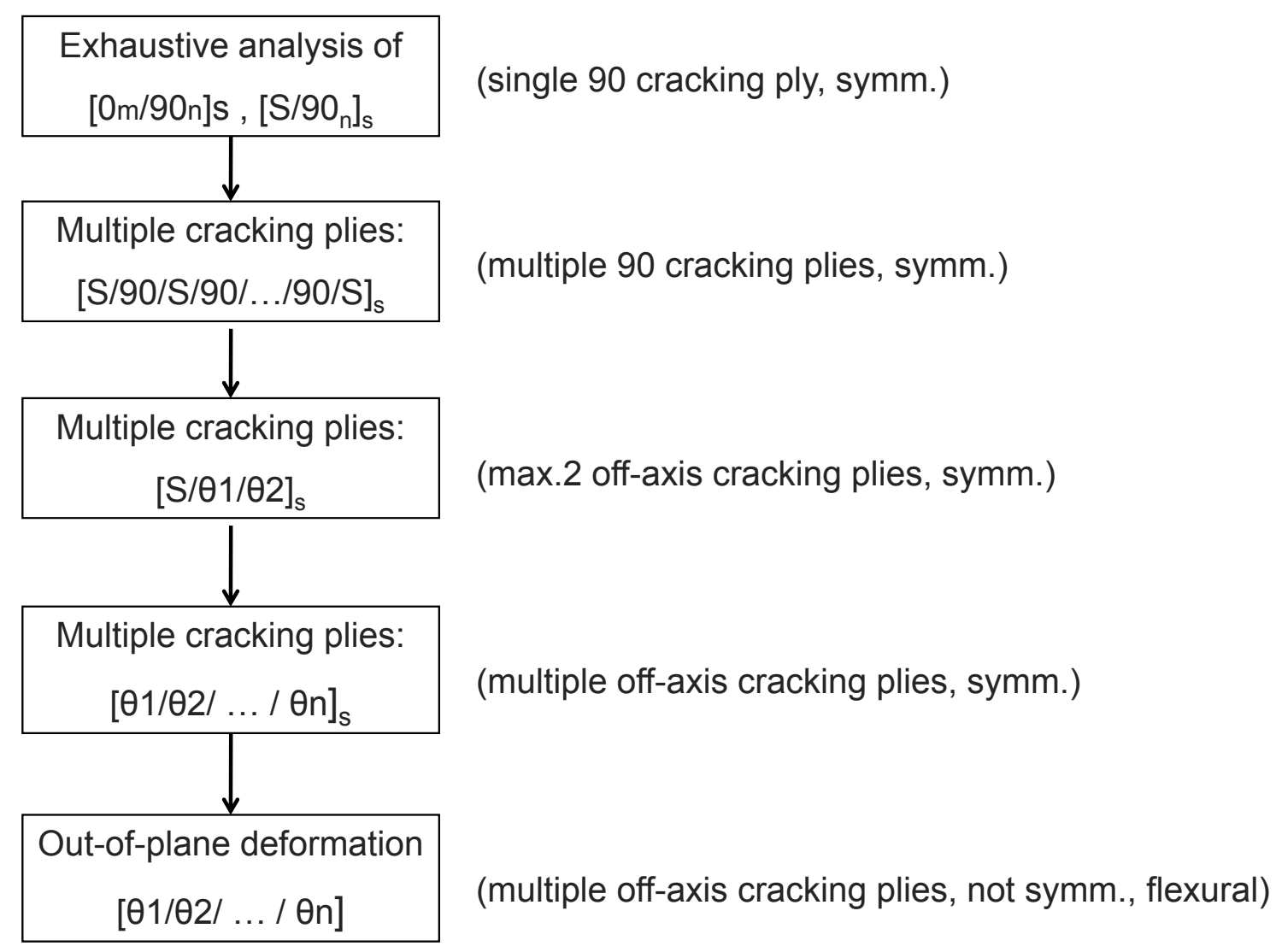

Figure 2.9: The general evolution, with respect to the modeling capabilities, of analytical models for matrix cracking in FRP laminates.

The above presentation is not meant to be merely a literature review on the given research topic - the characterization and evolution of damage in form of transverse cracks in general laminated composites under combined membarne/flexural loading - but it is also meant to offer a collection of available tools in order to approach the problem, and also to clearly state the limiting assumptions that each tool resorts to, in order provide a good level of awareness in using and further developing these tools for particular needs. 


\section{Chapter 3}

\section{Objectives}

Having set the above frame of possible theoretical paths and research stages regarding the stated problem, the main objectives of this dissertation can be listed as:

- Identify all available theoretical tools on approaching the problem of matrix cracking in laminated composites; clearly understand both the advantages and the drawbacks and limitations of each possible solution approach: limiting assumptions involved, the level of generality of the solution that can be reached by following different theoretical ways, the final reliance on experimental data required by different solutions.

- Asses the current stage of researching the problem of matrix cracking in laminated composites.

- Identify and analyze the limitations of the current models: limiting assumptions involved, experiments required in order to determine the analytical model parameters.

- Develop an analytical model of matrix cracking in laminated composites of general stacking sequence under generalized membrane/flexural deormation, including the effect of thermal residual stress.

The model aims to a release of the limiting assumptions used in previous models dealing with the same problem, and to a minimization of the necessary experimental data.

The present work makes use of

1. a material model (membrane-flexural thermo-elastic constants for given damage levels in any ply of the laminate) developed in [35]; 
2. a fracture mechanics inspired energy release rate damage evolution criterion;

3. a continuum damage mechanics inspired ply homogenization technique using plies crack densities as state variables.

The effect of thermal residual stress is included, which might be of critical importance in structural applications at very low temperatures, as it is the case of cryogenic fuel tanks. 


\section{Chapter 4}

\section{Model Formulation}

The present analytical model for progressive damage in composite laminates is developed and formulated considering some hypotheses and limits of applicability, as they are listed in the following (these hypotheses will be further discussed and detailed in the corresponding Sections where they are used and implemented):

- long aligned fiber reinforced composite materials; matrix cracking takes place in individual plies along the fiber direction.

- linear elastic material behavior up to damage onset; the material nonlinearity due to matrix plastic deformation is not implemented in the current formulation.

- brittle fracture material behavior; one matrix cracking suddenly develops and spans the whole width of the specimen (propagated cracks, see Section 4.4.1); the progressive damage growth is rather a discrete process characterized by discrete fracture events, than a continuum one.

- linear elastic nature of fracture events (see Fig. 4.14 in Section 4.7.1); the unloading path after a crack multiplication event follows a linear law with no residual plastic deformation; there is no energy released for the formation of plastic zones (see the energy balance in eq. (4.5)); the progressive damage growth is modeled under the frame of linear elastic fracture mechanics (LEFM).

- there is uniform spatial distribution of the propagated cracks in a laminate ply affected by matrix cracking; cracks are equally spaced between them. 
- there is perfect closure between separate faces of the matrix cracks when the crack closure condition is met (see Section 4.7.2); there is no material nonlinearity induced by friction or contact between opposite faces of the closed cracks.

- there is a strengthening effect ( $\mathrm{R}$-curve behavior, see Section 4.4.3) of the damage growth on the critical parameter (i.e., the critical ERR $G_{c}$ ) characterizing the material resistance to crack growth.

- the R-curve behavior might features an in-situ nature (i.e., ply thickness dependency) (see Section 4.4.3).

The main ingredients and modules of the model, and the basic principles of how they work and interact together are described in the following:

1. Reduced Material Properties - are approached through the material model in [35]. While overall laminate thermo-elastic properties for a given damage level (crack density) in each ply of the laminate are provided by the material model in [35], no information can be recovered with regard to individual plies properties (ply reduced stiffness and ply reduced thermal expansion coefficients). In this way, the material model features a laminate formulation (as opposed to ply formulation, when individual plies of the laminate are possible to be assigned reduced material properties according with their individual damage level). Due to this fact, while the model formulation in [35] is a very tempting tool due to its level of generality, close-form solution and easiness to be implemented, the laminate formulation works as a disadvantage for the model developed in this work, where individual reduced plies properties are needed in order to calculate the strain energy required by the energy release rate damage evolution function (point (4)). This fact is overcome by the iterative procedure of the present model (point (3)).

The background and the details of the formulation of the material model [35] used in the present model in oder to calculate the reduced material properties are presented in Section 2.1.2, 4.1, B.

2. A ply homogenization technique - inspired by the CDM. In this procedure, individual plies having a certain damage level (crack density) are regarded as fictitious homogenized materials having as effective thermo-elastic properties the real properties of the 
cracked ply (see Fig. 4.1). Once the reduced ply properties are determined, individual ply cracks are not physically regarded any more, but the ply cracks are rather considered as being smeared through the volume of the ply.

3. An iterative procedure - meant to detect the damaging ply (the (k0) ply in the present notation) at a certain level of loading, according to the damage evolution criterion (point (4)), and to allow the incremental increase in damage in this (k0) ply. Furthermore, after the $(k 0)$ ply is given one damage increment, the iterative procedure (also regarded as LSS iteration in the following, which stands for laminate stacking sequence iteration) repeatedly iterates over the stacking sequence of the laminate at the same load level, in order to check for possible multiple increments in damage level of the same damaging ply $(k 0)$, or to check for possible change of the $(k 0)$ ply at the same load level, due to stress redistribution after the damage level in the initial detected (k0) ply is increased and the ply stiffness is accordingly reduced.

Reduced material properties of individual ply can be calculated by applying the iterative procedure, based on the overall laminate reduced material properties provided by the material model (point (1)) and the fact that individual plies are considered one at a time for damage growth by the iterative procedure.

4. Damage Evolution - is approached through the fracture mechanics inspired Energy Release Rate criterion. The damaging ply $(k 0)$ is detected by the damage evolution criterion together with the iterative procedure, and the damage level is incrementally increased in this ply.

The background of damage growth formulation implemented in the present progressive damage model is presented in Section 2.2. Details of the implementation are presented in Section 4.4, 4.7.

5. Classical Laminate Theory (CLT) - provides the overall, effective constitutive behavior of the laminate containing cracks in individual plies, provided that the homogenized, effective ply properties are considered, according with point (2).

Details of the CLT as it is used in the present model are presented in Appendix A.

Further description is provided on the above model constituents, with an emphasis on material model and damage evolution criterion, as key elements of the present model. 


\subsection{Reduced Material Properties (Material Model)}

The effort of many researchers has been focused in developing models of reduction in thermo-elastic properties of fiber reinforced laminated composites, having individual plies affected by matrix cracking. The ideal model would be able to deal with a general stacking sequence laminate (plies at any orientation), featuring cracks in any ply, with no restriction on the number of cracking plies inside of the laminate. Both membrane stiffness and flexural stiffness of the laminate are of interest. The reduction in thermal expansion coefficient is also necessary to be assessed, in order to be able to implement the effect of manufacturing thermal residual stresses, and to have a complete description of the material behavior under matrix cracking. Yet, the desired level of generality proved to be a difficult goal to achieve, as discussed in Section 2.

Providing the whole set of both membrane and flexural material properties, due to its close form easy implementing formulation, and due to extensive model validation against numerical and experimental data [34, 35, 38], the COD based solution in [35] is considered in the present progressive damage model, as a material model for the reduced laminate thermo-elastic properties

$$
[C]_{6 \times 6}=\left[C\left(\rho^{(k)}\right)\right],\{\alpha\}_{6 \times 1}=\left\{\alpha\left(\rho^{(k)}\right)\right\}
$$

where $[C]$ is the reduced effective laminate stiffness matrix, $\{\alpha\}$ is the reduced effective laminate thermal expansion coefficients vector, and $\rho^{(k)}$ is the normalized crack density in each $(k)$ ply of the laminate $(k=1 \ldots N$, where $N$ is the total number of plies in the laminated composite). The normalized crack density is defined as $\rho^{(k)}=t^{(k)} / d^{(k)}$, where $t^{(k)}$ and $d^{(k)}$ are the thickness and the crack spacing for the ply (k), respectively (see Fig. 2.1 (a)). The normalized crack density is a measure of ply damage level equivalent to the crack density $\lambda$ in eq. (1.1). The $6 \times 6$ and $6 \times 1$ subscripts in (4.1) indicate that the reduced effective (laminate) material properties are for the case of combined membrane and flexural deformation.

The code implementation aspect of the material model in eq. (4.1) follows the following summarizing steps:

- the $\beta^{k}$ COD coefficients in the cracked ply (see eq. (2.9)) are calculated from eq. (B.17) (B.21).

- the reduction in laminate stiffness is calculated from eq. (B.29). 
- the reduction in laminate thermal expansion properties is calculated from eq. (B.33).

- the reduced laminate stiffness are calculated from eq. (B.28).

- the reduced laminate thermal expansion properties are calculated from eq. (B.32).

While the computer implementation of the material model does not pose special difficulties, the understanding of the background, the concepts, the elements, and the assumptions that are involved in deriving the aforementioned equations is more challenging. This is the reason why a comprehensive description of the background of the material model in [35], which is implemented in the present progressive damage model in order to calculate the reduced material properties (4.1), is presented in Appendix B.

\subsection{Ply homogenization technique}

The homogenization technique is meant to allow for the use of $C L T$, as it is described in Section 4.5. Individual plies featuring matrix cracking are regarded as fictitious homogeneous materials, where individual cracks are not physically considered, but the effect of the cracks on the ply materials properties is assessed for (see Fig. (4.1)), by combining the material model in Section 4.1 and the iterative procedure in Section 4.3. The material model allows for the calculation of overall (homogenized) properties of the laminate featuring matrix cracking in individual plies, and the iterative procedure allows for the calculation of overall (homogenized) properties of individual cracked plies. The homogenized thermo-elastic properties of the laminate are provided by eq. (4.1) (material model [35]), and the homogenized thermo-elastic properties of individual plies are provided by eq. (4.3).

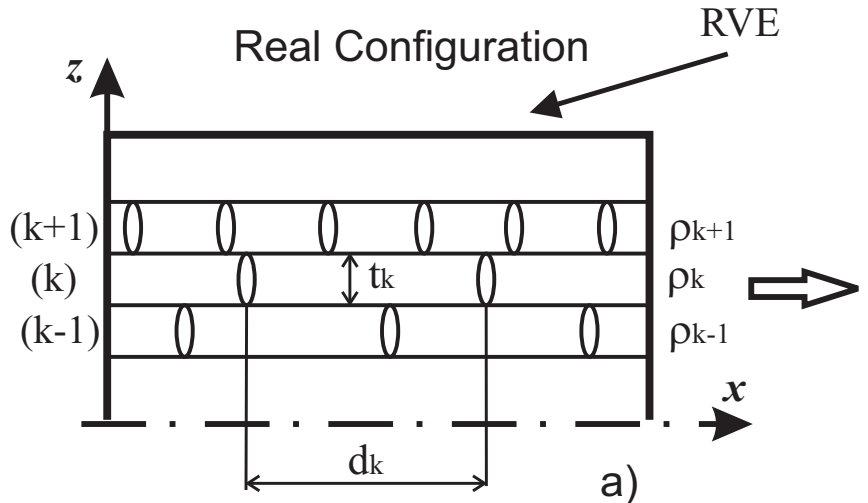

a)

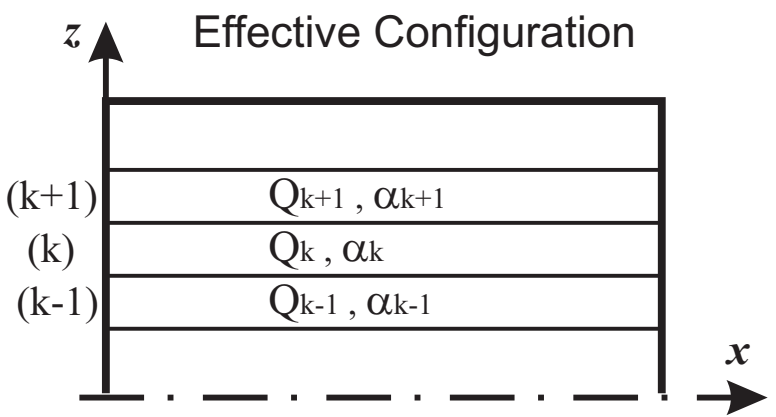

b)

Figure 4.1: CDM inspired ply homogenization technique. 
The homogenization technique brings two advantages: i) the release of the limitations of RUC based models with regard to the number of cracking plies or symmetric/balanced laminate stacking sequence, and ii) the use of CLT in order to calculate the effective stressstrain field in the damaged laminate (as presented in Section A.1), based on effective material properties. Then, the stored energy of deformation is calculated based on effective stressstrain components (as presented in Section A.2).

It is possible, in this way, to extend the level of generality of the problem by including the flexural deformation of the laminate.

\subsection{Iterative procedure}

The iterative procedure (also called the LSS iteration in the present model) has a double role. First, it checks for the cracking ply (noted $(k 0)$ ) inside of the laminate, at a given load level (see Fig. 4.13 in Section 4.6). The cracking ply is identified as being the ply which first meets the condition of the damage evolution criterion presented in Section 4.4.1. When a $(k 0)$ cracking ply is found, the crack density level in that ply is incrementally increased at a subsequent value

$$
\rho_{(k 0)}^{i+1}=\rho_{(k 0)}^{i}+\Delta \rho^{i}
$$

After a cracking ply $(k 0)$ is identified, and its crack density is accordingly increased, the new effective laminate properties are calculated by the material model as it is presented in Section 4.1, B. At this point, the iterative procedure provides a means of calculating the reduction in individual properties of the cracking ply $(k 0)$. Considering the fact that the reduction in effective laminate properties are only due to increasing the crack density in the $(k 0)$ ply (in other words, only due to reduction in material properties - ply stiffness and ply thermal expansion coefficients - of $(k 0)$ ply, without having affected the material properties of the other $(N-1)$ plies $)$, the new effective material properties of $(k 0)$ ply are calculated through $C L T$ as

$$
\begin{aligned}
& {\left[Q^{(k 0)}\right]=\frac{1}{t^{(k 0)}}\left[\left[A_{(k 0)}\right]-\sum_{k=1}^{N}\left(1-\delta_{k, k 0}\right) t^{(k)}\left[Q^{(k)}\right]\right]} \\
& \left\{\alpha^{(k 0)}\right\}=\frac{1}{t^{(k 0)}}\left[Q^{(k 0)}\right]^{-1}\left[-\left\{\delta_{E(k 0)}\right\}-\sum_{k=1}^{N}\left(1-\delta_{k, k 0}\right) t^{(k)}\left[Q^{(k)}\right]\left\{\alpha^{(k)}\right\}\right]
\end{aligned}
$$


where $\left[A_{(k 0)}\right]$ and $\left\{\delta_{E(k 0)}\right\}$ are respectively the effective laminate in-plane stiffness matrix and the effective laminate in-plane vector of thermal effect coefficients, provided by the material model in Section $4.1 ; \delta_{k, k 0}$ is the Kroneker-Delta function, which returns the value 1 if $k=k 0$, or value 0 if $k \neq k 0$. It has to be noted that the values $\left[A_{(k 0)}\right],\left\{\delta_{E(k 0)}\right\}$ are overall laminate properties; the subscript $(k 0)$ has been used for them rather to indicate that they are calculated due to a crack multiplication in $(k 0)$ ply only.

After both effective laminate properties (4.1), and the effective ply properties (4.3) are calculated, the iterative procedure is resumed in order to check for other cracking plies at the same level of loading, or in order to check for multiple crack increments (4.2) for the same cracking ply $(k 0)$. The iteration stops when no ply meets the condition in the damage evolution criterion (4.4).

Regarding the increment in crack density $\Delta \rho$ which is applied in (4.2) when the damage evolution criterion in (4.4) is satisfied, different modeling approaches can be considered: an infinitesimal increase in crack density at each cracking event, a discrete in crack density corresponding to the formation of one more crack, or a discrete doubling in crack density (see Section 4.4.2).

\subsection{Damage onset and damage evolution}

\subsubsection{Energy based damage growth criterion}

A damage evolution criterion is required in order to detect the conditions for matrix crack multiplication in individual plies of the laminate under loading. In the present formulation, a fracture mechanics inspired criterion (eq. (4.4)) is selected [54] in order to consider mixed modes crack formation for the case of matrix cracking under general in-plane and flexural deformation.

$$
g_{(k 0)}=(1-r) \sqrt{\frac{G_{I(k 0)}}{G_{I c}}}+r \frac{G_{I(k 0)}}{G_{I c}}+\frac{G_{I I(k 0)}}{G_{I I c}}-1 \geq 0, r=\frac{G_{I c}}{G_{I I c}}
$$

$G_{I(k 0)}, G_{I I(k 0)}$ are energy release rates due to modes $I, I I$ crack propagation in the ply $(k 0)$ of the laminate. $G_{I, I I(k 0)}$ are calculated at global level of the laminate, considering the contribution of all $(k)$ plies to the energy released due to crack growth in the current $(k 0)$ cracking ply (see eq. (4.7), (A.36)). Subscripts $(k 0)$ are used in (4.4) in order to emphasize that energy release rates are evaluated due to damage multiplication in the current cracking ply 
$(k 0)$ only, as imposed by the iterative procedure in Section 4.3. $G_{I c}, G_{I I c}$ are critical values of the energy release rates, or the material resistance to modes $I$ and $I I$ crack propagation; $G_{I c}$ and $G_{I I c}$ are regarded as material properties whose evaluation is based on experiments. A discussion of the nature of $G_{I c}, G_{I I c}$ material properties is presented in Section 4.4.3, where the possible in-situ $R$-curve behavior of the material resistance to crack multiplication is analyzed and analytically implemented. The notation $g_{(k 0)}$ is used for the damage evolution function in eq. (4.4).

Both modes of crack propagation (mode I for crack opening, and mode II for crack sliding shear) are considered in the combined formulation in (4.4). The damage evolution criterion in (4.4) is the mixed modes extension of the Griffith postulated energy release rate principle of damage mechanics, which states that the conditions for crack propagation are met if the rate of energy released $(G)$ by the elastic body due to formation of one unit surface of new crack reaches a certain critical value $\left(G_{c}\right)$, which is regarded as a material property.

While the mixed mode criterion in eq. (4.4) is casted and used in the present model due to its simplicity, a discussion on other mixed modes criteria proposed in the literature in performed in Appendix C.

For better understanding the Griffith principle and its extension to laminated composites as implemented in the present progressive damage model, let's imagine an elastic body under increasing external loading $F$ (see Fig. 4.2). Under the assumption of linear elastic material behavior, the load-deformation follows the linear path $O A$, as long as there is no new damage formation (i.e., as long as the stress-deformation field does not meet the criterion in eq. (4.4)). If $W$ is the work done by the external forces $F$ in order to achieve the elastic deformation $\delta$, and $U$ is the elastic strain energy stored in the body, then the equality $W=U$ and $\Delta W=\Delta U$ holds true at any moment $F$ or increment $\Delta F$ of the external loading, under the assumption of the linear elastic behavior.

Assuming that the point $A$ is the moment of crack growth, let's consider what happens at this moment, without further increase of the loading level. The moment right before the crack growth will be referred to as before, and the moment right after crack growth will be referred to as after. The operator $\Delta$ for some quantity will denote the difference of that quantity after crack growth and before crack growth, in the following. Due to new damage formation and the change in material stiffness, there will be both a change $\Delta W$ in the work done by the external loading and a change $\Delta U$ in the elastic strain energy stored in the body. The available energy $\Delta \Pi$ for the crack growth is the difference $\Delta \Pi=\Delta W-\Delta U$, which is 


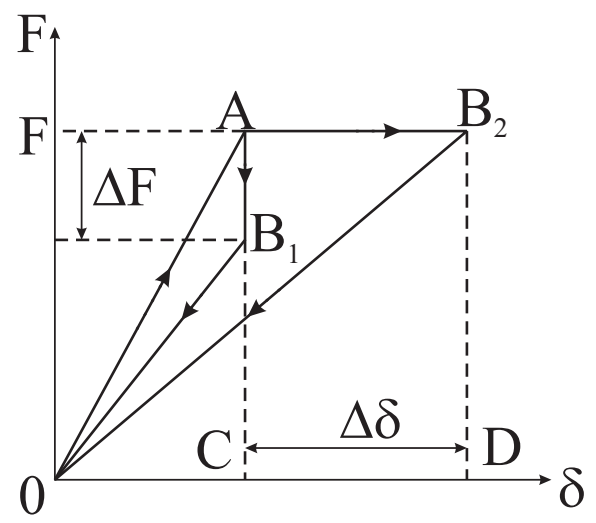

Figure 4.2: Load vs. Displacement control crack multiplication

the energy released for crack growth. Considering that some new crack area $\Delta A$ (only one face of the new crack!) is generated, the energy release rate for the formation of new crack is defined as $G=\frac{\Delta W-\Delta U}{\Delta A}$, and the Griffith principle postulates that the condition for crack growth is

$$
G=\frac{\Delta W-\Delta U}{\Delta A} \geq G_{c}
$$

Sometimes in fracture mechanics the available energy for crack growth is considered for the formation of both faces $2 \cdot \Delta A$ of the new crack. In that case the energy measure $\gamma$, which is called the fracture surface energy, is used instead of the energy release rate $G$ in eq. (4.5). The equality $G=2 \cdot \gamma$ exists between the two energy quantities.

Sometimes in fracture mechanics the finite discrete formulation $\Delta$ in eq. (4.5) is considered in its infinitesimal form $(\Delta \rightarrow \delta): G=\frac{\delta \Pi}{\delta A}$, and a continuum crack growth is modeled in this way. However, according to the assumption of the present model, the crack multiplication is regarded as discrete events, with one crack suddenly developing and spanning the whole width of the laminate (brittle cracking behavior, which is according with experimental observation of matrix cracking for composite systems used in structural applications), and the finite formulation $\Delta$ in eq. (4.5) is considered in this model. The finite formulation in eq. (4.5) has been label as finite fracture mechanics $[7,13,16]$.

As previously stated, the energy balance between the moments before and after crack growth has to be evaluated:

$$
\begin{aligned}
& \Delta U=U^{\text {after }}-U^{\text {before }} \\
& \Delta W=W^{\text {after }}-W^{\text {before }}=F \cdot \Delta \delta-0=F \cdot \Delta \delta
\end{aligned}
$$


where $F \cdot \Delta \delta$ is the work done by the external loading $F$ which is given the displacement $\Delta \delta$ due to the formation of new crack.

If displacement control conditions prevail in the moment of the new crack formation, then the displacement $\Delta \delta$ of the point of application of external loading becomes $\Delta \delta=0$ (which is the $A B_{1}$ segment in Fig. 4.2), and change in the work of external loading becomes $\Delta W=0$ in eq. (4.6). In this case, the Griffith energy balance in eq. (4.5) becomes

$$
G=-\frac{\Delta U}{\Delta A} \geq G_{c}
$$

A drop $\Delta F$ is recorded in the loading level in order to assure the displacement control conditions.

If load control conditions prevail in the moment of the new crack formation, then the load $F$ follows the path $A B_{2}$ in Fig. 4.2, and a change in displacement $\Delta \delta$ is recorded in between before and after moments of crack growth. In this case, the change in the work done by the external loading becomes $\Delta W=F \cdot \Delta \delta \neq 0$ in eq. (4.6). The change in the elastic strain energy corresponding to before and after moments of new crack formation is calculated as the difference in the areas beneath the loading and unloading before and after curves: $\Delta U=U^{\text {after }}-U^{\text {before }}=A_{O B_{2} D}-A_{O A C}=\frac{1}{2} F \cdot \Delta \delta$. Consequently, the relationship $\Delta W=2 \cdot \Delta U$ exists under load control conditions, and Griffith the energy balance in eq. (4.5) becomes

$$
G=\frac{\Delta U}{\Delta A} \geq G_{c}
$$

Due to the fact that most of the available experimental data is recorded under displacement control conditions, the energy balance in eq. (4.7) is considered and implemented in the present model. Additional disscusion on the displacement control case is provided in Section 4.7.1.

Because mixed modes of crack propagation appear in the case of general loading of the laminate (and they are accordingly considered by (4.4)), the variation of strain energy in (4.7), (4.8) is decomposed in components corresponding to modes $I$ and $I I$ crack formation, similar to $[24,29,30]$ (see Section A.2).

\subsubsection{Modeling crack multiplication in laminated composites}

In order to model and to evaluate the crack multiplication process, the following variables will be used for each ply ( $k$ ) of the laminate (see Fig. 2.1): 
- $d$ : crack spacing (the distance between two consecutive cracks);

$-\lambda$ : crack density; $\lambda=1 / d$;

- $\rho$ : normalized crack density; $\rho=t / d$, where $t$ is the thickness of ply $(k)$.

It can be noticed that the crack density $\lambda$ and the normalized crack density $\rho$ are equivalent quantities $(\rho=t \lambda)$, both of them giving a measure of the crack spacing $d$. While only one of them is enough in order to formulate the analytical model, both of them are kept and used in the following. The reason for this is that the material model ([35, 36], see Section 4.1) which is implemented in the present progressive damage model is formulated in terms of normalized crack density $\rho$, while the most of the experimental data is available in terms of crack density $\lambda$.

Also to be noticed that the quantities $d, \lambda, \rho$ are defined for each ply $(k)$ of the laminate. In order to emphasize this, the index $(k)$ would be proper to be used $\left(d_{(k)}, \lambda_{(k)}, \rho_{(k)}\right)$ for subsequent equations. However, in order to simplify the notations, the index $(k)$ is not attached, but it has to be considered that all subsequent equations regard a single ply $(k)$ of the laminate.

An arbitrary initial crack spacing $d_{0}$ corresponding to a very high distance between preexisting cracks is considered in the undamaged state of the material for the progressive damage model. The initial crack spacing has to be selected high enough in order to have a negligible influence on the virgin material properties. A value of $d_{0}=1000 \mathrm{~mm}$ has been used throughout the present model. The assumption of preexisting cracks in undamaged state and the associated initial crack spacing does not have any influence on the damage onset and evolution process. The preexisting cracks can be regarded as inherent flaws in the material as a result of manufacturing process.

Regarding the discrete increase in crack surface $\Delta A$ required in (4.5), the schematic representation of a pattern of equally spaced cracks in an arbitrary ply $(k)$ of the laminate is considered in Fig. 4.3, where $l_{i}$ is the length of one individual crack and $d$ is the crack spacing. The total crack length in the ply $(k)$ is then $l=\sum_{i} l_{i}$, and the total surface of the ply (which is the in-plane surface of the laminate, as well) can be evaluated as $S=l \cdot d$, which based on the definition of the normalized crack density $\rho=t / d$ becomes

$$
S=l \frac{t}{\rho}
$$

A finite increase in the crack length $\Delta l$ in the ply $(k)$ corresponds to a finite increase in crack 
density $\Delta \rho$, and (4.9) becomes

$$
S=(l+\Delta l) \frac{t}{(\rho+\Delta \rho)}
$$

By considering (4.9) and (4.10), the increase in the total crack length of the ply $(k)$ can be expressed as

$$
\Delta l=l \frac{\Delta \rho}{\rho}
$$

The new formed crack surface due to crack growth in ply $(k)$ can be written as $\Delta A=t \Delta l$, which after considering (4.11) and (4.9) becomes

$$
\Delta A=S \Delta \rho
$$

The relation (4.12) of the new formed crack surface is further used in (4.5) in order to apply the damage evolution criterion in (4.4).

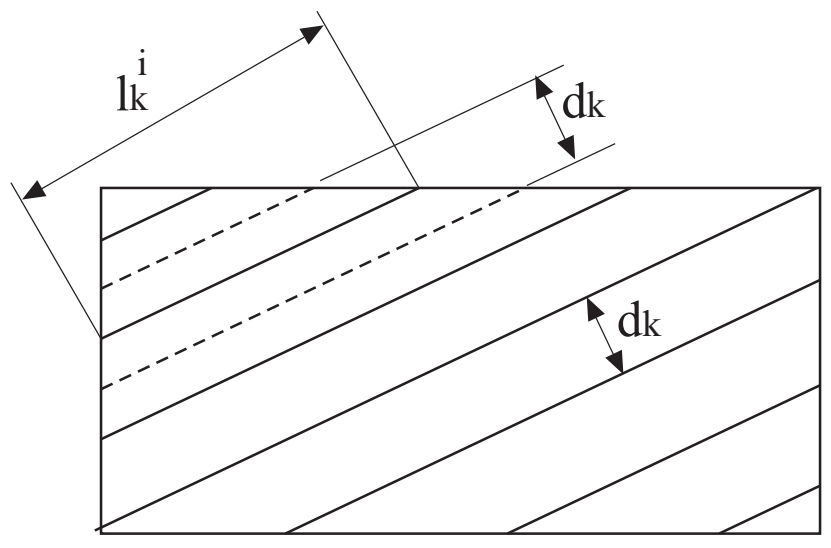

Figure 4.3: An equally spaced pattern of matrix cracks in one ply of a laminate.

The problem to solve next is to evaluate the increase in crack density $\rho$ according to the modeling strategy. Let's consider for this two consecutive instances of crack multiplication in ply $(k)$ : the instance $(i)$ corresponds to a given level of matrix cracking, and the instance $(i+1)$ corresponds to the moment following the discrete crack multiplication event in ply $(k)$. It can be said the the instance $(i)$ corresponds to the moment before crack growth, while the instance $(i+1)$ corresponds to the moment after crack growth. The following general equations can then be written:

$$
\begin{aligned}
& d^{i+1}=d^{i}+\underbrace{\Delta d^{i}}_{<0} \\
& \rho^{i+1}=\rho^{i}+\underbrace{\Delta \rho^{i}}_{>0}
\end{aligned}
$$


where $\Delta d^{i}, \Delta \rho^{i}$ represent discrete variations in crack spacing and normalized crack density, respectively, in order to shift from the damage state $(i)$ to the next damage state $(i+1)$ of the ply $(k)$. To be noted that for the crack multiplication process the quantity $\Delta d^{i}$ is a negative number, and the quantity $\Delta \rho^{i}$ is a positive number, as shown in eq. (4.13), (4.14).

A parameter $k_{d}^{i}$ of the crack spacing variation is introduced at this moment, such that the change in the crack spacing corresponding to the shift from instance $(i)$ to instance $(i+1)$ in eq. (4.13) can be expressed as

$$
\Delta d^{i}=-\frac{d^{i}}{k_{d}^{i}}
$$

To be noted that, in the most general case, the parameter $k_{d}^{i}$ can take different values for any two different instances $(i)-(i+1)$, this being the reason why it bears the superscript $i$. There could be different $k_{d}^{i}$ for different crack growth events.

The parameter $k_{d}^{i}$ expresses the change in crack spacing $d^{i}$ due to damage growth, and it is a first measure of the crack multiplication from damage state $(i)$ to the subsequent damage state $(i+1)$. It is considered that $k_{d}^{i} \geq 2$. The bounding case $k_{d}^{i}=2$ simulates a discrete doubling in the number of cracks from state $(i)$ to state $(i+1)$, which is the case of most of the analytical models based on RUC approach (see Section 2.1.1). A very big value of the $k_{d}^{i}$ parameter simulates a very small reduction in crack spacing from state $(i)$ to state $(i+1)$, which can be regarded as a continuum infinitesimal increase in crack density, as it is the case of the analytical models based on CDM approach (see Section 2.1.3). The goal here is to simulate a crack multiplication process that features 1 more crack from damage state $(i)$ to damage state $(i+1)$; the condition for this is set to be

$$
d^{i}=\frac{d_{0}}{i+1}
$$

The following quantities can be calculated based on (4.13), (4.14), (4.15)

$$
\begin{aligned}
d^{i+1} & =\frac{k_{d}^{i}-1}{k_{d}^{i}} d^{i}=\frac{1}{k k_{d}^{i}} d^{i} \\
\rho^{i+1} & =\frac{t}{d^{i+1}}=\frac{t}{d^{i}+\Delta d^{i}}=\frac{t}{d^{i}} \frac{k_{d}^{i}}{k_{d}^{i}-1}=\rho^{i} \cdot k k_{d}^{i} \\
\Delta \rho^{i} & =\left(k k_{d}^{i}-1\right) \rho^{i}
\end{aligned}
$$

where the notation $k k_{d}^{i}=k_{d}^{i} /\left(k_{d}^{i}-1\right)$ is used in the above equations.

If we set the $k k_{d}^{i}$ parameter to the value $k k_{d}^{i}=\frac{i+2}{i+1}$, the following relationship can be 
derived based on eq. (4.18)

$$
\begin{aligned}
\rho^{i} & =\rho^{i-1} \cdot k k_{d}^{i-1}=\frac{i+1}{i} \rho^{i-1}=\frac{i+1}{i} \frac{i}{i-1} \rho^{i-2}=\ldots=\frac{i+1}{i} \frac{i}{i-1} \ldots \frac{2}{1} \rho_{0} \\
& =(i+1) \rho_{0}
\end{aligned}
$$

Based on (4.20) the crack spacing at the instance $(i)$ can be calculated as

$$
d^{i}=\frac{t}{\rho^{i}}=\frac{t}{(i+1) \rho_{0}}=\frac{d_{0}}{i+1}
$$

which is exactly the 1 more crack damage growth condition in eq. (4.16). Consequently, the 1 more crack damage multiplication is assured by the value

$$
k k_{d}^{i}=\frac{i+2}{i+1}
$$

Similarly to eq. (4.20), the following equation is also true

$$
\rho^{i+1}=(i+2) \rho_{0}
$$

Based on (4.20) and (4.23), the increase in the normalized crack density from damage state (i) to damage state $(i+1)$ can be calculated as

$$
\Delta \rho^{i}=\rho^{i+1}-\rho^{i}=(i+2) \rho_{0}-(i+1) \rho_{0}=\rho_{0}=\text { ct. }
$$

which means that, in order to have assured the condition of 1 more crack, the increase in normalized crack density $\Delta \rho^{i}$ for each crack multiplication event has to be constant and equal to the initially considered crack density $\rho_{0}$.

In conclusion, the model setup in order to simulate the 1 more crack damage multiplication process is given by setting the parameter $k k_{d}^{i}$ to the value $k k_{d}^{i}=\frac{i+2}{i+1}$, which implies using the equations $(4.20),(4.21),(4.24)$. This is the setup of the present model. If another crack multiplication behavior is wanted to be simulated, the $k k_{d}^{i}$ parameter (or its equivalent $k_{d}^{i}$ ) has to be set accordingly. Letting $k k_{d}^{i}$ as in allows allows defining the crack multiplication as an 1 more crack discrete (finite) process. The corresponding energy released for the formation of one more crack (eq. (4.7)) is calculated at each model iteration.

\subsubsection{Modeling the in-situ $\mathrm{R}$-curve behavior of damage evolution in laminated composites}

The Griffith principle of classical fracture mechanics (eq. (4.5)) has been formulated for the case of a homogeneous material featuring brittle fracture. Based on this principle, the 
critical parameter for crack growth $\left(G_{c}\right.$ in eq. (4.5)) is a constant material property, see Fig. 4.4, where $a$ is the crack length at a certain load level, and $\Delta a$ is the crack growth which triggers the change in strain energy $\Delta U$ in eq. (4.5). The area of the new generated crack surface in eq. (4.5) is $\Delta A=t \cdot \Delta a$, where the thickness $t$ can be considered $t=1$ due to the similarity of the cracking mechanism over thickness, which allows the use of the plain strain assumption in classical fracture mechanics ( $\alpha$ being the plane of plane strain in Fig. 4.4). The exemplification in Fig. 4.4 is made for the case of mode $I$ (crack opening) loading case $P^{I}$; the Griffith principle is identical formulated for modes $I I$ and $I I I$ (in-plane shear and out-plane shear) crack propagation cases, having thus distinct values for the three $G_{I c}, G_{I I c}, G_{I I I c}$ critical material parameters corresponding to the three crack propagation modes. The Griffith principle represents an energy based crack growth law.

The energy based crack growth criterion in eq. (4.4) represents a proposed [54] mixed mode extension of the Griffith principle (which is formulated for the single mode case). Other mixed modes criteria are presented and discussed in Appendix C. The use of energy based criteria for modeling damage evolution in composites has been considered a better choice than the use of strength based criteria, as presented in Section 2.2. However, different micromechanics and damage mechanisms specific to UD composites and laminated composites have posed new challenges regarding the extension of classical fracture mechanics Griffith principle form the case of homogeneous material to the case of composite material, even if the brittle fracture is regarded in both cases.

The formulation of the present analytical model for intra-laminar matrix cracking in laminated composites takes into account and implements the in-situ $R$-curve behavior $G_{c}=$ $G_{c}(\lambda, t)$ (where $\lambda$ is the crack density of the ply, and $t$ is the ply thickness), as it was noticed in Section 2.2. The explanation of this behavior is based on the fiber bridging effect that has been experimentally shown for the case of intra-laminar matrix cracking in UD composites $[62,63,64,65,66,67]$. Based on this, extrapolation to the case of laminated composites is then modeled in the present work. The phenomenological explanation of the straightening effect $G_{c}=G_{c}(\lambda, t)$, and the analytical modeling in order to capture this effect, are presented in the following.

A multitude of test methods in order to experimentally measure the critical toughness values $G_{I c}$ and $G_{I I c}$ for mode $I$ and $I I$ intra-laminar crack propagation, as well as mixed mode fracture properties, have been designed, and the results of these experiments have been published in the technical literature $[55,62,63,64,65,66,67,68,69,70,71,72]$. A listing 
of these test methods reads:

- three point bending (TPB)

- four point bending (FPB)

- double edge notch (DEN)

- double torsion (DT)

- compact tension (CT)

- double cantilever beam (DCB)

- end notch flexure (ENF)

- mixed mode bending (MMB)

- end loaded split (ELS)

- compact tension shear (CTS)

- center notch $(\mathrm{CN})$

- three rail shear (TRS)

While studying the test methods for $G_{c}$ is not the objective of the present manuscript, some observations are required in order to phenomenologically explain the fracture behavior of laminated composites.

First, the test methods listed for determination of $G_{c}$ deal with UD composite specimens. According to the test method and the orientation of the material, there might be different directions for crack propagation for intra-laminar cracking (see Fig. 4.5 (a), (b)): longitudinal direction (with the crack front advancing along fibers direction in the plane of the laminate) and transverse direction (with the crack front advancing perpendicular to the plane of the laminate). For a complete picture of the directions of crack propagation, the case of inter-laminar cracking is also presented in Fig. 4.5 (c), (d), where another two different directions of crack propagation can be identified and labeled as $0 / 0$ direction (having the crack front perpendicular to the fibers direction) and 90/90 direction (having the crack front parallel to the fibers direction). The microscopic observations have shown that the morphology of the crack surface is different for different directions of crack propagation, this 
being the explanation why the $G_{c}$ 's might give different values dependent of the test method used and the direction of crack propagation.

Second, the stable or unstable crack propagation is dependent of the test method, as well. For some methods (TPB, FPB,CT,CTS,CN) there is sudden crack propagation, and the $G_{c}$ value is evaluated based on the total fracture surface. For other test methods (DT,DCB,ENF,MMB) stable crack growth can be achieved, and the critical ERR $G_{c}$ can be evaluated based on measurements of progressive crack growth $\Delta A$.

The case of interest is the last one, of stable crack propagation $[62,63,64,65,66,67]$. In this case a fibers bridging effect during crack propagation was noticed, see Fig. 4.6. The fibers bridging effect means that fibers connect the two faces of the crack once the crack is initiated, and due to this, stress transfer between the crack faces does exist. There is an initial value of the fracture toughness $G_{c}=G_{c}^{i}$ for crack initiation. While the crack length extends, the bridging fibers are pulled out of matrix and/or broken, which means an increase in the resistance to crack growth, i.e., a cumulative increase in $G_{c}$ with increasing crack length. At a certain crack length $a_{0}$ the bridging effect reaches a steady state, which means that while new bridging fibers appear at the crack tip, the same amount of fibers totally separate at the other end of the bridging zone, and the crack further propagates in a self-similar manner, at constant length $l^{s s}$ of the bridging zone. The energy released required to further propagate the crack will be a constant value $G_{c}^{s s}$ after the crack reaches the steady state length $a_{0}$. The variation of $G_{c}$ with the crack length is qualitatively presented in Fig. 4.6 (b).

It can be inferred that the main parameter governing the fiber bridging effect, and consequently the R-curve behavior in UD composite, is the fibers misalignment inside of the composite, which is dependent on the processing and manufacturing process of the composite material. A relevant experimental determination related to the connection between fiber misalignment/fiber bridging/R-curve behavior is presented in [63]: a double value of the steady state value $G_{c}^{s s}$ is recorded at an overall $2^{\circ}$ fiber misalignment inside of the UD composite, as compared to the case of well aligned fibers. By further increasing the degree of misalignment to $5^{\circ}$, a continuous increase in $G_{c}$ is recorded, without reaching a steady state value (i.e., self similar crack propagation). Another relevant experiment relative to the fiber bridging effect is presented in [67], where the $G_{c}^{s s}$ value is measured under fiber bridging effect, and then another measurements is performed when fibers in the bridging zone are cut with a sharp razor, and the measured $G_{c}$ value proves to be constant and equal to $G_{c}^{i}$ (see Fig. $4.6(\mathrm{~b}))$ 
The increase in critical ERR of the UD composite material with the crack length due to the bridging effect is referred to as an $\mathrm{R}$-curve behavior. For the phenomenological explanation that the present model relies on, this $\mathrm{R}$-curve behavior in UD composites is labeled as 1st level $R$-curve behavior.

It will be assumed and explained in the following that the $\mathrm{R}$-curve behavior in UD composites is further translated in a different $\mathrm{R}$-curve behavior in laminated composites, which is labeled as 2nd level $R$-curve in the present manuscript.

Having in mind the 1 st level $R$-curve behavior previously explained for the case of crack growth in an UD composite, let us imagine how things might work for a laminated composite (see Fig. 4.7). The loading mechanism for a laminated composite is different compared to the one encountered in experimental procedures for $G_{c}$ measurement in UD composites. The load $P^{I}$ (meaning for mode $I$ crack formation, as a study case) is applied so that the crack suddenly spans the whole width of the laminated composite specimen, for a brittle composite system. However, if we regard the moment of the first crack occurrence, there will be bridging fibers in between the two faces of the first crack. Function of some conditions, among which we can assume the elastic material properties, the thickness $t$ of the cracking ply, the crack COD, the fracture strength of the fibers, and especially the fibers misalignment inside the composite which basically governs the fibers bridging effect, there might be fractured and pulled out fibers from the very first crack occurrence in the laminate. There will be a corresponding critical ERR value for the formation of the first crack, which is noted $G_{c, 0}$ in Fig. 4.7.

While the crack density increases under loading (let's say, from state $\lambda_{i}$ to state $\lambda_{j}$ in Fig. 4.7 (a), with $\lambda_{j}>\lambda_{i}$ ), new fibers from the previous $\lambda_{i}$ bridging cracks will be broken and pulled off, while new bridging cracks corresponding to the $\lambda_{j}$ damage state are formed. The result is a cumulative fiber bridging effect at an overall scale of the laminated composites. The resistance to crack multiplication is continuously increasing due to the fact that increasing part of the stored elastic energy is released through breaking and pulling off fibers from the previously formed cracks. Probably there will be no steady state, because the bridging fibers in an existing crack will never be totally broken and the two faces of the crack will never be totally stress free, due to the expected small COD. It is expected that there is not going to be a self similar crack multiplication at the laminated composite level, and the steady state value of $G_{c}$ does not exist, but there is a continuous, probably linear increase in $G_{c}$, as shown in Fig. 4.7 (b). This model behavior is therefore labeled as 2nd level $R$-curve behavior, and 
it is specific to crack multiplication in laminated composites, as compared to the 1st level $R$-curve behavior specific to crack growth in UD composites. A $G_{c}=G_{c}(\lambda)$ variation can be modeled based on this phenomenological explanation, being thus in agreement with both the model back calculated values in Fig. 2.5, 2.6, 2.7, and with the experimentally measured values in Fig. 2.8.

The last aspect to consider is regarding the in-situ effect on the $\mathrm{R}$-curves in Fig. 2.5, 2.6, 2.7, 2 This can be explained based on the fact that the plain strain assumption in the case of traditional fracture mechanics Griffith principle (which is the plane $\alpha$ in Fig. 4.4 (a); a valid plain strain assumption can also be formulated in the case of UD composites, Fig. 4.6) is not satisfied any more for the case of laminated composites featuring a constraining effect on the COD of the cracking ply by the neighboring plies. The plane $\alpha$ in Fig. 4.8 can not be regarded as a plain strain plane any more. There is no self-similarity over the thickness of the cracking ply. There will probably be more broken and pulled off fibers in the thicker $t_{2}$ cracking ply than in the thinner $t_{1}$ ply, based on a higher COD, explaining the thickness dependence of the $\mathrm{R}$-curve behavior. Thus, this consideration allows for a modeling approach $G_{c}=G_{c}(\lambda, t)$ as schematically presented in Fig. 4.8 (b).

It is expected that both the critical ERR for the formation of the first crack in the laminate $G_{c}=G_{c, 0}$, and the slope $\beta$ of the subsequent crack evolution $G_{c}=G_{c}(\lambda)$ are function of the ply thickness $t$, as depicted in Fig. 4.8: $G_{c, 0}^{t 2} \geq G_{c, 0}^{t 1}$ and $\beta^{t 2} \geq \beta^{t 1}$ for $t_{2}>t_{1}$. Moreover, it is expected that the quantities $G_{c, 0}^{t}$ and $\beta^{t}$ have different values for different material systems. This is because the in-situ effect of the $R$-curve behavior shown in Fig. 4.8 (b) is also governed by the fiber bridging effect of the crack faces. How much energy is released for breaking and pulling off fibers in the bridging zones is function of fiber and matrix elastic and strength material properties, fiber-matrix interface properties, and the degree of fibers misalignment inside of the composite.

The in-situ $R$-curve behavior of the matrix cracking in laminated composites, phenomenologically explained previously and depicted in Fig. 4.7, 4.8, is analytically modeled in the following. It can be noticed that the in-situ $R$-curve behavior in Fig. 4.7, 4.8 features a linear characteristic, which is in agreement with the back-calculated data in Fig. 2.7 and with the experimental data in Fig. 2.8, but it is not in a very good agreement with the back-calculated data in Fig. 2.6. While the linear characteristic is implemented in the present analytical model, a higher order polynomial or an exponential variation might better describe some material systems. 

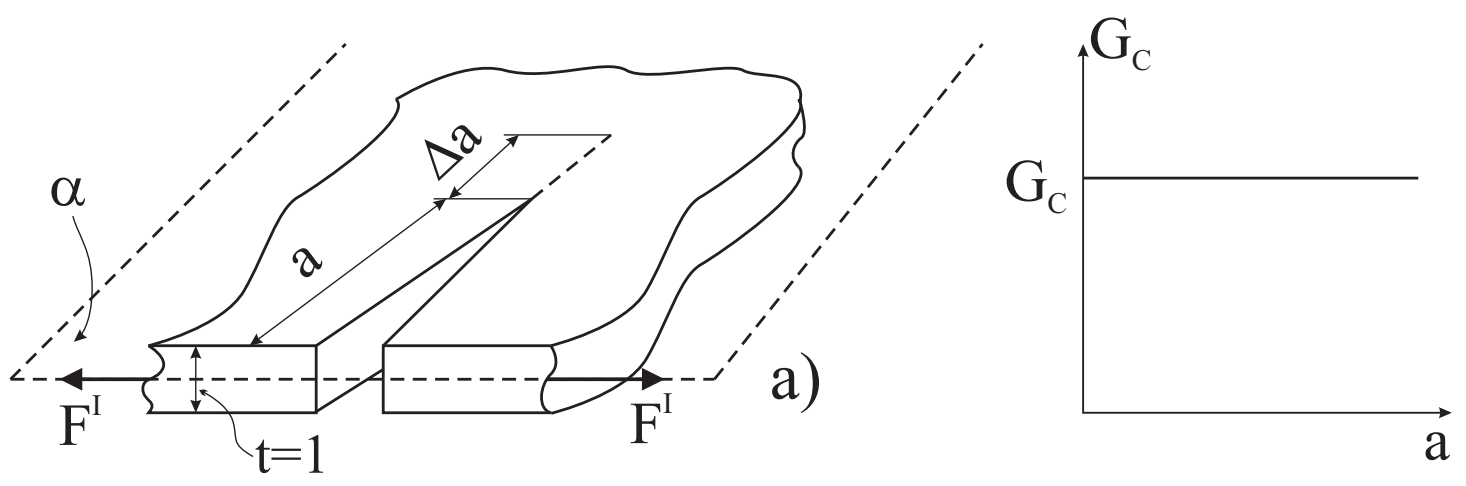

b)

Figure 4.4: a) Mode I crack extension for homogeneous material; b) Critical ERR for homogeneous material.

a) The $R$-curve effect on $G_{I c}$ at $t=t_{\text {ref }}$

A set of experimental data crack multiplication $\lambda=\lambda(\epsilon)$ or $\lambda=\lambda(\sigma)$ (similar to the schematic in Fig. 4.9), at a certain thickness $t=t_{\text {ref }}$ of the cracking ply is needed for the calibration of the $R$-curve behavior of a given composite material system. The reference thickness $t_{r e f}$ is arbitrary, and it will be further used as a base for the calibration of the in-situ behavior of the material system at thicknesses $t \neq t_{r e f}$. The needed $\lambda=\lambda(\epsilon)$ experimental data is preferable to come from a $\left[S / 90_{n}\right]_{S}$ laminate configuration under axial loading, so that only mode $I$ crack formation exists.

The $\mathrm{R}$-curve behavior $G_{I c}^{r e f}=G_{I c}(\lambda)$ at the reference thickness $t=t_{r e f}$ is linearly modeled by the equation

$$
G_{I c}\left(\lambda, t=t_{r e f}\right)=G_{I c}^{r e f}=G_{I c, 0}^{r e f}+\tan \left(\beta_{\lambda}^{r e f}\right) \cdot \lambda
$$

as shown in Fig. 4.10 (a).

The values of the critical ERR for the formation of the first crack $G_{I c, 0}^{r e f}$, and the slope angle $\beta_{\lambda}^{r e f}$ of $G_{I c}^{r e f}=G_{I c}^{r e f}(\lambda) \mathrm{R}$-curve variation at this arbitrary reference thickness $t=t_{r e f}$ are selected such that the set of experimental data $\lambda=\lambda(\epsilon)$ crack evolution in Fig. 4.9 is best fit. The critical ERR $G_{I c, 0}^{r e f}$ corresponds to matrix cracking onset (at the strain level $\epsilon_{0}^{r e f}$ in Fig. 4.9) in the reference experimental data set $\lambda=\lambda(\epsilon)$, while the angle parameter $\beta_{\lambda}^{\text {ref }}$ gives the slope of the $G_{I c}^{\text {ref }}=G_{I c}^{\text {ref }}(\lambda) \mathrm{R}$-curve in Fig. 4.10 (a), which further affects the inclination of the $\lambda=\lambda(\epsilon)$ curve for the matrix cracking evolution, as presented in Fig. 4.10 (b).

Two cases are exemplified in Fig. 4.10 :

$-\beta_{\lambda}^{r e f}=0^{\circ}$ : corresponds to no $\mathrm{R}$-curve effect during crack multiplication, which case 

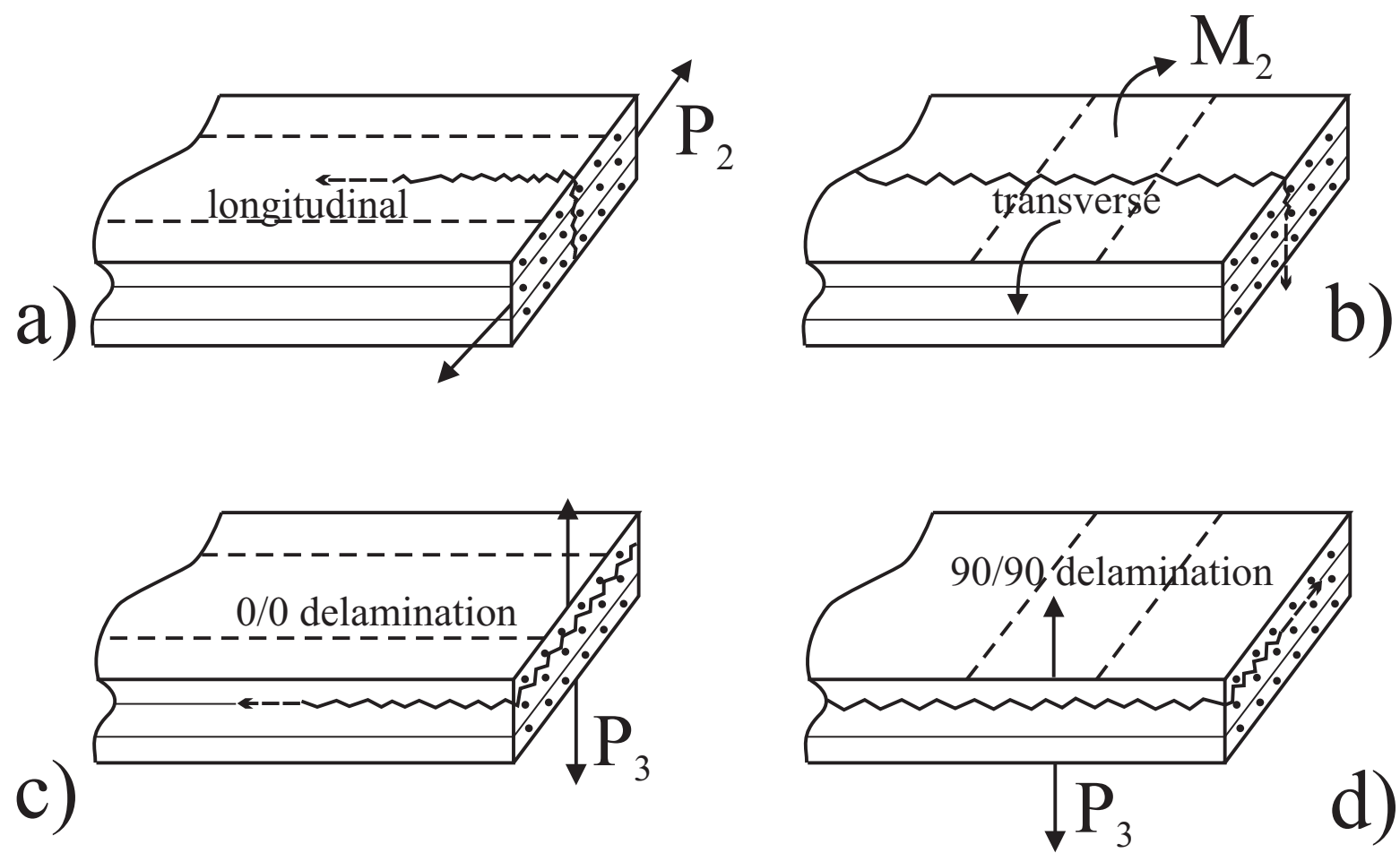

Figure 4.5: Different possible directions of mode I matrix cracking in laminated composites: a) intra-laminar, longitudinal; b) intra-laminar, transverse; c) inter-laminar 0/0; d) interlaminar 90/90.

could be regarded as no fibers misalignment, and consequently no fiber bridging effect inside of the composite material.

$-0^{\circ}<\beta_{\lambda}^{r e f}<90^{\circ}$ : corresponds to a certain degree of fibers misalignment inside of the composite, which triggers a certain level of $\mathrm{R}$-curve behavior.

The interval $\left[0^{\circ}, 90^{\circ}\right]$ mentioned above is an exaggerated one, in the sense that high values of the $\beta_{\lambda}^{\text {ref }}$ are not expected. Typical $\beta_{\lambda}^{\text {ref }}$ values are listed in Section 5.

b) The in-situ effect on $G_{I c}^{0}$ at $t \neq t_{\text {ref }}$

The in-situ effect describes the influence of the thickness of the cracking ply on the strengthening $R$-curve behavior of crack multiplication in laminated composites. Two parameters can be influenced by the thickness of the cracking ply: the critical ERR for the onset of the matrix cracking process $G_{I c, 0}$ and the slope $\beta$ of the $G_{c}=G_{c}(\lambda)$ variation, as presented in Fig. 4.8. 

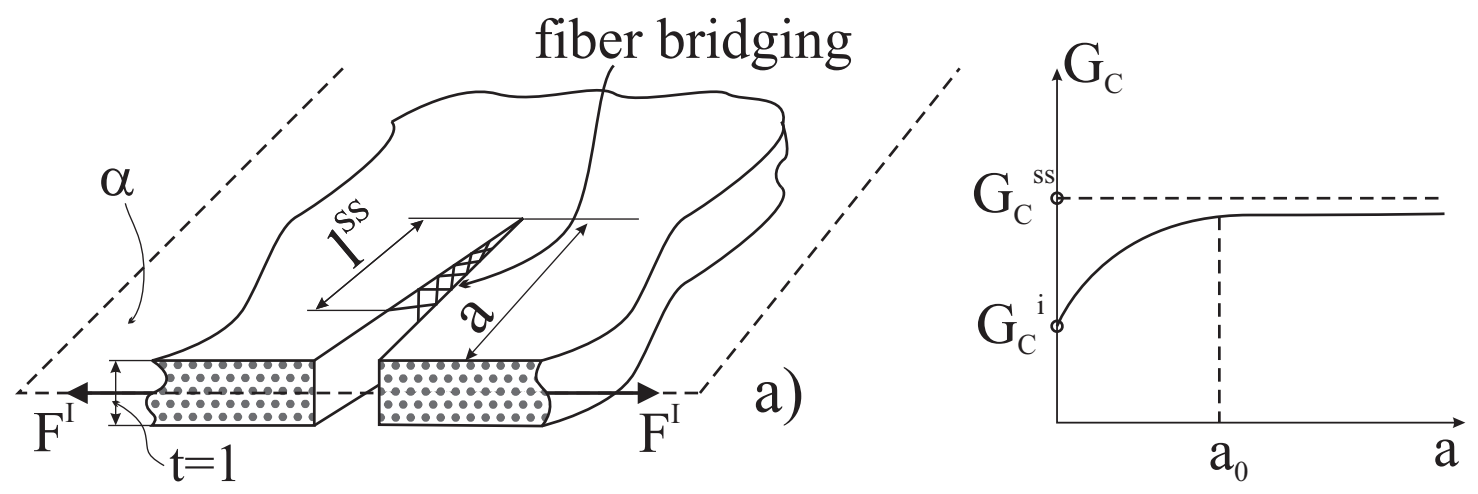

b)

Figure 4.6: a) Mode I crack extension for UD fiber-reinforced composite; b) Critical ERR for UD fiber-reinforced composite: $\mathrm{R}$-curve behavior.
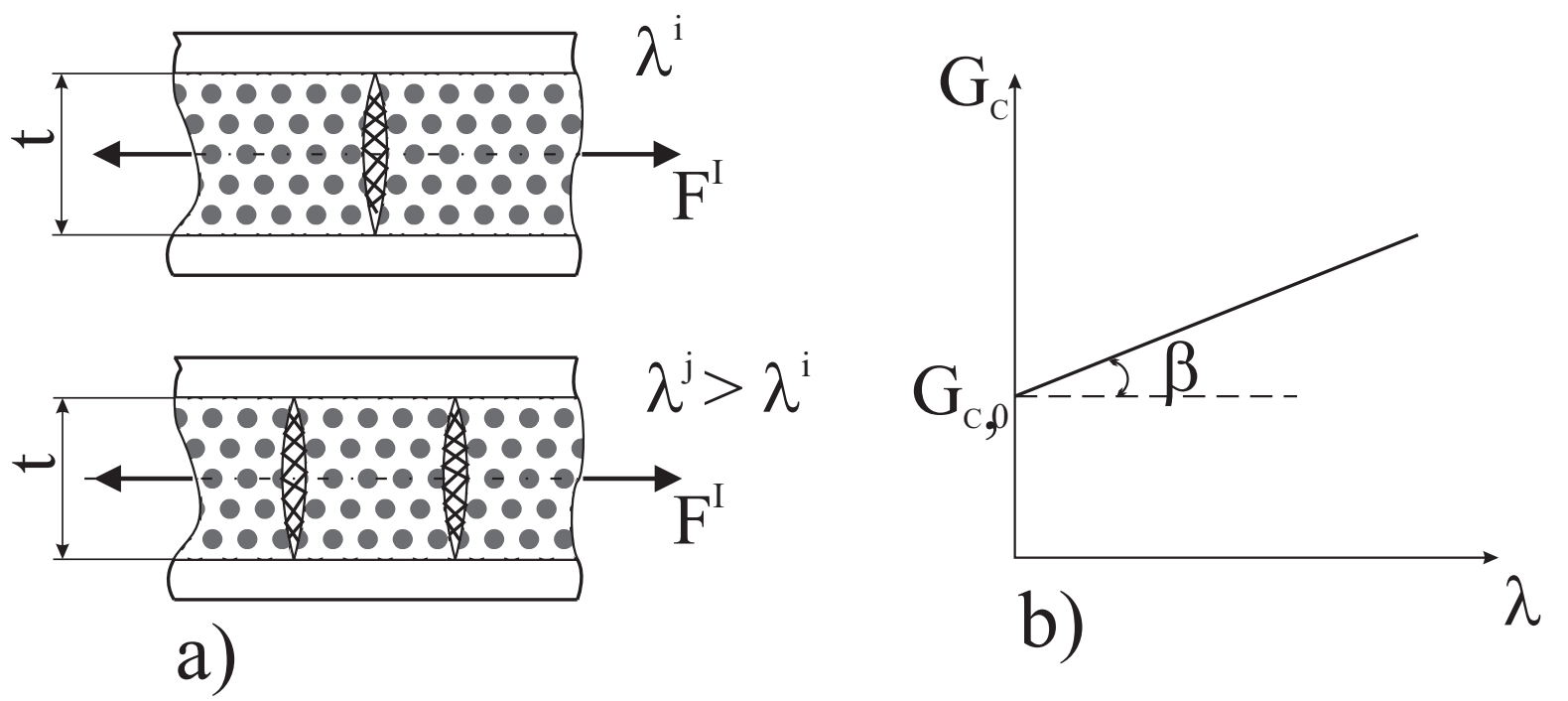

Figure 4.7: a) Mode I crack extension for laminated composite; b) Critical ERR for laminated composite: R-curve behavior.

The ply thickness coefficient $k$ is defined as

$$
k=k(t)=\frac{t}{t_{r e f}} \quad\left\{\begin{array}{lll}
>1 & \text { if } t>t_{r e f} \\
=1 & \text { if } t=t_{r e f} \\
<1 & \text { if } t<t_{r e f}
\end{array}\right.
$$

The thickness effect on the onset value of the critical ERR $G_{I c, 0}$ can be described in a linear manner by the following equation:

$$
G_{I c, 0}(t)=G_{I c, 0}^{r e f} \cdot\left[1+(k(t)-1)\left(\beta_{0}^{t}-1\right)\right]
$$

It can be noticed that for $t \neq t_{\text {ref }}$, the in-situ thickness effect on the onset value $G_{I c}^{0}$ is established by the fit parameter $\beta_{0}^{t}$, while for $t=t_{r e f}$ the equality $G_{I c, 0}=G_{I c, 0}^{r e f}$ is assured 


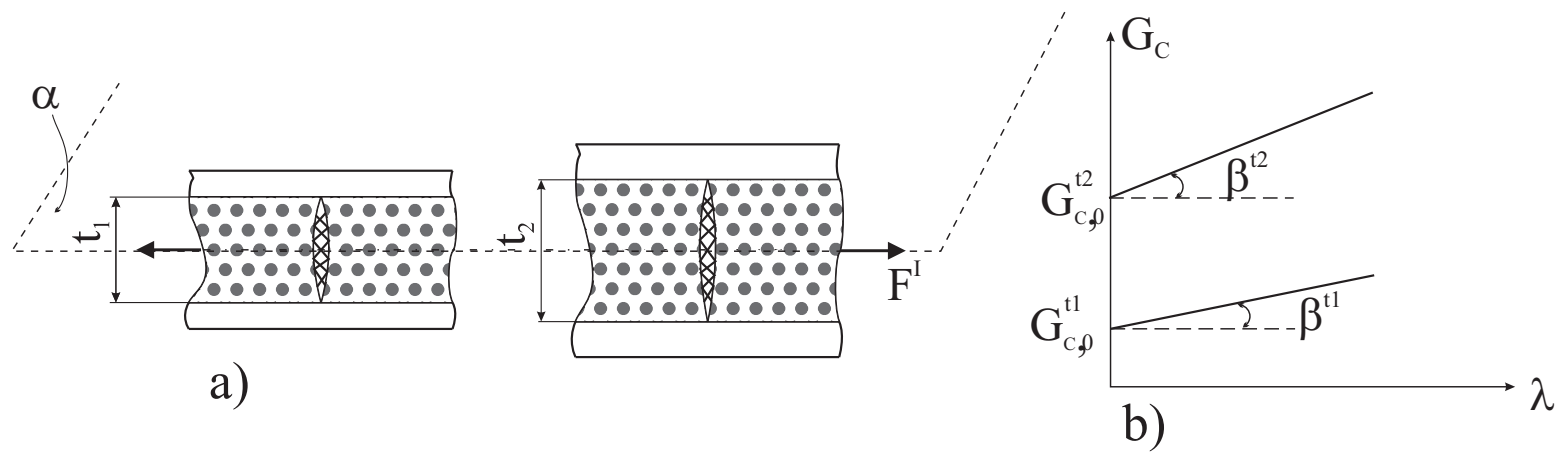

Figure 4.8: a) Mode I crack formation for cracking plies of different thickness; b) Critical ERR for cracking plies of different thickness: in-situ effect on the $\mathrm{R}$-curve behavior.

by eq. (4.27). The $\beta_{0}^{t}$ parameter is assumed to be bounded in the interval $1 \leq \beta_{0}^{t} \leq 2$. The linear influence of the ply thickness $t$ on the onset values of critical ERR $G_{I c, 0}$, for different values of $\beta_{0}^{t}$ parameter, is presented in Fig. 4.11 (a):

- for the bounding value $\beta_{0}^{t}=1: G_{I c, 0}(t)=G_{I c, 0}^{r e f}=$ ct., regardless the thickness $t$ of the cracking ply; there is no in-situ thickness influence on the critical ERR for damage onset $G_{I c, 0}$.

- for the bounding value $\beta_{0}^{t}=2: G_{I c, 0}(t)=k(t) \cdot G_{I c, 0}^{r e f}=\frac{t}{t_{r e f}} G_{I c, 0}^{r e f}$; there is a direct proportionality between the value of the critical ERR for damage onset $G_{I c, 0}$ at $t \neq t_{r e f}$ and the value of the critical ERR for damage onset $G_{I c, 0}^{r e f}$ at $t=t_{r e f}$; the proportionality factor is the thickness factor $k(t)=\frac{t}{t_{r e f}}$ itself.

- for intermediate values $1 \leq \beta_{0}^{t} \leq 2$ : the proportionality factor between the value of the critical ERR for damage onset $G_{I c, 0}$ at $t \neq t_{r e f}$ and the value of the critical ERR for damage onset $G_{I c, 0}^{r e f}$ at $t=t_{\text {ref }}$ becomes $\left[1+(k(t)-1)\left(\beta_{0}^{t}-1\right)\right]$, which is affected by the $\beta_{0}^{t}$ value.

The in-situ effect of the thickness of the cracking ply on the critical ERR for damage onset $G_{I c, 0}$ is presented in $\left(G_{c}-\lambda\right)$ coordinates in Fig. 4.11 (b) and in $(\lambda-\epsilon)$ coordinates in Fig. 4.11 (c). As it can be noticed from Fig. 4.11 (c), the influence of the $\beta_{0}^{t}$ parameter is on the onset value $\epsilon_{0}$ of matrix cracking for thickness of the cracking ply $t \neq t_{r e f}$.

It has to be noted that, even if the equality $G_{I c, 0}(t)=G_{I c, 0}^{r e f}$ is assured for $\beta_{0}^{t}=1$ in Fig. 4.11 (a), (b), this equality does not imply that $\epsilon_{0}=\epsilon_{0}^{r e f}$ in Fig. 4.11 (c), because the onset of matrix cracking is influenced not only by the critical ERR for onset $G_{I c, 0}$, but it is also influenced by the laminate configuration.

One extra experimental determination of the moment of damage onset $\left(\epsilon_{0}\right.$ in Fig. 4.9), at an arbitrary thickness of the cracking ply $t \neq t_{r e f}$ is needed in order to evaluate the fit 
parameter $\beta_{0}^{t}$.

c) The in-situ and $R$-curve effect on $G_{\text {Ic }}$ at $t \neq t_{\text {ref }}$

This effect regards the in-situ influence of the ply thickness on the slope of the $G_{I c}=$ $G_{I c}(\lambda) \mathrm{R}$-curve variation at $t \neq t_{r e f}$, and it is described by the equation

$$
G_{I c}(\lambda, t)=G_{I c, 0}(t)+\left[1+(k(t)-1)\left(\beta_{\lambda}^{t}-1\right)\right] \cdot \tan \left(\beta_{\lambda}^{r e f}\right) \cdot \lambda
$$

where the in-situ critical ERR for damage onset $G_{I c, 0}(t)$ is given by eq. (4.27), and $\beta_{\lambda}^{\text {ref }}$ is the slope angle that describes the $\mathrm{R}$-curve behavior at $t=t_{r e f}$, as it appears in eq. (4.25) and Fig. 4.10.

It can be observed that eq. (4.28) reduces to eq. (4.25) if the thickness factor $k(t)=1$, i.e., $t=t_{r e f}$. Fort he case of $t \neq t_{r e f}$, the slope of the $G_{I c}=G_{I c}(\lambda, t) \mathrm{R}$-curve variation in eq. (4.28) is set by the fit parameter $\beta_{\lambda}^{t}$, which is considered bounded by the limit values $1 \leq \beta_{\lambda}^{t} \leq 2$. The influence of the $\beta_{\lambda}^{t}$ parameter on the in-situ $\mathrm{R}$-curve behavior of the $G_{I c}(\lambda, t)$ variation is pictured in Fig. $4.12(\mathrm{a})$ :

- for the bounding value $\beta_{\lambda}^{t}=1$ : the slope angle $\beta_{\lambda}$ of the $G_{I c}(\lambda, t)$ variation at $t \neq t_{r e f}$ remains equal to the slope $\beta_{\lambda}^{r e f}$ of the $G_{I c}\left(\lambda, t=t_{r e f}\right)$ given by eq. (4.25); there is no in-situ thickness effect on the $G_{I c}(\lambda) \mathrm{R}$-curve behavior;

- for the bounding value $\beta_{\lambda}^{t}=2$ : there is a direct proportionality between the slope value $\beta_{\lambda}$ of the $G_{I c}(\lambda, t)$ variation at $t \neq t_{r e f}$ and the slope $\beta_{\lambda}^{r e f}$ of $G_{I c}\left(\lambda, t=t_{r e f}\right)$ variation; the proportionality factor is the thickness factor $k(t)=\frac{t}{t_{r e f}}$ itself.

- for intermediate values $1 \leq \beta_{\lambda}^{t} \leq 2$ : the proportionality factor between the slope value of the $G_{I c}(\lambda, t)$ variation at $t \neq t_{r e f}$ and the slope of $G_{I c}\left(\lambda, t=t_{r e f}\right)$ variation becomes $\left[1+(k(t)-1)\left(\beta_{\lambda}^{t}-1\right)\right]$, which is affected by the $\beta_{\lambda}^{t}$ value.

The influence of the $\beta_{\lambda}^{t}$ parameter on the $\lambda-\epsilon$ crack evolution is presented in Fig. 4.12 (b). This influence is on the inclination of the $\lambda-\epsilon$ crack evolution curve, after the moment of onset $\epsilon_{0}$ has been set by eq. (4.27) (see Fig. 4.11).

A whole extra experimental curve $\lambda=\lambda(\epsilon)$ at a cracking ply thickness $t \neq t_{\text {ref }}$ is needed in order to evaluate the fit parameter $\beta_{\lambda}^{t}$.

Some final observations on the above implementation of the in-situ $\mathrm{R}$-curve behavior of crack multiplication in laminated composites:

It is interesting to notice that the critical value of ERR for matrix cracking onset, $G_{I c, 0}^{r e f}$, is not an unique material property (as usually the value $G_{I c}$ from fracture mechanics experiments on UD composites is), but the $G_{I c, 0}^{r e f}$ depends on the thickness of the cracking ply in 
that laminate which is selected as the reference laminate (i.e., the laminate that provides the experimental data in Fig. 4.9). If a different laminate configuration is selected as reference, a different $G_{I c, 0}^{r e f}$ is obtained. This might be surprising at first, but it is normal considering that an in-situ (thickness dependent) effect is regarded by the $G_{I c, 0}^{r e f}$ value, so different values are expected for different cracking ply thicknesses.

If the fit parameters $\beta_{\lambda}^{\text {ref }}, \beta_{0}^{t}, \beta_{\lambda}^{t}$ in eq, (4.25), (4.27), (4.28) are set to the values $\beta_{\lambda}^{\text {ref }}=$ $0^{\circ}, \beta_{0}^{t}=1, \beta_{\lambda}^{t}=1$, then a model with no $\mathrm{R}$-curve behavior and no in-situ influence is obtained.

All the above modeling approach regards only the mode $I$ critical ERR, $G_{I c}$. While a similar extension to the mode $I I$ critical ERR value $G_{I I c}$ is trivial, there is no experimental data based on which to assume that the fiber bridging effect has a similar influence for mode $I I$ crack propagation as it has for mode $I$. Because of this, the in-situ $\mathrm{R}$-curve behavior for $G_{I I c}$ is not implemented in the present model, and there is an unique $G_{I I c}$ value for damage onset and for damage growth as well (no R-curve behavior), regardless the thickness of the cracking ply (no in-situ effect). Additional experimental investigation and research is needed in order to decide if this is an oversimplification or it is the real mode $I I$ material behavior. Under these conditions and assumptions, the mixed modes crack multiplication criterion in eq. (4.4) turns into

$$
\begin{aligned}
& g(\lambda, t)=(1-r(\lambda, t)) \sqrt{\frac{G_{I}}{G_{I c}(\lambda, t)}}+r(\lambda, t) \frac{G_{I}}{G_{I c}(\lambda, t)}+\frac{G_{I I}}{G_{I I c}}-1 \geq 0 \\
& r(\lambda, t)=\frac{G_{I c}(\lambda, t)}{G_{I I c}}
\end{aligned}
$$

where is has to be remembered that the energy release rates (not critical ones!) $G_{I}, G_{I I}$ are functions of the damage level $\lambda$ and laminate configuration $t$, as well. 


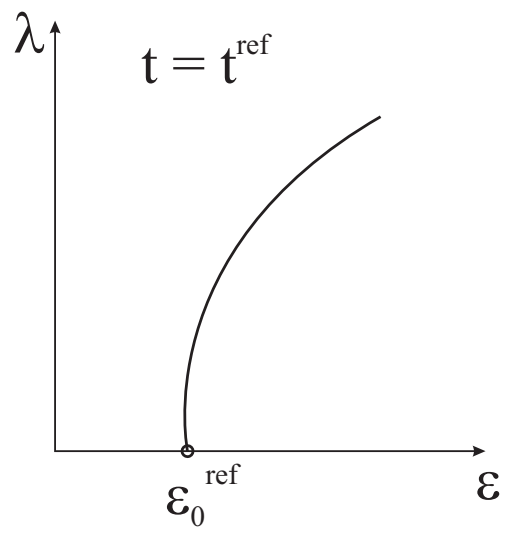

Figure 4.9: Typical matrix cracking multiplication data in laminated composites.
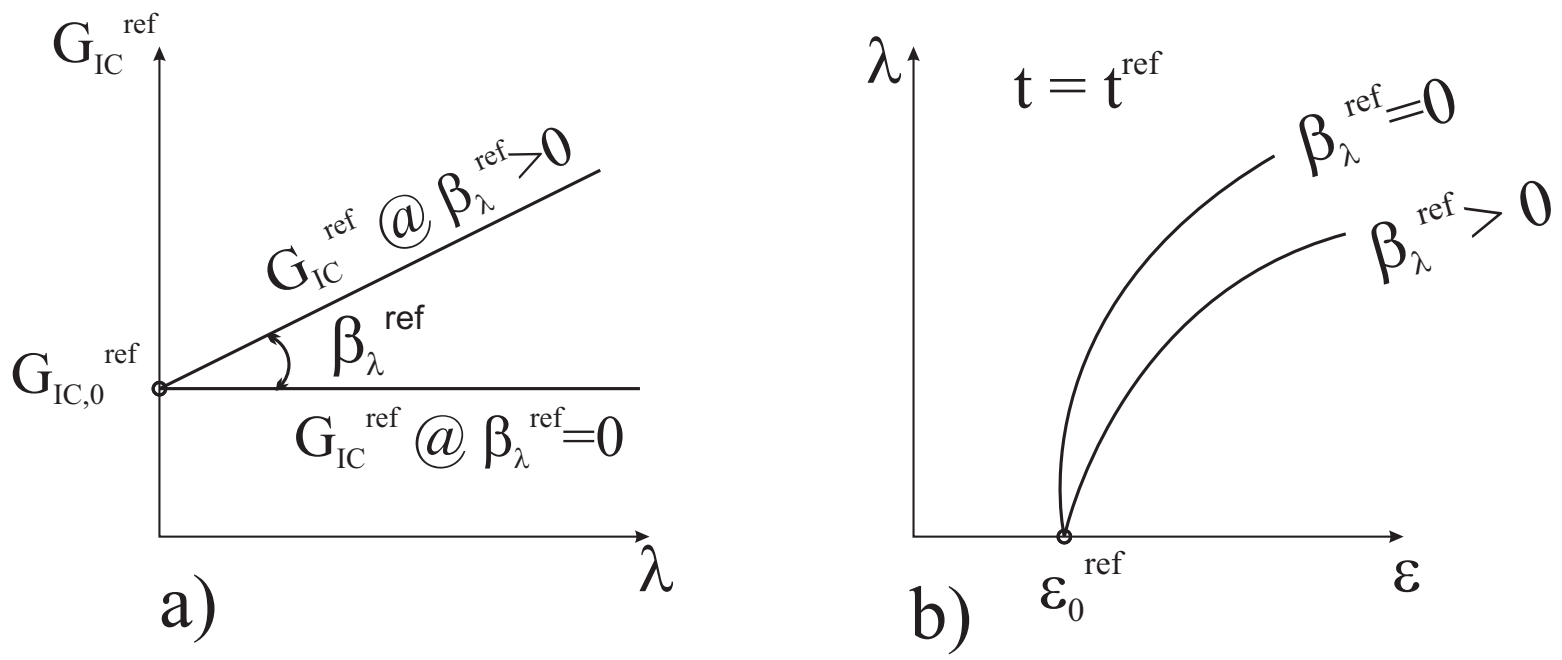

Figure 4.10: a) Model of $\mathrm{R}$-curve behavior $G_{I c}=G_{I c}(\lambda)$ at $t=t_{r e f}$; b) The effect of $\mathrm{R}$-curve behavior on damage evolution $\lambda=\lambda(\epsilon)$ at $t=t_{\text {ref }}$. 


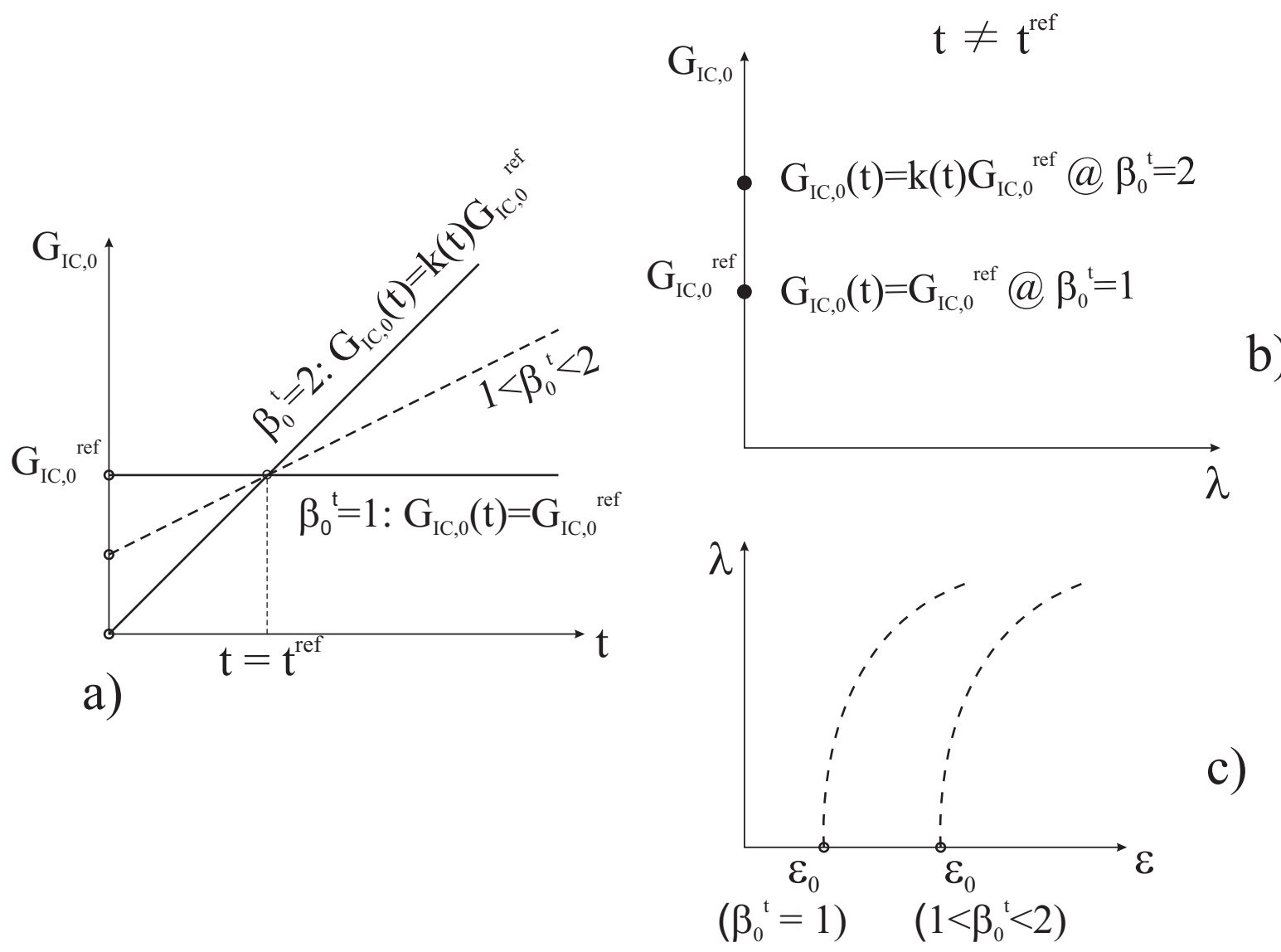

Figure 4.11: a) Model of in-situ effect on the critical ERR for damage onset $\left(t \neq t_{r e f}\right)$; b) In-situ effect on the critical ERR for damage onset in $\left(G_{I c, 0}-\lambda\right)$ coordinates; c) In-situ effect on the critical ERR for damage onset in $(\lambda-\epsilon)$ coordinates.
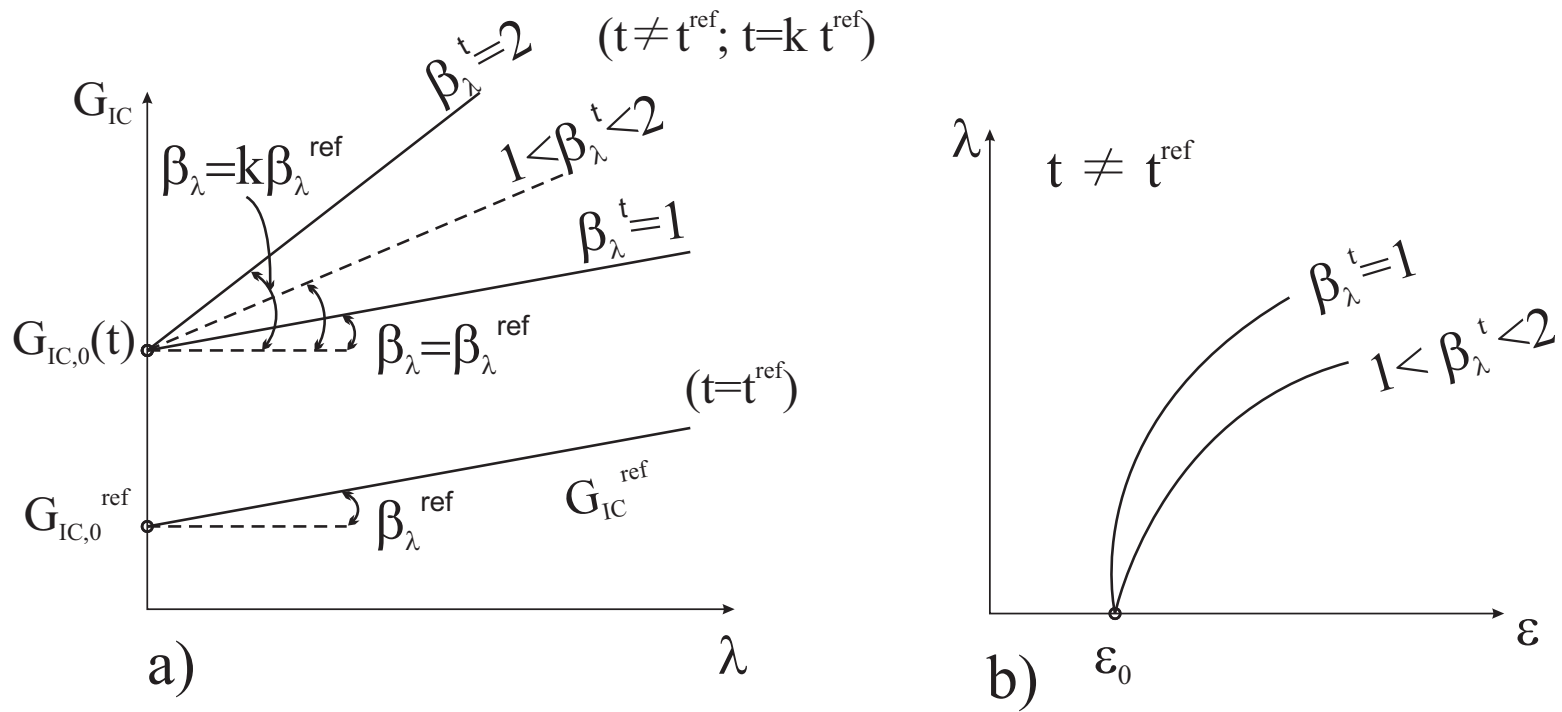

Figure 4.12: a) Model of in-situ R-curve behavior; b) The effect of in-situ R-curve behavior on damage evolution data. 


\subsection{Classical Laminate Theory}

Classical Laminate Theory is used here with a double scope. First, it provides the overall load-deformation behavior of the laminate. Second, it is used to derive the strain energy stored upon loading, including the effect of thermal residual stresses which are inherent in laminated composites. The stated double scope can be accomplished if:

a) the effective laminate thermo-elastic properties (reduced by the presence of matrix cracking in individual plies) are known, as they are offered by the material model in Section 4.1, and

b) the individual effective ply thermo-elastic properties (reduced by the presence of matrix cracking) are known, as they are offered by the iterative procedure in Section 4.3.

Having known the aforementioned laminate and ply effective material properties, the CLT provides the well-known load-deformation relationship for the laminate [56]

$$
\{\epsilon\}^{t o t}=\left\{\begin{array}{l}
\epsilon^{0} \\
\kappa
\end{array}\right\}^{t o t}=[A B D]^{-1} \cdot\left\{\begin{array}{l}
N \\
M
\end{array}\right\}+\left\{\begin{array}{l}
\alpha_{E} \\
\alpha_{B}
\end{array}\right\} \cdot \Delta T
$$

where $\left\{\epsilon^{0}, \kappa\right\}^{\text {tot }}$ is the overall laminate deformation, due to both mechanical loading $\{N, M\}$ applied on laminate and laminate thermal deformation (given by the temperature difference $\Delta T$ between the processing temperature and the service temperature); $[A B D]$ is the generalized (membrane and flexural) stiffness matrix of the laminate; $\left\{\alpha_{E}, \alpha_{B}\right\}$ is the generalized (membrane and flexural) vector of thermal expansion coefficients of the laminate.

Equation (4.30) represents the constitutive relationship of the laminate for the case of both mechanical and thermal loading. A detailed derivation of the whole set of equations of CLT (including the effect of temperature difference $\Delta T$ ) is provided in Appendix A.

\subsection{Analytical model formulation}

The way how the previous described components are connected and work together is described in the following, and it is schematically presented in Fig. 4.13. The simplified notations $\{F\}_{(6 \times 1)},\{\epsilon\}_{(6 \times 1)},[C]_{(6 \times 6)},\{\alpha\}_{(6 \times 1)}$ in Fig. 4.13 refer to the constitutive equation

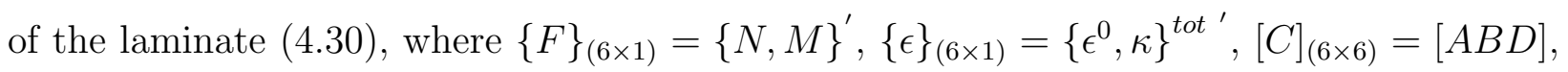
and $\{\alpha\}_{(6 \times 1)}=\left\{\alpha_{E}, \alpha_{B}\right\}^{\prime}$ in eq. (4.30) (the superscript \{\}$^{\prime}$ denotes the transpose of a vector in the present notation). 


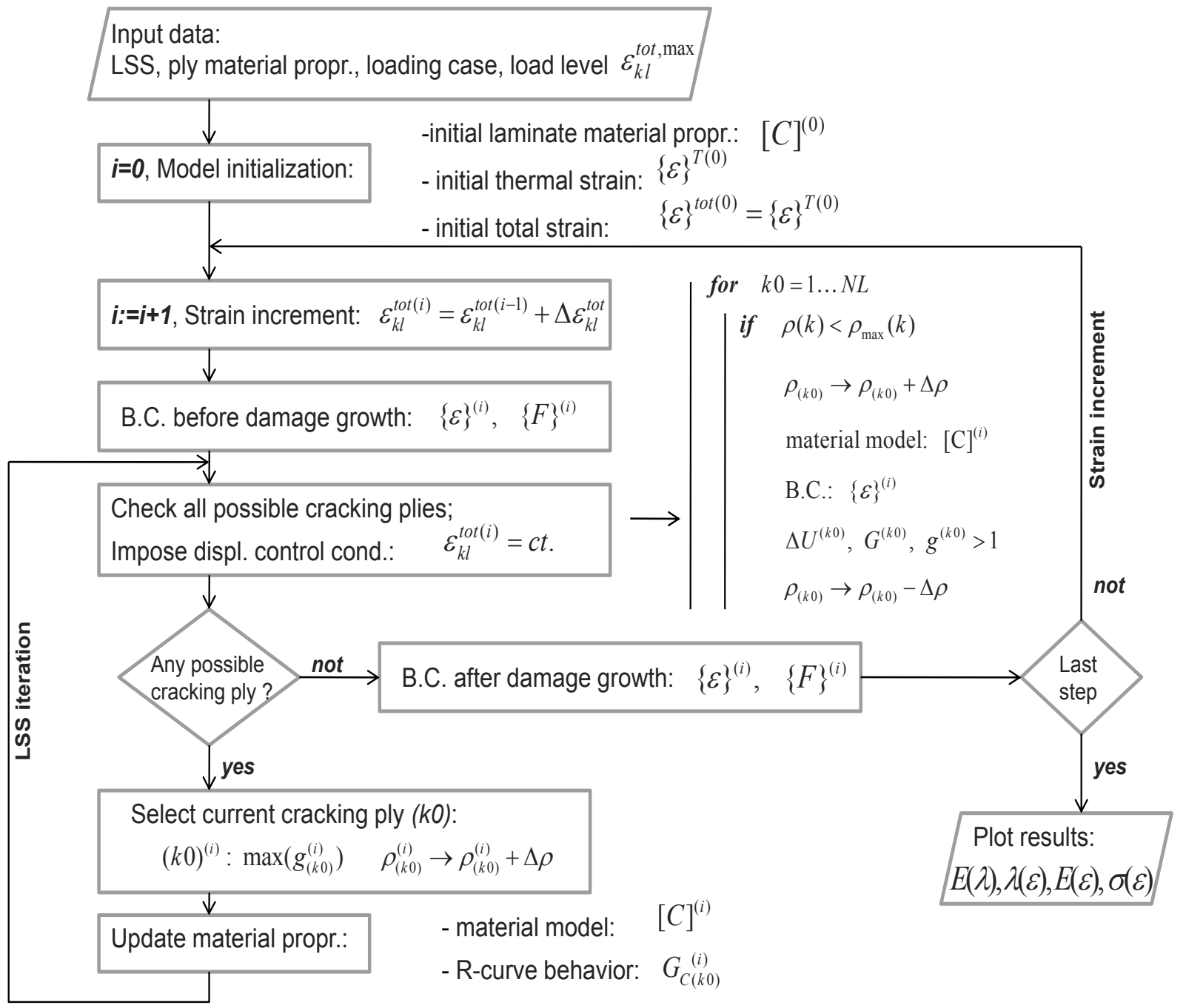

Figure 4.13: The flow-chart of the membrane-flexural model for composited laminates subjected to damage in form of matrix cracking.

The present analytical formulation is meant to be a progressive damage model for matrix cracking in general laminated composites under in-plane (membrane) and out-plane (flexural) deformation. The model formulation and implementation will be compared against experimental results in Section 5. However, all the experimental measurements are performed under either displacement or load control. Actually, most of the available experimental data in the literature and all of the experimental data used for model validation in Section 5 is under displacement control case. This is the reason why the algorithmic implementation of the present model is for the case of displacement control loading of the laminate. Similar implementation can be easily done for the case of load control loading of the laminate. For 
more details on displacement control vs. load control implementation, see Section 4.7.1.

In order to be consistent with the displacement control case, the loading of the laminate is made by strain increments $\left\{\epsilon^{i}\right\}$; more precisely by increments $\epsilon_{k l}^{i}$ of that component of the strain vector $\{\epsilon\}$ that corresponds to the loading case considered: for example, $\epsilon_{x}^{i}$ strain increments will be applied for the uniaxial extension along $x$ direction of the laminate; $\epsilon_{y}^{i}$ strain increments will be applied for the uniaxial extension along $y$ direction of the laminate; $\kappa_{x}^{i}$ curvature increments will be applied for the uniaxial bending along $x$ direction, s.o. The other strain components of the vector $\left\{\epsilon^{i}\right\}$ will result according to the boundary conditions specific to each loading case, see Section 4.7.1. The strain increments $\left\{\epsilon^{i}\right\}$ can be regarded as being provided by the loading machine, according to each loading case desired, as it is described in Section 4.7.1. There is an initial deformation state on top of each the "loading machine" strain increments $\left\{\epsilon^{i}\right\}$ are applied: this is the initial laminate deformation due to thermal difference $\Delta T$.

The algorithmic procedure for progressive matrix cracking in laminated composites is described by the following steps:

- at each load step $(i)$, which for the displacement control case is the laminate strain increment $\left\{\epsilon^{i}\right\}$, the state of stress/strain in individual plies of the laminate can be evaluated based on material thermo-elastic properties of individual plies $Q_{(k)}^{i-1}, \alpha_{(k)}^{i-1}$ corresponding to the previous load step $(i-1)$, and implicitly corresponding to the damage state $\lambda_{(k)}^{i-1}$ for each ply $(k)$, at the previous load step $(i-1)$. The elastic strain energy in the laminate is then calculated as presented in Appendix A. This elastic strain energy is labeled as $U^{\text {before }}$, which means the stored strain energy before a crack multiplication event at load step $(i)$.

- next, the iterative procedure (labeled as LSS iteration in Fig. 4.13) comes into picture and checks for the possibility of crack multiplication in each ply $(k)$ of the laminate: each ply $(k)$ is given in turn the role of the possible cracking ply $(k 0)$, and the damage level in the current $(k 0)$ ply $\lambda_{(k 0)}^{i}$ is increased by a finite increment $\Delta \lambda_{(k 0)}^{i}$ corresponding to one more $\operatorname{crack}\left(\right.$ see Section 4.4.2): $\lambda_{(k 0)}^{i}:=\lambda_{(k 0)}^{i}+\Delta \lambda_{(k 0)}^{i}$. Based on this new damage level in the $(k 0)$ ply, the reduced material properties of the laminate $C^{i}, \alpha^{i}$ are updated by the material model (see Section 4.1), and the new reduced material properties of the cracking ply $(k 0)$ can be evaluated by eq. (4.3). Based on these updated material properties, and based on imposing displacement control condition on the $\epsilon_{k l}^{i}$ strain component corresponding to the considered loading case (see Section 4.7.1), the new stresses in individual plies of the laminate and the new strain energy in the laminate $U^{\text {after }}$ can be calculated, which is the elastic strain energy 
after a crack multiplication event.

- then, the energy released rate for crack multiplication in ply $(k 0)$ at the load step $(i)$ is calculated according to eq. (4.7): $G_{(k 0)}^{i}=-\Delta U / \Delta A$, where $\Delta U=U^{\text {after }}-U^{\text {before }}$ is the release in elastic strain energy due to crack growth, and $\Delta A$ is the new formed crack area corresponding to 1 more crack inside of the cracking ply $(k 0)$, i.e., satisfying eq.(4.22). The elastic strain energy released due to damage growth is calculated as corresponding to both modes $I$ and $I I$ crack formation (see Section A.2), and the crack growth criterion in eq. (4.4) can now be evaluated. If the condition in eq. (4.4) is met, it means that the ply $(k 0)$ really is a possible cracking ply at the load increment $(i)$. If the condition in eq. (4.4) is not met, it means that the ply $(k 0)$ is not possible to undergo crack multiplication at the load increment $(i)$, and the damage level is set back to the initial crack density $\lambda_{(k 0)}^{i}:=\lambda_{(k 0)}^{i}-\Delta \lambda_{(k 0)}^{i}$.

- it is possible that, according to the laminate configuration and the increment size of the laminate loading $\left\{\epsilon^{i}\right\}$, more than one ply of the laminate is detected as a possible cracking ply (i.e., the condition in eq. (4.4) is met) during the LSS iteration. In that case, the cracking ply at a moment is selected as the ply with the maximum ERR, which is the maximum damage coefficient $g_{(k 0)}^{i}$ in eq. (4.4).

- the iterative procedure (LSS iteration) is repeated until there is no more cracking ply at the loading level $(i)$. It is possible that many cracking plies or repeated cracking events in the same ply are detected at the same load level $(i)$.

- the new decreased force load $F^{i}$ required on the laminate, due to reducing the laminate properties $C^{i}, \alpha^{i}$ is calculated based on the constitutive equation (4.30) and the specific boundary conditions of the loading case (see Section 4.7.1). The load drop during crack multiplication is specific to the displacement control case, when a certain component $\epsilon_{k l}^{i}$ of the laminate deformation $\left\{\epsilon^{i}\right\}$ is kept constant during crack multiplication.

- the calculation at the load increment $(i)$ is done; all the new global quantities $\epsilon^{i}, F^{i}, C^{i}, \alpha^{i}$ corresponding to the laminate, as well local quantities $Q_{(k)}^{i}, \alpha_{(k)}^{i}, \lambda_{(k)}^{i}$ corresponding to the individual ply $(k)$ are known and updated as for the load increment $(i)$

- the next load increment $(i+1)$, which for the displacement control case is the laminate strain increment $\left\{\epsilon^{i+1}\right\}$, can be applied.

This organization of the model allows for a good control of the model out-put. For example, the model is able to select in between two plies with high but close orientation angle, as it is the case of a 90 ply vs. an 85 ply, at the same level of loading. The most susceptible to damage evolution (the 90 ply) is treated first, and if this meets the condition 
for damage growth, the corresponding amount of strain energy is released; this fact might mean that the remaining stored strain energy is no longer enough to trigger a damage growth in the 85 ply.

The increase in crack density for a given ply is allowed until the so called Characteristic Damage State $(C D S)$ is reached (according to experimental observations, the crack density is saturated when the crack spacing is approximately equal to the ply thickness, $d^{(k)}=$ $\left.t^{(k)}, \rho^{(k)}=1\right)$.

Progressive damage in form of matrix cracking in laminated composites is assessed by following the above described procedure. The output of the analytical model is:

- the curve of damage multiplication under external loading for each ply $(k)$ of the laminate: $\lambda=\lambda(\epsilon)$;

- the curve of reduction in thermo-elastic properties of the laminated composite under external loading: $E=E(\epsilon)$ (where $E$ is a generic notation for any material property);

- the curve of reduced material properties of the laminate as a function of damage level in any ply $(k): E=E(\lambda)$;

- the nonlinear (due to damage evolution) constitutive stress-strain behavior of the material: $\sigma=\sigma(\epsilon)$.

Model output for various laminate configurations, and validation against experimental data, are presented in Section 5.

\subsection{Additional comments on some modeling aspects}

\subsubsection{Displacement vs. Load control case}

Most of the available crack multiplication experimental data in the literature are provided for the case of displacement control experiments: known displacement (stretch) is progressively applied on the composite specimen until the loading level of crack multiplication is reached. The stretch of the specimen is kept constant during crack multiplication. Due to crack multiplication the material stiffness properties are reduced, this implying a reduction of the force load while the specimen displacement is kept constant. This further implies a reduction in the stored strain energy in the specimen $(\Delta U<0)$ during crack multiplication, and the ERR due to new crack formation under displacement control is calculated as $G=-\Delta U / \Delta A$ (see eq. (4.7)). 
A similar process takes place if the experiment was conducted under load control case: known force load is progressively applied on the composite specimen until the loading level of crack multiplication is reached. The force load on the specimen is kept constant during crack multiplication. Due to crack multiplication the material stiffness properties are reduced, this implying an increase in the specimen deformation while the force load is kept constant. This further implies an increase in the stored strain energy in the specimen $(\Delta U>0)$ during crack multiplication, and the ERR due to new crack formation under load control is calculated as $G=\Delta U / \Delta A$ (see eq. (4.8)).

Due to the fact that most of the available experimental data is under displacement control case, the analytical implementation in the present model is also for the case of displacement control. It has to be mentioned that the crack multiplication experiments usually reproduce the behavior of one material point: the composite specimen is a rectangular one without stress concentrators, and under the homogenization assumption there is an uniform displacement/strain/stress field at any point of the specimen. While the displacement control implementation of the analytical model is proper in order to reproduce this kind of uniform field experiments and compare analytical model output against experimental results, this would not be the case for comparing analytical results against non-uniform field experiments due to stress concentrators. For example, let us consider the case of an experiment specimen with a central hole. In this case the specimen itself is not any longer representative for any material point inside of the specimen, and we have to distinguish between the structural (specimen) level and material point level. Even if the structure (the specimen) is under known displacement control (the case of structural load control is similar), we can not say that a particular material point is under constant deformation during crack multiplication, but it can be rather considered under constant load. The load/deformation at structural (specimen) level and the load/deformation at material point level are now different, there is a non-uniform field of the load/deformation of material points inside of the specimen due to the stress concentrator that is the central hole. In this case, a classical Newton-Raphson incremental-iterative procedure is required at structural level, due to material nonlinearity generated by the crack multiplication process.

However, as long as the main goal of the present manuscript is to asses the predictive capabilities of the analytical model by comparison against available experimental crack multiplication data, the displacement control case is implemented as described in Section 4.6, and the Newton-Raphson algorithm is not needed in this case. 
If we further extend the comparison displacement vs. load control case, we can infer that an experimental data set under displacement control would be different compared to an experimental data set under load control. This is based on the fact that the strain energy variation under displacement control $\left(\Delta U_{d c}\right.$ in Fig. 4.14, the area of the oab triangle) is different from the strain energy variation under load control ( $\Delta U_{l c}$ in Fig. 4.14, the area of the oac triangle), during the crack multiplication process.

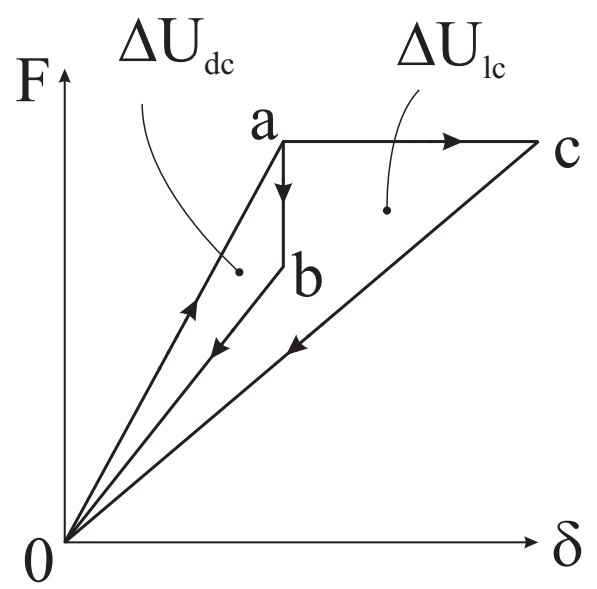

Figure 4.14: Elastic strain energy released in the case of Displacement control vs. Load control.

Moreover, the stress redistribution process, and consequently the crack growth mechanism inside of the composite laminate is different for the two cases:

- under displacement control: the crack multiplication is initiated in the cracking ply $(k 0)$, which first meets the conditions in eq. (4.4). Due to crack growth in the ply $(k 0)$ the stiffness of the laminate is reduced, and the stiffness $[Q]_{(k 0)}$ of the cracking ply is accordingly reduced. The stiffness $[Q]_{(k)}$ of the other $(k)$ plies is not affected by the crack growth in ply $(k 0)$, and due to the fact that the deformation level on the laminate is kept constant during crack multiplication (displacement control), the stresses $\sigma_{k}$ in the non-cracking plies $(k)$ will be constant during crack multiplication under displacement control.

- under load control: the crack multiplication is similarly initiated in the cracking ply $(k 0)$, which has its stiffness $\left[Q_{k 0}\right]$ reduced, while the stiffness $\left[Q_{k}\right]$ of the other $(k)$ plies is not changed. However, due to constant load level on the laminate during crack multiplication (load control), the laminate deformation is increased, and consequently the deformation of the $(k)$ plies is increased, which implies an increase in the stresses $\sigma_{k}$ in the non-cracking plies $(k)$ (stress redistribution) during crack multiplication under load control. 
Another aspect considered for the implementation of the displacement control case in the present model is the contribution of both the mechanical $\left(\epsilon^{M}\right)$ and thermal $\left(\epsilon^{T}\right)$ strain components to the total strain: $\epsilon^{t o t}=\epsilon^{M}+\epsilon^{T}$, as presented in Appendix A. If we consider, as an example, the most common case of uniaxial loading under displacement control, then the condition $\epsilon_{x}=$ ct. has to be imposed in the moment of crack multiplication. A similar condition on curvature, $\kappa_{x}=$ ct., has to be imposed in order to simulate bending experiments under curvature control, as it is the case in [36]. The question is on what component (mechanical, thermal, total) of the deformation the displacement control condition has to be applied. While this question does not arise in the case of neglecting the thermal influence $\Delta T$ because there is only one strain component $\epsilon^{t o t}=\epsilon^{M}$, the question has to be considered in order to properly model the influence of the thermal residual stresses/deformation and to correlate with experimental measurements. It is thus considered that during displacement control experiments the strain component that is actually maintained constant by the loading machine during crack multiplication process is the $\epsilon^{\text {tot }}$ component. Both the material stiffness properties $\left([C]\right.$ for laminate, $[Q]_{(k 0)}$ for individual ply) and the material thermal expansion coefficients ( $\{\alpha\}$ for laminate, $\{\alpha\}_{(k 0)}$ for individual ply) change during crack multiplication, and consequently both mechanical and thermal strain components change their values during crack multiplication, while there is the total strain $\left(\epsilon_{x}^{t o t}=\right.$ ct. for extension experiments, $\kappa_{x}^{\text {tot }}=$ ct. for bending experiments) which is kept constant by the loading machine during displacement control experiments.

Considering the displacement control case as it has been previously described, boundary conditions consistent with the experimental procedures has to be considered in the analytical implementation. Theoretical results for four different loading cases, namely uniaxial extension $\left(\epsilon_{x}\right)$, uniaxial bending $\left(\kappa_{x}\right)$, combined uniaxial extension - uniaxial bending $\left(\epsilon_{x}-\kappa_{x}\right)$, and biaxial extension $\left(\epsilon_{x}-\epsilon_{y}\right)$ are presented in Section 5. The corresponding considered boundary conditions for these loading cases are presented in the following. For each loading case and corresponding b.c., a linear system of $6 \times 6$ equations (eq. (4.30)) has to be solved at each load increment and each iteration in order to reproduce the desired experimental procedure.

1) uniaxial extension loading $\epsilon_{x}$

This case corresponds to a classical uniaxial extension loading experiment under displacement control ( $\epsilon_{x}=$ ct. during crack growth) in an extension type machine, see Fig. 4.15 (a). 


$$
\epsilon^{t o t}=\left\{\begin{array}{c}
\epsilon_{x} \\
\epsilon_{y} \\
\gamma_{x y} \\
\kappa_{x} \\
\kappa_{y} \\
\kappa_{x y}
\end{array}\right\} \begin{aligned}
& \text { imposed } \\
& =0, \text { grips constraint } \\
& =0, \text { grips constraint } \\
& =0, \text { grips constraint }
\end{aligned} \quad N M=\left\{\begin{array}{c}
N_{x} \\
N_{y} \\
N_{x y} \\
M_{x} \\
M_{y} \\
M_{x y}
\end{array}\right\} \begin{aligned}
& \neq 0, \text { applied, unknown } \\
& \neq 0, \text { grips reaction } \\
& \neq 0, \text { grips reaction } \\
&
\end{aligned}
$$

2) uniaxial bending loading $\kappa_{x}$

This case corresponds to an uniaxial bending experiment under curvature control $\left(\kappa_{x}=\right.$ ct. during crack growth), as it was designed and described in [36], see Fig. 4.15 (b).

$$
\epsilon^{t o t}=\left\{\begin{array}{c}
\epsilon_{x} \\
\epsilon_{y} \\
\gamma_{x y} \\
\kappa_{x} \\
\kappa_{y} \\
\kappa_{x y}
\end{array}\right\} \begin{aligned}
& \neq 0, \text { free } \\
& \neq 0, \text { free } \\
& \text { imposed } \\
& =0, \text { grips constraint } \\
& \neq 0, \text { free }
\end{aligned} \quad N M=\left\{\begin{array}{l}
N_{x} \\
N_{y} \\
N_{x y} \\
M_{x} \\
M_{y} \\
M_{x y}
\end{array}\right\} \begin{aligned}
& =0 \\
& \text { applied, unknown } \\
& \neq 0
\end{aligned}
$$

3) combined uniaxial extension - uniaxial bending loading $\epsilon_{x}-\kappa_{x}$

This case corresponds to a combined extension-bending experiment, under displacement control case ( $\epsilon_{x}=$ ct. during crack growth), as it is schematically presented in Fig. 4.15 (c).

$$
\epsilon^{t o t}=\left\{\begin{array}{c|c}
\epsilon_{x} \\
\epsilon_{y} \\
\gamma_{x y} \\
\kappa_{x} \\
\kappa_{y} \\
\kappa_{x y}
\end{array}\right\} \begin{aligned}
& \text { imposed } \\
& \neq 0, \text { free } \\
& \text { imposed } \\
& =0, \text { grips constraint } \\
& =0, \text { free }
\end{aligned} \quad N M=\left\{\begin{array}{c}
N_{x} \\
N_{y} \\
N_{x y} \\
M_{x} \\
M_{y} \\
M_{x y}
\end{array}\right\} \begin{aligned}
& \text { applied, unknown } \\
& =0 \\
& =0
\end{aligned}
$$

The imposed bending deformation $\kappa_{x}$ is considered as a fraction $f$ of the extension deformation $\epsilon_{x}: \kappa_{x}=f \cdot \epsilon_{x}$.

There is no available experimental data for this loading case.

4) biaxial extension loading $\epsilon_{x}-\epsilon_{y}$

This case corresponds to a biaxial extension experiment, under displacement control case ( $\epsilon_{x}=$ ct. during crack growth), as it is schematically presented in Fig. 4.15 (d). 


$$
\epsilon^{t o t}=\left\{\begin{array}{c}
\epsilon_{x} \\
\epsilon_{y} \\
\gamma_{x y} \\
\kappa_{x} \\
\kappa_{y} \\
\kappa_{x y}
\end{array}\right\} \begin{aligned}
& \text { imposed } \\
& \text { imposed } \\
& \neq 0, \text { free } \\
& \neq 0, \text { free } \\
& \neq 0, \text { free }
\end{aligned} \quad N M=\left\{\begin{array}{c}
N_{x} \\
N_{y} \\
N_{x y} \\
M_{x} \\
M_{y} \\
M_{x y}
\end{array}\right\} \begin{aligned}
& \text { applied, unknown } \\
& \text { applied, unknown } \\
& =0
\end{aligned}
$$

The imposed extension deformation $\epsilon_{y}$ is considered as a fraction $f$ of the extension deformation $\epsilon_{x}: \epsilon_{y}=f \cdot \epsilon_{x}$.

There is no available experimental data for this loading case.

a)

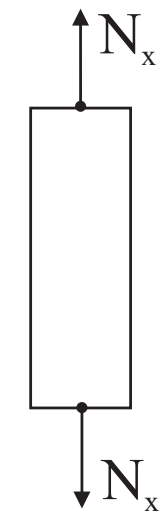

c)

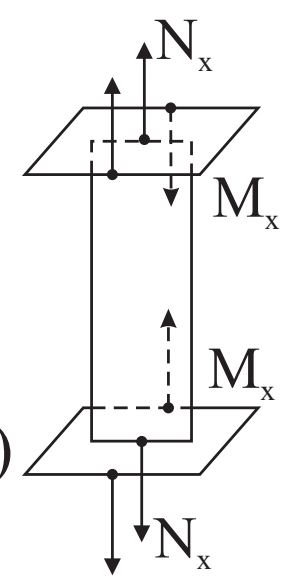

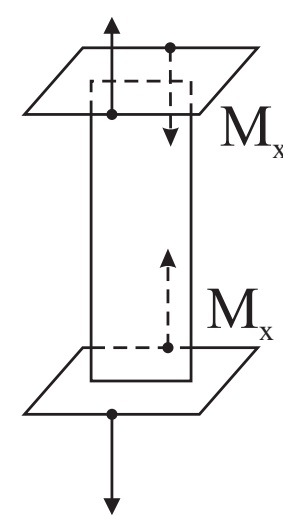

b)

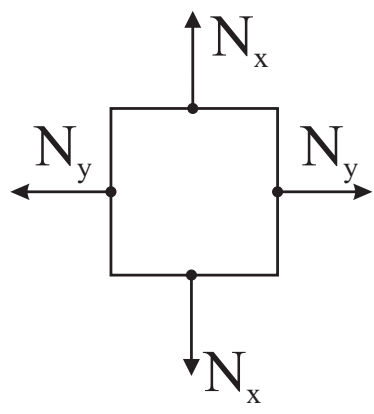

d)

Figure 4.15: Loading cases and B.C. considered for numerical simulation: a) uniaxial extension; b) uniaxial bending; c) combined uniaxial extension - uniaxial bending; d) biaxial extension. 


\subsubsection{Crack Closure model}

Two problems related to the material model in $[35,36]$ have been identified and required special treatment, as it is briefly presented in the following.

First, the material model $[35,36]$ provides the reduced material properties for an arbitrary laminate configuration $\left[\theta_{1} / \theta_{2} / \ldots / \theta_{n}\right]$ featuring matrix cracking damage of $\lambda_{1}, \lambda_{2} \ldots \lambda_{n}$ crack density in its individual plies. When reduced thermoelastic material properties are calculated based on $[35,36]$, it is supposed that each cracked ply is under crack opening loading conditions, which means that each individual ply $(k)$ brings a contribution to the reduction in material properties through its crack density $\lambda_{(k)}$. This assumption is true and requires $\mathrm{nu}$ further attention if the loading conditions on the laminate are such that each cracked ply is under tension conditions (see Fig. 4.16 (a)), i.e., there is a positive crack opening displacement (COD) for each cracked ply, which brings a contribution to reducing the material properties of the laminate. However, if the loading conditions on the laminate are such that individual cracked plies are under compression (see Fig. 4.16 (b)), we can suppose that the existing crack in that ply is closed, there is a zero COD even if the crack density $\lambda \neq 0$. This might be, for example, the case of the laminate under bending loading, when same of the plies are under tension loading and some of them are under compression. This problem has to be accounted for, and a crack closure procedure has to be implemented in the global model of the progressive damage analysis of the composite laminate.

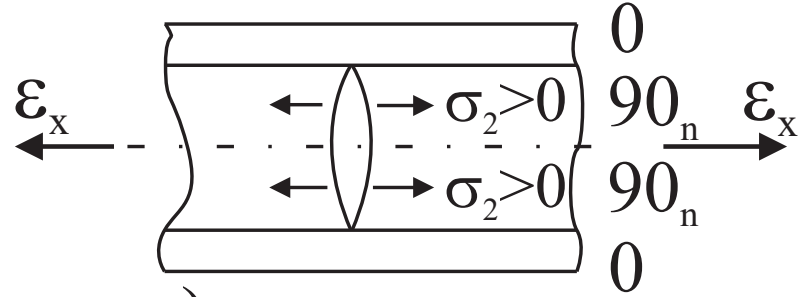

a)

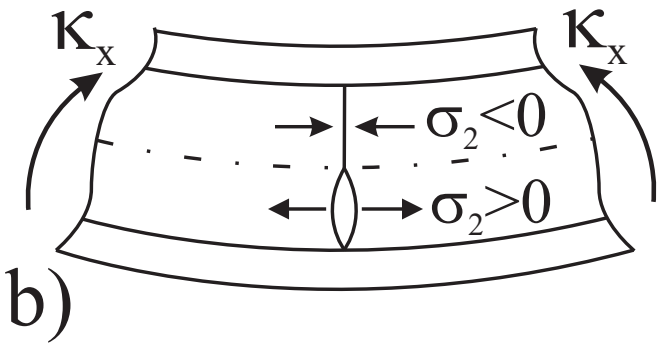

Figure 4.16: Crack closure effect: a) positive COD in all cracking plies; b) both open and closed cracks in individual plies.

The crack closure procedure is implemented here based on checking the sign of transverse stresses $\sigma_{2(k)}$ in each ply $(k)$ of the laminate, at each load level. If $\sigma_{2(k)}>0$, the reduced material properties are calculated by considering the crack density $\lambda_{(k)}$ of ply $(k)$. If $\sigma_{2(k)} \leq 0$, the damage level in ply $(k)$ is set as $\lambda_{(k)}=0$, and the existing crack density in ply $(k)$ does not bring a contribution to the reduced material properties of the laminate, due to the crack 
closure effect. However, the assignment $\lambda_{(k)}=0$ is a temporary one, and it holds as long as the ply stress $\sigma_{2(k)} \leq 0$. The real crack density without considering the crack closure effect is recorded, and it is assigned back to the cracked ply $(k)$ as soon as the stress in ply $(k)$ becomes $\sigma_{2(k)}>0$ under global laminate loading conditions. This approach assures the possibility of considering combined extension-bending loading cases (see Section 5) when the sign of transverse stress $\sigma_{2(k)}$ in an individual ply $(k)$ can change between tensioncompression, according to the combined loading considered. This approach also assures the possibility of extending the capabilities of the model such that loading history (for example, bending after extension) is accounted for.

It can be noticed that the assumption of the crack closure model is the perfect closure of the existing crack under ply compression conditions. It is supposed that there are no material nonlinearities induced by the contact and friction of the closing faces of the crack.

Second problem has already been introduced in Fig. 4.16: due to extending the capabilities of the model to bending deformation, the laminate stacking sequence has to be input as "split" around the middle plan of the laminate. For example, the $\left[0 / 90_{2 n} / 0\right]$ LSS in Fig. 4.16 (a) has to actually be input as $\left[0 / 90_{n} / 90_{n} / 0\right]$ (Fig. 4.16 (b)) in order to accurately model the possible crack closure in the middle $90^{\circ}$ plies. However, this brings another issue related to the formulation of the material model in $[35,36]$ : due to the fact that this material model is a COD based model, and the COD in some $(k)$ ply of the laminate is proportional to the ply thickness (see eq. (2.9) (B.2)), the crack evolution results for a $\left[0 / 90_{2 n} / 0\right]$ input LSS will be different compared to the results for the same laminate but using a $\left[0 / 90_{n} / 90_{n} / 0\right]$ input LSS, as it is presented in Fig. 4.17. Considering the case of uniaxial extension loading of the laminate, the calculated reduced properties for the $\left[0 / 90_{2 n} / 0\right]$ LSS will be lower than for the $\left[0 / 90_{n} / 90_{n} / 0\right]$ LSS at the same crack level $\lambda$ in both cases (see Fig. 4.17 (c)). The rate of reducing material properties for the $\left[0 / 90_{2 n} / 0\right]$ LSS will be faster than the rate for the $\left[0 / 90_{n} / 90_{n} / 0\right]$ LSS, which will have an effect on the $\lambda-\epsilon$ crack evolution.

The problem presented in Fig. 4.17 (c) is solved in the following manner (exemplification is made for the $\left[0 / 90_{2 n} / 0\right] \mathrm{LSS}$ in Fig. 4.17): the user input of the laminate configuration is the expanded $\left[0 / 90_{n} / 90_{n} / 0\right]$ one, in order to be able to address the possible bending associated crack closure effect in the middle plies of the laminate. If crack closure is detected in the middle plies, the reduced material properties are calculated on the expanded LSS. If there is no crack closure at the given load level, the LSS is switched to the collapsed $\left[0 / 90_{2 n} / 0\right]$ one, and reduced material properties are calculated on this laminate configuration. In this 

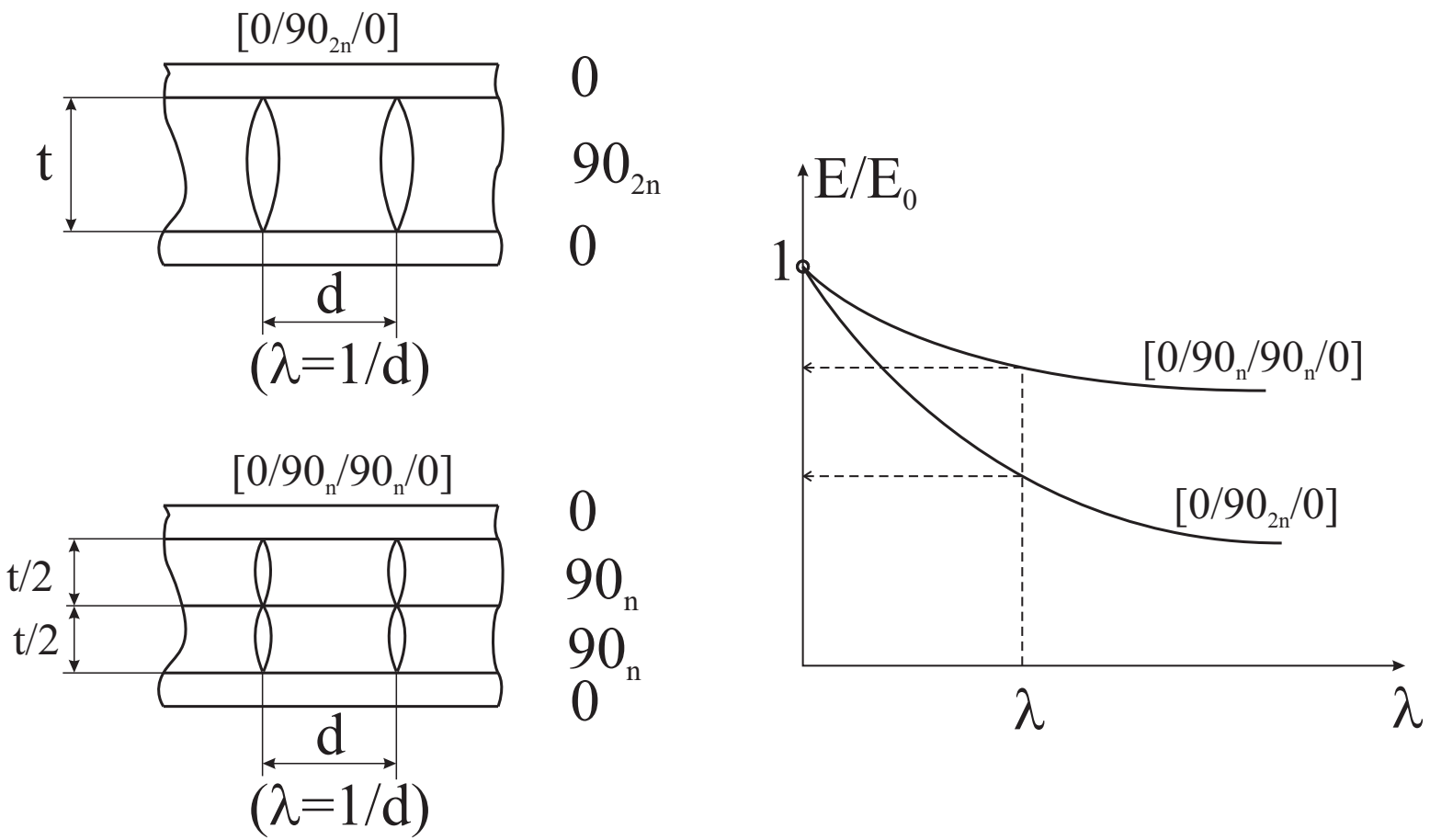

Figure 4.17: The behavior of the analytical material model $[35,36]$ for different input LSS.

way both the crack closure effect and the consistency with the COD formulation of the material model in $[35,36]$ can be assured. The check for crack closure at the middle plies of the laminate and the possible switch expanded-collapsed LSS is performed at each load level during the incremental loading of the laminate. The continuously check and shift expanded-collapsed LSS in order to correctly apply the material model brings the need for the accordingly redistribution of all the characteristic measures of the laminate over the LSS: plies thicknesses $t_{(k)}$, plies orientations $\theta_{(k)}$, plies thermo-elastic properties $Q_{(k)}, \alpha_{(k)}$, crack spacing $d_{(k)}$, crack density $\lambda_{(k)}$. 


\section{Chapter 5}

\section{Results and Discussion}

The current section presents the output of the analytical model for progressive damage in form of intra-laminar matrix cracking in laminated composites. A comprehensive testing program of the model has been considered in order to proper understand the predictive capabilities of the model and verify its output. The Results section is organized in two parts.

In the first part (Section 5.1) the model is run for a variety of test cases, and the output is analyzed and verified against the expected trends and engineering judgment. This first part can be regarded as a testing program of the analytical model, in order to verify the expected trends of the model output.

In the second part (Section 5.2) the model is run for a variety of cases for which experimental data is available from the literature. The second part can be regarded as a testing program of the analytical model, in order to verify the model output against experimental data.

The damage process is analyzed under its aspects of damage onset, damage evolution, and reduction in thermo-elastic properties of the material. According to eq. (4.1), the model output regarding the reduced material properties is: i) laminate reduced stiffness matrix $[C]=[A B D]$ (or its equivalent laminate compliance matrix $[S]=[C]^{-1}$ ), and ii) laminate reduced CTE coefficients $\{\alpha\}$ (or its equivalent laminate thermal load coefficients $\{\delta\}$ ). The laminate stiffness matrix $[C]$ is defined in eq. (A.1), the laminate CTE coefficients are defined in eq. (A.3), and the laminate thermal loads coefficients are defined in eq. (A.2). Both membrane and flexural thermo-elastic properties are addressed in the previous equations, this being the cause why mixed units are encountered in the expressions of $[C]$ and $\{\alpha\}$. The 
$[C],[S]$ matrices have dimension $6 \times 6$, and the $\{\alpha\},\{\delta\}$ vectors have dimension $6 \times 1$.

In order to define the equivalent engineering constants of the laminate (which will be part of the analysis in the present Results section), the following notations are used [35]:

$$
\begin{array}{r}
{[C]=\left[\begin{array}{ll}
C_{E E} & C_{E B} \\
C_{B E} & C_{B B}
\end{array}\right]} \\
{[S]=[C]^{-1}=\left[\begin{array}{ll}
S_{E E} & S_{E B} \\
S_{B E} & S_{B B}
\end{array}\right]}
\end{array}
$$

where the subscripts $E E, B B, E B$ denote the $3 \times 3$ submatrices separately defining the membrane $(E E)$, flexural $(B B)$, and membrane-flexural coupling $(E B, B E)$ laminate behavior.

The separate membrane and bending CTE have been defined using the $E, B$ subscript notation in eq. (A.2), (A.3).

Based on these equivalent notations, consistent units-wise compliance matrix and CTE vector of the laminate are defined as

$$
\begin{aligned}
& {[\hat{S}]=\left[\begin{array}{cc}
t \cdot S_{E E} & \frac{t^{2}}{2} \cdot S_{E B} \\
\frac{t^{2}}{2} \cdot S_{B E} & \frac{t^{3}}{12} \cdot S_{B B}
\end{array}\right]} \\
& \{\hat{\alpha}\}=\left\{\begin{array}{c}
\alpha_{E} \\
\frac{t}{2} \cdot \alpha_{B}
\end{array}\right\}
\end{aligned}
$$

and the equivalent engineering moduli of the laminate characterizing both the membrane and flexural deformation are defined as

$$
\hat{E}_{i i}=\left\{\begin{array}{l}
\frac{1}{\hat{S}_{i j}} \text { for } i, j=1 \ldots 6, i=j \\
-\frac{\hat{S}_{i j}}{\hat{S}_{i i}} \text { for } i, j=1 \ldots 6, i \neq j, \text { no sum over } i
\end{array}\right.
$$

The terms $\hat{E}_{i i}, \hat{\nu}_{i j}$ with $i, j=1 \ldots 3$ represent the membrane elastic properties of the laminate, with $i, j=3 \ldots 6$ represent the flexural elastic properties, and with $i=1 \ldots 3, j=$ $3 \ldots 6$ represent the membrane/flexural coupling terms. For example, the laminate Young modulus $E_{x}$ is the $\hat{E}_{11}$ term in eq. (5.3), $E_{y}$ is $\hat{E}_{22}$ term, and the laminate shear modulus $G_{x y}$ is the $\hat{E}_{33}$ term in eq. (5.3). Similarly, the laminate Poisson $\nu_{x y}$ is the $\hat{E}_{12}$ term, and $\nu_{y x}$ is the $\hat{E}_{21}$ term in eq. (5.3). The laminate bending stiffness along the $x$ direction, $B_{x}$, is the $\hat{E}_{44}$ term, and along $y$ direction, $B_{y}$, is the $\hat{E}_{55}$ term. The laminate twisting stiffness, $T_{x y}$, is the $\hat{E}_{66}$ term in eq. (5.3). The direct extension-bending coupling term $E B_{x}$, between applied extension $\epsilon_{x}$ and resulting curvature $\kappa_{x}$, is the term $\hat{E}_{14}$ in eq. (5.3). Al the other laminate coupling terms can be similarly identified in eq. (5.3). 
The equivalent engineering moduli of the laminate in eq. (5.3) will be analyzed in the following sections.

\subsection{Analytical model output: analysis of expected trends}

The following cases are considered in this section regarding the analysis of the model output trends:

- the influence of the laminate configuration on the damage process (Section 5.1.1); by laminate configuration is understood the properties of the cracking ply $(k 0)$ (ply material properties, ply thickness, ply orientation) and the properties of the neighboring plies $(k)$, as well.

- extension vs. bending loading cases: comparison of the damage process. (Section 5.1.2)

- damage under combined Extension-Bending loading (Section 5.1.3)

- the influence of the In-situ $R$-curve behavior of the critical damage parameter $\left(G_{I C}\right)$ for laminated composites (Section 5.1.4)

- the influence of thermal residual stresses on damage process (Section 5.1.5)

A Glass-Epoxy material system, featuring the individual lamina material properties listed in the following, is considered for the analysis of the analytical model output:

$$
\begin{aligned}
& E_{1}=35 \mathrm{GPa} ; \quad E_{2}=9 \mathrm{GPa} ; \quad G_{12}=4 \mathrm{GPa} ; \quad \nu_{12}=0.28 ; \quad \nu_{23}=0.42 \\
& \alpha_{1}=6.7 \cdot 10^{-} 6^{\circ} \mathrm{C}^{-1} ; \quad \alpha_{2}=29.3 \cdot 10^{-} 6^{\circ} \mathrm{C}^{-1}
\end{aligned}
$$

The processing temperature (stress free temperature) of the laminated composite is considered $S F T=130{ }^{\circ} \mathrm{C}$. The operating temperature (room temperature) is considered $R T=21^{\circ} \mathrm{C}$.

The critical energy release rates of the material are considered $G_{I C, 0}^{r e f}=0.20 \mathrm{~N} / \mathrm{mm}, G_{I I C}=$ $1 \mathrm{~N} / \mathrm{mm}$, where $G_{I C, 0}^{r e f}$ is the initiation value of mode $I$ critical ERR, $G_{I C}$, at the reference ply thickness (see Section 4.4.3), and $G_{I I C}$ is the value of mode $I I$ critical ERR, which is considered constant for the present model.

The individual ply thickness is $t_{k}=0.125 \mathrm{~mm}$.

When one of the above listed laminate properties is parametrically considered for the present study, the range of variation of that property will be explicitly specified. In order 
to asses only the influence of the considered parameters, the in-situ $\mathrm{R}$-curve behavior (see Section 4.4.3) is not considered for the following study cases, unless explicitly specified.

\subsubsection{The influence of the laminate configuration}

Both the properties of the cracking ply $(k 0)$ and the properties of the neighboring plies $(k)$ are analyzed in this section. The following possible influences are considered and investigated:

- The influence of the ply elastic properties

- The influence of the properties of the cracking ply $(k 0)$ :

- the influence of the $(k 0)$ ply thickness

- the influence of the $(k 0)$ ply orientation

- interior vs. surface $(k 0)$ ply

- The influence of the properties of the neighboring plies $(k)$ :

- the influence of the $(k)$ plies orientation

- the influence of the $(k)$ plies thickness

A testing laminate configuration is defined for some of the following verifications under this section, in order to assure consistency and comparability between the model output for different testing cases. This laminate configuration is $\left[ \pm \theta_{m} / 90_{n} / \pm \theta_{m}\right]$, where $\pm \theta_{m}$ represents an uncracking sublaminate. When this testing configuration is not able to catch the material behavior for particular testing cases, particular laminate configurations are defined and used, as appropriate.

i1) The influence of the ply material properties

The testing configuration for this case is $\theta=0, m=1 / 2, n=2$, which means a $\left[0 / 90_{2} / 0\right]$ laminate.

The study case is run by considering the elastic properties $E_{1}, E_{2}, G_{12}$ of the constituent plies as parameters. To be noticed that the the parametric material properties are assigned simultaneously to the cracking ply $(k 0)$ and the neighboring plies $(k)$ for the present study. Another possibility of studying the influence of ply material properties on damage process 
would be to assign parametric material properties to only the cracking ply $(k 0)$, and to only the neighboring plies $(k)$.

The loading case for this study case is uniaxial tension.

The parametric study is run for the following values of the ply elastic properties: $E_{1}=$ $[25,35,45] G P a ; E_{2}=[6,9,12] G P a ; G_{12}=[3,4,5] G P a$.

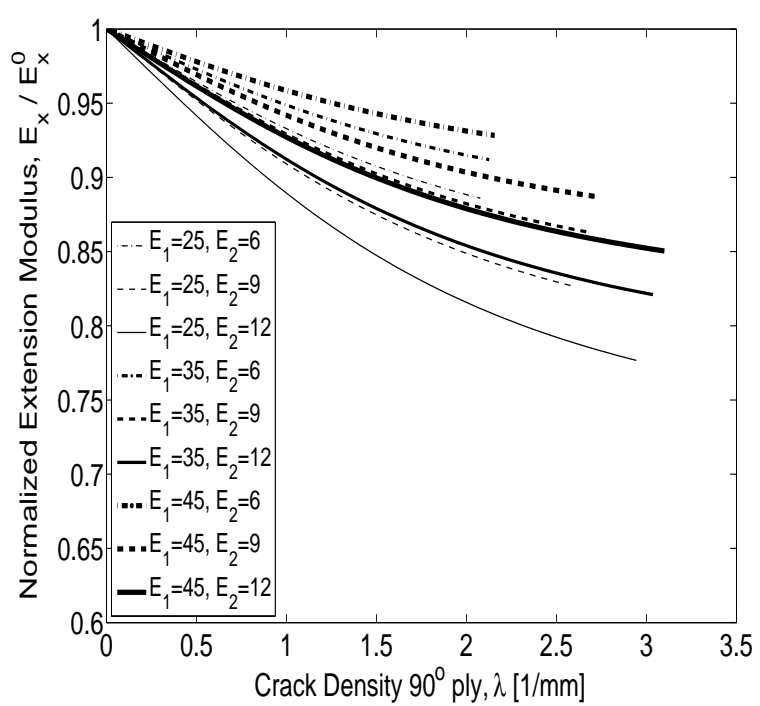

Figure 5.1: The influence of ply stiffness on the normalized extension stiffness $E_{x} / E_{x}^{0}$ of the laminate.

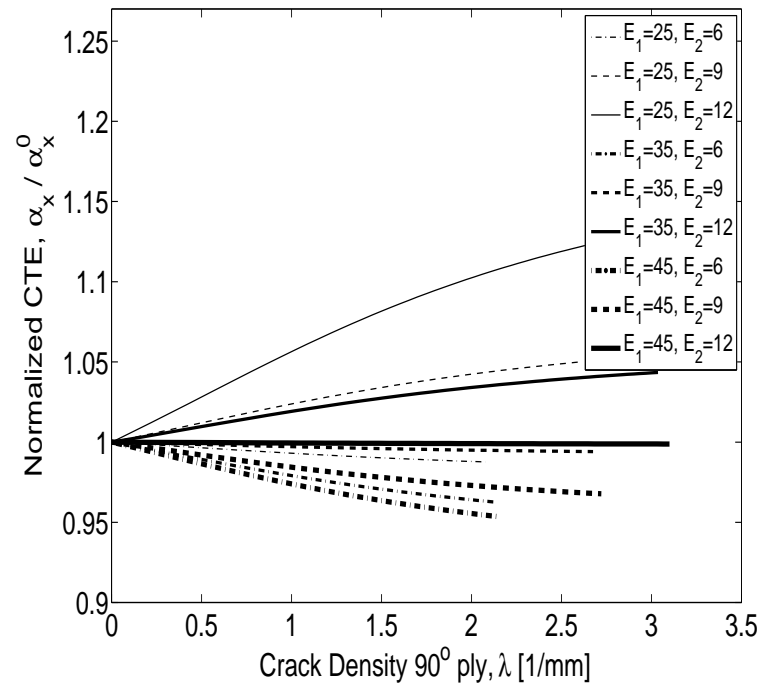

Figure 5.2: The influence of ply stiffness on the normalized CTE $\alpha_{x} / \alpha_{x}^{0}$ of the laminate.

The degradation in laminate material properties is presented in Fig. 5.1 - 5.4. The crack onset and evolution of the laminate for various values of the aforementioned parameters are presented in Fig. 5.5, 5.6. The corresponding nonlinear stress-strain curves due to progressive damage are shown in Fig. 5.7.

It can be observed in Fig. 5.5, 5.6 that both the longitudinal $\left(E_{1}\right)$ and transverse $\left(E_{2}\right)$ Young moduli of the cracking ply influence the damage process, both onset and evolution.

If the $\lambda-\epsilon$ damage evolution in Fig. 5.5 is regarded, the influence of the transverse elastic modulus $E_{2}$ can be appreciated as considerable (a considerable difference in crack density $\lambda$ is recorder for a given value of the applied strain $\epsilon$ ), and the influence of longitudinal elastic modulus $E_{1}$ can be appreciated as negligible (a negligible difference in crack density $\lambda$ is recorder for a given value of the applied strain $\epsilon$ ). Surprisingly, higher values of the transverse elastic modulus $E_{2}$ trigger an earlier onset of damage, and consequently a higher crack density for a given level of the applied loading $\epsilon_{x}$. 


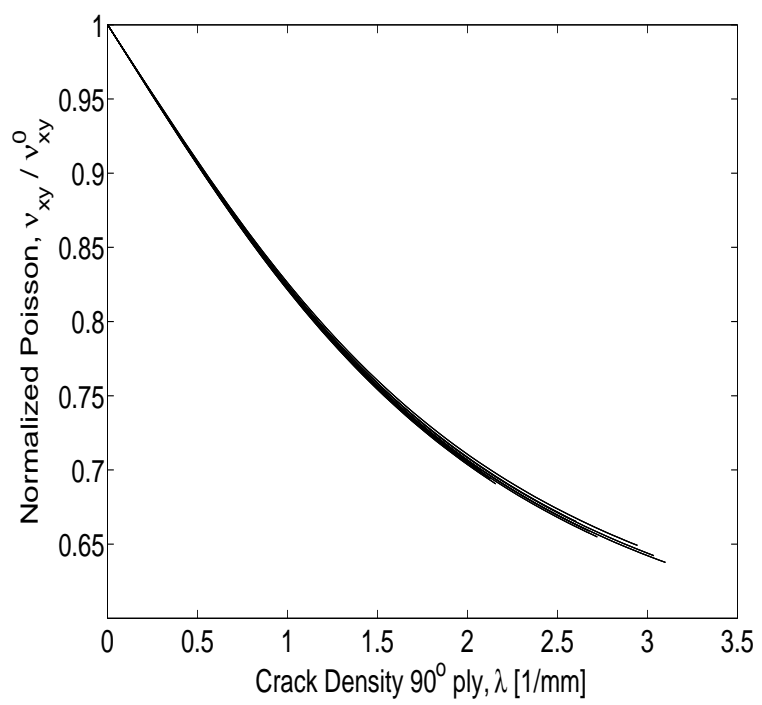

Figure 5.3: The influence of ply stiffness on the normalized Poisson $\nu_{x y} / \nu_{x y}^{0}$ of the laminate.

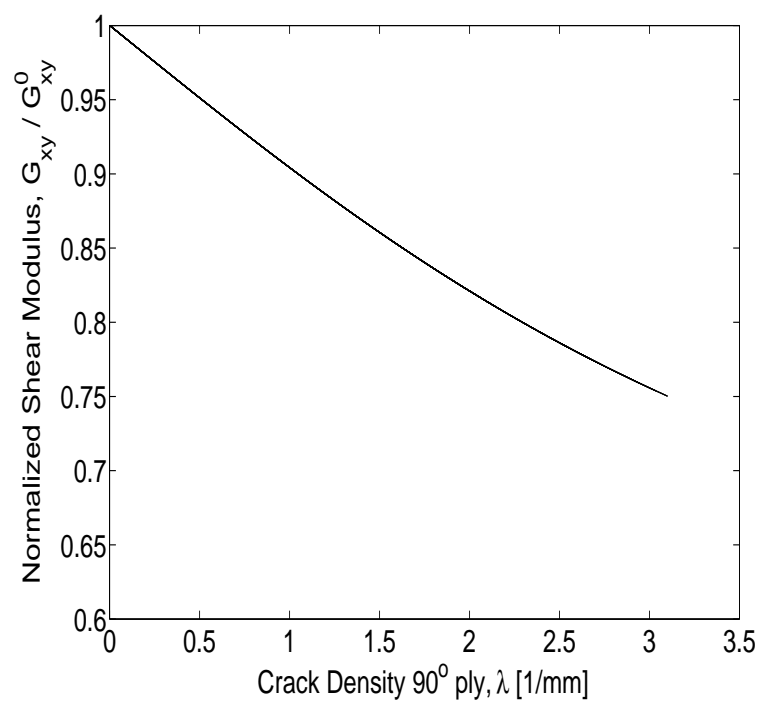

Figure 5.4: The influence of ply stiffness on the normalized shear modulus $G_{x y} / G_{x y}^{0}$ of the laminate.

However, if the $\lambda-\sigma$ damage evolution in Fig. 5.6 is regarded, both $E_{1}$ and $E_{2}$ material properties can be appreciated as having a considerable influence on damage onset, and consequently on damage evolution. It is usual, as it will be shown during the model validation process in the current section and in Section 5.2, that damage evolution curves that are clustered together in $\lambda-\epsilon$ coordinates, are actually spread apart in $\lambda-\sigma$ coordinates. Both $\lambda-\epsilon$ and $\lambda-\sigma$ curves need to be analyzed in order to fully understand the damage evolution behavior of the laminated composite.

Another insight and useful information regarding damage evolution is provided by the stress-strain curve presented in Fig. 5.7. The clustering of these curves according to the values of $E_{1}$ elastic modulus, and the small variations inside of each cluster group according to the values of $E_{2}$ elastic modulus are expected for the considered loading case, due to the fact that $E_{1}$ is the major modulus along the loading direction for the $0^{\circ}$ plies, which are not affected by matrix cracking in the $90^{\circ}$ plies. However, if we are looking at only one cluster (group) of curves, for example the group of thin dotted, dashed and solid lines (i.e., $E_{1}=25 G P a$ and $E_{2}=6,9,12 G P a$, respectively), we can see that while the initial (for undamaged material, up to damage onset) slopes of the material are order by the increasing value of $E_{2}$, these slopes became parallel after damage onset, which means a damage process more severe for high values of $E_{2}$. Actually this conclusion is identical with the one from 


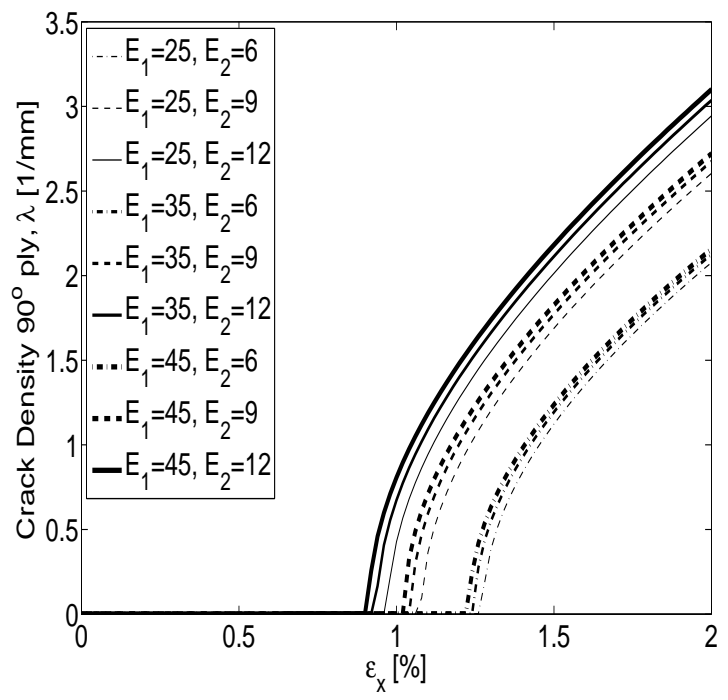

Figure 5.5: The influence of ply stiffness on the damage process, $\lambda=\lambda\left(\epsilon_{x}\right)$.

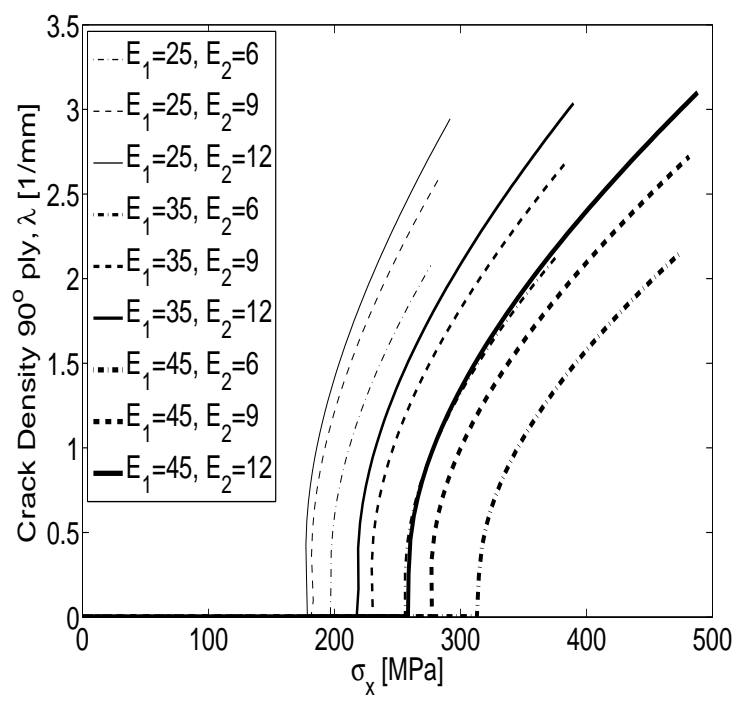

Figure 5.6: The influence of ply stiffness on the damage process, $\lambda=\lambda\left(\sigma_{x}\right)$

analyzing damage in $\lambda-\epsilon$ coordinates, where it has been shown that higher values of $E_{2}$ trigger earlier damage onset and higher crack density for a given loading $\epsilon$.

As consequences of the damage evolution presented in Fig. 5.5, 5.7, the reduction in thermo-elastic material properties is shown in Fig. $5.1-5.2$.

It has to be noted that the plotted quantities in these graphs are normalized with respect to their initial (undamaged) properties, this being the way how the reduced material properties will be plotted in most of the cases. The scope of the normalization is that comparison can be made between different cases, since the normalized quantity is actually a measure of the gradient of the property reduction, as compared to its undamaged state.

It can be noticed in Fig. 5.1 that the reduction in longitudinal elastic modulus of the laminate, $E_{x}$, is influenced by both longitudinal $\left(E_{1}\right)$ and transverse $\left(E_{2}\right)$ moduli of the cracking ply. A higher transverse modulus $E_{2}$ of the cracking ply triggers a more severe reduction in the laminate modulus $E_{x}$, which is consistent with the findings from analyzing $\lambda-\epsilon$ and $\sigma-\epsilon$ curves. However, the reduction in laminate modulus can be attenuated by an increase of the longitudinal modulus $E_{1}$ of the cracking ply.

It can be observed in Fig. 5.2 that the trends recorded for the laminate CTE $\alpha_{x}$ are exactly opposite compared to the trends for the laminate longitudinal Young modulus $E_{x}$. What is interesting to be noted in Fig. 5.2 is the fact that the laminate CTE can either decrease, remain constant, or increase with increasing damage level, in function of the material 


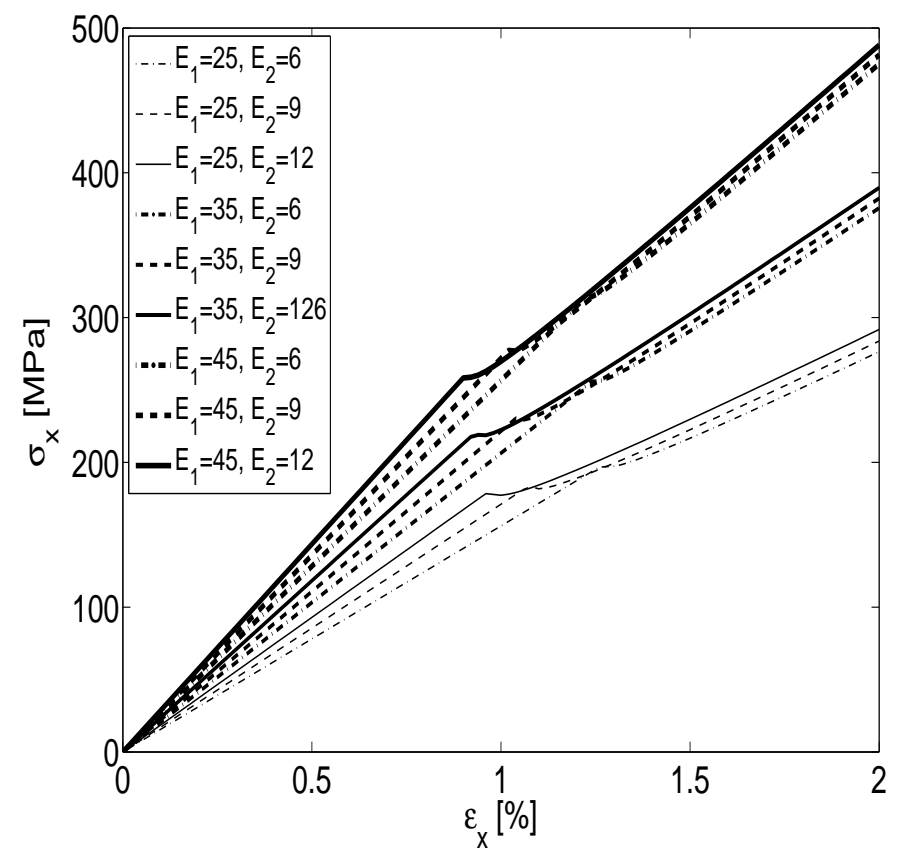

Figure 5.7: The influence of ply stiffness on the stress-strain behavior of the laminate.

properties of the plies.

In Fig. 5.3, 5.4 it can be observed that the elastic moduli of the cracking ply have no or very little influence on the reduction of Poisson coefficient $\nu_{x y}$ and shear modulus $G_{x y}$ of the laminate.

The results of the parametric analysis with the shear elastic modulus of the cracking ply $\left(G_{12}\right)$ are not presented here. This is because the $G_{12}$ parameter has no influence on none of the laminate properties (similar to the cases in Fig. 5.3, 5.4). Not even reduction of the normalized shear modulus of the laminate, $G_{x y} / G_{x y}^{0}$, is influenced by different values of the ply shear modulus $G_{12}$. 
i2) The influence of the properties of the cracking ply $(k 0)$

\section{i2-1) The influence of the (k0) ply thickness}

The testing configuration for this case is $\theta=0, m=1 / 2$, which means a $\left[0 / 90_{n} / 0\right]$ laminate family.

The study case is run by considering the thickness (which is given by the $n$ parameter) of the cracking ply as a varying parameter.

The loading case for this study case is uniaxial tension.

The parametric study is run for the following values of number of ply making the cracking $90_{n}$ stack: $n=[1,2,3,4,12]$.

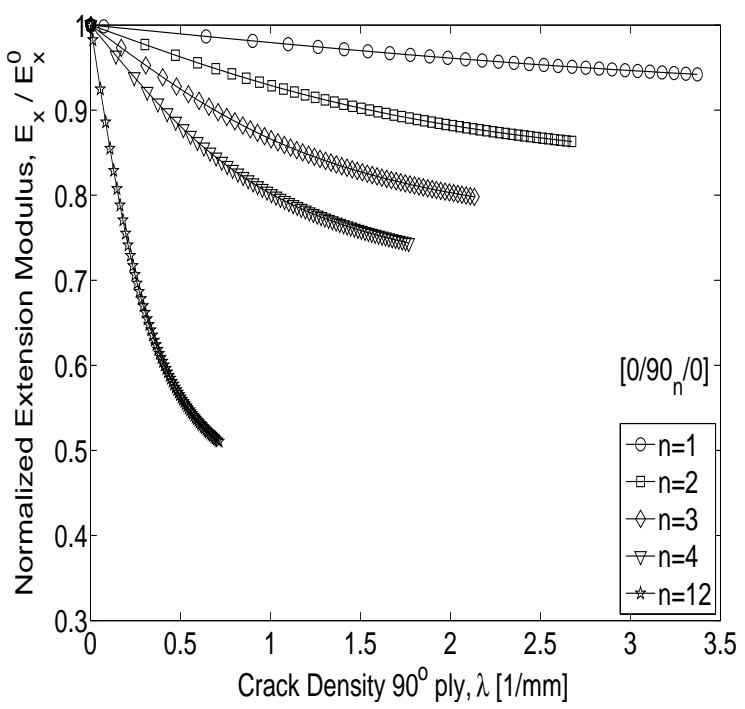

Figure 5.8: The influence of the cracking ply thickness on the normalized extension stiffness $E_{x} / E_{x}^{0}$ of the laminate.

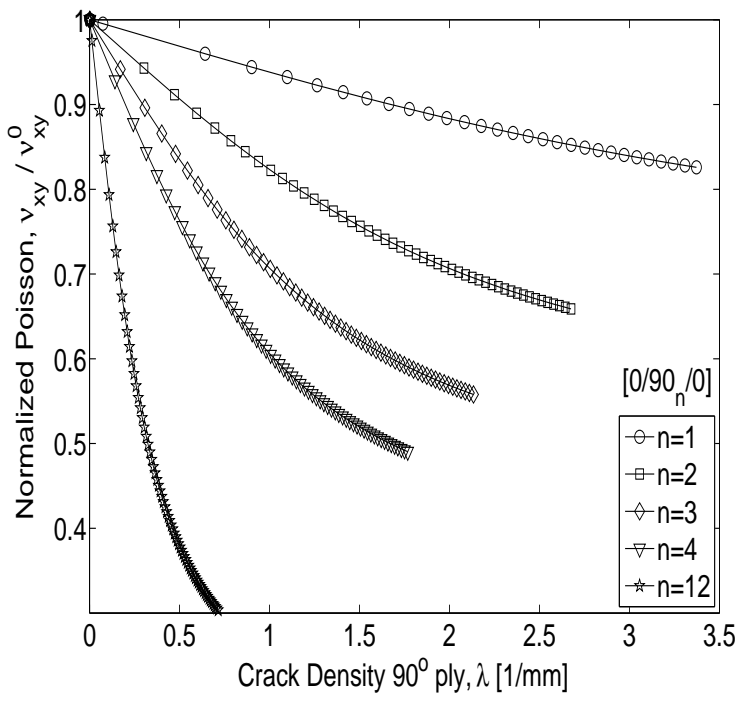

Figure 5.9: The influence of the cracking ply thickness on the normalized Poisson $\nu_{x y} / \nu_{x y}^{0}$ of the laminate.

The degradation of laminate thermo-elastic material properties is presented in Fig. 5.8 5.11. Damage evolution under increasing loading for varying thickness of the cracking ply is presented in Fig. 5.12, 5.13. The equivalent stress-strain curves are presented in Fig. 5.14.

It can be noticed in Fig. 5.12 the expected trend of decreasing strain level required for damage onset, which is according with experimental observations (see Fig. 2.4). This predictive capability of the model comes from the energy formulation of the damage growth criterion (see Section 2.2,4.4), and it is an advantage of the energy based formulation over the strength based models. The disadvantage of the energy based criterion is that 


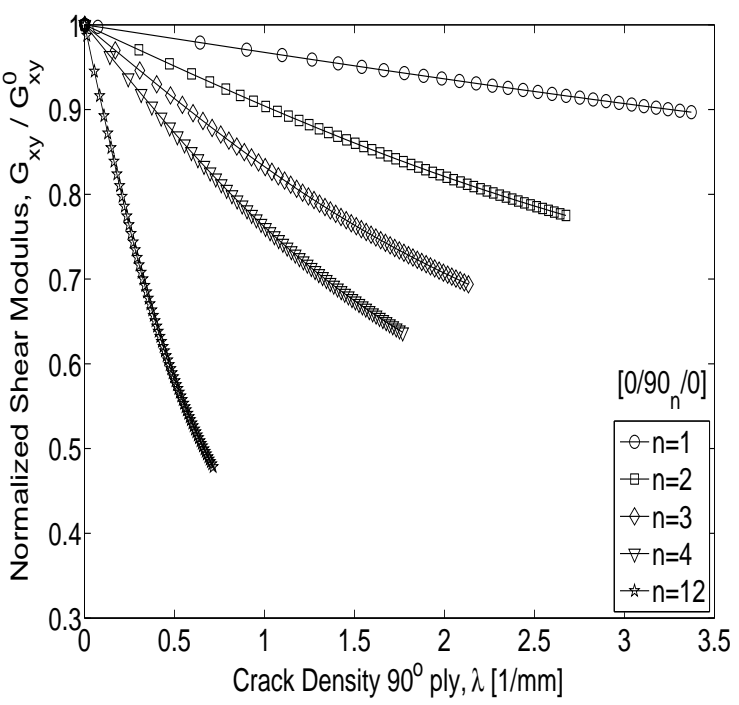

Figure 5.10: The influence of the cracking ply thickness on the normalized shear modulus $G_{x y} / G_{x y}^{0}$ of the laminate.

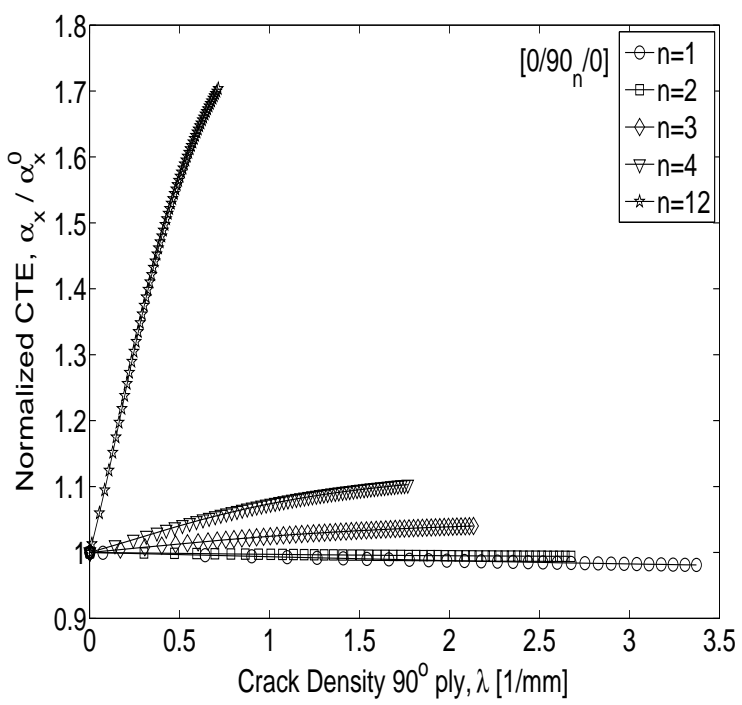

Figure 5.11: The influence of the cracking ply thickness on the normalized CTE $\alpha_{x} / \alpha_{x}^{0}$ of the laminate.

it predicts a continuously decrease in strain to damage onset for a continuously increase in ply thickness. This fact is exemplified by the high thickness case $n=12$ in Fig. 5.12. According with experimental observation, the strain to damage onset does not decrease any more with increasing ply thickness. However, it can be clearly observed in Fig. 5.12 that the reduction in strain to damage onset is not linear but rather an asymptotic one, which might counterbalance, at some extent, the aforementioned disadvantage of the energy based model. A thin-thick transition thickness implementation (see Section 2.2) might be required in order to more accurately capture the material behavior.

Another aspect interesting to notice in Fig. 5.12 is the fact that damage develops more abrupt for thinner plies than for thicker plies. This behavior is confirmed by experimental validation in Section 5.2.

Regarding comparing the equivalent damage evolution curves $\lambda-\epsilon$ in Fig. 5.12 and $\lambda-\sigma$ in Fig. 5.13, it can be noticed that they have a different appearance. The abrupt damage propagation for thiner plies is not so obvious in the $\lambda-\sigma$ coordinates.

The stress-strain curves in Fig. 5.14 reveal that the reduction in the initial (undamaged) elastic modulus of the laminate increases with the ply thickness. This fact can also be noticed in Fig. 5.8, where the normalized reduction in the Young modulus of the laminate is plotted as function of crack density. 


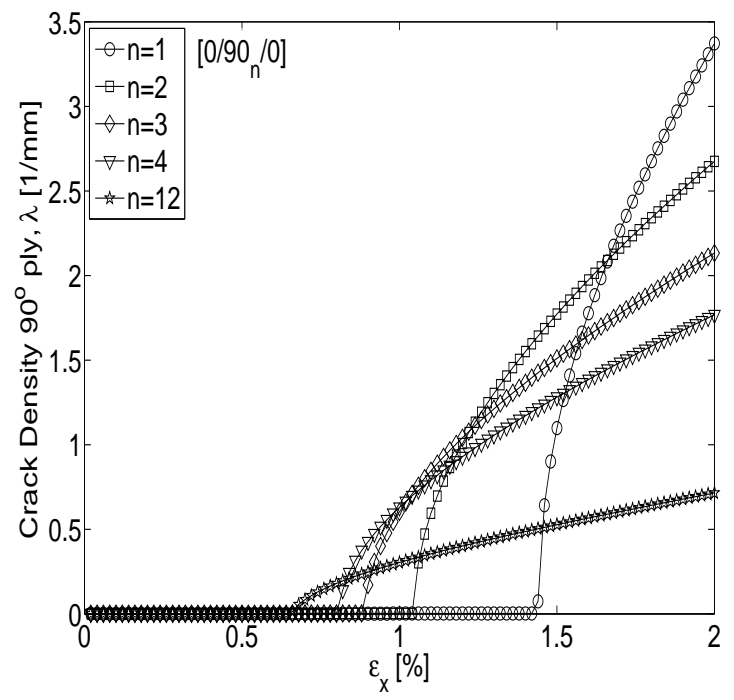

Figure 5.12: The influence of the cracking ply thickness on the damage process, $\lambda=$ $\lambda\left(\epsilon_{x}\right)$.

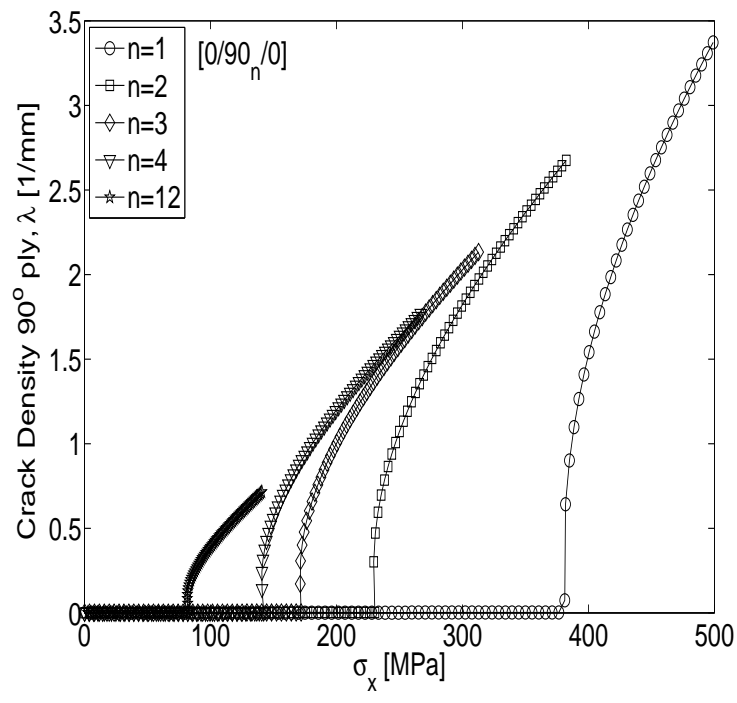

Figure 5.13: The influence of the cracking ply thickness on the damage process, $\lambda=$ $\lambda\left(\sigma_{x}\right)$.

The reduction in the normalized reduced elastic properties of the laminate as a function of crack density is shown in Fig. 5.8 - 5.10. A higher reduction in the $E_{x}, \nu_{x y}, G_{x y}$ elastic properties for a given level of matrix cracking can be noticed here. The physical explanation of this is due to the crack opening displacement (COD) of the crack faces, which is higher for higher ply thickness, translating thus in a higher drop in elastic properties. The COD formulation of the present analytical model allows for this predictive capability of the model.

Contrary to the trends for elastic properties in Fig. 5.8 - 5.10, an increase of the laminate CTE with ply thickness can be noticed in Fig. 5.11, which also can be physically explained based on the COD concept. 


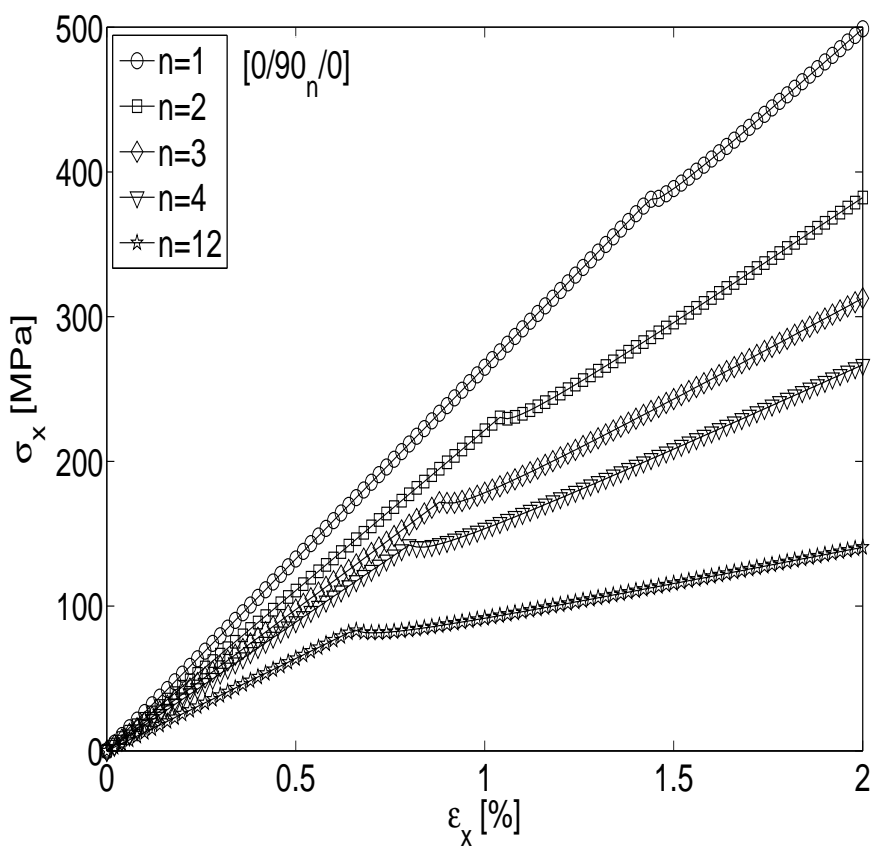

Figure 5.14: The influence of the cracking ply thickness on the stress-strain behavior of the laminate. 
i2-2) The influence of the $(k 0)$ ply orientation

The testing configuration for this case is a $[0 / \pm \theta / 0 / \pm \theta / 0]$ laminate.

The study case is run by considering the orientation $\theta$ of the off-axis ply as parameter.

The loading case for this study case is uniaxial tension.

The parametric study is run for the following values of the orientation of the cracking ply: $\theta=[80,70,60]$ deg.

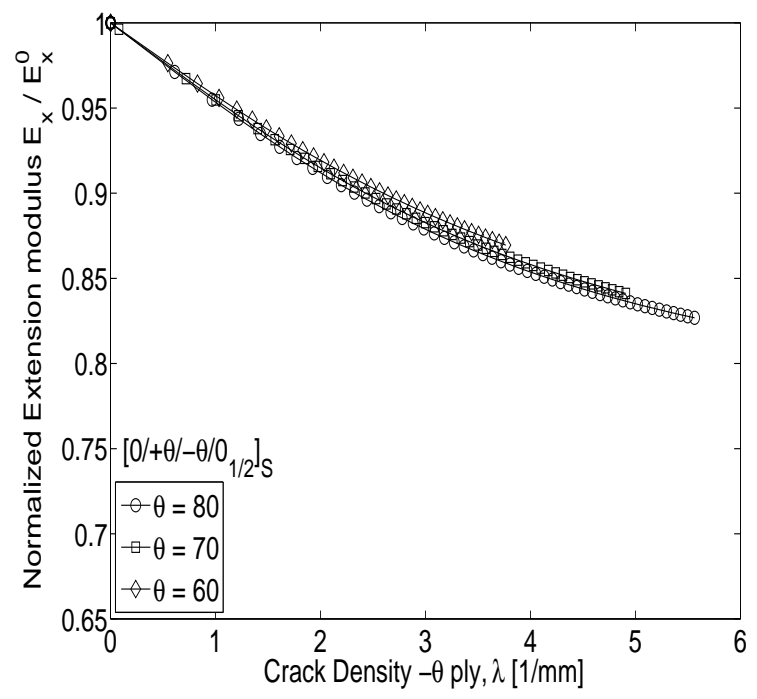

Figure 5.15: The influence of the cracking ply orientation on the normalized extension stiffness $E_{x} / E_{x}^{0}$ of the laminate.

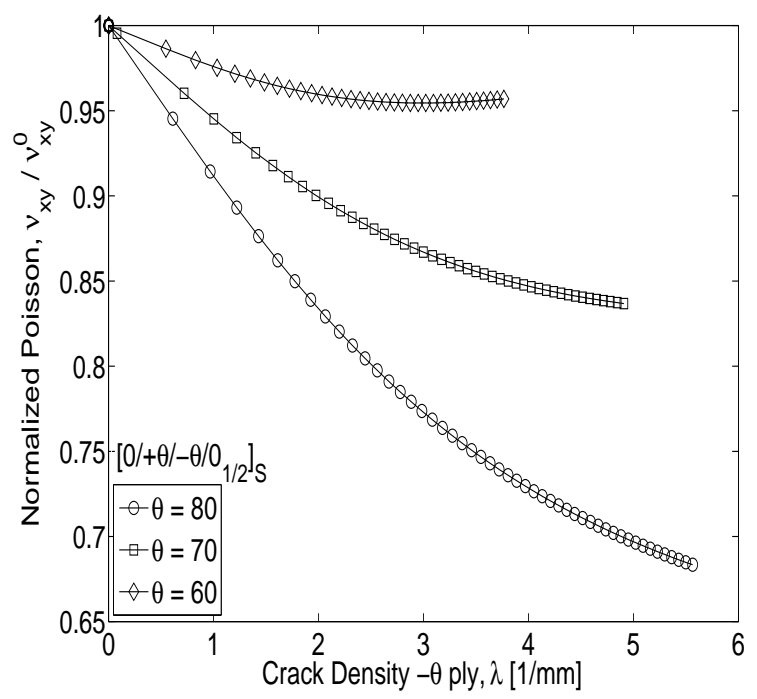

Figure 5.16: The influence of the cracking ply orientation on the normalized Poisson $\nu_{x y} / \nu_{x y}^{0}$ of the laminate.

The evolution of the thermo-elastic properties of the laminate is presented in Fig. 5.15 5.18. The almost insensitivity of the normalized Young modulus $E_{x} / E_{x}^{0}$ to the orientation $\theta$ of the cracking ply can be noticed in Fig. 5.15. Unlike the Young modulus, the influence of the $\theta$ off-axis orientation on the Poisson coefficient and the shear modulus is shown in Fig. 5.16, 5.17, where a higher drop of $\nu_{x y}, G_{x y}$ values for a given level of crack density $\lambda$ can be noticed for higher values of the off-axis angle $\theta$. The reversed trend, as compared to $\nu_{x y}, G_{x y}$, of the laminate CTE $\alpha_{x}$ is presented in Fig. 5.18. Again, the possibility of either decreasing or increasing in the laminate CTE can be noticed in Fig. 5.18, in function of the configuration of the laminate.

The evolution of matrix cracking under the considered loading case is presented in Fig. 5.19, 5.20, where the expected trend of decreasing strain to damage onset with increasing off-axis orientation of the cracking ply can be noticed. The earlier damage onset 


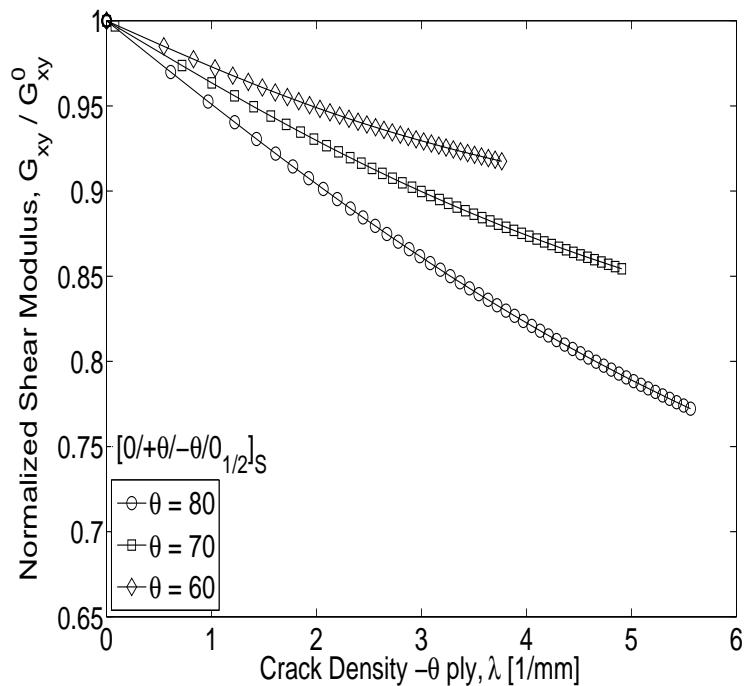

Figure 5.17: The influence of the cracking ply orientation on the normalized shear modulus $G_{x y} / G_{x y}^{0}$ of the laminate.

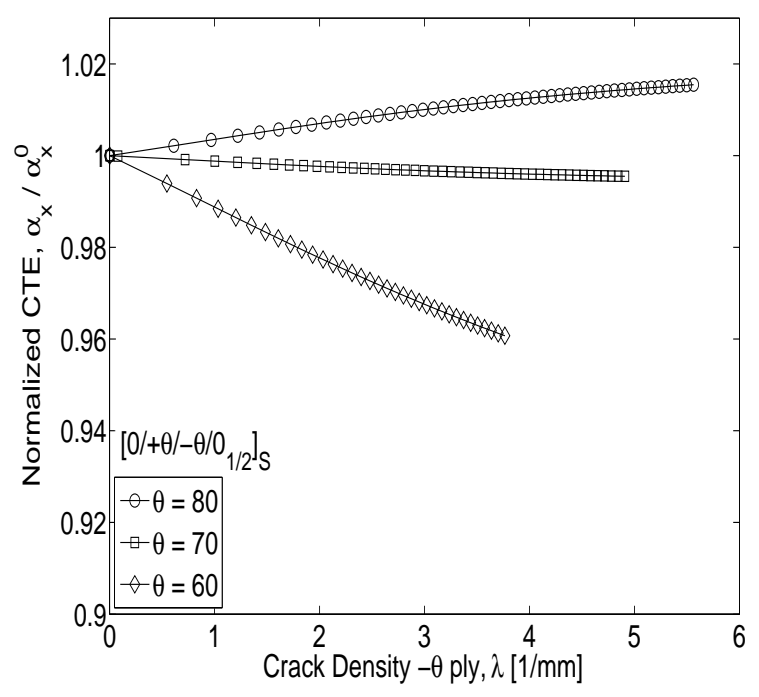

Figure 5.18: The influence of the cracking ply orientation on the normalized CTE $\alpha_{x} / \alpha_{x}^{0}$ of the laminate.

triggers a higher damage level $\lambda$ (crack density) for a given load level $\epsilon$, for the whole loading sequence (unlike the aspect of the damage progression curves in Fig. 5.12).

The stress-strain curves in Fig. 5.21 shows parallel lines before and after damage onset, even if the moment of onset is different. This reveals a similar reduction of the extension modulus of the laminate due to matrix cracking, regardless the orientation $\theta$ of the cracking ply. This fact is also confirmed by the plot in Fig. 5.15, where the reduction of the normalized Young modulus of the laminate is presented. 


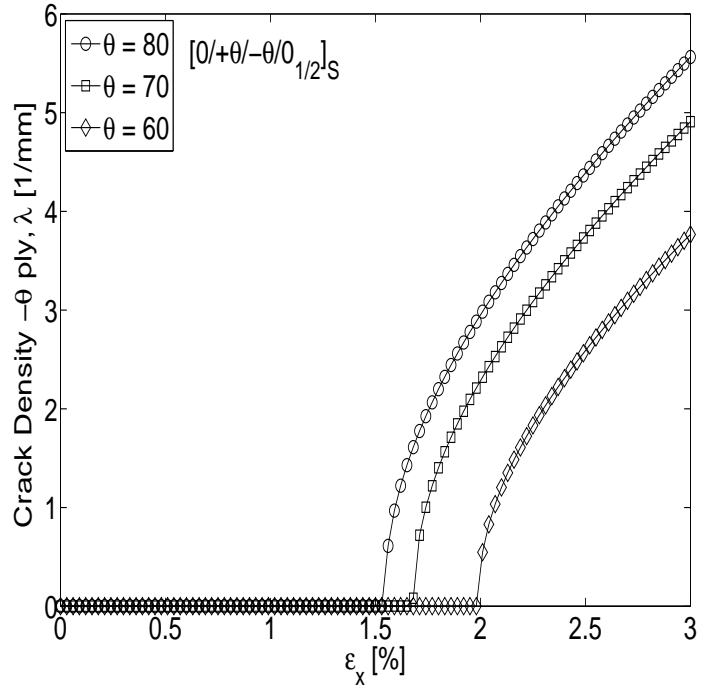

Figure 5.19: The influence of the cracking ply orientation on the damage process, $\lambda=\lambda\left(\epsilon_{x}\right)$.

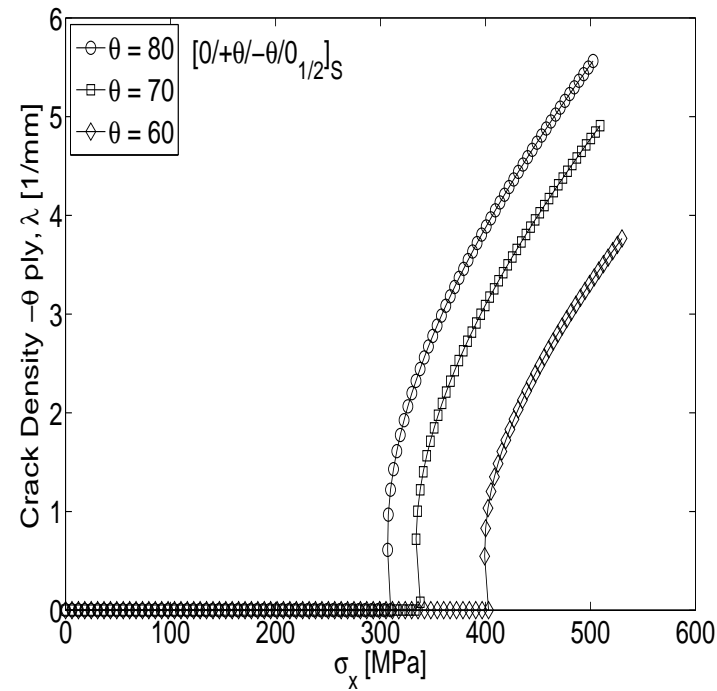

Figure 5.20: The influence of the cracking ply orientation on the damage process, $\lambda=\lambda\left(\sigma_{x}\right)$.

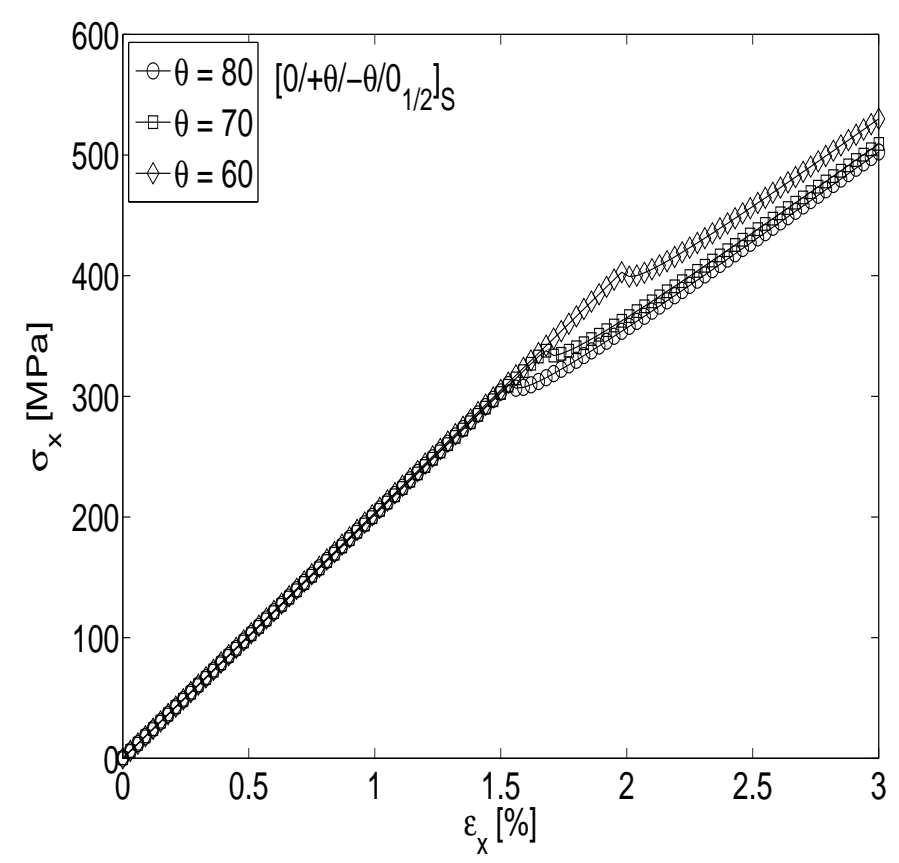

Figure 5.21: The influence of the cracking ply orientation on the stress-strain behavior of the laminate. 
i2-3) Interior vs. Surface (k0) ply

For this case is a $\left[90 / 0_{4} / 90\right]$ laminate is compared against a $\left[0 / 90 / 0_{2} / 90 / 0\right]$ laminate. The cracking ply is a surface one for the first laminate configuration, and an interior one for the second configuration. The two laminates are equivalent from the equivalent thermo-elastic properties point of view. The loading case is uniaxial tension.

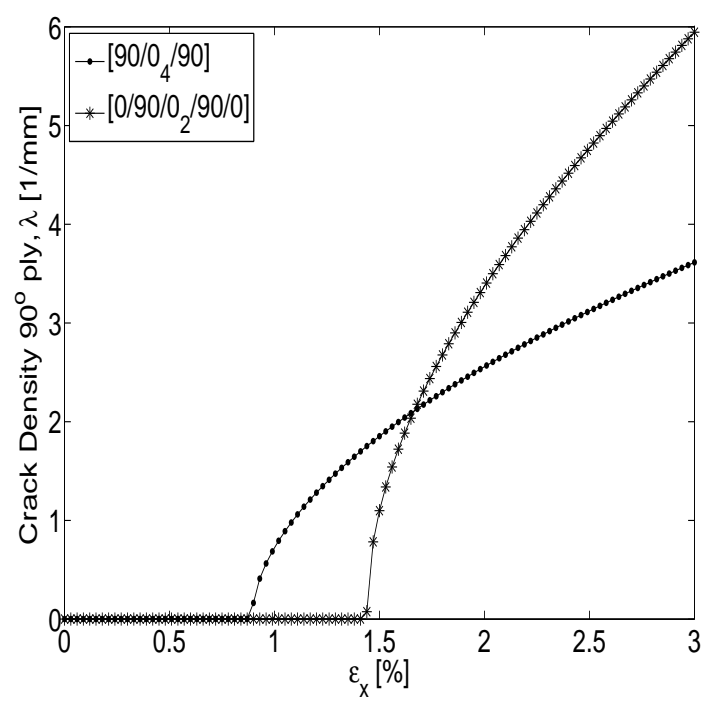

Figure 5.22: Damage evolution in interior vs. surface cracking plies, $\left.\lambda=\lambda\left(\epsilon_{x}\right)\right)$.

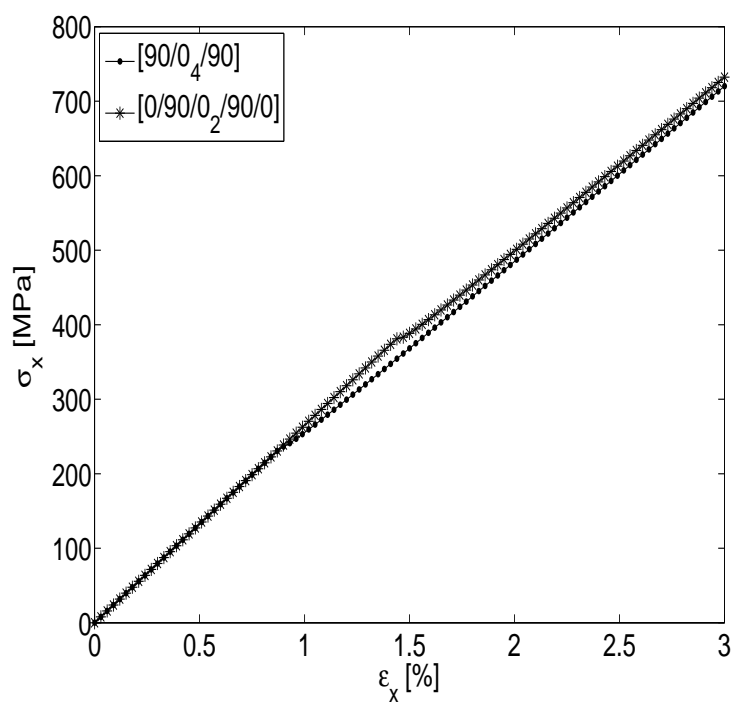

Figure 5.23: Stress-strain behavior of the laminate for interior vs. surface cracking plies.

Due to the formulation of the material model (see Section 2.1.2, 4.1,B) used in the present progressive damage model, the difference in damage onset and evolution for an interior ply, as compared to a surface ply, can be made. This can be seen in Fig. 5.22. As expected, damage starts latter in the interior cracking plies. This is because there is an increase constraining effect of the neighboring plies for the case of the interior cracking plies. Also damage evolution is more abrupt for the interior cracking plies.

The corresponding stress-strain evolution is presented in Fig. 5.23, where again the earlier moment of damage onset for the surface cracking plies can be noticed. The two curves in Fig. 5.23 run parallel after damage onset, which means that the reduction in the elastic modulus of the laminate is not affected by the position of the cracking ply. This fact is confirmed by the $E_{x}-\lambda$ evolution (not shown here) for the two laminate configurations. 
i3) The influence of the properties of the neighboring plies $(k)$

Two cases are considered in this section, in order to analyze the influence of the neighboring $(k)$ plies to damage process in the cracking $(k 0)$ ply:

- the influence of the orientation of the neighboring plies $(k)$

- the influence of the thickness of the neighboring plies $(k)$

Both of these cases gives a measure of the constraining effect of the neighboring plies on damage process in the cracking ply.

\section{i3-1) The influence of the $(k)$ plies orientation}

The testing configuration for this case is $m=1, n=2$ in the originally defined testing LSS, which means a $\left[ \pm \theta / 90_{2} / \pm \theta /\right]$ laminate.

The case is run by considering the orientation $\theta$ of the uncracking plies as parameter. The $\theta$ value can be regarded as a measure of the stiffness of the neighboring plies. Low values of the $\theta$ off-axis angle have been considered for this study case, in order to avoid inducing cracks in the $\pm \theta$ plies.

The loading case for this study case is uniaxial tension.

The parametric study is run for the following values of the orientation angle of the uncracking plies: $\theta=[0,15,30] \mathrm{deg}$.

The influence of the off-axis angle of the uncracking plies on the thermo-elastic properties of the laminate is shown in Fig. 5.24 - 5.27. The $\theta$ angle has a slight influence on the reduction of the normalized Young modulus in Fig. 5.24, a medium influence on the shear modulus in Fig. 5.26, and a considerable influence on the laminate Poisson coefficient and CTE in Fig. 5.25 and Fig. 5.27. It is interesting to notice the reversed effect of the $\theta$ angle on Poisson and shear modulus in Fig. 5.25, 5.26 (decreased reduction with increasing value of $\theta$ ), as compared to the effect on Young modulus in Fig. 5.24 (increased reduction with increasing value of $\theta$ ). Also to be noticed that the rate of reduction (i.e., the slope of the reduction curves) has a linear evolution for the Poisson ration in Fig. 5.25, but is has a nonlinear evolution for the reduction of the Young modulus in Fig. 5.24, for a considered linear variation of the $\theta$ parameter. The effect of $\theta$ angle on the Young modulus seems to be rather exponentially increasing for linearly increasing $\theta$ values. 


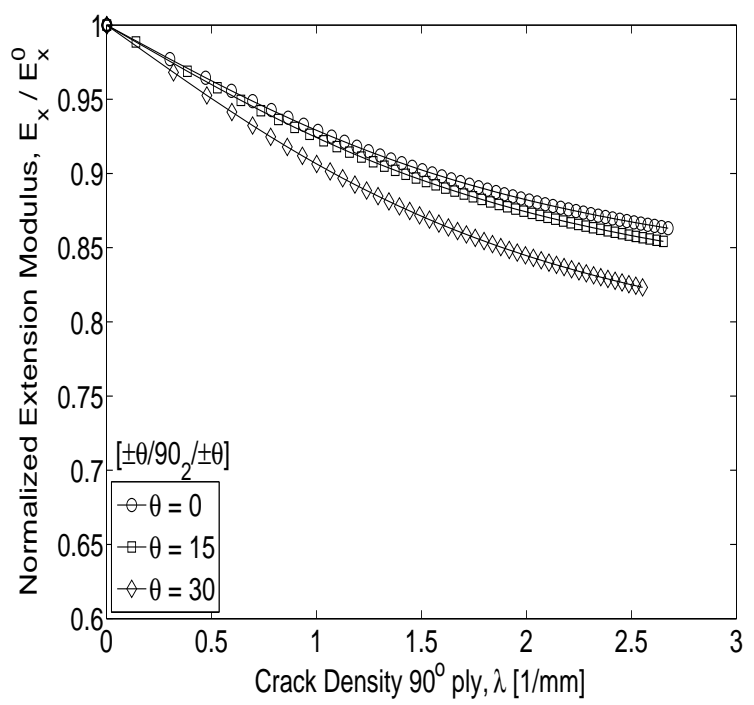

Figure 5.24: The influence of the neighboring plies orientation on the normalized extension stiffness $E_{x} / E_{x}^{0}$ of the laminate.

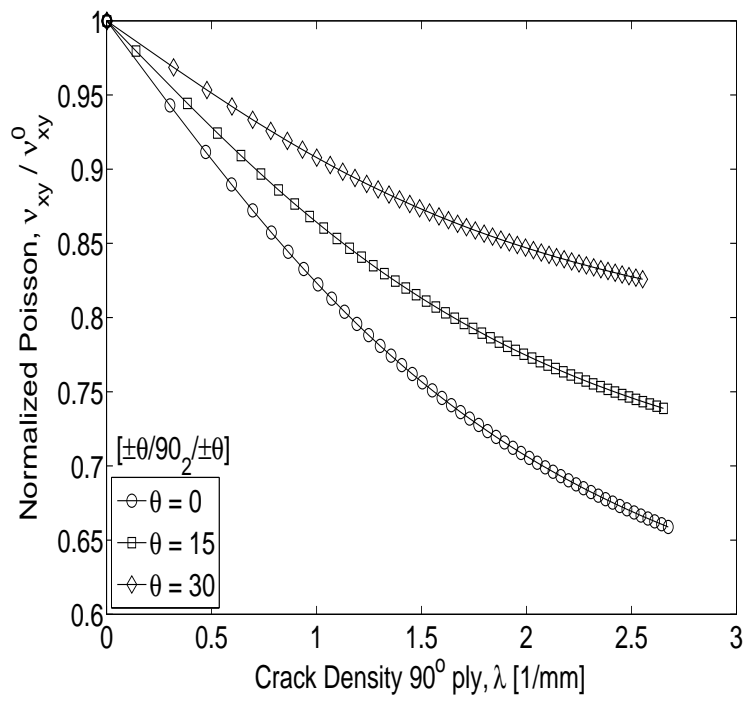

Figure 5.25: The influence of the neighboring plies orientation on the normalized Poisson $\nu_{x y} / \nu_{x y}^{0}$ of the laminate.

The possibility of either increase or decrease of the laminate CTE with damage evolution, in function of the laminate configuration, can be again noticed in Fig. 5.27.

The influence of the orientation $\theta$ of the neighboring plies on damage onset and evolution is presented in Fig. 5.28, 5.29. It can be noticed that the crack evolution curves are clustered in $\lambda-\epsilon$ coordinates in Fig. 5.28, which means a negligible effect of the orientation $\theta$ of the neighboring plies $(k)$ on damage process in the cracking ply $(k 0)$. However, the corresponding damage evolution curves in $\lambda-\sigma$ coordinates in Fig. 5.29 are not clustered together, and this appearance might be misleading if only $\lambda-\sigma$ damage evolution data is analyzed. Actually even the curves in Fig. 5.29 are generated at very close levels of the incrementally applied strain $\epsilon$, and they should be regarded as 'clustered' on the scale of the applied loading. The separation of the curves in Fig. 5.29 in governed not only by the level of the load increment, but also by the material properties and laminate configuration.

The corresponding stress-strain curves are presented in Fig. 5.30, where the very close moment of damage onset is again noticed. A slightly greater reduction in the elastic modulus of the laminate with greater off-axis angle of the uncracking plies can also be observed. This behavior is confirmed by the plot in Fig. 5.24. 


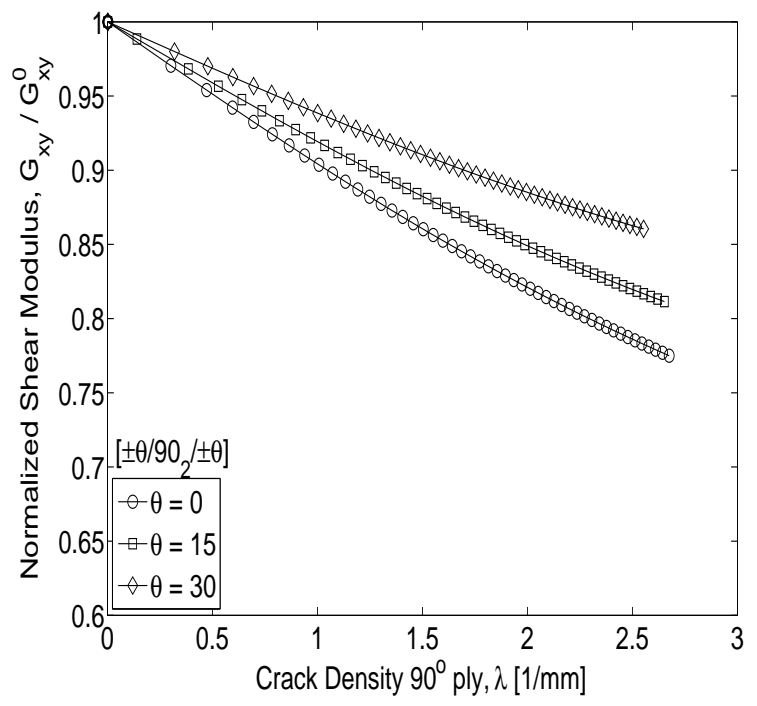

Figure 5.26: The influence of the neighboring plies orientation on the normalized shear modulus $G_{x y} / G_{x y}^{0}$ of the laminate.

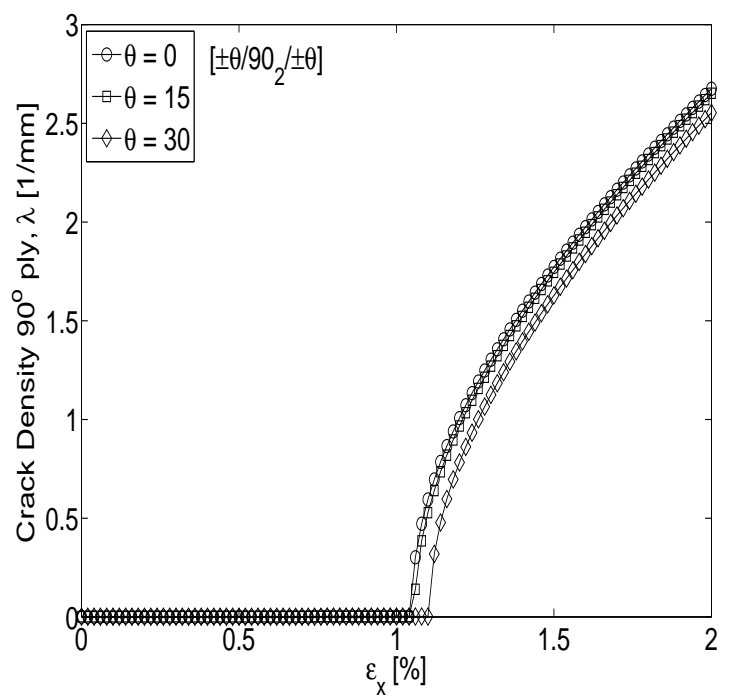

Figure 5.28: The influence of the neighboring plies orientation on the damage process, $\lambda=\lambda\left(\epsilon_{x}\right)$.

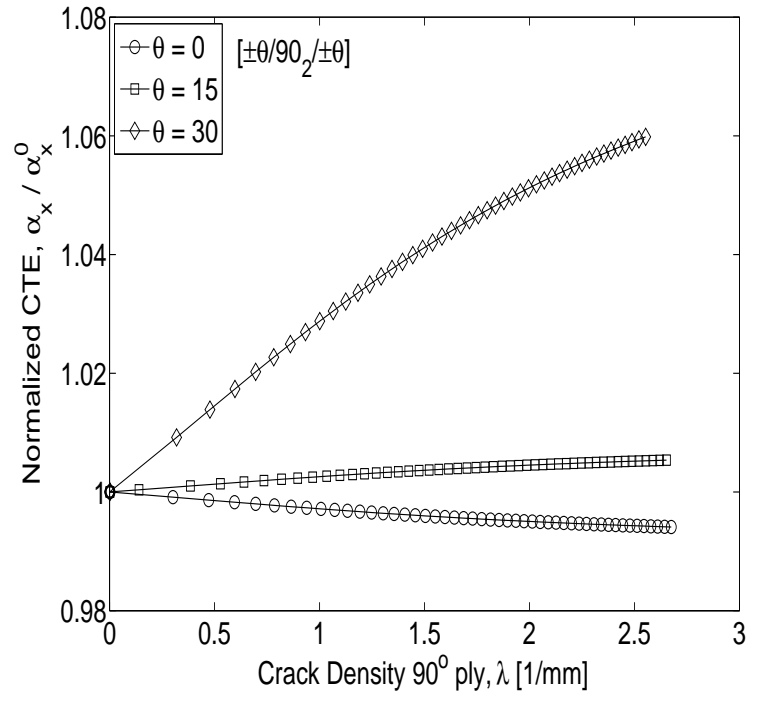

Figure 5.27: The influence of the neighboring plies orientation on the normalized CTE $\alpha_{x} / \alpha_{x}^{0}$ of the laminate.

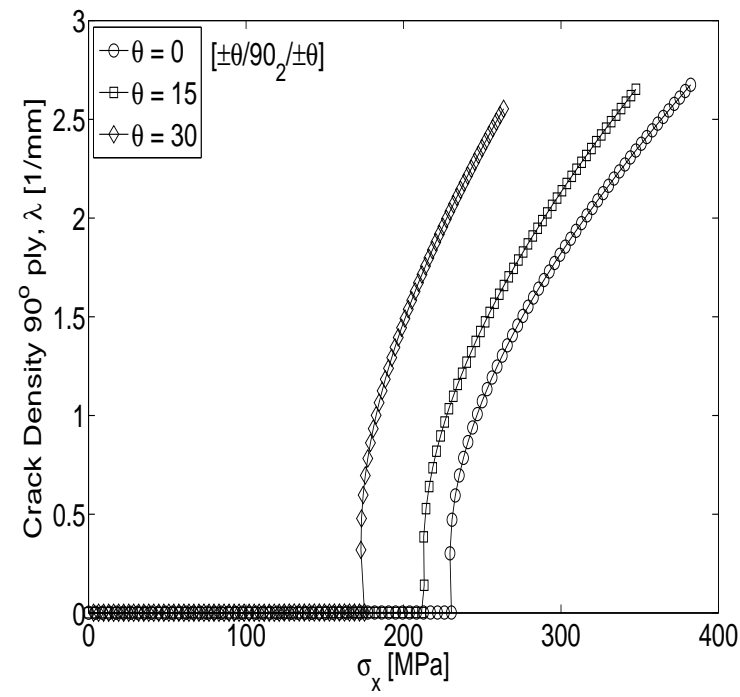

Figure 5.29: The influence of the neighboring plies orientation on the damage process, $\lambda=\lambda\left(\sigma_{x}\right)$. 


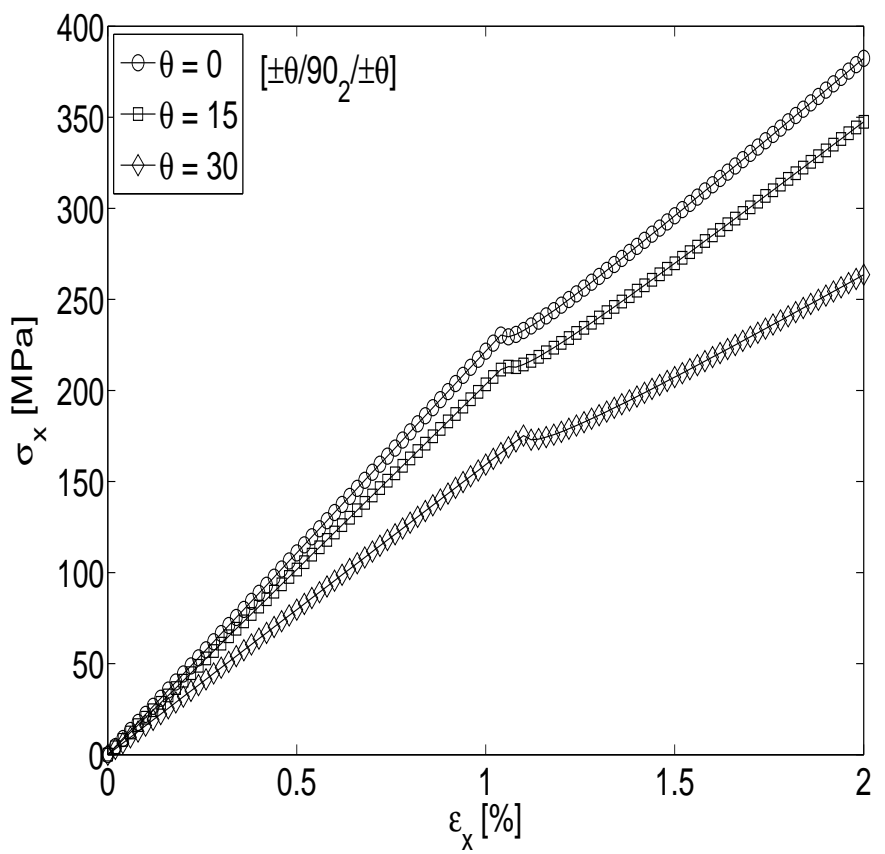

Figure 5.30: The influence of the neighboring plies orientation on the stress-strain behavior of the laminate. 
i3-2) The influence of the (k) plies thickness

The testing configuration for this case is $\theta=0, n=2$, which means a $\left[0_{m} / 90_{2} / 0_{m}\right]$ laminate.

The study case is run by considering the thickness of the cracking ply (i.e., $m$ in the definition of LSS) as parameter.

The loading case for this study case is uniaxial tension.

The parametric study is run for the following values of number of plies making the uncracking $0_{m}$ stack: $m=[1,2,3]$.

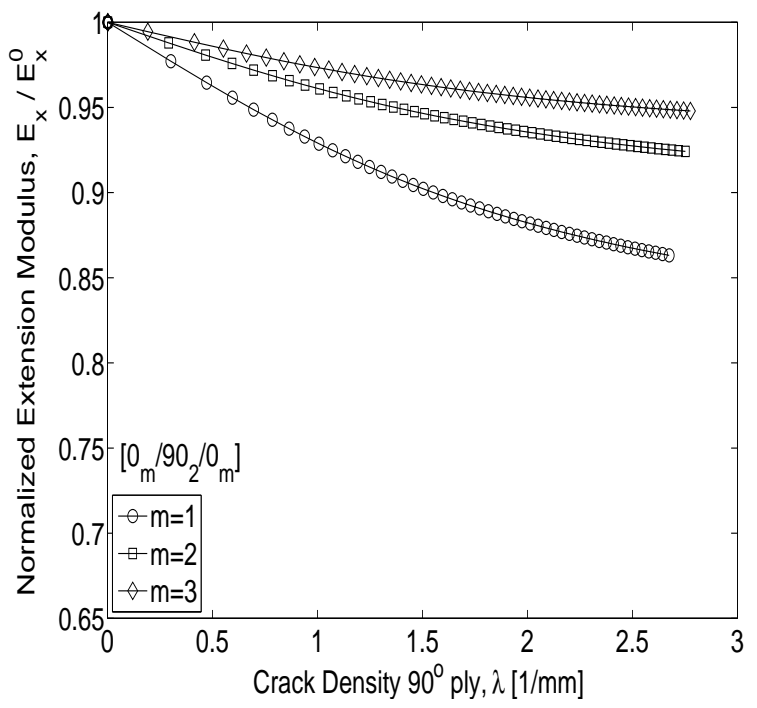

Figure 5.31: The influence of the neighboring plies thickness on the normalized extension stiffness $E_{x} / E_{x}^{0}$ of the laminate.

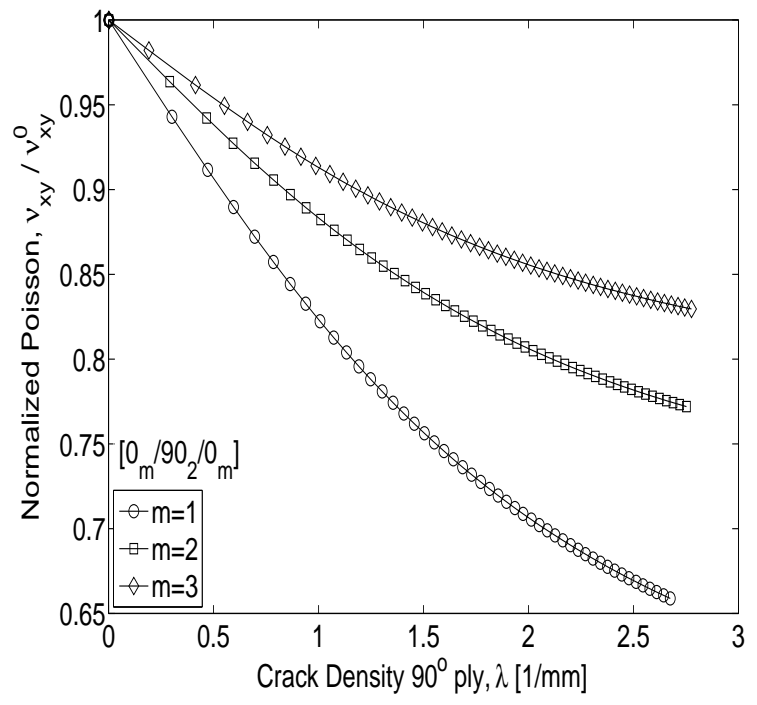

Figure 5.32: The influence of the neighboring plies thickness on the normalized Poisson $\nu_{x y} / \nu_{x y}^{0}$ of the laminate.

The general trends and behavior noticed for the previous case of the constraining effect of the neighboring plies for varying $\theta$ angle also apply in the present case for varying thickness of the neighboring plies. A decreasing drop in elastic properties with increasing thickness of the neighboring plies can be noticed in Fig. 5.31 - 5.33. Unlike the case of the angle $\theta$, where opposite influence could be noticed for the case of $E_{x}$ as compared to $\nu_{x y}, G_{x y}$, all three elastic constants $E_{x}, \nu_{x y}, G_{x y}$ in Fig. 5.31 - 5.33 are influenced in the same way by the thickness of the uncracking plies. Another noticeable difference is with regard to the behavior of the laminate CTE in Fig. 5.34, where only decreasing values of $\alpha_{x}$ are recorded. A negligible influence of neighboring plies thickness on damage evolution can be noticed in Fig. 5.35. 


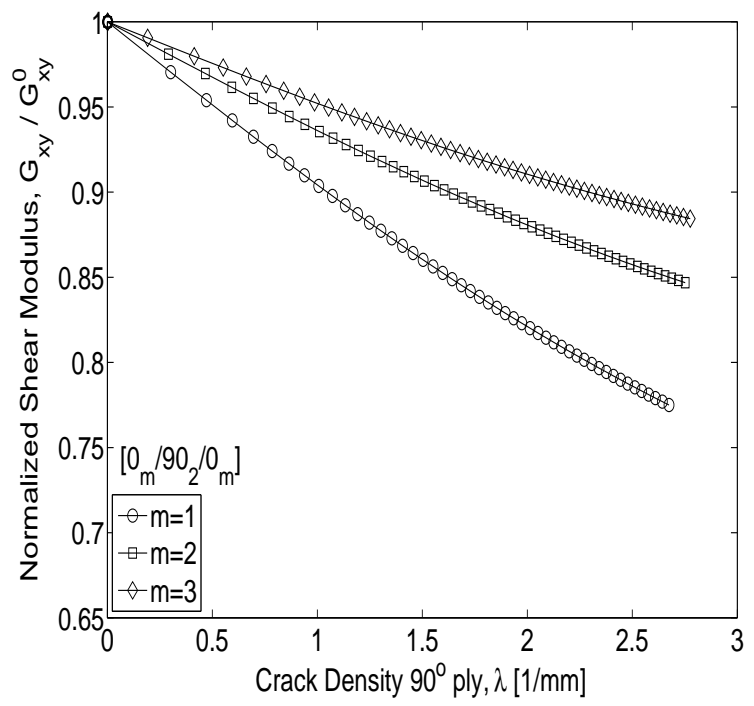

Figure 5.33: The influence of the neighboring plies thickness on the normalized shear modulus $G_{x y} / G_{x y}^{0}$ of the laminate.

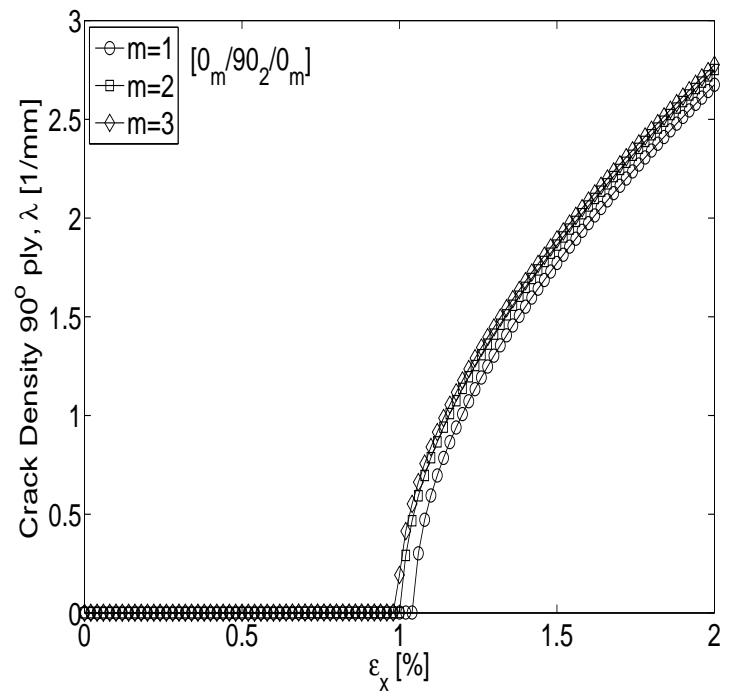

Figure 5.35: The influence of the neighboring plies thickness on the damage process, $\lambda=\lambda\left(\epsilon_{x}\right)$.

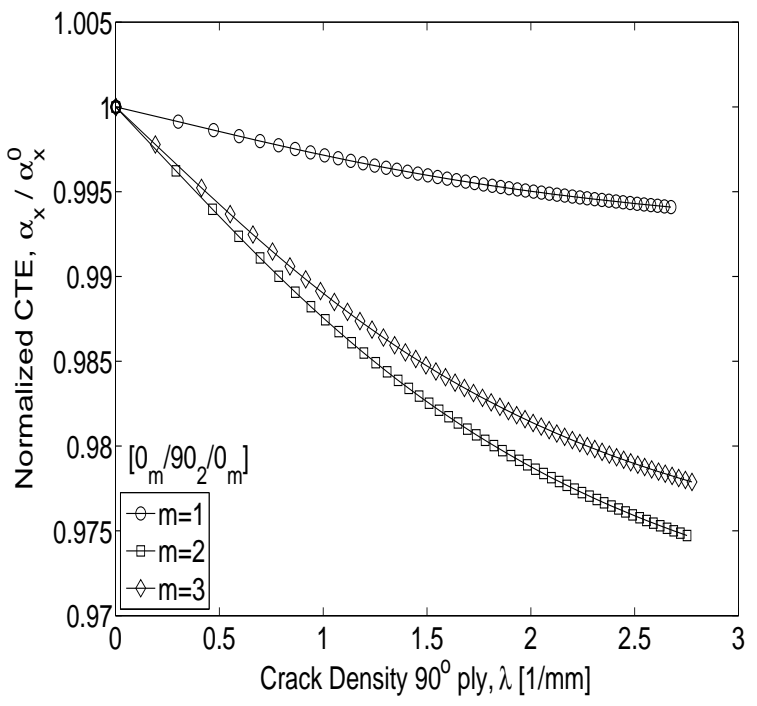

Figure 5.34: The influence of the neighboring plies thickness on the normalized CTE $\alpha_{x} / \alpha_{x}^{0}$ of the laminate.

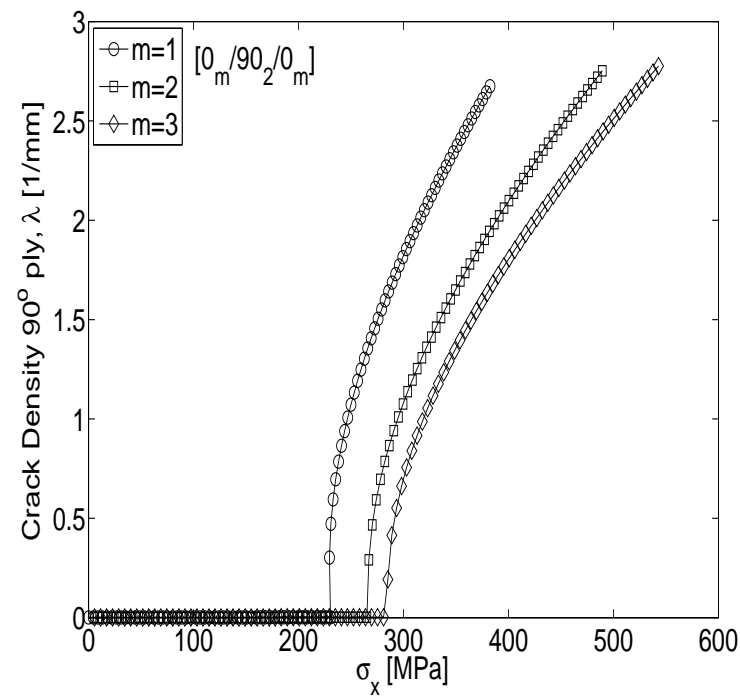

Figure 5.36: The influence of the neighboring plies thickness on the damage process, $\lambda=\lambda\left(\sigma_{x}\right)$. 


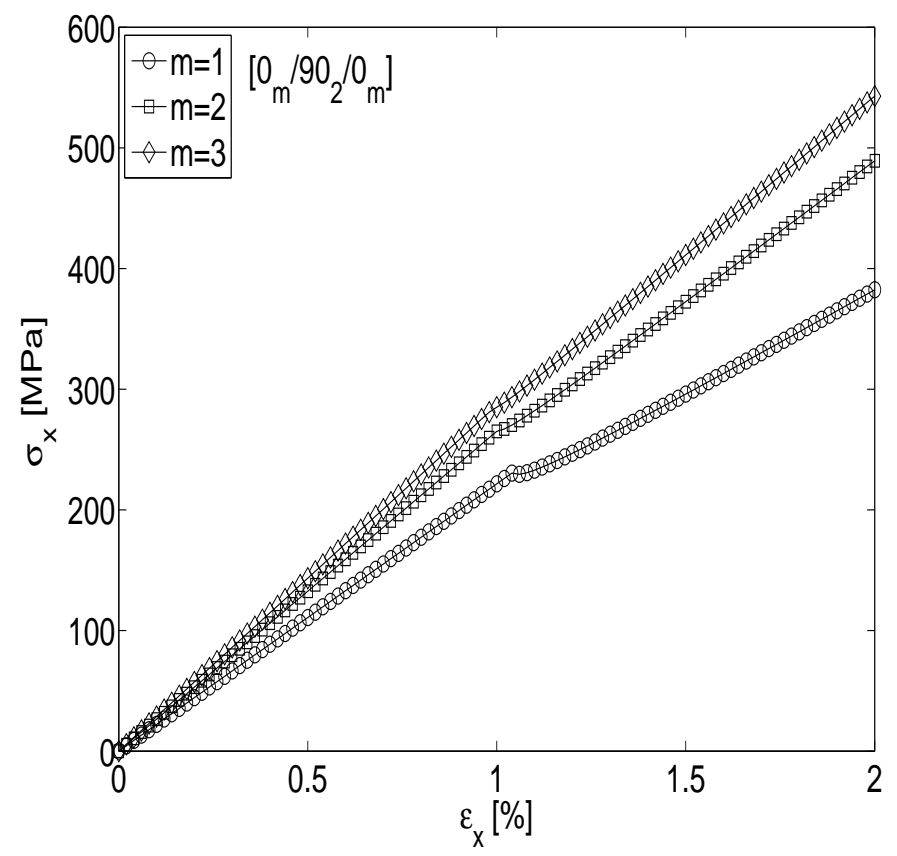

Figure 5.37: The influence of the neighboring plies thickness on the stress-strain behavior of the laminate. 


\subsubsection{Extension vs. Bending loading}

A first analysis of the model output for the flexural deformation case is performed in this section. Comparative studies for the same LSS separately loaded in extension and bending are performed. The analytical model trends are recorded and analyzed.

The comparative extension-bending has two sections:

- first, damage evolution for the considered extension and bending cases is analyzed, and

- second, the reduction in the thermo-elastic material properties relevant to both membrane and flexural behavior of the laminate is analyzed.

i1) Comparative analysis of damage evolution for extension and bending loading

The LSS for this study is $\left[0 / 90_{2} / 0 / 90_{3} / 0\right]_{S}$, selected such that to provide relevant information regarding the capabilities of the analytical model.

The loading for the two cases is of displacement control type (see Section 4.7.1): incremental strain $\epsilon_{x}$ for extension, and incremental curvature $\kappa_{x}$ for bending. Due to the selected LSS and considered loading scenarios, only matrix cracking in the $90^{\circ}$ plies of the laminate develops.

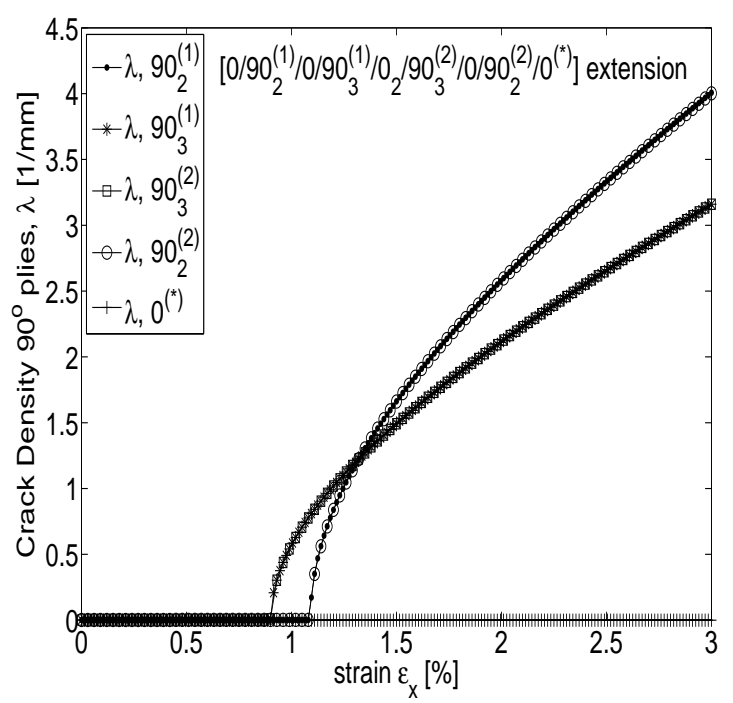

Figure 5.38: Damage evolution under extension loading, $\lambda=\lambda\left(\epsilon_{x}\right)$

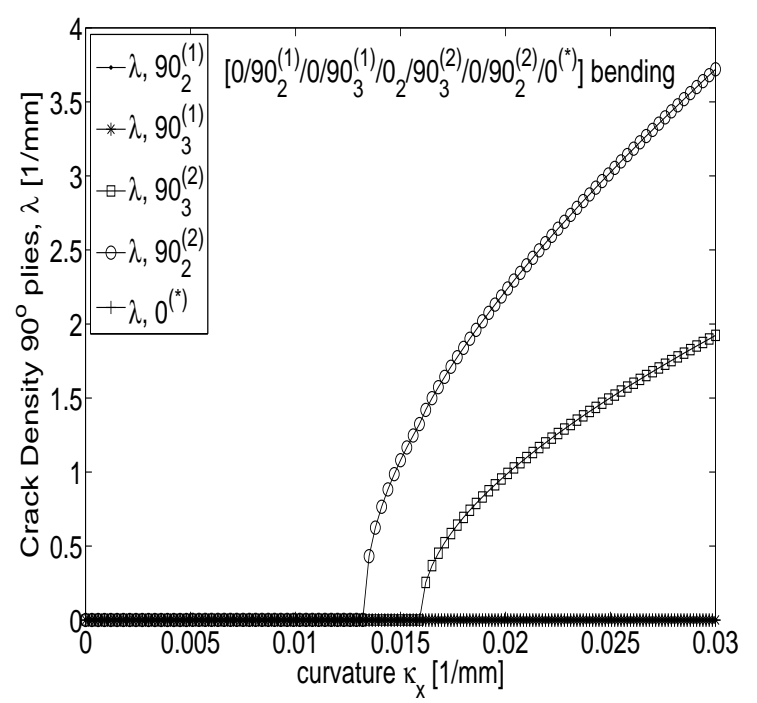

Figure 5.39: Damage evolution under bending loading, $\lambda=\lambda\left(\kappa_{x}\right)$

The damage process for the extension loading is presented in Fig. 5.38, and for bending loading is presented in Fig. 5.39. It can be observed that, for extension loading case, matrix 
cracking first initiates in the thicker $90_{3}$ ply, and then in the thinner $90_{2}$ ply, this model prediction being according with the expected physical behavior of the material. Also as expected, the damage process in the top $90_{2}$ and $90_{3}$ plies (on the symmetric part of the laminate) is identical to the bottom $90_{2}$ and $90_{3}$ plies. The cracking process in one of the 0 plies is additionally plotted in Fig. 5.38, which shown no matrix cracking during extension loading, as expected.

On the contrary, for bending loading case damage initiates first in the thinner $90_{2}$ ply, and then in the thicker $90_{3}$ ply. This is because of the outer position of the $90_{2}$ ply inside of the laminate under bending deformation, which translates in higher ply strain for the same applied curvature $\kappa$ on the laminate. According with the expected behavior of the damage process, matrix cracking is not triggered at all in the mirror $90_{2}$ and $90_{3}$ plies, due to the fact that these plies are under compressive strain under the considered bending loading on the laminate. As in the case of extension loading, matrix cracking does not take place in the 0 ply for bending loading.

i2) Comparative analysis of reduced material properties for extension and bending loading

A simpler LSS, namely $\left[0 / 90_{2} / 0\right]_{S}$ has been selected for this comparative analysis. Similarly to the previous case, matrix cracking develops only in the $90_{2}$ plies. A direct correspondence between extension-bending loading can not be done because different plies of the laminate loaded in bending will experience different strain levels according with their position inside of the laminate. Two levels of loading have been considered for the study in this section: $\epsilon_{x}=0.03$ and $\kappa_{x}=0.06 \mathrm{~mm}^{-1}$, such that the level of matrix cracking in the cracking $90_{2}$ is almost the same fort he two loading cases. Qualitative and quantitative comparison is done based on the results obtain from the two loading cases.

The evolution of thermo-elastic properties for the two loading cases is presented in Fig. $5.40-5.47$. It can be seen in Fig. 5.40, 5.41 that the reduction in the normalized extension and bending moduli along $x$ direction of the laminate $\left(E_{x} / E_{x}^{0}\right.$ and $B_{x} / B_{x}^{0}$, respectively) is double for the extension loading case $\epsilon_{x}$ as compared to the bending loading case $\kappa_{x}$. This is expected because of the fact that both top and bottom $90_{2}$ plies of the laminate are affected by matrix cracking for $\epsilon_{x}$ loading, and only the top $90_{2}$ ply is affected for $\kappa_{x}$ loading. Regarding the evolution of the normalized extension and bending moduli along $y$ direction of the laminate $\left(E_{y} / E_{y}^{0}\right.$ and $B_{y} / B_{y}^{0}$, respectively), it can be observed that these are 


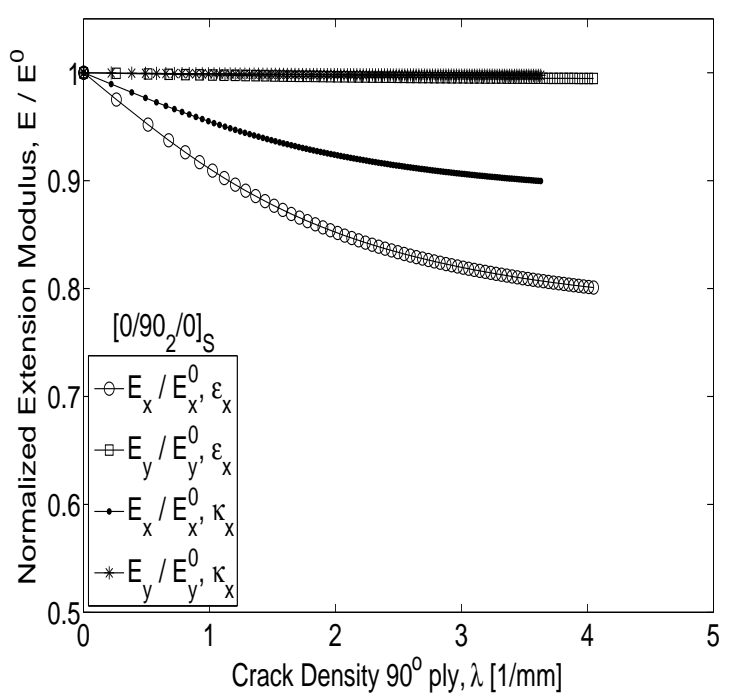

Figure 5.40: Extension vs. bending loading: reduction of the normalized extension stiffness $E / E^{0}$ of the laminate.

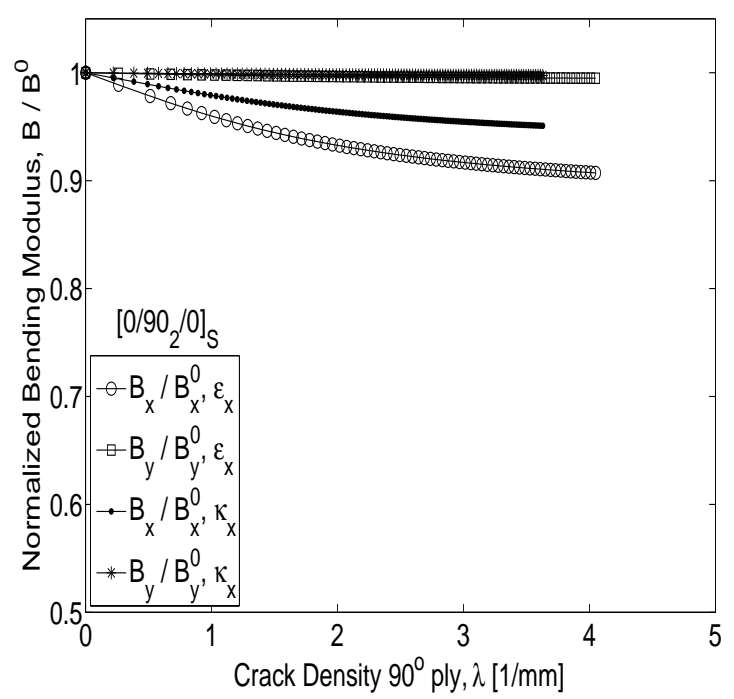

Figure 5.41: Extension vs. bending loading: reduction of the normalized bending stiffness $B / B^{0}$ of the laminate.

not affected by matrix cracking in the $90_{2}$ plies, regardless the loading case. This is expected as well, due to the fact that for the considered particular LSS matrix cracking affects the stiffness properties only along that direction of the laminate where cracks are transverse to (i.e., the $x$ direction of the laminate), and the COD of the cracks plays a role in reducing the material stiffness along that direction. For the considered loading cases there is no COD along $y$ direction, and consequently the is no stiffness reduction along $y$ direction, even if the $90_{2}$ plies have cracks.

However, for the laminate Poisson evolution in Fig. 5.42, both $\nu_{x y}$ and $\nu_{y x}$ are reduced by damage in the $90_{2}$ plies. The greater reduction is for the direct Poisson coefficient $\nu_{x y}$, corresponding to the direction of the applied loading that produces matrix cracking.

Similar trends can be noticed for the reduction in the shear modulus $G_{x y}$ in Fig. 5.44 and torsional modulus in Fig. 5.45. The reduction in properties for extension loading as double the reduction in properties for bending loading.

Regarding the coupling extension-bending modulus in Fig. 5.43, this material properties in not plotted normalized to the initial (undamaged) value, because this initial value is zero due to the symmetric LSS considered for this study case. It can be observed that the coupling modulus remains zero for extension loading $\epsilon_{x}$, even if there is damage inside of the $90_{2}$ plies of the laminate. This is because matrix cracking develops consecutively in 


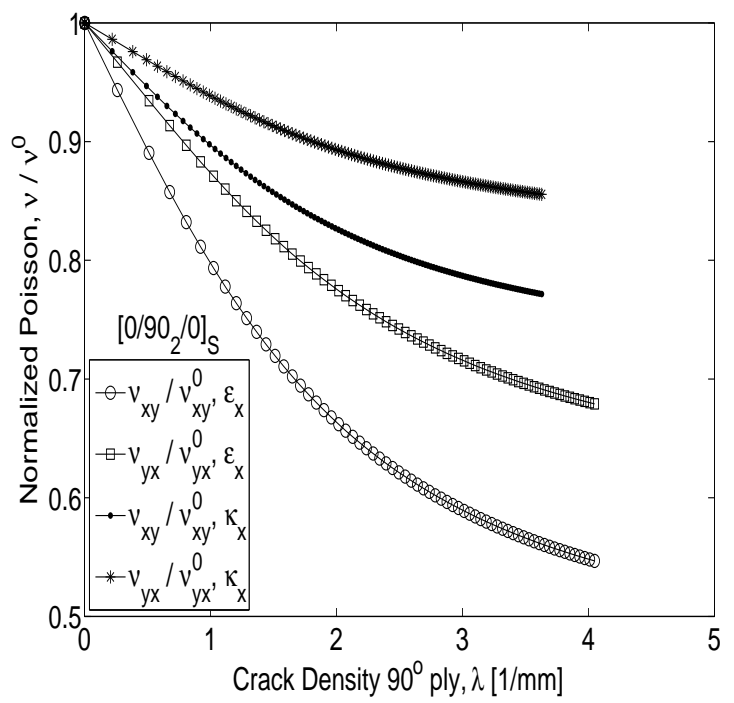

Figure 5.42: Extension vs. bending loading: reduction of the normalized Poisson $\nu / \nu^{0}$ of the laminate.

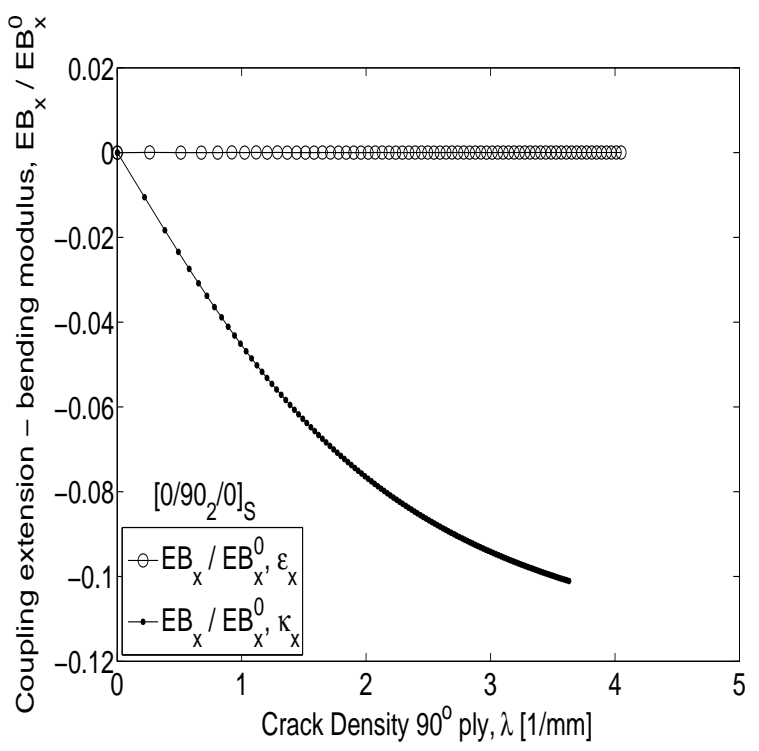

Figure 5.43: Extension vs. bending loading: evolution of the extensionbending coupling term, $E B_{x} / E B_{x}^{0}$, of the laminate.

both top and bottom $90_{2}$ plies, and the laminate preserves its material symmetry. However, non-zero extension-bending coupling appears for the case of bending loading $\kappa_{x}$. This is because matrix cracking develops only in the top $90_{2}$ plies and the laminate loses its material symmetry.

The same kind of comparative reasoning applies for the case of laminate CTE in Fig. 5.46, 5.47 The change in the extension CTE is more pronounced for the case of extension loading and for the case of CTE along $x$ direction of the laminate, $\alpha_{x}$. The change in laminate CTE along $y$ direction of the laminate, $\alpha_{y}$, is negligible, at least for the laminate configuration and properties considered in this example.

Regarding the change in bending CTE $\alpha_{B}$ in Fig. 5.47, it can be noticed that it remains zero for extension loading (due to preserving the material symmetry of the laminate), while it becomes different from zero for bending loading (due to losing the material symmetry of the laminate). A more pronounced change in the bending CTE is recorded for the $\alpha_{B x}$ corresponding to the loading direction $\kappa_{x}$. 


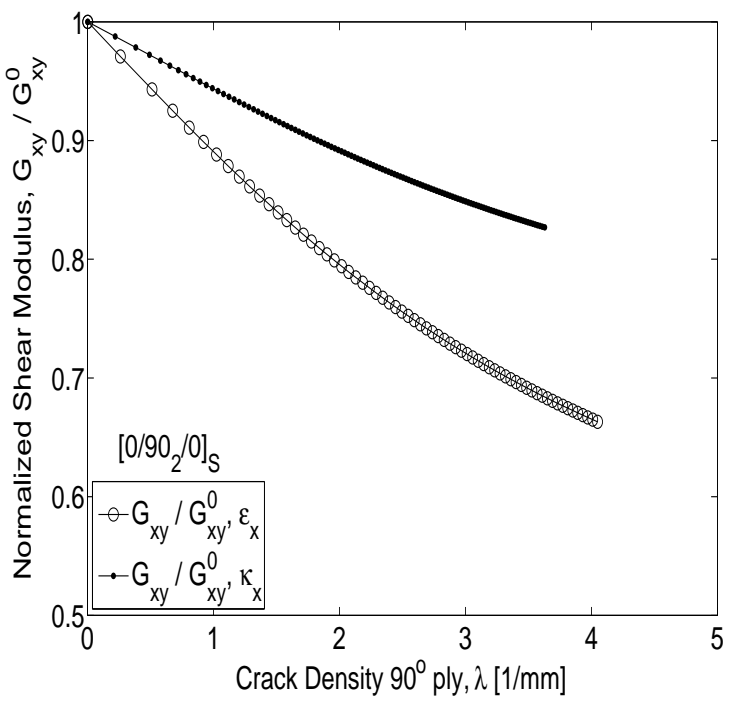

Figure 5.44: Extension vs. bending loading: reduction of the normalized shear modulus $G_{x y} / G_{x y}^{0}$ of the laminate.

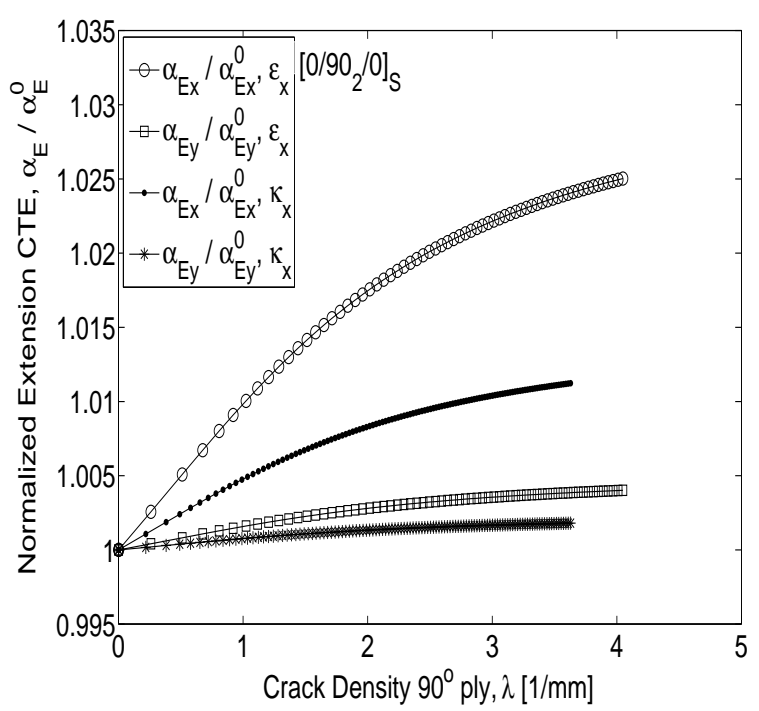

Figure 5.46: Extension vs. bending loading: reduction of the normalized extension $\mathrm{CTE}, \alpha_{E} / \alpha_{E}^{0}$, of the laminate.

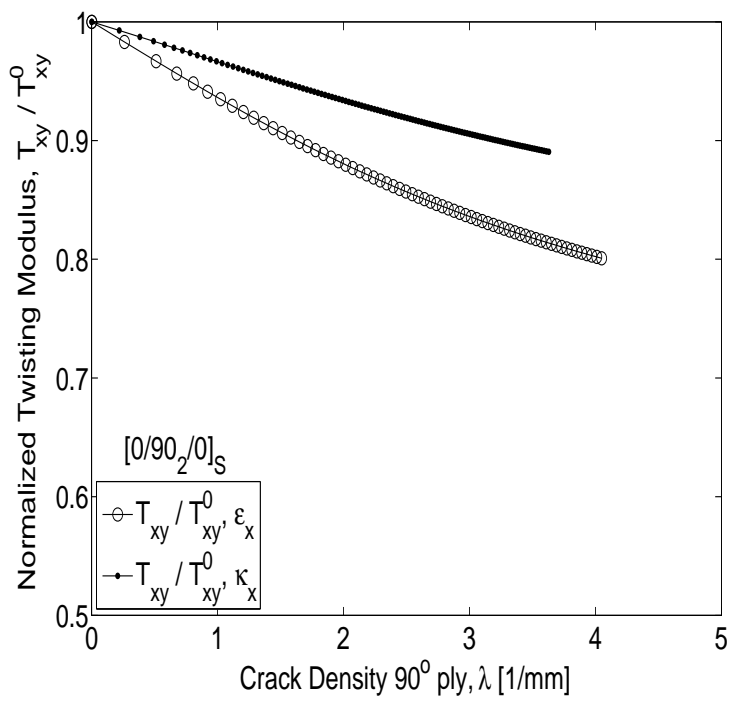

Figure 5.45: Extension vs. bending loading: reduction of the normalized twisting modulus $T_{x y} / T_{x y}^{0}$ of the laminate.

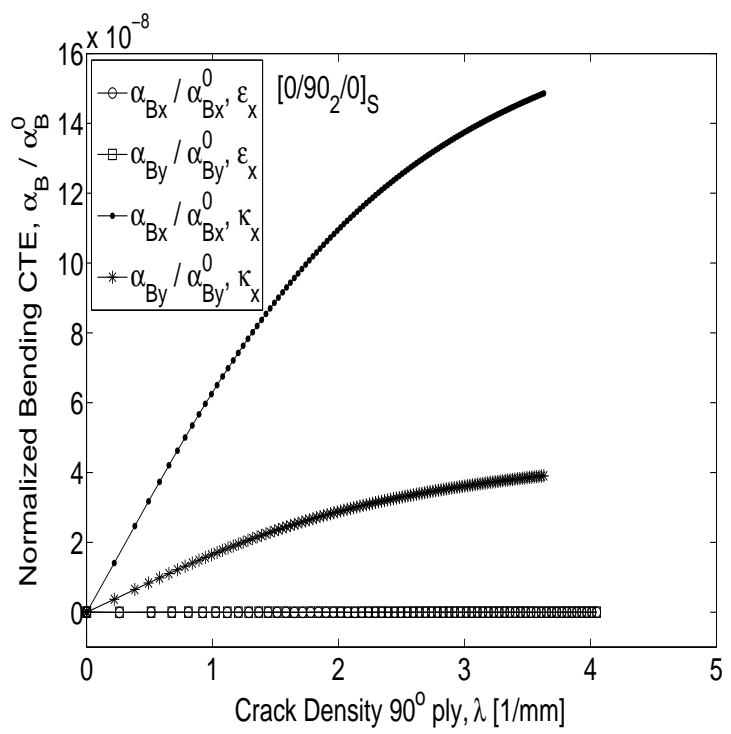

Figure 5.47: Extension vs. bending loading: evolution of the bending $\mathrm{CTE}, \alpha_{B}$, of the laminate. 


\subsubsection{Combined loading}

After separate cases of extension and bending loading have been analyzed in the previous sections, combined loading is regarded in the present section. Two cases of combined loading are considered and analyzed in the following:

- combined extension-bending loading $\left(\epsilon_{x}-\kappa_{x}\right)$

- biaxial extension loading $\left(\epsilon_{x}-\epsilon_{y}\right)$

i1) The case of extension-bending combined loading $\left(\epsilon_{x}-\kappa_{x}\right)$

The LSS considered for this combined loading case is $\left[0 / 90_{2} / 0 / 90_{3} / 0\right]_{S}$, identical with the one consider for the case analyzed in Section 5.1.2 i1). The loading case is simultaneous extension $\epsilon_{x}$ and bending $\kappa_{x}$, under displacement control, as it is described in Section 4.7.1. The ration of the two applied incremental deformations is $\kappa_{x}=0.5 \cdot \epsilon_{x}$ for the present study. Any other loading ratio can be considered.

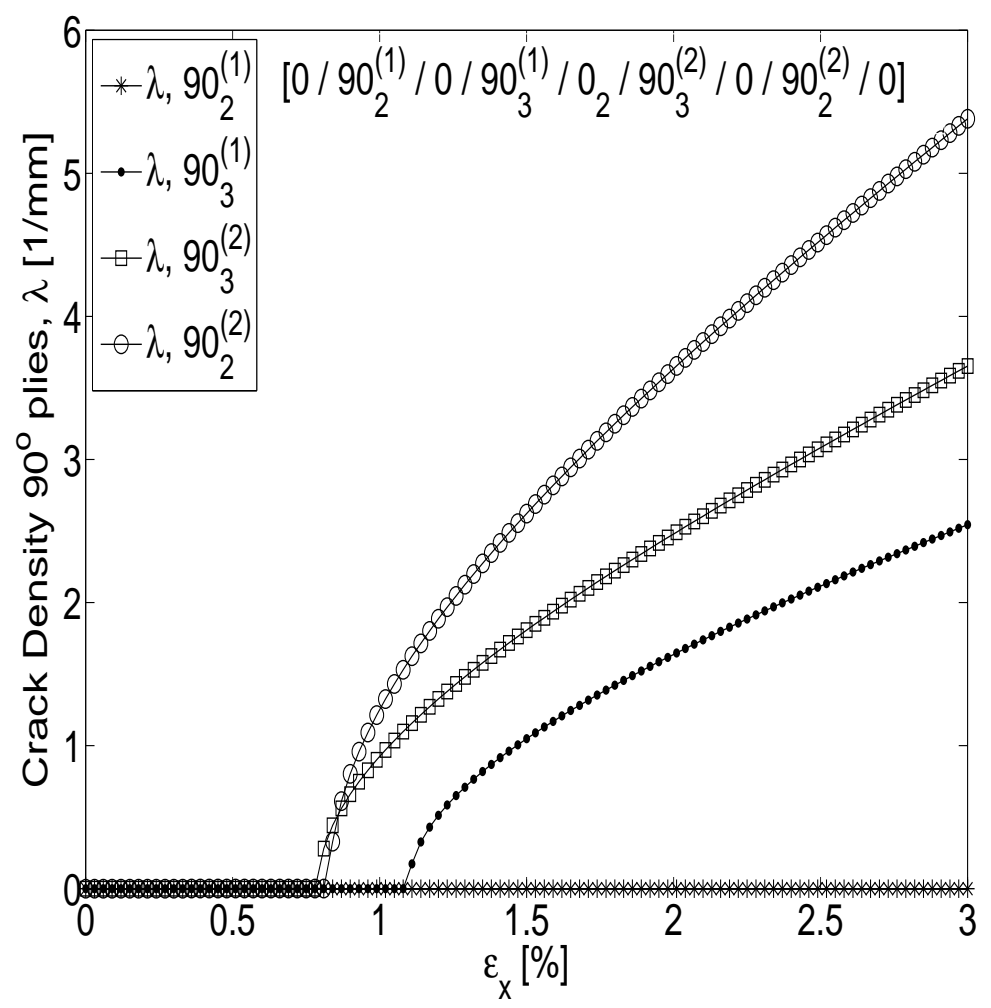

Figure 5.48: Damage process for the case of extension-bending combined loading, $\left(\epsilon_{x}-\kappa_{x}\right)$. 
The matrix cracking evolution under combined extension-bending loading is presented in Fig. 5.48. The plies succession $\left[0 / 90_{2}^{(1)} / 0 / 90_{3}^{(1)} / 0_{2} / 90_{2}^{(2)} / 0 / 90_{3}^{(2)}\right]$ represents the considered LSS written without using the symmetry of the plies stacking. The superscripts (1) denotes plies at the bottom of the LSS (which would be under compression if only bending deformation was applied), and the superscripts (2) denotes plies at the top of the LSS (which would be under tension if only bending deformation was applied).

It can be observed that the cracking process initiates almost simultaneous in the top $90_{2}^{(2)}$ and $90_{3}^{(2)}$ plies. It is interesting at this moment to make a qualitative comparison with the damage onset for the cases of separate extension and separate bending presented in Fig. 5.38 and Fig. 5.39, respectively. For the pure extension case in Fig. 5.38 damage initiated first in the $90_{3}$ plies followed by the $90_{2}$ plies, due to the thickness effect of the cracking plies. For the pure bending case in Fig. 5.39 damage initiated first in the outer $90_{2}^{(2)}$ ply followed by the $90_{3}^{(2)}$ ply, due to the position of the plies inside of the laminate under bending. A combination of the two effects can be noticed in Fig. 5.48 for the combined extension-bending deformation, when damage in the top plies initiates almost simultaneous.

Regarding the damage process in the bottom $90_{2}^{(1)}$ and $90_{3}^{(1)}$ plies, it can be observed that there is matrix cracking in the $90_{3}^{(1)}$ ply, and there is no matrix cracking in the $90_{2}^{(1)}$ ply. Even if the bottom plies experience negative strains due to the bending deformation, they also experience positive strain due to the extension deformation. This combination is more dominated by the extension deformation for the $90_{3}^{(1)}$ ply, and it is more dominated by the bending deformation for the $90_{2}^{(1)}$, because of the position these plies have inside of the laminate. This is the reason why matrix cracking appears in the $90_{3}^{(1)}$ ply and it does not appear in the $90_{2}^{(1)}$ ply.

The ability of the analytical model to reproduce the damage process in Fig. 5.38, 5.39 and 5.48 according to the expected physical material behavior proved the predictive capabilities of the analytical progressive damage model.

i2) The case of biaxial extension loading $\left(\epsilon_{x}-\epsilon_{y}\right)$

Two laminate configurations are considered for this combined loading case: [0/90/0] and $[0 / 90 / 0 / 90 / 0]$. The biaxial loading is consider under displacement control case (see Section 4.7.1), with a loading ratio $\epsilon_{y}=1 \cdot \epsilon_{x}$. It is expected that, due to biaxial loading $\epsilon_{x}-\epsilon_{y}$, matrix cracking will develop in all plies of each laminate. 


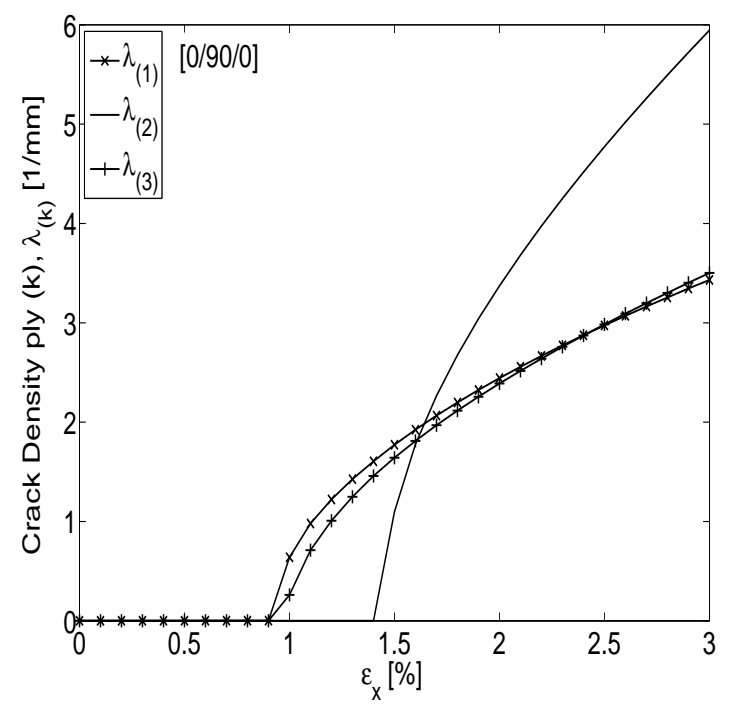

Figure 5.49: Damage process for the case of biaxial extension loading, $\left(\epsilon_{x}-\epsilon_{y}\right)$, [0/90/0] LSS.

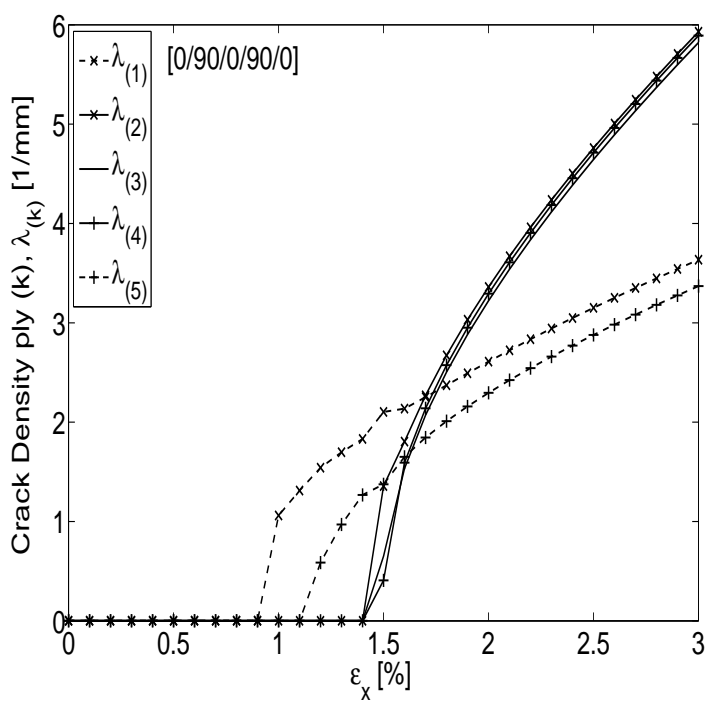

Figure 5.50: Damage process for the case of biaxial extension loading, $\left(\epsilon_{x}-\epsilon_{y}\right)$, [0/90/0/90/0] LSS.

The predicted damage evolution for the two laminate configurations is presented in Fig. 5.49, 5.50. The plies are numbered started with (1) to the number of plies in each laminate, according to the regular convention in CLT.

For the $[0 / 90 / 0]$ laminate in Fig. 5.49, it can be seen that damage starts first in the surface $0^{\circ}$ plies, and then in the interior $90^{\circ}$ ply. This is due to the constraining effect of the neighboring plies undergone by the $90^{\circ}$ ply, which is not active for the surface $0^{\circ}$ plies.

It can be also noticed that the crack evolution in the surface $0^{\circ}$ plies is almost identical. The crack evolution in these two plies has to be identical, because they are subjected to the same conditions. However, the slight difference between the two curves corresponding to the $0^{\circ}$ plies in Fig. 5.49 are due to the searching algorithm for the cracking plies, and due to the increment in the applied loading $\epsilon_{x}-\epsilon_{y}$. The iterative procedure (see Section 4.3, 4.6) searches for one cracking ply at a loading moment, and when this cracking ply is identified, the damage level is increased in this ply. For the [0/90/0] laminate configuration both top and bottom plies have the same conditions for matrix cracking, but only one of them is identified as a cracking ply at a certain moment. It is nevertheless true that after one of the plies is identified as a cracking ply, the iterative procedure further searches for other cracking plies at the same load level, or for repeated matrix cracking in the same ply at the same load level. However, once the damage increment is applied for one single ply among the two 
plies equally meeting the conditions for damage growth at a given load level, a momentary unbalance appear between the two identical plies. For the laminate configuration in Fig. 5.49 this unbalance is small and it is adjusted by the iterative procedure at subsequent load increments.

For the $[0 / 90 / 0 / 90 / 0]$ laminate in Fig. 5.50, the same observation of damage initiating first in the surface $0^{\circ}$ plies stands. The interior $90 / 0 / 90$ plies are under the same conditions, and they undergo identical damage process. However, the spurious difference between the surface $0^{\circ}$ plies is more obvious for this case. It is expected that the two surface $0^{\circ}$ plies also undergo identical damage process. 


\subsubsection{In-situ R-curve behavior}

The model results and tendencies presented in Section 5.1.1, 5.1.2, 5.1.3 do not include the in-situ $R$-curve behavior of the laminated composite, which can appear due to the fiber bridging effect during crack multiplication process. The $\mathrm{R}$-curve behavior has been phenomenologically explained and analytically modeled in Section 2.2.1, 4.4.3. If, according to the factors presented in Section 4.4.3 among which the governing one is the fibers misalignment, the fiber bridging effect takes place during matrix cracking, the induced $\mathrm{R}$-curve behavior can modify the tendencies presented in the previous sections.

As a reminder, there are three material parameters describing the in-situ $\mathrm{R}$-curve behavior of intra-laminar matrix cracking in the present model:

- $\beta_{\lambda}^{\text {ref }}$ : describes the $\mathrm{R}$-curve effect of progressive damage, for a certain reference thickness $\left(t=t_{r e f}\right)$ of the cracking ply, on the critical ERR for matrix cracking $G_{I C}$.

The influence of $\beta_{\lambda}^{\text {ref }}$ parameter is modeled by eq. (4.25), and is is schematically depicted in Fig. 4.10.

- $\beta_{\mathbf{0}}^{\mathbf{t}}$ : describes the in-situ effect (i.e., the influence of the thickness of the cracking ply) on the initiation value of the critical ERR for matrix cracking, $G_{I C, 0}$, for thickness of the cracking ply other than the reference thickness $\left(t \neq t_{\text {ref }}\right)$.

The influence of $\beta_{0}^{t}$ parameter is modeled by eq. (4.27), and is is schematically depicted in Fig. 4.11.

- $\beta_{\lambda}^{\mathbf{t}}$ : describes both the in-situ influence of the ply thickness and $\mathrm{R}$-curve influence of the progressive damage on the critical ERR for matrix cracking $G_{I C}$, for thickness of the cracking ply other than the reference thickness $\left(t \neq t_{r e f}\right)$.

The influence of $\beta_{\lambda}^{t}$ parameter is modeled by eq. (4.28), and is is schematically depicted in Fig. 4.12.

A model setup with $\beta_{\lambda}^{\text {ref }}=0, \beta_{0}^{t}=1, \beta_{\lambda}^{t}=1$ simulates a progressive damage without any effect of the in-situ $\mathrm{R}$-curve effect, which corresponds to a perfect longitudinal alignment of the reinforcing fibers inside of individual plies of the laminate.

The influence of the in-situ $\mathrm{R}$-curve effect, i.e., the influence of the $\beta_{\lambda}^{\text {ref }}, \beta_{0}^{t}, \beta_{\lambda}^{t}$ material parameter, on the output of the analytical model is the focus of this section. For this, two simple laminate configurations are considered in the following. First, a [0/90/0] LSS is used as reference configuration $\left(t=t_{r e f}\right.$ for the cracking $90^{\circ}$ ply), in order show the influence 
of $\beta_{\lambda}^{\text {ref }}$ parameter. The initiation value of the critical ERR at the reference thickness (see eq. (4.25)) is considered $G_{I C, 0}^{r e f}=0.2 \mathrm{~N} / \mathrm{mm}$ for the following study.

Second, a $\left[0 / 90_{4} / 0\right]$ LSS is used to show the influence of the $\beta_{0}^{t}$ and $\beta_{\lambda}^{t}$ parameters, at thickness of the cracking ply $t \neq t_{r e f}$. The loading case is uniaxial tension $\epsilon_{x}$ for both laminates.

The study is run for the following values of the in-situ $\mathrm{R}$-curve parameters: $\beta_{\lambda}^{\text {ref }}=$ $[0,4,8]$ deg., $\beta_{0}^{t}=[1,2], \beta_{\lambda}^{t}=[1,2]$.

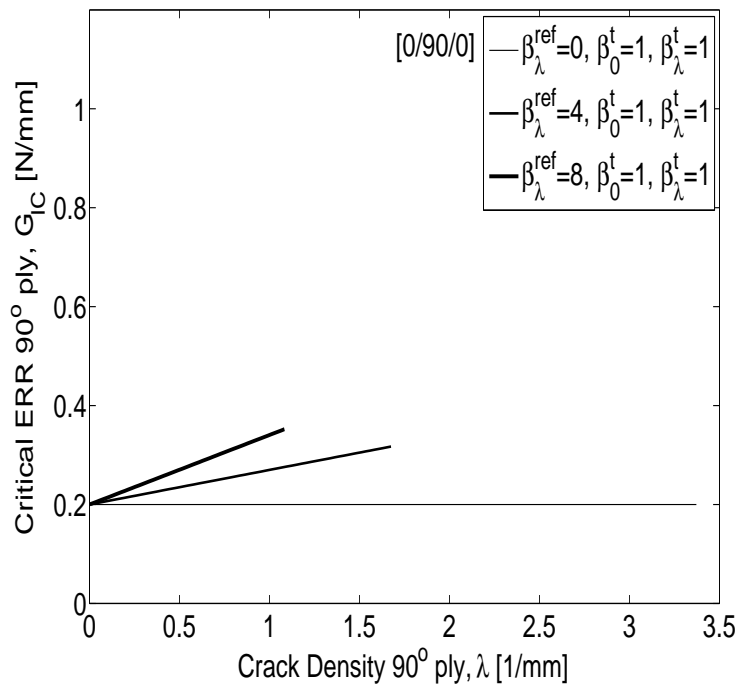

Figure 5.51: The influence of $\beta_{\lambda}^{\text {ref }}$ parameter on the $\mathrm{R}$-curve behavior at the reference thickness $\left(t=t_{r e f}\right), G_{I C}(\lambda)$ coordinates.

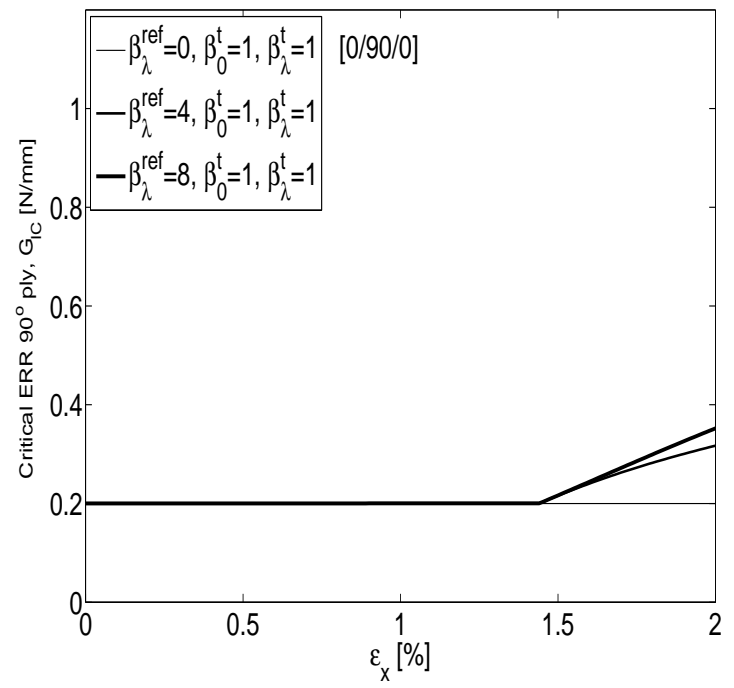

Figure 5.52: The influence of $\beta_{\lambda}^{\text {ref }}$ parameter on the $\mathrm{R}$-curve behavior at the reference thickness $\left(t=t_{\text {ref }}\right), G_{I C}\left(\epsilon_{x}\right)$ coordinates.

The influence of $\beta_{\lambda}^{\text {ref }}$ parameter on the model output, for the reference thickness of the cracking ply $t=t_{r e f}$, is presented in Fig. 5.51 - 5.54. The $\mathrm{R}$-curve behavior, also regarded as the strengthening behavior of the material, i.e., the increase in the critical strength value $G_{I C}$ for increasing damage level, can be observed in Fig. 5.51. The equivalent information is the increase in the critical ERR $G_{I C}$ for increasing load level, which is presented in Fig. 5.52. The influence of the $\mathrm{R}$-curve effect on matrix cracking evolution is presented in Fig. 5.53, where the strengthening effect for increasing $\beta_{\lambda}^{\text {ref }}$ values results in decreasing crack densities $\lambda$ for the same level of the applied deformation $\epsilon_{x}$. The corresponding stress-strain plot of the laminate is presented in Fig. 5.54, where lower reduction in the slope of $\sigma-\epsilon$ curve can be noticed for higher values of the $\beta_{\lambda}^{\text {ref }}$ parameter. 


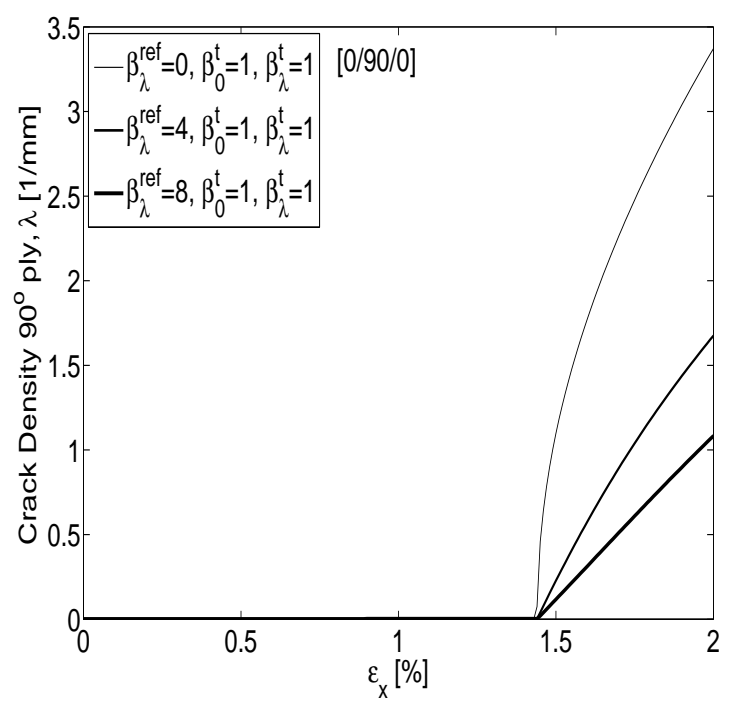

Figure 5.53: The influence of $\beta_{\lambda}^{\text {ref }}$ parameter on damage evolution at the reference thickness $\left(t=t_{\text {ref }}\right)$.

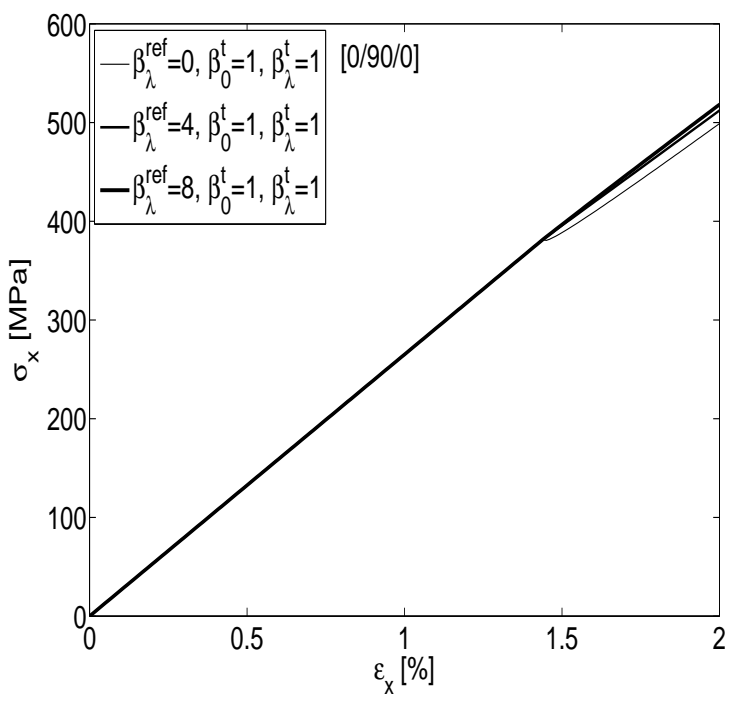

Figure 5.54: The influence of $\beta_{\lambda}^{\text {ref }}$ parameter on the stress-strain behavior of the laminate at the reference thickness $\left(t=t_{r e f}\right)$.

Next, the influence of $\beta_{0}^{t}$ and $\beta_{\lambda}^{t}$ material parameters on the model output, for thickness of the cracking ply other than the reference thickness $\left(t \neq t_{r e f}\right)$, is presented in Fig. $5.55-5.58$.

It can be observed in Fig. 5.55 that, for the case of $\beta_{\lambda}^{\text {ref }}=0$, the $G_{I C}$ curves might feature or not in-situ behavior (i.e., greater $G_{I C}$ value for greater ply thickness), according to the value of $\beta_{0}^{t}$ parameter. If $\beta_{0}^{t}=1$, there is no induced in-situ effect. If $\beta_{0}^{t}>1$, there is in-situ effect. However, the $G_{I C}$ curves do not feature R-curve behavior regardless the value of $\beta_{0}^{t}$ and $\beta_{\lambda}^{t}$. This is because it is considered that if no $\mathrm{R}$-curve behavior (i.e., $\beta_{\lambda}^{\text {ref }}=0$ ) is recorded at $t=t_{r e f}$, then it is most likely that no $\mathrm{R}$-curve behavior will be recorded at $t \neq t_{\text {ref }}$.

Then, it can be observed that for the cases of $\beta_{\lambda}^{r e f}>0$, besides the in-situ effect, the strengthening $G_{I C}$ curves will definitely feature an $\mathrm{R}$-curve effect. This is because it is considered that if $\mathrm{R}$-curve behavior (i.e., $\beta_{\lambda}^{\text {ref }}>0$ ) is recorded at $t=t_{\text {ref }}$, then it is most likely that $\mathrm{R}$-curve behavior will be recorded at $t \neq t_{r e f}$, as well.

However, there might be an influence of the thickness of the cracking ply on the level of the R-curve behavior, i.e., the slope of the $G_{I C}=G_{I C}(\lambda)$ curve. This thickness influence on the $\mathrm{R}$-curve behavior is set by the $\beta_{\lambda}^{t}$ parameter. It can be observed in Fig. 5.55 that a value $\beta_{\lambda}^{t}=1$ preserves the same slope level as for the case of $t=t_{\text {ref }}$ in Fig. 5.51, and a 


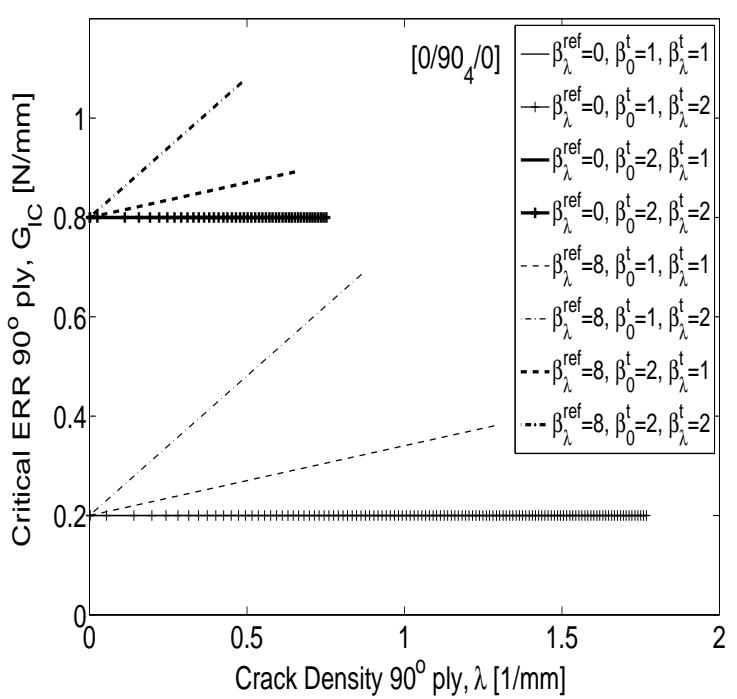

Figure 5.55: The influence of $\beta_{0}^{t}, \beta_{\lambda}^{t}$ parameters on the in-situ $\mathrm{R}$-curve behavior at $t \neq t_{r e f}, G_{I C}(\lambda)$ coordinates.

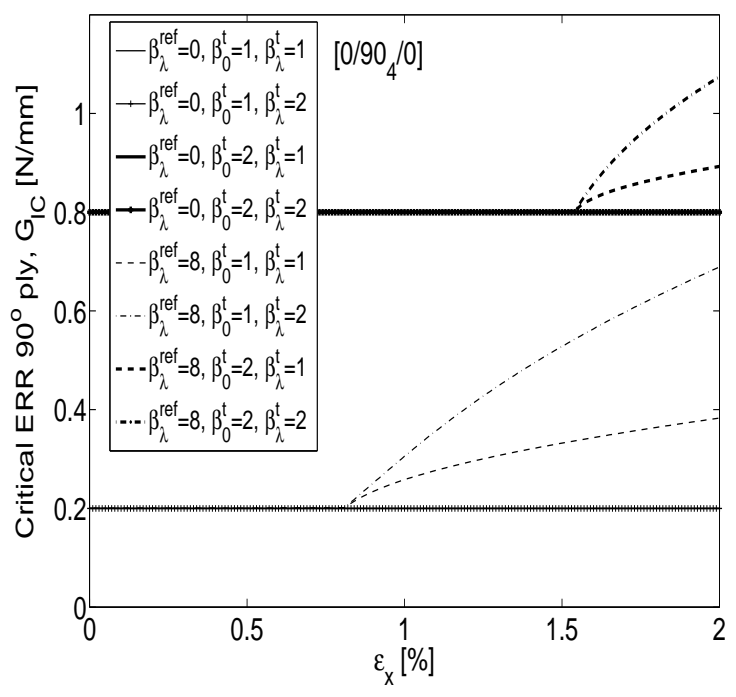

Figure 5.56: The influence of $\beta_{0}^{t}, \beta_{\lambda}^{t}$ parameters on the in-situ $\mathrm{R}$-curve behavior at $t \neq t_{\text {ref }}, G_{I C}\left(\epsilon_{x}\right)$ coordinates.

value $\beta_{\lambda}^{t}>1$ modifies the slope level according to the thickness of the cracking ply.

Information similar to the $G_{I C}=G_{I C}(\lambda)$ in-situ R-curve behavior in Fig. 5.55 is provided in the $G_{I C}=G_{I C}(\epsilon)$ plots in Fig. 5.56. The corresponding crack evolution taking into account the in-situ R-curve behavior in Fig. 5.55, 5.56, is presented in Fig. 5.57. Here it can be noticed that the values of the $\beta_{\lambda}^{r e f}, \beta_{0}^{t}, \beta_{\lambda}^{t}$ material parameter can considerable influence the damage process in the composite laminate. The corresponding stress-strain curves are presented in Fig. 5.58. 


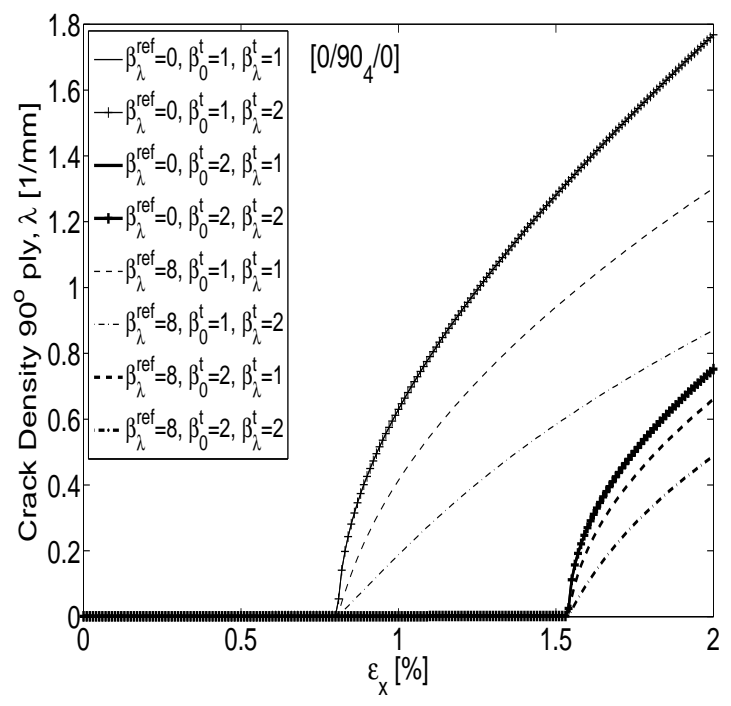

Figure 5.57: The influence of $\beta_{0}^{t}, \beta_{\lambda}^{t}$ parameters on damage evolution at $t \neq t_{\text {ref }}$.

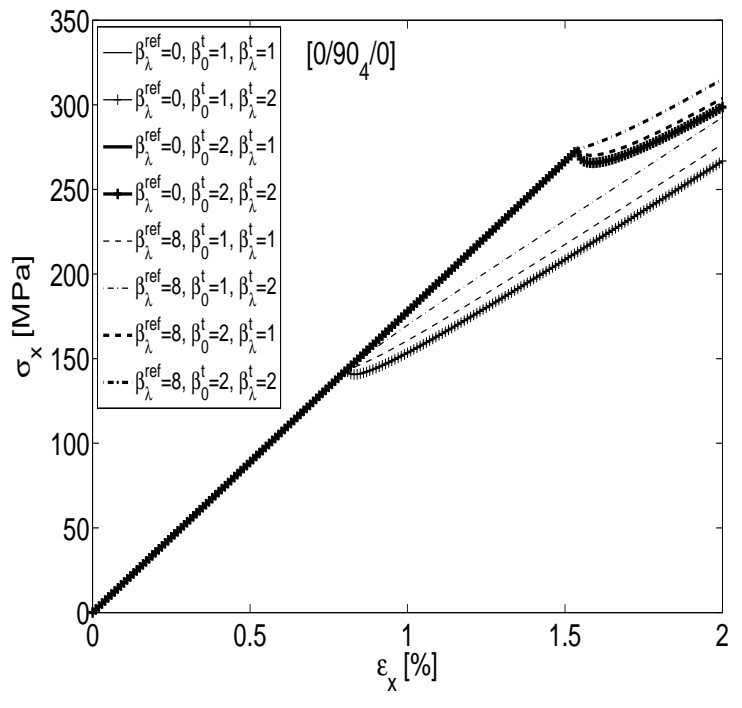

Figure 5.58: The influence of $\beta_{0}^{t}, \beta_{\lambda}^{t}$ parameters on the stress-strain behavior of the laminate at $t \neq t_{\text {ref }}$. 


\subsubsection{The influence of thermal residual stresses}

Thermal residual stresses due to the difference between the processing (curing) temperature and the operating (service) temperature act on individual plies of the laminates (see Appendix A), in addition to the stresses induced by the mechanical loading. The effect of these thermal stresses is analyzed in the present section. Two materials systems are considered for this: i) a Carbon-Epoxy composite in a $\left[0_{2} / 90_{4} / 0_{2}\right]$ laminate configuration, and ii) a Glass-Epoxy composite in a [0/90/0] laminate configuration.

The thermo-elastic properties of the Glass-Epoxy material system are the ones listed at the beginning of the present chapter. The material properties of the Carbon-Epoxy material system are the following:

$$
\begin{aligned}
& E_{1}=116.5 \mathrm{GPa} ; \quad E_{2}=9 \mathrm{GPa} ; \quad G_{12}=5 \mathrm{GPa} ; \quad \nu_{12}=0.3 ; \quad \nu_{23}=0.5 \\
& \alpha_{1}=-0.09 \cdot 10^{-} 6{ }^{o} \mathrm{C}^{-1} ; \quad \alpha_{2}=28.8 \cdot 10^{-} 6{ }^{\circ} \mathrm{C}^{-1} \\
& G_{I C, 0}^{r e f}=0.20 \mathrm{~N} / \mathrm{mm} ; \quad G_{I I C}=1 \mathrm{~N} / \mathrm{mm}
\end{aligned}
$$

The parametric study is performed considering various operating temperatures, denotes as room temperature $(R T)$ in the subsequent plots. The range of variation of the RT parameter is in from the processing temperature down to cryogenic temperatures. The considered processing temperatures are $225^{\circ} \mathrm{C}$ for the Carbon-Epoxy material system and $120^{\circ} \mathrm{C}$ for the Glass-Epoxy system. The processing temperature is denoted as stress free temperature $(S F T)$ in the subsequent plots. The loading case is uniaxial extension $\epsilon_{x}$.

The parametric study is run for the following values of the operating temperatures: $R T=[120,0,-120,-300]{ }^{\circ} C$ for the Glass-Epoxy material system, and $R T=[225,100,20,-100,-225]^{\circ} C$ for the Carbon-Epoxy material system.

The output of the analytical model for the case of Carbon-Epoxy laminate is presented in Fig. 5.59. First it can be observed that the level of the mechanical loading at damage onset is decreased with decreasing operating temperature $R T$. This is because of the higher thermal residual stresses that develop at higher temperature differences $\Delta T=R T-S F T$. At $R T=20^{\circ} \mathrm{C}$, the crack density in both 90 and 0 plies are plotted in Fig. 5.59; matrix cracking does not develop in the 0 ply. Lowering the operating temperature at $R T=$ $-100{ }^{\circ} \mathrm{C}$, matrix cracking initiates in both 90 and 0 plies without even applying mechanical loading, but exclusively due to thermal residual stresses. The level of initial damage due to exclusively thermal stresses is increased as the operating temperature is further decreased at $R T=-225^{\circ} \mathrm{C}$. This model results are conform with the expected physical behavior of 


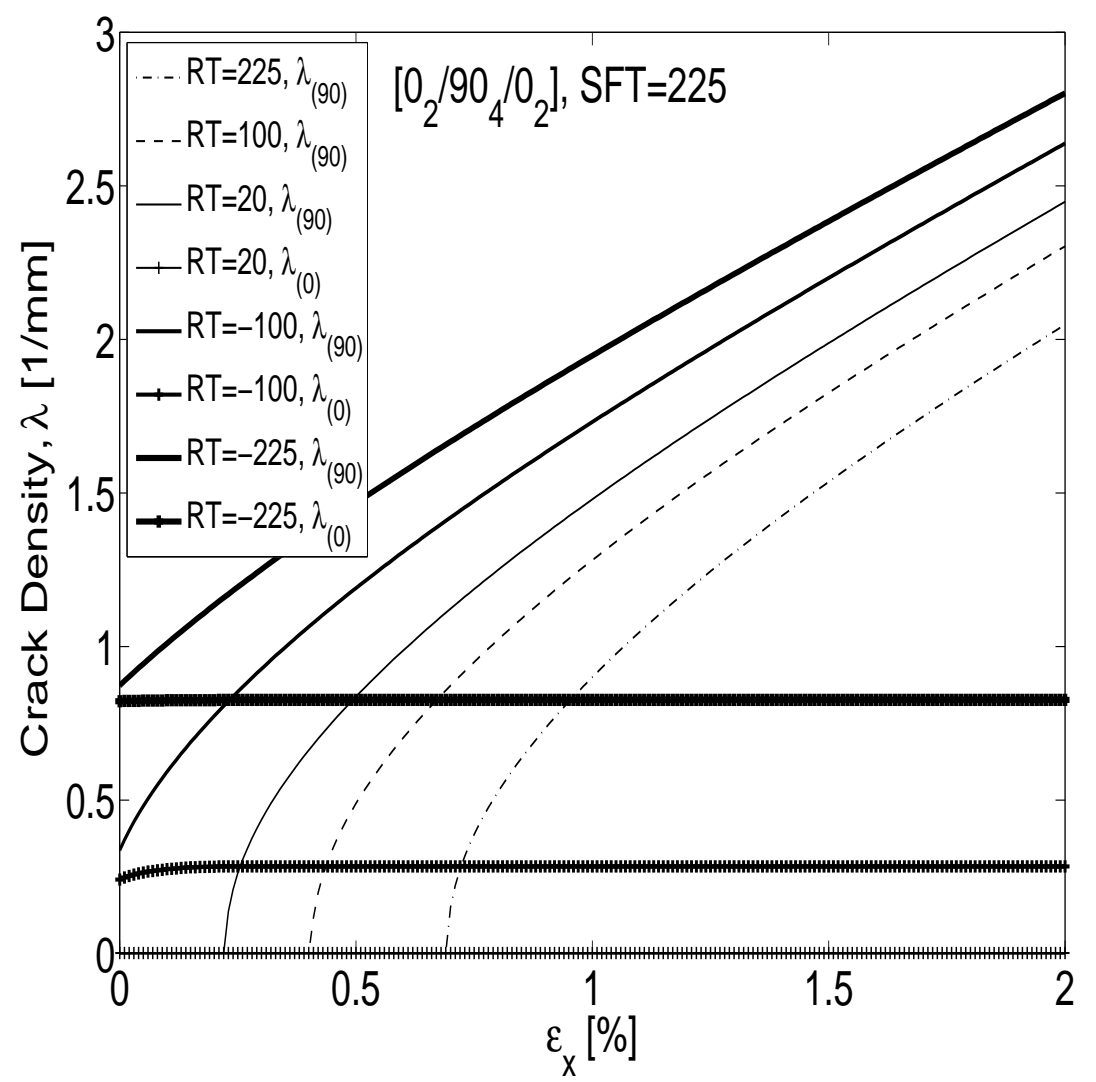

Figure 5.59: The influence of thermal residual stresses on damage process, Carbon-Epoxy material system.

the material, and prove the predictive capabilities of the analytical model.

What is interesting to notice is the predicted behavior of the Glass-Epoxy laminate in Fig. 5.60, as compared to the Carbon-Epoxy laminate in Fig. 5.59. First, the influence of the temperature difference between the processing and operating temperature is much more reduced for the Glass-Epoxy material system: the values of mechanical loading at damage onset are close to each other for the whole range of $R T$ parameter. Second, matrix cracking at zero mechanical loading does not develop, not even at very low cryogenic operating temperature $R T=-300{ }^{\circ} \mathrm{C}$ considered in Fig. 5.60. Then, the effect of $\Delta T$ for the Glass-Epoxy material is surprisingly reversed, although reduced, as compared to the Carbon-Epoxy material. This result can be due to the highly different CTE of the individual plies for the two material systems, and especially due to the negative longitudinal ply CTE, $\alpha_{1}$, in the case Carbon-Epoxy material. The reversed behavior of the two material systems regarding the influence of the thermal stresses requires further investigation. 


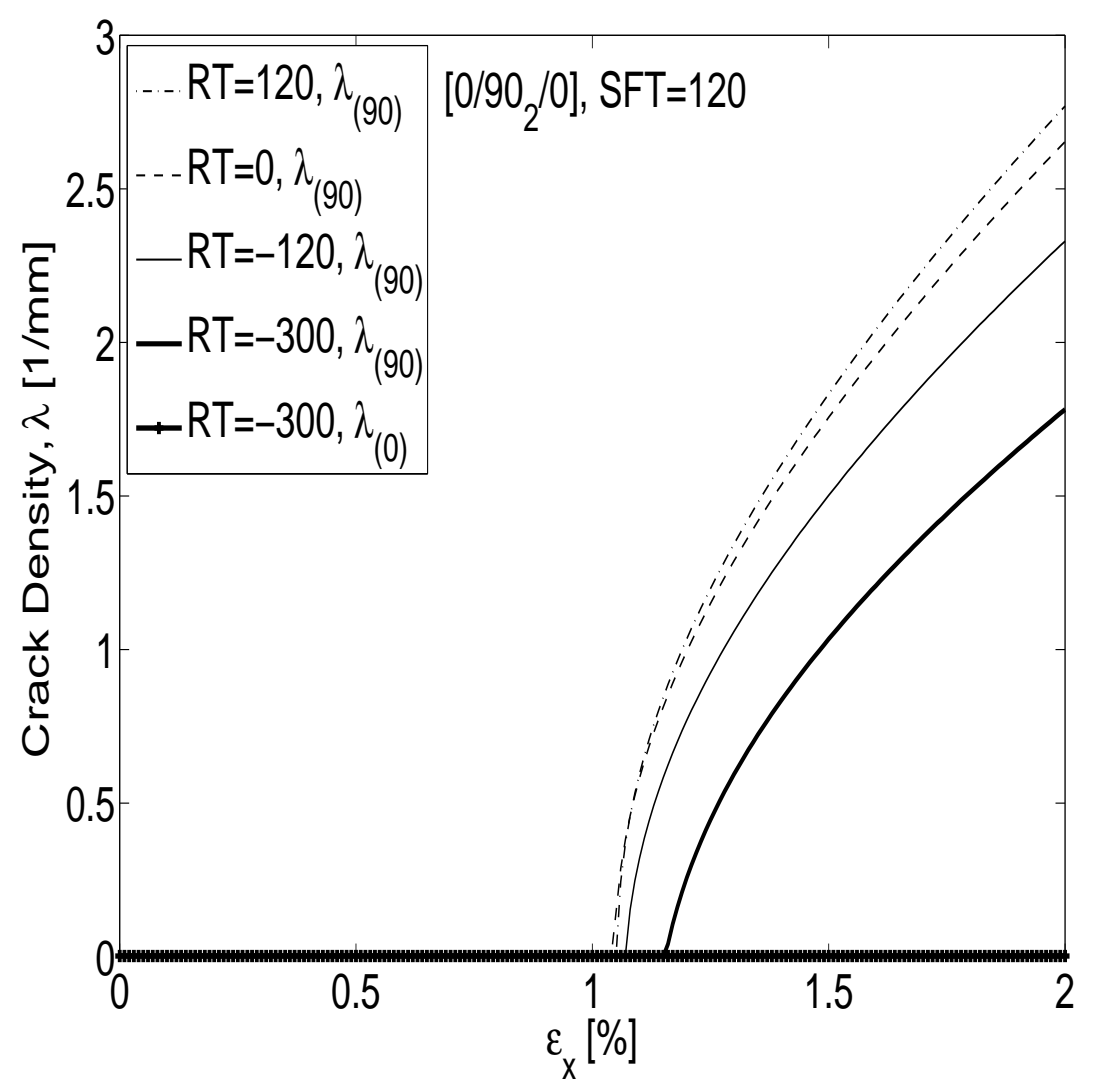

Figure 5.60: The influence of thermal residual stresses on damage process, Glass-Epoxy material system.

One aspect that is wort being noticed with regard to the influence of thermal residual stresses on intra-laminar matrix cracking in fiber-reinforced laminated composites. The thermal stresses are generated due to the CTE mismatch between individual plies of the laminate, and along different directions of the orthotropic plies. This source of residual stresses is considered in the present analytical model (see Appendix A), similarly to any formulation based on the CLT which considers the ply level as the smallest constituent unit of the laminate (i.e., fiber and matrix constituents are not regarded separately, but the homogenized properties of the orthotropic ply are calculated and used for stress analysis). However, there is the deeper level of individual fiber and matrix constituents, with their own values of the CTE's, CTE mismatch, and resulting thermal residual stresses. It is claimed in $[73,74,75]$ that, based on the higher CTE mismatch at fiber/matrix level, the thermal residual stresses should be calculated at this level of individual constituents. Thus, a stress calculation in individual fiber and matrix constituents is proposed $[76,77,78,79,80]$ based 
on the Multi-Continuum Theory (MCT), which is a modeling strategy of stress calculation at the level of fiber and matrix constituents.

The behavior and tendencies presented in Fig. 5.59, 5.60 consider the thermal residual stresses calculated the level of homogenized orthotropic ply. It is possible that, due different thermal stresses at the fiber and matrix level, different matrix cracking behavior to be recorded for the laminated composite. However, an accurate analysis at fiber/matrix level should consider the fiber/matrix interface as a very probable location of the matrix cracking initiation due to thermal stresses. This would bring an additional complication to the modeling effort, because the properties of the interface has to be evaluated and considered (for example, the influence of interface in not considered in the MCT modeling strategy in $[76,77,78,79,80]$. A full validation of the analytical tendencies in of the present model in Fig. 5.59, 5.60 requires comparison against experimental data of progressive damage for the two material systems, at varying operating temperatures. 


\subsection{Analytical model output: validation against exper- iments}

A comprehensive research and selection of experimental data available in the literature regarding the phenomenon of progressive matrix cracking in laminated composites has been conducted. Five experimental programs, listed in the following, could be identified and selected for the porpouse of the present chapter, which is the validation of the analytical model against experimental data.

- experimental data set 1: $[31,61,81,82]$

- experimental data set $2:[8,9]$

- experimental data set 3: $[1,2,58,83,84]$

- experimental data set 4: [51, 85]

- experimental data set 5: [36]

The first three entries of the above list offer data regarding the progressive damage process under uniaxial extension only. The last two offer experimental data regarding both uniaxial extension and uniaxial flexural loading.

\subsubsection{Model validation, experimental data set 1: $[31,61,81,82]$}

The laminate configurations considered under this testing program are $\left[ \pm 25 / 90_{n}\right]_{S}$ and $\left[0_{m} / 90_{n}\right]_{S}$. The values of $m, n$ parameters are specified in the corresponding progressive damage plots presented in the following.

The loading case is uniaxial extension $\epsilon_{x}$.

The material is a Carbon/Epoxy system, T300/934, with thermo-elastic properties of individual laminae listed in the following, as they are offered in [31, 61, 81, 82]:

$E_{1}=163.4 \mathrm{GPa}, \quad E_{2}=11.9 \mathrm{GPa}, \quad G_{12}=6.5 \mathrm{GPa}, \quad \nu_{12}=0.3, \quad \nu_{23}=0.5$ $\alpha_{1}=0.35 \cdot 10^{-6}{ }^{\circ} \mathrm{C}, \quad \alpha_{2}=28.8 \cdot 10^{-6}{ }^{\circ} \mathrm{C}$

The ply thickness is $t_{k}=0.132 \mathrm{~mm}$. The processing temperature (stress free temperature) is $S F T=150{ }^{\circ} \mathrm{C}$.

The in-situ R-curve material parameters (see Section 4.4.3) used in the analytical model in order to closest reproduce the experimental data are 


$$
G_{I C, 0}^{r e f}=0.2 \mathrm{~N} / \mathrm{mm}, \quad \beta_{\lambda}^{r e f}=6 \mathrm{deg} ., \quad \beta_{0}^{t}=1.2, \quad \beta_{\lambda}^{t}=2
$$

The selected reference LSS configuration (see the concept of reference thickness $t_{r e f}$ in Section 4.4.3) is $\left[ \pm 25 / 90_{2}\right]_{S}$.

Due to the fact that, according to the tested laminate configurations and loading case, matrix cracking takes place only in mode $I$, the value of the critical ERR for mode $I I, G_{I I, C}$ does not matter for the present data set.

Analytical model prediction of matrix cracking process (onset and evolution) are compared against experimental results in Fig. 5.61 - 5.64, for various thicknesses of the cracking 90 ply stack of the $\left[ \pm 25 / 90_{n}\right]_{S}$ laminate configuration. A centralized plot of the whole data set of samples featuring different thicknesses of the cracking 90 stack is presented in Fig. 5.65.

It can be noticed that the previous progressive damage plots are in $(\lambda-\sigma)$ coordinates, as they are offered in $[31,61,81,82]$. The corresponding damage evolution plots in $(\lambda-\epsilon)$ coordinates are presented in Fig. 5.66. A different appearance of damage evolution plots in $(\lambda-\epsilon)$ coordinates in Fig. 5.66, as compared to the ones in $(\lambda-\sigma)$ coordinates in Fig. 5.65 can be noticed. Not only are the curves in $(\lambda-\epsilon)$ coordinates closer spaced as compared to their equivalents in $(\lambda-\sigma)$ coordinates, but even the relative position between curves corresponding to different $90_{n}$ thickness are different. This implies that damage evolution plots in both $(\lambda-\sigma)$ and $(\lambda-\epsilon)$ coordinates has to be considered in order to gain a complete picture of the progressive damage process.

For the $\left[0_{m} / 90_{n}\right]_{S}$ laminate configuration, results of progressive damage are presented in Fig. 5.67 - 5.70, for different thicknesses of the $90_{n}$ cracking stack, and for different thicknesses of the constraining $0_{m}$ sublaminate. A centralized damage evolution plot is presented in Fig. 5.71 in $(\lambda-\sigma)$ coordinates, and the equivalent plot of the model predictions in $(\lambda-\epsilon)$ coordinates is presented in Fig. 5.72.

It can be observed in Fig. 5.61 - 5.65 that the analytical model predicts the matrix cracking process in good agreement with experimental data for different thicknesses of the $90_{n}$ cracking stack of the $\left[ \pm 25 / 90_{n}\right]_{S}$ laminate configuration. The expected trend of earlier damage initiation in the thicker plies is confirmed by both experimental and analytical results.

Regarding the results for the $\left[0_{m} / 90_{n}\right]_{S}$ laminate configuration in Fig. $5.67-5.71$, it can be observed that the main trends of damage evolution are well predicted by the analytical model, but the degree of correlation between analytical-experimental results are different for different samples of this data set. A good correlation of both damage onset and evolution can 
be noticed for the $\left[0 / 90_{n}\right]_{S}$ laminate configuration in Fig. $5.67-5.69$. For the $\left[0_{2} / 90_{n}\right]_{S}$ laminate configuration in Fig. 5.70, the analytical-experimental correlation is good for damage onset, but it is poorer for damage evolution at high values of the crack density $\lambda$, where the analytical model predicts a slower increase in crack density than the experimental results.

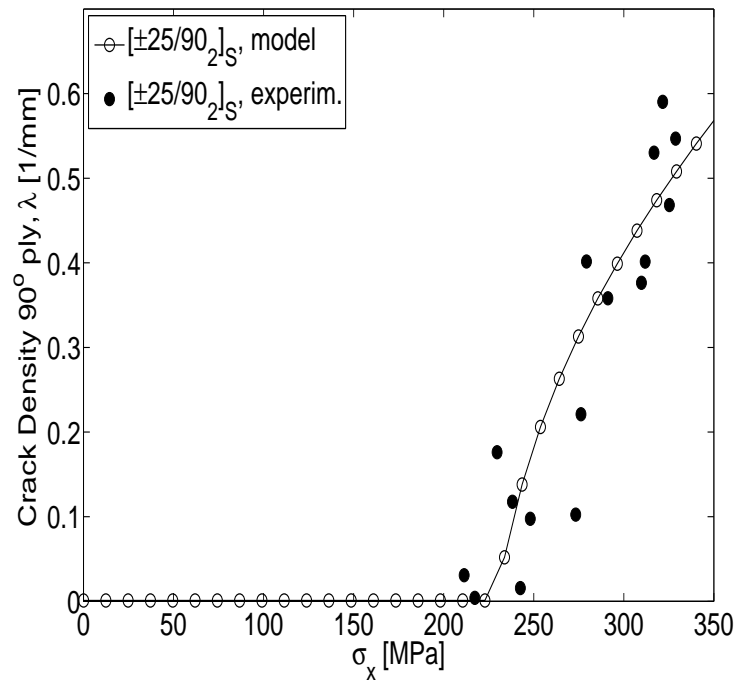

Figure 5.61: Matrix cracking progression: analytical curve vs. experimental data, Wang $[81,82]$. $\left[ \pm 25 / 90_{2}\right]_{S}$ LSS.

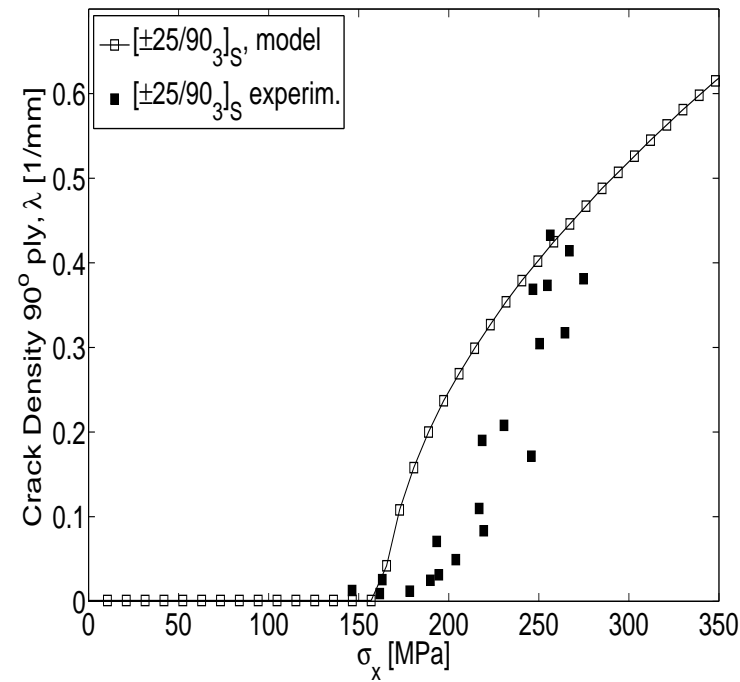

Figure 5.62: Matrix cracking progression: analytical curve vs. experimental data, Wang $[81,82] .\left[ \pm 25 / 90_{3}\right]_{S}$ LSS. 


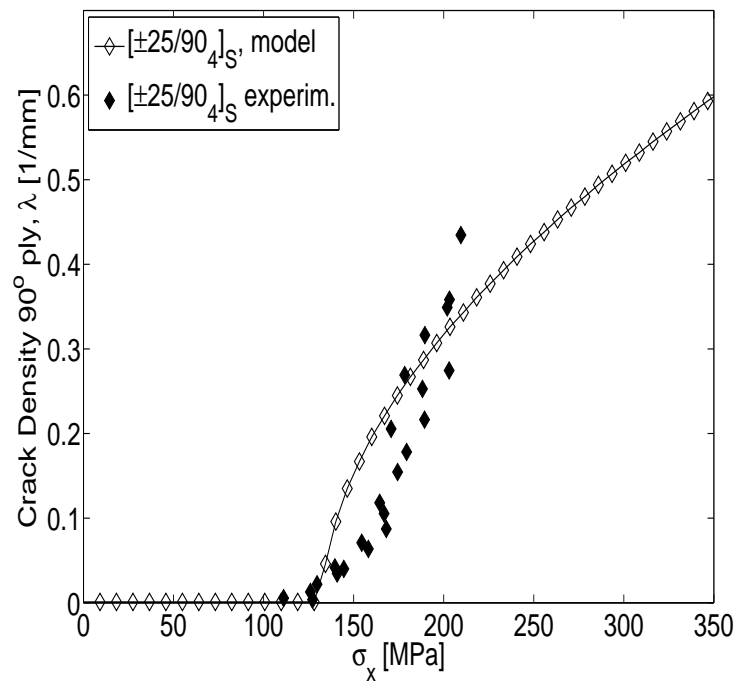

Figure 5.63: Matrix cracking progression: analytical curve vs. experimental data, Wang $[81,82]$. $\left[ \pm 25 / 90_{4}\right]_{S}$ LSS.

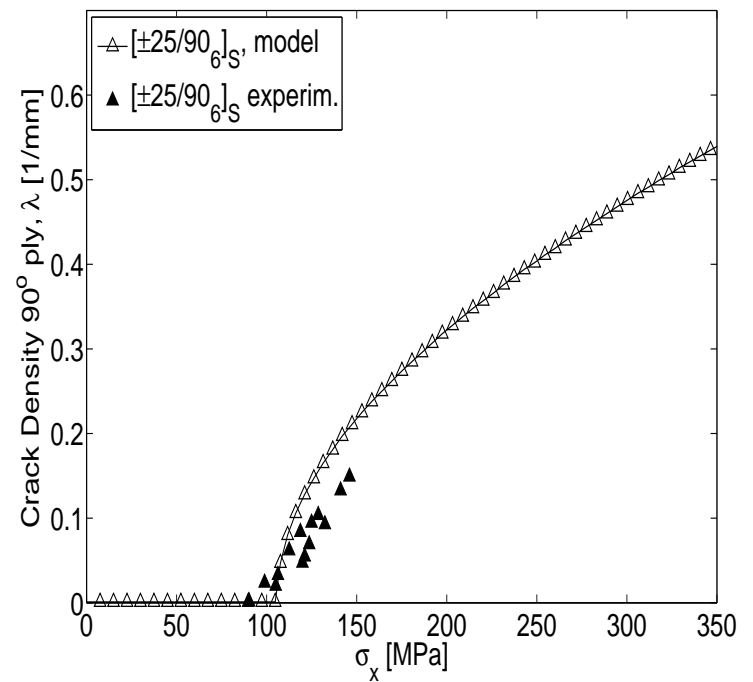

Figure 5.64: Matrix cracking progression: analytical curve vs. experimental data, Wang $[81,82] .\left[ \pm 25 / 90_{6}\right]_{S}$ LSS.

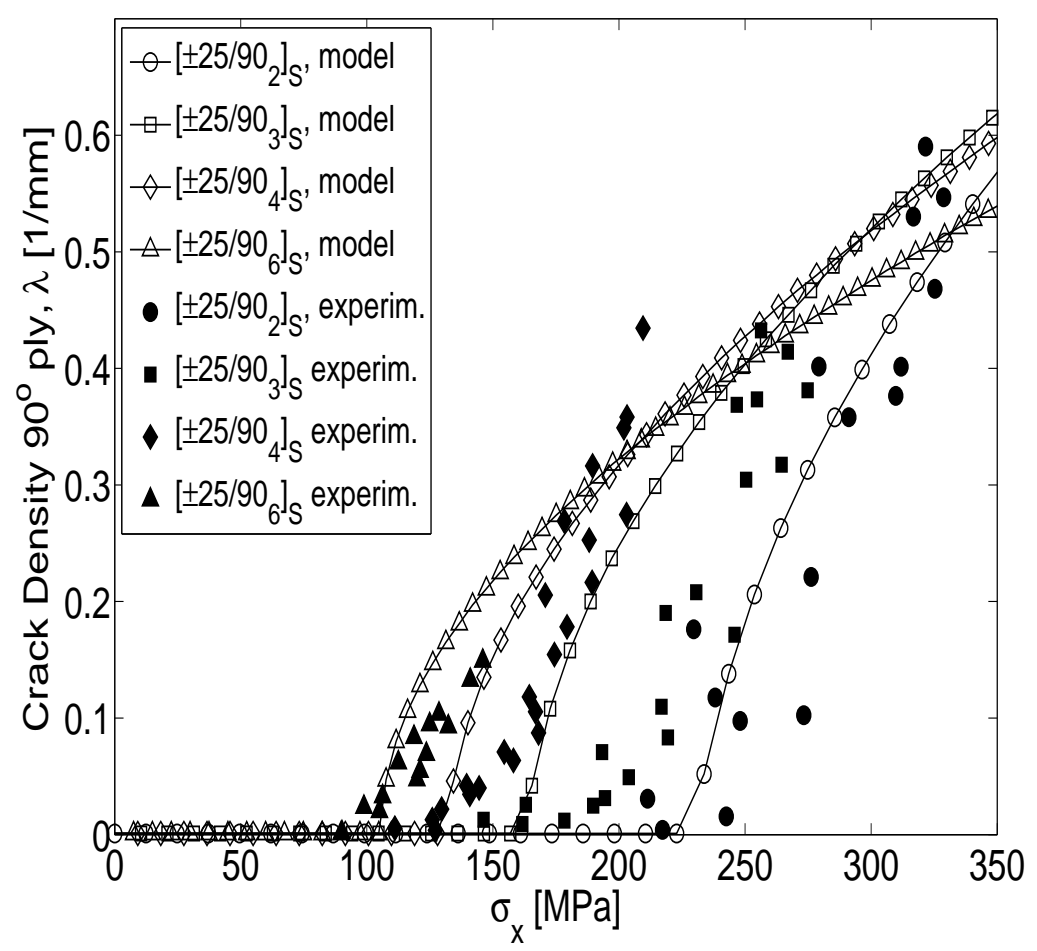

Figure 5.65: Matrix cracking progression: analytical curves vs. experimental data, Wang $[81,82] .\left[ \pm 25 / 90_{n}\right]_{S}$ LSS. 


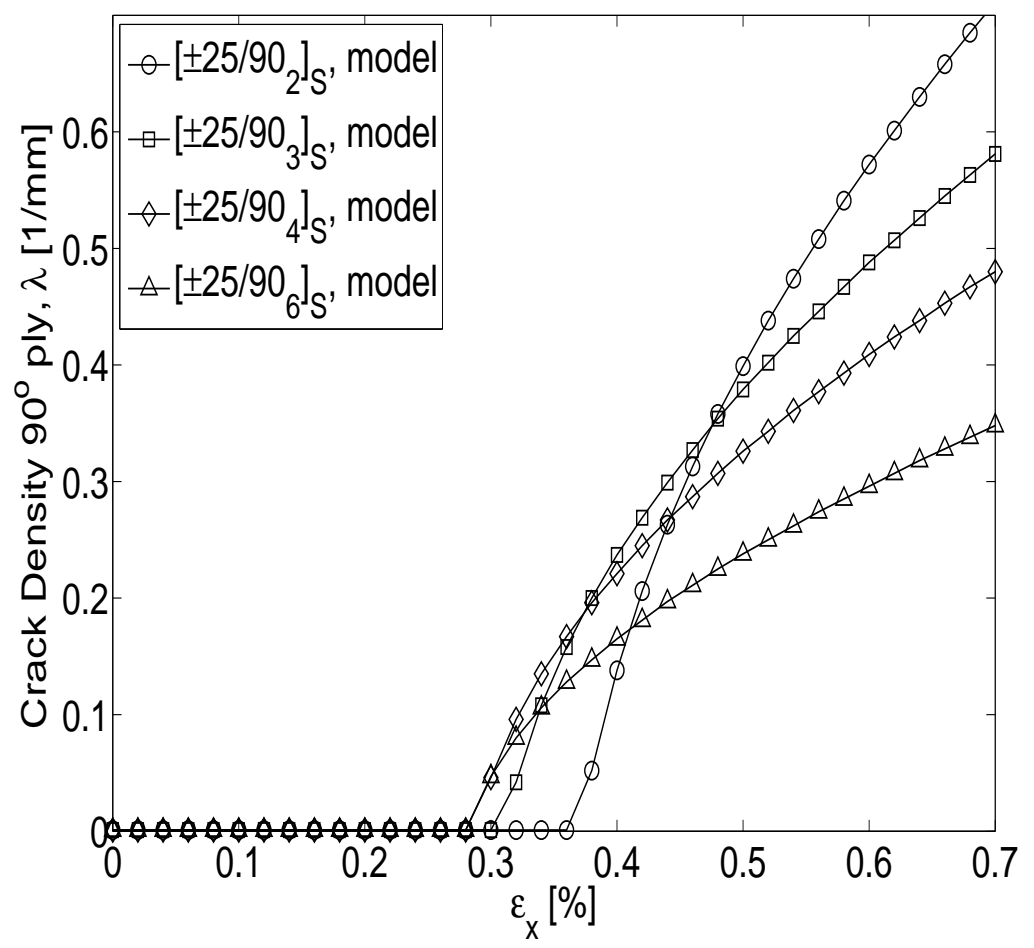

Figure 5.66: Matrix cracking progression: analytical curves. $\left[ \pm 25 / 90_{n}\right]_{S}$ LSS.

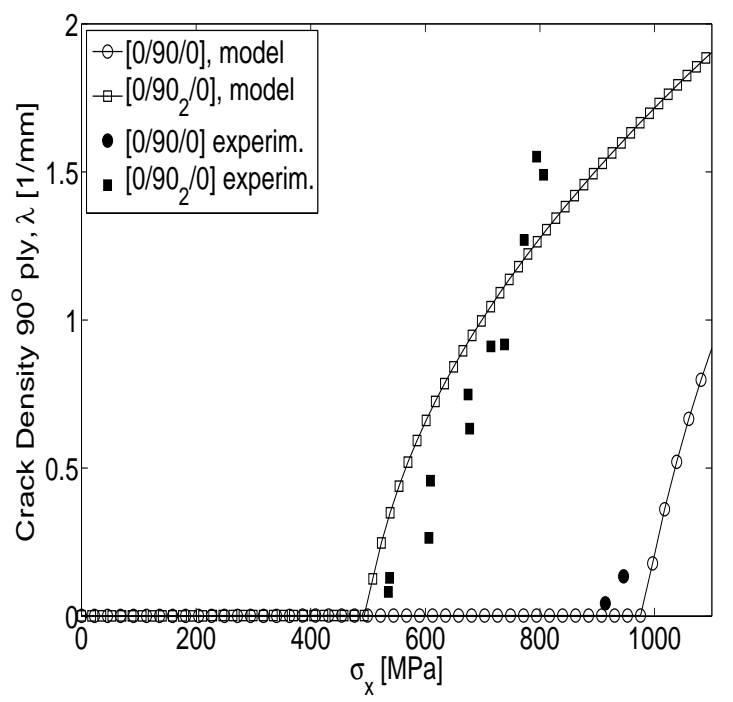

Figure 5.67: Matrix cracking progression: analytical curve vs. experimental data, Wang [31, 61]. [0/90/0] and $\left[0 / 90_{2} / 0\right]$ LSS.

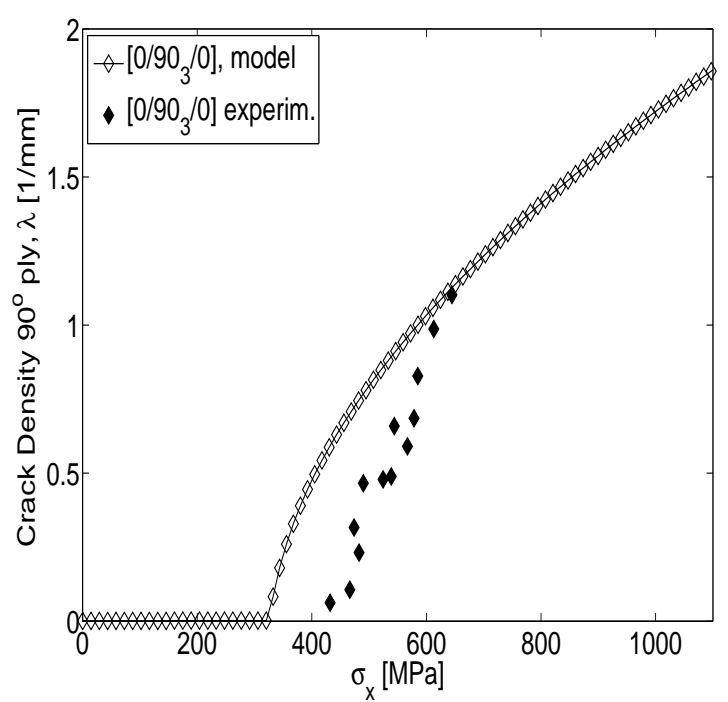

Figure 5.68: Matrix cracking progression: analytical curve vs. experimental data, Wang [31, 61]. [0/90 $/ 0]$ LSS. 


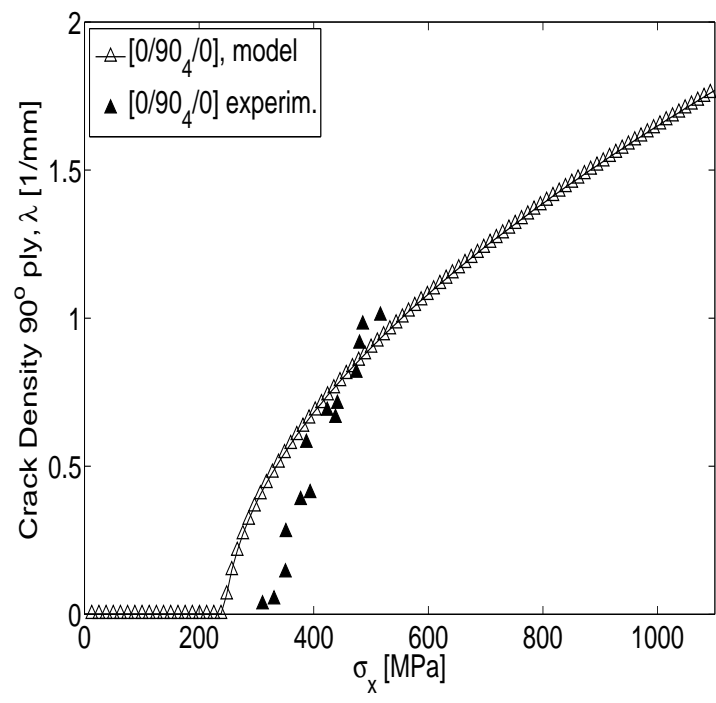

Figure 5.69: Matrix cracking progression: analytical curve vs. experimental data, Wang [31, 61]. [0/904/0] LSS.

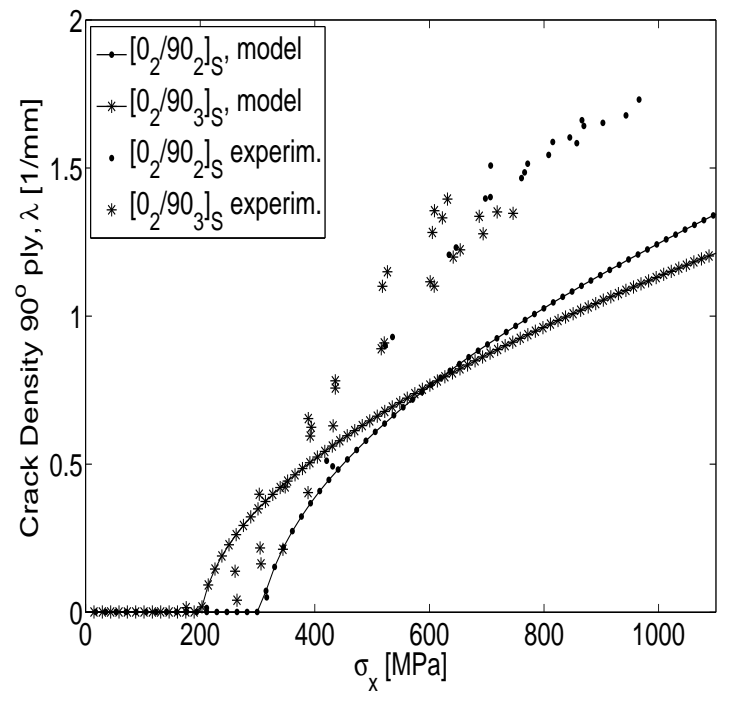

Figure 5.70: Matrix cracking progression: analytical curve vs. experimental data, Wang $[31,61] . \quad\left[0_{2} / 90_{2}\right]_{S}$ and $\left[0_{2} / 90_{3}\right]_{S}$ LSS.

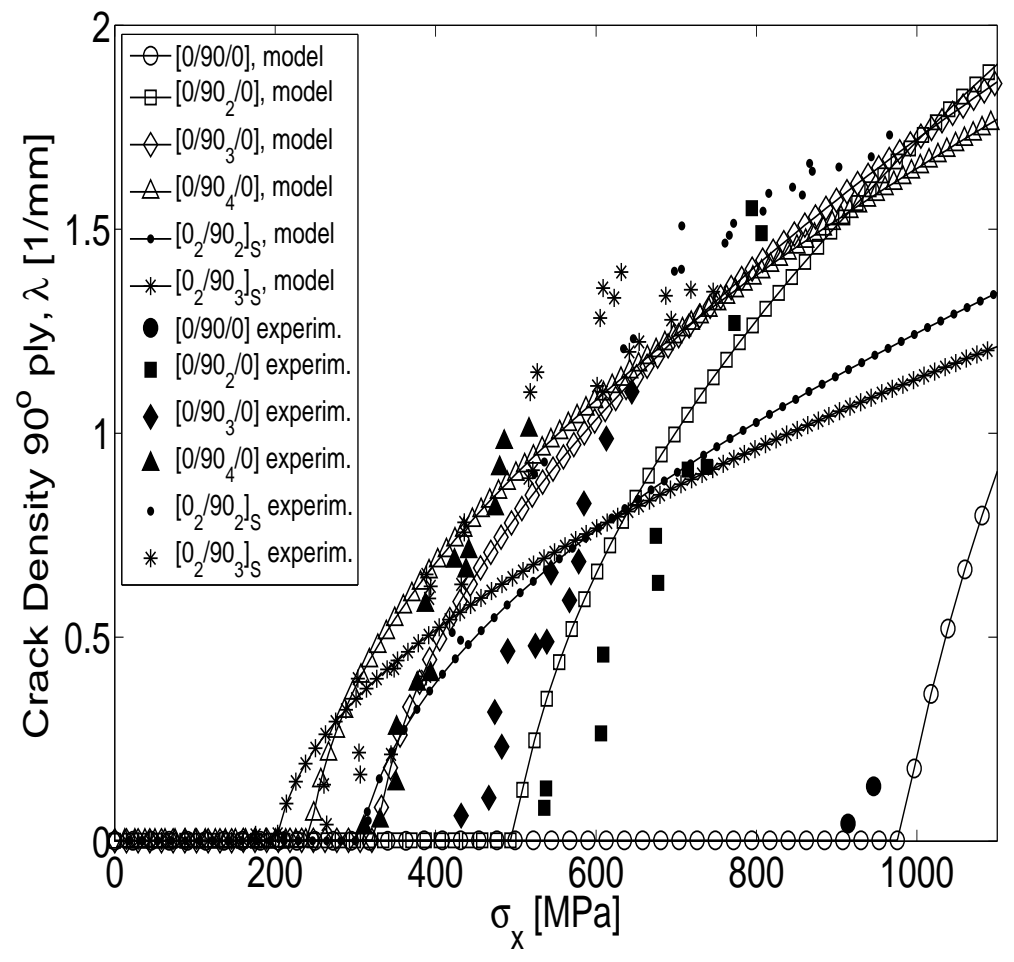

Figure 5.71: Matrix cracking progression: analytical curves vs. experimental data, Wang $[31,61] .\left[0_{m} / 90_{n}\right]_{S}$ LSS. 


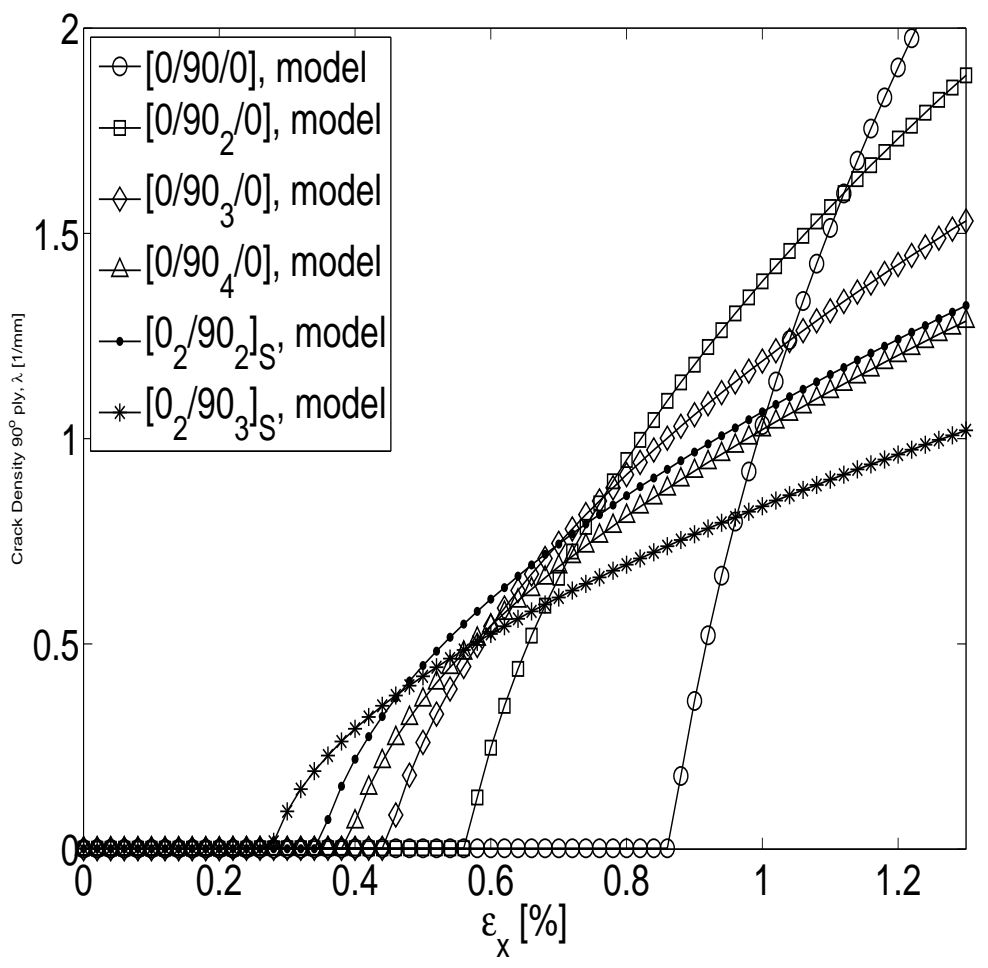

Figure 5.72: Matrix cracking progression: analytical curves. $\left[0_{m} / 90_{n}\right]_{S}$ LSS. 


\subsubsection{Model validation, experimental data set 2: [8,9]}

The laminate configurations considered under this testing program are of $\left[0_{m} / 90_{n}\right]_{S}$ and $\left[90 / 0_{m}\right]_{S}$ type. The second one represents some of the very few experimental data in the literature dealing with matrix cracking in surface plies of the laminate. The values of $m, n$ parameters are specified in the corresponding progressive damage plots presented in the following.

The loading case is uniaxial extension $\epsilon_{x}$.

Two Carbon/Epoxy material systems considered for the testing program in [8, 9] are regarded in the present section: AS4/Hercules 3501-6 and IM6/Avimid-K. The thermoelastic properties of individual laminae are listed in the following, as they are offered in $[8,9]$ :

For AS4/Hercules 3501-6 material system:

$E_{1}=130 G P a, \quad E_{2}=9.7 G P a, \quad G_{12}=5 G P a, \quad \nu_{12}=0.3, \quad \nu_{23}=0.5$

$\alpha_{1}=-0.09 \cdot 10^{-6}{ }^{\circ} \mathrm{C}, \quad \alpha_{2}=28.8 \cdot 10^{-6}{ }^{\circ} \mathrm{C}$

For IM6/Avimid-K material system:

$E_{1}=134 G P a, \quad E_{2}=9.8 G P a, \quad G_{12}=5.5 G P a, \quad \nu_{12}=0.3, \quad \nu_{23}=0.5$

$\alpha_{1}=-0.09 \cdot 10^{-6}{ }^{\circ} \mathrm{C}, \quad \alpha_{2}=28.8 \cdot 10^{-6}{ }^{\circ} \mathrm{C}$

The ply thickness is $t_{k}=0.125 \mathrm{~mm}$. The processing temperature (stress free temperature) is $S F T=125^{\circ} \mathrm{C}$ for AS4/Hercules 3501-6 and $S F T=225^{\circ} \mathrm{C}$ for IM6/Avimid-K.

The in-situ R-curve material parameters (see Section 4.4.3) used in the analytical model in order to closest reproduce the experimental data are:

For AS4/Hercules 3501-6 material system:

$G_{I C, 0}^{r e f}=0.14 \mathrm{~N} / \mathrm{mm}, \quad \beta_{\lambda}^{r e f}=1.5 \mathrm{deg} ., \quad \beta_{0}^{t}=1.1, \quad \beta_{\lambda}^{t}=2$

For IM6/Avimid-K material system:

$G_{I C, 0}^{r e f}=0.53 \mathrm{~N} / \mathrm{mm}, \quad \beta_{\lambda}^{r e f}=10 \mathrm{deg} ., \quad \beta_{0}^{t}=1, \quad \beta_{\lambda}^{t}=1.4$

The selected reference LSS configuration (see the concept of reference thickness $t_{r e f}$ in Section 4.4.3) is $[0 / 90]_{S}$ for AS4/Hercules 3501-6 and $\left[0 / 90_{2}\right]_{S}$ for IM6/Avimid-K.

Due to the fact that, according to the tested laminate configurations and loading case, matrix cracking takes place only in mode $I$, the value of the critical ERR for mode $I I, G_{I I, C}$ does not matter for the present data set.

The progressive damage analytical vs. experimental results for the AS4/Hercules 3501-6 material are presented in Fig. 5.73, 5.74 (a centralized plot is shown in Fig. 5.75), and for 
the IM6/Avimid-K material are presented in Fig. 5.77, 5.78 (a centralized plot is shown in Fig. 5.79). It can be observed in these plots that the laminate configurations tested cover the family of both $\left[0_{m} / 90_{n}\right]_{S}$ with $m$ fixed and $n$ variable, where the effect of the cracking ply thickness can be assessed, and $\left[0_{m} / 90_{n}\right]_{S}$ with $n$ fixed and $m$ variable, where the constraining effect of the neighboring plies can be assessed. The corresponding analytical predictions of damage progression in $(\lambda-\epsilon)$ coordinates are presented in Fig. 5.76 for AS4/Hercules 3501-6 and in Fig. 5.80 for IM6/Avimid-K.

Good correlation of the analytical model output with experimental results, for both damage onset and damage progression, can be noticed for the AS4/Hercules 3501-6 material in Fig. 5.73, 5.75. Some differences between the predicted and the experimental results seem to appear at high values of crack density $\lambda$, where the analytical model predicts a steeper increase in crack density than the experimental results.

The discrepancy between analytical and experimental results at high level of damage has also been encountered for the data set in Section 5.2.1. However, the outcome for the data set presented in this section is different compared to the outcome in Section 5.2.1, in the sense that in the present section the analytical model prediction is steeper than experimental data, while in Section 5.2.1 the situation is reversed, the analytical model prediction is slower than experimental data. Among the possible causes for the recorded analytical vs. experiment discrepancies might be one or a combination of the following factors:

i) finding the best combination of $\beta_{\lambda}^{\text {ref }}, \beta_{\lambda}^{t}$ material parameters which are responsible for modeling the $R$-curve behavior for a given material system;

ii) modeling the $R$-curve behavior by higher order polynomial or exponential lows, as opposed to linear lows implemented in the present model (see Section 4.4.3);

iii) the ability of the analytical material model (see Section 4.1) to accurately predict the reduced material properties at high crack densities;

iv) the accuracy of the experimental measurements.

The thickness effect of the cracking ply is well predicted in Fig. 5.73, 5.75 by the analytical model. A constraining thickness effect of the neighboring plies can be concluded if the damage curves corresponding to $\left[0 / 90_{2}\right]_{S}$ and $\left[0_{2} / 90_{2}\right]_{S}$ laminate configurations in Fig. 5.75 are compared: damage initiates earlier in the $\left[0 / 90_{2}\right]_{S}$ LSS featuring a thiner 0 neighboring ply than compared to $\left[0_{2} / 90_{2}\right]_{S}$ LSS. However, if the same damage curves are regarded in $(\lambda-\epsilon)$ coordinates as in Fig. 5.76, it can be observed that the constraining effect of the neighboring plies is almost zero. 
The same observations and comments apply for the analytical vs. experimental results corresponding to the IM6/Avimid-K material system in Fig. 5.77 - 5.80. The noticeable difference for this material system is that very good correlation can be observed even at high values of the damage level $\lambda$.

Experimental results for the process of matrix cracking in the case of cracking plies situated at the surface of the composite laminate are presented in Fig. 5.81 - 5.83, where the $\left[90 / 0_{m}\right]_{S}$ LSS featuring cracks in the surface 90 plies is regarded. The material system is AS4/Hercules 3501-6. This data set are the only experimental results that could be identified in the literature regarding matrix cracking in surface plies. It can be observed in these plots that the analytical predictions are too steep as compared to experimental data. The cause for this behavior of the analytical model could be in the selected $\beta_{\lambda}^{r e f}, \beta_{\lambda}^{t}$ parameters governing the material $\mathrm{R}$-curve behavior. Another cause could be the ability of the material model (see Section 4.1) to accurately predict the reduction in material properties for the case of surface cracking plies. The material model has not been especially validated for this case, due to lack of experimental data. Yet another cause could be the accuracy of the experimental determination of damage progression in Fig. 5.81 - 5.83. Comparison against another set of experimental data for the case of surface cracking plies has not been performed, due to lack of such data. Again, if the damage curves in $(\lambda-\sigma)$ coordinates in Fig. $5.81-5.83$ are regarded in $(\lambda-\epsilon)$ coordinates (Fig. 5.84), they come almost identical, with no influence of the thickness of the middle uncracking $0_{m}$ ply.

The analytical variation of the critical ERR, $G_{I C}$, corresponding to the simulated in-situ $R$-curve behavior of the laminated composite material is presented in Fig. 5.85 in $\left(G_{I C}-\lambda\right)$ coordinates, and in Fig. 5.86 in $\left(G_{I C}-\sigma\right)$ coordinates. This plots represent the strengthening behavior of the material as it was described and modeled in Section 4.4.3, and it is governed by the $G_{I C, 0}^{r e f}, \beta_{\lambda}^{r e f}, \beta_{0}^{t}, \beta_{\lambda}^{t}$ material parameters. 


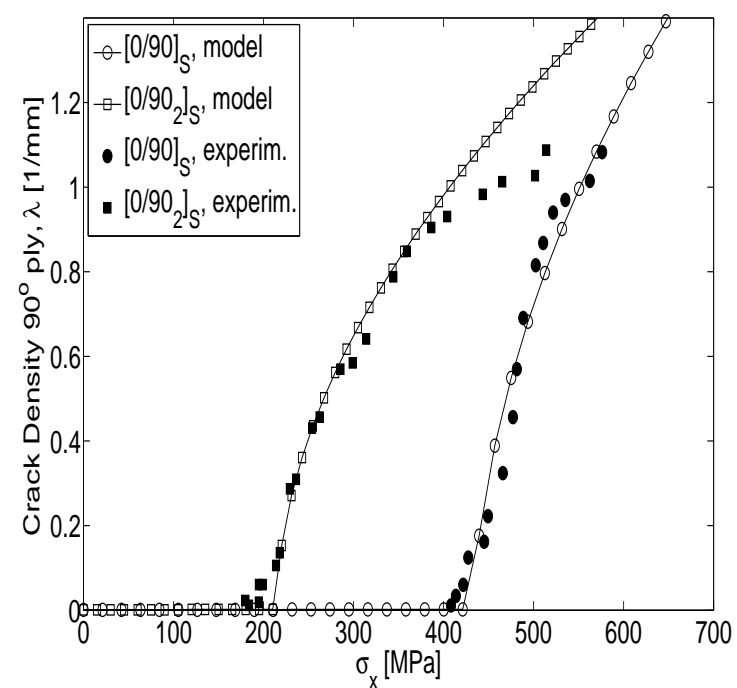

Figure 5.73: Matrix cracking progression: analytical curve vs. experimental data, Nairn $[8,9] . \quad[0 / 90]_{S}$ and $\left[0 / 90_{2}\right]_{S}$ LSS, AS4/Hercules 3501-6 material system.

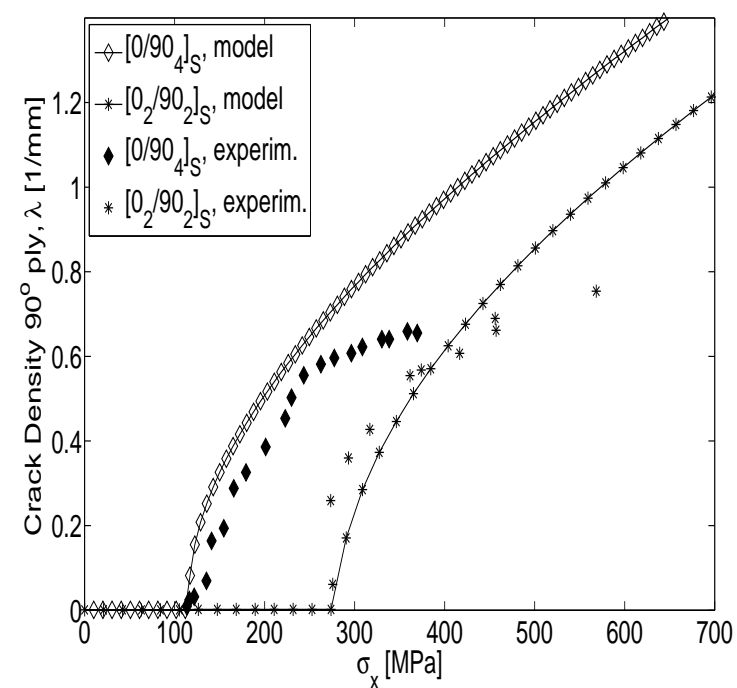

Figure 5.74: Matrix cracking progression: analytical curve vs. experimental data, Nairn $[8,9] .\left[0 / 90_{4}\right]_{S}$ and $\left[0_{2} / 90_{2}\right]_{S}$ LSS, AS4/Hercules 3501-6 material system.

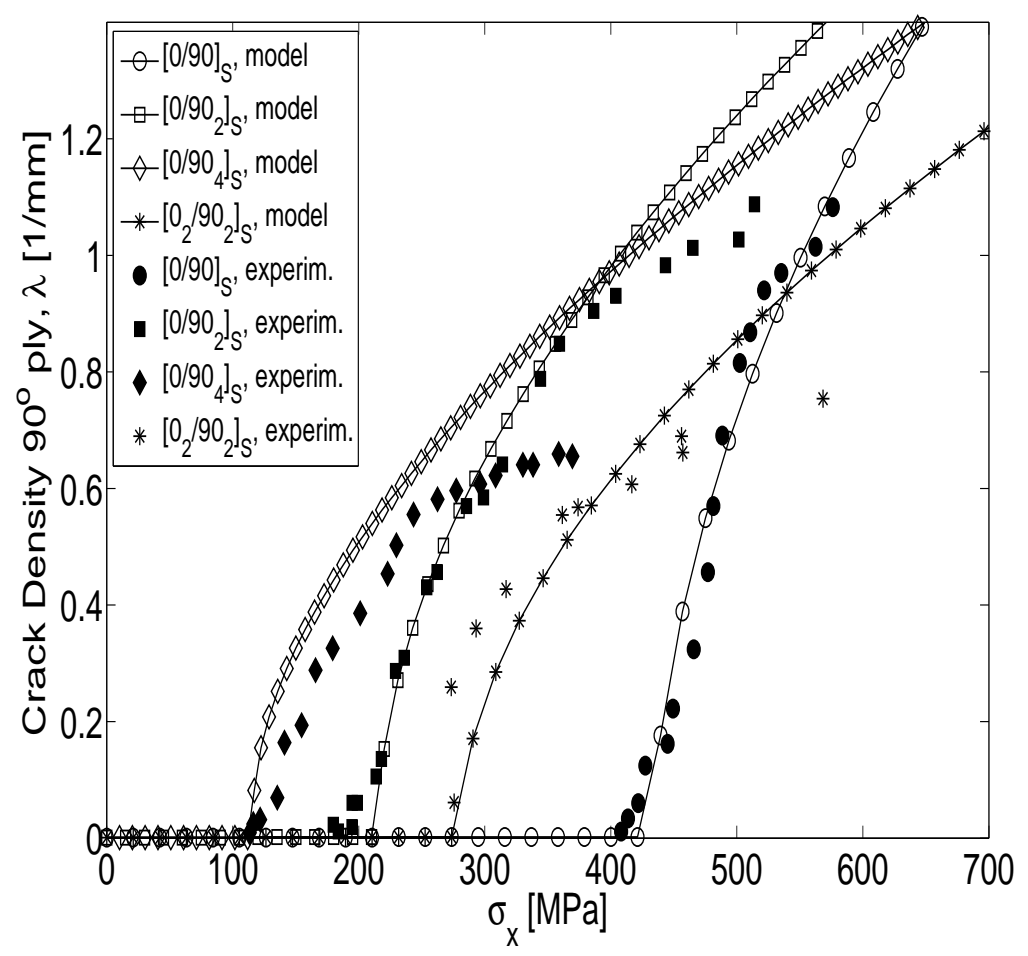

Figure 5.75: Matrix cracking progression: analytical curves vs. experimental data, Nairn $[8,9] .\left[0_{m} / 90_{n}\right]_{S}$ LSS, AS4/Hercules 3501-6 material system. 


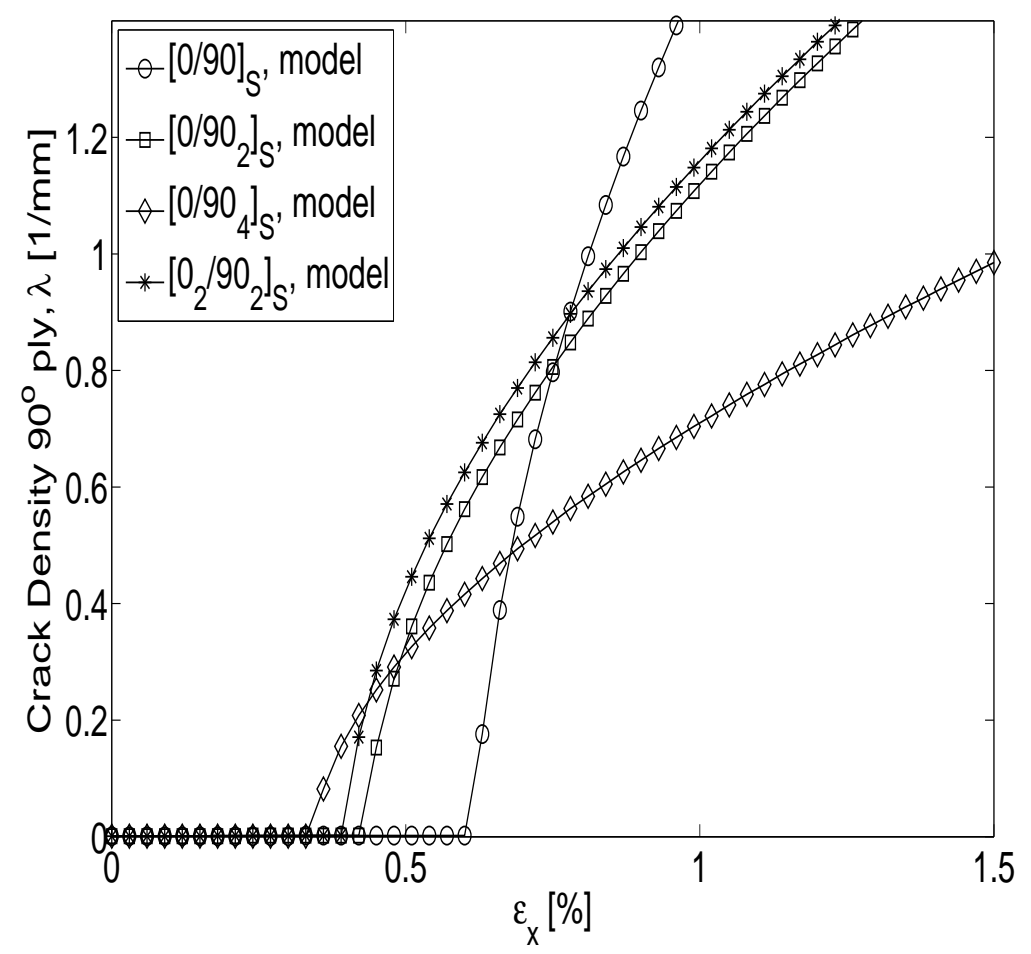

Figure 5.76: Matrix cracking progression: analytical curves. $\left[0_{m} / 90_{n}\right]_{S}$ LSS, AS4/Hercules 3501-6 material system.

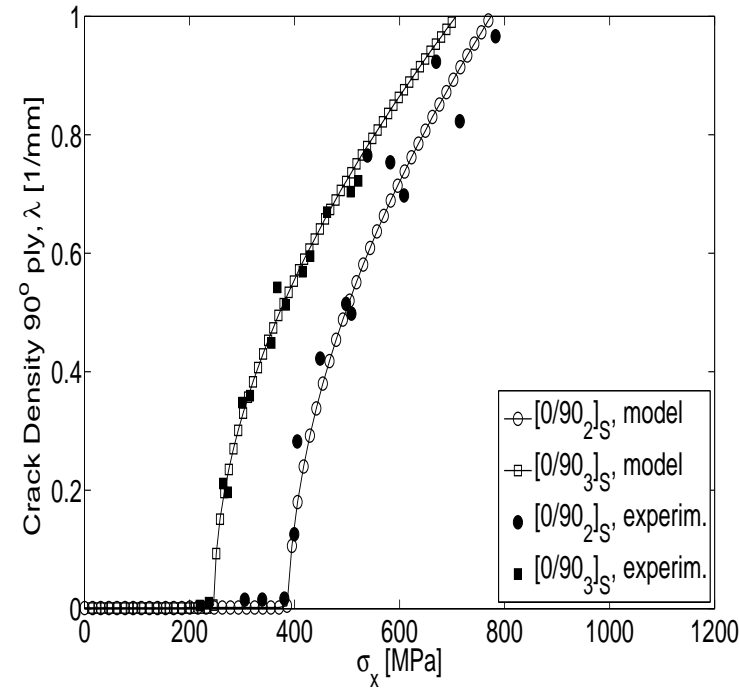

Figure 5.77: Matrix cracking progression: analytical curve vs. experimental data, Nairn [8]. $\quad\left[0 / 90_{2}\right]_{S}$ and $\left[0 / 90_{3}\right]_{S}$ LSS, IM6/Avimid-K material system.

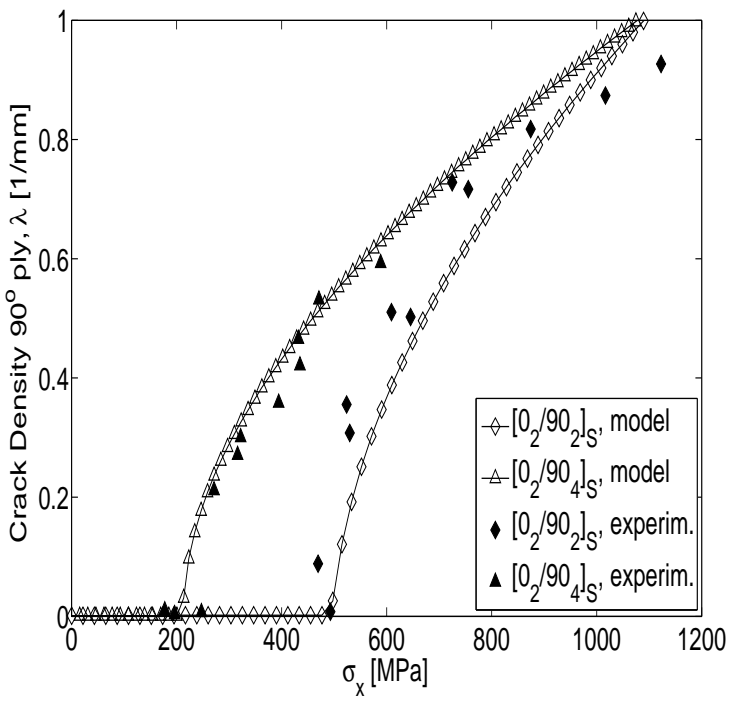

Figure 5.78: Matrix cracking progression: analytical curve vs. experimental data, Nairn [8]. $\quad\left[0_{2} / 90_{2}\right]_{S}$ and $\left[0_{2} / 90_{4}\right]_{S}$ LSS, IM6/Avimid- $K$ material system. 


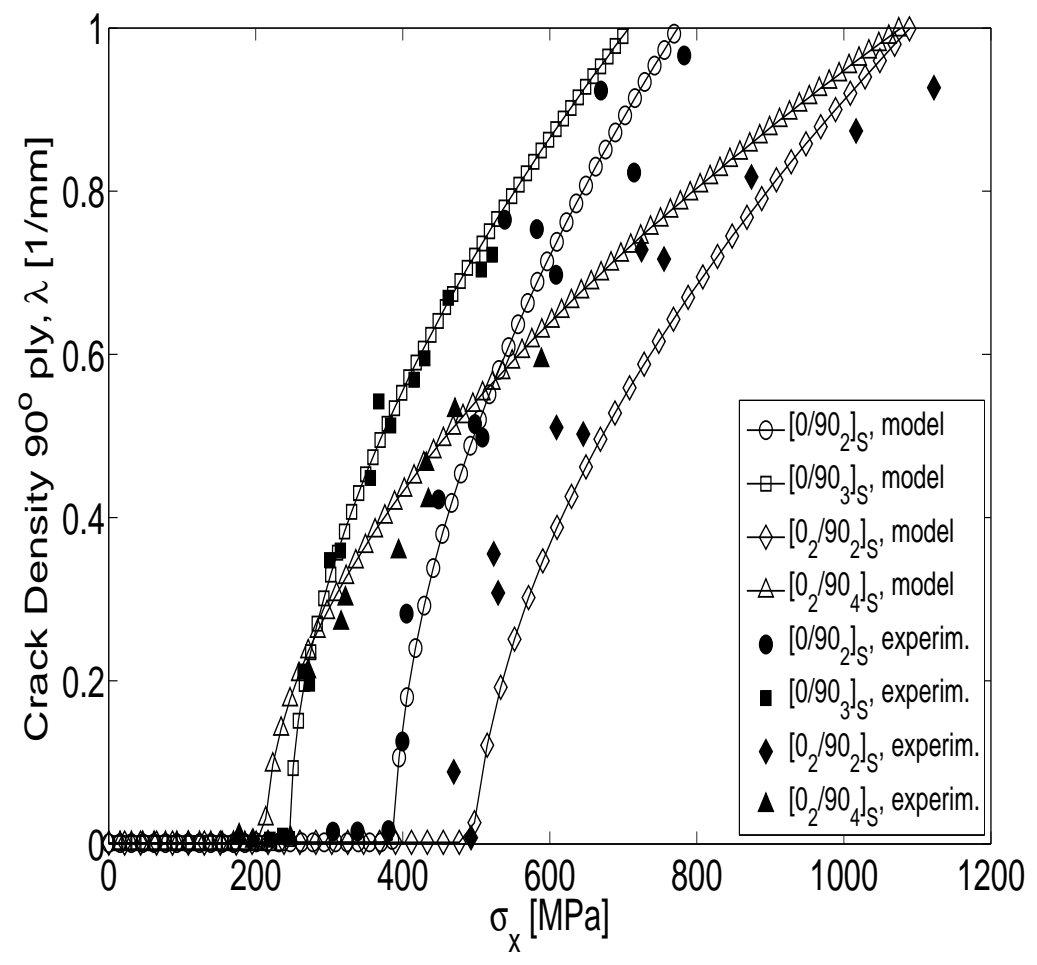

Figure 5.79: Matrix cracking progression: analytical curves vs. experimental data, Nairn [8]. $\left[0_{m} / 90_{n}\right]_{S}$ LSS, IM6/Avimid-K material system. 


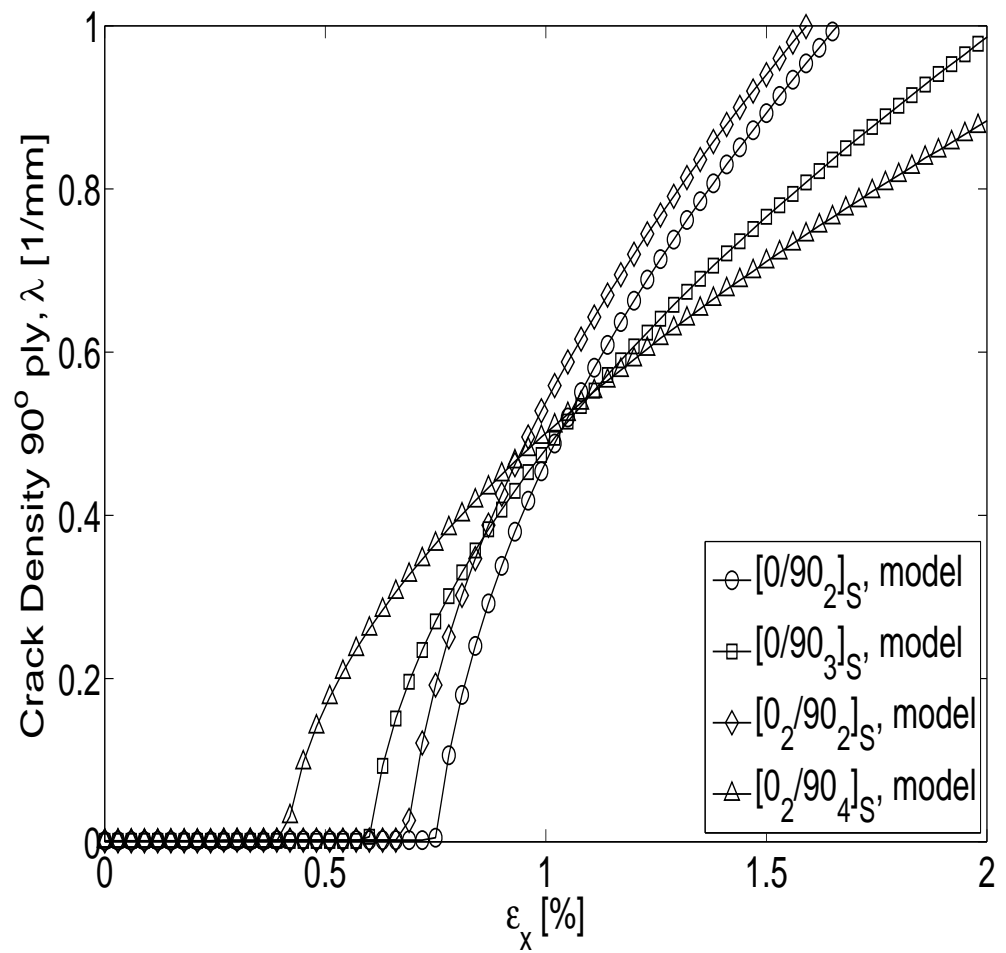

Figure 5.80: Matrix cracking progression: analytical curves. $\left[0_{m} / 90_{n}\right]_{S}$ LSS, IM6/Avimid-K material system.

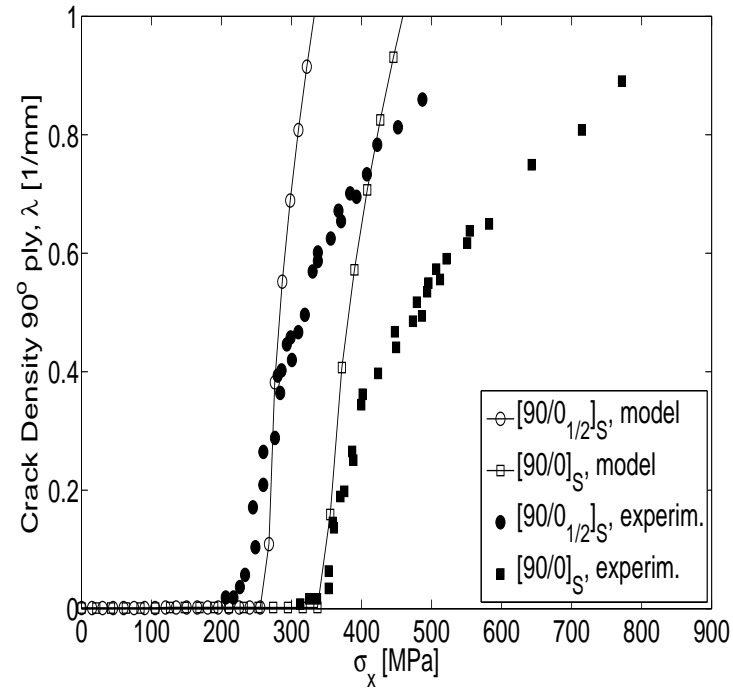

Figure 5.81: Matrix cracking progression: analytical curve vs. experimental data, Nairn $[8,9] .\left[90 / 0_{1 / 2}\right]_{S}$ and $[90 / 0]_{S}$ LSS, AS4/Hercules 3501-6 material system.

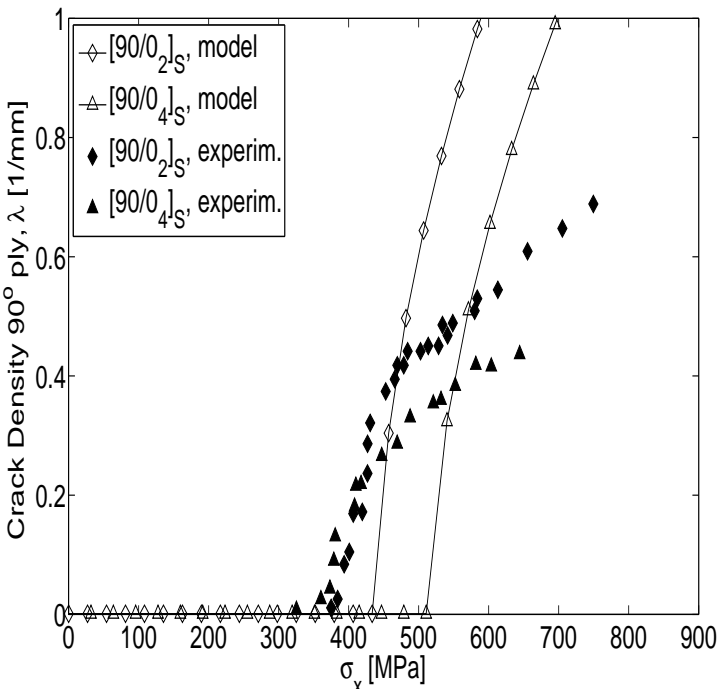

Figure 5.82: Matrix cracking progression: analytical curve vs. experimental data, Nairn $[8,9] . \quad\left[90 / 0_{2}\right]_{S}$ and $\left[90 / 0_{4}\right]_{S}$ LSS, AS4/Hercules 3501-6 material system. 


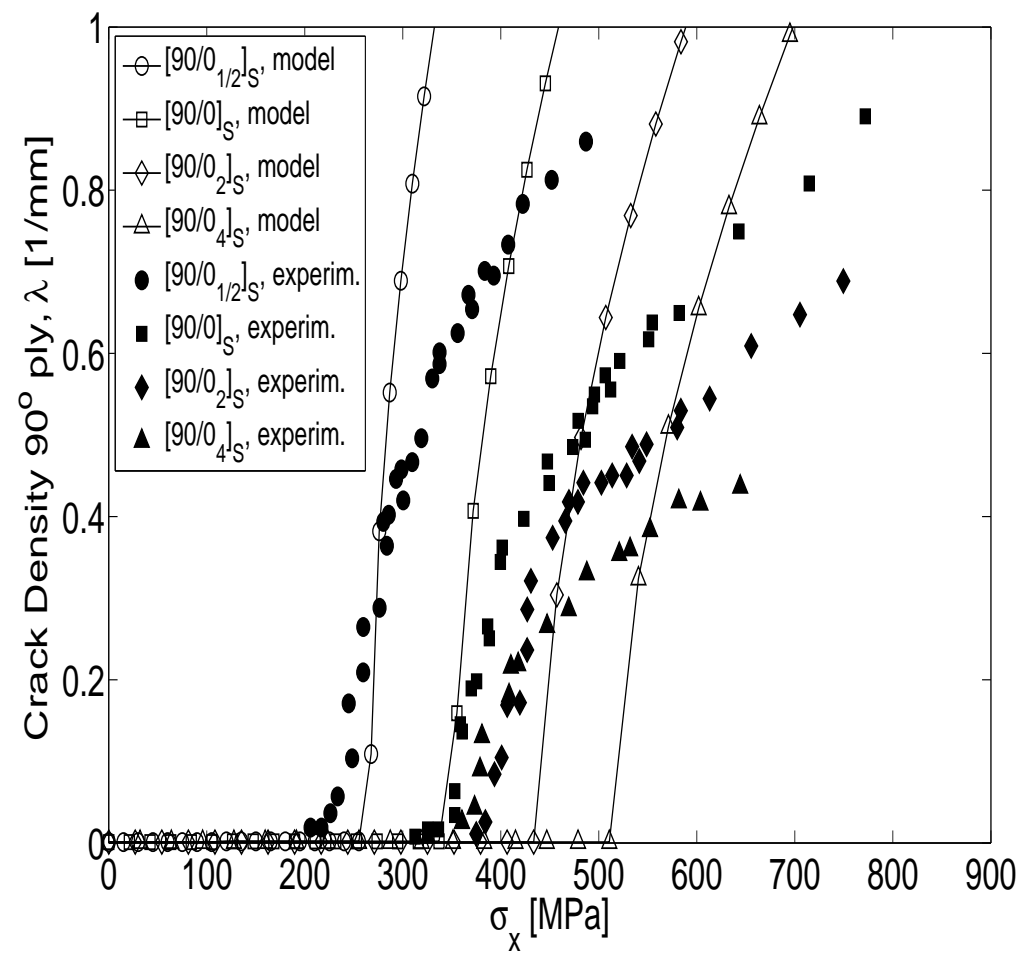

Figure 5.83: Matrix cracking progression: analytical curves vs. experimental data, Nairn $[8,9] .\left[90 / 0_{m}\right]_{S}$ LSS, AS4/Hercules 3501-6 material system. 


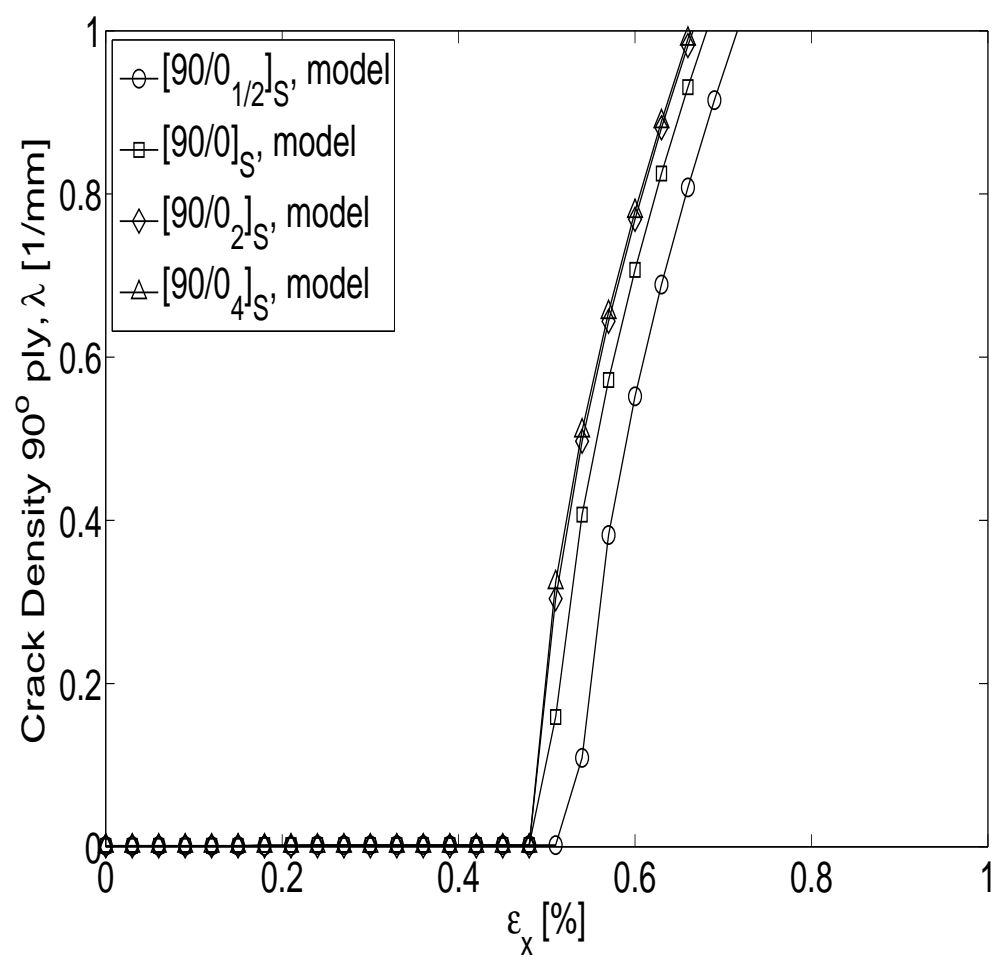

Figure 5.84: Matrix cracking progression: analytical curves. [90/0 $]_{S}$ LSS, AS4/Hercules 3501-6 material system.

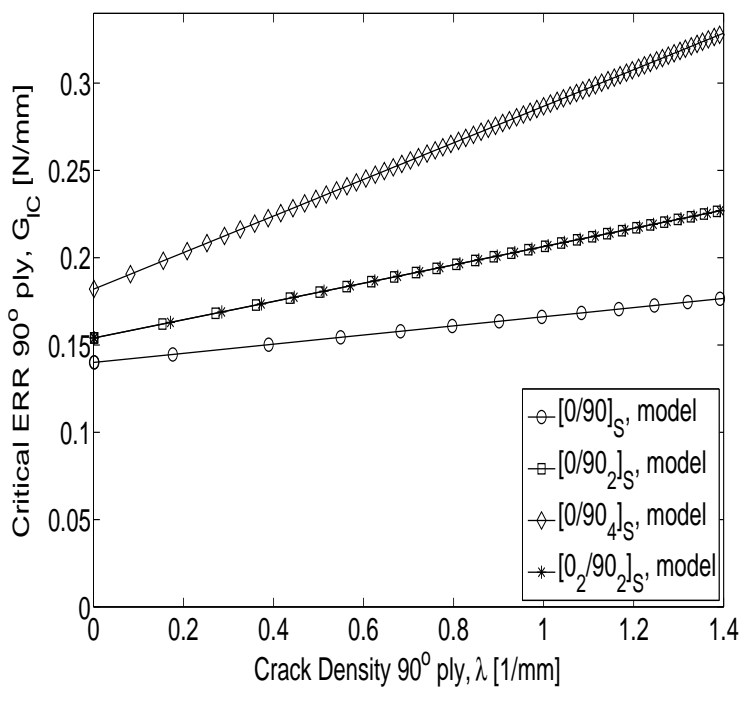

Figure 5.85: The $G_{I C}=G_{I C}(\lambda)$ in-situ $\mathrm{R}$-curve evolution of the critical ERR for the AS4/Hercules 3501-6 material system, $\left[0_{m} / 90_{n}\right]_{S}$ LSS.

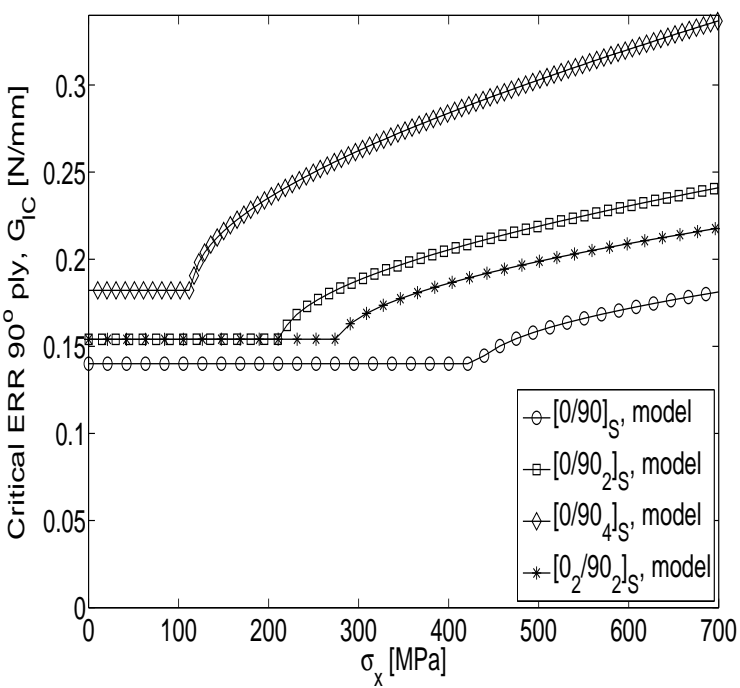

Figure 5.86: The $G_{I C}=G_{I C}(\sigma)$ in-situ $\mathrm{R}$-curve evolution of the critical ERR for the AS4/Hercules 3501-6 material system, $\left[0_{m} / 90_{n}\right]_{S}$ LSS. 


\subsubsection{Model validation, experimental data set 3: $[1,2,58,83,84]$}

A variety of laminate configurations are considered under this testing program: $\left[ \pm \theta / 90_{4}\right]_{S}$ featuring matrix cracking in the 90 ply stack $[1,83],\left[0 / \pm \theta_{4} / 0_{1 / 2}\right]_{S}$ featuring cracks in the $\pm \theta$ off-axis plies [2], and $\left[0_{m} / 90_{n}\right]_{S}$ featuring matrix cracking in the 90 ply stack $[58,84]$.

The loading case is uniaxial extension $\epsilon_{x}$ for all laminate configurations.

Two material systems are used: Glass/Epoxy $H y E$ 9082Af/Fiberite in $[1,2,83]$ and Glass/Epoxy Vicotex 913/28\%/192/EC9756 in [58,84]. The thermo-elastic properties of individual laminae for the two material systems are listed in the following, as they are offered in $[1,2,58,83,84]$ :

For Glass/Epoxy HyE 9082Af/Fiberite:

$E_{1}=44.73 G P a, \quad E_{2}=12.76 G P a, \quad G_{12}=5.8 G P a, \quad \nu_{12}=0.297, \quad \nu_{23}=0.42$ $\alpha_{1}=2 \cdot 10^{-6}{ }^{o} \mathrm{C}, \quad \alpha_{2}=29.8 \cdot 10^{-6}{ }^{o} \mathrm{C}$

For Glass/Epoxy Vicotex 913/28\%/192/EC9756:

$E_{1}=42.5 G P a, \quad E_{2}=13.3 G P a, \quad G_{12}=5.8 G P a, \quad \nu_{12}=0.29, \quad \nu_{23}=0.42$ $\alpha_{1}=2 \cdot 10^{-6}{ }^{o} \mathrm{C}, \quad \alpha_{2}=29.8 \cdot 10^{-6}{ }^{\circ} \mathrm{C}$

The ply thickness and the stress free temperature are $t_{k}=0.144 \mathrm{~mm}, S F T=125^{\circ} \mathrm{C}$ for HyE 9082Af/Fiberite, and $t_{k}=0.135 \mathrm{~mm}, S F T=120^{\circ} \mathrm{C}$ for Vicotex 913/28\%/192/EC9756.

The in-situ R-curve material parameters (see Section 4.4.3) used in the analytical model in order to closest reproduce the experimental data are

For Glass/Epoxy HyE 9082Af/Fiberite:

$G_{I C, 0}^{r e f}=0.5 \mathrm{~N} / \mathrm{mm}, \quad \beta_{\lambda}^{\text {ref }}=15$ deg. $, \quad \beta_{0}^{t}=2, \quad \beta_{\lambda}^{t}=2$

For Glass/Epoxy Vicotex 913/28\%/192/EC9756:

$G_{I C, 0}^{r e f}=0.43 \mathrm{~N} / \mathrm{mm}, \quad \beta_{\lambda}^{r e f}=10 \mathrm{deg} ., \quad \beta_{0}^{t}=1.3, \quad \beta_{\lambda}^{t}=1$

The selected reference LSS configurations (see the concept of reference thickness $t_{r e f}$ in Section 4.4.3) are $\left[0_{2} / 90_{4}\right]_{S}$ for HyE 9082Af/Fiberite, and $\left[0 / 90_{2}\right]_{S}$ for Vicotex 913/28\%/192/EC9

For those laminate configurations where matrix cracking takes place only in mode $I$ (i.e., $\left[ \pm \theta / 90_{4}\right]_{S}$ and $\left.\left[0_{m} / 90_{n}\right]_{S}\right)$, the value of the critical ERR for mode $I I, G_{I I, C}$ does not matter. For the $\left[0 / \pm \theta_{4} / 0_{1 / 2}\right]_{S}$ laminate configuration featuring cracks in the $\pm \theta$ off-axis plies under mixed mode loading, the value of mode $I I$ critical ERR is required by the analytical model, and this value is considered $G_{I I, C}=5 \mathrm{~N} / \mathrm{mm}$.

A first set of analytical vs. experimental $[1,83]$ results regards the $\left[ \pm \theta / 90_{4}\right]_{S}$ laminate configurations and Glass/Epoxy HyE 9082Af/Fiberite material system, and it is presented 
in Fig. 5.87 - 5.113.

The reduction of laminate material properties with increasing damage level is presented in Fig. 5.87 - 5.96. Reduced Young modulus of the laminate is shown in Fig. 5.87 - 5.90, and reduced Poisson ration is shown in Fig. 5.91 - 5.94. Centralized plots are shown in Fig. 5.95, 5.96.

A good agreement between analytical and experimental reduced elastic properties of the laminate can be noticed in Fig. 5.87 - 5.94. However, if the slope of the analytical predictions is compared against the slope of the experimental results, differences can be noticed for this data set. The slope of the analytical curve decreases at high crack densities, while the slope of the experimental results seems to be almost constant. Taking into account that, according to eq. (4.7), the gradient of the reduced material properties is a parameter governing the damage growth, this might be a cause for the observed discrepancy of the previous discussed damage growth curves (Section 5.2.1, 5.2.2) at high crack densities $\lambda$ (the gradient of the elastic strain energy $\Delta U$ of the laminate appears in eq. (4.7), which implies the gradient of the elastic properties of the laminate during matrix cracking).

Damage growth curves in terms of reduced material properties as function of the applied strain $\epsilon_{x}$ are presented in Fig. $5.97-5.104$ for each of the $\left[ \pm \theta / 90_{4}\right]_{S}$ laminate configuration. Centralized plots are shown in Fig. 5.105, 5.106. Good agreement of model vs. experiments is found.

Damage growth curves in $(\lambda-\epsilon)$ coordinates are presented in Fig. $5.107-5.110$, and the centralized plot is shown in Fig. 5.111. Good agreement of the analytical damage evolution curves with experimental data can be noticed in the listed plots for low angles of the off-axis uncracking $\theta$ plies. The least agreement is obtain for the highest off-axis angle, $\theta=40 \mathrm{deg}$., where the model prediction is steeper than the trend of the experimental data points. It is possible that other damage mechanisms, like delamination, could take place at this high value $\theta=40 \mathrm{deg}$. of the off-axis angle. If this is the case, elastic energy is released for the formation of these additional damage, and the resulting experimental level of matrix cracking in the monitored $90_{4}$ ply will be lower that the analytical predicted value, which does not consider the possible delamination.

The strengthening curves of the modeled in-situ $\mathrm{R}$-curve behavior, corresponding to the selected $G_{I C, 0}^{r e f}, \beta_{\lambda}^{r e f}, \beta_{0}^{t}, \beta_{\lambda}^{t}$ material parameters are presented in Fig. 5.112, 5.113. The modeled R-curve behavior (i.e., the increasing value of the critical $\mathrm{ERR} G_{I C}$ with increasing crack density $\lambda$ ) can be noticed in Fig. 5.112, with no in-situ effect (i.e., thickness effect 
of the cracking ply), due to the fact that the whole set of samples are characterized by the same thickness of the cracking ply. However, slight differences between the $G_{I C}=G_{I C}(\epsilon)$ curves can be noticed in Fig. 5.113, due to the fact that the damage growth process is also a function of the laminate configuration.

A second set of analytical vs. experimental [2] results regards the $\left[0 / \pm \theta_{4} / 0_{1 / 2}\right]_{S}$ laminate configurations and Glass/Epoxy HyE 9082Af/Fiberite material system, and it is presented in Fig. 5.114 - 5.127. The particular feature of this set of experimental data is that matrix cracking takes place in the off-axis $\pm \theta$ plies, under combined $I$ and $I I$ modes.

Reduced Young as function of crack density is presented in Fig. 5.114 - 5.116 with a centralized plot in Fig. 5.117, and reduced Poisson is presented in Fig. 5.118 - 5.120 with a centralized plot in Fig. 5.121. The same observations as in the case of the previous data set holds: the agreement between the analytical and experimental results is good. However, if the slope of the reduced material properties is regarded, differences can be noticed between the analytical curve and the experimental trend at high values of the crack density $\lambda$. These can affect the accuracy of the crack evolution predictions at high crack densities.

It is interesting to notice the evolution of the Poisson ratio of the laminate at the lowest value of the off-axis orientation of the cracking ply, $\theta=55$, in Fig. 5.121. A slightly increasing trend of the Poisson coefficient with increasing damage level is recorded for this particular laminate configuration, which is opposed to the trends recorded for higher values of the off-axis ply orientation. This fact suggests that there is a threshold value of the off-axis orientation of the cracking ply at which the Poisson coefficient remains constant regardless the matrix cracking process. At off-axis angles higher than the threshold value the Poisson coefficient decreases with damage level, and at off-axis angles lower than the threshold value the Poisson coefficient increases with damage level. This acknowledge of the material behavior can be useful in assessing the damage tolerance of the composite and even tailoring the desired response of the material under matrix cracking conditions.

Damage evolution curves for the considered laminate configurations are presented in Fig. 5.122 - 5.124, with a centralized plot in Fig. 5.125. The ability of the analytical model to predict the experimental trends can be observed in these plots. The least accuracy of the analytical predictions is for the $\left[0 / 90_{8} / 0_{1 / 2}\right]_{S}$ laminate.

To be noticed that the $\mathrm{R}$-curve parameters $G_{I C, 0}^{r e f}=0.5$ and $\beta_{\lambda}^{r e f}=15$ have been used, as they were set for the $\left[0_{2} / 90_{4}\right]_{S}$ laminate configuration from the previous $\left[ \pm \theta / 90_{4}\right]_{S}$ data set of the same Glass/Epoxy HyE 9082Af/Fiberite material system. The observation to be 
made here is that even if the material system is the same in the two cases, this is not a guarantee that the $\mathrm{R}$-curve parameters are the same. It has been shown in Section 2.2.1 that the $\mathrm{R}$-curve behavior is mainly governed by the degree of fiber misalignment in longitudinal direction, and the fiber misalignment can be different for the same material system, if the processing conditions are different. Experimental data regarding the two laminate configurations were published in different years, and it is not know if the composite samples were manufactured under the same processing conditions. A different set of $\mathrm{R}$-curve parameters might be required for the present $\left[0 / \pm \theta_{4} / 0_{1 / 2}\right]_{S}$ laminate configurations.

The modeled in-situ R-curve evolution of the mode $I$ critical ERR, $G_{I C}$, is resented in Fig. 5.126 - 5.127. A higher slope and initial value can be noticed for the $G_{I C}(\lambda) \mathrm{R}$-curve corresponding to the $\left[0 / 90_{8} / 0_{1 / 2}\right]_{S}$ laminate configuration, as compared to the other two $\left[0 / \pm 70_{4} / 0_{1 / 2}\right]_{S}$ and $\left[0 / \pm 55_{4} / 0_{1 / 2}\right]_{S}$ laminates. This is because is it considered that the thickness of the cracking ply for the $\pm \theta_{4}$ configuration is half of the thickness of the cracking ply for the $90_{8}$ configuration. It is considered that matrix cracking takes place separately in the $+\theta_{4}$ plies and in the $-\theta_{4}$ plies, and cracks are arrested at the interface between the $\pm \theta_{4}$ ply stacks. This fact brings the in-situ thickness effect into picture, as it is governed by the $\beta_{0}^{t}$ and $\beta_{\lambda}^{t}$ parameters (see Section 4.4.3). Consequently, the $\mathrm{R}$-curve behavior will be different for the $90_{8}$ configuration and the $\pm \theta_{4}$ configuration, since they feature different thicknesses of cracking ply.

While the in-situ $\mathrm{R}$-curve behavior is implemented for the mode $I$ critical ERR, $G_{I C}$, there is no information regarding the behavior of the node $I I$ critical ERR, $G_{I I C}$, which is considered constant in the present model (see Section 4.4.3). Damage evolution for the present $\left[0 / \pm \theta_{4} / 0_{1 / 2}\right]_{S}$ data set is influenced by both $G_{I C}$ and $G_{I I C}$ critical parameters, due to the mixed mode conditions in the off-axis plies.

A third set of experimental results $[58,84]$ regards the $\left[0 m / 90_{n}\right]_{S}$ laminate configurations and Glass/Epoxy Vicotex 913/28\%/192/EC9756 material system. The comparison of analytical output vs. experimental data is presented in Fig. 5.128 - 5.135. Very good agreement of the predicted reduced material properties can be noticed in Fig. 5.128, 5.129 for all laminate configurations. Regarding the damge evolution curves in Fig. 5.130 - 5.133, the correlation between analytical and experimental results is very good for the $\left[0 / 90_{2}\right]_{S}$ and $\left[0_{2} / 90_{2}\right]_{S}$ laminate configurations, but it is less satisfying for the $\left[0 / 90_{4}\right]_{S}$ laminate.

The modeled evolution of the critical ERR $G_{I C}$ is presented in Fig. 5.134, 5.135, as it is set by the $G_{I C, 0}^{r e f}, \quad \beta_{\lambda}^{r e f}, \quad \beta_{0}^{t}, \quad \beta_{\lambda}^{t}$ in-situ R-curve parameters. 


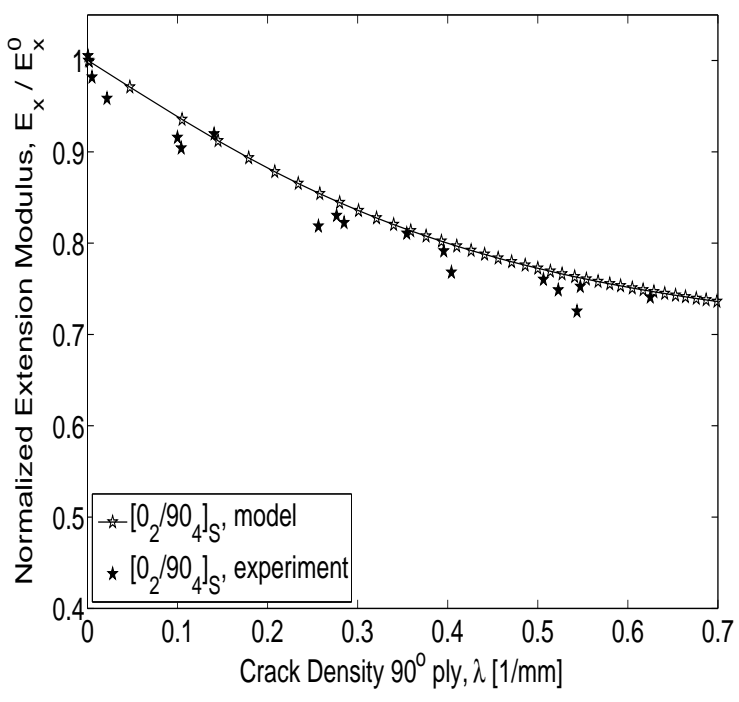

Figure 5.87: Reduced Young modulus $E_{x}(\lambda)$ : analytical curve vs. experimental data, Varna [1]. $\left[0_{2} / 90_{4}\right]_{S}$ LSS, HyE 9082Af/Fiberite material system.

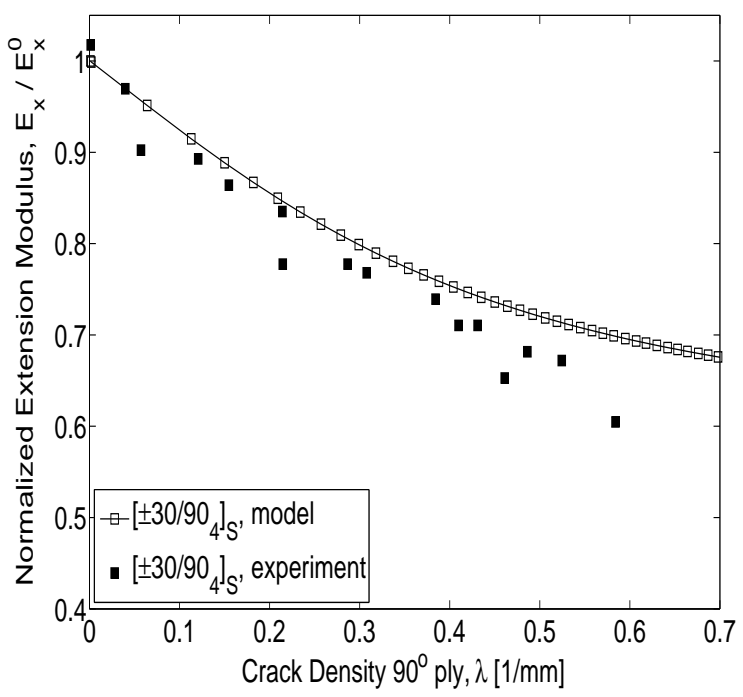

Figure 5.89: Reduced Young modulus $E_{x}(\lambda)$ : analytical curve vs. experimental data, Varna [1]. $\left[ \pm 30 / 90_{4}\right]_{S}$ LSS, $H y E$ 9082Af/Fiberite material system.

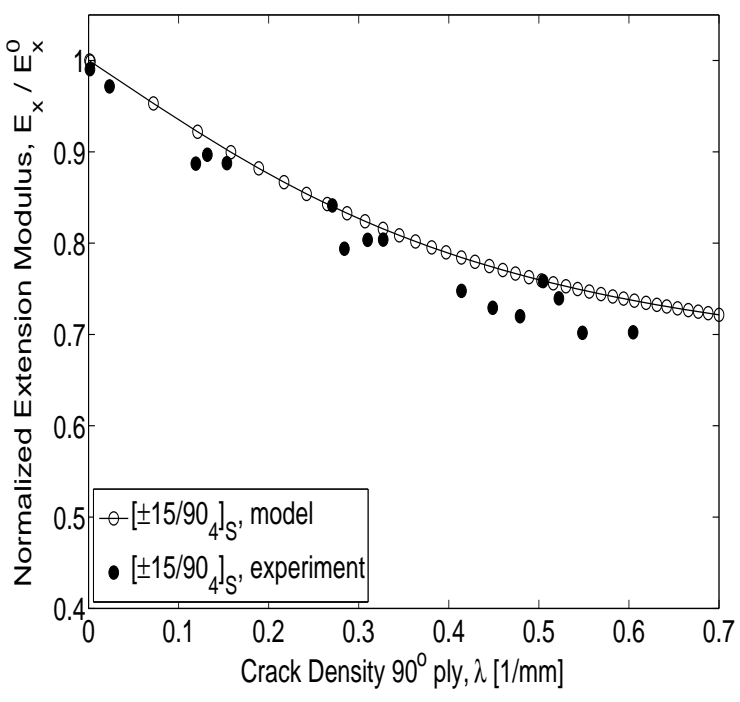

Figure 5.88: Reduced Young modulus $E_{x}(\lambda)$ : analytical curve vs. experimental data, Varna [1]. $\left[ \pm 15 / 90_{4}\right]_{S}$ LSS, HyE 9082Af/Fiberite material system.

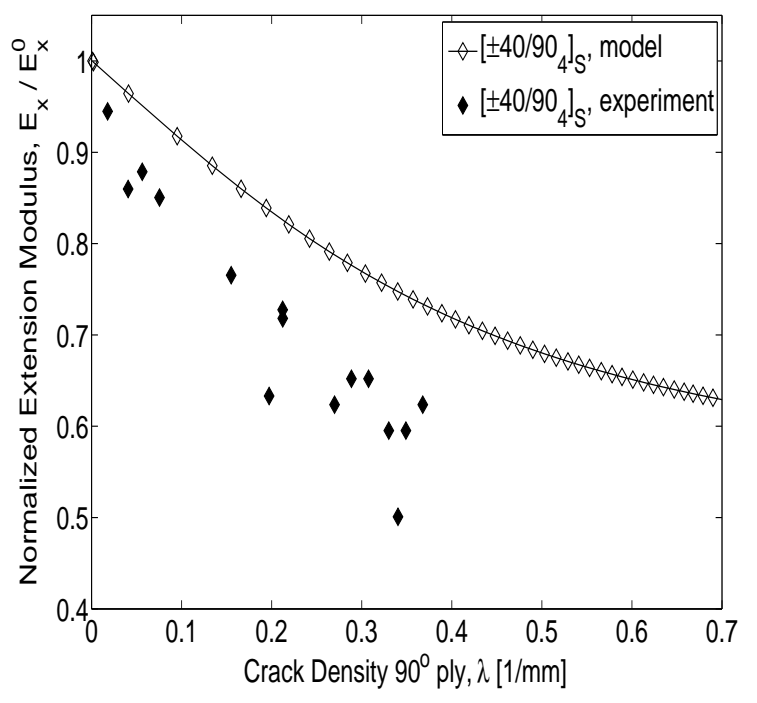

Figure 5.90: Reduced Young modulus $E_{x}(\lambda)$ : analytical curve vs. experimental data, Varna [1]. $\left[ \pm 40 / 90_{4}\right]_{S} \mathrm{LSS}, H y E$ 9082Af/Fiberite material system. 


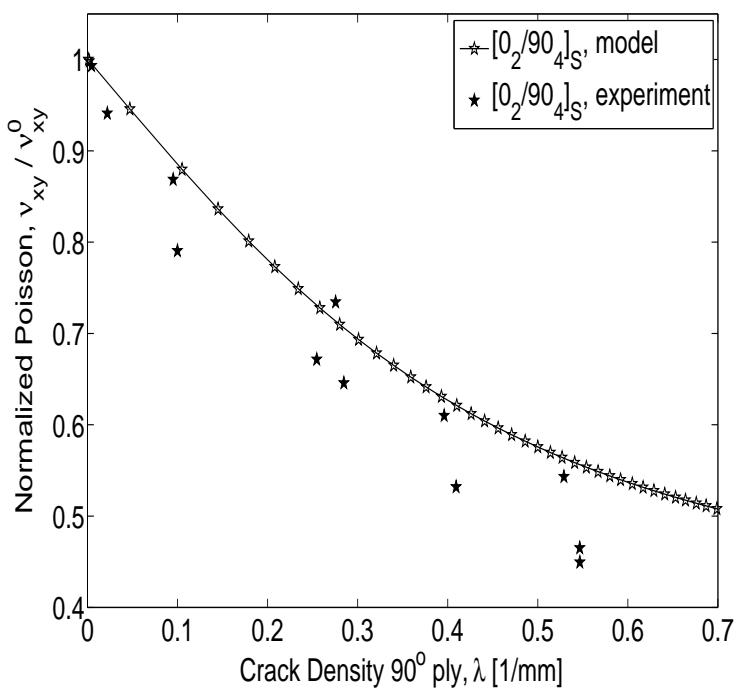

Figure 5.91: Reduced Poisson coefficient $\nu_{x y}(\lambda)$ : analytical curve vs. experimental data, Varna [1]. $\left[0_{2} / 90_{4}\right]_{S}$ LSS, HyE 9082Af/Fiberite material system.

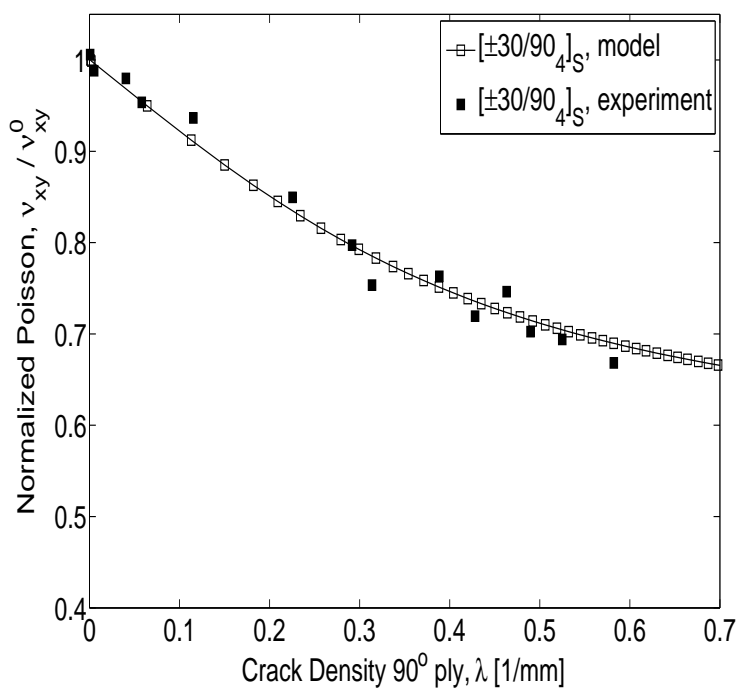

Figure 5.93: Reduced Poisson coefficient $\nu_{x y}(\lambda)$ : analytical curve vs. experimental data, Varna [1]. $\left[ \pm 30 / 90_{4}\right]_{S}$ LSS, $H y E$ 9082Af/Fiberite material system.

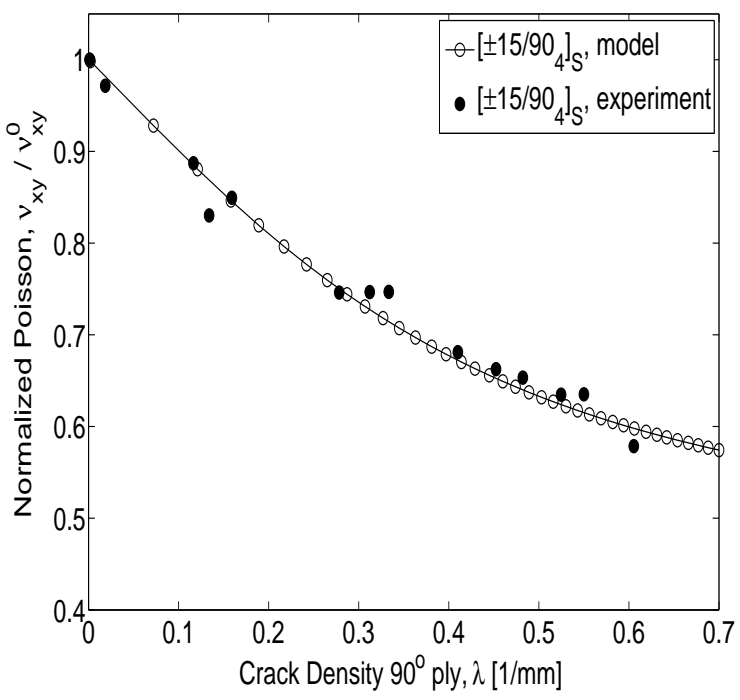

Figure 5.92: Reduced Poisson coefficient $\nu_{x y}(\lambda)$ : analytical curve vs. experimental data, Varna [1]. $\left[ \pm 15 / 90_{4}\right]_{S}$ LSS, HyE 9082Af/Fiberite material system.

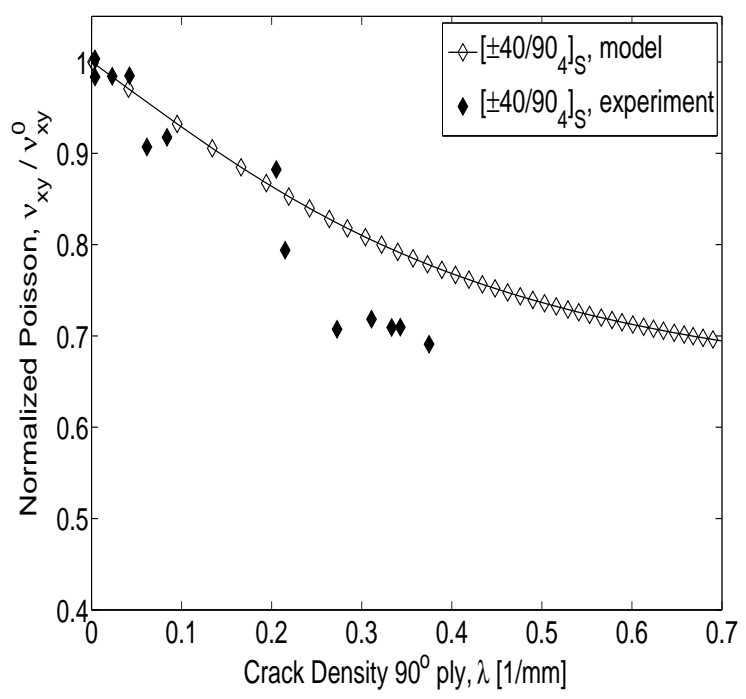

Figure 5.94: Reduced Poisson coefficient $\nu_{x y}(\lambda)$ : analytical curve vs. experimental data, Varna $[1] .\left[ \pm 40 / 90_{4}\right]_{S}$ LSS, HyE 9082Af/Fiberite material system. 


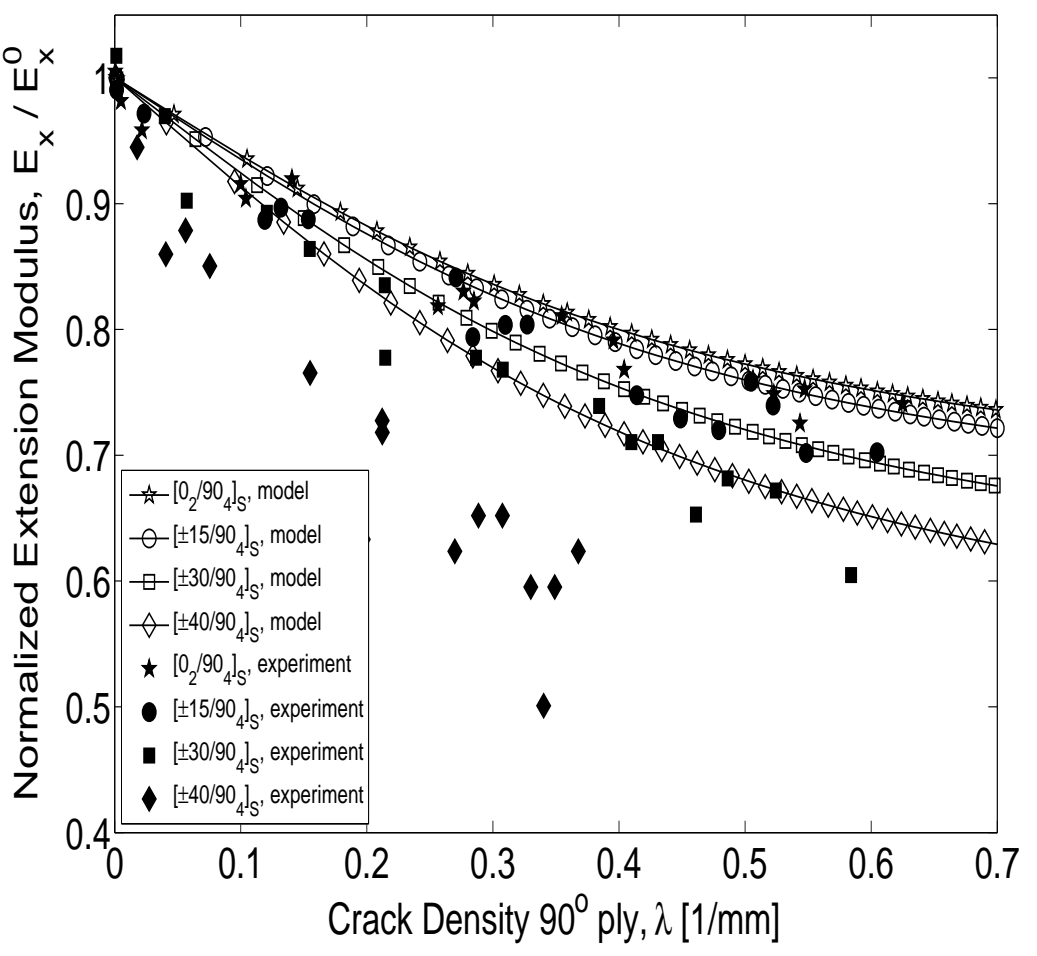

Figure 5.95: Reduced Young modulus $E_{x}(\lambda)$ : analytical curves vs. experimental data, Varna [1]. $\left[ \pm \theta / 90_{4}\right]_{S}$ LSS, HyE 9082Af/Fiberite material system. 


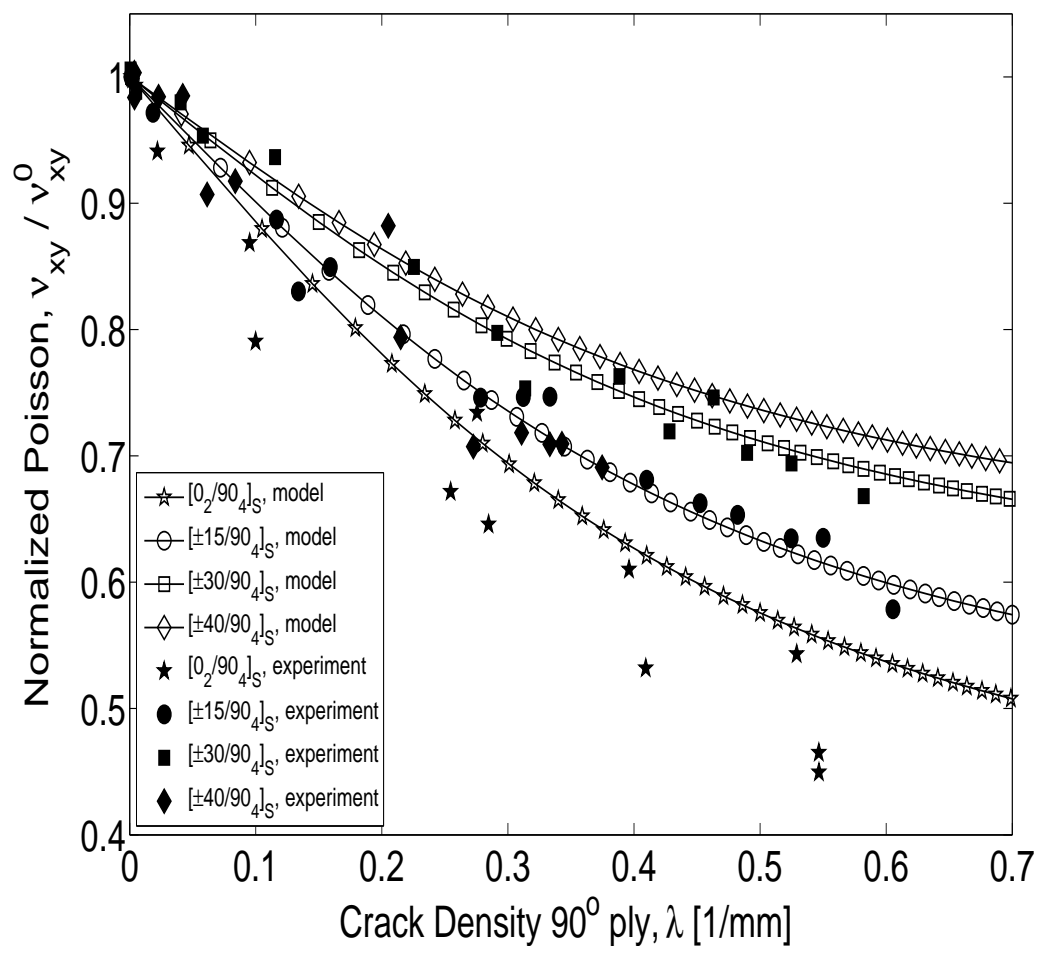

Figure 5.96: Reduced Poisson coefficient $\nu_{x y}(\lambda)$ : analytical curves vs. experimental data, Varna [1]. $\left[ \pm \theta / 90_{4}\right]_{S}$ LSS, HyE 9082Af/Fiberite material system.

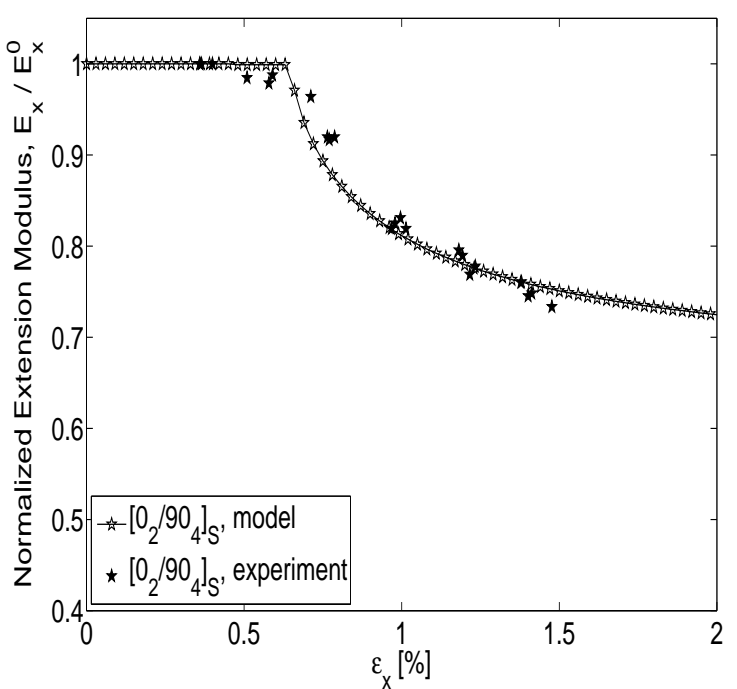

Figure 5.97: Reduced Young modulus $E_{x}\left(\epsilon_{x}\right)$ : analytical curve vs. experimental data, Varna [1]. $\left[0_{2} / 90_{4}\right]_{S}$ LSS, HyE 9082Af/Fiberite material system.

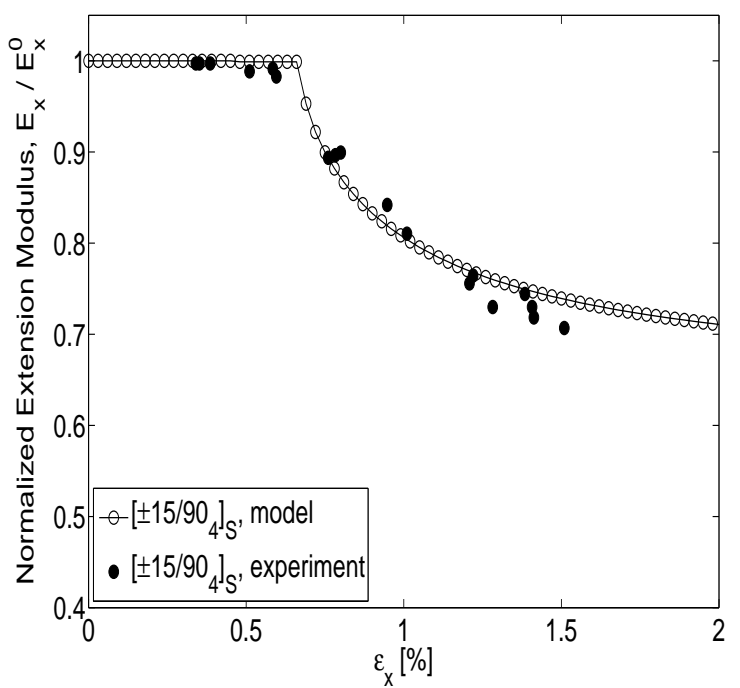

Figure 5.98: Reduced Young modulus $E_{x}\left(\epsilon_{x}\right)$ : analytical curve vs. experimental data, Varna $[1] .\left[ \pm 15 / 90_{4}\right]_{S}$ LSS, $H y E$ 9082Af/Fiberite material system. 


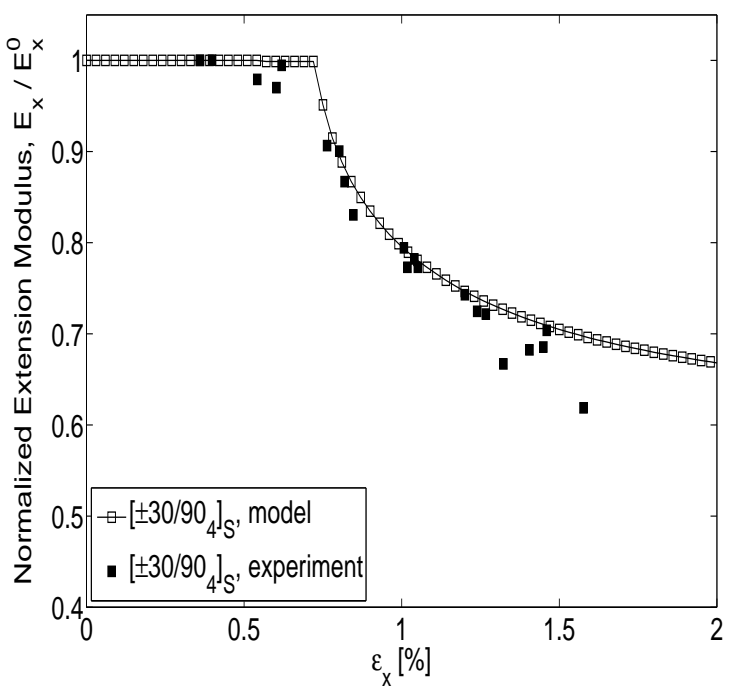

Figure 5.99: Reduced Young modulus $E_{x}\left(\epsilon_{x}\right)$ : analytical curve vs. experimental data, Varna [1]. $\left[ \pm 30 / 90_{4}\right]_{S}$ LSS, HyE 9082Af/Fiberite material system.

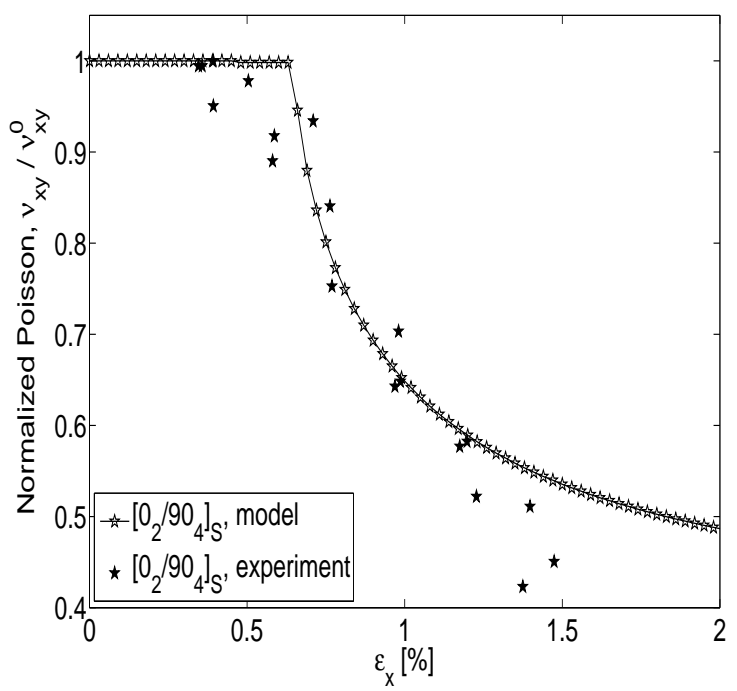

Figure 5.101: Reduced Poisson coefficient $\nu_{x y}\left(\epsilon_{x}\right)$ : analytical curve vs. experimental data, Varna [1]. $\left[0_{2} / 90_{4}\right]_{S}$ LSS, HyE 9082Af/Fiberite material system.

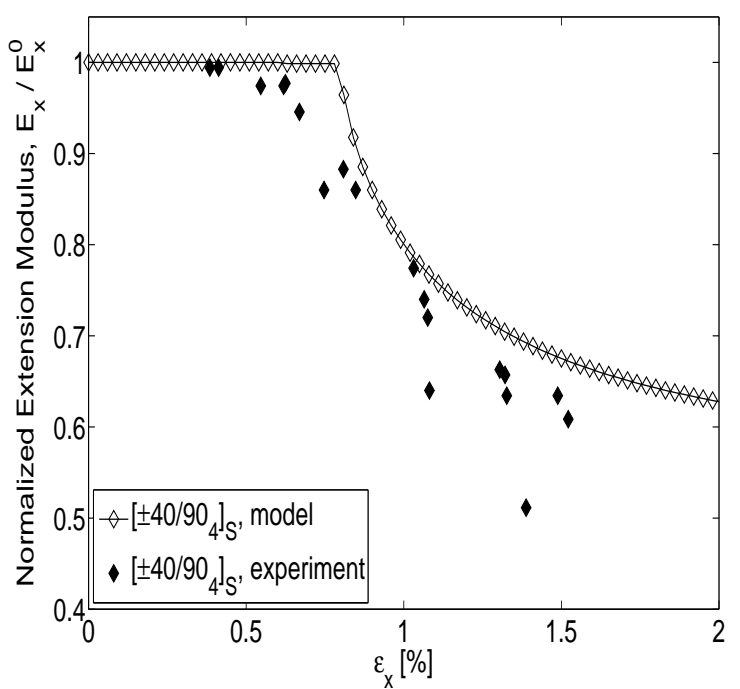

Figure 5.100: Reduced Young modulus $E_{x}\left(\epsilon_{x}\right)$ : analytical curve vs. experimental data, Varna [1]. $\left[ \pm 40 / 90_{4}\right]_{S}$ LSS, HyE 9082Af/Fiberite material system.

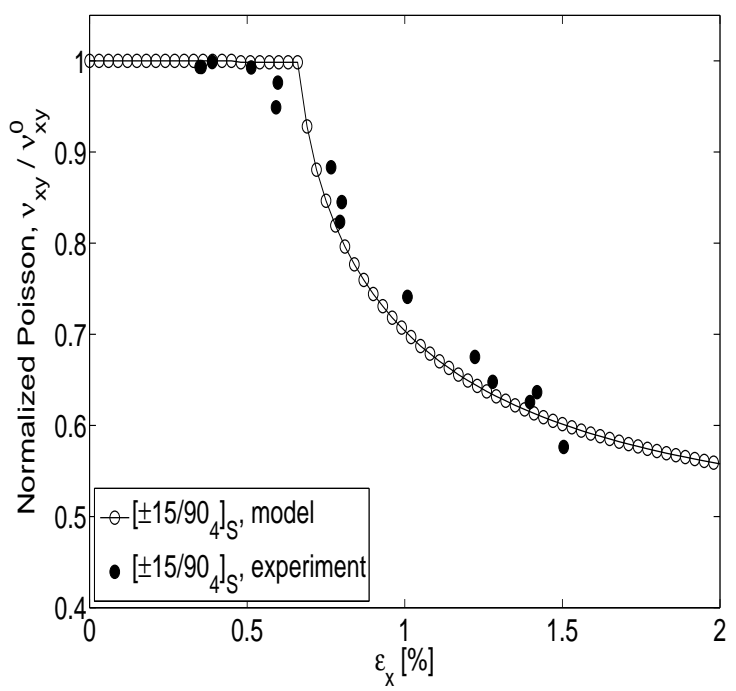

Figure 5.102: Reduced Poisson coefficient $\nu_{x y}\left(\epsilon_{x}\right)$ : analytical curve vs. experimental data, Varna $[1] .\left[ \pm 15 / 90_{4}\right]_{S}$ LSS, $H y E$ 9082Af/Fiberite material system. 


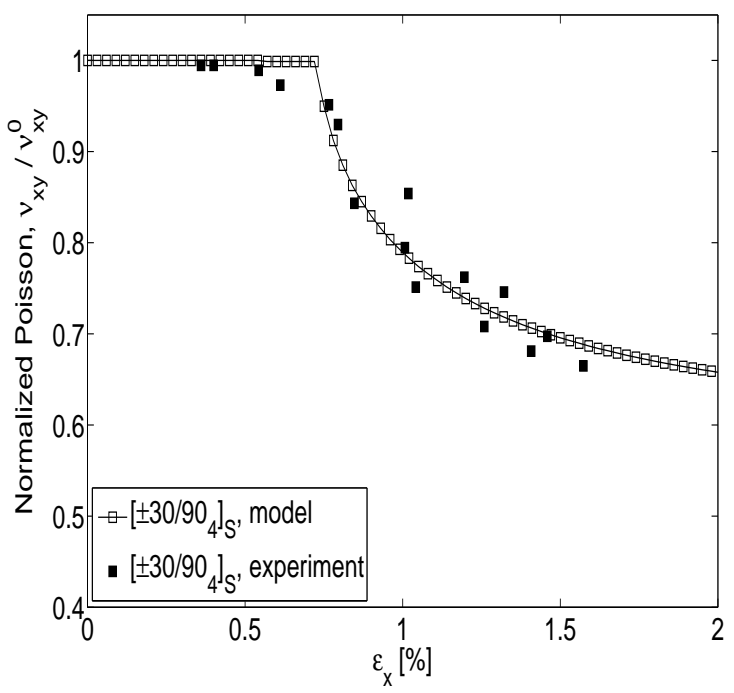

Figure 5.103: Reduced Poisson coefficient $\nu_{x y}\left(\epsilon_{x}\right)$ : analytical curve vs. experimental data, Varna $[1] .\left[ \pm 30 / 90_{4}\right]_{S}$ LSS, $H y E$ 9082Af/Fiberite material system.

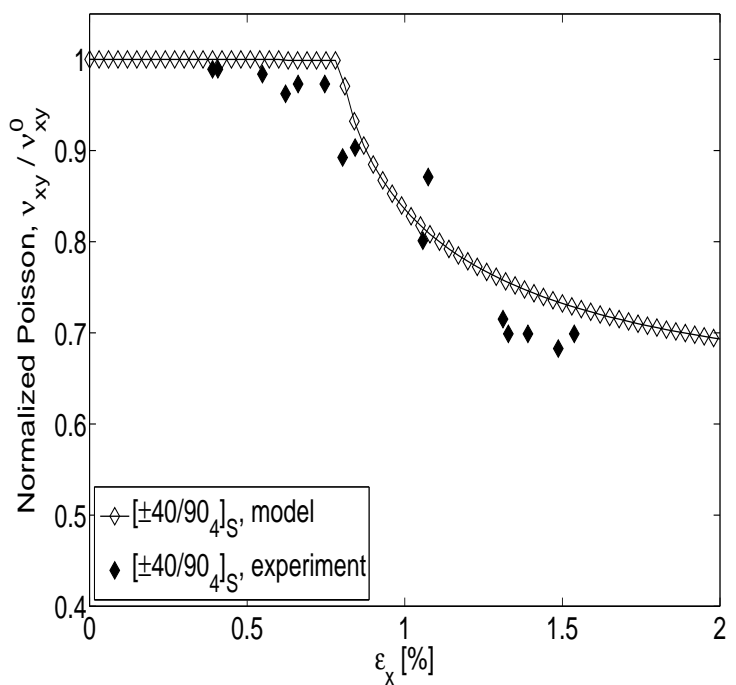

Figure 5.104: Reduced Poisson coefficient $\nu_{x y}\left(\epsilon_{x}\right)$ : analytical curve vs. experimental data, Varna [1]. $\left[ \pm 40 / 90_{4}\right]_{S}$ LSS, HyE 9082Af/Fiberite material system.

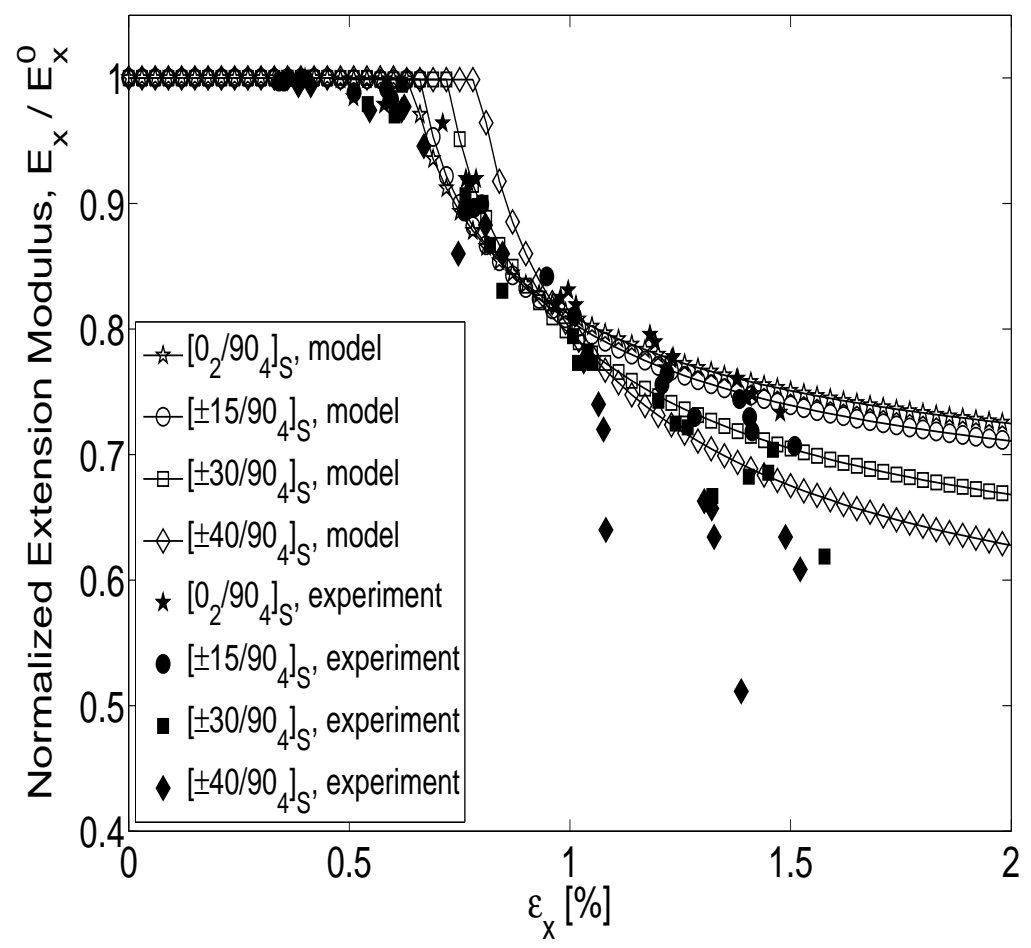

Figure 5.105: Reduced Young modulus $E_{x}\left(\epsilon_{x}\right)$ : analytical curves vs. experimental data, Varna [1]. $\left[ \pm \theta / 90_{4}\right]_{S}$ LSS, HyE 9082Af/Fiberite material system. 


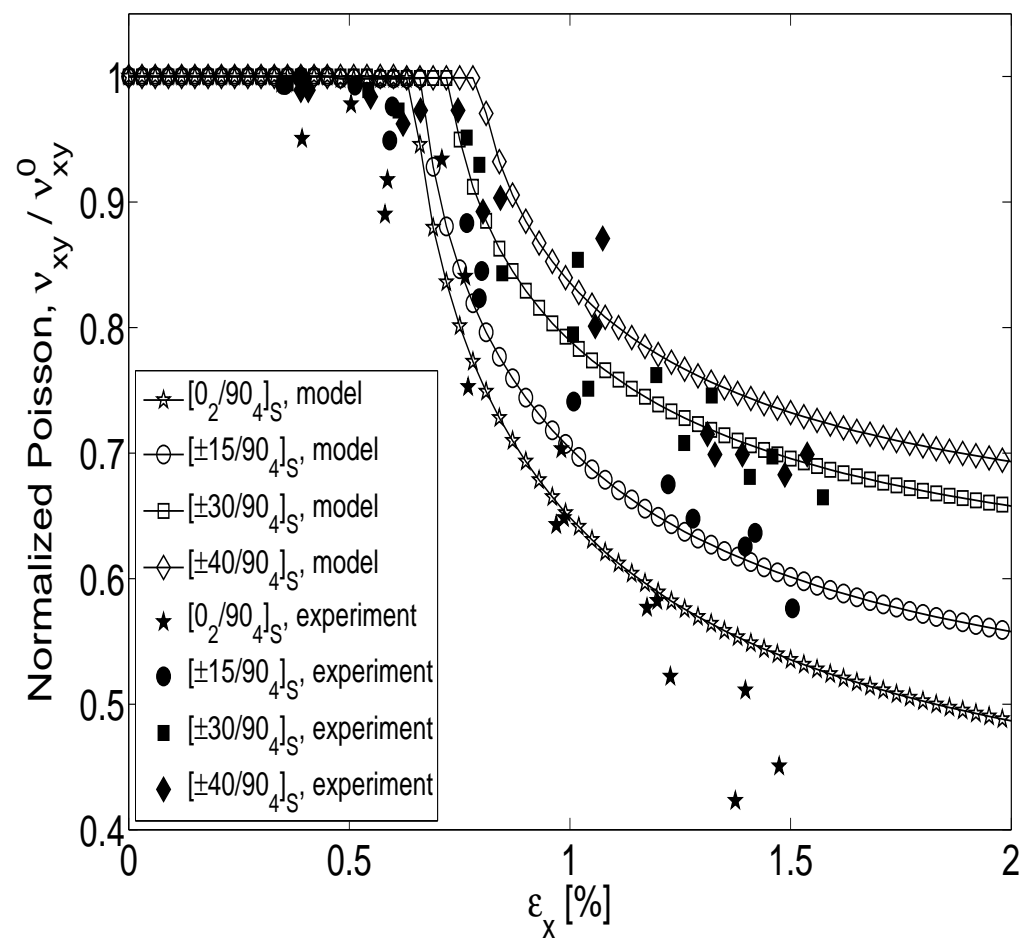

Figure 5.106: Reduced Poisson coefficient $\nu_{x y}\left(\epsilon_{x}\right)$ : analytical curves vs. experimental data, Varna [1]. $\left[ \pm \theta / 90_{4}\right]_{S}$ LSS, HyE 9082Af/Fiberite material system.

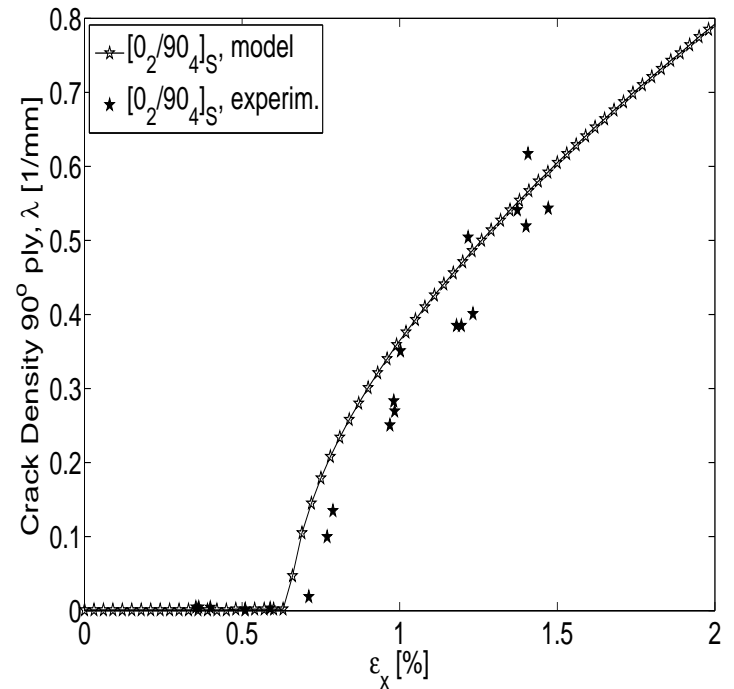

Figure 5.107: Matrix cracking progression: analytical curve vs. experimental data, Varna [83]. $\quad\left[0_{2} / 90_{4}\right]_{S}$ LSS, HyE 9082Af/Fiberite material system.

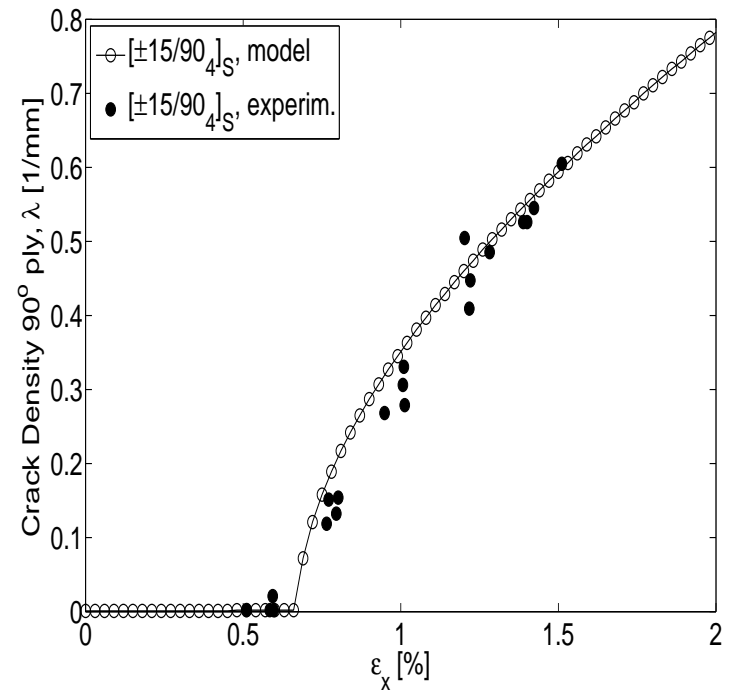

Figure 5.108: Matrix cracking progression: analytical curve vs. experimental data, Varna [83]. $\left[ \pm 15 / 90_{4}\right]_{S}$ LSS, HyE 9082Af/Fiberite material system. 


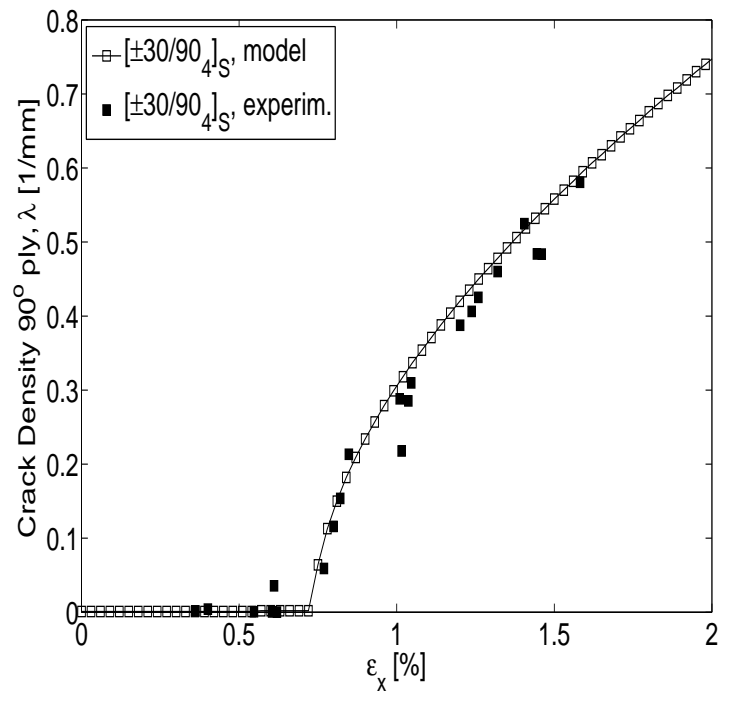

Figure 5.109: Matrix cracking progression: analytical curve vs. experimental data, Varna [83]. $\left[ \pm 30 / 90_{4}\right]_{S}$ LSS, HyE 9082Af/Fiberite material system.

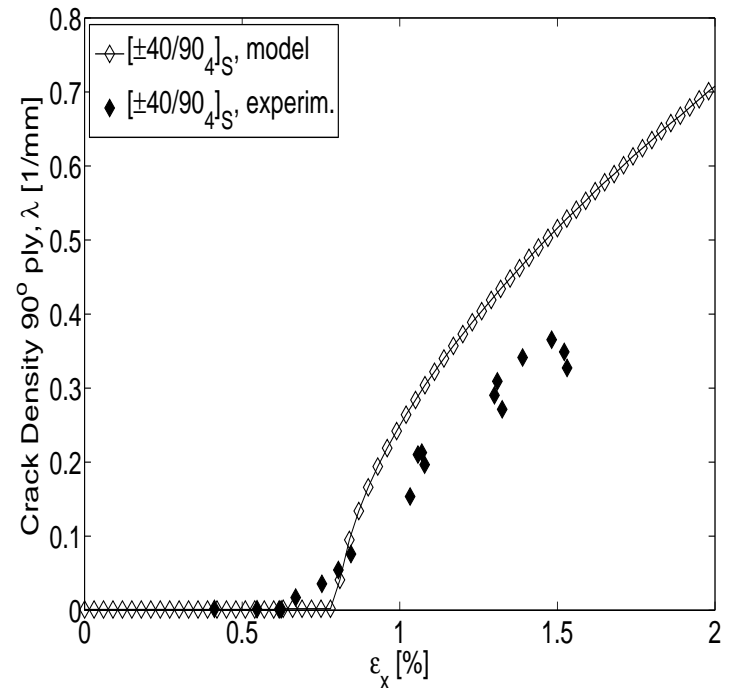

Figure 5.110: Matrix cracking progression: analytical curve vs. experimental data, Varna [83]. [ $\left[ \pm 40 / 90_{4}\right]_{S}$ LSS, HyE 9082Af/Fiberite material system.

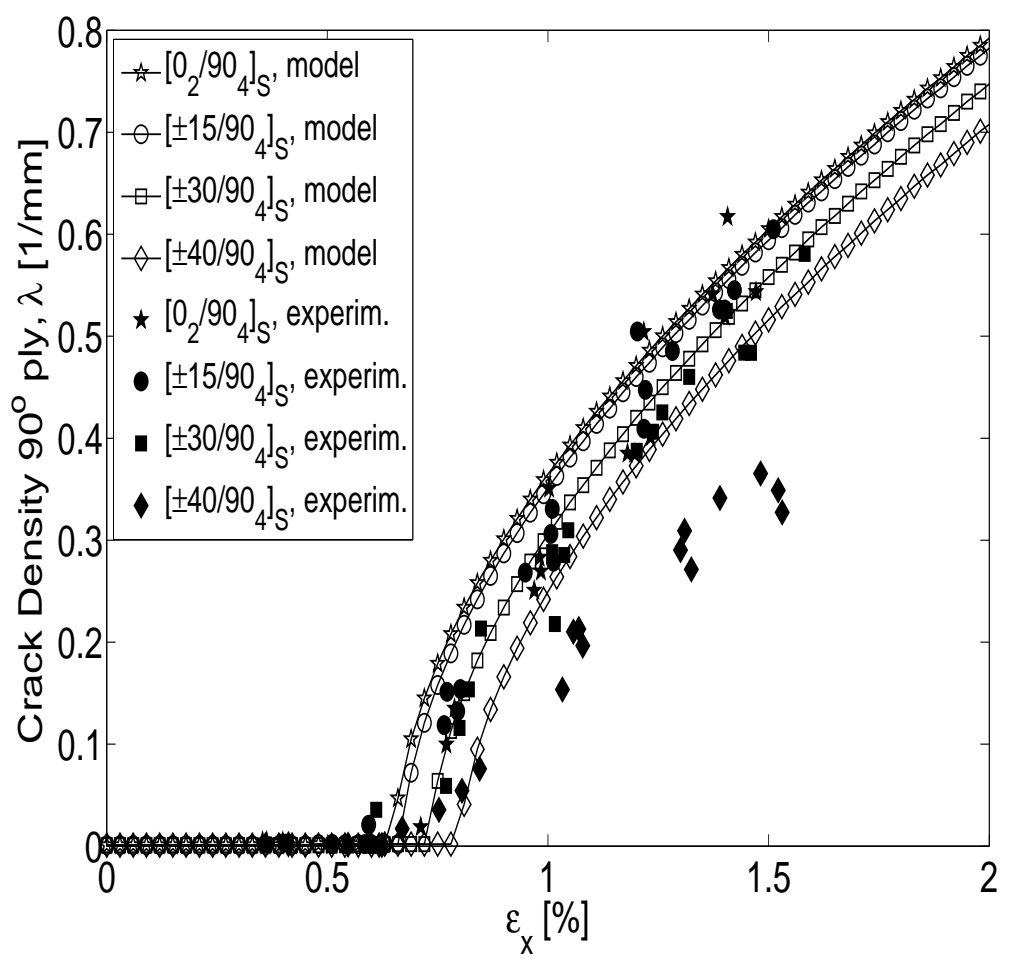

Figure 5.111: Matrix cracking progression: analytical curves vs. experimental data, Varna [83]. $\left[ \pm \theta / 90_{4}\right]_{S}$ LSS, HyE 9082Af/Fiberite material system. 


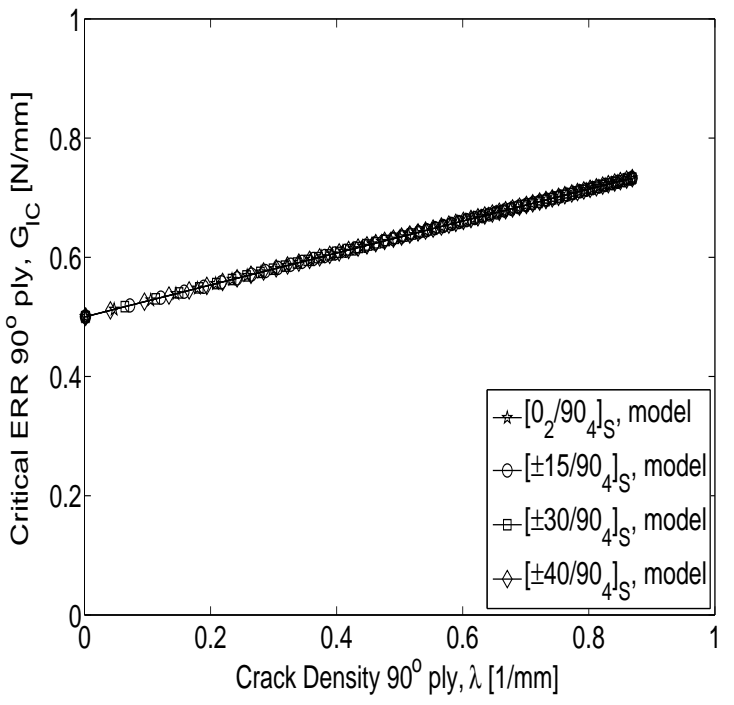

Figure 5.112: The $G_{I C}=G_{I C}(\lambda)$ insitu R-curve evolution of the critical ERR for the HyE 9082Af/Fiberite material system, $\left[ \pm \theta / 90_{4}\right]_{S}$ LSS.

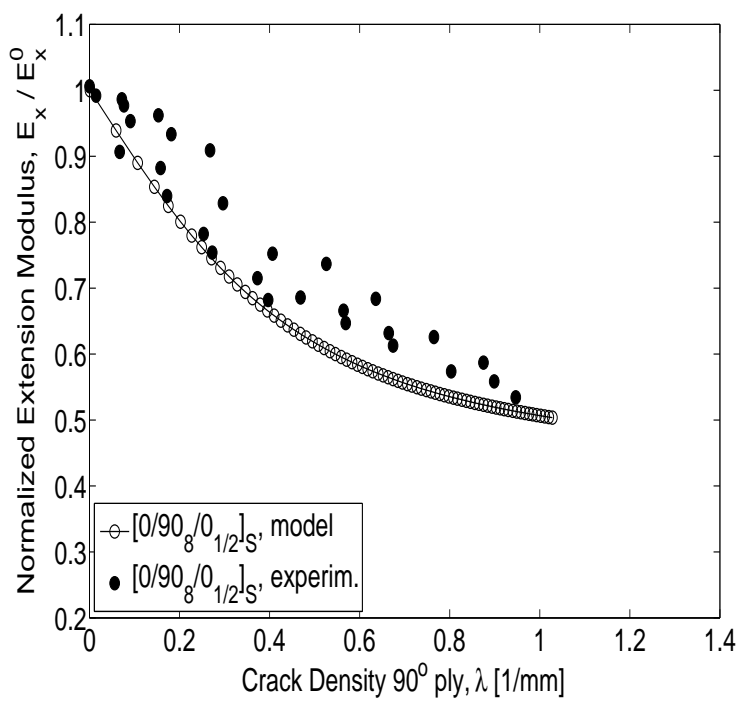

Figure 5.114: Reduced Young modulus $E_{x}(\lambda)$ : analytical curve vs. experimental data, Varna [2]. [0/908 $\left./ 0_{1 / 2}\right]_{S}$ LSS, $H y E$ 9082Af/Fiberite material system.

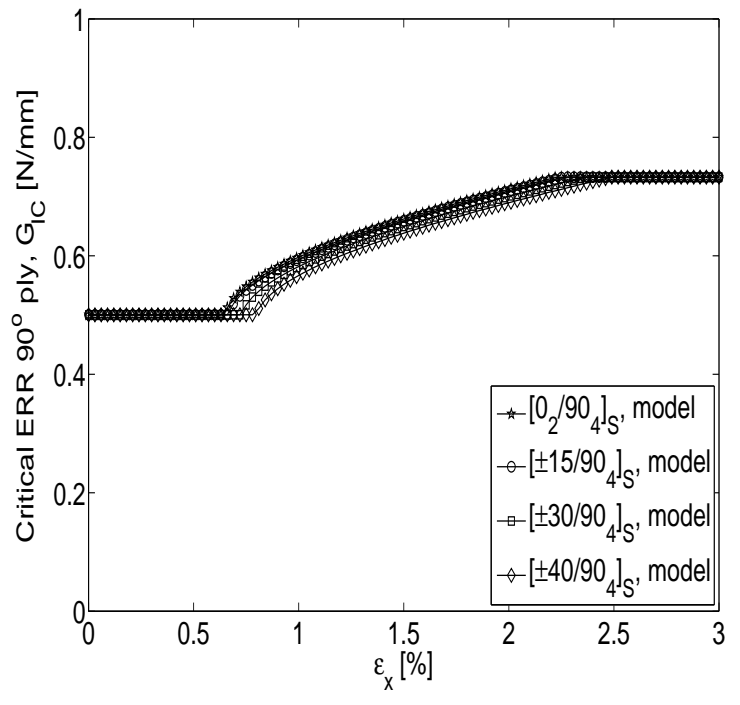

Figure 5.113: The $G_{I C}=G_{I C}(\epsilon)$ insitu R-curve evolution of the critical ERR for the HyE 9082Af/Fiberite material system, $\left[ \pm \theta / 90_{4}\right]_{S}$ LSS.

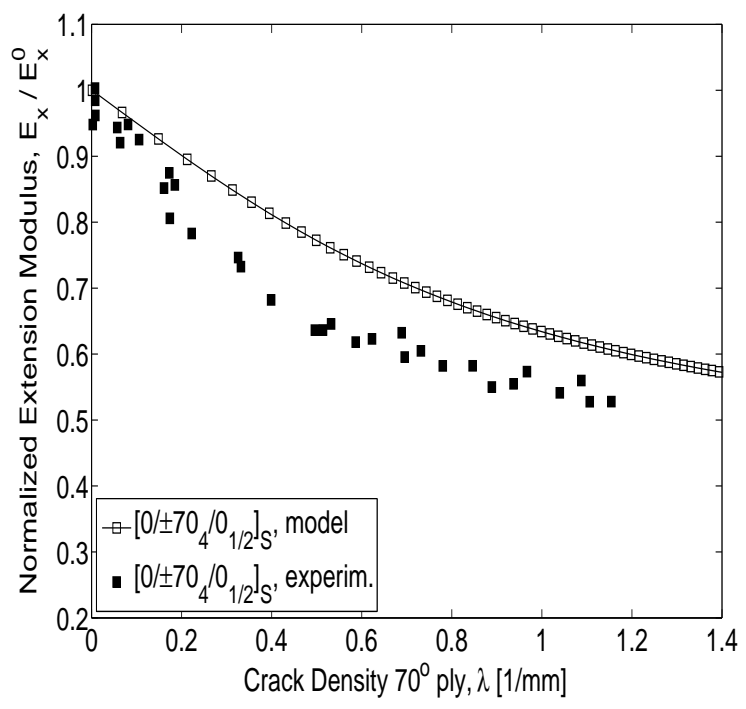

Figure 5.115: Reduced Young modulus $E_{x}(\lambda)$ : analytical curve vs. experimental data, Varna [2]. $\left[0 / \pm 70_{4} / 0_{1 / 2}\right]_{S}$ LSS, HyE 9082Af/Fiberite material system. 


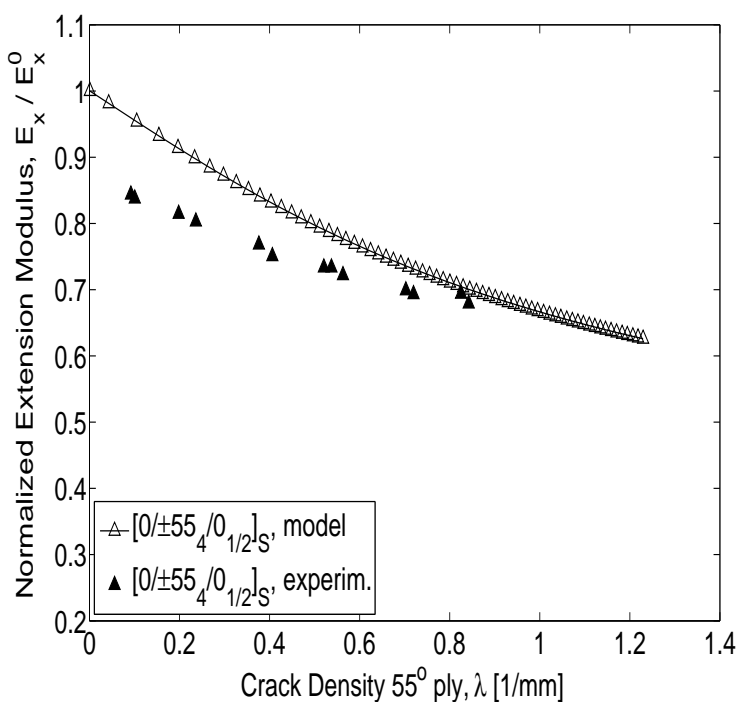

Figure 5.116: Reduced Young modulus $E_{x}(\lambda)$ : analytical curve vs. experimental data, Varna $[2] .\left[0 / \pm 55_{4} / 0_{1 / 2}\right]_{S}$ LSS, HyE 9082Af/Fiberite material system.

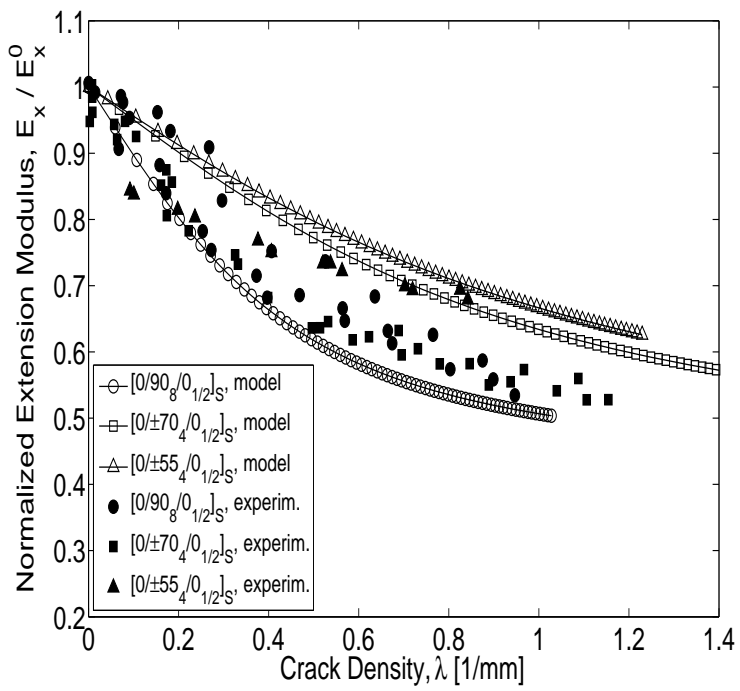

Figure 5.117: Reduced Young modulus $E_{x}(\lambda)$ : analytical curves vs. experimental data, Varna [2]. $\left[0 / \pm \theta_{4} / 0_{1 / 2}\right]_{S}$ LSS, HyE 9082Af/Fiberite material system. 


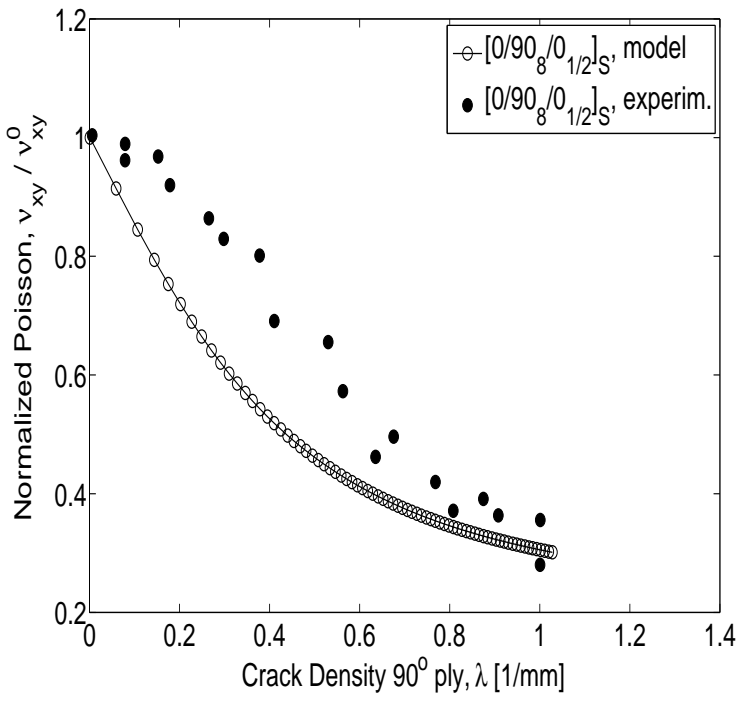

Figure 5.118: Reduced Poisson coefficient $\nu_{x y}(\lambda)$ : analytical curve vs. experimental data, Varna [2]. [0/908 $\left./ 0_{1 / 2}\right]_{S}$ LSS, HyE 9082Af/Fiberite material system.

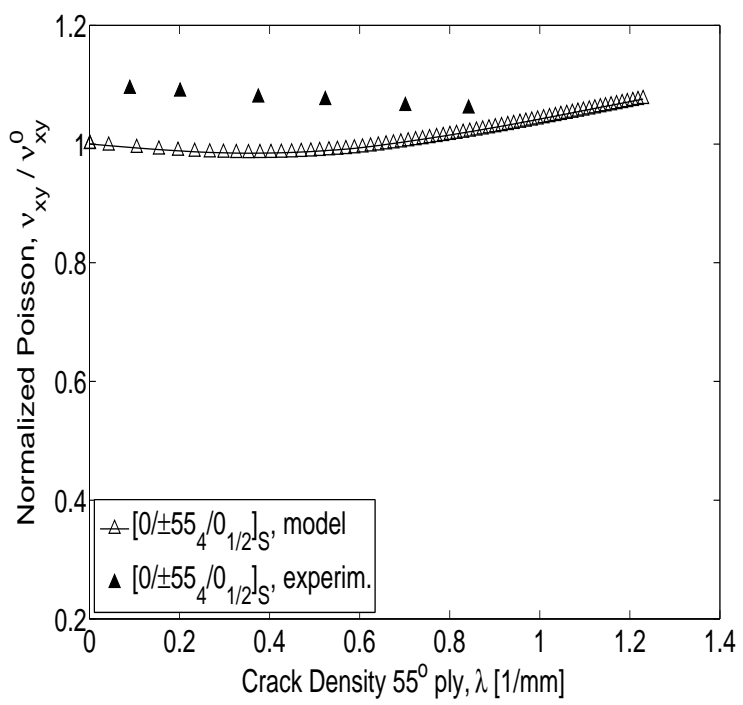

Figure 5.120: Reduced Poisson coefficient $\nu_{x y}(\lambda)$ : analytical curve vs. experimental data, Varna [2]. $\left[0 / \pm 55_{4} / 0_{1 / 2}\right]_{S}$ LSS, $H y E$ 9082Af/Fiberite material system.

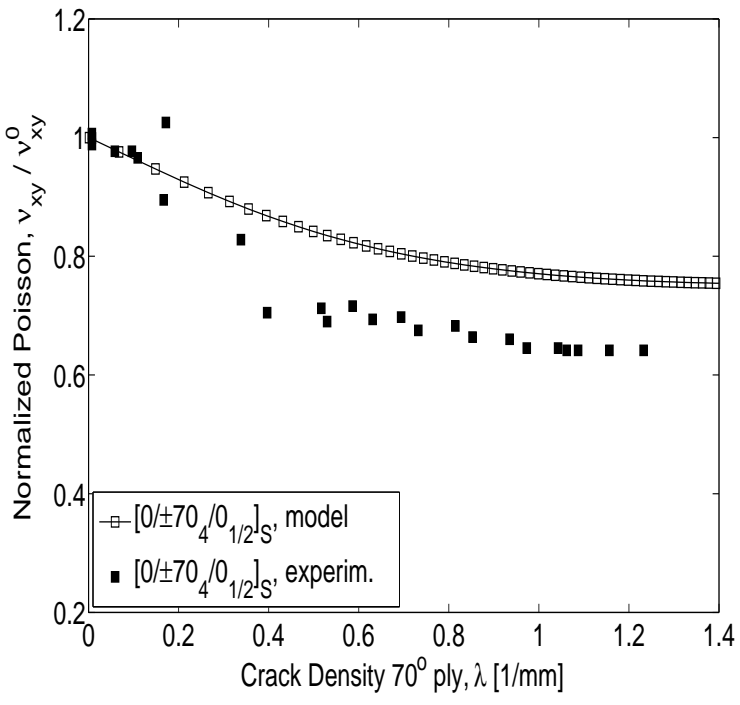

Figure 5.119: Reduced Poisson coefficient $\nu_{x y}(\lambda)$ : analytical curve vs. experimental data, Varna [2]. $\left[0 / \pm 70_{4} / 0_{1 / 2}\right]_{S} \mathrm{LSS}, H y E$ 9082Af/Fiberite material system.

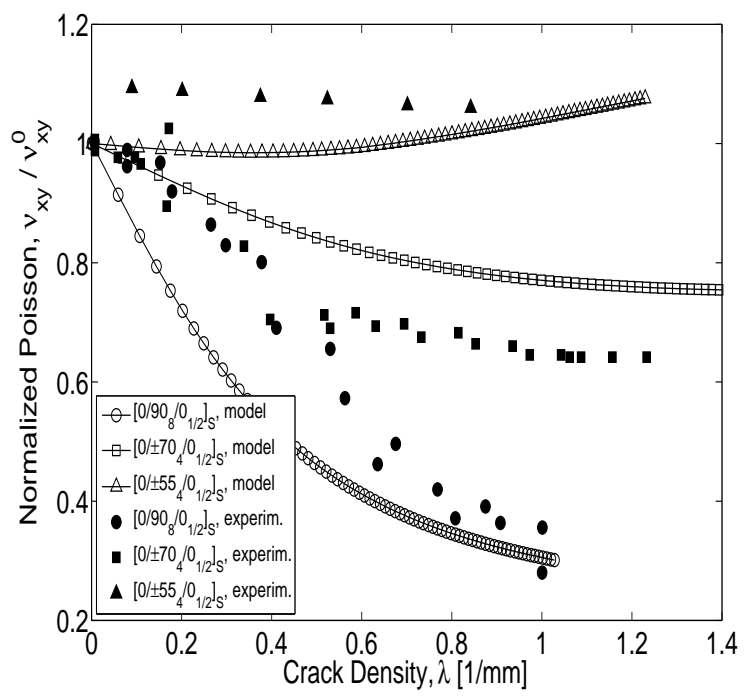

Figure 5.121: Reduced Poisson coefficient $\nu_{x y}(\lambda)$ : analytical curve vs. experimental data, Varna [2]. $\left[0 / \pm \theta_{4} / 0_{1 / 2}\right]_{S}$ LSS, $H y E$ 9082Af/Fiberite material system. 


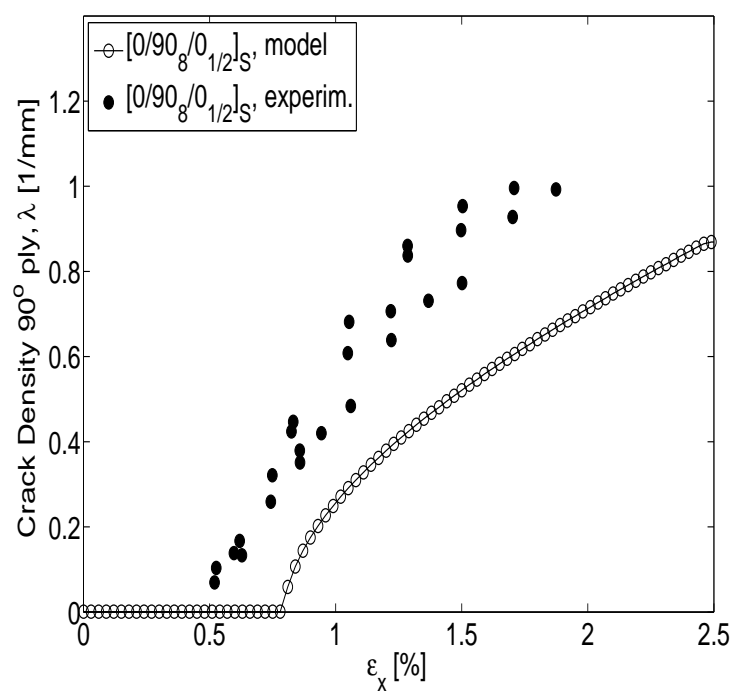

Figure 5.122: Matrix cracking progression: analytical curve vs. experimental data, Varna [2]. [0/908 $\left./ 0_{1 / 2}\right]_{S}$ LSS, HyE 9082Af/Fiberite material system.

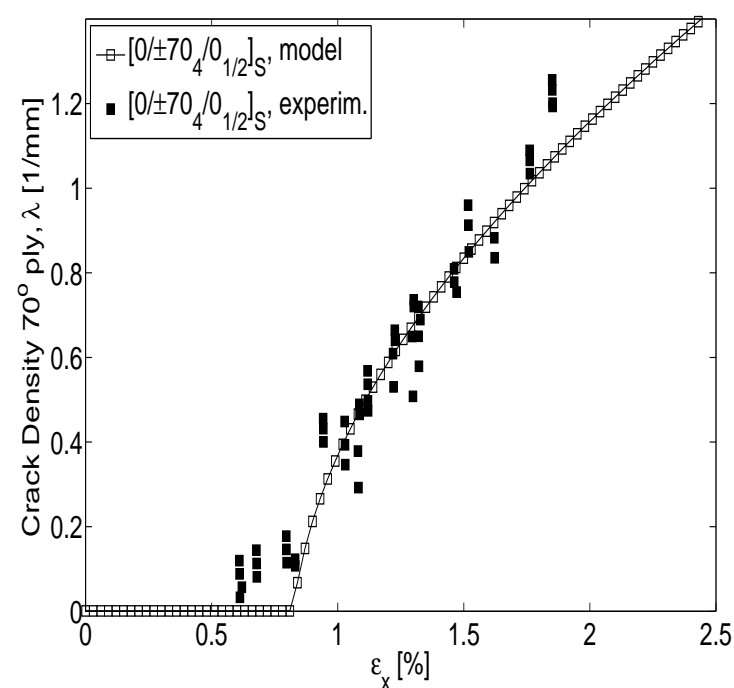

Figure 5.123: Matrix cracking progression: analytical curve vs. experimental data, Varna [2]. $\left[0 / \pm 70_{4} / 0_{1 / 2}\right]_{S}$ LSS, HyE 9082Af/Fiberite material system.

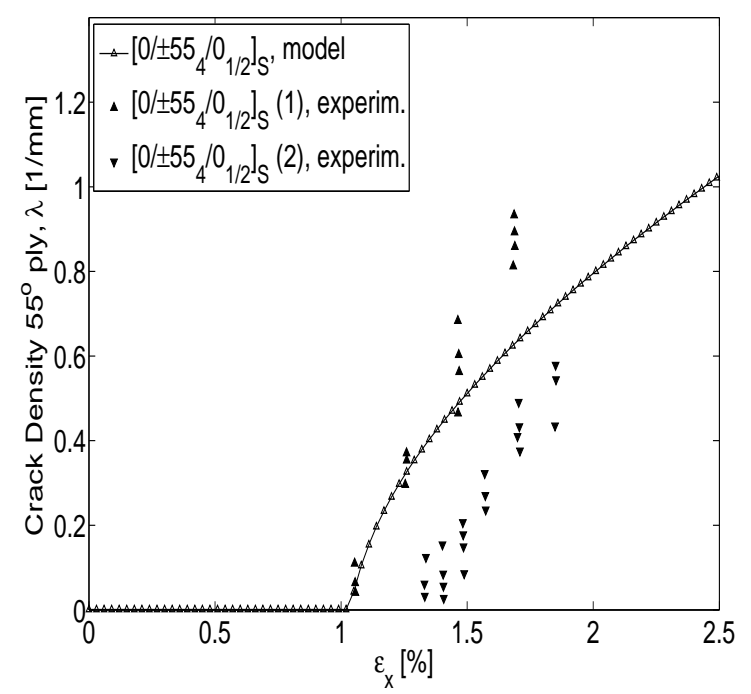

Figure 5.124: Matrix cracking progression: analytical curve vs. experimental data, Varna [2]. $\left[0 / \pm 55_{4} / 0_{1 / 2}\right]_{S}$ LSS, HyE 9082Af/Fiberite material system. 


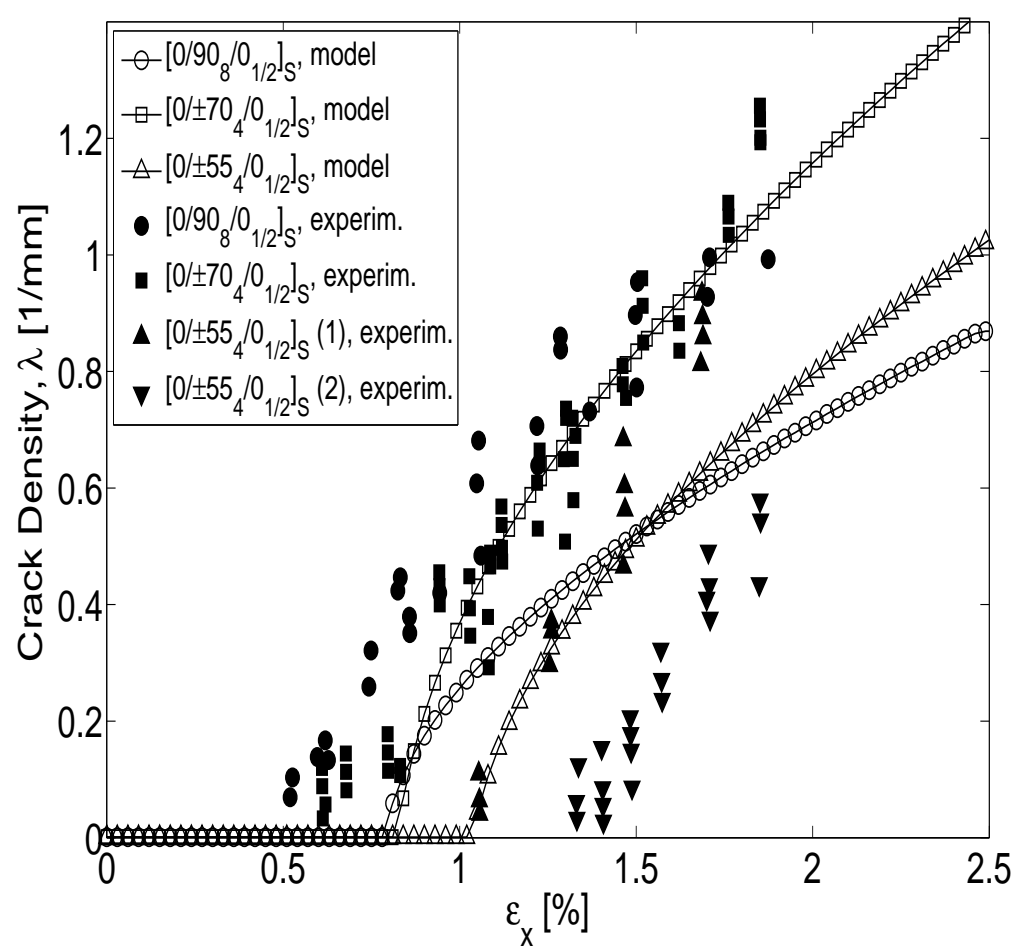

Figure 5.125: Matrix cracking progression: analytical curves vs. experimental data, Varna [2]. $\left[0 / \pm \theta_{4} / 0_{1 / 2}\right]_{S}$ LSS, HyE 9082Af/Fiberite material system.

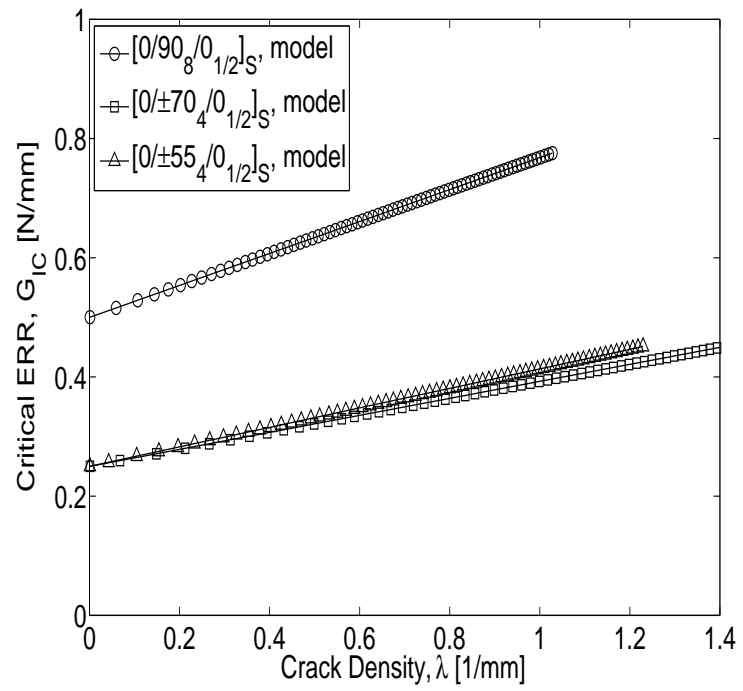

Figure 5.126: The $G_{I C}=G_{I C}(\lambda)$ insitu R-curve evolution of the critical ERR for the HyE 9082Af/Fiberite material system, $\left[0 / \pm \theta_{4} / 0_{1 / 2}\right]_{S}$ LSS.

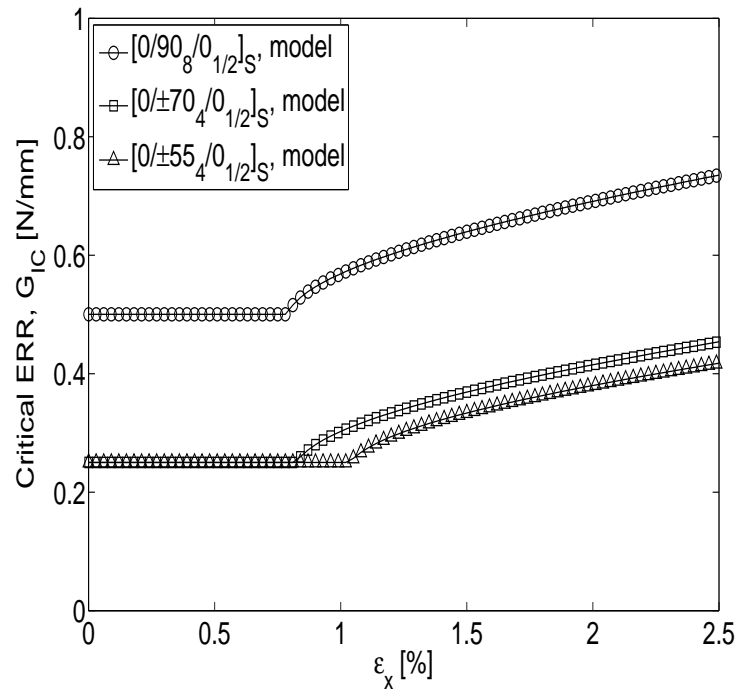

Figure 5.127: The $G_{I C}=G_{I C}(\lambda)$ insitu R-curve evolution of the critical ERR for the HyE 9082Af/Fiberite material system, $\left[0 / \pm \theta_{4} / 0_{1 / 2}\right]_{S}$ LSS. 


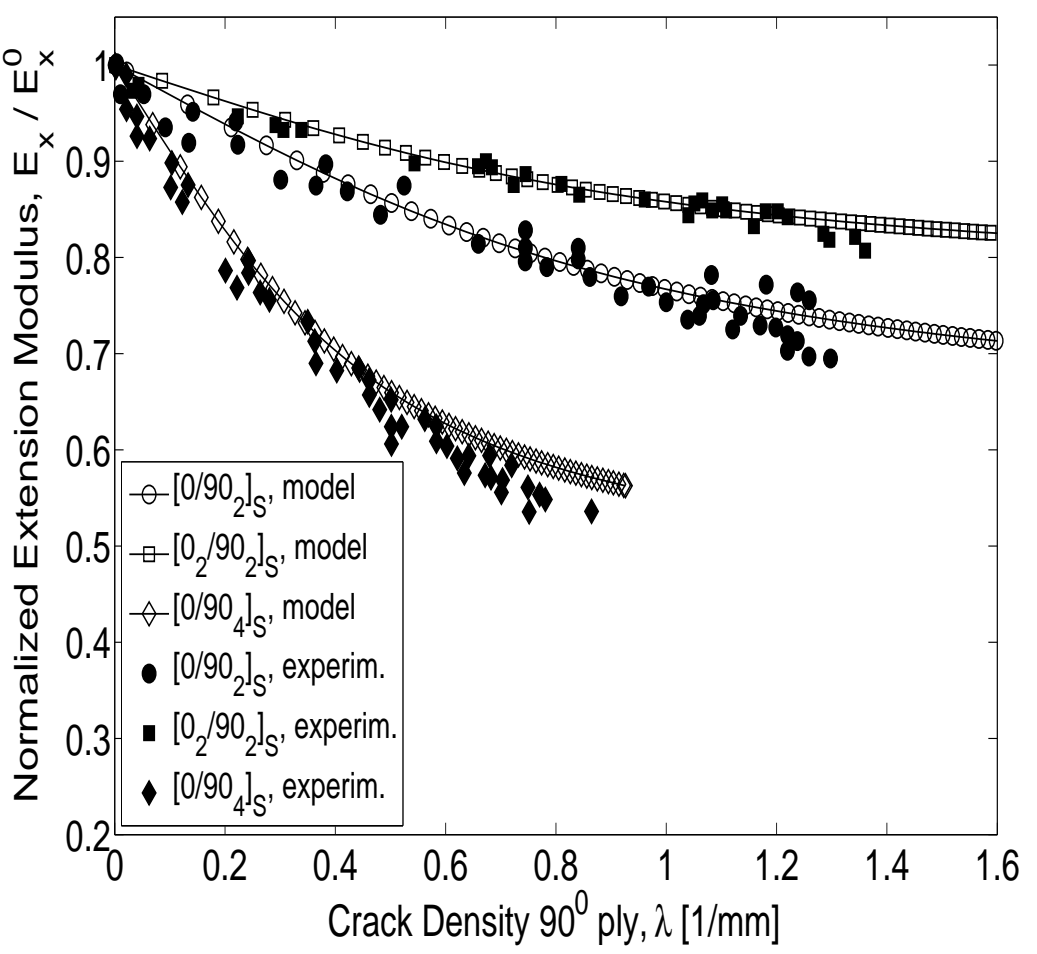

Figure 5.128: Reduced Young modulus $E_{x}(\lambda)$ : analytical curves vs. experimental data, Rubenis [84]. $\left[0_{m} / 90_{n}\right]_{S}$ LSS, Vicotex 913/28\%/192/EC9756 material system. 


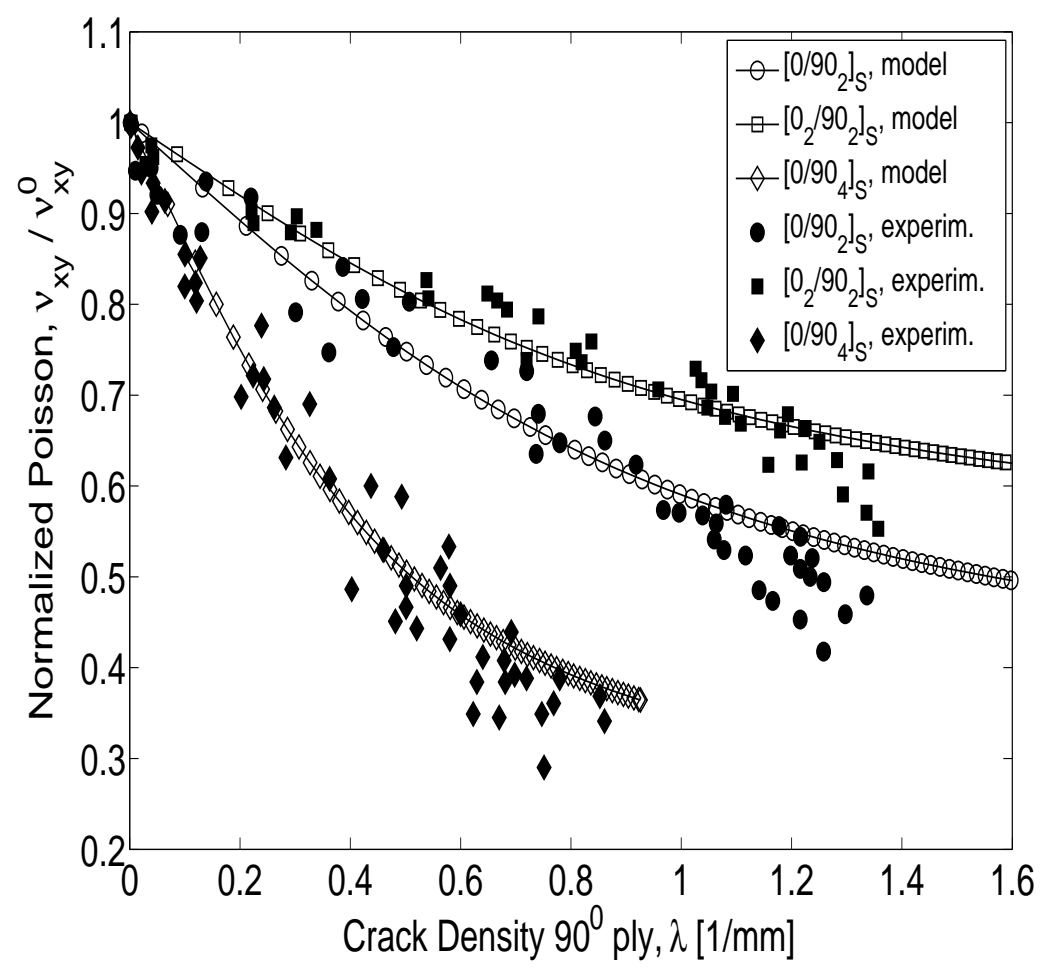

Figure 5.129: Reduced Poisson coefficient $\nu_{x y}(\lambda)$ : analytical curves vs. experimental data, Rubenis [84]. $\left[0_{m} / 90_{n}\right]_{S}$ LSS, Vicotex 913/28\%/192/EC9756 material system.

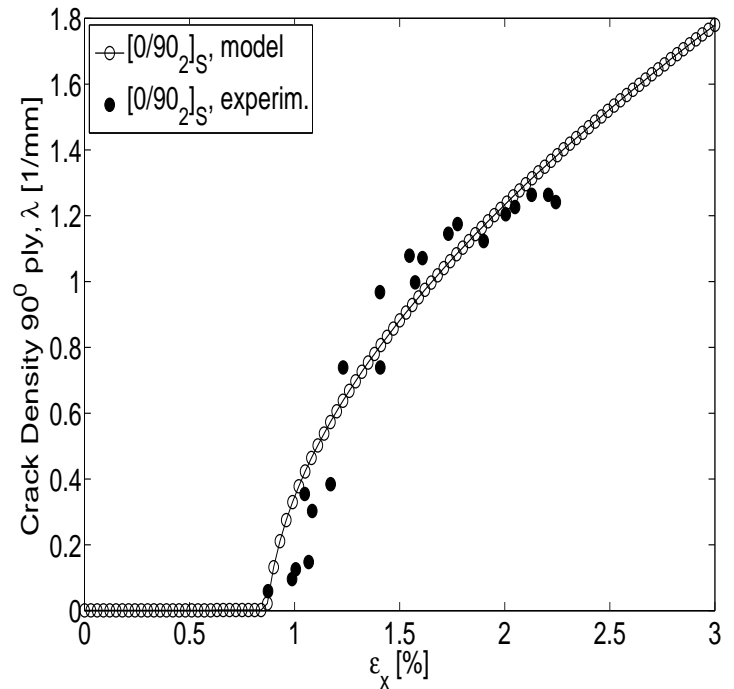

Figure 5.130: Matrix cracking progression: analytical curve vs. experimental data, Varna [58]. $\left[0 / 90_{2}\right]_{S}$ LSS, Vicotex 913/28\%/192/EC9756 material system.

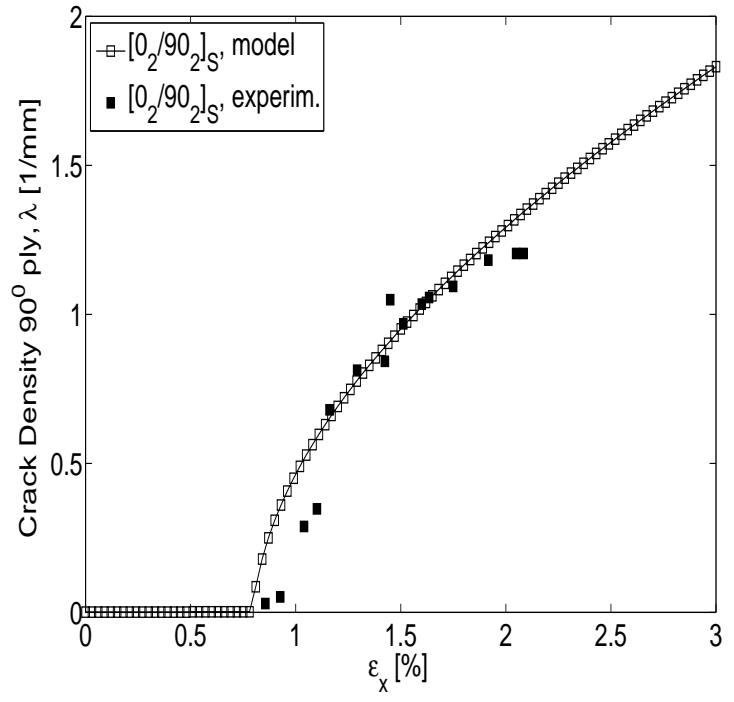

Figure 5.131: Matrix cracking progression: analytical curve vs. experimental data, Varna [58]. $\left[0_{2} / 90_{2}\right]_{S}$ LSS, Vicotex 913/28\%/192/EC9756 material system. 


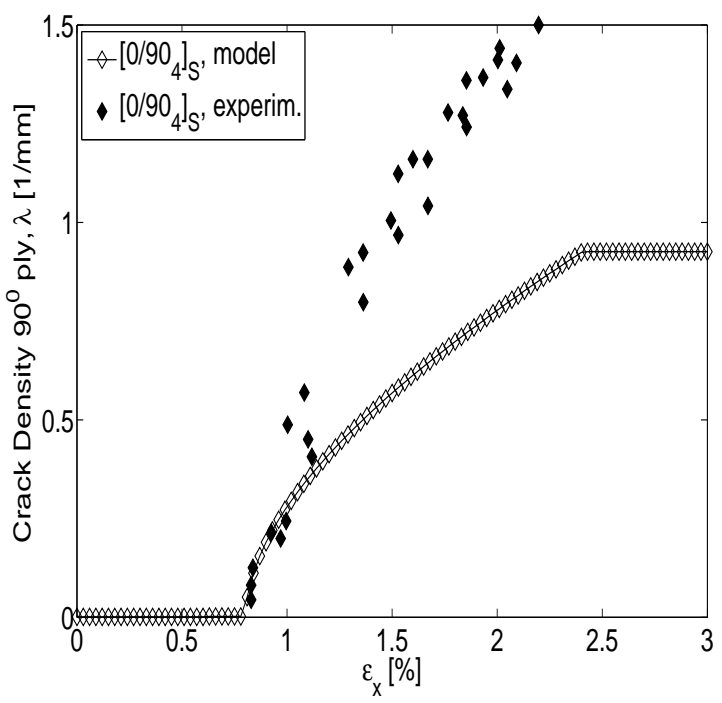

Figure 5.132: Matrix cracking progression: analytical curve vs. experimental data, Varna [58]. [0/904 $]_{S}$ LSS, Vicotex 913/28\%/192/EC9756 material system.

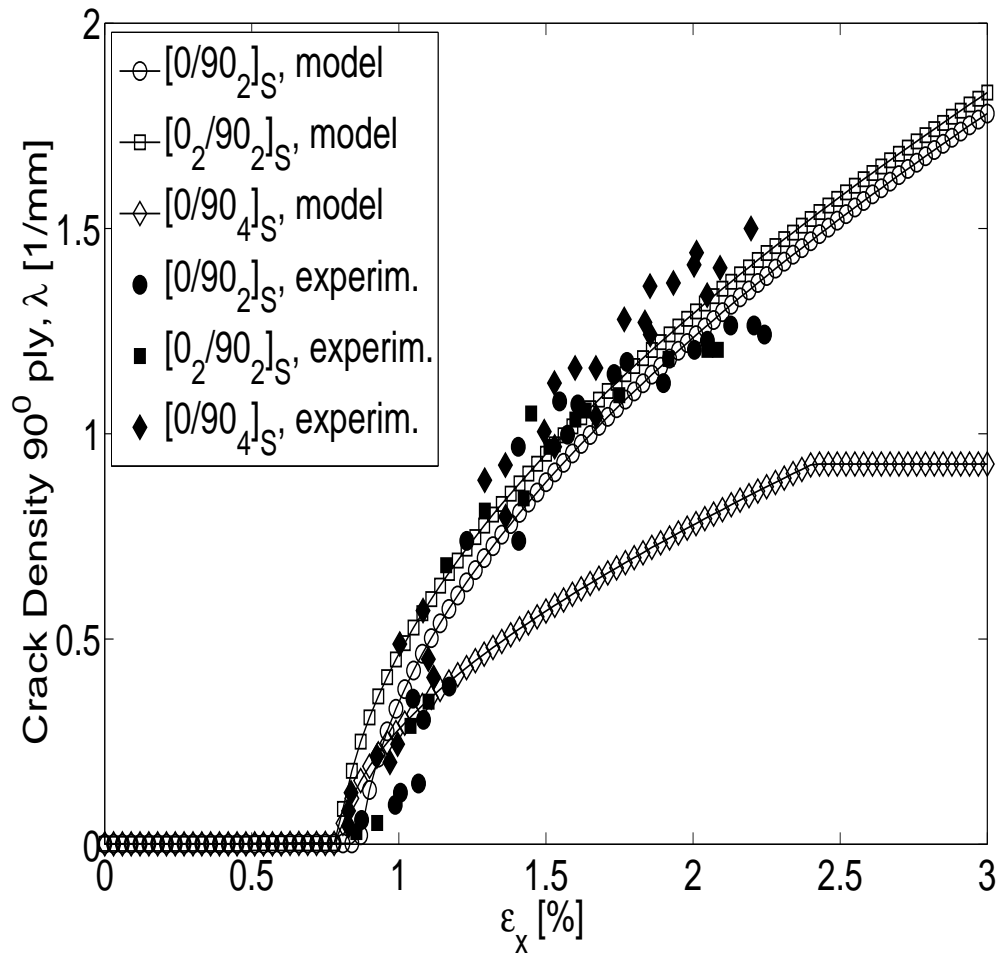

Figure 5.133: Matrix cracking progression: analytical curves vs. experimental data, Varna [58]. $\left[0_{m} / 90_{n}\right]_{S}$ LSS, Vicotex 913/28\%/192/EC9756 material system. 


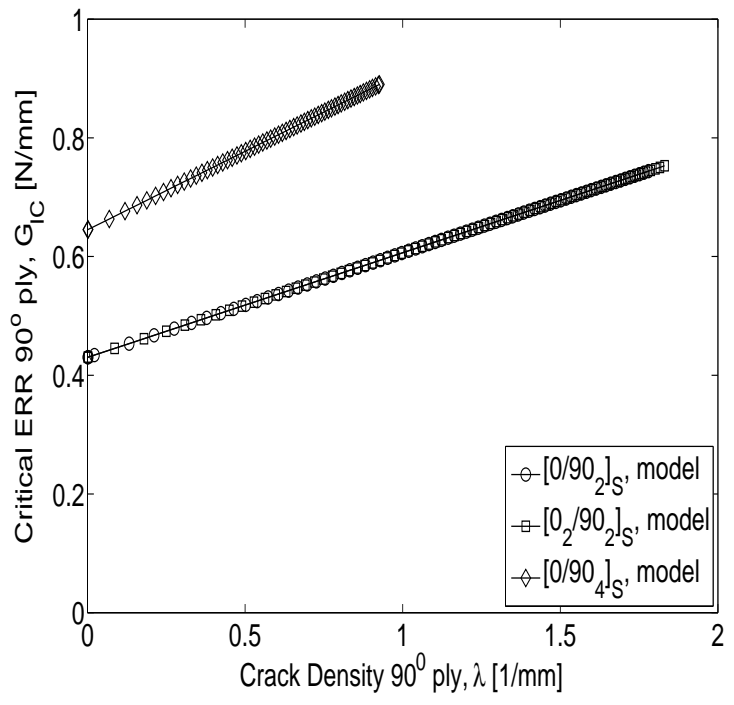

Figure 5.134: The $G_{I C}=G_{I C}(\lambda)$ in-situ $\mathrm{R}$-curve evolution of the critical ERR for the Vicotex 913/28\%/192/EC9756 material system, $\left[0_{m} / 90_{n}\right]_{S}$ LSS.

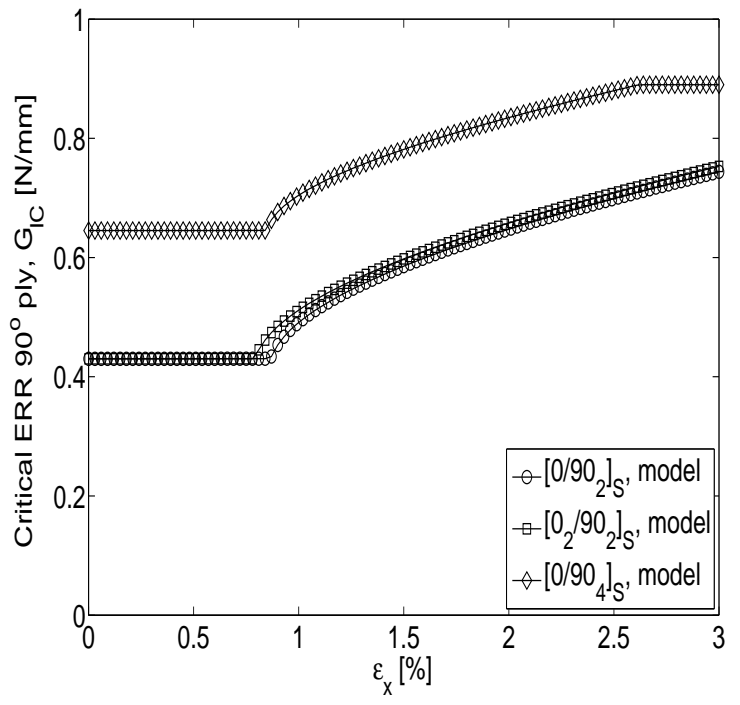

Figure 5.135: The $G_{I C}=G_{I C}(\epsilon)$ in-situ $\mathrm{R}$-curve evolution of the critical ERR for the Vicotex 913/28\%/192/EC9756 material system, $\left[0_{m} / 90_{n}\right]_{S}$ LSS. 


\subsubsection{Model validation, experimental data set 4: $[51,85]$}

The laminate configurations considered under this testing program are $[0 / 90]_{S}$ and $[0 / 90 /-$ $45 /+45]_{S}$ for the case of uniaxial extension loading $\epsilon_{x}[85]$, and $[0 / 90]_{2 S}$ for the case of uniaxial bending loading $\kappa_{x}[51]$.

The material used is an E-Glass/Epoxy Epikote 828/NMA/BDMA system with thermoelastic properties of individual laminae listed in the following, as they are offered in [51, 85]:

For the samples used in uniaxial extension testing:

$E_{1}=46 G P a, \quad E_{2}=13 G P a, \quad G_{12}=5 G P a, \quad \nu_{12}=0.3, \quad \nu_{23}=0.5$

$\alpha_{1}=6.7 \cdot 10^{-6}{ }^{o} \mathrm{C}, \quad \alpha_{2}=29.3 \cdot 10^{-6}{ }^{o} \mathrm{C}$

For the samples used in uniaxial bending testing:

$E_{1}=37 G P a, \quad E_{2}=9.5 G P a, \quad G_{12}=4 G P a, \quad \nu_{12}=0.28, \quad \nu_{23}=0.42$

$\alpha_{1}=6.7 \cdot 10^{-6}{ }^{o} \mathrm{C}, \quad \alpha_{2}=29.3 \cdot 10^{-6}{ }^{\circ} \mathrm{C}$

The ply thickness is $t_{k}=0.55 \mathrm{~mm}$. The processing temperatures (stress free temperature) are $S F T=150^{\circ} \mathrm{C}$ for extension samples, and $S F T=130^{\circ} \mathrm{C}$ for bending samples.

The in-situ R-curve material parameters (see Section 4.4.3) used in the analytical model in order to closest reproduce the experimental data are

$G_{I C, 0}^{r e f}=0.1 \mathrm{~N} / \mathrm{mm}, \quad \beta_{\lambda}^{r e f}=28 \mathrm{deg} ., \quad \beta_{0}^{t}=1.2, \quad \beta_{\lambda}^{t}=1.5$

The selected reference LSS configuration (see the concept of reference thickness $t_{r e f}$ in Section 4.4.3) is $[0 / 90]_{S}$.

Due to the fact that there is a laminate configuration $\left([0 / 90 /-45 /+45]_{S}\right)$ featuring matrix cracking in off-axis plies under mixed mode loading, the value of mode $I I$ critical ERR is needed for the analytical model, and it is considered $G_{I I, C}=2 \mathrm{~N} / \mathrm{mm}$.

Analytical and experimental results for reduced material properties under uniaxial extension loading $\epsilon_{x}$ are presented in Fig. 5.136, 5.137. While the agreement is good for both laminate configurations, the same before noticed tendency of the analytical model regarding the slope of the predicted curve at high values of the crack density can be observed for the $[0 / 90]_{S}$ laminate configuration.

The kink points in the analytical prediction of the elastic modulus for the $[0 / 90 /-45 /+$ 45] $]_{S}$ laminate in Fig. 5.136 has to be noticed. This is because initially the reduced elastic modulus is due to matrix cracking in the transverse 90 ply only, and then matrix cracking in the \pm 45 off-axis plies starts at a certain level of the external loading $\epsilon_{x}$, which triggers the change in slope of the $E_{x}(\lambda)$ plot. 
Matrix crack evolution curves under uniaxial extension loading are presented in Fig. 5.138 for $[0 / 90]_{S}$ laminate in $(\lambda-\sigma)$ coordinates, and in Fig. 5.139 for both $[0 / 90]_{S}$ and $[0 / 90 /-$ $45 /+45]_{S}$ laminates in $(\lambda-\epsilon)$ coordinates. Only information about the matrix cracking in the 90 ply is provided by the experimental data for the $[0 / 90 /-45 /+45]_{S}$ laminate configuration. It can be seen in Fig. 5.139 that the analytical model also predicts matrix cracking in the off-axis \pm 45 plies. According to the analytical prediction, matrix cracking in the +45 ply takes place first, followed then by matrix cracking in the -45 ply. This is according to the expected behavior, because the thickness of the +45 ply is double the thickness of the -45 ply. This succession of cracking events in different plies of the laminates is the cause of the kink points in Fig. 5.136 for the $[0 / 90 /-45 /+45]_{S}$ laminate configuration.

Results for the matrix cracking process under uniaxial bending $\kappa_{x}$ are presented in Fig. 5.140, 5.141 for the case of $[0 / 90]_{2 S}$ laminate. This $[0 / 90]_{2 S}$ LSS can also be written as an $\left[0^{(1)} / 90^{(2)} / 0^{(3)} / 90^{(4)} / 90^{(5)} / 0^{(6)} / 90^{(7)} / 0^{(8)}\right]$ laminate, where ply numbering is indicated by superscript indexes. Experimental information about matrix cracking under bending loading is provided for the uppermost $90^{(7)}$ ply, in Fig. 5.140 in $\lambda-M_{x}$ coordinates, and in Fig. 5.141 in $\lambda-\kappa_{x}$ coordinates $\left(\kappa_{x}\right.$ is the applied uniaxial curvature, and $M_{x}$ is the equivalent applied uniaxial distributed bending moment). It ca be observe from the two figures that the analytical model catches the tendency of the experimentally recorded matrix cracking process, but the agreement between analytical and experimental results is not the best. More experimental validation for the bending case is required in order to conclude if the differences are because of the predictive capabilities of the analytical model, or because of the accuracy of the experimental results. Analytical predictions for matrix cracking in the middle-upper $90^{(5)}$ ply is also shown in Fig. 5.140, 5.141, without knowing if damage did take place in the $90^{(5)}$ ply during experiments. 


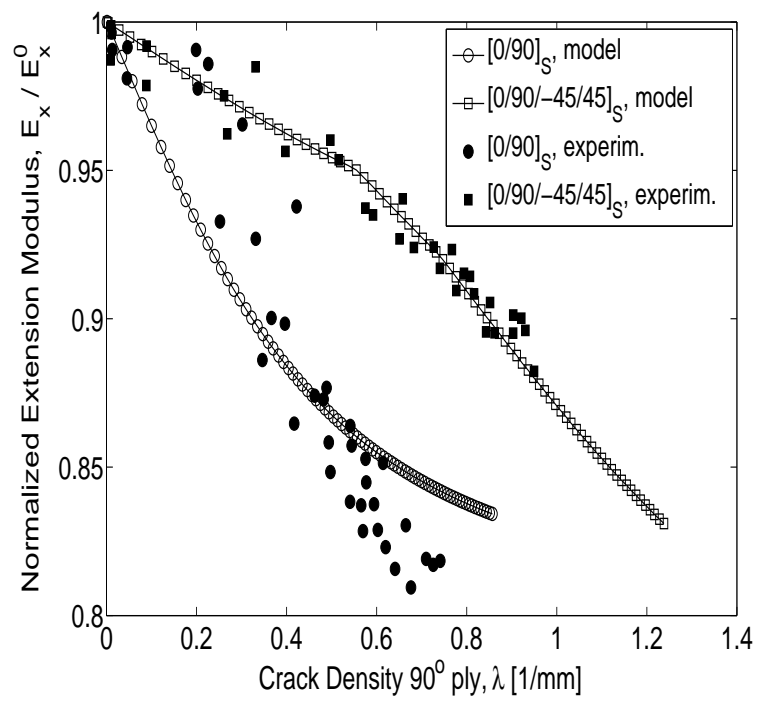

Figure 5.136: Reduced Young modulus: analytical curves vs. experimental data, Ogin $[85] .[0 / 90]_{S}$ and $[0 / 90 /-45 /+45]_{S}$ LSS, $\epsilon_{x}$ loading.

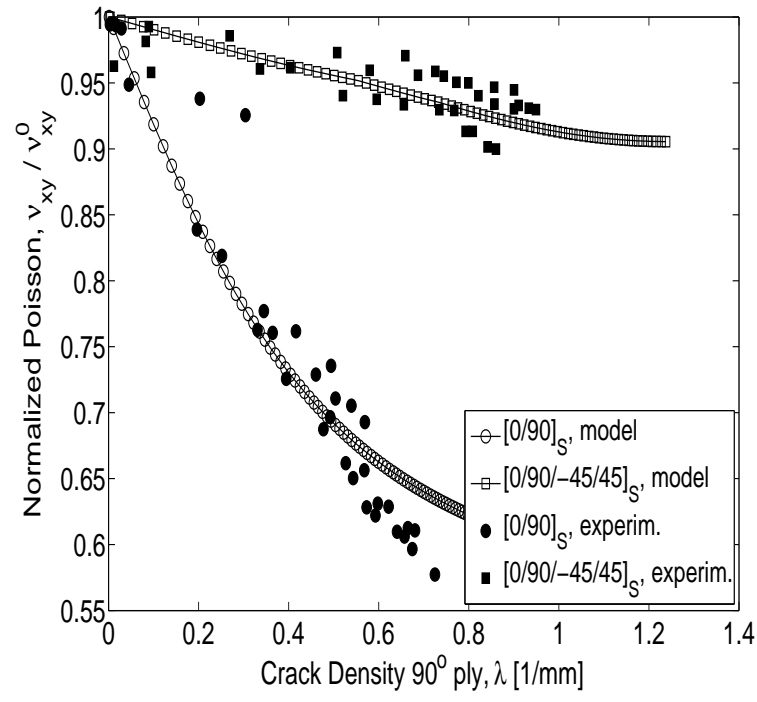

Figure 5.137: Reduced Poisson coefficient: analytical curves vs. experimental data, Ogin [85]. [0/90 $]_{S}$ and $[0 / 90 /-$ $45 /+45]_{S}$ LSS, $\epsilon_{x}$ loading.

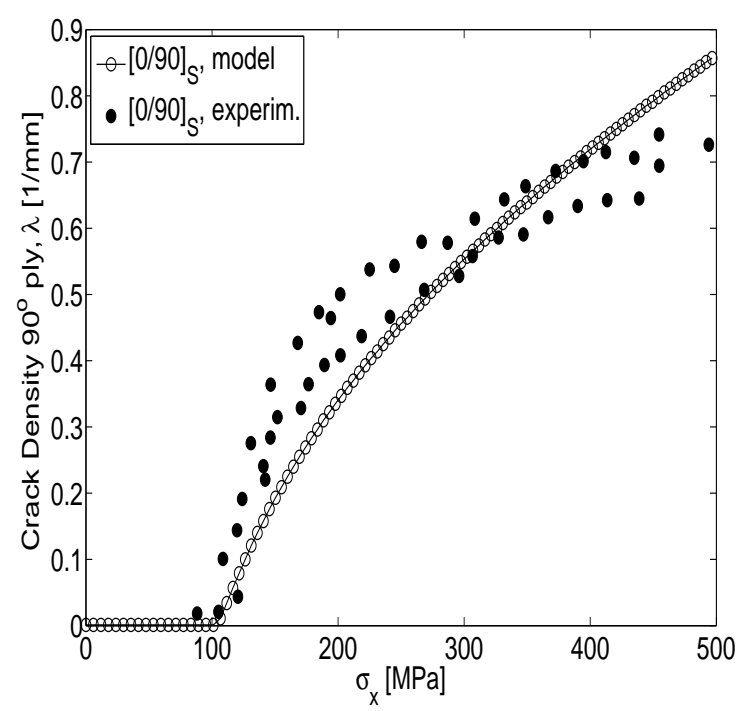

Figure 5.138: Matrix cracking progression: analytical curve vs. experimental data, Ogin [85]. [0/90] $]_{S}$ LSS, $\epsilon_{x}$ loading. 


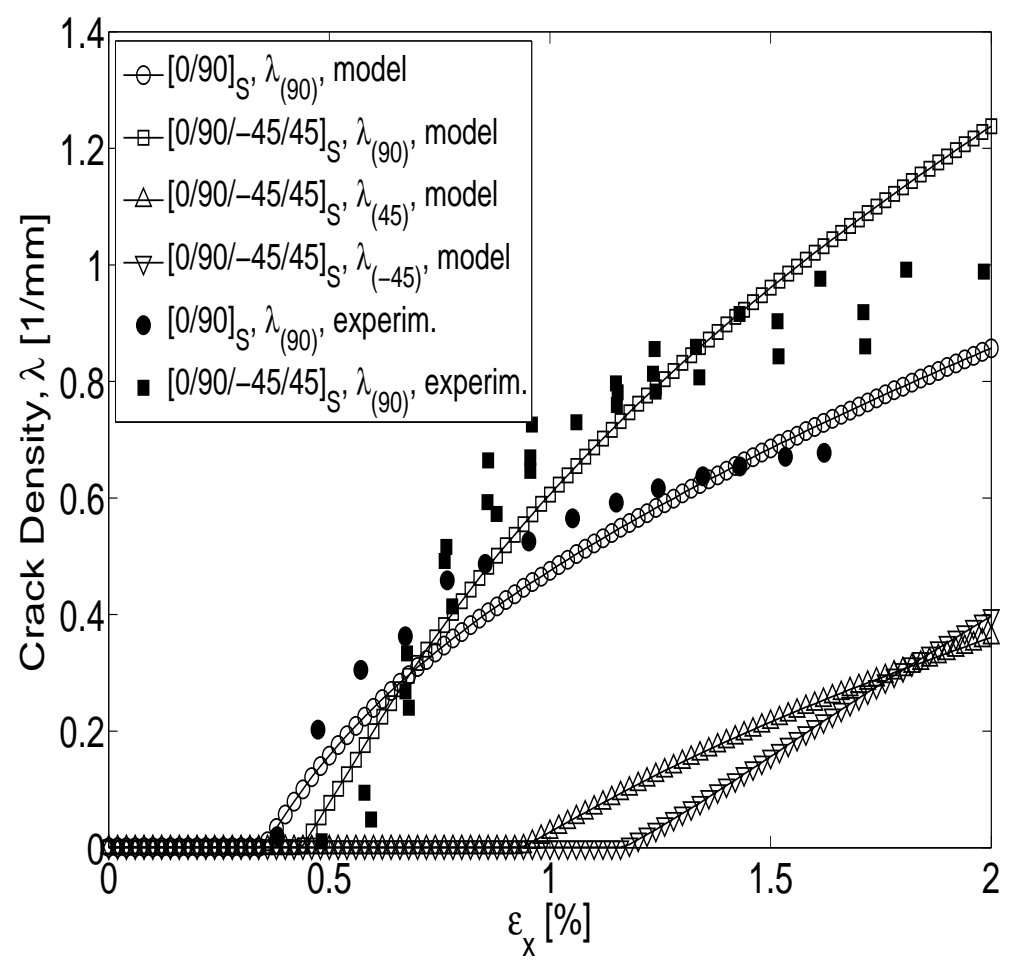

Figure 5.139: Matrix cracking progression: analytical curve vs. experimental data, Ogin [85]. $[0 / 90]_{S}$ and $[0 / 90 /-45 /+45]_{S}$ LSS, $\epsilon_{x}$ loading.

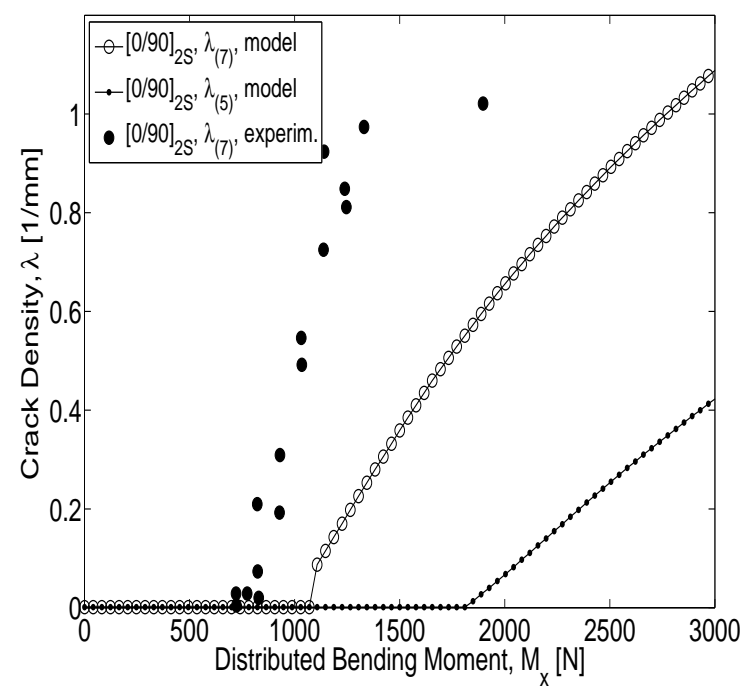

Figure 5.140: Matrix cracking progression: analytical curve vs. experimental data, Ogin [51]. [0/90] $]_{2 S}$ LSS, $M_{x}$ loading.

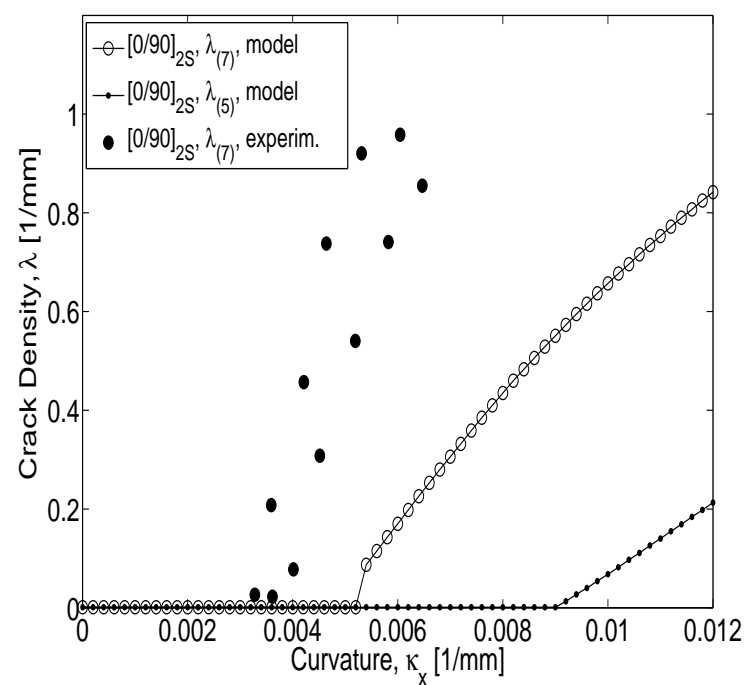

Figure 5.141: Matrix cracking progression: analytical curve vs. experimental data, Ogin [51]. [0/90 $]_{2 S}$ LSS, $\kappa_{x}$ loading. 


\subsubsection{Model validation, experimental data set 5: [36]}

A variety of laminate configurations and loading cases are considered under this testing program:

- $\left[0 / 90_{n} / 0\right]$ featuring cracks in the $90_{n}$ ply stack under uniaxial extension loading $\epsilon_{x}$, - $\left[0_{n} / 90_{n} /+45_{n} /-45_{n}\right]_{S}$ and $\left[0_{n} /+45_{n} /-45_{n}\right]_{S}$ featuring cracks in the $90_{n},+45_{n}$, and $-45_{n}$ stacks under uniaxial extension loading $\epsilon_{x}$,

- $\left[90_{n} / 0_{n} /-45_{n} /+45_{n}\right]_{S}$ under uniaxial bending $\kappa_{x}$.

The material is a Glass/Epoxy Fibredux 913G-E-5-30\% system with thermo-elastic properties of individual laminae listed in the following, as they are offered in [36]

$E_{1}=46 \mathrm{GPa}, \quad E_{2}=18 \mathrm{GPa}, \quad G_{12}=7.9 \mathrm{GPa}, \quad \nu_{12}=0.29, \quad \nu_{23}=0.41$ $\alpha_{1}=6.72 \cdot 10^{-6}{ }^{o} \mathrm{C}, \quad \alpha_{2}=29.3 \cdot 10^{-6}{ }^{o} \mathrm{C}$

The ply thickness is $t_{k}=0.125 \mathrm{~mm}$. The processing temperature (stress free temperature) is $S F T=125^{\circ} \mathrm{C}$.

The in-situ R-curve material parameters (see Section 4.4.3) used in the analytical model in order to closest reproduce the experimental data are

$$
G_{I C, 0}^{r e f}=0.2 \mathrm{~N} / \mathrm{mm}, \quad \beta_{\lambda}^{r e f}=0 \text { deg. }, \quad \beta_{0}^{t}=1, \quad \beta_{\lambda}^{t}=1
$$

The selected reference LSS configuration (see the concept of reference thickness $t_{r e f}$ in Section 4.4.3) is $[0 / 90 / 0]$.

Due to the fact that there are laminate configurations $\left(\left[0_{n} / 90_{n} /+45_{n} /-45_{n}\right]_{S}\right)$ featuring cracks in off-axis plies under mixed mode loading, the value of mode $I I$ critical ERR is needed for the analytical model, and it is considered $G_{I I, C}=1 \mathrm{~N} / \mathrm{mm}$.

As a first observation to start with is the fact that the above mentioned value $E_{1}=$ $46 G P a$ of the ply longitudinal Young modulus has been modified to $E_{1}=34 G P a$ for all analytical results. The reason for this is that using the cited value of $E_{1}=46 G P a$, the stress-strain experimental points in Fig. 5.148 - 5.151 could not be very close reproduced by the analytical curves, on their undamaged portion, before the matrix cracking onset. The analytical curves before damage onset are calculated exclusively based on CLT, without having superposed any other modeling features, and the reason of mismatch between the experimental and analytical results could not be identified other than the input value of the ply material properties. By modifying the ply longitudinal Young modulus from $E_{1}=$ $46 \mathrm{GPa}$ to $E_{1}=34 \mathrm{GPa}$ for the Glass/Epoxy Fibredux 913G-E-5-30\% material system, closer agreement could be obtained for the stress-strain behavior before damage onset, as it 
is shown in Fig. $5.148-5.151$.

A first set of experimental results is offered in [36] for the $\left[0 / 90_{n} / 0\right]$ LSS under uniaxial extension $\epsilon_{x}$. Reduced material properties of the laminate are presented in Fig. 5.142, 5.143, for the Young modulus and Poisson ratio, respectively. Good agreement between analytical and experimental results can be noticed in the two plots. However, the model tendency of predicting a lower slope of the analytical trend compared to the experimental trend, at high values of the crack density $\lambda$, can be again noticed, especially for high thicness of the cracking ply $\left(90_{4}\right.$ and $\left.90_{8}\right)$.

The matrix cracking evolution curves are presented in Fig. 5.144 - 5.147. The analytical vs. experimental correlation is very good for the 90 and $90_{2}$ thicknesses of the cracking ply, and it becomes less good for the $90_{4}$ and $90_{8}$ thicknesses at high crack densities. This can be correlated to the previous observation about the slope prediction of the reduced material properties at high crack densities and high ply thickness. Centralized plots of the damage evolution curves are presented in Fig. 5.152.

Stress-strain curves featuring nonlinearities due to matrix cracking are shown in Fig. 5.148 5.151, with a centralized plot presented in Fig. 5.153. Good agreement between analytical and experimental results can be noticed in these plots. The least level of correlation is obtained for the $\left[0 / 90_{8} / 0\right]$ laminate configuration in Fig. 5.151, featuring the thickest cracking ply, which is in connection with the least level of correlation of damage evolution curve in Fig. 5.147 for the same laminate configuration. This observation might be related to an observation from the beginning of the present manuscript (see the transition thickness effect in Section 2.2), where the hypothesis that the predicting ability of an energy based model is reduced at high thickness of the cracking ply has been formulated.

A second set of experimental data in [36] regards the $\left[0_{n} / 90_{n} /+45_{n} /-45_{n}\right]_{S}$ (with $n=1,2$ ) laminate configuration, under uniaxial extension $\epsilon_{x}$. Analytical vs. experimental damage evolution curves are presented in Fig. 5.154, considering as variable the crack density in the $90_{n}$ ply of the laminate. It ca be noticed that the analytical model is able to predict the main experimental trend of damage evolution, namely close values of damage onset, and higher values of crack density $\lambda$ for the $n=1$ LSS as compared to $n=2$, at high level of the applied deformation $\epsilon_{x}$. However, analytical prediction for both $n=1$ and $n=2$ LSS are supraevaluated as compared to experimental measurements. This might be due to the fact that other forms of damage, namely delamination, are observed during laminate loading [36], due to high \pm 45 off-axis angles of the constituent plies. Part of the available elastic energy 
is released for the formation of delamination, and less energy is available for the formation of matrix cracking, which translates in lower experimental recorded levels of matrix cracking than it would be the case without delamination. The analytical model does not consider other damage mechanisms than intra-laminar matrix cracking.

Comparative analytical damage evolution curves for the $\left[0_{n} / 90_{n} /+45_{n} /-45_{n}\right]_{S}$ LSS considering as variable the crack density in all $90_{n},+45_{n}$ and $-45_{n}$ plies are presented in Fig. 5.155. The model predictions are according to the expected material behavior. Matrix cracking initiates first in the $90_{n}$ plies, followed in order by the $-45_{n}$ and $+45_{n}$ plies for each value of the ply thickness $n=1,2$. Damage initiates first in the $-45_{n}$ plies and then in the $+45_{n}$ plies due to the fact that the thickness of the $-45_{n}$ plies is double the thickness of $+45_{n}$ plies, do to the central position of the $-45_{n}$ inside of the symmetric laminate. Based on the same reason of ply thickness, matrix cracking initiates earlier in the $90_{n}$ plies of the $n=2$ laminate configuration than $n=1$.

Analytical and experimental stress-strain plots under progressive matrix cracking conditions for the $\left[0_{n} / 90_{n} /+45_{n} /-45_{n}\right]_{S}$ LSS are presented in Fig. 5.156, 5.157, and for $\left[0_{n} /+45_{n} /-45_{n}\right]_{S}$ LSS in Fig. 5.158, 5.159. The kink point in the stress-strain curves can be noticed as corresponding to successive matrix cracking onset in the $90,-45$ and +45 plies of the laminate.

Flexural deformation $\kappa_{x}$ under displacement control conditions is applied to $\left[90_{2} / 0_{2} /-\right.$ $\left.45_{2} / 45_{2}\right]_{S}$ and $\left[90_{2} /-45_{2} / 45_{2}\right]_{S}$ laminate configurations. Matrix cracking is recorded for the surface $90_{2}$ plies on the extension side of the bending specimen. Analytical and experimental matrix cracking evolution curves are presented in Fig. 5.160, where it can be seen that the analytical model predicts the steep increase in crack density $\lambda$ with the applied deformation $\kappa_{x}$, although the analytically predicted damage onset takes place earlier compared to the experimental measurements. The bending moment-curvature plots (which are the equivalent of the stress-strain plots for the case of extension loading) for the considered LSS are presented in Fig. 5.161, where good agreement between analytical predictions and experiments can be noticed. 


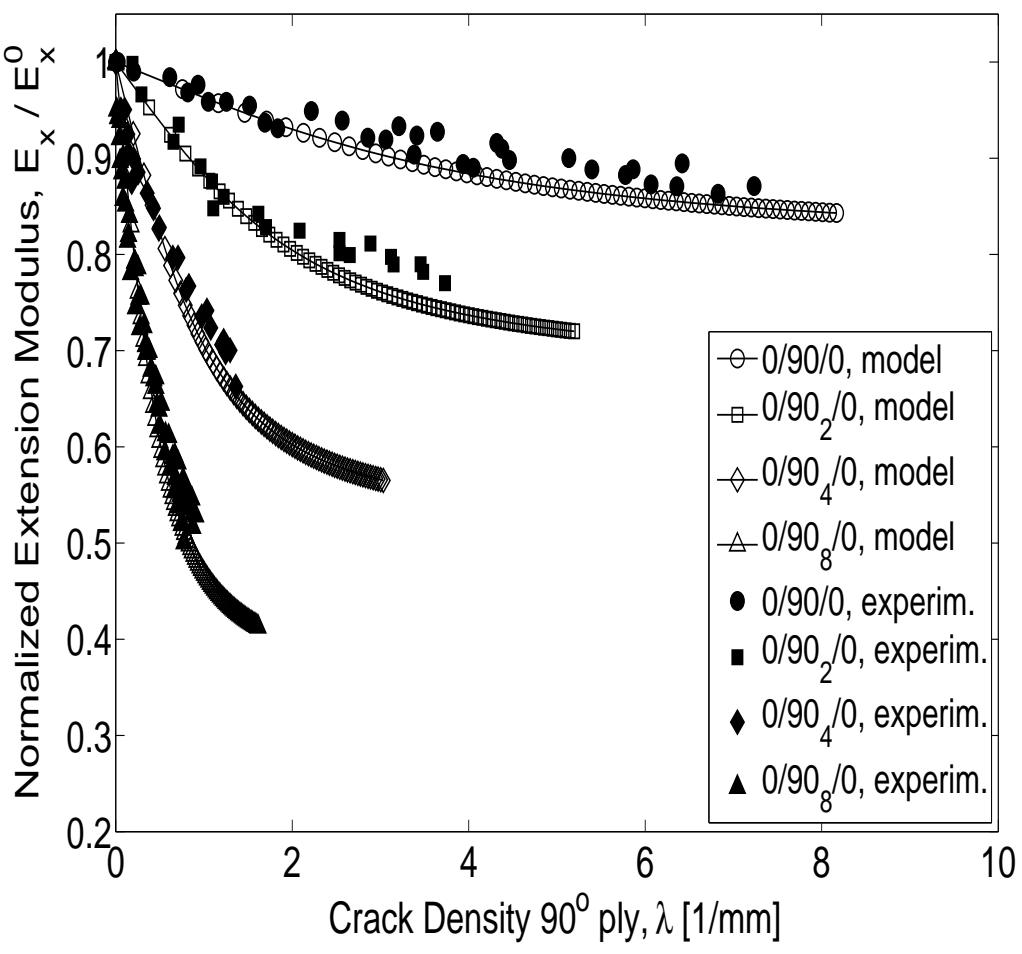

Figure 5.142: Reduced Young modulus: analytical curves vs. experimental data, Gudmundson [36]. [0/90 $/ 0]$ LSS, $\epsilon_{x}$ loading. 


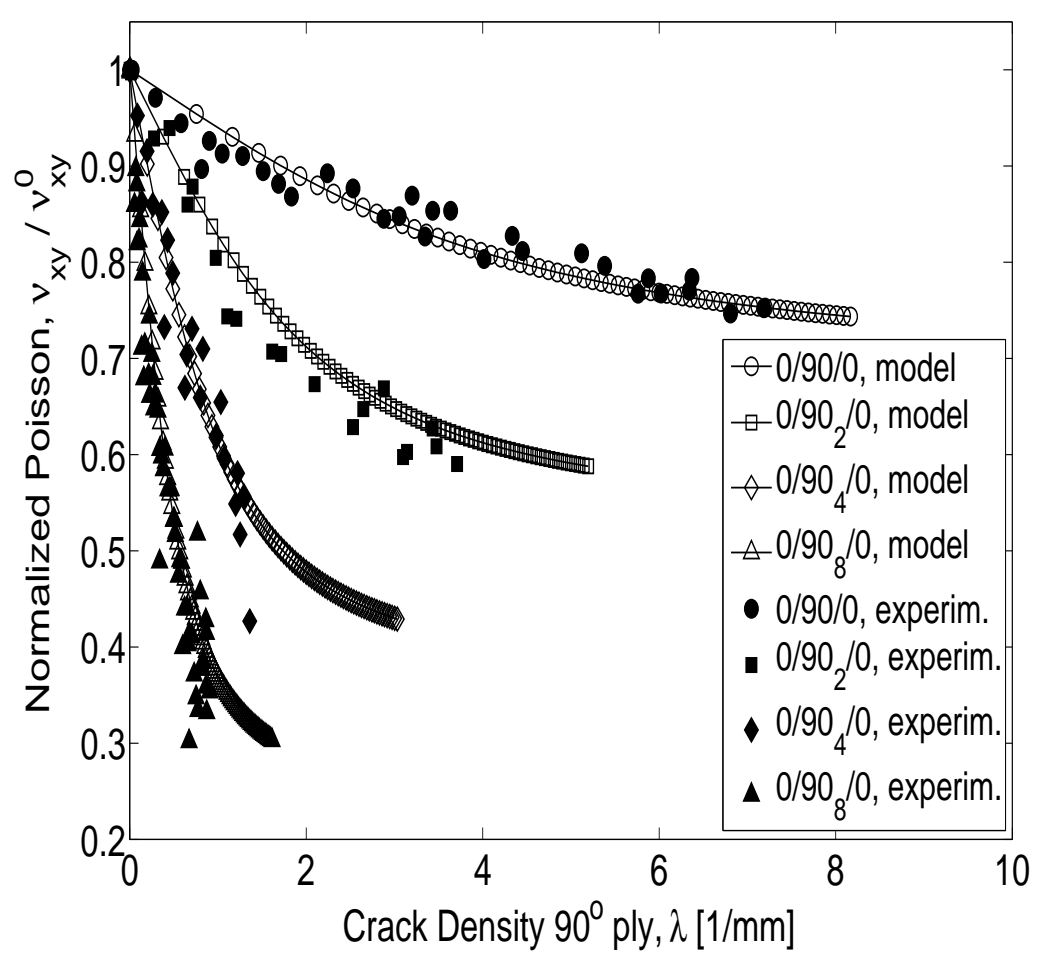

Figure 5.143: Reduced Poisson coefficient: analytical curves vs. experimental data, Gudmundson [36]. [0/90n $/ 0]$ LSS, $\epsilon_{x}$ loading.

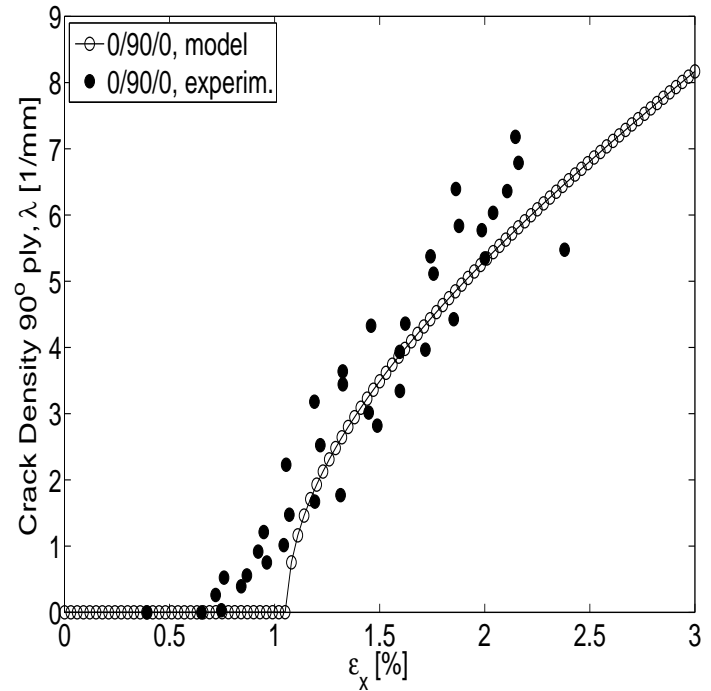

Figure 5.144: Matrix cracking progression: analytical curve vs. experimental data, Gudmundson [36]. [0/90/0] LSS, $\epsilon_{x}$ loading.

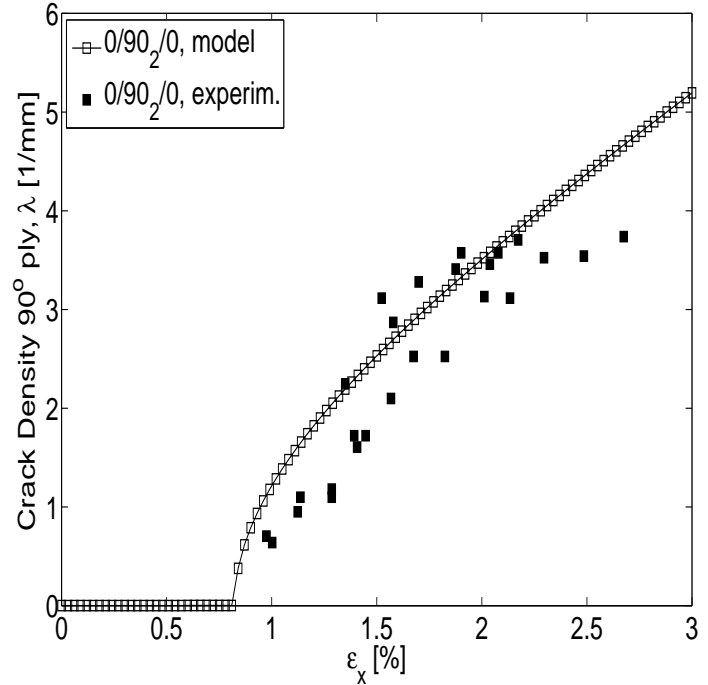

Figure 5.145: Matrix cracking progression: analytical curve vs. experimental data, Gudmundson [36]. [0/902/0] LSS, $\epsilon_{x}$ loading. 


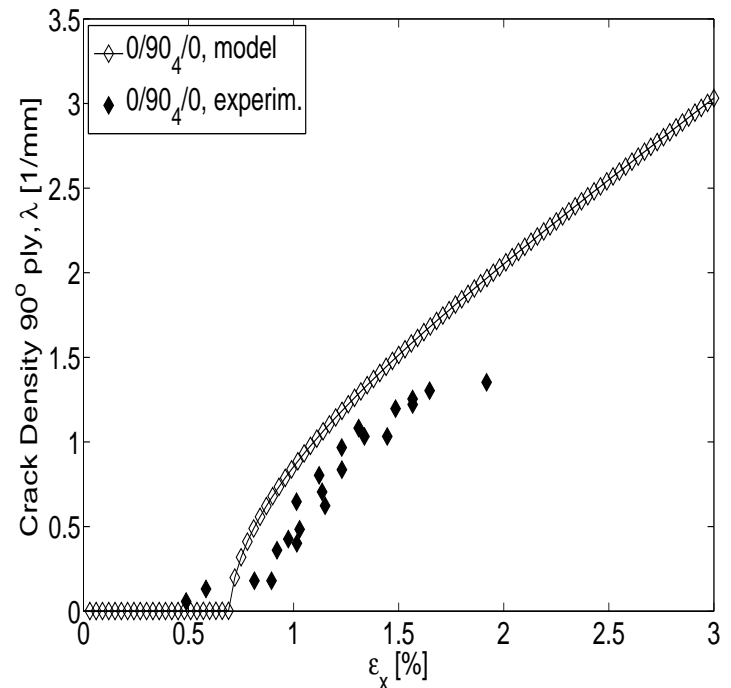

Figure 5.146: Matrix cracking progression: analytical curve vs. experimental data, Gudmundson [36]. [0/904/0] LSS, $\epsilon_{x}$ loading.

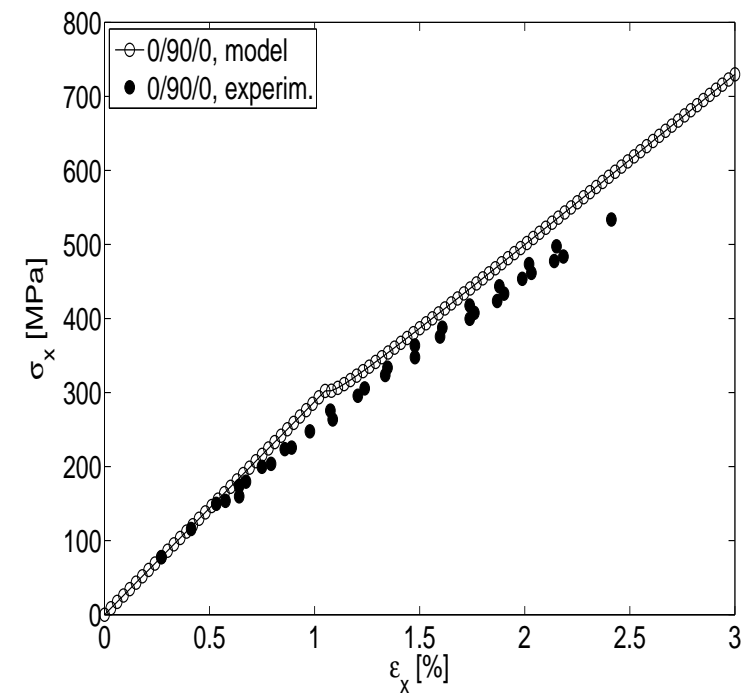

Figure 5.148: Stress-strain evolution: analytical curve vs. experimental data, Gudmundson [36]. [0/90/0] LSS, $\epsilon_{x}$ loading.

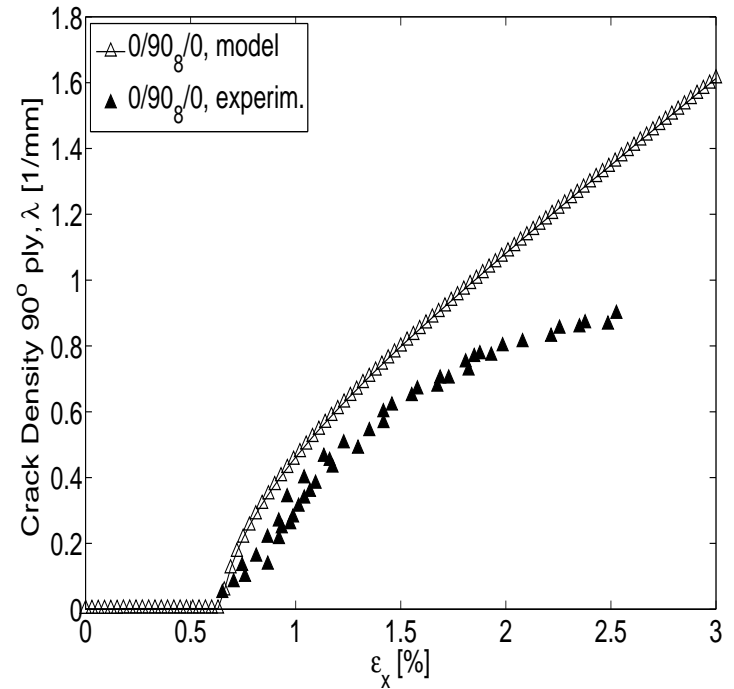

Figure 5.147: Matrix cracking progression: analytical curve vs. experimental data, Gudmundson [36]. [0/908/0] LSS, $\epsilon_{x}$ loading.

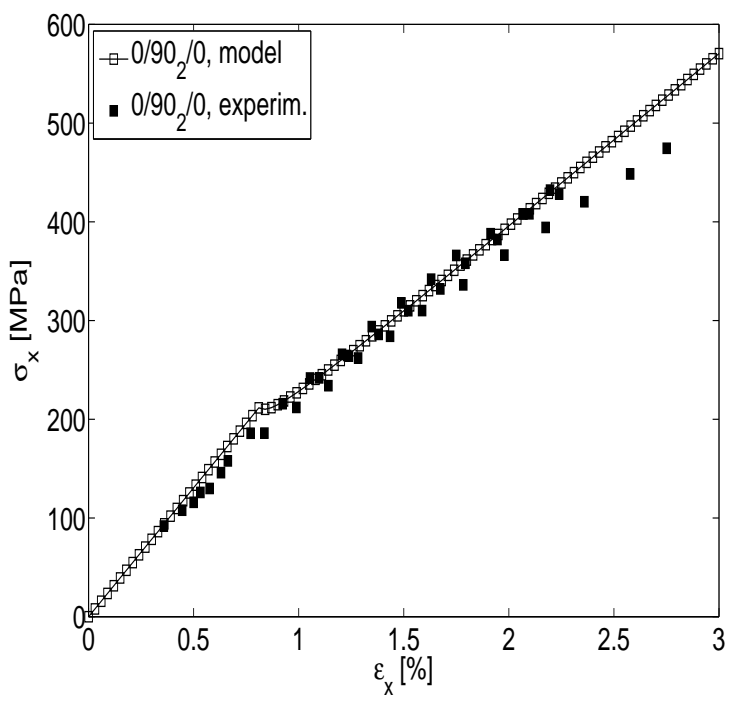

Figure 5.149: Stress-strain evolution: analytical curve vs. experimental data, Gudmundson [36]. [0/902/0] LSS, $\epsilon_{x}$ loading. 


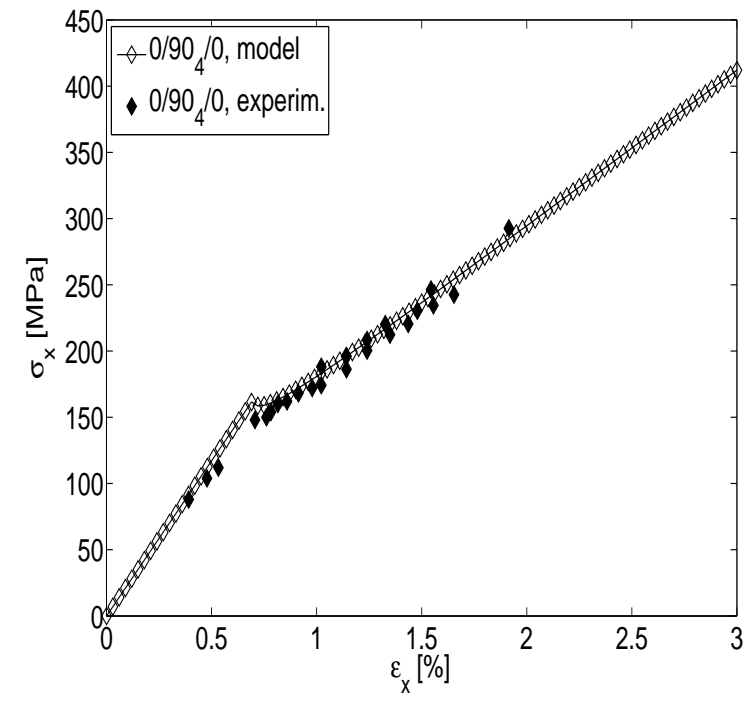

Figure 5.150: Stress-strain evolution: analytical curve vs. experimental data, Gudmundson [36]. [0/904/0] LSS, $\epsilon_{x}$ loading.

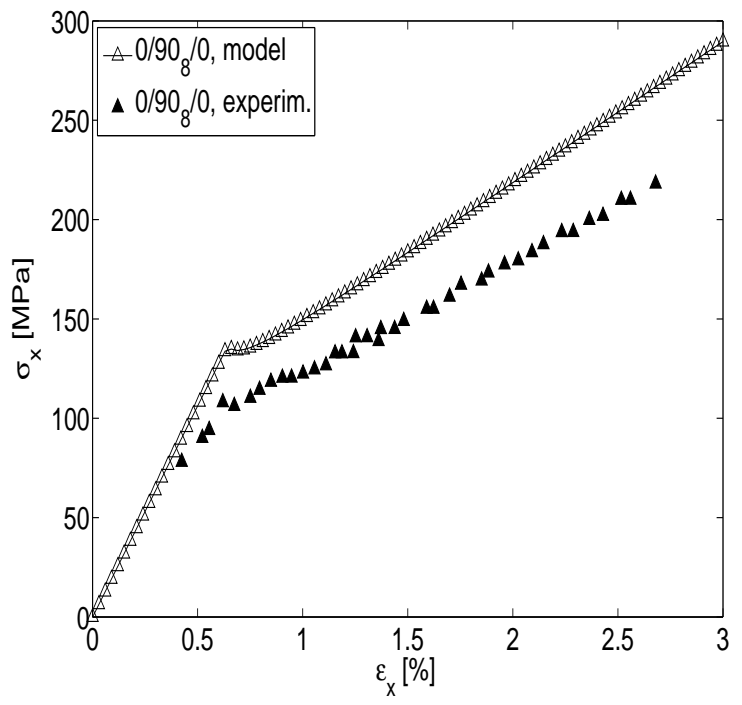

Figure 5.151: Stress-strain evolution: analytical curve vs. experimental data, Gudmundson [36]. [0/908/0] LSS, $\epsilon_{x}$ loading.

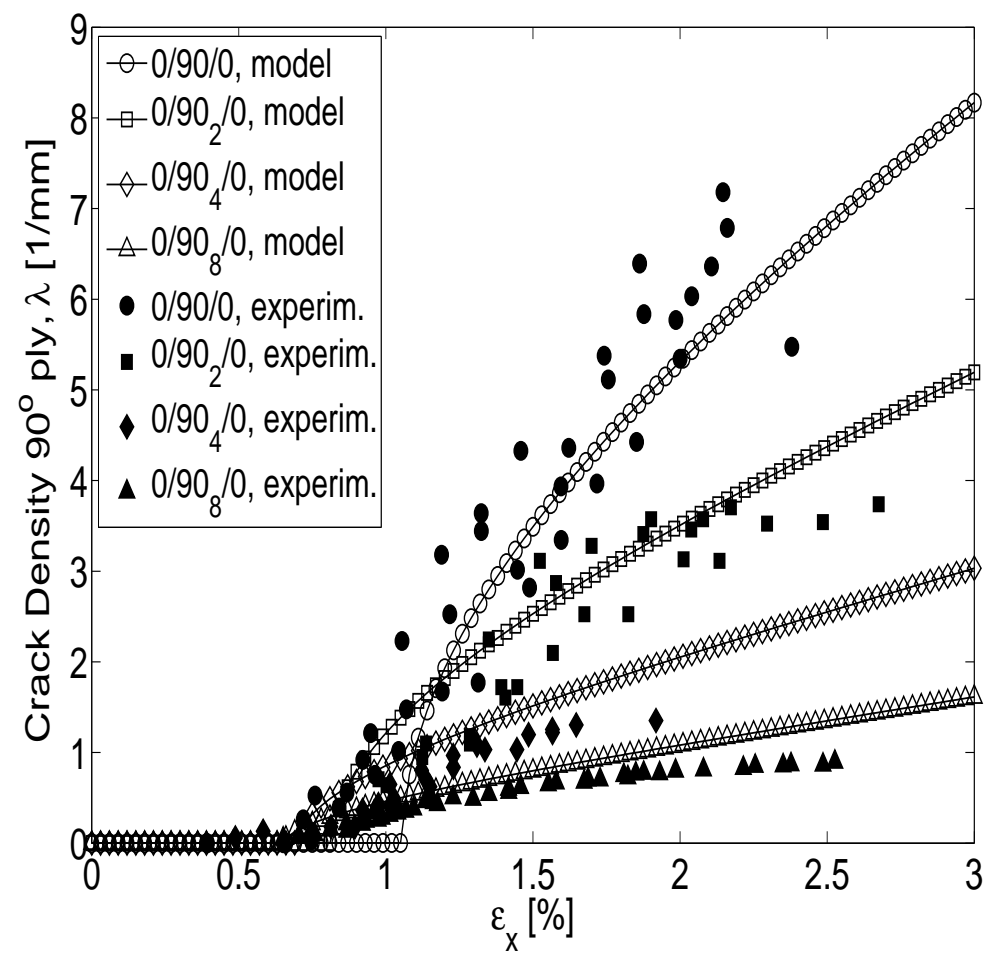

Figure 5.152: Matrix cracking progression: analytical curves vs. experimental data, Gudmundson [36]. [0/90n $/ 0]$ LSS, $\epsilon_{x}$ loading. 


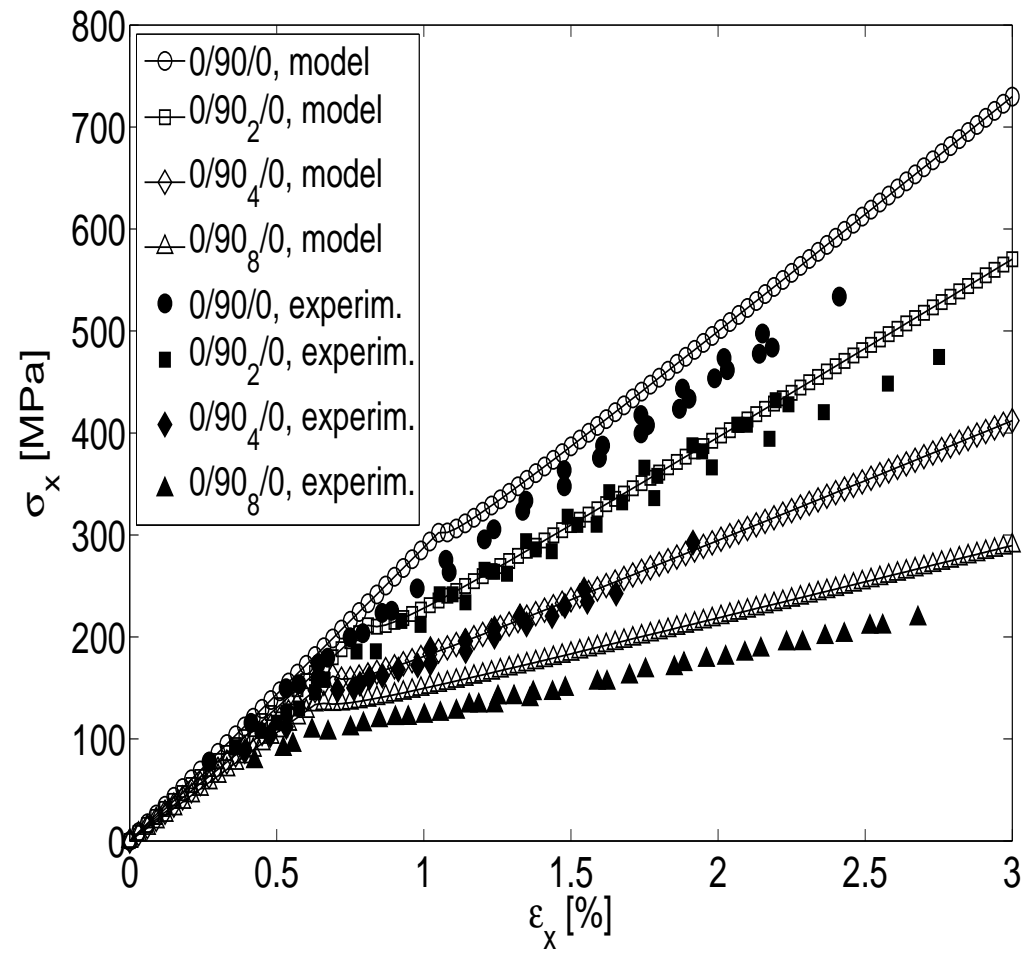

Figure 5.153: Stress-strain evolution: analytical curves vs. experimental data, Gudmundson [36]. [0/90n $/ 0]$ LSS, $\epsilon_{x}$ loading. 


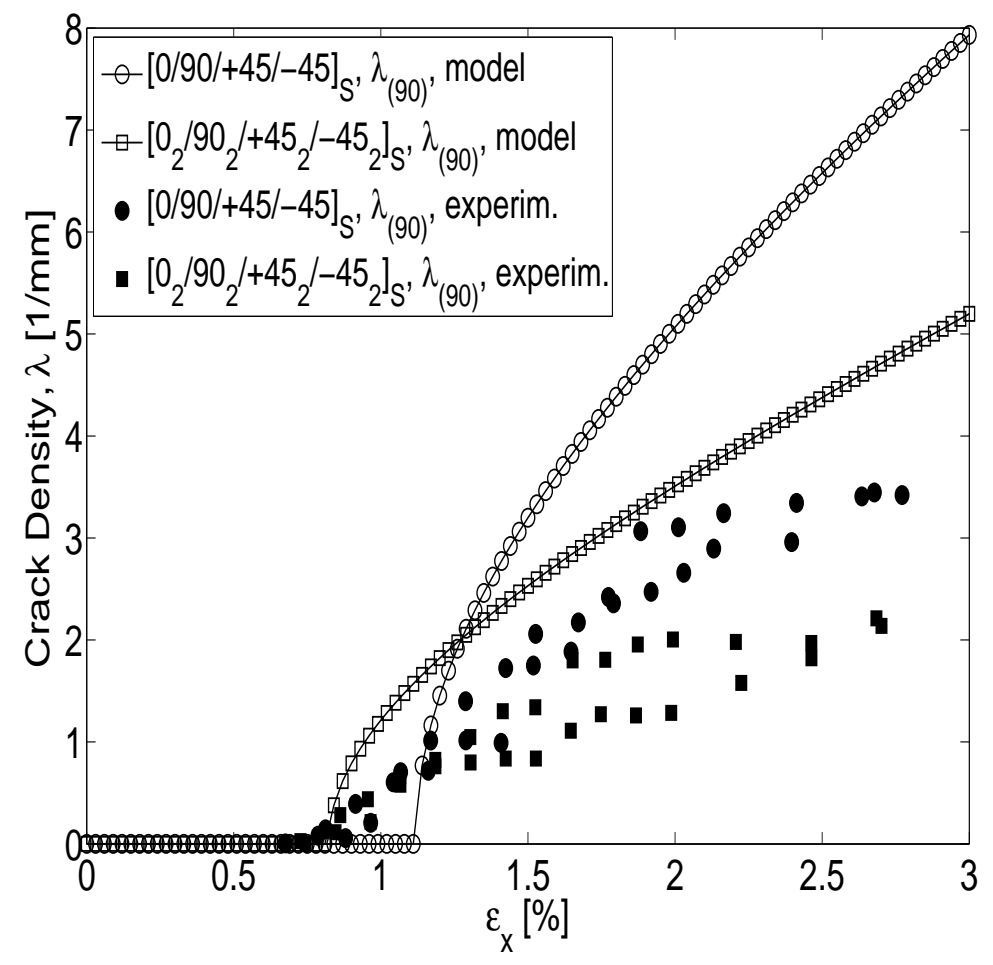

Figure 5.154: Matrix cracking progression: analytical curves vs. experimental data, Gudmundson $[36] .\left[0_{n} / 90_{n} /+45_{n} /-45_{n}\right]_{S}$ LSS, $\epsilon_{x}$ loading. 


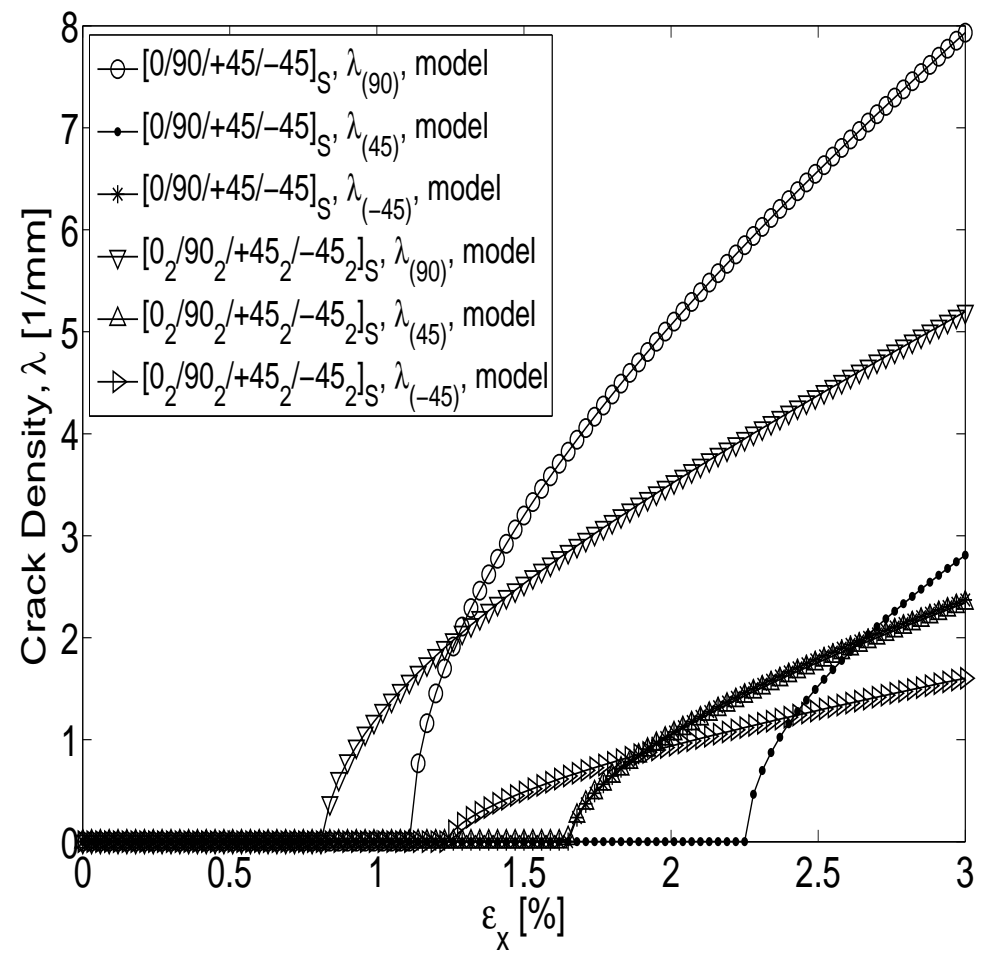

Figure 5.155: Matrix cracking progression: analytical curve. $\left[00_{n} / 90_{n} /+45_{n} /-45_{n}\right]_{S}$ LSS, $\epsilon_{x}$ loading.

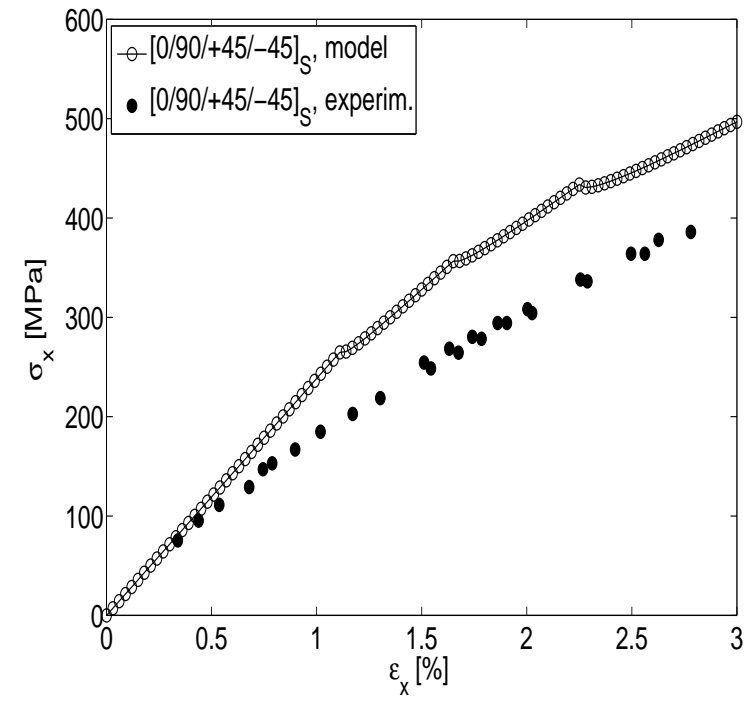

Figure 5.156: Stress-strain evolution: analytical curve vs. experimental data, Gudmundson [36]. $[0 / 90 /+45 /-45]_{S}$ LSS, $\epsilon_{x}$ loading.

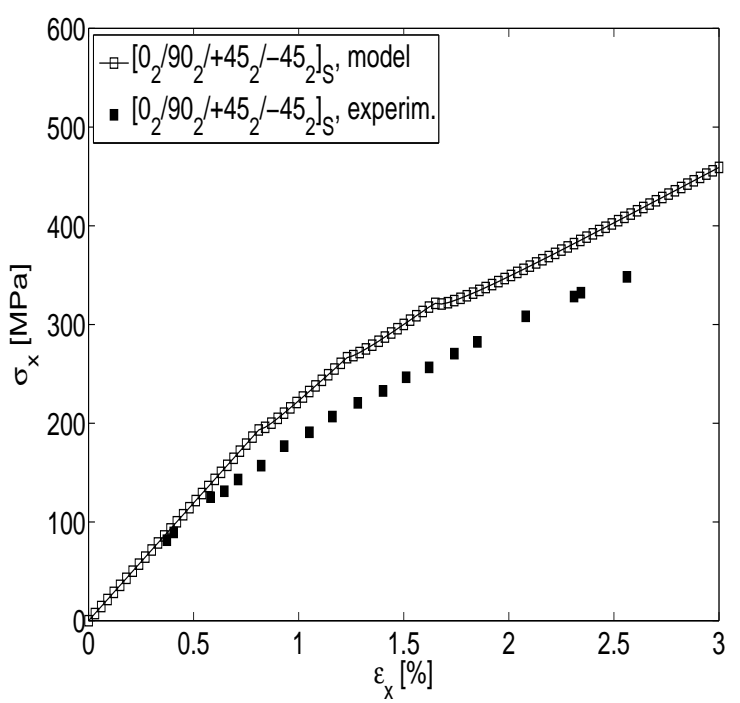

Figure 5.157: Stress-strain evolution: analytical curve vs. experimental data, Gudmundson [36]. $\left[0_{2} / 90_{2} /+45_{2} /-45_{2}\right]_{S}$ LSS, $\epsilon_{x}$ loading. 


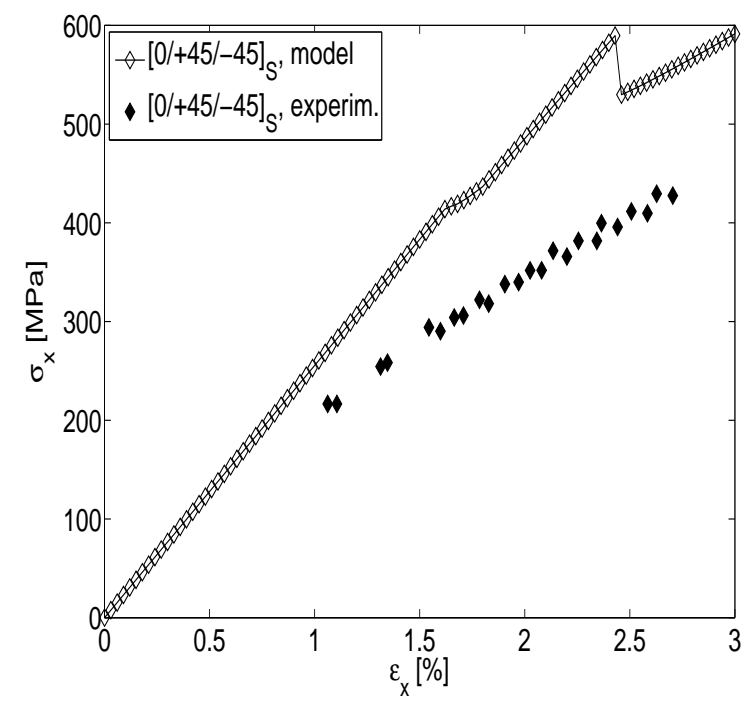

Figure 5.158: Stress-strain evolution: analytical curve vs. experimental data, Gudmundson [36]. $[0 /+45 /-45]_{S}$ LSS, $\epsilon_{x}$ loading.

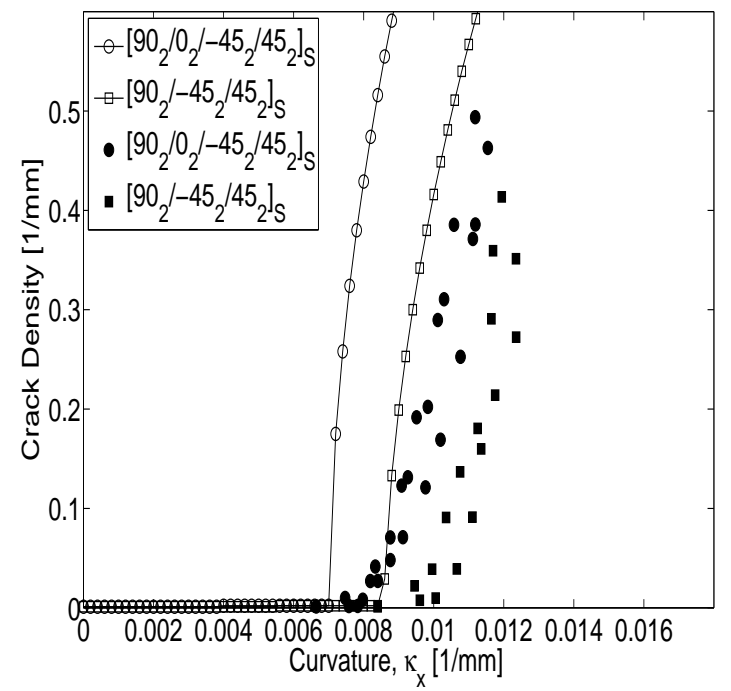

Figure 5.160: Matrix cracking progression: analytical curve vs. experimental data, Gudmundson [36]. $\left[90_{2} / 0_{2} /-45_{2} /+\right.$ $\left.45_{2}\right]_{S}$ and $\left[90_{2} /-45_{2} /+45_{2}\right]_{S}$ LSS, $\kappa_{x}$ loading.

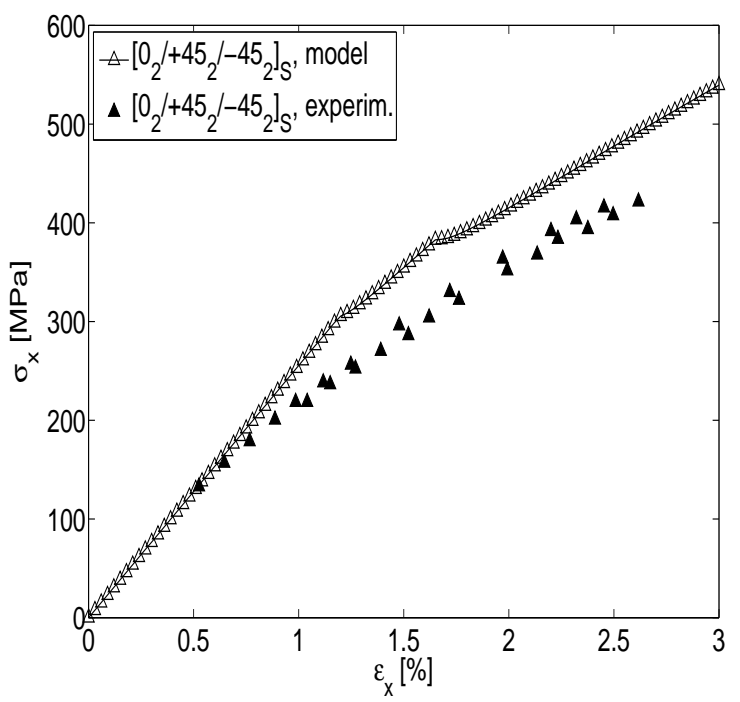

Figure 5.159: Stress-strain evolution: analytical curve vs. experimental data, Gudmundson $[36]$. $\left[0_{2} /+45_{2} /-45_{2}\right]_{S}$ LSS, $\epsilon_{x}$ loading.

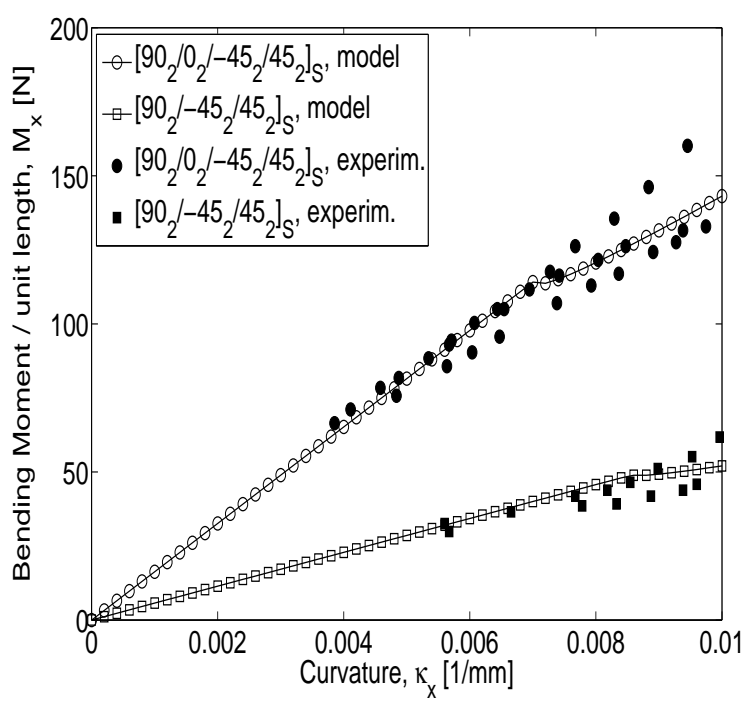

Figure 5.161: Moment-curvature evolution: analytical curves vs. experimental data, Gudmundson [36]. $\left[90_{2} / 0_{2} /-45_{2} /+\right.$ $\left.45_{2}\right]_{S}$ and $\left[90_{2} /-45_{2} /+45_{2}\right]_{S}$ LSS, $\kappa_{x}$ loading. 


\section{Chapter 6}

\section{Conclusions}

The problem of intra-laminar matrix cracking as a damage mechanism in laminated FRP is being approached by the present analytical model formulation. The laminate configuration can be a general one, without being limited to symmetric or balanced stacking sequence, as it is the case of most analytical models available in the literature. The deformation of the laminate can be either membrane, flexural or combination of the two. Matrix cracking under mode $I, I I$, or $I-I I$ mixed mode conditions in included in the present model. All these features confer a high level of generality to the analytical model, making it applicable to large classes of engineering applications.

The multiple aspects of degradation in the material thermo-elastic constants, damage onset and damage growth under increasing external loading are treated in this work. This is accomplished by combining and integrating the following components into an unitary predictive model: i) a material model able to predict the degradation of the thermo-elastic properties of the laminate for known levels of damage in individual plies; ii) the homogenization concept inspired by CDM, according to which physical cracks are not considered inside of the laminate, but the effect of the physical cracks on the overall thermo-elastic properties of the laminate is assessed; iii) an energy based damage evolution law inspired by Fracture Mechanics, taking the mixed $I$ - II modes of crack formation into account; iv) an iterative procedure meant to search for the cracking ply among the all plies of the laminate, at a given level of the external loading; v) Classical Laminate Theory meant to describe the overall constitutive behavior of the laminate having individual plies affected by matrix cracking.

The material model [35] provides the whole set of the membrane and flexural reduced 
thermo-elastic properties of the laminate, and predicts very well the experimental matrix cracking data. However, when the slope of the analytical prediction curves is compared against the slope of the experimental data trends, discrepancies seem to consistently appear at high values of crack densities. These discrepancies might be responsible for the poorer correlation between the analytical predictions and experimental data regarding the damage evolution curves $\lambda=\lambda$ (loading), as presented and commented in Section 5.2.

The homogenization technique is a modeling approach based on equivalent (effective, homogenized) material properties of the cracked laminate which is inspired by $C D M$, and it is consequently present in any CDM model. It is nevertheless true that the calculation of the out-of-plane (involving $z$ direction) stress and strain components that appear as a result of the perturbation field induced by the presence of cracks is not possible based on homogenization technique. From this point of view, the micromechanics of damage approach is able to claim higher accuracy. On the other hand, the micromechanics of damage solution based on RUC (see Section 2.1.1) proved to feature strong limitations regarding the level of generality of the problems that can be solved (e.g., cracks developing in only one ply; cracks developing in at most two plies; solution applying only to particular LSS configurations, like balanced and symmetric; solution applying only to in-plane membrane deformation). Furthermore, even solutions based on micromechanics of damage rely on simplifications and assumptions regarding the out-of-plane stress/strain components in the cracked ply, these assumptions being sometimes extended to the extreme case of neglecting the mentioned out-of-plane perturbation components.

The release of the aforementioned limitations of the RUC models proved to be possible by using the homogenization technique in conjunction with CLT. Based on the review and the analysis of previous models in the literature resorting to this type of approach (e.g., Li [6], Barbero [30], Soutis [57, 86], McCartney [87, 88, 89]) it can be expected that the combination between the CDM inspired homogenization technique and the CLT is a solution to be considered for the problem of matrix cracking in laminated composites. The effective stress-strain field is calculated based on effective material properties and CLT, and the stored energy of deformation is calculated based on in-plan effective stress-strain components. It is possible, in this way, to extend the level of generality of the problem by including the flexural deformation of the laminate.

The damage evolution law (eq. (4.4)) used in the present model is based on the so called " $K_{I}$ failure criterion" developed in [54], which considers mixed mode conditions (mode $I$ 
crack opening and mode $I I$ crack shearing) for intra-laminar crack propagation in UD composites. By extending the idea of intra-laminar matrix cracking from UD to laminated composites, the equation in (4.4) turns to be the conditions for matrix cracking multiplication in one ply of the laminate at a certain loading moment, under mixed mode loading conditions. However, the damage process for single crack growth in UD composites might be different as compared to crack multiplication in laminated composite (see the concept of in-situ $R$ curve behavior due to fiber bridging effect, as it has been explained in Section 4.4.3) and the certainty of the damage evolution law in eq. (4.4) is difficult to asses.

Since various mixed mode crack growth criteria have been proposed in the literature (see Appendix $\mathrm{C}$ ), the open question is what damage evolution law might be the best choice in order to accurately reproduce matrix cracking results for composite laminates under mixed modes loading. A specific study of various mixed modes damage evolution laws for the case of intra-laminar matrix cracking might be required in order to answer to this question. This specific study is not performed in the present manuscript, but the damage evolution law in eq. (4.4) is casted and used as a mixed mode damage criterion. This might bring a degree of uncertainty and lack of accuracy in predicting damage evolution results for those cases (laminate configurations and/or loading conditions) where mixed $I$ and $I I$ mode loading prevails for individual plies of the laminate. The problem of the best choice for the damage evolution criterion is left as an open issue. However, various other mixed modes damage criteria are listed and discussed in Appendix $\mathrm{C}$, in order to get an overall qualitative idea of the prediction capabilities of different choices for the damage evolution law.

Furthermore, due to the observed in-situ $R$-curve behavior in laminated composites, even the idea that a damage evolution law based on critical ERR $\left(G_{I C}, G_{I I C}\right)$ is inappropriate in order to describe the progressive matrix cracking in laminated composites has been formulated in some studies (Hahn [17], Gudmundson and Adolfsson [36], Soutis [57], Varna [58]). However, the formulation proposed in the present manuscript first has modeled and implemented the in-situ $R$-curve behavior as expected to be encountered in the case of laminated composites, and then the damage evolution law will consequently have this effect built-in (see eq. (4.29)). The in-situ R-curve behavior is phenomenologically explained based on the fiber bridging effect during matrix cracking in laminated composites, and based on experimental observations it is implemented only for the mode $I$ critical parameter of matrix cracking, $G_{I C}$ (see Section 4.4.3). There is no available experimental data regarding the mode $I I$ critical parameter, $G_{I I C}$, which is considered constant in the present formulation. 
The advantage of an ERR based damage evolution law is the fact that the experimentally noticed influence of the laminate configuration on the damage process can be accounted for (see Section 5.1.1, 5.2). In order to consider mixed $I-I I$ modes of crack formation, the separate contribution of the elastic strain energy to each mode has to be calculated, as presented in Section A.2.

The iterative procedure not only allows for finding the plies inside of the laminate that satisfy the condition of matrix crack multiplication, but also allows for distinguishing between those plies that might simultaneously satisfy the crack growth condition. This could happen for the case of laminated composites containing plies orientated at close values of the offaxis angle, while considering high increments of the applied load. In this case, the current cracking ply is selected as the one featuring the highest value of the ERR coefficient $g$ in eq. (4.4). After the damage level is increased in the current cracking ply, the iterative procedure reiterates over the whole LSS in order to check for multiple damage increments in the current cracking ply, or for multiple cracking plies undergoing damage at the same level of the external loading. The load redistribution process between individual plies of the laminate can thus be accounted for, function of the simulated testing conditions (see Section 4.7.1).

Crack densities in individual plies are used as damage variables of the present progressive damage model. This approach brings the advantage of effectively calculating and knowing the matrix crack density at every level of loading, which can be valuable information when the permeability of the laminate is of interest, as it is the case of the composite pressure vessels, for example. Most of the analytical models for progressive damage incorporated in commercial FEA packages do not provide this information due to their CDM type stress softening formulation (see Appendix E).

The simulation of the crack multiplication process is based on the discrete 1 more crack behavior of the laminated composite, which according to experimental observations of matrix cracking in brittle composites is considered as being the closest modeling approach to the real behavior of the material. Most of the RUC based models consider a doubling of the number of cracks when the crack multiplication conditions are met, while the CDM models consider a continuously infinitesimal increase of the damage variable. However, the present model is able to adopt any of these crack multiplication strategies, as describes in Section 4.4.2.

The model incorporates the effect of the thermal residual stresses due to the difference between the processing and the operating temperature, which confers higher accuracy of the 
analytical results and extends the predictive capabilities of the model to a high range of operating temperatures, including structural applications at cryogenic temperatures.

Special modeling features are incorporated in order to capture the behavior of the composite material developing matrix cracking under flexural deformation. The crack closure model (see Section 4.7.2) allows for the possibility to consider the effects of the loading history on the properties and the behavior of the composite material.

The analytical output of the model is compared with available experimental data for the case of both membrane and flexural loading. However, while a large quantity of experimental data could be identified in the literature regarding the membrane loading case, the available data regarding the flexural case is yet scarce. A testing program specially designed for the case of flexural deformation could provide additional useful data for the validation of the proposed analytical model. Another niche where additional experimental data can be useful for the validation of the model is the change in the laminate CTE due to the effect of matrix cracking. 


\section{Appendix A}

\section{Classical Laminate Theory considering the effect of thermal residual stresses}

\section{A.1 General constitutive formulation}

Even if the Classical Laminate Theory $(C L T)$ equations can be found in a textbook of laminated composite materials, their derivation will be considered in the following, in order to make an unitary presentation of all the supporting concept and equations required in the previous sections, and in order to achieve completeness and correctness in considering the effect of the thermal residual stresses due to a temperature difference $\Delta T=T_{s}-T_{p}$, where $T_{s}$ is the service temperature ( $T_{s}$ is usually the room temperature for most of the experiments), and $T_{p}$ is the processing temperature (which is a function of the manufacturing process and the type of matrix used, usually between $(125-225)^{\circ} \mathrm{C}$ for most of the curing processes). The right derivation and use of the equations including the thermal residual stress is important in the energy formulation of this model, where the strain energy due to thermal residual stresses has to be considered, as presented in Section A.2.

The following dedicated CLT relations and notations will be used throughout this chapter:

- $[A],[B]$, and $[D]$ are the well known $3 \times 3$ laminate stiffness matrices. They describe the composite laminate behavior under in-plane membrane loading $([A])$, under flexural loading $([D])$, and the coupling between in-plane and flexural loading $([B])$. The laminate stiffness 
matrices are calculated as

$$
\begin{aligned}
& {[A]=\sum_{k=1}^{N}[\bar{Q}]_{k} \int_{z_{k-1}}^{z_{k}} d z=\sum_{k=1}^{N} t_{k}[\bar{Q}]_{k}} \\
& {[B]=\sum_{k=1}^{N}[\bar{Q}]_{k} \int_{z_{k-1}}^{z_{k}} z d z=\sum_{k=1}^{N} t_{k} \bar{z}_{k}[\bar{Q}]_{k}} \\
& {[D]=\sum_{k=1}^{N}[\bar{Q}]_{k} \int_{z_{k-1}}^{z_{k}} z^{2} d z=\sum_{k=1}^{N} t_{k}\left[\bar{z}_{k}^{2}+\frac{t_{k}^{2}}{12}\right][\bar{Q}]_{k}}
\end{aligned}
$$

where $t_{k}, \bar{z}_{k}$ and $[\bar{Q}]_{k}$ are the thickness, the middle coordinate and the stiffness matrix of ply $(k)$, respectively, and $N$ is the total number of plies in the laminate.

The $6 \times 6$ laminate stiffness matrix including both the effect of in-plane and flexural loadings is compactly noted as $[A B D]$ throughout this section. The inverse of the $6 \times 6$ laminate stiffness matrix represents the laminate compliance matrix and it is noted $[S]$ : $[S]=[A B D]^{-1}$.

- $\left\{\delta_{E}\right\}$ and $\left\{\delta_{B}\right\}$ are $3 \times 1$ vectors and they are called laminate thermal load coefficients in this chapter. They describe the laminate in-plane $\left(\left\{\delta_{E}\right\}\right)$ and flexural $\left(\left\{\delta_{B}\right\}\right)$ behavior under thermal loading $\Delta T$ (see Section A.1.2, A.1.3). The laminate thermal load coefficients are function of thermo-elastic properties of individual plies $(k)$ and laminate configuration, and they are calculated as

$$
\begin{aligned}
& \left\{\delta_{E}\right\}=-\sum_{k=1}^{N}[\bar{Q}]_{k}\{\alpha\}_{k} \int_{z_{k-1}}^{z_{k}} d z=-\sum_{k=1}^{N} t_{k}[\bar{Q}]_{k}\{\alpha\}_{k} \\
& \left\{\delta_{B}\right\}=-\sum_{k=1}^{N}[\bar{Q}]_{k}\{\alpha\}_{k} \int_{z_{k-1}}^{z_{k}} z d z=-\sum_{k=1}^{N} t_{k} \bar{z}_{k}[\bar{Q}]_{k}\{\alpha\}_{k}
\end{aligned}
$$

where $\{\alpha\}_{k}$ is the vector of thermal expansion coefficients of ply $(k)$.

- $\left\{\alpha_{E}\right\}$ and $\left\{\alpha_{B}\right\}$ are $3 \times 1$ vectors and they represent the laminate thermal expansion coefficients. They describe the laminate in-plane $\left(\left\{\alpha_{E}\right\}\right)$ and flexural $\left(\left\{\alpha_{B}\right\}\right)$ deformation under thermal loading $\Delta T$ (see Section A.1.2, A.1.3). The laminate thermal expansion coefficients are calculated as

$$
\left\{\begin{array}{c}
\alpha_{E} \\
\alpha_{B}
\end{array}\right\}=-[A B D]^{-1}\left\{\begin{array}{c}
\delta_{E} \\
\delta_{B}
\end{array}\right\}=-[S]\left\{\begin{array}{c}
\delta_{E} \\
\delta_{B}
\end{array}\right\}
$$

As it can be observed, the above defined overall laminate properties are function of individual ply materials properties, individual ply thickness/orientation, and laminate stacking sequence (LSS). 
The loading cases considered in the following are:

- the distributed mechanical membrane $\left(\{N\}=\left\{N_{x}, N_{y}, N_{x y}\right\}\right)$ and flexural $(\{M\}=$ $\left.\left\{M_{x}, M_{y}, M_{x y}\right\}\right)$ loading. In compacted notation, the $6 \times 1$ loading vector is noted as

$$
\{N, M\}=\left\{N_{x}, N_{y}, N_{x y}, M_{x}, M_{y}, M_{x y}\right\}
$$

- the thermal loading $\Delta T$

The following notations are introduced and used throughout this section: $M$-mechanical component (due to mechanical loading only); $T$-thermal component (due to temperature difference $\Delta T$ only); tot-total component (due to both mechanical and thermal effect).

\section{A.1.1 The case of only mechanical loading}

In this case, the mechanical loading acting on laminate is given by (A.4), and the thermal loading $\Delta T=0$. The resulting mechanical deformation of the laminate is noted as $\{\epsilon\}^{M}=$ $\left\{\epsilon^{0}, \kappa\right\}^{M}$, where $\left\{\epsilon^{0}\right\}^{M}$ is the $3 \times 1$ vector of the laminate middle-plane membrane (inplane) mechanical deformation, and $\{\kappa\}^{M}$ is the $3 \times 1$ vector of laminate flexural mechanical deformation (curvatures).

The resulting mechanical deformation of the individual $(k)$ ply is noted $\{\epsilon\}_{k}^{M}$ and it is calculated as

$$
\{\epsilon\}_{k}^{M}=\left\{\epsilon^{0}\right\}^{M}+z \cdot\{\kappa\}^{M}
$$

The resulting mechanical stress induced in each ply is calculated as

$$
\{\sigma\}_{k}^{M}=[\bar{Q}]_{k} \cdot\{\epsilon\}_{k}^{M}
$$

The through-thickness equilibrium equations of the laminate, between the applied external loads (A.4) and the resulting internal stresses (A.6), reads

$$
\int_{-t / 2}^{t / 2}\{\sigma\}_{k}^{M} d z=\{N\}, \quad \int_{-t / 2}^{t / 2}\{\sigma\}_{k}^{M} \cdot z d z=\{M\}
$$

Based on the equilibrium equations in (A.7), after replacing the ply stresses (A.6) and the ply strains (A.5), and after performing all the calculations by considering the notations in (A.1), the following two equations can be written:

$$
\begin{aligned}
\{N\} & =\left\{\epsilon^{0}\right\}^{M} \cdot[A]+\{\kappa\}^{M} \cdot[B] \\
\{M\} & =\left\{\epsilon^{0}\right\}^{M} \cdot[B]+\{\kappa\}^{M} \cdot[D]
\end{aligned}
$$


from where the laminate mechanical deformation can be calculated as

$$
\{\epsilon\}^{M}=\left\{\begin{array}{l}
\epsilon^{0} \\
\kappa
\end{array}\right\}^{M}=[A B D]^{-1} \cdot\left\{\begin{array}{l}
N \\
M
\end{array}\right\}
$$

Equation (A.9) represents the constitutive relationship of the laminate for the case of mechanical loading only. It provides the laminate deformation $\{\epsilon\}^{M}$ for the applied mechanical loading $\{N, M\}$. Based on the laminate deformation in (A.9), the individual ply strains (A.5) and ply stresses (A.6) can be further calculated.

\section{A.1.2 The case of only thermal loading $\Delta T$}

In this case, the laminate is subjected to the thermal difference $\Delta T=T_{s}-T_{p}$ between the processing $\left(T_{p}\right)$ and the service $\left(T_{s}\right)$ temperatures, without any mechanical loading acting on laminate $(\{N, M\}=0$ in (A.4)). The resulting thermal deformation of the laminate is noted as $\{\epsilon\}^{T}=\left\{\epsilon^{0}, \kappa\right\}^{T}$, where $\left\{\epsilon^{0}\right\}^{T}$ is the $3 \times 1$ vector of laminate middle-plane membrane (in-plane) thermal induced deformation, and $\{\kappa\}^{T}$ is the $3 \times 1$ vector of laminate flexural thermal induced deformation (curvatures).

It has to be noted that the case of laminate free thermal deformation is considered in the derivation of all the following equations. It is nevertheless true that cases of laminate constraint thermal deformation can be encountered in different structural applications, and those cases has to be treated as separate problems, yet based on the physics described here for the case of the laminate free thermal deformation. The goal here is to consider the effect of thermal residual stresses due to the temperature difference $\Delta T$. Even if the thermal deformation of the laminate is free, inter-plies thermal deformation constraints appear, due to the fact that different layers have different values of the thermal expansion coefficients on different directions. This inter-plies constraints on the free plies thermal deformation is the cause of thermal residual stresses in the individual plies of the laminate. It can be inferred in this way that the problem of thermal effect on the laminated composites is a combined one, by considering the free thermal deformation of the laminate, and by considering in the same time the inter-plies constraints on the free thermal deformation of individual plies. This problem is depicted in Fig. A.1, where the simplest case of a symmetric [0/90/0] laminate is presented. This simple laminate configuration was selected in order to introduce the physics of the behavior. The results will be extended then to the case of a general laminate. 


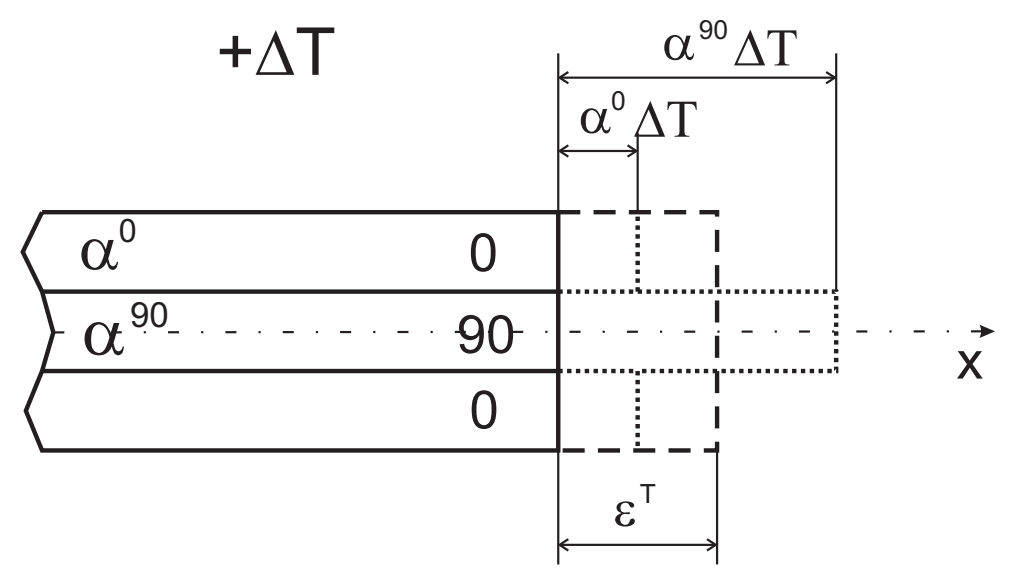

Figure A.1: The influence of different CTE of different plies of the laminate.

Let us consider first only the case of a positive temperature change $+\Delta T$. In Figure A.1 the initial state $\Delta T=0$ is represented by continuous lines, the resulting laminate thermal dilatation due to applied $+\Delta T$ is represented by dashed lines, and the hypothetical individual plies thermal dilatation due to applied $+\Delta T$, if the plies were not glued together into a laminate, is represented by dotted lines. Here the specific behavior of laminated materials comes into picture. The whole laminate is free to expand, but individual plies constrain each other from totally free thermal expansion, due to their different thermal expansion coefficients. Furthermore, not only are the thermal expansion coefficients different between plies, but they are also different on different directions of the same ply (for a typical unidirectional fiber reinforced ply, the thermal expansion along the fiber direction is less than the thermal expansion along the transverse to fibers direction). Thus, while the thermal expansion coefficient of $0^{\circ}$ ply on $x$ direction (which is the fiber direction denoted as 1 direction) is less than the thermal expansion coefficient of $90^{\circ}$ ply on $x$ direction (which is the transverse to fiber direction denoted as 2 direction), the situation reverses along $y$ direction of the laminate. Because of these differences on different directions (which is the basic definition of a composite material), the term constraints between plies enlarges its meaning. In Figure A.1, along the laminate $x$ direction, the $90^{\circ}$ are really constrained from totally free thermal dilatation by the $0^{\circ}$ plies, while the $0^{\circ}$ plies are forced by the $90^{\circ}$ plies to expand more than their own free thermal dilatation.

Bearing these in mind, it can be now proceeded with analyzing the state of deformation in the particular case of the symmetric laminate in Fig. A.1. Let's note the thermal deformation of the laminate $\left\{\epsilon^{T}\right\}=\left\{\epsilon^{0}, k\right\}^{T}$. Based on the fact that the laminate is symmetric, there 
will be no thermal induced curvatures $\left(\{k\}^{T}=0\right)$, the thermal deformation of the laminate has only the in-plane components $\left\{\epsilon^{0}\right\}^{T}$. In this case, the thermal deformation of the ply $(k)$ is

$$
\{\epsilon\}_{k}^{T}=\left\{\epsilon^{0}\right\}^{T}
$$

It has to be noted at this moment that not all of the ply thermal deformation $\{\epsilon\}_{k}^{T}$ induces ply stresses, because same of the ply thermal deformation actually represents free thermal deformation. What induces ply stresses is the amount of constrained thermal deformation of ply $(k)$, which based on the reasoning related to Fig. A.1 can be expressed as

$$
\{\epsilon\}_{k}^{T, c}=\{\epsilon\}_{k}^{T}-\{\alpha\}_{k} \cdot \Delta T
$$

where the superscript $c$ stands for "constrained".

Thus, the thermal induced stresses in ply $(k)$ due to inter-ply constraints (in turn due to the CTE mismatch between individual plies) can be calculated as

$$
\{\sigma\}_{k}^{T}=[\bar{Q}]_{k} \cdot\{\epsilon\}_{k}^{T, c}=[\bar{Q}]_{k} \cdot\left(\{\epsilon\}_{k}^{T}-\{\alpha\}_{k} \cdot \Delta T\right)
$$

If all the calculations are performed, it can be seen that the above equation stands for both $0^{\circ}$ and $90^{\circ}$ plies inside of the laminates. If further calculations are performed, it can be seen that the above equation can be applied for both $+\Delta T$ and $-\Delta T$ temperature change. Moreover, if we leave the particular case of a symmetric laminate presented in Figure A.1, and consider the case of a general laminate, bending deformations (curvatures) appear as a result of temperature change. In this case, the overall free thermal deformation of the laminate is described by both in-plane deformation of the laminate mid-surface $\left\{\epsilon^{0}\right\}^{T}$ and bending laminate curvatures $\{\kappa\}^{T}:\{\epsilon\}^{T}=\left\{\epsilon^{0}, k\right\}^{T}=\left\{\epsilon_{x}^{0}, \epsilon_{y}^{0}, \epsilon_{x y}^{0}, \kappa_{x}, \kappa_{y}, \kappa_{x y}\right\}^{T}$ In this case (of the general laminate), the thermal deformation of the ply $(k)$ becomes

$$
\{\epsilon\}_{k}^{T}=\left\{\epsilon^{0}\right\}^{T}+z \cdot\{k\}^{T}
$$

which replaces the expression in equation (A.10) (which applies only for the case of symmetric laminate), and it is further used in (A.11) and (A.12) in order to calculate the constrained thermal deformation and the resulting thermal stress in ply $(k)$.

At this moment, the state of thermal stress in individual plies of the laminate is determined as expressed in (A.12), based on laminate overall free thermal deformation $\{\epsilon\}^{T}=$ $\left\{\epsilon^{0}, k\right\}^{T}$, and individual plies properties: ply stiffness matrix $[\bar{Q}]_{k}$ and ply thermal expansion coefficients vector $\{\alpha\}_{k}$ (both of these expressed in laminate global coordinate system). 
The ply thermal expansion coefficients vector $\{\alpha\}_{k}$ in (A.12) is expressed in global laminate coordinate system, and can be calculated using the regular in-plane strain transformation matrix (noted $[T]_{k}$ here for strain rotation from the laminate coordinate system $(x-y)$ to ply coordinate system $(1-2))$. Since $\{\alpha\} \Delta T$ is a strain, the $\{\alpha\}_{k}$ vectors transform like strains

$$
\left\{\begin{array}{l}
\alpha_{x} \\
\alpha_{y} \\
\frac{1}{2} \alpha_{x y}
\end{array}\right\}_{k}=[T]_{k}^{-1}\left\{\begin{array}{l}
\alpha_{1} \\
\alpha_{2} \\
0
\end{array}\right\}_{k}
$$

where $\alpha_{1}, \alpha_{2}$ are the ply thermal expansion coefficients in its own material coordinate system $(1-2)$ (with 1 axis along fiber direction, and 2 axis in-plane perpendicular to fiber direction).

The thermal induced stress in (A.12) is called residual thermal stress. It is interesting to note that these stresses are present in each ply of the laminate, but they equilibrate each other at the laminate level. This is because the case considered here is that of zero external mechanical loading $(\{N, M\}=0)$, combined with free overall thermal expansion of the whole laminate, so there are no applied forces and moments at laminate level. In other words, wherever is necessary, the following relations, which represent the force and momentum equilibrium equations through the laminate thickness, stand true

$$
\int_{-t / 2}^{t / 2}\{\sigma\}_{k}^{T} d z=0, \quad \int_{-t / 2}^{t / 2}\{\sigma\}_{k}^{T} \cdot z d z=0
$$

Based on the equilibrium equations in (A.15), after replacing the ply stresses (A.12) and the ply strains (A.13), and after performing all the calculations by considering the notations in (A.1) and (A.2), the following two equations can be written:

$$
\begin{aligned}
& 0=\left\{\epsilon^{0}\right\}^{T} \cdot[A]+\{\kappa\}^{T} \cdot[B]+\left\{\delta_{E}\right\} \cdot \Delta T \\
& 0=\left\{\epsilon^{0}\right\}^{T} \cdot[B]+\{\kappa\}^{T} \cdot[D]+\left\{\delta_{B}\right\} \cdot \Delta T
\end{aligned}
$$

from where the laminate thermal deformation can be calculated as

$$
\{\epsilon\}^{T}=\left\{\begin{array}{l}
\epsilon^{0} \\
\kappa
\end{array}\right\}^{T}=-[A B D]^{-1} \cdot\left\{\begin{array}{l}
\delta_{E} \\
\delta_{B}
\end{array}\right\} \cdot \Delta T
$$

Equation (A.17) represents the constitutive relationship of the laminate for the case of thermal loading only. It provides the laminate deformation $\{\epsilon\}^{T}$ for the applied temperature difference $\Delta T$. Based on the laminate deformation in (A.17), the individual ply strains (A.13) and ply stresses (A.12) can be further calculated. 
As previously stated when eq.(A.2) was introduced, the terms $\left\{\delta_{E}\right\},\left\{\delta_{B}\right\}$ are called laminate thermal load coefficients. The reason of using the label "load" is that the thermal deformation in (A.17) can be regarded as the result of an equivalent (fictitious mechanical) loading

$$
\left\{\begin{array}{l}
N \\
M
\end{array}\right\}^{T}=-\left\{\begin{array}{l}
\delta_{E} \\
\delta_{B}
\end{array}\right\} \cdot \Delta T
$$

which are called thermal loads in some text books. Using this notation, the constitutive relationship in (A.17) can be written as

$$
\{\epsilon\}^{T}=\left\{\begin{array}{l}
\epsilon^{0} \\
\kappa
\end{array}\right\}^{T}=[A B D]^{-1} \cdot\left\{\begin{array}{l}
N \\
M
\end{array}\right\}^{T}
$$

However, as explained before, the terms $\{N\}^{T},\{M\}^{T}$ in the right hand side of (A.19) are not real forces acting on the laminate, because the laminate is under free thermal expansion and the mechanical loads $\{N\}^{M},\{M\}^{M}$ are zero in the case studied here.

Using the notation in eq.(A.3), the constitutive relation in (A.17) can be alternatively written as

$$
\{\epsilon\}^{T}=\left\{\begin{array}{l}
\epsilon^{0} \\
\kappa
\end{array}\right\}^{T}=\left\{\begin{array}{l}
\alpha_{E} \\
\alpha_{B}
\end{array}\right\} \cdot \Delta T
$$

The generalized thermal expansion coefficients $\left\{\alpha_{E}, \alpha_{B}\right\}$ in (A.20) describe the overall laminate behavior under temperature change $\Delta T$, for both in-plane (membrane) and bending (flexural) deformations. The thermal expansion coefficients $\{\alpha\}_{E},\{\alpha\}_{B}$ in (A.3) and (A.20) depend on plies thermo-elastic properties, plies thickness, plies orientation and LSS. Another particular behavior of the laminates as heterogeneous materials can be noted here: for the case of general laminate configuration, change in temperature induces both a change in the linear dimensions and change in curvatures.

\section{A.1.3 The case of both mechanical and thermal loading}

If both mechanical loading $(\{N\},\{M\} \neq 0)$ and temperature difference $(\Delta T \neq 0)$ act on laminate, the state of deformation and the state of stress of the laminate can be calculated based on the superposition principle, by adding the deformation-stress states presented in Section A.1.1 (for mechanical loading only) and Section A.1.2 (for thermal loading only), as follows: 
- the total deformation of the laminate is calculated based on (A.9) and (A.17)

$$
\{\epsilon\}^{t o t}=\left\{\begin{array}{l}
\epsilon^{0} \\
\kappa
\end{array}\right\}^{t o t}=\left\{\begin{array}{l}
\epsilon^{0} \\
\kappa
\end{array}\right\}^{M}+\left\{\begin{array}{l}
\epsilon^{0} \\
\kappa
\end{array}\right\}^{T}=[A B D]^{-1} \cdot\left(\left\{\begin{array}{l}
N \\
M
\end{array}\right\}-\left\{\begin{array}{l}
\delta_{E} \\
\delta_{B}
\end{array}\right\} \cdot \Delta T\right)
$$

Equation (A.21) represents the constitutive relationship of the laminate for the case of mechanical and thermal loading.

- the total deformation of ply $(k)$ is calculated based on (A.5) and (A.13)

$$
\begin{aligned}
\{\epsilon\}_{k}^{t o t} & =\{\epsilon\}_{k}^{M}+\{\epsilon\}_{k}^{T}=\left\{\epsilon^{0}\right\}^{M}+z \cdot\{\kappa\}^{M}+\left\{\epsilon^{0}\right\}^{T}+z \cdot\{\kappa\}^{T} \\
& =\left\{\epsilon^{0}\right\}^{t o t}+z \cdot\{\kappa\}^{\text {tot }}
\end{aligned}
$$

As previously explained in Section A.1.2, not all deformation in (A.22) induces ply stresses, since part of it actually represents free thermal deformation.

- the total stress of ply $(k)$ is calculated based on (A.6) and (A.12)

$$
\begin{aligned}
\{\sigma\}_{k}^{t o t} & =\{\sigma\}_{k}^{M}+\{\sigma\}_{k}^{T}=[\bar{Q}]_{k} \cdot\left(\{\epsilon\}_{k}^{M}+\{\epsilon\}_{k}^{T}-\{\alpha\}_{k} \cdot \Delta T\right) \\
& =[\bar{Q}]_{k} \cdot\left(\{\epsilon\}_{k}^{t o t}-\{\alpha\}_{k} \cdot \Delta T\right)
\end{aligned}
$$

As an observation it can be noted that the same constitutive relation in (A.21) can be obtained by considering the through the thickness laminate equilibrium, between the external applied loading $\{N, M\}$ and the internal generated stresses (eq.(A.23))

$$
\{N\}=\int_{-t / 2}^{t / 2}\{\sigma\}_{k}^{t o t} d z \quad\{M\}=\int_{-t / 2}^{t / 2}\{\sigma\}_{k}^{t o t} \cdot z d z
$$

\section{A.2 Laminate Strain Energy: effect of thermal residual stresses and damage mode separation}

As a notation convention, single and double subscript indices will be used throughout this section, in order to properly identify the location and the coordinate system of the stress/deformation components. When the second subscript index is missing it implicitly means that the quantity is in laminate c.s. For example: $\epsilon_{(k)}$ denotes the strain of ply $(k)$ in laminate c.s.; $\epsilon_{(k, k 0)}$ denotes the strain of ply $(k)$ rotated in the c.s. of ply $(k 0)$. 
As a generic equation, the strain energy density stored in ply $(k)$ of the laminate, for the linear-elastic case, is

$$
\begin{aligned}
U_{0(k)} & =\frac{1}{2}\left(\sigma_{(k)}\right)^{\prime} \cdot \epsilon_{(k)}=\frac{1}{2}\left(\sigma_{(k)}\right)^{\prime} \cdot\left[\bar{Q}_{(k)}\right]^{-1} \cdot \sigma_{(k)}= \\
& =\frac{1}{2}\left(\epsilon_{(k)}\right)^{\prime} \cdot \sigma_{(k)}=\frac{1}{2}\left(\epsilon_{(k)}\right)^{\prime} \cdot\left[\bar{Q}_{(k)}\right] \cdot \epsilon_{(k)}
\end{aligned}
$$

where $\sigma_{(k)}, \epsilon_{(k)}$ are the in-plane stress/strain components of the ply $(k)$ for the plane stress formulation:

$$
\begin{aligned}
\sigma_{(k)} & =\left(\sigma_{11}, \sigma_{22}, \sigma_{12}\right)_{(k)} \\
\epsilon_{(k)} & =\left(\epsilon_{11}, \epsilon_{22}, \epsilon_{12}\right)_{(k)}
\end{aligned}
$$

The equation in terms of strain (the last part of eq. (A.25)) will be considered in the following. The superscript \{\}$^{\prime}$ used in eq. (A.25) and throughout of this section denotes the transpose of a vector quantity, instead of the dedicated notation \{\}$^{T}$, which might bring confusion with the thermal components defined in Section A.1.

To be reminded that, in the case of considering the effect of the thermal residual stresses, there is a combination of mechanical and thermal stress/strain components acting on the laminate (see Section A.1.3), and the strain component that effectively gives strain energy of deformation in the ply $(k)$ is (see eq. (A.23))

$$
\epsilon_{(k)}=\epsilon_{(k)}^{t o t}-\alpha_{(k)} \cdot \Delta T
$$

The goal is to split the strain energy density $U_{0(k)}$ in (A.25) so that separate components for mode $I$ (crack opening mode, i.e., matrix cracking under transverse tension) and mode II (crack shear mode, i.e., matrix cracking under shear) can be identified. The separate mode components are needed in order to express the modes $I, I I \mathrm{ERR}$ in the mised modes crack growth criteria eq. (4.4). For doing this, the strain component that gives strain energy (eq. (A.27)) has to be accordingly separated in its $I, I I$ mode components.

Before proceeding to the modes separation of the ply strain, it has to be noted that eq. (A.27) represents the deformation of any ply $(k)$ of the laminate, and it is written in laminate c.s. What we are eventually looking for (See Section 4.4.1) is the ERR of the laminate due to crack multiplication in the ply $(k 0)$, which ply is denoted as the cracking ply at a certain moment during loading. The strain energy contribution from all plies $(k)$ to the crack multiplication in ply $(k 0)$ has to be taken into account. For this, the strain in ply 
$(k)$ is first rotated in the c.s. of the cracking ply $(k 0)$

$$
\epsilon_{(k, k 0)}=\epsilon_{(k, k 0)}^{t o t}-\alpha_{(k, k 0)} \cdot \Delta T=\epsilon_{(0, k 0)}^{t o t}+z \cdot \kappa_{(k 0)}^{t o t}-\alpha_{(k, k 0)} \cdot \Delta T
$$

where $\epsilon_{(0, k 0)}, \kappa_{(k 0)}$ are laminate deformation (middle plane strain $\epsilon_{(0)}$ and curvature $\kappa$ ) expressed in c.s. of $(k 0)$ ply.

Then, $I$ and $I I$ mode components of strain are separated as corresponding to the ERR for modes $I$ and $I I$ crack multiplication in ply $k 0$.

$$
\begin{aligned}
\epsilon_{(k, k 0)} & =\left(\epsilon_{11}, \epsilon_{22}, \epsilon_{12}\right)_{(k, k 0)} \\
& \Rightarrow\left\{\begin{array}{l}
\epsilon_{(k, k 0)}^{I}=\left(0, \epsilon_{22}, 0\right)_{(k, k 0)} \\
\epsilon_{(k, k 0)}^{I I}=\left(0,0, \epsilon_{12}\right)_{(k, k 0)}
\end{array}\right.
\end{aligned}
$$

Consequently to the strain damage modes separation in (A.29), the strain energy density of ply $(k)$ corresponding to modes $I$ and $I I$ crack formation in ply $(k 0)$ can be expressed as

$$
\begin{aligned}
& U_{0(k, k 0)}^{I}=\frac{1}{2}\left(\epsilon_{(k, k 0)}^{I}\right)^{\prime} \cdot\left[\bar{Q}_{(k, k 0)}\right] \cdot \epsilon_{(k, k 0)}^{I} \\
& U_{0(k, k 0)}^{I I}=\frac{1}{2}\left(\epsilon_{(k, k 0)}^{I I}\right)^{\prime} \cdot\left[\bar{Q}_{(k, k 0)}\right] \cdot \epsilon_{(k, k 0)}^{I I}
\end{aligned}
$$

The strain energy of the whole ply $(k)$ which contributes to modes $I$ and $I I$ crack formation in the $(k 0)$ ply can then be written as

$$
\begin{aligned}
& U_{(k, k 0)}^{I}=\int_{V_{k}} U_{0(k, k 0)}^{I} d V_{k}=S \int_{z_{k}} U_{0(k, k 0)}^{I} d z_{k} \\
& U_{(k, k 0)}^{I I}=\int_{V_{k}} U_{0(k, k 0)}^{I I} d V_{k}=S \int_{z_{k}} U_{0(k, k 0)}^{I I} d z_{k}
\end{aligned}
$$

where $V_{k}$ is the volume of ply $(k)$ and $S$ is the in-plane surface of the laminate.

By replacing (A.29), (A.30) into (A.31), the strain energy of the ply $(k)$ which contributes to modes $I$ and $I I$ crack formation in ply $(k 0)$ can be further written as

$$
\begin{aligned}
U_{(k, k 0)}^{I} & =\frac{S}{2} \int_{z_{k}}\left(\epsilon_{(k, k 0)}^{I}\right)^{\prime} \cdot\left[\bar{Q}_{(k, k 0)}\right] \cdot \epsilon_{(k, k 0)}^{I} d z_{k}= \\
& =\frac{S}{2} \bar{Q}_{22(k, k 0)} \underbrace{\int_{z_{k}}\left(\epsilon_{22(0, k 0)}^{t o t}+z \cdot \kappa_{22(k 0)}^{t o t}-\alpha_{22(k, k 0)} \Delta T\right)^{2} d z_{k}}_{J^{I}} \\
U_{(k, k 0)}^{I I} & =\frac{S}{2} \int_{z_{k}}\left(\epsilon_{(k, k 0)}^{I I}\right)^{\prime} \cdot\left[\bar{Q}_{(k, k 0)}\right] \cdot \epsilon_{(k, k 0)}^{I I} d z_{k}= \\
& =\frac{S}{2} \bar{Q}_{66(k, k 0)} \underbrace{\int_{z_{k}}\left(\epsilon_{12(0, k 0)}^{t o t}+z \cdot \kappa_{12(k 0)}^{t o t}-\alpha_{12(k, k 0)} \Delta T\right)^{2} d z_{k}}_{J^{I I}}
\end{aligned}
$$


The terms $J^{I}, J^{I I}$ will be calculated in the following. For this, the subscripts 22,12 in the expressions of $J^{I}$ and $J^{I I}$ in eq. (A.32) will be temporarily dropped in order to calculate the $J^{I}$ and $J^{I I}$ in only one equation:

$$
\begin{aligned}
J^{I, I I} & =\int_{z_{k}}\left(\epsilon_{(0, k 0)}^{t o t}+z \cdot \kappa_{(k 0)}^{t o t}-\alpha_{(k, k 0)} \Delta T\right)^{2} d z_{k}= \\
& =\int_{z_{k-1}}^{z^{k}}\left[\left(\epsilon_{(0, k 0)}^{t o t}\right)^{2}+z^{2}\left(\kappa_{(k 0)}^{t o t}\right)^{2}+\left(\alpha_{(k, k 0)}\right)^{2}(\Delta T)^{2}+\right. \\
& \left.+2 \epsilon_{(0, k 0)}^{t o t} \kappa_{(k 0)}^{t o t} z-2 \epsilon_{(0, k 0)}^{t o t} \alpha_{(k, k 0)} \Delta T-2 \kappa_{(k 0)}^{t o t} \alpha_{(k, k 0)} z \Delta T\right] d z= \\
& =\left[\left(\epsilon_{(0, k 0)}^{t o t}\right)^{2}+\left(\alpha_{(k, k 0)}\right)^{2}(\Delta T)^{2}-2 \epsilon_{(0, k 0)}^{t o t} \alpha_{(k, k 0)} \Delta T\right] \underbrace{\int_{z_{k-1}}^{z^{k}} d z}_{A}+ \\
& +\left[2 \epsilon_{(0, k 0)}^{t o t} \kappa_{(k 0)}^{t o t}-2 \kappa_{(k 0)}^{t o t} \alpha_{(k, k 0)} \Delta T\right] \underbrace{\int_{z_{k-1}}^{z^{k}} z d z}_{B}+ \\
& +\left[\kappa_{(k 0)}^{t o t}\right]^{2} \underbrace{\int_{z_{k-1}}^{z^{k}} z^{2} d z}_{C}
\end{aligned}
$$

After performing the calculations in the expressions of $A, B, C$ terms in eq. (A.33), these can be written as

$$
\begin{aligned}
& A=\int_{z_{k-1}}^{z^{k}} d z=t_{k} \\
& B=\int_{z_{k-1}}^{z^{k}} z d z=t_{k} \bar{z}_{k} \\
& C=\int_{z_{k-1}}^{z^{k}} z^{2} d z=t_{k}\left(\bar{z}_{k}+\frac{t_{k}^{2}}{12}\right)
\end{aligned}
$$

and the $J^{I}, J^{I I}$ terms in eq. (A.33) are determined. Then, the $J^{I}, J^{I I}$ in (A.33) are replaced in (A.32): 


$$
\begin{aligned}
U_{(k, k 0)}^{I, I I} & =\frac{S}{2} \bar{Q}_{i i(k, k 0)} \cdot J^{I, I I}= \\
& =\frac{S}{2} \bar{Q}_{i i(k, k 0)}\left[\left(\left(\epsilon_{(0, k 0)}^{t o t}\right)^{2}+\left(\alpha_{(k, k 0)}\right)^{2}(\Delta T)^{2}-2 \epsilon_{(0, k 0)}^{t o t} \alpha_{(k, k 0)} \Delta T\right) t_{k}+\right. \\
& +\left(2 \epsilon_{(0, k 0)}^{t o t} \kappa_{(k 0)}^{t o t}-2 \kappa_{(k 0)}^{t o t} \alpha_{(k, k 0)} \Delta T\right) t_{k} \bar{z}_{k}+ \\
& \left.+\left(\kappa_{(k 0)}^{t o t}\right)^{2} t_{k}\left(\bar{z}_{k}+\frac{t_{k}^{2}}{12}\right)\right]= \\
& =\frac{S}{2} \bar{Q}_{i i(k, k 0)}[\left(\epsilon_{(0, k 0)}^{t o t}\right)^{2} \underbrace{t_{k}}_{S 1}+(\Delta T)^{2} \underbrace{\left(\alpha_{(k, k 0)}\right)^{2} t_{k}}_{S 2}-2 \epsilon_{(0, k 0)}^{t o t} \Delta T \underbrace{\alpha_{(k, k 0)} t_{k}}_{S 3}+ \\
& +\left(\left(\kappa_{(k 0)}^{t o t}\right)^{2}+2 \epsilon_{(0, k 0)}^{t o t} \kappa_{(k 0)}^{t o t}\right) \underbrace{t_{k} \bar{z}_{k}}_{S 4}+\frac{1}{12} \kappa_{(k 0)}^{\left(\kappa^{t o t}\right)} \underbrace{t_{k}^{3}}_{S 5}-2 \kappa_{(k 0)}^{t o t} \Delta T \underbrace{\alpha_{(k, k 0)} t_{k} \bar{z}_{k}}_{S 6}]
\end{aligned}
$$

where the ply stiffness component $\bar{Q}_{i i(k, k 0)}$ represents the $\bar{Q}_{22(k, k 0)}$ term for mode $I$, and the $\bar{Q}_{66(k, k 0)}$ term for mode $I I$, according to eq. (A.32).

Finally, strain energy of the laminate for modes $I$ and $I I$ crack formation in the cracking ply $(k 0)$ is expressed by the summation of the available strain energy in each ply $(k)$ :

$$
U_{(k 0)}^{I, I I}=\sum_{k=1}^{N L} U_{(k, k 0)}^{I, I I}
$$

where $N L$ is the total number of plies in the laminate.

The terms labeled as $S 1 \ldots S 6$ in eq. (A.35) have been separately grouped because these are the terms that have to be summed over the total number of plies in the laminate in order to calculate the summation in eq. (A.36): $\sum_{k=1}^{N L}\left(\bar{Q}_{i i(k, k 0)} \cdot S j\right), \quad j=1 \ldots 6$. It can be observed that the other terms besides $S j$ in eq. (A.35) are not ply $(k)$ quantities, but they are laminate quantities, and consequently they are constant terms for the summation in eq. (A.36).

The modes separation of ply strain in eq. (A.29) might require some discussion. The general expression of ply strain energy in (A.25) supposes a quadratic combination of all $\epsilon_{i j(k)}$ terms, with $i, j=1,2,3$. Using only the $\epsilon_{22(k, k 0)}, \epsilon_{12(k, k 0)}$ ply strain components in eq. (A.29), (A.30) implies that some terms from eq. (A.25) are not recovered in eq. (A.30), namely all the terms involving $\epsilon_{11(k)}$ and the term involving the cross-combination $\epsilon_{22(k)}$. $\epsilon_{12(k)}$. The separation of ply strain in eq. (A.29) is considered by the author of the present manuscript as the right way to separate components corresponding to the strain energies of 
mode $I$ and $I I$ crack formation. This way of strain components separation for calculating strain energies corresponding to mode $I$ and $I I$ crack formation can also be found in [24]. Moreover, the damage modes strain separation in (A.29) is consistent with the equation of the work done for the elastic closure of the crack in classical fracture mechanics ([90, eq.(16)]), which equation is the base of the Virtual Crack Closure Technique in FEA crack propagation problems. Neither in [90, eq.(16)] the terms corresponding to $\epsilon_{11(k)}$ and $\epsilon_{22(k)} \cdot \epsilon_{12(k)}$ are used for calculating the elastic work required for crack closure, which is equal to the strain energy released during crack formation. The elastic work for crack closure in [90, eq.(16)] is calculated as the product between modes $I$ and $I I$ stress components (transverse to the crack for mode $I$ and shearing the crack for mode $I I$ ) and their corresponding displacements, fact that makes the mode separation in (A.29) consistent with the equation in [90, eq.(16)].

It can be noticed that the terms $\epsilon_{(0, k 0)}^{t o t}, \kappa_{(k 0)}^{t o t}, \alpha_{(k, k 0)}$ in eq. (A.32) require a rotation from the laminate c.s. to ply $(k 0)$ c.s. The strain rotation in order to obtain $\epsilon_{(0, k 0)}^{t o t}$ is based on the regular strain rotation relation of the stress/strain analysis. The rotation of the CTE in order to obtain $\alpha_{(k, k 0)}$ is also based on the strain rotation formula, based on $\{\epsilon\}=\{\alpha\} \cdot \Delta T$ relationship. Intuitively, the curvature $\kappa_{(k 0)}^{t o t}$ should be obtained based on a strain rotation, as well, even if a relationship for rotating curvatures is not offered by the stress analysis textbooks. This relationship for rotating curvatures will be derived in the following:

The laminate deformation (i.e., the laminate mid-plane strain and curvature), in laminate c.s., is $\epsilon_{(0)}^{t o t}=\left(\epsilon_{11(0)}, \epsilon_{22(0)}, \epsilon_{12(0)}\right)^{t o t}$ and $\kappa^{t o t}=\left(\kappa_{11}, \kappa_{22}, \kappa_{12}\right)^{t o t}$

The ply $(k)$ strain, in laminate c.s. is

$$
\epsilon_{(k)}^{t o t}=\epsilon_{(0)}^{t o t}+z \kappa^{t o t}
$$

which allow to calculate the laminate curvature, in laminate c.s., as

$$
\kappa^{t o t}=\frac{1}{z}\left(\epsilon_{(k)}^{t o t}-\epsilon_{(0)}^{t o t}\right)
$$

The classical relationship for rotating strain between the laminate c.s. and the ply $(k 0)$ c.s. can be written for the ply $(k)$ as

$$
\epsilon_{(k, k 0)}^{t o t}=R \cdot T(\theta) \cdot R^{-1} \cdot \epsilon_{(k)}^{t o t} \quad \Rightarrow \quad \epsilon_{(k)}^{t o t}=R \cdot \underbrace{[T(\theta)]^{-1}}_{T(-\theta)} \cdot R^{-1} \cdot \epsilon_{(k, k 0)}^{t o t}
$$

where $T(\theta)$ is the stress rotation matrix, and $R$ is the Reuter matrix. By replacing (A.39) 
in (A.38), the laminate curvature, in laminate c.s. can be expressed as

$$
\kappa^{t o t}=R \cdot T(-\theta) \cdot R^{-1} \cdot \underbrace{\frac{1}{z}\left(\epsilon_{(k, k 0)}^{t o t}-\epsilon_{(0, k 0)}^{t o t}\right)}_{\kappa_{(k 0)}^{t o t}}
$$

where the term $\kappa_{(k 0)}^{\text {tot }}$ can be identified as the laminate curvature, in $(k 0)$ ply c.s.

This allows writing the relationship for rotating the laminate curvature between the laminate c.s. and the $(k 0)$ ply c.s. as

$$
\begin{aligned}
\kappa^{t o t} & =R \cdot T(-\theta) \cdot R^{-1} \cdot \kappa_{(k 0)}^{t o t} \\
\kappa_{(k 0)}^{t o t} & =R \cdot T(\theta) \cdot R^{-1} \cdot \kappa^{t o t}
\end{aligned}
$$




\section{Appendix B}

\section{Analytical model for the reduced}

thermo-elastic properties of composite laminates with matrix cracks

\section{(The Gudmundson-Adolfsson}

\section{approach $[32,33,34,35,36,37,38])$}

A first brief conceptual presentations of the background elements used in Gudmundson and Adolfsson $[32,33,34,35,36]$ to derived the reduced material properties of the composite laminate featuring matrix cracks (eq. (2.14)) was done in Section 2.1.2. Further details are offered in the present section in order to understand and clarify the components and concepts involved in the expression of eq. (2.14). The physical meaning of the parameters involved in the aforementioned equations, and the principles leading to the derivation of these equations are explained during the present chapter.

A detailed presentation of the modeling approach based on COD and the energy balance expressed in eq. (2.12) will be presented in the following. This is because the model based on eq. (2.12) provides the complete set of reduced membrane and flexural thermo-elastic properties of the laminated composite, as it is implemented in the present manuscript.

A short presentation of the modeling approach based on COD and the theory of elastic body with voids, as expressed in eq. (2.10), will be presented in Appendix B.5 for the sake of completeness. The model based on eq. (2.10) provides reduced material properties without 
considering the flexural properties.

The Gudmundson-Adolfsson (GA) modeling approach is based on the concept of COD combined with fracture mechanics principles (e.g., crack propagation under the action of surface tractions on the prospective crack surface, stress intensity factors). The detailes of this modeling approach are presented during the present section.

The main notations used throughout this section are:

- $k$ superscript denotes quantities at ply level.

- (c) subscript denotes quantities corresponding to the cracked (damaged) material.

- (a) superscript denotes average quantities.

- overbar $(\bar{\alpha})$ denotes effective quantities, i.e., quantities at macro (overall, global scale) level. The effective quantities can be defined at both ply $\left(\bar{\alpha}^{k}\right.$, superscript $k$ used) and laminate $(\bar{\alpha}$, no superscript used) level.

The main unknown of the model is the average crack opening displacement (COD) $\Delta u$ (see eq. (2.8)) which appears at the prospective crack location under the action of surface tractions in the uncracked material at that location (see eq. (2.9)). It has to be noted that the term average is applied in two different ways in the present model: i) when superscript $(a)$ is used, the average is considered over the material volume, as it is given by eq. (2.2), (2.11) for the case of average strain in the cracked material (similar average stress can be defined); ii) when it refers to the average COD, the average is defined over the ply thickness according to eq. (2.8).

\section{B.1 Details of the modeling approach based on COD and energy balance $[35,36]$ : in-plane and bending deformation}

The modeling approach based on energy balance in eq. (2.12) in used in [35] in order to calculate the the whole set (membrane and flexural) of the reduced material properties of the cracked laminated. More details are presented in the following regarding the calculation of the released energy due to matrix cracking, $\Delta W$ in (2.12). 
For the uncracked material, a stress state $\sigma_{i j}^{k}, i, j=1 \cdots 3$ exists in each ply $(k)$ of the laminate, for a given deformation state of the laminate. The stress state $\sigma_{i j}^{k}$ results in a corresponds vector $\tau_{i}^{k}, i=1 \cdots 3$ of surface tractions at the prospective location of the crack in each ply of the laminate, according to the Couchy stress resultant formula of the classical elasticity:

$$
\tau_{i}=\sigma_{i j} \cdot n_{j}
$$

where $n_{j}$ is the vector of the direction cosines of the normal to the crack surface (see Fig. B.2). Due to the fact that cracks always appear along the fiber direction (i.e., the 1 direction in the c.s. of the ply), the normal direction $n$ is always along the 2 transverse direction of the cracking ply.

The resulting surface traction $\tau_{i}$ is responsible for the corresponding COD $\Delta u_{i}$. According to eq. (2.9), the COD in one ply $(k)$ is considered as a linear effect of the surface tractions in all $(i)$ plies of the laminate $(i=1 \cdots N)$ :

$$
\Delta u_{i}^{k}=t^{k} \sum_{l=1}^{N} \beta_{i j}^{k l} \tau_{j}^{l}
$$

The $\beta_{i j}^{k l}$ coefficients are regarded as COD coefficients and they become the new unknowns of the problem; if the $\beta_{i j}^{k l}$ are known, the COD $\Delta u_{i}^{k}$ are also known, according to eq. (B.2). The $\beta_{i j}^{k l}$ coefficients act as a compliance between the surface traction $\tau_{j}^{l}$ and the resulting COD $\Delta u_{i}^{k}$. The subscripts $i, j$ in eq. (B.2) regard components of the tensor/vector quantities $(i, j=1 \cdots 3)$, and the superscripts $k, l$ regard plies numbering $(k, l=1 \cdots N$, where $N$ is the number of plies in the laminate).

A first assumption is that the surface tractions in plies $(l)$ do not influence COD in ply $(k)$, there is no coupling between COD in different plies, and eq. (B.2) of the COD in ply $(k)$ is simplified written as

$$
\Delta u_{i}^{k}=t^{k} \cdot \beta_{i j}^{k} \cdot \tau_{j}^{k}
$$

A second assumption is that there is no coupling between $I, I I, I I I$ modes of damage, and eq. (B.3) can be further written as

$$
\Delta u_{i}^{k}=t^{k} \cdot \beta_{i i}^{k} \cdot \tau_{i}^{k}
$$

The surface tractions for a ply inside of a composite laminate are depicted in Fig. 2.3 (a). The surface traction $\tau_{1}$ induces mode $I I$ in-plane shearing matrix cracking, $\tau_{2}$ induces mode $I$ opening matrix cracking, and $\tau_{3}$ induces mode III out-plane shearing matrix cracking, in 
the conventional c.s. of the laminated composite. The $\tau_{3}$ out-plane component of surface traction is not regarded in the conventional plate theory. Eq.(B.4) can be further expressed in matrix form as

$$
\Delta u^{k}=t^{k} \cdot \beta^{k} \cdot \tau^{k}
$$

where, after dropping the out-plane shear component, $\tau^{k}$ and $\Delta u^{k}$ become $2 \times 1$ vectors, and $\beta^{k}$ is a $2 \times 2$ matrix expressed as

$$
\beta=\left[\begin{array}{cc}
\beta_{11} & 0 \\
0 & \beta_{22}
\end{array}\right]
$$

It can be noticed up to this moment that the COD in one ply $(k)$ of the laminate (eq. (B.4)) is a function of:

- ply thickness $t^{k}$

- laminate configuration (i.e., orientation, thickness, and material properties of each ply of the laminate) through the stress resultant $\tau^{k}$;

- crack density in ply $(k)$ through $\beta^{k}$.

The required energy released for the crack formation $(\Delta W$ in eq. (2.12)) is calculated based on the Irwin's principle which states that the energy released equals the mechanical work for the crack closure. Considering one thickness cross-section of the crack (Fig. 2.3 (b)), the mechanical work for the crack closure over the crack length can be expressed as

$$
\Delta w^{k}=\frac{1}{2} \cdot t^{k} \cdot \tau_{i}^{k} \cdot \Delta u_{i}^{k}=\frac{1}{2} \cdot\left(t^{k}\right)^{2} \cdot \tau_{i}^{k} \cdot \beta_{i i}^{k} \cdot \tau_{i}^{k}
$$

If the total length of cracks in the ply $(k)$ of the laminate is $l^{k}$, the corresponding energy released for the formation of that cracks system is

$$
\Delta W^{k}=l^{k} \cdot \Delta w^{k}=\frac{1}{2} \cdot l^{k} \cdot\left(t^{k}\right)^{2} \cdot \tau_{i}^{k} \cdot \beta_{i i}^{k} \cdot \tau_{i}^{k}
$$

The material model assumes the equally spaced cracks in each ply $(k)$ of the laminate, and the normalized crack density of the ply is denoted as $\rho^{k}=\frac{t^{k}}{d^{k}}$, where $d^{k}$ is the crack spacing. The total length of the cracks in ply $(k)$ can then be expressed as

$$
l^{k}=A \cdot \frac{\rho^{k}}{t^{k}}
$$


where $A$ is the in-plane surface of the laminate. Based on this, the energy released for the formation of the cracks system in ply $(k)$ is

$$
\Delta W^{k}=\frac{1}{2} \cdot A \cdot \rho^{k} \cdot t^{k} \cdot \tau_{i}^{k} \cdot \beta_{i i}^{k} \cdot \tau_{i}^{k}
$$

The total energy released by the laminate for the formation of the particular crack system in each ply of the laminate is calculated as

$$
\Delta W=\sum_{k=1}^{N} \Delta W^{k}
$$

All the above reasoning is based on knowing the COD $\Delta u_{i}$, or knowing the equivalent COD coefficients $\beta_{i i}$, for each damage mode, in each ply of the laminate. At this point, an approximation is involved in the analytical model of the reduced material properties, by considering the fracture mechanics solution of similar problems (Fig. 2.3 (b), (c)) for the problem of matrix cracking in laminated composites (Fig. 2.3 (a)). The similar problems for which a fracture mechanics solution exists are:

- the problem of a row of cracks in an infinite homogeneous transversely isotropic body (Fig. $2.3(\mathrm{~b})$ ), which translates in matrix cracks in interior plies of the laminate (denoted with superscript $(i))$;

- the problem of a row of cracks in a semi-infinite homogeneous transversely isotropic body (Fig. 2.3 (c)), which translates in matrix cracks in surface plies of the laminate (denoted with superscript $(s))$;

In this way, there will be specific COD solutions for interior plies and for surface plies: $\beta^{(i)}$ and $\beta^{(s)}$, respectively.

Moreover, if the surface traction $\tau$ in Fig. 2.3 (a), (b) features an uniform (constant) distribution over the ply thickness, then it corresponds to membrane loading of the laminate. In this case, a fracture mechanics solution for the specific case of the uniform surface traction has to be used, and the resulting COD will be denoted as $\beta_{(E E)}$ for the membrane case. If the surface traction $\tau$ features a linear distribution over the ply thickness, then it corresponds to flexural loading of the laminate. In this case, a fracture mechanics solution for the specific case of the linear surface traction has to be used, and the resulting COD will be denoted as $\beta_{(B B)}$ for the flexural case.

For the case of combined membrane-flexural deformation of the laminate, the stresses in each ply of the laminate, and consequently corresponding surface tractions, feature the 
linear distribution in Fig. B.1. In this case, the ply stresses and the corresponding surface traction are decomposed in the uniform $\tau_{(E)}$ and linear $\tau_{(B)}$ components, as in Fig. B.1. The reason for this specific decomposition of the surface traction is to apply the available fracture mechanics solutions. While there could be many ways to decompose the surface traction $\tau$ into uniform and linearly varying components, the fracture mechanics solution for the linear distribution of the surface traction is offered for the linear profile $\tau_{(B)}$ as it is presented in Fig. B.1.

One aspect to be noted is that the surface traction $\tau_{1}$ in Fig. $2.3(\mathrm{~b})$ is responsible for mode III of damage, as opposed to the previous statement corresponding to Fig. 2.3 (a), where it was said that $\tau_{1}$ corresponds to mode $I I$. The mode $I I$ of Fig. 2.3 (a) is when the conventional c.s. of the laminated composite is regarded, but the COD derivation corresponding to $\tau_{1}$ in Fig. 2.3 (b) is made considering the mode $I I I$ of damage. In this way, the COD model gets a through the thickness crack propagation feature.

Summarizing, the whole set of the needed COD solutions in each ply of the laminate, for the case of combined membrane-flexural deformation ca be expressed as $\beta_{m(l)}^{(p)}$, where the superscript $(p)$ denotes the position of the ply (either interior $(i)$ or surface $(s)$ ), the subscript $m$ denotes the damage mode (22 for mode $I$ and 11 for mode $I I I$ ), and the the subscript $(l)$ denotes the deformation case $((E E)$ for membrane and $(B B)$ for flexural).

The analytical fracture mechanics solutions of the problems in Fig. 2.3 (b), (c) (row of cracks) implies the fact that the neighboring cracks interact with each other, affecting each other's COD. An earlier solution of only one crack in an infinite/semi-infinite body was used in $[32,33]$, and the resulting model of the reduced material properties was confined to the "dilute crack density" case, where the cracks are far spaced from each other. It was shown in $[32,33]$, by comparison against experimental data, that the dilute crack density solution provides good results for the reduced material properties up to a crack density $\rho^{k} \approx 0.5$. However, by using the fracture mechanics solution of rows of cracks (Fig. 2.3 (b), (c)), the dilute crack density limitation of the model is lifted in [34, 35, 36, 37].

The approximate solution is validated through extensive comparison against FEA and experimental results of reduced material properties in $[32,34,35,36,37,38]$. Moreover, it is shown in [32] by FEA parametric studies that the COD parameter $\beta$ is a robust one, not being influenced too much by the laminate configuration, and it is concluded this way that the approximate solution (i.e., using the analytical fracture mechanics solution of the problem in Fig. 2.3 (b), (c) to the problem in Fig. 2.3 (a)) is acceptable. 
Adi Adumitroaie Appendix B. Analytical model for reduced material properties

\section{B.2 Calculation of COD $\beta$ coefficients from analytical fracture mechanics and numerical methods}

The fracture mechanics solutions $[90,91]$ to the problems depicted in Fig. 2.3 (b), (c) offer the stress intensity factors for the modes $I$ and $I I I$, corresponding to $\tau_{2}$ and $\tau_{1}$ surface traction loading conditions at the prospective crack location:

$$
\begin{aligned}
K_{I} & =f_{2} \cdot \tau_{2} \\
K_{I I I} & =f_{1} \cdot \tau_{1}
\end{aligned}
$$

where the functions $f_{1}, f_{2}$ include the effect of the crack length ( $a$ in Fig. 2.3 (b)) and crack spacing (i.e., the reciprocal influence of the cracks on their own COD).

In order to calculate COD based on stress intensity factors, the fracture mechanics relation between energy released and stress intensity factors, for each damage mode, is called upon:

$$
\begin{gathered}
\Delta W_{I}=\int_{0}^{t} \gamma_{2} \cdot K_{I}^{2} d a=\gamma_{2} \cdot \tau_{2}^{2} \int_{0}^{t} f_{2}^{2} d a \\
\Delta W_{I I I}=\int_{0}^{t} \gamma_{1} \cdot K_{I I I}^{2} d a=\gamma_{1} \cdot \tau_{1}^{2} \int_{0}^{t} f_{1}^{2} d a
\end{gathered}
$$

where $\gamma_{1}, \gamma_{2}$ are function of transversely isotropic material properties

$$
\begin{aligned}
\gamma_{1} & =\frac{1}{2 G_{T L}} \\
\gamma_{2} & =\frac{1-\nu_{L T} \nu_{T L}}{E_{T}}
\end{aligned}
$$

and $a$ is the crack length, which evolves from zero to ply thickness $t$.

By the use of eq. (B.7), the energy released is expressed as

$$
\begin{aligned}
\Delta W_{I} & =\frac{1}{2} \cdot t^{2} \cdot \tau_{2} \cdot \beta_{22} \cdot \tau_{2} \\
\Delta W_{I I I} & =\frac{1}{2} \cdot t^{2} \cdot \tau_{1} \cdot \beta_{11} \cdot \tau_{1}
\end{aligned}
$$

and the COD coefficients can be further calculated from(B.13), (B.15) as

$$
\begin{aligned}
& \beta_{22}=\frac{2 \gamma_{2}}{t^{2}} \int_{0}^{t} f_{2}^{2} d a \\
& \beta_{11}=\frac{2 \gamma_{1}}{t^{2}} \int_{0}^{t} f_{1}^{2} d a
\end{aligned}
$$


The expression of the $f_{1}, f_{2}$ functions are offered in $[91,90]$ for some geometry cases (i.e., interior $(i)$ and surface $(s)$ row of cracks, see Fig. 2.3 (b), (c)) and loading cases (i.e., uniform $(E)$ and linear distribution $(B)$ of the surface tractions, see Fig. B.1). Thus, the following COD coefficients can be calculated based on the analytical fracture mechanics solution of stress intensity factors in given in $[90,91]: \beta_{11(E E)}^{(i)}, \beta_{22(E E)}^{(i)}, \beta_{11(E E)}^{(s)}, \beta_{22(E E)}^{(s)}, \beta_{22(B B)}^{(i)}$

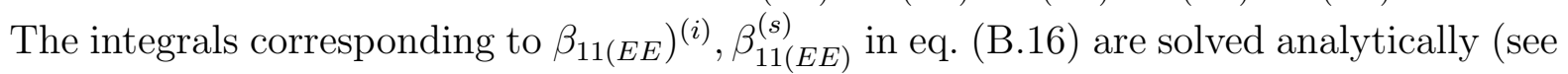
their final expression in eq. (B.17), (B.19)), while the integrals corresponding to $\beta_{22(E E)}^{(i)}, \beta_{22(E E)}^{(s)}, \beta$ are solved numerically (see their final expression in eq. (B.17), (B.18), (B.19)), this bringing the coefficients $a_{i}, c_{i}, d_{i}$ in their expressions (see Table B.1), due to the numerical integration scheme involved.

However, analytical solution for the remaining $\beta_{11(B B)}^{(i)}, \beta_{11(B B)}^{(s)}, \beta_{22(B B)}^{(s)}$ is not found in fracture mechanics papers. Because of this fact, the remaining COD coefficients are evaluated by Gudmundson and Adolfsson $[35,36]$ by numerically (FEA) evaluating the energies released for the required geometry cases and loading conditions. The FEA calculation is performed for the geometry cases like presented in Fig. 2.3 (b), (c) and for various crack spacings. The resulting analytical expressions of the stress intensity factors as function of crack density is then the least squares curve fit of the point results for various crack densities. The integrals corresponding to the resulting least squares curve fit analytical expressions of the stress intensity factors are then numerically evaluated (see their final expression in eq. (B.18), (B.21)), this bringing the coefficients $b_{i}, g_{i}, h_{i}$ in their expressions (see Table B.1), due to the numerical integration scheme involved.

A coupling exists for surface $(s)$ cracks between the extension/bending tractions and COD (for example, a $\tau_{1(E)}$ surface traction induces a change in the $\Delta u_{2(B)}$ COD). This coupling results in nonzero coupling terms $\beta_{11(E B)}^{(s)}$ and $\beta_{22(E B)}^{(s)}$. The corresponding extension/bending coupling terms for the case of the interior $(i)$ cracks are zero. These coupling COD coefficients are also evaluated by the FEA method as previously described, and they are presented in eq. (B.20), with the corresponding numerical integration coefficients $e_{i}, f_{i}$ given in Table B.1.

Interior cracks (superscript $(i))$ : 
- components associated with extension:

$$
\begin{aligned}
& \beta_{11(E E)}^{k(i)}=\frac{\pi}{2} \gamma_{1}^{k} \frac{8}{\left(\pi \rho^{k}\right)^{2}} \ln \left[\cosh \left(\frac{\pi \rho^{k}}{2}\right)\right] \\
& \beta_{22(E E)}^{k(i)}=\frac{\pi}{2} \gamma_{2}^{k} \sum_{j=1}^{10} \frac{a_{j}}{\left(1+\rho^{k}\right)^{j}}
\end{aligned}
$$

- components associated with bending:

$$
\begin{aligned}
& \beta_{11(B B)}^{k(i)}=\frac{\pi}{16} \gamma_{1}^{k} \sum_{j=1}^{10} \frac{b_{j}}{\left(1+\rho^{k}\right)^{j}} \\
& \beta_{22(B B)}^{k(i)}=\frac{\pi}{16} \gamma_{2}^{k} \sum_{j=1}^{10} \frac{c_{j}}{\left(1+\rho^{k}\right)^{j}}
\end{aligned}
$$

Surface cracks (superscript $(s)$ ):

- components associated with extension:

$$
\begin{aligned}
& \beta_{11(E E)}^{k(s)}=\pi \gamma_{1}^{k} \frac{8}{\left(2 \pi \rho^{k}\right)^{2}} \ln \left[\cosh \left(\pi \rho^{k}\right)\right] \\
& \beta_{22(E E)}^{k(s)}=1.1215^{2} \pi \gamma_{2}^{k} \sum_{j=1}^{10} \frac{d_{j}}{\left(1+\rho^{k}\right)^{j}}
\end{aligned}
$$

- components associated with extension-bending coupling:

$$
\begin{aligned}
& \beta_{11(E B)}^{k(s)}=-\frac{3 \pi-8}{3} \gamma_{1}^{k} \sum_{j=1}^{10} \frac{e_{j}}{\left(1+\rho^{k}\right)^{j}} \\
& \beta_{22(E B)}^{k(s)}=-0.2364 \pi \gamma_{2}^{k} \sum_{j=1}^{10} \frac{f_{j}}{\left(1+\rho^{k}\right)^{j}}
\end{aligned}
$$

- components associated with bending:

$$
\begin{aligned}
& \beta_{11(B B)}^{k(s)}=\frac{3 \pi^{2}-16 \pi+24}{3 \pi} \gamma_{1}^{k} \sum_{j=1}^{10} \frac{g_{j}}{\left(1+\rho^{k}\right)^{j}} \\
& \beta_{22(B B)}^{k(s)}=0.1481 \pi \gamma_{2}^{k} \sum_{j=1}^{10} \frac{h_{j}}{\left(1+\rho^{k}\right)^{j}}
\end{aligned}
$$


Table B.1: The values of $a_{j}, b_{j}, c_{j}, d_{j}, e_{j}, f_{j}, g_{j}, h_{j}$ coefficients in eq. (B.17) - (B.21) (from Gudmundson and Adolfsson [35, 36]).

\begin{tabular}{rrrrrrrrr}
\hline $\mathrm{j}$ & $a_{j}$ & $b_{j}$ & $c_{j}$ & $d_{j}$ & $e_{j}$ & $f_{j}$ & $g_{j}$ & $h_{j}$ \\
\hline 1 & 0.63666 & 3.40409 & 1.65364 & 0.25256 & -0.29208 & -0.035206 & 2.00624 & 0.86388 \\
2 & 0.51806 & -1.50821 & 0.87842 & 0.27079 & 8.70026 & 0.97809 & -2.57041 & -2.87984 \\
3 & 0.51695 & -0.37842 & 10.61342 & -0.49814 & -82.11858 & -7.58709 & 31.41892 & 34.19264 \\
4 & -1.04897 & -3.62256 & -135.67488 & 8.62962 & 479.23609 & 44.52217 & -175.60882 & -167.57885 \\
5 & 8.95572 & -101.24283 & 747.53392 & -51.24655 & -1633.39371 & -150.85828 & 547.80573 & 458.21598 \\
6 & -33.09444 & 481.87306 & -2236.32476 & 180.96305 & 3497.22398 & 333.94609 & -1114.56953 & -667.14207 \\
7 & 74.32002 & -916.59087 & 3772.21227 & -374.29813 & -4751.26789 & -487.71444 & 1494.40862 & 400.51994 \\
8 & -103.06411 & 898.56902 & -3604.17159 & 449.59474 & 3954.62876 & 471.87242 & -1256.18363 & 109.75154 \\
9 & 73.60337 & -452.85541 & 1827.29629 & -286.51016 & -1835.18896 & -273.59771 & 594.64188 & -252.27544 \\
10 & -20.34326 & 93.35216 & -383.01680 & 73.84223 & 363.47237 & 69.47435 & -120.34912 & 87.33293 \\
\hline
\end{tabular}




\section{B.3 Calculation of membrane and flexural reduced ma- terial properties}

The reduced thermo-elastic properties of the laminate containing cracked plies is performed based on the COD solution described in Section B.2.

The elastic stored energy in the undamaged laminate is calculated based on the deformation state and the material properties at the laminate level:

$$
W_{0}=\frac{A}{2}(\bar{\epsilon}-\alpha \Delta T)^{`} \cdot C \cdot(\bar{\epsilon}-\alpha \Delta T)
$$

where $\bar{\epsilon}=\left\{\epsilon_{0}, \kappa\right\}$ is the laminate deformation, including the membrane and flexural deformation, and $C, \alpha$ are the thermoelastic properties of the undamaged laminate (here the superscript "denotes the transpose of an array).

Based on eq. (2.12), (B.10), and (B.22) the elastic stored energy after the energy released due to matrix cracking can be calculated as

$$
W_{(c)}=W_{0}-\Delta W=\frac{A}{2}\left[(\bar{\epsilon}-\alpha \Delta T)^{c} \cdot C \cdot(\bar{\epsilon}-\alpha \Delta T)-\sum_{k} \frac{1}{2} \cdot A \cdot \rho^{k} \cdot t^{k} \cdot \tau^{k} \cdot \beta^{k} \cdot \tau^{k}\right]
$$

On the other hand, the elastic stored energy in the laminate containing cracked plies can also be calculated similarly to eq. (B.22), based on the imposed deformation state $\bar{\epsilon}$ and reduced thermoelastic properties $C_{(c)}, \alpha_{(c)}$ of the cracked material:

$$
W_{(c)}=\frac{A}{2}\left(\bar{\epsilon}-\alpha_{(c)} \Delta T\right)^{\natural} \cdot C_{(c)} \cdot\left(\bar{\epsilon}-\alpha_{(c)} \Delta T\right)
$$

The reduced thermoelastic properties $C_{(c)}, \alpha_{(c)}$ of the cracked material are expressed as the reduction effect of the matrix cracking $\Delta C, \Delta \alpha$ to the virgin material properties $C, \alpha$

$$
\begin{aligned}
& C_{(c)}=\left[\begin{array}{ll}
C_{(c)(E E)} & C_{(c)(E B)} \\
C_{(c)(B E)} & C_{(c)(B B)}
\end{array}\right]=C+\Delta C \\
& \alpha_{(c)}=\left\{\begin{array}{l}
\alpha_{(c)(E)} \\
\alpha_{(c)(B)}
\end{array}\right\}=\alpha+\Delta \alpha
\end{aligned}
$$

and eq. (B.24) can be further written as

$$
W_{(c)}=\frac{A}{2}[\bar{\epsilon}-(\alpha+\Delta \alpha) \Delta T]^{\cdot} \cdot(C+\Delta C) \cdot[\bar{\epsilon}-(\alpha+\Delta \alpha) \Delta T]
$$


By identification of elastic energy in the cracked material given by equations (B.23) and (B.26), the reduction in thermoelastic properties of the cracked laminate can be calculated as function of the crack density in each ply of the laminate

$$
\begin{aligned}
\Delta C & =f\left(\rho^{k}\right) \\
\Delta \alpha & =f\left(\rho^{k}\right)
\end{aligned}
$$

and the reduced thermoelastic properties of the laminate are calculated based on eq. (B.25).

The final expressions of the laminate reduced stiffness $C_{(c)}$ are presented in eq. (B.28), (B.29), and the final expression of the laminate reduced thermal coefficients are presented in eq. (B.32), (E

The reduced material properties of the laminate are listed in the following. In order to simplify the notations, the middle coordinate of ply $(k)$ which is usually noted as $\bar{z}^{k}$, will be regarded simply as $z^{k}$ in the next equations.

- The reduced in-plane and flexural stiffness coefficients of the laminate:

$$
\begin{aligned}
& C_{(c) E E}=C_{E E}+\Delta C_{E E} \\
& C_{(c) E B}=C_{E B}+\Delta C_{E B} \\
& C_{(c) B B}=C_{B B}+\Delta C_{B B}
\end{aligned}
$$

where the stiffness reduction due to a certain crack density $\rho^{k}=t^{k} / d^{k}$ in each ply $(k)$ of the laminate is given by

$$
\begin{aligned}
& \Delta C_{E E}=-\sum_{k=1}^{N} \sum_{l=1}^{N} \sqrt{t^{k} \rho^{k} t^{l} \rho^{l}} A_{E E}^{k l} \\
& \Delta C_{E B}=-\sum_{k=1}^{N} \sum_{l=1}^{N} \sqrt{t^{k} \rho^{k} t^{l} \rho^{l}}\left[z^{l} A_{E E}^{k l}+\frac{t^{l}}{2} A_{E B}^{k l}\right] \\
& \Delta C_{B B}=-\sum_{k=1}^{N} \sum_{l=1}^{N} \sqrt{t^{k} \rho^{k} t^{l} \rho^{l}}\left[z^{k} z^{l} A_{E E}^{k l}+\frac{z^{k} t^{k}}{2} A_{E B}^{k l}+\frac{z^{l} t^{k}}{2} A_{B E}^{k l}+\frac{t^{k} t^{l}}{4} A_{B B}^{k l}\right]
\end{aligned}
$$

The $3 \times 3$ matrices $A_{m}^{k l}$ (with $k, l$ being ply indexes, and $m$ being either $E E, E B, B E, B B$ ) are

$$
A_{m}^{k l}=\bar{Q}^{k}\left(N^{k}\right)^{T} \beta_{m}^{k l} N^{l} \bar{Q}^{l}
$$


where the $N$ matrices give the orientation of the crack surface (which is the same as fiber orientation) of the ply $(k)$, in laminate coordinate system

$$
N^{k}=\left(\begin{array}{ccc}
n_{1}^{k} & 0 & n_{2}^{k} \\
0 & n_{2}^{k} & n_{1}^{k}
\end{array}\right)
$$

with $n_{1}^{k}, n_{2}^{k}$ being the direction cosines between the crack c.s. (the same with fiber orientation c.s. of ply $k$ ) and laminate c.s.

- The reduced in-plane and flexural thermal load coefficients of the laminate:

$$
\begin{aligned}
& \delta_{(c) E}=\delta_{E}+\Delta \delta_{E} \\
& \delta_{(c) B}=\delta_{B}+\Delta \delta_{B}
\end{aligned}
$$

where the reduction $\Delta \delta_{E}, \Delta \delta_{B}$ of thermal loads coefficients due to a certain crack density $\rho^{k}$ in each ply $(k)$ of the laminate is given by

$$
\begin{aligned}
& \Delta \delta_{E}=\Delta C_{E E} \cdot \alpha_{E}+\Delta C_{E B} \cdot \alpha_{B}+\sum_{k=1}^{N} \sum_{l=1}^{N} \sqrt{t^{k} \rho^{k} t^{l} \rho^{l}} A_{E E}^{k l} \alpha^{l} \\
& \Delta \delta_{B}=\Delta C_{B E} \cdot \alpha_{E}+\Delta C_{B B} \cdot \alpha_{B}+\sum_{k=1}^{N} \sum_{l=1}^{N} \sqrt{t^{k} \rho^{k} t^{l} \rho^{l}}\left[z^{k} A_{E E}^{k l}+\frac{t^{k}}{2} A_{B E}^{k l}\right] \alpha^{l}
\end{aligned}
$$

The meaning of $2 \times 2$ matrices $\beta_{m}^{k l}$ in (B.30) is briefly described in Section 2.1.2, then more detailed explained in the first part of the current Appendix B. They provide the relationship between the surface tractions $\tau^{k}$ on the prospective crack surface of ply $(k)$ and the resulting average crack opening and crack sliding displacement $\Delta u^{k}$. As explained in Section 4.1 and Appendix B, the coefficients $\beta_{m}^{k l}$ are provided based on both extrapolation of FE analyses, and extrapolation of known fracture mechanics solutions for similar problems, as presented in $[35]$.

A brief comment is required by the double summation in eq. (B.29), (B.33). The two equations are listed here exactly as they are presented in $[35,36]$, in order not to alter their form as they are given in the cited sources. The double summation suggests the general form $\beta^{k l}$ of the COD coefficients as in eq. (2.9) ([36]), which implies the coupling effect between the surface tractions in ply $(l)$ and COD in ply $(k)$. However, as explained at the beginning of the current section, the assumption of no coupling is further used in the model in [35], which 
brings the COD coefficients to their $\beta^{k}$ form in eq. (B.3). Due to the no coupling assumption, the $k l$ superscript reduces to $k k$, and the use of a double superscript is not needed any more, the $\beta^{k l}$ coefficient becomes $\beta^{k}$ now. It can be observed though that the COD coefficient in eq. (B.30) is used in its general $\beta^{k l}$ expression, without considering the aforementioned assumption of no COD coupling between different plies of the laminate. This fact triggers the general double summation expressions expressions in eq. (B.29), (B.33). However, the numerical implementation is done using the $\beta^{k}$ coefficients as they are presented in their final form in eq. (B.17) - (B.21), and the double summation in eq. (B.29), (B.33) becomes a simple summation over the $k$ index $(l=k)$.

\section{B.4 Observations on the mathematical manipulation of the needed quantities of the material model}

- The extension-bending decomposition of the stresses in Fig. B.1 requires a rearrangement of the well known equations of the CLT, in order to emphasize the needed $\tau_{(E)}, \tau_{(B)}$ components, as presented in the following (as opposed to the previous section, the overbar notation will be be reserved in this section in order to denote stress-strain quantities at the mid-surface of the laminate and at the mid-coordinates of each ply, as presented in the following).

The effective (overall, laminate level) of the load and deformation of the whole laminate are noted as

$$
F=\left(\begin{array}{l}
N \\
M
\end{array}\right), \quad \epsilon=\left(\begin{array}{l}
\epsilon_{0} \\
\kappa
\end{array}\right)
$$

where $\epsilon_{0}$ are the $3 \times 1$ in-plane strains of the laminate mid-surface (describing the membrane deformation), and $\kappa$ are the laminate curvatures (describing the flexural deformation).

The constitutive relationship at the laminate level is

$$
\epsilon=S \cdot P+\alpha \Delta T
$$

where $S=C^{-1}$ is the laminate compliance matrix, $C$ is the laminate stiffness matrix (traditionally noted as $C=[A B D]$ in CLT), and $\alpha$ is the vector of the laminate CTE. Expressions for the $C, \alpha$ thermoelastic properties of the laminate can be calculated based on ply material properties and laminate configuration, and can be found in any composite materials 
text-book. In the current notation, the $S, \alpha$ quantities are written as

$$
S_{6 \times 1}=\left(C_{6 \times 1}\right)^{-1}=\left[\begin{array}{ll}
C_{(E E)} & C_{(E B)} \\
C_{(B E)} & C_{(B B)}
\end{array}\right]^{-1}, \quad \alpha_{6 \times 1}=\left\{\begin{array}{l}
\alpha_{E} \\
\alpha_{B}
\end{array}\right\}
$$

The in-plane state of stress in ay ply of the laminate is given by

$$
\sigma^{k}=Q^{k} \cdot\left(\epsilon^{k}-\alpha^{k} \Delta T\right)=
$$

where the linearly varying (due to combined membrane-flexural deformation) ply strain is

$$
\epsilon^{k}=\epsilon_{0}+z^{k} \cdot \kappa
$$

Here the rearranging of the typical CLT equations comes into picture, in order to describe the stress decomposition in Fig. B.1. Thus, based on eq. (B.38), the ply strain $\bar{\epsilon}^{k}$ at the mid-coordinate $\bar{z}^{k}$ of the ply $(k)$ can be written as

$$
\bar{\epsilon}^{k}=\epsilon_{0}+\bar{z}^{k} \cdot \kappa
$$

and the mid-surface strain of the laminate is calculated from (B.38) as

$$
\epsilon_{0}=\bar{\epsilon}^{k}-\bar{z}^{k} \cdot \kappa
$$

which is then replaced in (B.38)

$$
\epsilon^{k}=\bar{\epsilon}^{k}-\bar{z}^{k}+z^{k} \cdot \kappa
$$

The ply strain $\epsilon^{k}$ as expressed in (B.41) allows to rewrite the ply stress in (B.37) as

$$
\begin{aligned}
\sigma^{k} & =Q^{k} \cdot\left(\bar{\epsilon}^{k}-\bar{z}^{k}+z^{k} \cdot \kappa-\alpha^{k} \Delta T\right)= \\
& =Q^{k} \cdot\left(\bar{\epsilon}^{k}-\bar{z}^{k}-\alpha^{k} \Delta T\right)+Q^{k} \cdot z^{k} \cdot \kappa= \\
& =\sigma_{(E)}^{k}+\sigma_{(B)}^{k}
\end{aligned}
$$

The ply stress in eq. (B.42) is of course equivalent to the traditional formulation in eq. (B.37). The purpose of the final rearrangement in (B.42) is to calculate the needed uniform $\left(\sigma_{(E)}^{k}\right.$, corresponding to membrane loading $)$ and linearly varying $\left(\sigma_{(B)}^{k}\right.$, corresponding to flexural loading) ply stress components, based on which the surface traction decomposition is done as depicted in Fig. B.1, by the use of eq. (B.1).

- Eq. (B.10) of the energy release requires considering the whole set of the extension/bending and II/III modes (after disregarding the mode II out plane shearing mode 

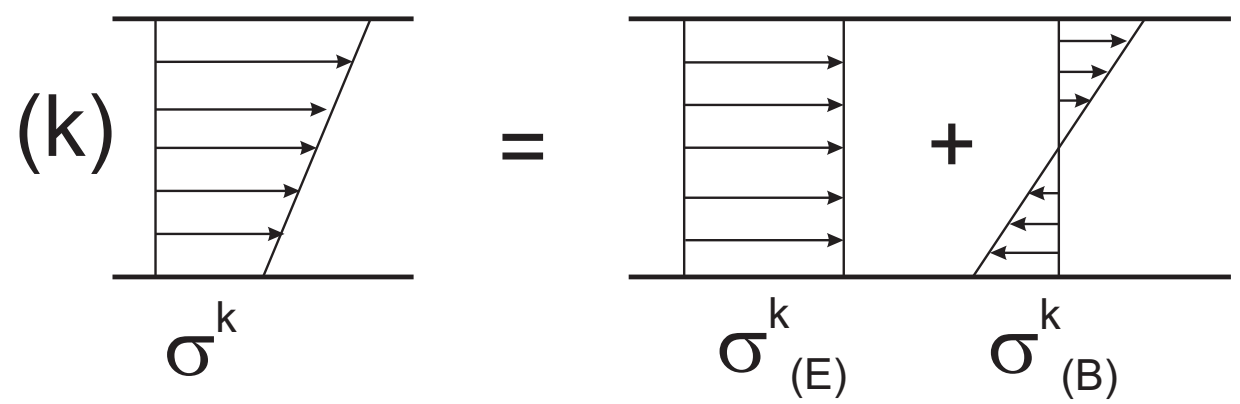

Figure B.1: Decomposition of ply stresses in uniform and linear components

given by $\tau_{3}$ in Fig. 2.3 (b)) components of the $\tau^{k}$ and $\beta^{k}$ quantities. For this, a matrix notation is used as follows:

The Couchy stress formula in eq. (B.1) is expressed as

$$
\begin{aligned}
& \tau_{(E)(2 \times 1)}^{k}=N_{(2 \times 3)}^{k} \cdot \sigma_{(E)(3 \times 1)}^{k} \\
& \tau_{(B)(2 \times 1)}^{k}=N_{(2 \times 3)}^{k} \cdot \sigma_{(B)(3 \times 1)}^{k}
\end{aligned}
$$

where the $N^{k}$ matrix contains the director cosines of the crack surface, which is given by the orientation of the ply (cracks always follow the longitudinal fiber direction).

$$
N^{k}=\left[\begin{array}{ccc}
n_{1}^{k} & 0 & n_{2}^{k} \\
0 & n_{2}^{k} & n_{1}^{k}
\end{array}\right]
$$

The needed $\tau^{k}$ and $\beta^{k}$ quantities in eq. (B.10) are then expressed in matrix form as

$$
\tau_{(4 \times 1)}^{k}=\left(\begin{array}{c}
\tau_{(E)(2 \times 1)}^{k} \\
\tau_{(B)(2 \times 1)}^{k}
\end{array}\right), \quad \beta_{(4 \times 4)}^{k}=\left[\begin{array}{ll}
\beta_{(E E)(2 \times 2)}^{k} & \beta_{(E B)(2 \times 2)}^{k} \\
\beta_{(B E)(2 \times 2)}^{k} & \beta_{(B B)(2 \times 2)}^{k}
\end{array}\right],
$$

The corresponding whole set of the COD's in eq. (B.2) would be expressed as

$$
\Delta u_{(4 \times 1)}^{k}=\left(\begin{array}{c}
\Delta u_{(E)(2 \times 1)}^{k} \\
\Delta u_{(B)(2 \times 1)}^{k}
\end{array}\right)
$$

However, the COD's in (B.46) are not needed to be explicitly calculated, since they are just replaced in the expression of the mechanical work in eq. (B.7).

- The whole calculations in Section B.3 to derive the reduced material properties in (B.27) are performed in laminate c.s. However, the derivation of the COD coefficients $\beta$ in Section B.2 is made in the c.s. of individual plies. Because of this, a rotation of the COD 
coefficients given by eq. (B.17) - (B.21) (and arranged in matrix form in eq. (B.45)) from the ply c.s. to laminate c.s. is required.

The expression for the $\beta$ rotation is based on the following considerations:

The surface traction components $\tau$ and the corresponding COD components $\Delta u$ in eq. (B.5) can be expressed in either ply c.s. (denoted as $y$ axes in Fig. B.2) or laminate c.s. (denoted as $x$ axes in Fig. B.2). The correspondence between the components in the two coordinate systems is given by the classical relations

$$
\begin{gathered}
\tau_{y}=\left\{\begin{array}{l}
\tau_{y 1} \\
\tau_{y 2}
\end{array}\right\} \underbrace{\left[\begin{array}{cc}
\cos \theta & \sin \theta \\
-\sin \theta & \cos \theta
\end{array}\right]}_{R}\left\{\begin{array}{l}
\tau_{x 1} \\
\tau_{x 2}
\end{array}\right\} \\
\Delta u_{y}=\left\{\begin{array}{l}
\Delta u_{y 1} \\
\Delta u_{y 2}
\end{array}\right\}\left[\begin{array}{cc}
\cos \theta & \sin \theta \\
-\sin \theta & \cos \theta
\end{array}\right]\left\{\begin{array}{l}
\Delta u_{x 1} \\
\Delta u_{x 2}
\end{array}\right\}
\end{gathered}
$$

Eq. (B.5) written in ply c.s. reads

$$
\Delta u_{y}=t \cdot \beta_{y} \cdot \tau_{y}
$$

where $\beta_{y}$ are the COD coefficients as given by eq. (B.17) - (B.21). By substituting eq. (B.47) into (B.48) the expression of the same eq. (B.5) written in laminate c.s. is obtained as

$$
\Delta u_{x}=t \cdot R^{-1} \cdot \beta_{y} \cdot R \cdot \tau_{x}
$$

Equations (B.48) and (B.49) allow for writing the rotation relationship for $\beta$ as

$$
\beta_{x}=R^{-1} \cdot \beta_{y} \cdot R
$$

\section{B.5 Details of the modeling approach based on COD and theory of elastic body with voids $[32,33,34]$ : only in-plane deformation}

As previously mentioned, this method provides the reduced material properties of the laminated composite without considering the bending effect. The method is presented here for the sake of completeness. The method is not implemented in the analytical model proposed in the present manuscript, which is based on the reduced material properties for both membrane and flexural deformation, as it was presented during Appendix B.1 - B.4. 


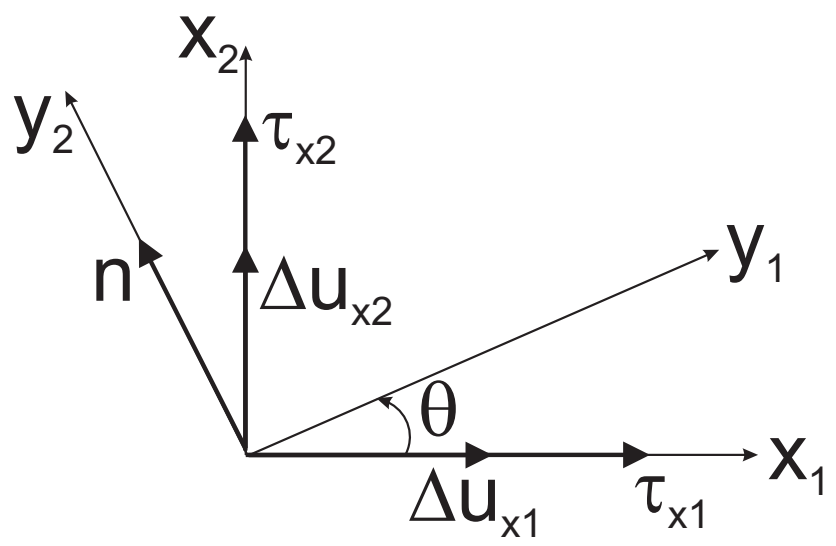

Figure B.2: Rotation of surface tractions and COD.

The model algorithm in order to determine the thermo-elastic properties of the composite laminate containing matrix cracks in any of its plies is the following:

- the effective laminate strain $\bar{\epsilon}_{(c)}$ is imposed;

- effective (overall) strain in each cracked ply $(k)$ inside of the laminate is expressed according to eq. (2.10):

$$
\bar{\epsilon}_{(c)}^{k}=\epsilon_{(c)}^{(a) k}+\Delta \epsilon^{k}
$$

- the iso-strain condition is imposed on the effective strain of the cracked ply $\bar{\epsilon}_{(c)}^{k}$ :

$$
\bar{\epsilon}_{(c)}^{k}=\bar{\epsilon}_{(c)}
$$

- the incremental ply strain $\Delta \epsilon^{k}$ is calculated based on ply $(k)$ average COD in (B.2):

$$
\Delta \epsilon_{i j}^{k}=\frac{\rho^{k}}{2 t^{k}}\left(\Delta u_{i}^{k} n_{j}^{k}+\Delta u_{j}^{k} n_{i}^{k}\right)
$$

- the average ply strain $\epsilon_{(c)}^{(a) k}$ is calculated from (B.51):

$$
\epsilon_{(c)}^{(a) k}=\bar{\epsilon}_{(c)}^{k}-\Delta \epsilon^{k}
$$

- having $\epsilon_{(c)}^{(a) k}$ known, the average ply stress $\sigma_{(c)}^{(a) k}$ is calculated by considering the constitutive equation of the ply in average sense

$$
\epsilon_{(c)}^{(a) k}=S^{k} \sigma_{(c)}^{(a) k}+\alpha^{k} \Delta T
$$

where $S^{k}, \alpha^{k}$ are material properties of the undamaged material in ply $(k)$ (due to the fact that eq. (B.55) applies for intervals of material between cracks). 
- having $\sigma_{(c)}^{(a) k}$ known, the effective laminate stress $\bar{\sigma}_{(c)}$ can be calculated based on the equilibrium equation

$$
\bar{\sigma}_{(c)}=\sum_{k=1}^{N} v^{k} \sigma_{(c)}^{(a) k}
$$

where $v^{k}$ is volume fraction of ply $(k)$ inside of the laminate.

- having the effective laminate strain $\bar{\epsilon}_{(c)}$ and the effective laminate stress $\bar{\sigma}_{(c)}$ known, the effective properties $S_{(c)}, \alpha_{(c)}$ of the cracked laminate are identified based on the effective constitutive equation

$$
\bar{\epsilon}_{(c)}=S_{(c)} \bar{\sigma}_{(c)}+\alpha_{(c)} \Delta T
$$

To be remembered that the first stage of model development addresses only the in-plane case. Bending deformation is eliminated from the model due to eq. (B.52) (imposed in-plane iso-strain). To be noted that, corresponding to the first stage of model development, FEA method in not used in order to provide a solution, but it is used in order to validate the approximate analytical solution. 


\section{Appendix C}

\section{Various mixed modes crack growth criteria}

In order to simplify the notations, the subscript $(k 0)$ (indicating damage evolution in a certain ply $(k 0)$ of the laminate where the condition for matrix cracking multiplication is met) is dropped, and equation (4.4) is rewritten as

$$
g=(1-r) \sqrt{\frac{G_{I}}{G_{I c}}}+r \frac{G_{I}}{G_{I c}}+\frac{G_{I I}}{G_{I I c}}-1 \geq 0
$$

where $G_{I c}, G_{I I c}$ are critical ERR corresponding to crack multiplication due to pure $I, I I$ modes of loading, and $r=G_{I c} / G_{I I c}$. It has to be reminded that $G_{I c}, G_{I I c}$ can be regarded as material properties, but they are not constant numbers in the formulation of the present model, but they might feature an in-situ $R$-curve behavior, as described in Section 4.4.3.

The $G_{I}, G_{I I}$ quantities in (C.1) are ERR by individual $I, I I$ modes of loading as part of the mixed mode loading. Experimentally assessing the individual participation of each of the $I$ and $I I$ modes of loading as parts of the mixed mode loading is not a trivial task. This has been attempted by designing special test methods, as it is the case of off-axis axial loading for mixed mode intra-laminar matrix cracking, where the ratio of individual $I$ and II modes can be evaluated as part of the mixed mode loading. However, most of the experimental mixed mode data in the literature is not for intra-laminar crack growth, but it is for inter-laminar crack growth, i.e., delamination. In this case, the mixed mode loading by the use of Mixed Mode Bending (MMB) test method, which is a combination of Double Cantilever Beam (DCB) test method for pure mode $I$ loading and End Notch Flexure (ENF) test method for pure mode $I I$ loading, has become the common test method for mixed mode 
loading, Reeder [92, 93], Benzeggagh and Kenane [94], Camanho and Dávila [95], Rikards [96] (see Fig. 4.5 in order to review the possible directions of crack propagation for the two cases of inter/intra-laminar matrix cracking).

Furthermore, comparative studies on the ability of various damage evolution laws to reproduce experimental mixed mode data was also performed mostly for the case of interlaminar crack growth [92, 93, 94, 95]. This is the reason why the available information on inter-laminar damage will be considered as an qualitative indicator of what kind of behavior might be expected for the case of intra-laminar damage, even though the damage mechanisms in the two cases (inter-laminar vs. intra-laminar) might be different, as it has been shown in $[61,62,63,64,71]$.

A comprehensive study on various mixed mode damage criteria for the case of interlaminar crack growth, for material systems based on carbon fibers in combination with different thermoset and thermoplastic types of matrix was performed in [92, 93, 94, 95, 96], based on comparison against mixed mode loading experimental data. As for the case of intralaminar crack growth, the literature data is more scarce. Studies on various intra-laminar crack growth laws for mixed mode loading conditions can be found in Hahn [54], Donaldson [70]. Some of the references regard the inter-laminar and intra-laminar crack growth laws as being interchangeable for a given material system, as based on similar fracture mechanisms. However, there is no hard proof regarding the generalization of a crack growth criterion between the two inter/intra-laminar directions of crack propagation. On the contrary, counterarguments are presented in [61] based on microscopic observation of the crack surfaces for the two inter/intra-laminar cases.

The various mixed modes crack propagation criteria proposed in the previously cited references ale listed and briefly discussed in the following.

A suggestive visualization of how each criterion predicts the mixed mode crack propagation can be obtained by plotting the $G_{I}=f\left(G_{I I}\right)$ variation for each criterion, where $G_{I}, G_{I I}$ are values satisfying the damage criterion. The $G_{I}, G_{I I}$ values can be regarded as separate contributions of the mixed mode loading to individual $I$ and $I I$ modes for crack growth. Another suggestive graph equivalent to $G_{I}=f\left(G_{I I}\right)$ can be obtained by plotting $G_{T}=f(\beta)$, where $G_{T}=G_{I}+G_{I I}$ is the total energy released by both $I$ and $I I$ loading modes in the moment of crack growth, and $\beta=\frac{G_{I I}}{G_{I}+G_{I I}}$ is a measure of mode mixity.

It can be observed that $0 \leq \beta \leq 1$, where $\beta=0$ for pure mode $I$ loading (i.e., $G_{I I}=0$ ), and $\beta=1$ for pure mode $I I$ loading (i.e., $\left.G_{I}=0\right)$. Also from the $G_{I}=f\left(G_{I I}\right)$ and $G_{T}=f(\beta)$ 
plots presented in the following it can be observed that each crack growth criterion assures a loading variation from $G_{I c}$ (i.e., pure mode $I$ loading, $\left.G_{I I}=0\right)$ to $G_{I I c}$ (i.e., pure mode $I I$ loading, $\left.G_{I}=0\right)$. However, the predictive characteristics of different criteria can be highly different, as it results from the plots presented in the following.

The minimum required material properties for all of the following crack growth criteria are the critical values $G_{I c}, G_{I I c}$ ERR, corresponding to pure mode $I$ and pure mode $I I$ loading. Some of the proposed criteria require additional material parameters.

- the simple non-interacting criteria $[92,93]$

$$
\begin{aligned}
& g=G_{I}-G_{I c} \geq 0 \\
& g=G_{I I}-G_{I I c} \geq 0
\end{aligned}
$$

which supposes that there is no interaction between separate modes $I$ and $I I$ ERR components of the mixed mode loading. The corresponding $G_{I}=f\left(G_{I I}\right)$ and $G_{T}=f(\beta)$ variations are presented in Fig. C.1 and Fig. C.2, respectively.

- the linear criterion $[92,93]$

$$
g=\frac{G_{I}}{G_{I c}}+\frac{G_{I I}}{G_{I I c}}-1 \geq 0
$$

which assumes a linear variation between $G_{I}, G_{I I}$ loading components at the moment of crack growth. The corresponding $G_{I}=f\left(G_{I I}\right)$ and $G_{T}=f(\beta)$ variations are presented in Fig. C.1 and Fig. C.2, respectively.

- the power law criterion [70, 92, 93, 95, 96]

$$
g=\left(\frac{G_{I}}{G_{I c}}\right)^{m}+\left(\frac{G_{I I}}{G_{I I c}}\right)^{n}-1 \geq 0
$$

which can be regarded as a generalization of the linear criterion (C.4). Two additional material parameters ( $m$ and $n$ ) are required, which are selected as fit parameters to the experimental data. Usually, the two fit parameters are considered $m=n$ in order to reduce the number of needed material parameters. The corresponding $G_{I}=f\left(G_{I I}\right)$ and $G_{T}=f(\beta)$ variations are presented in Fig. C.1 and Fig. C.2, respectively, where it can be observed that a wide range of material behaviors can be simulated, from convex to concave $G_{I}=f\left(G_{I I}\right)$ and $G_{T}=f(\beta)$ variations. 


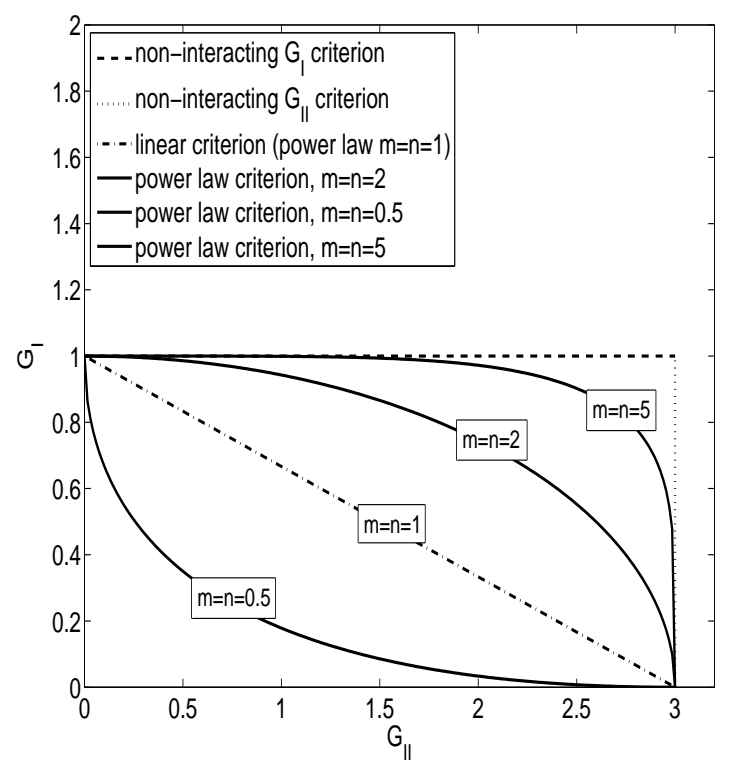

Figure C.1: Mixed modes damage growth criteria: simple non-interacting; linear; power law $\left(G_{I}=f\left(G_{I I}\right)\right.$

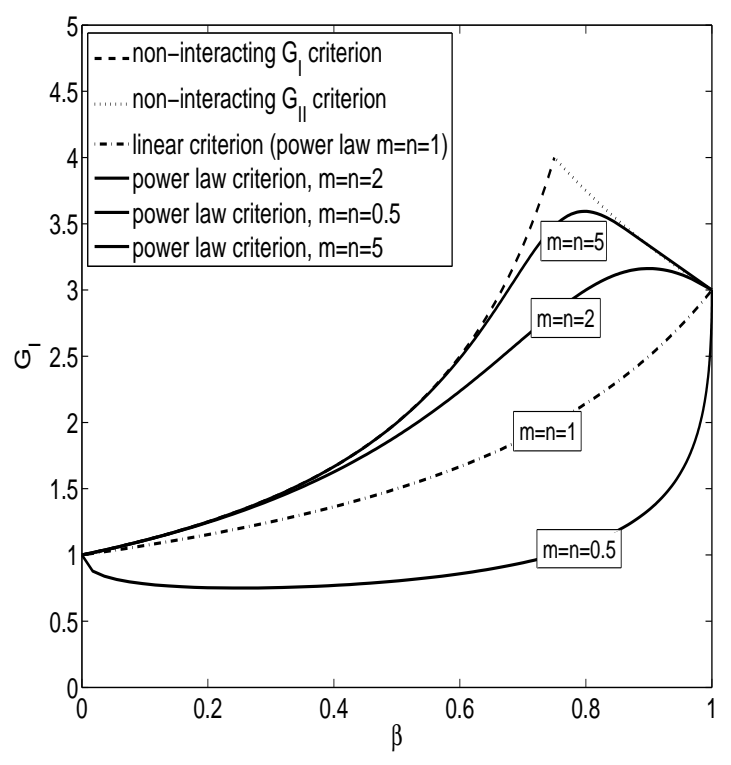

Figure C.2: Mixed modes damage growth criteria: simple non-interacting; linear; power law $\left(G_{T}=f(\beta)\right)$.

- the exponential hackle criterion $[92,93,96]$

$$
g=G_{I}+G_{I I}-\left(G_{I c}-G_{I I c}\right) \cdot \exp [\gamma \cdot(1-N)]-G_{I I c} \geq 0
$$

where $N=\sqrt{1+\frac{G_{I I}}{G_{I}} \sqrt{\frac{E_{11}}{E_{22}}}}$. This criterion can also reproduce a wide variety of material responses by varying the extra material parameter $\gamma$, as it can be seen in the corresponding $G_{I}=f\left(G_{I I}\right)$ and $G_{T}=f(\beta)$ variations which are presented in Fig. C.3 and Fig. C.4. What it is interesting to notice to this criterion as compared to the previous ones, is the fact that the exponential hackle criterion eq. (C.6) is able to predict an initial increase in the required $G_{I}$ while applying an initial mode $I I$ loading $G_{I I}$, which is in conformance with mixed mode experimental observation for some materials systems. This behavior encountered in some experimental observations could be explained based on the fact that, for some materials, an initial mode $I I$ shear type loading induces an internal "friction" effect in the material, which translates in a higher mode $I$ crack opening type loading required in order to generate cracking.

- the exponential $K_{I} / K_{I I}$ criterion $[92,93]$ 


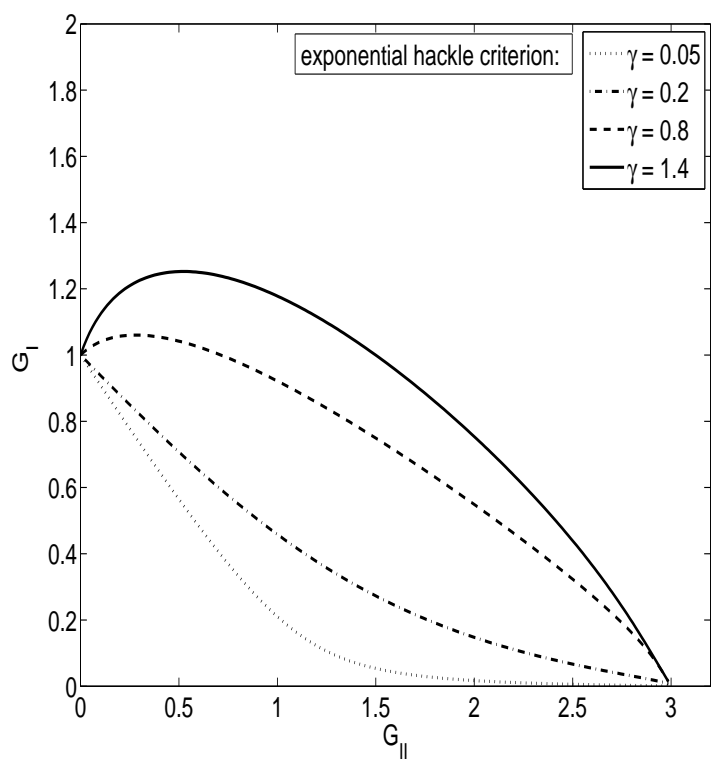

Figure C.3: Exponential hackle mixed modes damage growth criterion $\left(G_{I}=\right.$ $\left.f\left(G_{I I}\right)\right)$.

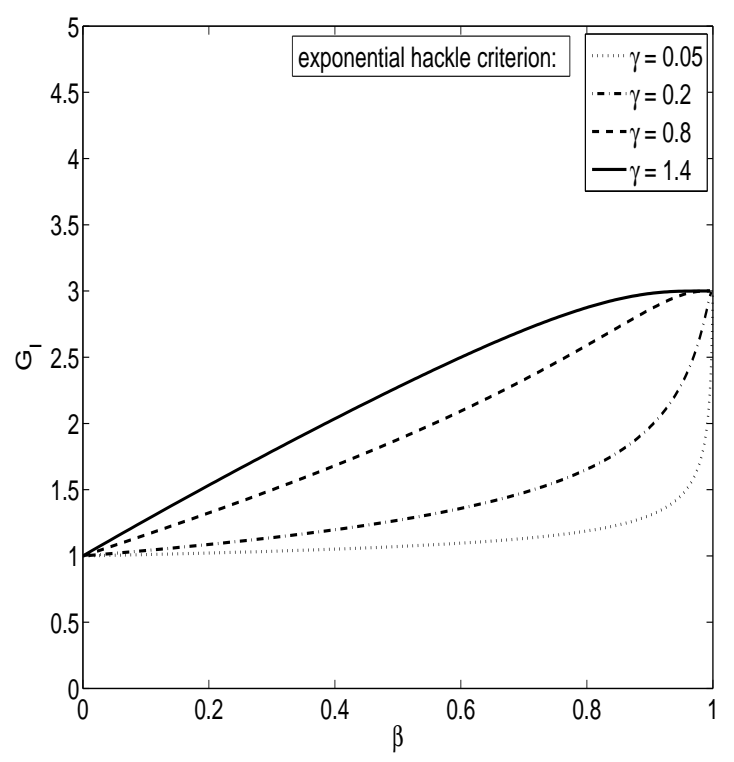

Figure C.4: Exponential hackle mixed modes damage growth criterion $\left(G_{T}=\right.$ $f(\beta))$.

$$
g=G_{I}+G_{I I}-\left(G_{I I c}-G_{I c}\right) \cdot \exp \left(-\eta \cdot \sqrt{\frac{G_{I}}{G_{I I}}}\right)-G_{I c} \geq 0
$$

also requires one extra material parameter $\eta$, based on which a large variety of material responses can be modeled, among which the initial increase in the required $G_{I}$ while applying an initial mode $I I$ loading $G_{I I}$. The corresponding $G_{I}=f\left(G_{I I}\right)$ and $G_{T}=f(\beta)$ variations are presented in Fig. C.5 and Fig. C.6, respectively.

- the interaction criterion $[92,93]$

$$
g=\left(\frac{G_{I}}{G_{I c}}-1\right)\left(\frac{G_{I I}}{G_{I I c}}-1\right)-i\left(\frac{G_{I}}{G_{I c}}\right)\left(\frac{G_{I I}}{G_{I I c}}\right) \geq 0
$$

where the interaction parameter $i$ is defined as

$$
i=\left[\kappa+\phi\left(\frac{G_{I}}{G_{I}+G_{I I}}\right)\right]
$$

takes into account the ratio of $I$ and $I I$ modes under mixed mode conditions. Two additional $\kappa$ and $\phi$ material parameters are required by the crack growth criterion in (C.8). Based on these two extra material parameters, the interaction criterion can be adjusted to match 


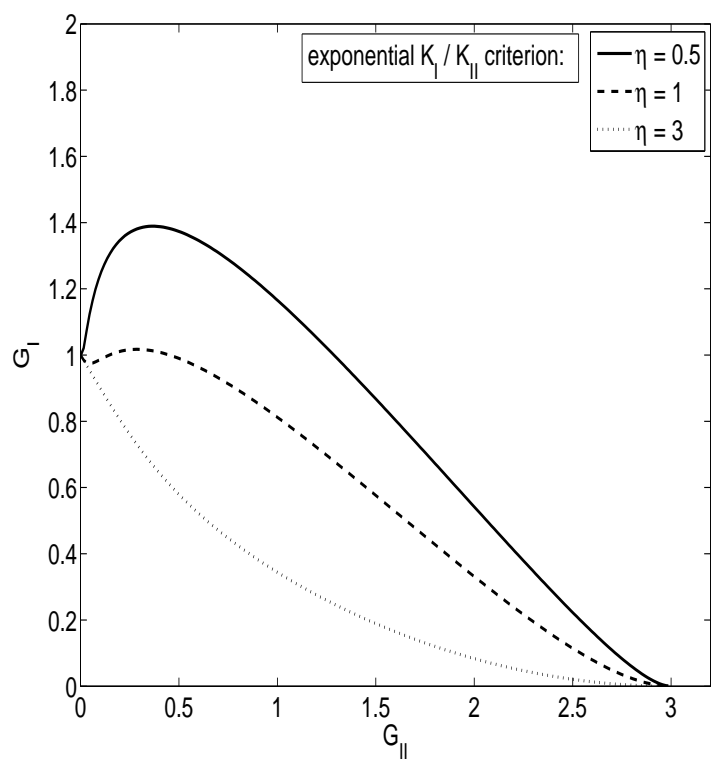

Figure C.5: Exponential $K_{I} / K_{I I}$ mixed modes damage growth criterion $\left(G_{I}=\right.$ $\left.f\left(G_{I I}\right)\right)$.

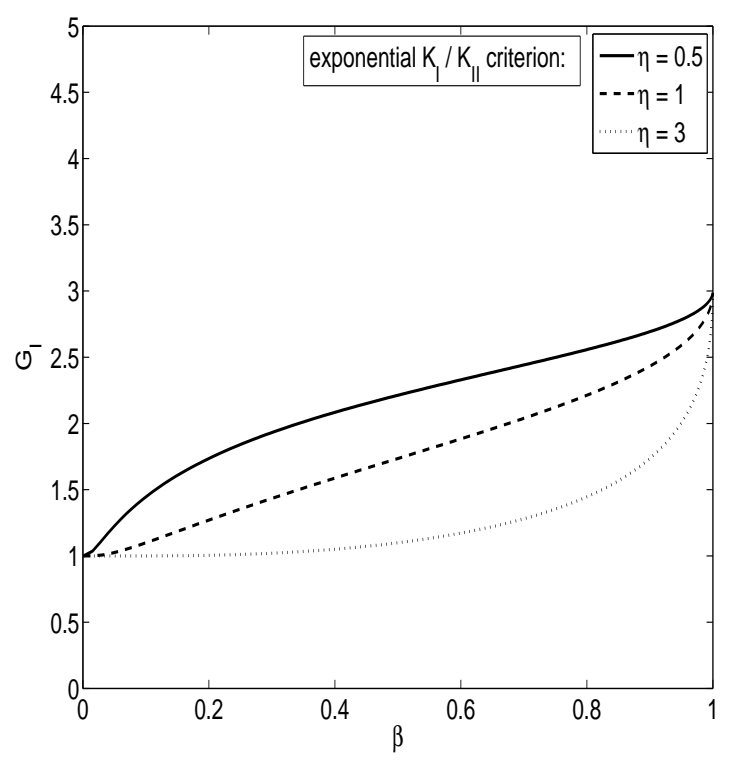

Figure C.6: Exponential $K_{I} / K_{I I}$ mixed modes damage growth criterion $\left(G_{T}=\right.$ $f(\beta))$.

various material responses, as presented in the corresponding $G_{I}=f\left(G_{I I}\right)$ and $G_{T}=f(\beta)$ variations, Fig. C.7 and Fig. C.8, respectively.

- the BK criterion $[94,95]$

$$
g=G_{I}+G_{I I}-\left[G_{I c}+\left(G_{I I c}-G_{I c}\right)\left(\frac{G_{I I}}{G_{I}+G_{I I}}\right)^{\eta}\right] \geq 0
$$

is also based on the pure modes critical ERR $\left(G_{I c}\right.$ and $\left.G_{I I c}\right)$, but it also requires an additional fit parameter $\eta$ is needed in order to reproduce a variety of material responses. The total energy release at the moment of mixed mode crack propagation, $G_{I}+G_{I I}$, is a function of the mixed mode ratio $\beta$.

- the $K_{I}$ criterion $[54,70,92]$

is the one used in the present model, eq. (C.1). No additional parameters are required besides the critical ERR for pure $I$ and $I I$ loading cases, but the price paid for its simplicity is the fact that only a limited type of material response can be modeled, as presented in the corresponding $G_{I}=f\left(G_{I I}\right)$ and $G_{T}=f(\beta)$ plots, Fig. C.9 and Fig. C.10. While 


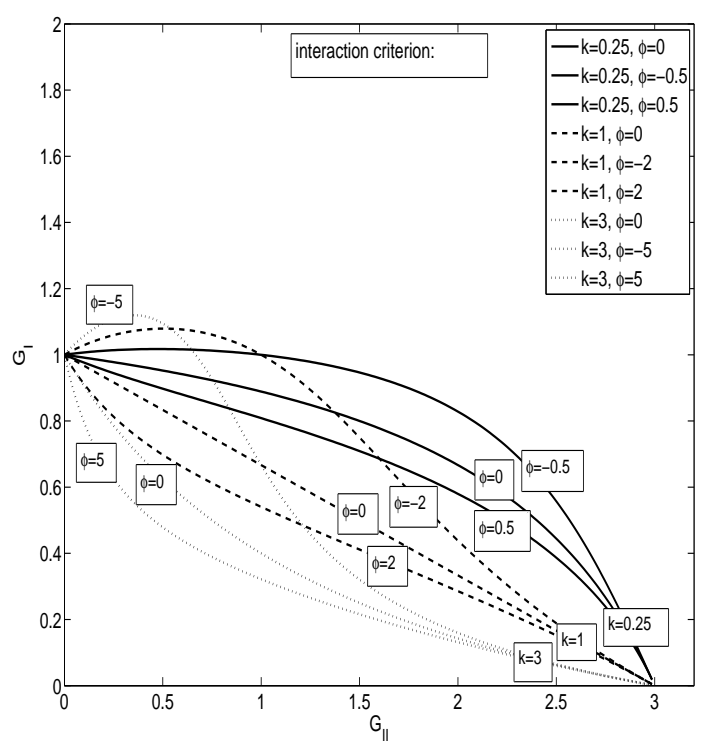

Figure C.7: Interaction mixed modes damage growth criterion $\left(G_{I}=f\left(G_{I I}\right)\right)$.

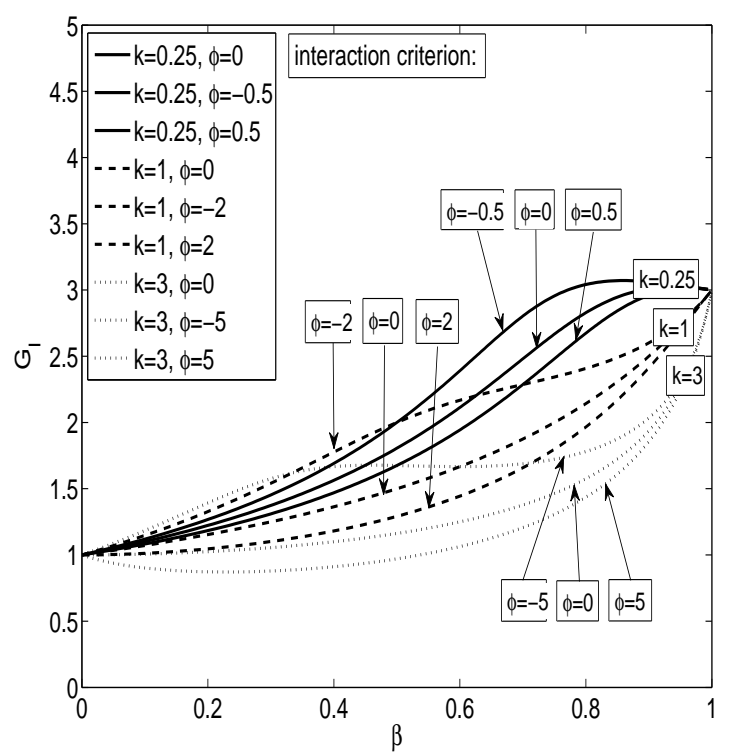

Figure C.8: Interaction mixed modes damage growth criterion $\left(G_{T}=f(\beta)\right)$.

good agreement to experimental data was found in [54] based on comparison with limited available data for intra-laminar type of damage for UD composites, a poor agreement with experimental data was found in [92] for inter-laminar type of damage.

The criterion in eq. (C.1) is casted in the present model mainly due to its simplicity (no extra experiment-fit material parameters required), but there is no certitude on how proper the criterion is for the material systems analyzed in Section 5. It has been shown in $[70,92,93,94,95,96]$ that different crack evolution laws might be required for different material systems. Furthermore, the same criterion might require different values of the fit parameters $(m, n, \gamma, \eta, \kappa, \varphi$ in eq. (C.5), (C.6), (C.7), (C.8), (C.10)) for different material systems. The conclusion of the studies in $[70,92,93,94,95,96]$ is that each material system has to have its own crack growth criterion adjusted to it, based on experimental mixed mode loading data.

Based on mixed mode experimental data (not shown in the present manuscript) presented in $[92,93,94,96]$ for the case of inter-laminar crack propagation and in [70] for the case of intra-laminar crack propagation, for different material systems, it might be concluded that the general shape of the $G_{I}=f\left(G_{I I}\right)$ plot is expected to have a convex trend, possible to feature an initial increase in the required $G_{I}$ value for an increase in the mode mixity (the $G_{I I}$ value). The convex shape can be simulated by the eq. (C.6), (C.7), (C.8) criteria (as 


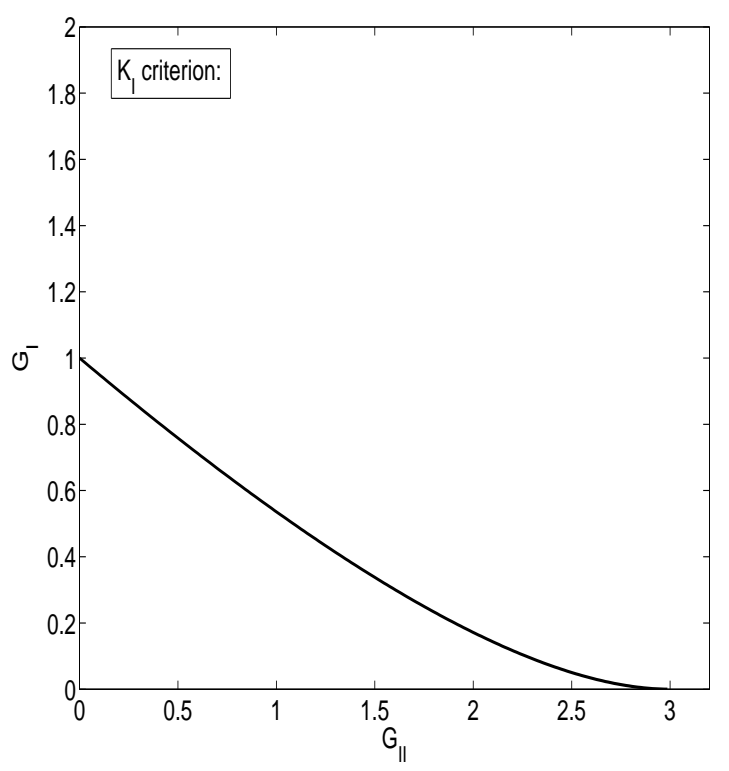

Figure C.9: $K_{I}$ mixed modes damage growth criterion $\left(G_{I}=f\left(G_{I I}\right)\right)$.

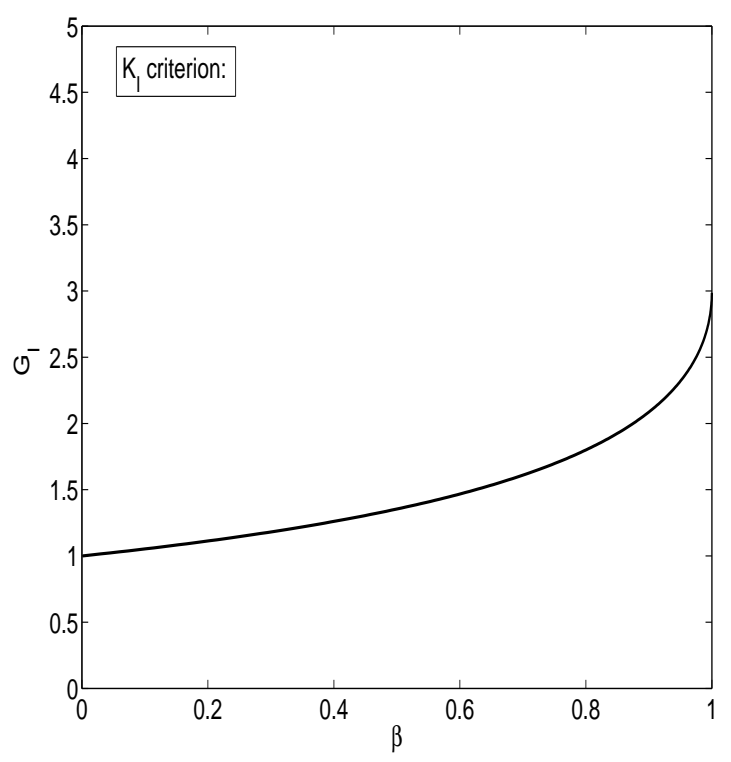

Figure C.10: $K_{I}$ mixed modes damage growth criterion $\left(G_{T}=f(\beta)\right)$.

shown in Fig. C.3, C.5, C.7), but it can not be simulated by the currently used eq. (C.1) criterion, which features a slightly concave shape (as shown in Fig. C.9). However, there is not evidence that the conclusion of the convex shape of the $G_{I}=f\left(G_{I I}\right)$ plot is a general one, applicable to any material system.

The various damage laws in $[54,70,92,93]$ are actually proposed for both cases of inter/intra-laminar cracking (see Fig. 4.5), even if the validation against experimental data is done for only one case out of the two. However, based on other studies, for example Wang [61], it is expected that the two inter/intra-laminar modes of matrix cracking evolution feature different damage mechanisms, and caution should be practiced in generalizing some crack growth criteria between inter/intra-laminar cracking. The inter-laminar cracking mechanism might be governed by the pure matrix toughness properties in the matrix enriched layers in between different plies of the composite laminate, while the intra-laminar cracking mechanism might be highly influenced by the properties of the fiber-matrix interface, in addition to the toughness properties of pure matrix inside of the composite ply. It results that, for the case of intra-laminar damage, even the manufacturing and processing conditions can have an influence on the cracking behavior of the material. For example, the same material system could feature different intra-laminar matrix cracking behavior if the same fibers have undergone different treatment that can influence the properties of the fiber-matrix 
interface.

Furthermore, additional to the above observations, the possible $R$-curve material behavior with respect to the critical ERR material parameter $G_{c}$ has to be considered, as it was presented in Section 4.4.3. Some of the studies regarding the cracking criteria (for example [93]) deal with the stage of cracking initiation, without considering the further stage of crack development that brings fiber bridging, which in turn generates the $R$-curve material behavior. It has been shown in [94] that, even if the same criteria is used for the crack initiation and propagation stages, the required material parameters are different, which actually is equivalent to two different criteria for the two crack propagation stages. The $R$-curve material behavior could be more pronounced in the case of intra-laminar cracking (see Beaumont [62], Truss [63], Hansen [64], Jacobsen [65], Ni [67]) than in the case of inter-laminar cracking, since the $R$-curve mechanism is generated by the fiber misalignment which shows up more for matrix cracking inside of the ply (intra-laminar) than for matrix cracking between plies (inter-laminar). 


\section{Appendix D}

\section{Alternative approaches to crack multiplication in laminated composites}

Most of the predictive analytical models for matrix cracking in laminated composites in the literature that are based on energy balance damage growth criteria (see eq. (2.17)), rely on the assumption of constant values of the critical ERR $G_{I C}, G_{I I C}$ corresponding to modes $I$ and $I I$ crack formation. The $G_{I C}$ and $G_{I I C}$ values are considered constant material parameters under this assumption. They are determined either from mode $I$ and $I I$ testing of UD composite samples, or from adjusting them as input of the analytical model such that the analytical output fits the experimental data of matrix cracking process in laminated composites.

As an example of this constant $G_{I C}, G_{I I C}$ modeling approach can be cited the work in Nairn $[7,8,9,10,11,12,13,14,15,16]$. Usually, the output of the analytical model is represented by $\lambda=\lambda(\sigma)$ or $\lambda=\lambda(\epsilon)$ damage evolution curves, similar to the ones schematically presented in Fig. D.1, where the matrix cracking process for two laminate configurations, $\left[0 / 90_{n}\right]_{S}$ and $\left[0 / 90_{m}\right]_{S}$ with $n>m$ is depicted. Based on its energy formulation, the analytical model is able to catch the thickness effect of the cracking ply, as presented in Fig. D.1. However, the close fit of the experimental data for progressive matrix cracking multiplication under external loading corresponding to various laminate configurations and material systems seems to be a difficult task based on constant $G_{I C}, G_{I I C}$ parameters. Even in the previous cited papers $[7,8,9,10,11,12,13,14,15,16]$, where the RUC model based on 
constant $G_{I C}$ has been continuously improved and refined, the values used to fit the experimental data are always presented as $G_{I C}=G_{I C} \pm$ tolerance, which shows that a constant $G_{I C}$ values can not closely reproduce different laminate configurations. Moreover, an adjusting $f$ factor in used in [8] in order to correct the analytical predictions at high crack densities.

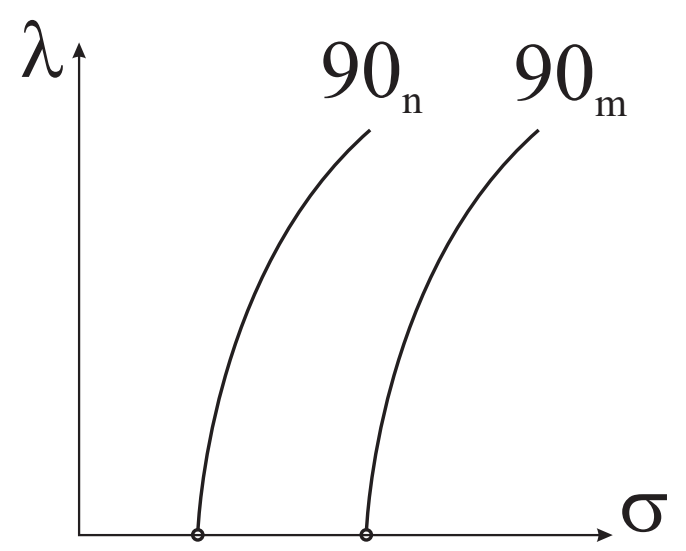

Figure D.1: Damage evolution curves for different thicknesses of the cracking ply, $(\lambda-\sigma)$ coordinates.

Observations regarding the possibility of a strengthening $R$-curve behavior in laminated composites are formulated in those models claiming that the critical material parameter $G_{c}$ is not a constant number, but it actually increases with increasing crack density $\lambda$, Hahn [17], Gudmundson and Adolfsson [36], Soutis [57], Varna [58], Yalvac [59], Zhang [60]. In this way, the critical parameter for crack growth becomes $G_{c}=G_{c}(\lambda)$ (see Fig. $2.5-2.8$ ).

Furthermore, due to the observed in-situ $R$-curve behavior in laminated composites, even the idea that a damage evolution law based on critical ERR $\left(G_{I C}, G_{I I C}\right)$ is inappropriate in order to describe the progressive matrix cracking in laminated composites has been formulated in some studies $[17,36,57,58]$.

Two alternative approaches, Gudmundson and Adolfsson [36] and Varna [58], to the problem of matrix crack multiplication in laminated composites are briefly describe din the present section.

The progressive damage model in [36] takes the advantage of closely spaced damage evolution curves $\rho=\rho(\epsilon)$ for a family of $\left[0 / 90_{n}\right]_{S}$ laminates, when plotted in $(\rho-\epsilon)$ coordinates, as schematically presented by the dashed curves in Fig. D.2. $\rho$ represents the normalized crack density in any ply of the laminate, $\rho=t / d$, where $d$ is the matrix crack spacing in the respective ply, and $t$ is the ply thickness. Based on this observation, a central unique fit curve is selected for the whole family of the $\left[0 / 90_{n}\right]_{S}$ laminates, which is the solid curve in 
Fig. D.2, and this unique $\rho=\rho(\epsilon)$ curve is considered as being the damage growth criterion. It is supposed that the solid curve $\rho=\rho(\epsilon)$ makes the correspondence between the strain transverse to the fiber direction $\epsilon$ in any ply of the laminate and the damage level $\rho$ in that ply.

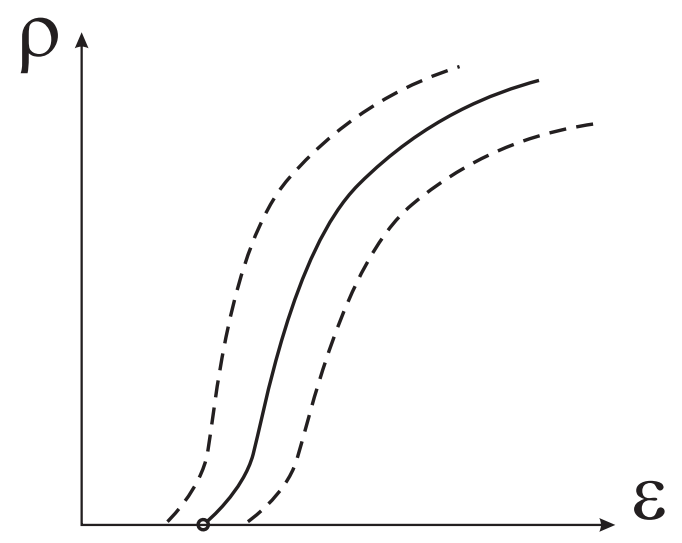

Figure D.2: Closely spaced damage evolution curves for different thicknesses of the cracking ply in $(\rho-\epsilon)$ coordinates. Selection of the $\rho(\epsilon)$ damage evolution criterion, Gudmundson [36].

In order to select the $\rho=\rho(\epsilon)$ damage evolution curve in Fig D.2, an experimental data set for the $\left[0 / 90_{n}\right]_{S}$ family of laminates, corresponding to a certain material system, is needed. The $\rho=\rho(\epsilon)$ curve is considered a material property.

However, it can be inferred that, based on the concept of the unique $\rho=\rho(\epsilon)$ damage evolution curve, the ply thickness effect on the damage onset presented in Fig. 2.4 can not be simulated. There is an unique value of the applied strain in Fig. D.2 when the damage onset takes place for every particular laminate of the $\left[0 / 90_{n}\right]_{S}$ family, regardless the thickness $n$ of the cracking ply.

Moreover, the strain considered in the damage evolution curve in Fig. D.2 (the solid line) represents the strain transverse to the fiber direction for individual plies. When the damage evolution law is applied to off-axis cracking plies or when shear loading acts on the laminate, only the transverse strain is considered for the crack multiplication process. The effect of the shear strain which is responsible for the mode $I I$ crack formation is not considered at all. In this way, the damage evolution criterion in [36] schematically represented by the solid line in Fig. D.2 does not consider the mixed modes conditions for matrix cracking formation, but it is a pure mode $I$ criterion. These might be some of the reason why poor agreement to experimental data is obtained in [36] for matrix cracking in the off-axis plies of the laminate. 
Another modeling approach that considers the in-situ $\mathrm{R}$-curve effect of the laminate configuration on the matrix cracking process is proposed in [58], based on the following experimental data and reasoning. Two laminate configurations, $\left[0 / 90_{n}\right]_{S}$ and $\left[0 / 90_{m}\right]_{S}$, with $n>m$, are considered. According to experimental measurements [58], each of the two laminate configurations features its own damage evolution curve, as schematically depicted in Fig. D.3 (b). Based on specific fracture mechanics tests (successive loading-unloading, while measuring the change in the compliance of the composite sample and keeping track of the increase in crack density, see [58]), the effective experimental in-situ $\mathrm{R}$-curves for the two laminate configurations can be obtained, as they are schematically depicted in Fig. D.3 (a). By the combination of the two set of experimental curves corresponding to the two considered $\left[0 / 90_{n}\right]_{S}$ and $\left[0 / 90_{m}\right]_{S}$ LSS, an unique curve $G_{C}=G_{C}(\epsilon)$ can be obtained, as it is depicted in Fig. D.3 (c). The unique $G_{C}=G_{C}(\epsilon)$ is proposed as an R-curve damage evolution criterion, for any laminate configuration of the considered material system.

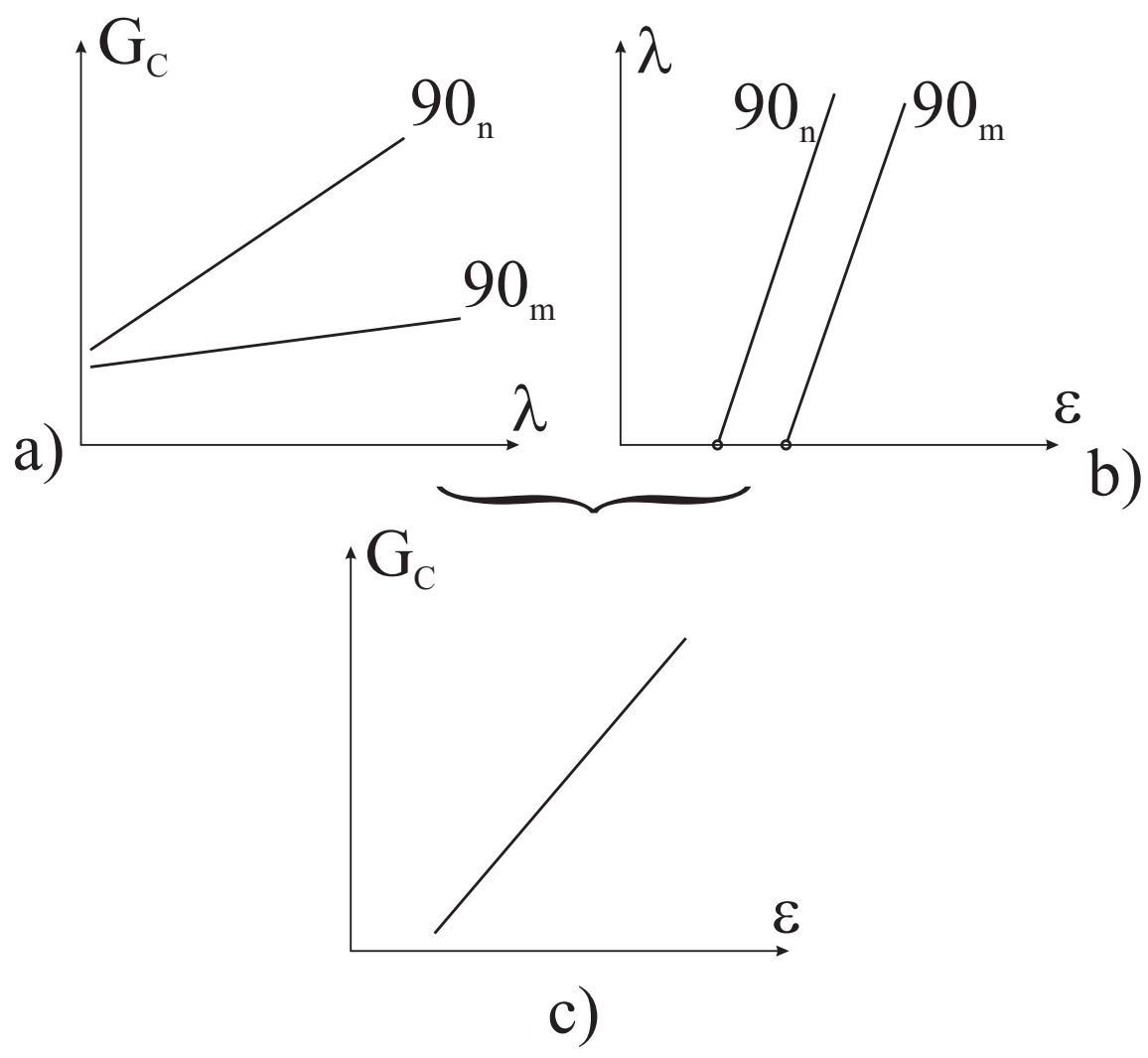

Figure D.3: Combination of $G_{C}(\lambda)$ and $\lambda(\epsilon)$ experimental data in order to obtain the $G_{C}(\epsilon)$ R-curve, Varna [58].

It is interesting to note that the reduction of the two sets of two curves in Fig. D.3 (a), (b) 
to an unique curve in Fig. D.3 (c) is possible only if the two cures in Fig. D.3 (b) feature a certain appearance, as it is depicted in Fig. D.4. Only in this case of intersecting trends of the experimental data for the crack multiplication process is possible to obtain an unique curve in Fig. D.3 (c) based on the diverging trends in Fig. D.3 (a). If the damage evolution curves are parallel, as depicted in Fig. D.3 (b), or they are diverging, then the curves in Fig. D.3 (a), (b) do not superpose in an unique curve in Fig. D.3 (c).

The question is now if the $\lambda(\epsilon)$ curves are always intersecting as presented in Fig. D.3 (b) for different thickness of the cracking ply, regardless the laminate configuration and the material system. A clear proof for this assumption is not presented in [58]. However, a review of the analytical and experimental data collected from various sources presented in the present manuscript seems to support the needed assumption. It can be observed that the intersecting trend of the $\lambda(\epsilon)$ damage evolution plots appears in Fig. 5.12, 5.66, 5.72, 5.76, 5.80, 5.152, 5.154, 5 . The trend of the experimental data in these plots is as required by the $G_{C}=G_{C}(\epsilon) \mathrm{R}$-curve damage evolution law in [58], which might impose this modeling approach as a viable alternative to modeling matrix cracking evolution in laminated composites.

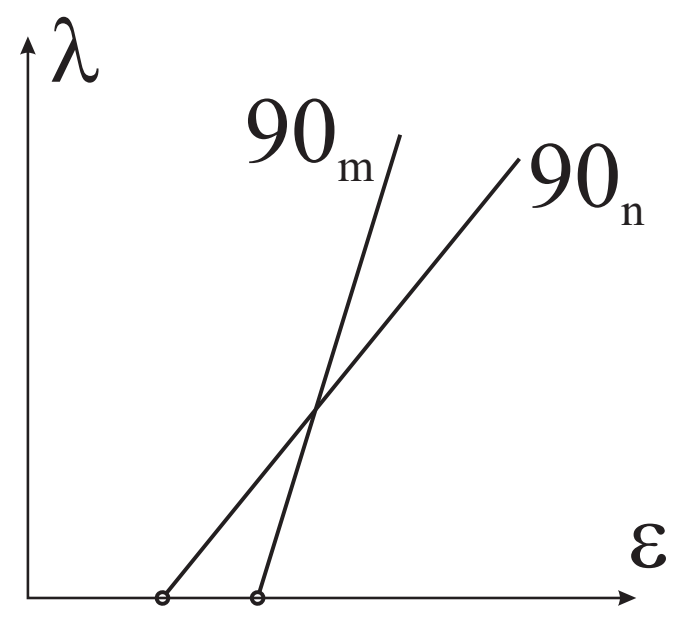

Figure D.4: Required intersecting trends of the $\lambda(\epsilon)$ damage evolution curves for different thicknesses of the cracking ply.

The ability of the analytical model proposed in the present manuscript to reproduce the needed trend in [58] (i.e., the intersecting $\lambda(\epsilon)$ damage evolution curves for different ply thicknesses in Fig. D.4) might be regarded as an advantage of the present formulation.

The implementation of the method in [58] is difficult due to the required experimental data. The $G_{C}(\lambda)$ measurements (Fig.D.3 (a)) are difficult to perform and are prone to high variability, resulting in both expensive experiments and reduced reliability of the generated 
data. 


\section{Appendix E}

\section{Overview of progressive matrix cracking implementation in commercial FEA packages}

A survey of the progressive damage capabilities for composite materials implemented in popular commercial FEA packages, focused on transverse matrix cracking damage mode, has been conducted. The goal of this survey is to asses the performances of the current FEA products, by analyzing some aspects of the theoretical models implemented into these products (e.g., the embedded assumptions, the advantages and drawbacks of the the model formulation, s.o.). The background assumptions of the theoretical models are usually not transparent for the user of the FEA product, setting limitations which are often passed over by the FEA analyst. Based on their theoretical implementation, the predictive capabilities and some of the the limitations of commercial FEA software are assessed and discussed. The present survey is meant as a frame in which the model formulation proposed in the present manuscript can be compared and evaluated.

Great professional performances have been achieved by the engineering R\&D departments of the very well known companies who developed the commercial FEA packages under the present survey. Their theoretical models are always backed and supported by academic research. Sometimes, in-house modifications and improvements are added to the original formulation in order to facilitate the numerical implementation, to extend the level of generality, or to improve the user friendlyness of the model (for example, by simplifying the user input of the needed material properties, which are not always trivial to be found or 
determined).

By implementing progressive damage models into some of the FEA packages, the FEA user has been provided great numerical tools in order to analyze and asses the behavior of the composite structures, especially damage tolerance capabilities of structures made of composite materials. These tools opened new, long wanted possibilities regarding material analysis and optimization, weight reduction and cost savings. The new implemented progressive damage models are the result of cutting edge research in composite materials engineering, and they were totally inexistent just a few years before. However, it is nevertheless true that the new advanced features with regard to progressive damage in composite materials could be difficult to understand in the right way and applied with the maximum of awareness by the FEA analyst. It is the analyst's task to thoroughly study and understand the implemented theoretical models into the FEA codes.

It is well known that, due to their heterogeneous nature, composite materials feature different damage mechanisms, Dávila [53], Hashin [97], Puck [98]:

- fiber dominated failure modes, which can in turn have different forms, like fiber breakage in tension, fiber buckling/kinking in compression, or even fiber crushing under hydrostatic pressure and fiber shearing under transverse punch loading;

- matrix dominated failure modes, like matrix cracking under transverse extension, matrix crushing under transverse compression, inter-ply delamination/debonding.

Current FEA software tackle the problem of all or of some of the above listed damage mechanisms. A combination of different damage modes is difficult to approach at analytical theoretical level and to implement at numerical level, due to the fact that each damage mode represents a difficult problem by itself, and different damage mechanisms influence and trigger each other. This is why the research effort of academia and industry corporates that develop analytical and numerical products oriented toward offering comprehensive tools regarding damage in composites is highly remarkable. This survey is focused on mode I-II transverse matrix cracking inside of the composite ply (also called intra-laminar matrix cracking) as damage mechanism, in order to make a comparison with alternative solutions proposed in the present manuscript and in previous work, Barbero [29, 30, 99, 100].

The preset survey is intended to briefly present the main advantages and disadvantages of various progressive damage models implemented in commercial FEA software, as of today. Behind each commercial implementation there is an academia level research. These academia 
roots will be presented and briefly discussed. The consistency from the academia model to the commercial model will be analyzed, and the differences will be discussed, when they appear. Based on some of the possible shortcomings identified in the implementation of the theoretical formulation into the FEA packages, an alternative transverse matrix cracking progressive damage formulation is proposed, which is based on the work in $[29,30,99,100]$.

Usually, a progressive damage model operates by reducing the stiffness of the damaging ply, according to some stiffness reduction scheme. The stiffness reduction parameter $d$ is a function of the damage variable $\omega$ (which accounts for the damage level, for example the matrix crack density in some models), and the reduction in material properties is expressed as

$$
E=(1-d) E^{0}
$$

where $E$ is the reduced material property due to damage, $E^{0}$ is the undamaged material property, and $d$ is the stiffness reduction parameter. The reduction parameter $d$ (and consequently, the reduced material property $E$ ) is a function of damage variable $\omega$.

Under this conceptual setup of progressive damage, there are modeling approaches (Soutis [101], Tan and Nuismer [26], Yalvac [27, 28], Barbero [29, 30], Abdi [102]) that show a dependence of the damage parameter $d$ not only on the damage level $\omega$, but also on the laminate configuration, like the thickness of the damaging ply, the properties of the neighboring plies, or the position of the ply inside of the laminate (LSS). Usually, CDM models neglect this influence, and material degradation laws (softening laws) are set based on experimental observations on particular laminate configurations, which are then considered valid for any laminate configuration. When laminate configuration is accounted for, separate experiments for separate LSS are required. The damage variable $\omega$ is usually set as the strain level by CDM based models, since crack density is not accounted for under this modeling approach.

A list of the main developers on the commercial FEA market (as of today) included in this study is presented in the following (in alphabetical order):

- Abaqus (Simulia)

- Ansys (Ansys Inc.)

- Adina (Adina R\&D Inc.)

- Comsol (Comsol Group) 
- Genoa (Alpha Star Corporation)

- Helius (Firehole Composites)

- Hypersizer (Collier Research Corporation)

- LS-Dyna (Livermore Software Technology Corp.)

- (MD) MSC Nastran (MSC.Software Corporation)

- NEI Nastran (NEi Software)

- NX Nastran (Siemens PLM Software)

Among them, the following packages do not have their own developed model, but can use another FEA product (as add-on) in order to run composite materials progressive damage analyses

- Ansys - can interface with the model implementation from Helius and Genoa;

- (MD) MSC Nastran - can interface with the model implementation from Genoa;

- NEI Nastran - can interface with the model implementation from Helius;

and the following have their own in-house developed damage models for composite materials

- Abaqus

- LS-Dyna

- Genoa

- Helius

It has to be noted with regard to the previous list that Abaqus and LS-Dyna are stand alone FEA solvers, while Genoa and Helius are add-on products special developed for tackling the problem of progressive damage behavior of the material, and they interface with the host FEA solvers. The progressive damage implementation of the four products in the last list will be discussed in the following. 


\section{E.1 The progressive damage model in Genoa}

It has to be stated first that the discussion in here is based on the information presented in [103], where the theoretical source of the progressive damage model in Genoa is cited as being Abdi [102, 104]. Further on, according to [102, 104], the formulation in these two papers is based on the analytical model originally developed in Soutis $[57,101]$. In conclusion, all the references $[57,101,102,103,104]$ have been studied and are discussed in this section. It is not completely clear if these sources represent the last implementation into the Genoa PDA algorithm, or if the implementation is exactly as presented into the cited sources; this is because the Theoretical Manual of the Genoa product has not been available.

A few comments on the Genoa progressive damage model are follows next:

- the Genoa progressive damage model for matrix cracking [102, 104] is based on a micromechanics of damage approach (see Section 2.1.1), initially developed in [57, 101] as a RUC shear lag analysis. The damage state variable is represented by the ply crack density. One advantage of a model based on crack density as damage state variable is the fact that the crack density is effectively calculated, and this information can be used in applications where, besides the reduction in material thermo-elastic properties, the permeability of the structure is of interest, as well (e.g., composite structures used to contain liquids or gases). This advantage can be considered as opposed to the formulation of CDM modes, where the crack density is not a state variable, and information about the permeability of the structure can not be retrieved.

- based on its RUC micromechanics formulation, the progressive damage model in Genoa is able to predict the influence of the laminate configuration (i.e., the thickness of the cracking ply or the stiffness of the neighboring plies, see Section 5.1.1) on the stiffness reduction of the cracking ply.

- as a common disadvantage of the micromechanics based models is the fact that their level of applicability is limited to simple laminate configurations and loading cases. This is also the case of the model in $[57,101]$, which is valid only for balanced symmetric laminate of the type $[S / 90]_{S}$ featuring matrix cracks only in the middle $90^{\circ}$ ply (see Fig. E.1). Such case scenario of only one cracking ply is very limiting with regard to the types of laminate configurations and loading cases that can be approached. For example, the thermal loading is equivalent to a biaxial mechanical loading which is prone to induce matrix cracking in both $0^{\circ}$ and $90^{\circ}$ plies of a $[0 / 90]_{S}$ laminate configuration (see Fig. 5.59 in Section 5.1.5). The 
same is the case of a hole notched laminate under uniaxial loading: even if the applied load on the laminate is uniaxial, the state of stress sin the vicinity of the hole is a biaxial one, and matrix cracking in multiple plies of the laminate, along different directions, is possible.

The case of multiple cracking plies is over the possibilities of the analytical model in $[57,101]$, limitation which is transfered to $[102,104]$, and further on to Genoa capabilities. However, this single central cracking ply limitation in the original formulation of the model in $[57,101]$ is disregarded, and the model is applied in order to analyze multiple damaging plies configurations, as is the case of the analysis performed on a $[0 / 45 / 90 /-45]_{S}$ laminate configuration in [104] featuring cracks in all three off-axis plies, or the case of a $\left[0_{2} /-60_{2} /+\right.$ $\left.60_{2}\right]_{S}$ laminate configuration in [105]. Even if the comparison with experimental data is good in the two cases, yet it has to be remembered that the original analytical model was not designed for these two cases.

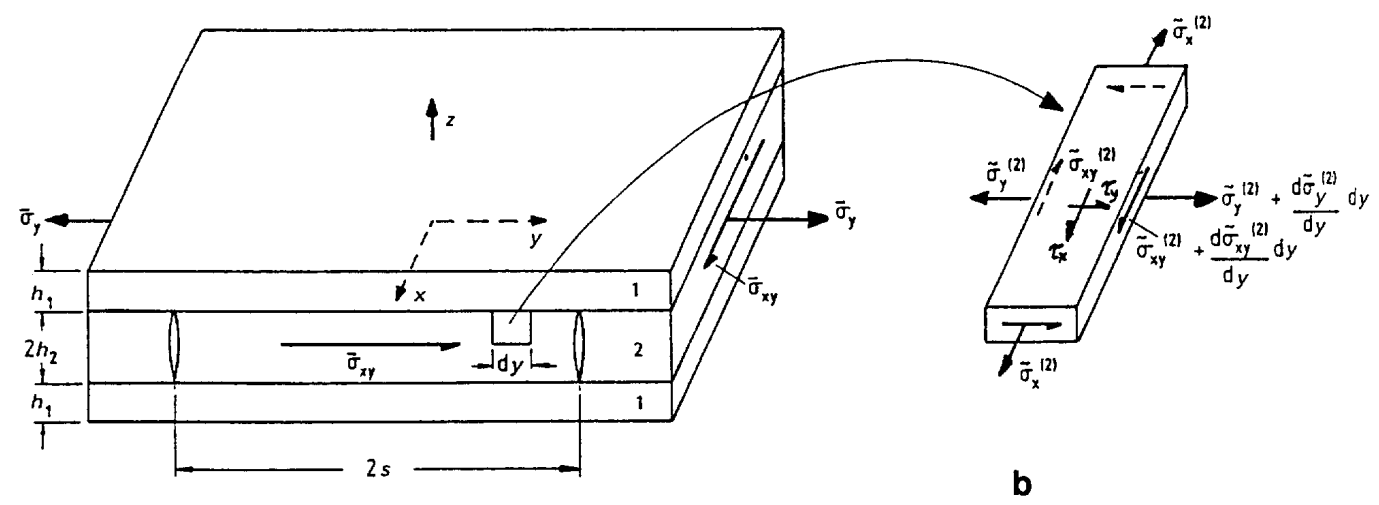

$\mathbf{a}$

Figure E.1: Micromechanics RUC model developed for symmetric laminate featuring matrix cracks in the central ply, Soutis [101].

A homogenization technique (i.e., considering the effective, equivalent, homogenized 
properties of the damaged ply) seems to be applied in Genoa, in order to release the limitation of only one damaging ply in the original micromechanics model. Even so, it has to be remembered that the micromechanics equations in the original model still impose restrictions on the configuration of the laminate, which has to feature cracks in the central ply only, and the above examples of $[0 / 45 / 90 /-45]_{S}$ and $\left[0_{2} /-60_{2} /+60_{2}\right]_{S}$ represent a departure from the assumption in the analytical model.

- damage onset in $[102,104]$ is based on strength based FC, namely the maximum stress of the composite ply in transverse tension. As explained in Section 2.2, a strength based FC is not able to consider damage initiation and evolution as function of laminate configuration. Moreover, the ply transverse tensile strength FC for damage onset in $[102,104]$ regards only the mode I matrix cracking, without considering the effect of mode II shear stresses.

- damage evolution is implemented as discrete events intended to be approached by an energy release rate criterion, but instead of using the critical energy release rate $\left(G_{C}\right)$ as the material property governing damage growth, the matrix strength is substituted as critical value in the energy balance in $[102,104]$. Thus, the damage evolution criterion is reduced from an ERR one to a strength based one. The substitution from ERR to matrix strength also introduces a spurious conversion factor $\alpha$ in the energy balance equation $([102,104$, eq.2]), which is considered $\alpha=10$ in the examples in $[102,104]$. This kid of parameter would be difficult to understand and set by the user. Moreover, it is not specified if the matrix strength in the energy balance should be the value corresponding to bulk matrix material or to in-situ material (matrix inside of the composite). It is well known that the two values are different.

By substituting the matrix strength to the critical energy release rate into the energy balance for damage growth, the experimental observation of the dependence of the critical parameter on the damage level (see Section 2.2.1) can not be considered in the model in $[102,104]$. This dependence was originally considered in the initial formulation in $[57,101]$, where a resistance curve ( $\mathrm{R}$-curve) behavior of the critical parameter $G_{c}$ is considered.

Moreover, the energy equation for damage growth in $[102,104]$ considers only the mode I matrix cracking.

- out of plane (flexural) deformation can not be approached under the limitations of the analytical model in $[57,101]$.

- the effect of matrix cracking on the thermal expansion coefficients of the laminated composite is not implemented in Genoa. 
Additional damage mechanisms besides transverse matrix cracking are not implemented in Genoa.

\section{E.2 The progressive damage model in Abaqus}

A brief description of the intra-laminar progressive damage model for laminated composites in Abaqus is provided in the following:

The Abaqus progressive damage model for composite materials is based on the smeared crack concept (see Chapter 21.3 Damage and Failure for Fiber-Reinforced Composites in [106] and Chapter 4.5.3 A Cracking Model for Concrete and Other Brittle Materials in [107]). According to the smeared crack concept, cracks are not physically represented and tracked, but cracks are considered by the effect they have on the stiffness and the load carrying capacity of the material. As a parenthesis here, even the micromechanics (RUC based) FEA implementations (e.g.,Genoa, see Section E.1) use the concept of the smeared crack: although the matrix crack is physically considered at the RUC level in order to derive the equivalent reduced properties of the composite material, then the cracked ply is considered at its reduced stiffness value for further calculations, and the physical presence of the cracks is not needed any more for structural calculations. While the cracks are physically present at at the RUC level, the RUC calculation is only a subroutine called in order to evaluate the reduced material properties; cracks are not considered at structural level, they become smeared, considered by their effect on the material properties.

What is specific at the progressive damage implementation in Abaqus is the formulation labeled as the fictitious crack concept, originally developed for damage in concrete and other brittle materials, Hillerborg [108, 109, 110, 111, 112]. According to this concept, two constitutive laws are defined in order to characterize the material behavior before and after damage onset. Before onset the material is characterized by the regular engineering $(\sigma-\epsilon)$ constitutive law (linear elastic in the simplest case). After the crack onset at a material point, the material is not considered immediately split in two separate faces of the crack, but there is still stress transfer in between the two faces of the crack. The stress transfer capacity at the crack location is considered proportional to the relative distance between the two separating (but not separated) faces at the crack location (Fig. E.2), and the constitutive law $(\sigma-\epsilon)$ before crack onset is replaced by a $(\sigma-\delta)$ law after crack onset, where $\delta$ represents the relative displacement between the two faces of the propagating crack, while still reduced 
stress transfer capacity exists at the crack location. The $(\sigma-\delta)$ law after onset is a measure of degradation in the material property at the crack location. Once this degradation in material properties is established, the physical crack representation is not needed any more, the crack can be considered as fictitious, but the crack effect on the material properties is accounted for. By combining the two constitutive laws, the pre- and post- damage onset the material behavior can be represented as depicted in Fig. E.3 (a).
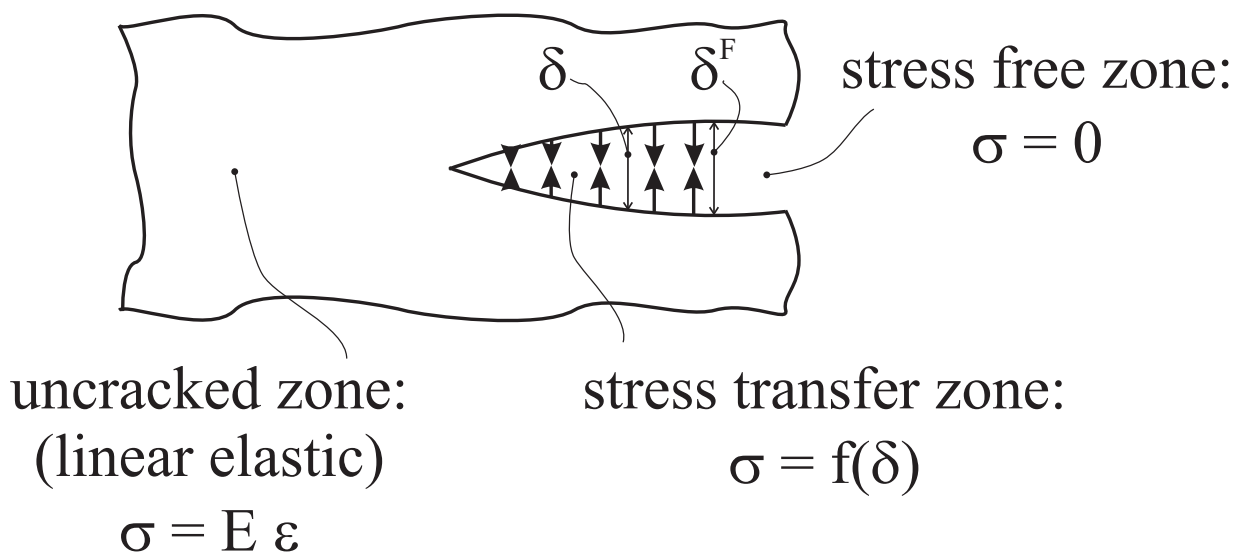

Figure E.2: Model of stress transfer (cohesive zone) during crack propagation.

The constitutive $(\sigma-\delta)$ stress softening law in depicted in Fig. E.3 (a) was successfully applied to FE Cohesive Zone Models (CZM), in order to model inter-ply delamination in composites, Dávila and Camanho $[95,113]$. When using cohesive elements in CZM delamination modeling, the $(\sigma-\delta)$ stress softening formulation is well fit to the FE modeling because the required displacement $\delta$ in the softening law (which, in a CZM model, represents the relative displacement between the debonding plies) is provided by the FE solution. The softening law in Fig. E.3 (a) represents a good approach for FE modeling of inter-laminar damage mode (i.e., delamination, debonding). The base assumption [95, 108, 113] of the cohesive law in Fig. E.3 (a) is that the area beneath the $(\sigma-\delta)$ plot represents the critical energy released rate $G_{C}$ of the material, which is a material constant parameter according to the Griffith fracture mechanics principle [90].

When it comes to modeling intra-laminar progressive damage in Abaqus (i.e., modes of damage taking place inside of the individual plies of the laminate, as it is the case of transverse matrix cracking), the concept of $(\sigma-\delta)$ constitutive stress softening law is applied. This approach represents an extension of the CZM inter-laminar damage modeling to intralaminar damage. The problem that arises is the fact that the damage level calculated 
at the integration point of the finite element is not governed by the relative displacement between two distinct locations, as it was the case of modeling delamination, where damage level at the integration point of the cohesive element was consider a function of the relative displacement between the two distinct faces of the cohesive element. Because of this, the required $\delta$ displacement in the $(\sigma-\delta)$ constitutive law can not be calculated based on the displacement FE solution. In order to overcome this problem, a characteristic length $L_{c}$ of the element (which is proportional to the element size) is used in order to calculate an equivalent displacement [106], which in the case of transverse matrix cracking is

$$
\delta_{e q}=L^{c} \cdot \epsilon_{e q}
$$

where $\epsilon_{e q}$ is an equivalent strain calculated based on the FE state of strain solution at the material (integration) point. The equivalent strain in (E.2) is calculated as

$$
\epsilon_{e q}=\sqrt{\left\langle\epsilon_{22}\right\rangle^{2}+\epsilon_{12}^{2}}
$$

An equivalent stress is also calculated based on the tensorial stress state at the material point

$$
\sigma_{e q}=\frac{\sigma_{22} \epsilon_{22}+\sigma_{12} \epsilon_{12}}{\epsilon_{e q}}
$$

The material behavior up to damage onset $\left(\delta_{e q} \leq \delta_{e q}^{0}\right)$, see Fig. E.3 (a)) is considered linear elastic, and the damage onset is evaluated in Abaqus [106] based on Hashin ply strength FC [97]. Based on the equivalent displacement in eq.(E.2), the softening material behavior after damage onset $\left(\delta_{e q}>\delta_{e q}^{0}\right)$ is modeled as linear $(\sigma-\delta)$ softening, Fig. E.3 a).

The stiffness degradation parameter is approximated as varying according to the equation

$$
d=\frac{\delta_{e q}^{F}\left(\delta_{e q}-\delta_{e q}^{0}\right)}{\delta_{e q}\left(\delta_{e q}^{F}-\delta_{e q}^{0}\right)}
$$

which allows a variation of the damage parameter $0 \leq d \leq 1$ for $\delta_{e q}^{0} \leq \delta_{e q} \leq \delta_{e q}^{F}$, which in turn allows a stiffness degradation according to eq. (E.1). The relationship $d=d\left(\delta_{e q}\right)$ in eq. (E.5) is presented in Fig. E.3 (b).

The final failure (i.e., the total stiffness degradation) corresponds to the moment $\delta_{e q}^{F}$ in Fig. E.2 and Fig. E.3, which is determined by the value of critical ERR of the material, $G_{c}$, which is considered a material constant:

$$
\delta_{e q}^{F}=\frac{2 G_{c}}{\sigma_{e q}^{0}}
$$




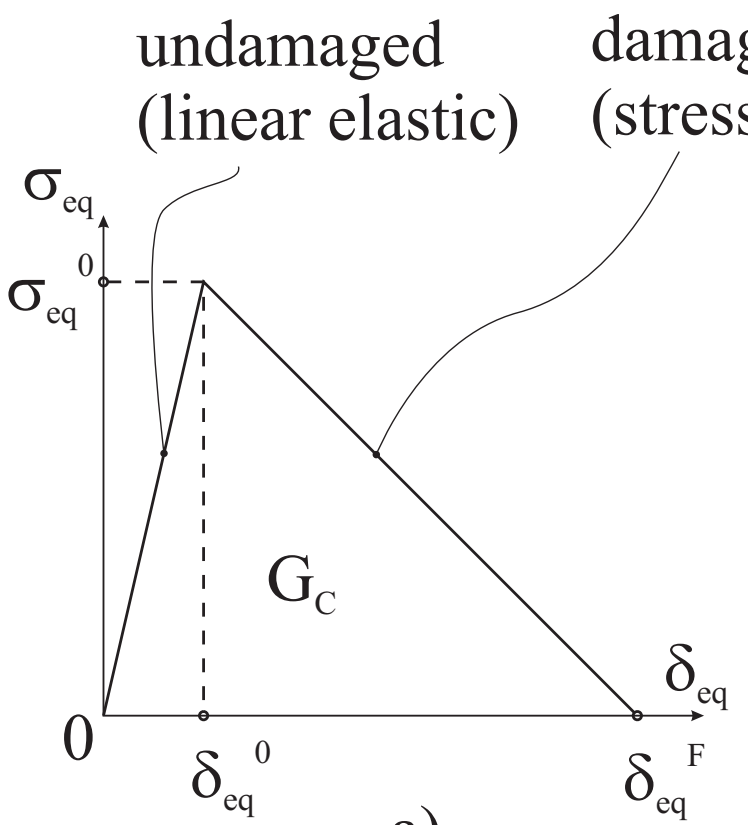

a)

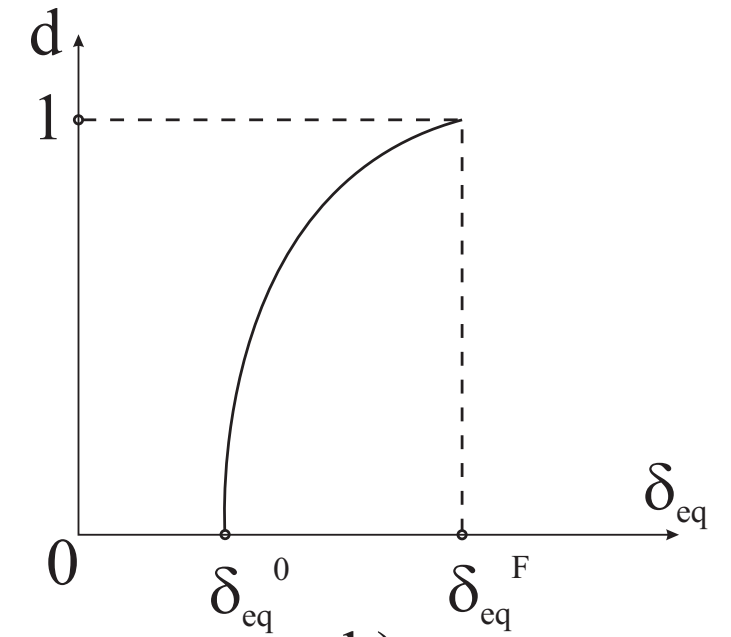

b)

Figure E.3: a) Linear softening $(\sigma-\delta)$ stress transfer model; b) The variation of the damage parameter in the $(\sigma-\delta)$ softening model.

Some comments on the formulation of the Abaqus model of progressive damage for composite materials follows next:

- a damage onset FC based on ply strength is not able to predict the experimentally observed in-situ constraining effect of the laminate configuration on damage initiation (see Fig. 2.4).

- by considering the softening law in Fig E.3 (a), the Abaqus progressive damage model falls into the category of continuum damage mechanics models (CDM), which is applied here at ply level. If we consider the experimental observations of the in-situ effect of the laminate configuration on the stiffness reduction of the damaging ply, Tan and Nuismer [26], [102], then the Abaqus formulation is not able to predict this effect, because the $(\sigma-\delta)$ softening law in Fig E.3 (a) is independent of the laminate configuration.

- the softening behavior due to damage growth is based on the concept of critical ERR as constant material parameter $\left(G_{C}\right.$ in Fig. E.3 (a)). When matrix cracking in laminated composites is regarded, there are evidences, Gudmundson [36, 37], Soutis92 [57], Hahn [17], Varna [58] of the fact that the $G_{C}$ material parameter is not a constant, but it follows an $\mathrm{R}$-curve behavior, featuring a dependence on both damage level and ply thickness. This behavior could be explained based on the bridging effect of the fibers along the cracking 
surface during crack multiplication (see Section 4.4.3). This aspect is not considered under the present Abaqus model.

- as opposed to RUC based micromechanics damage models, the crack density is not a state variable in the Abaqus model, due to its CDM nature. Because of this, the present model can not be used in order to directly asses the permeability properties of composite materials in structural applications where this is of interest.

- it is a well known fact that the critical ERR parameter has different values for different crack propagation modes: $G_{C}^{I} \neq G_{C}^{I I}$ for transverse matrix cracking in laminated composites, and mode mixity also has its own effect on crack formation, Beaumont [62], Donaldson [70], Reeder92 [92, 93], Benzeggagh and Kenane [94]. This effect is not implemented in the Abaqus model. It is not specified if the $G_{C}$ user input parameter corresponds to mode $I$ (opening) or mode II (shear sliding) cracking. It is the most probable that the user will regard it as the mode $I$ crack propagation, according to the most available material data. This single mode $I$ implementation might affect the results as the mode $I I$ (the shear therm $\epsilon_{12}$ in eq. (E.3)) increases, resulting into a total mismatch between the input mode $I$ critical ERR, $G_{I C}$, and mode $I I$ loading $\epsilon_{12}$ for the case of pure shear.

- the reduced material properties of the orthotropic ply after damage onset are based on the stiffness reduction scheme in Matzenmiller [114]. However, contrary to [114], the $E_{22}$ and $G_{12}$ material parameters of the damaged ply are reduced at the same rate in Abaqus, if we consider the case of only transverse matrix cracking due to tension loading. There is no concrete experimental evidence that the two material parameters has to be equally degraded, and this approach could be consider as an approximation.

- the progressive damage model in Abaqus does not provide information regarding the change in thermal expansion coefficients of the laminate, for those applications where this aspect is of interest.

An additional comment has to be made with regard to the mesh dependency aspect of the problem. It is claimed in $[106,107]$ that the characteristic length parameter $\left(L_{c}\right.$ in eq. (E.2)) is meant to alleviate the mesh dependency of the progressive damage model, which is an inherent drawback of any energy based FE implementation. This explanation is considered not accurate by the author of this section. It is true that a strong mesh dependency appears in those FE models where the ERR (which is the energy dissipated for the formation of new unit surface of the crack, see eq. (4.7)) is calculated from the FE solution of crack evolution. In this case (which is, for example, the case of Virtual Crack Closure Technique 
(VCCT)) the ERR continuously decreases upon the mesh refinement, and special techniques, like introducing a characteristic length of the element, are required in order to alleviate the mesh dependency. But this is not the case of the intra-laminar progressive damage model under discussion in this section. In the progressive damage formulation in Fig. E.3 the ERR (distinction to be made between energy release rate ERR, $G$, and critical ERR, $G_{C}$ ) is not calculated from the FE solution. Moreover, the ERR is not calculated at all in order to be used in the stress softening formulation of the model. Only the critical ERR $G_{C}$ is used in order to define the softening law in Fig. E.3. The progressive damage model in Abaqus is not an ERR based model (even if the critical ERR value $G_{C}$ is used in the formulation of the model), and consequently there is no mesh dependency introduced by the energy calculation.

It seems that the $L_{c}$ parameter in eq.(E.2) does not alleviate the mesh dependency, but it might introduce mesh dependency. The need of the $L_{C}$ parameter is to provide a way of calculating a relative displacement $\delta$ at the material point of the damaged material (since there are no defined faces of the fictitious crack, as it is the case of CZM applied to delamination). The need of calculating the displacement $\delta$ is in order to use the $(\sigma-\delta)$ stress softening model based on critical ERR value, $G_{C}$. The use of stress softening model based on $G_{c}$ brings the price of introducing a mesh dependent parameter, $L_{c}$, and thus the mesh dependency is introduced into the progressive damage model.

This affirmation can be checked following the model formulation in [106] (the equations (E.2) - (E.6)) for the case of different mesh densities, fine $(f)$ and coarse $(c)$, corresponding to characteristic lengths $L_{c}^{f}<L_{c}^{c}$. One more instance of the model is exemplified for more clarity: the case of extreme fine mesh, when $L_{c}^{f} \rightarrow 0$, noted as $L_{c}^{0}$. The schematic of the model for these three meshing cases are presented in Fig. E.4. In Fig. E.4 (a) the $(\sigma-\delta)$ softening law, which is the central idea of the progressive damage model in Abaqus, is presented for the three mesh cases. The $\delta_{e q}$ variable, including the moment of damage onset $\delta_{e q}^{0}$, is a function of $L_{c}$ according to eq. (E.2). However, in terms of $\delta$, the moment of final failure $\left(\delta^{F}\right.$ in eq. (E.6)) is a constant. The variation of the damage parameter $d$ for the three mesh densities is presented in Fig. E.4 (b), and the back-translation in terms of material strain $\epsilon$ is presented in Fig. E.4 (c).

If we consider, as an example, the simplest case of a composite laminate plate without stress concentrations (uniform stress-strain field) under imposed strain state, we can see that, according to Fig. E.4 (c), different levels of stiffness reduction correspond to the same strain level $\epsilon$ for different mesh densities $\left(d^{c}<d^{f}<d^{0}\right)$. Equivalently, the imposed strain state 
on the composite plate in order to achieve the final failure (total degradation of stiffness) is different for the considered mesh cases $\left(\epsilon_{e q}^{F, c}<\epsilon_{e q}^{F, f}<\epsilon_{e q}^{F, 0}\right)$, approaching infinity for the case where the element size approaches zero. This behavior means mesh sensitivity of the progressive damage model.

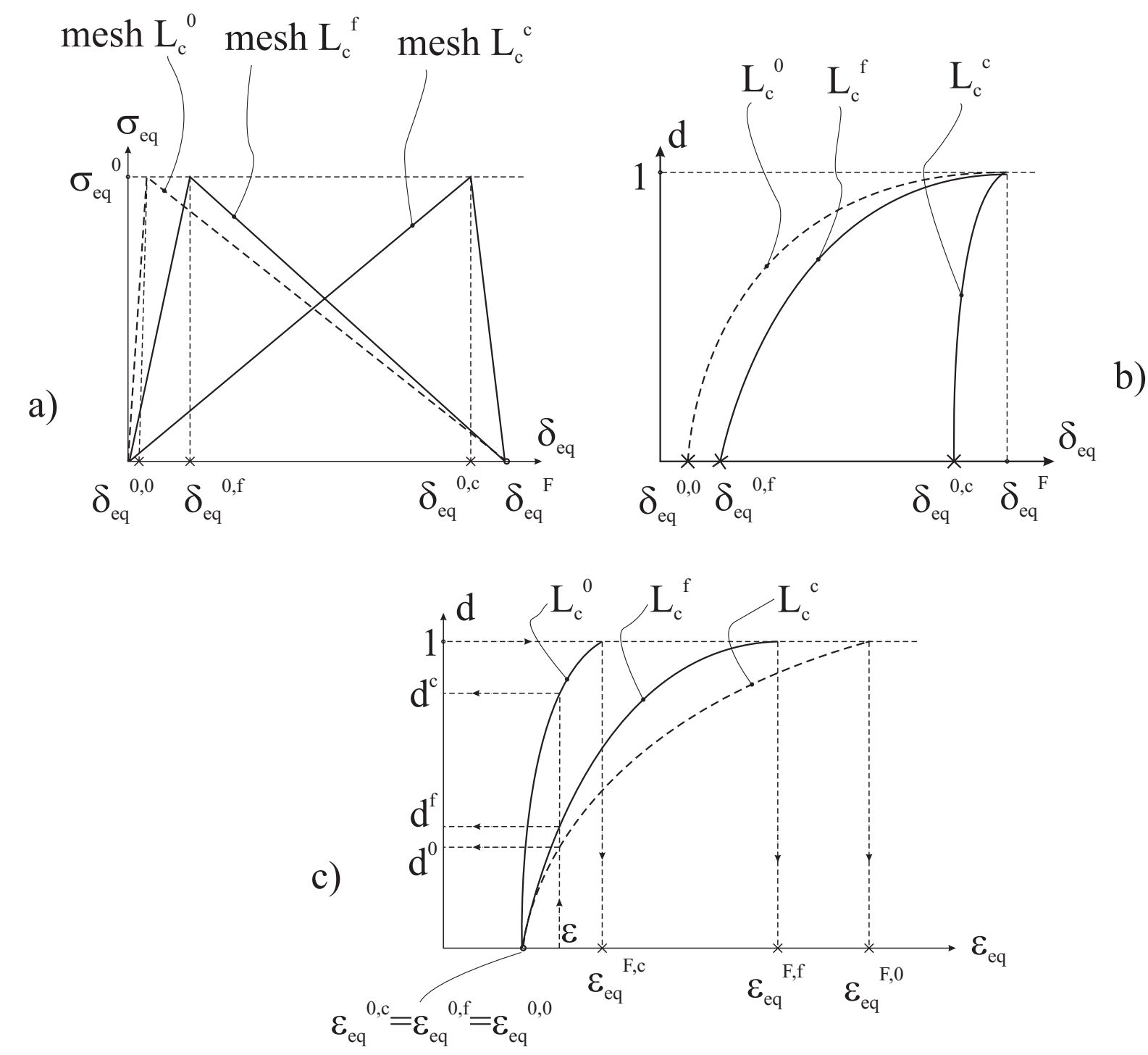

Figure E.4: Mesh sensitivity in the $(\sigma-\delta)$ softening model

An observation has to be made here: the above analyzed mesh sensitivity is not the same with the classical FEA mesh sensitivity of the displacement based solution. The solution in the case of classical instance of mesh sensitivity converges upon mesh refinement, while the results in the case of the present model continuously diverge upon mesh refinement (see Fig. E. 4 c)) due to the $L_{c}$ parameter. The same is the case of mesh sensitivity introduced by 
calculating ERR from FEA solution, as it is the case of VCCT techniques. The two instances of mesh sensitivity can overlap each other into a FEA model. Special counteraction measures are needed to alleviate the continuously diverging mesh sensitivity, which are usually called characteristic length in the literature (see, for example Oliver [115]), as well.

\section{E.3 The progressive damage model in Helius}

Brief description of the progressive damage modeling approach in Helius MCT (MultiContinuum Theory):

Two modeling concepts are introduced in Helius MCT terminology [73, 74]: Multiscale , which means that the whole dimensional spectrum from the laminate (structural) level, to ply level, and to fiber and matrix constituents level is covered by the analysis; MultiContinuum, which means that the ply level analysis is not treated under the homogenization assumption as in Classical Laminate Theory (CLT), but it is considered as two continuum constituents, namely the fiber and the matrix (Fig. E.5). The MCT method for failure analysis of composites, Mayes [76, 77, 78, 80], Hansen [116], implies calculating stresses in fiber and matrix constituents and applying progressive damage theories at the constituent level. The Helius MCT product for damage analysis can virtually interact with any FEA solver:

- the ply stresses at the material point are known from the FEA solver and are passed to Helius;

- the MCT method is applied by Helius in order to resolve the ply stresses into fiber and matrix stresses, and damage theory is applied at constituent level. Damage onset is detected by quadratic strength based FC, and degradation of material stiffness is considered if the damage onset criterion is met;

- the reduced material properties are passed back to the EFA solver;

- the above steps are iterated until equilibrium is achieved.

In order to calculate stresses and apply failure theory at constituent level, the constituent elastic and strength properties are needed. The elastic properties are back-calculated by Helius based on an internal FEA micromechanics model (i.e., at fiber and matrix level) of 


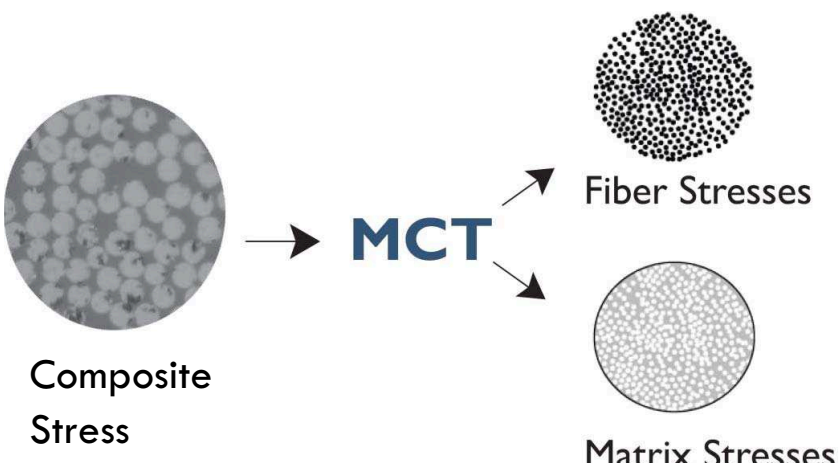

Figure E.5: Fiber-matrix averaging approach of MCT (from [73]).

the UD composite, having the experimentally determined homogenized elastic properties of the UD composite as input data. The FEA micromechanics model is iteratively solved for the elastic properties of fiber and matrix constituents until the UD ply properties are fit. In this way the in-situ elastic properties of the fiber and matrix are evaluated and used for modeling. The starting values for the iterative FEA procedure are the bulk properties of the fiber and matrix constituents.

The constituent strength properties are analytically back-calculated by considering the stress state in fiber and matrix at the moment of the UD ply failure, based on the homogenized ply strength values. Having known the constituent strength values, separate quadratic failure criteria for fiber and matrix are applied in order to detect the damage onset in constituents at the material point of the UD ply inside of the laminate. Fiber and matrix are supposed to simultaneously exist at any material point.

Some observations on the Helius MCT formulation are the following:

- the fiber and matrix stresses are evaluated by the MCT approach in an average sense, according to each constituent volumetric participation inside of the ply. The fiber-matrix interaction is not considered in detail. Strength values of individual constituents are evaluated based on homogenized UD ply strengths. Based on these observations, it is not obvious which is the advantage of applying the stress and failure analysis at the micro level of fiber and matrix, since the ply homogenized stress and strength govern the analysis.

On the contrary, there might be disadvantages in taking the modeling at micro level, due 
to the fact that the usual $2 D$ plane stress analysis at ply level is now inevitable replaced by a $3 D$ analysis at fiber and matrix level, which implies the need of $3 D$ stress evaluation for the failure analysis. At this moment the problem might arise from the fact that weight is given to out of plane elastic and strength parameters, which are difficult to evaluate. For example, even if the advantage of transverse isotropy are invoked for defining the $E_{33}$ out of plane elastic modulus equal to the $E_{22}$ in-plane one, this might not be true in the case of strength values. It is possible that the strength value are different on the 3 and 2 directions due to different damage mechanisms on these directions. While on 3 direction damage is of inter-laminar type (supposing fracture between laminated plies, parallel to the plan of the laminate), on 2 direction damage if of intra-laminar type (supposing fracture transverse to the plan of the laminate). This difference between the inter-laminar and intra-laminar critical values of the UD composite has been experimentally proved for the case of critical energy release rate strength parameter, Wang [82]. The same reasoning applies in the case of the damage evolution: for example, there is no guideline if the out of plane stiffness of the UD ply should be degraded at the same rate as the $E_{22}$ elastic modulus, in the case of transverse matrix cracking. It is thus considered that replacing the plane stress $2 D$ analysis at ply level with a $3 D$ constituent level one might bring more complication and uncertainty into analysis than bringing the desired accuracy.

- one advantage of the constituent level analysis is the fact that residual thermal stresses inside of fiber and matrix, due to the CTE mismatch at fiber-matrix level, can be evaluated. It is reasonable to suppose that the residual stresses at constituent level are higher than those at ply level (which are considered by the CLT), due to higher CTE mismatch at constituents level compared to ply level. However, the change in thermal expansion coefficients of the constituents/composite due to ply damage is not evaluated by the Helius model.

- both ply elastic properties and bulk fiber/matrix elastic properties are needed in order to apply the iterative FEA micromechanic procedure to determine the in-situ elastic constants of the constituents. This is an extra requirement for the user. It is true that the values corresponding to the bulk constituent are only starting values for the iterative procedure, but it is not clear from the available documentation if the iterative procedure is sensitive to the start values. If this is the case, close to reality $3 D$ elastic constants of the constituents are needed, thus doubling quantity of elastic input data required from the user.

- due to the fact that failure criteria are applied at constituent level, yet based on the UD ply strength values, the in-situ effect of the laminate configuration on damage initiation 
(as explained at the beginning of this section) can not be considered.

- three different stiffness reduction strategies are considered in conjunction with the FEA solver that Helius can be added on $[75,117]$. The stifness reduction strategy is an user's choice when the analysis is set up. The three stiffness reduction methods are:

1. Progressive Failure Analysis - implemented for both Ansys and Abaqus interfaces;

2. Include Post Failure Nonlinearity - implemented for both Ansys and Abaqus interfaces;

3. Energy Based Degradation - implemented only for Abaqus interface.

The Progressive Failure Analysis user option applies an instantaneously stiffness reduction scheme when the constituent FC condition is met. Despite its name of progressive, the stiffness degradation is not progressive at the material point level, but it is of ply discount type, based on sudden stiffness reduction. The level of discount (degradation) of the stiffness values at the material point of the ply is user selected. This discount level ca be important with regard to the post damage response of the structure, Fertig [118], and the user might not have guidelines for setting this parameter. It is explained in the Helius technical documentation $[75,117]$ that the damage analysis becomes progressive while the damage spreads over a structural component under loading. However, this is due to the stress concentrations and variable stress field over the structural component, not due to the progressive damage nature of the method itself.

The Include Post Failure Nonlinearity and Energy Based Degradation options are truly progressive damage at the material point level. The importance of implementing real progressive damage (as opposed to ply discount damage) in Helius was underlined in Mayes [79], where the material point progressive stiffness reduction is called "2nd generation MCT failure analysis". However, the method in [79] is based on experimental curves presented in Knops [119], which are expected to be dependent on the laminate configuration. The last Helius implementation is not based on the stiffness reduction method presented in [79].

The Include Post Failure Nonlinearity option is a CDM like softening techniques based on strain level as damage variable. It requires the matrix normal failure strain as a measure of the end of the progressive damage process (final failure). This is another failure parameter which can not be a-priori known by the user and most likely dependent on the configuration of the composite material, as well. The method is based on gradual stiffness reduction of the matrix by a series of four discrete reduction events (see Fig. E.6). 


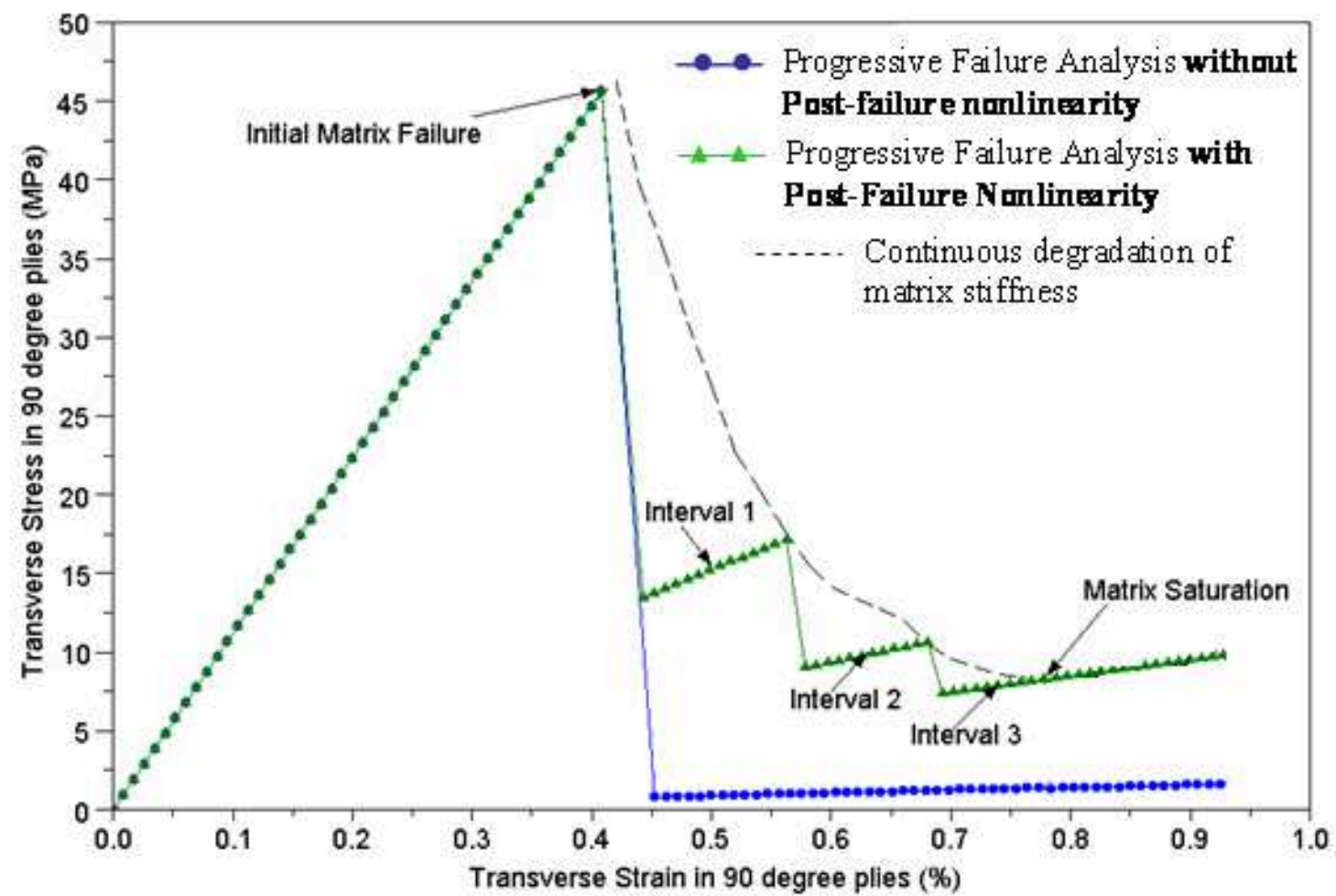

Figure E.6: Discrete (4 step) matrix stiffness degradation, Helius progressive damage model (from [75]).

The Energy Based Degradation option is available only under Abaqus and requires the critical energy release rate $G_{C}$ as damage parameter (see Fig. E.3 (a)). Conceptually, this stiffness reduction method is entirely similar to the previously described progressive damage model implemented in Abaqus (see Section. E.2). The difference resides in how damage onset is detected: Helius is based on $3 D$ quadratic FC at fiber and matrix level, while Abaqus is based on $2 D$ quadratic FC (Hashin) at UD ply level. After damage initiation is detected, stiffness reduction is applied at ply level (as opposed to constituent level in the previous two Helius stiffness reduction methods, Progressive Failure Analysis and Include Post Failure Nonlinearity). The ply stiffness reduction in the case of matrix cracking is of isotropic type, which means that all $E_{22}, E_{33}, G_{12}, G_{13}, G_{23}$ elastic constants of the damaging ply are degraded at the same rate. As shown in $\mathrm{Li}[6]$, there is no reason to consider the same rate of degradation for $G_{12}$ shear modulus as for $E_{22}$ extension modulus. Moreover, the $G_{12}$ is more prone to be LSS dependent, and there is no clear intuitive guideline for the degradation of out of plane stiffnesses. In this regard, the isotropic stiffness reduction can 
be considered a rough approximation.

All the observations made in case of Abaqus progressive damage method with regard to softening law are also applicable here. The Energy Based Degradation method is actually based on the characteristic length $L_{c}$ of the element (see eq. (E.2)) which is provided by Abaqus to Helius. The mesh dependency aspect of the results due to the use of $L_{c}$ was discussed din Section E.2.

A basic but interesting case (see Fig. E.7) is presented in [120], as an argument that the mesh sensitivity is reduced by the present Energy Based Degradation (EBD) progressive damage implementation. If the model formulation and the discussion related to mesh sensitivity in Section E.2 is reviewed at this moment, it seems that the argument of alleviated mesh sensitivity which is made in the example in [120] presented in Fig. E.7 does not hold entirely. An overlap of the two kinds of mesh sensitivities (the converging one introduced by the FEA solution, and the diverging one introduced by the progressive damage model) actually happens in the example in Fig. E.7. It is true that, for the case presented as example and for the mesh densities used there, the progressive damage model mesh sensitivity alleviates the FEA solution mesh sensitivity. But, considering that the FEA solution mesh dependence is converging by itself upon mesh refinement, while the progressive damage mesh dependence is continuously diverging, bigger difference in results can be expected upon continuously mesh refinement. Moreover, it is difficult to compare the cases presented in the example in [120], because the mesh sensitivity is not the only variable to account for in the cases compared in Fig. E.7, but the cases presented as without EBD are actually ply discount based, while the cases with EBD are progressive damage based. An example to keep in mind with regard to mesh dependency is the one discussed in SectionE.2: progressive damage for the case of a specimen without central hole, where due to the uniform stress-strain field, only the influence of mesh size on the progressive damage behavior can be evaluated.

\section{E.4 The progressive damage model in LS-Dyna}

Damage in UD laminated composites has been implemented in LS-Dyna starting with early materials models (MAT22, MAT54, MAT55) where damage onset is based on 2D stress quadratic FC and damage propagation is based on ply discount methods $[121,122]$. Extension to the $3 D$ formulation is done in MAT161, in order to approach modeling of damage under impact loading. This $3 D$ formulation brings into picture the complication 

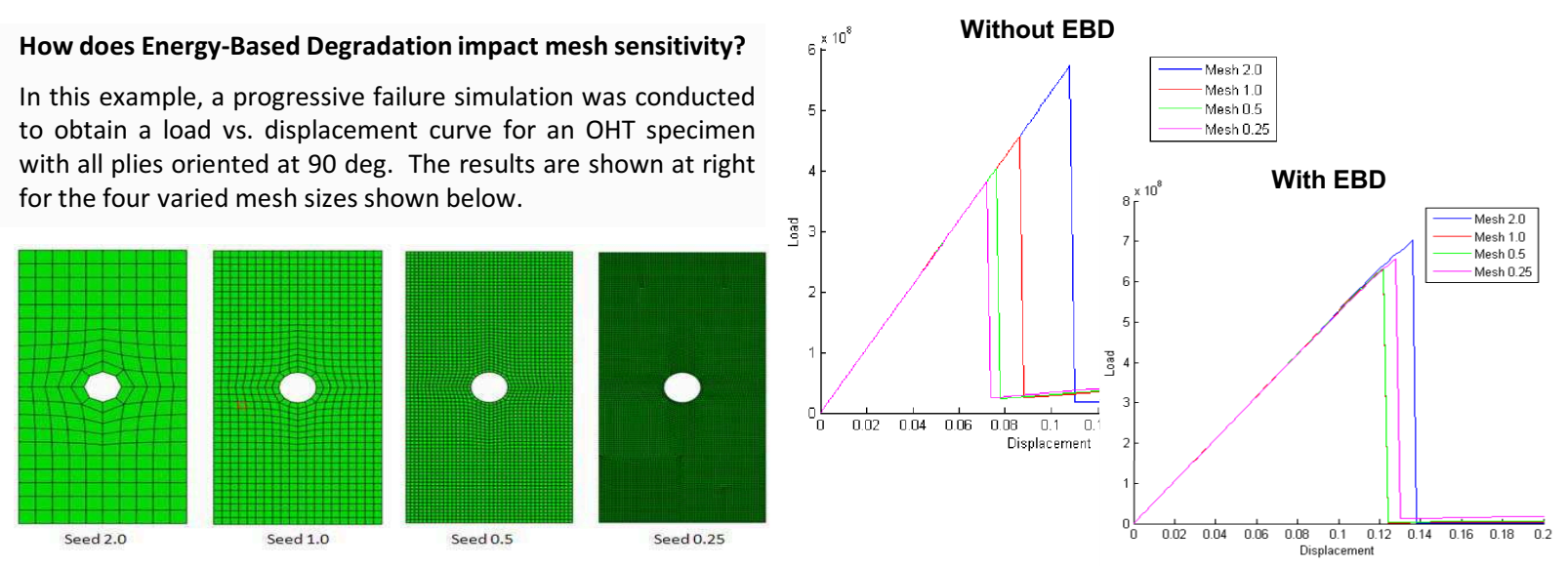

Figure E.7: Mesh sensitivity study for Helius progressive damage model (from [120]).

imposed by the need of $3 D$ elastic and strength material properties, this being the price paid for generalizing the model from $2 D$ in-plane damage modes to $3 D$ damage modes, as it is the case of impact loading. Regarding the in-plane damage mode of intra-laminar matrix cracking which is the focus of this chapter, these material models features the limitation of any strength based FC for damage onset and ply discount method for damage propagation discussed in the previous subsections.

Progressive damage at the material point is implemented starting with the material model MAT162 [121, 123]. Damage onset in this model is based on the 3D strength FC first implemented in MAT161, and progressive damage is based on the CDM approach originally developed for the $2 D$ case in Matzenmiller [114], and then extended to $3 D$ progressive damage in Yen [124]. According to these theoretical references, the progressive damage for composite materials in LS-Dyna is a strain controlled CDM softening law, conceptually expressed in 
terms of $(\sigma-\epsilon)$ stress-strain state at the material point as

$$
\sigma=\exp \left[\frac{1}{m}\left(1-r^{m}\right)\right] E^{0} \epsilon
$$

where $E^{0}$ is the undamaged material property and $(\sigma, \epsilon)$ is an equivalent stress-strain state corresponding to each damage mode of the UD ply: longitudinal (fiber) tension-compression, transverse (matrix) tension-compression, and in-plane shear (matrix). $r$ is a function of the strain state formulated according to each damage mode, called damage threshold, which takes the value $r=1$ at damage onset (when the condition in the quadratic stress FC is met), then increases continuously with the strain state at the material point. $m$ is a damage parameter which has to be fit based on experimentally determined damage behavior of the material. The material behavior up to damage threshold $(0<r<1)$ is linear elastic, corresponding to the virgin material elastic properties. After damage initiation $(r \geq 1)$, the strain controlled softening behavior is according to the exponential law supposed in eq. (E.7). The pre- and post- damage behavior is depicted in Fig. E.8. There is a separate graph as the one presented in Fig. E.8 for each damage variable $d$ (see eq. (E.8)), corresponding to each elastic material property of the UD composite ply.

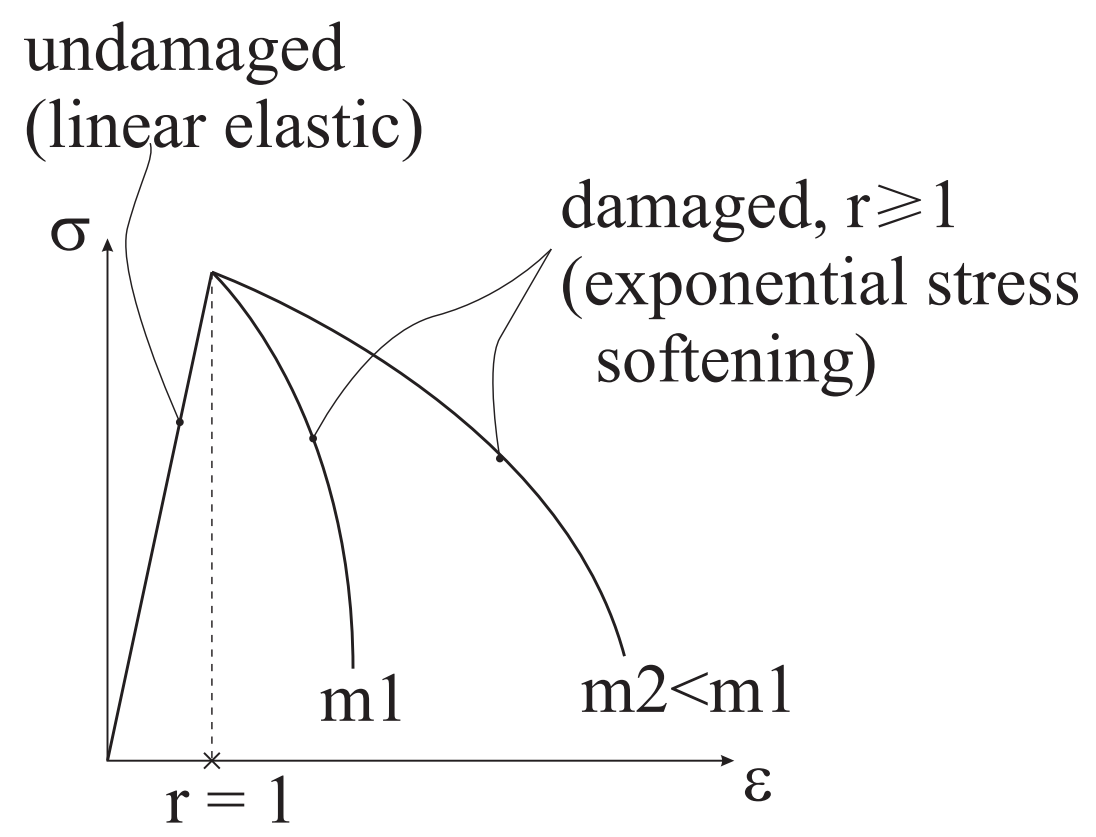

Figure E.8: Progressive damage model, $(\sigma-\epsilon)$ exponential softening law.

The softening law in eq. (E.7) allows for expressing the damage parameter in eq. (E.1) 
as

$$
d_{i j}=1-\exp \left[\frac{1}{m_{i j}}\left(1-r^{m_{i j}}\right)\right]
$$

where there is a stiffness reduction parameter $d_{i j}$ corresponding to each elastic modulus $E_{i j}$ in the $3 \mathrm{D}$ stiffness matrix of the UD composite ply $(i, j=1 \ldots 3)$. Here $E_{i j}$ are the diagonal terms of the ply 3D stiffness matrix, namely the Young and shear moduli; the off diagonal Poisson therms of the ply are considered not affected by damage, based on the model in [114].

It can be observed that the softening law $(\sigma-\epsilon)$ in Fig. E.8 might be regarded as similar to the softening law $(\sigma-\delta)$ in Fig. E.3 (a), Section E.2. However, the two formulations are not equivalent. Even if both of them are strain controlled softening law, the one in Fig. E.3 (a) is set by the critical ERR material constant and requires a characteristic length of the element $L_{c}$, while the one in Fig. E.8 is set by the material parameter $m$ and does not require a characteristic length of the element $L_{c}$. Even if, from the mesh dependency point of view, it seems to be an advantage of the $(\sigma-\epsilon)$ law in Fig. E.8 (because it does not introduce the mesh size dependent parameter $L_{c}$ ), it is yet very difficult to obtain the softening parameter $m$ from experiments. As a general rule, low values of $m$ correspond to a more ductile softening behavior (see the $m_{2}$ curve in Fig. E.8), while high values correspond to a more brittle behavior (see the $m_{1}$ curve in Fig. E.8).

As expressed in $[123,124]$, the right determination of the damage parameter $m$ is an "open issue". According to the original model in [114], the damage parameter $m$ is supposed to be determined from uniaxial tests of the UD ply, which are representative to each damage mode and stiffness degradation parameter. In both $[114,124]$ references, exemplification is done for the case of $d_{11}$, which corresponds to progressive damage of the UD ply due to fiber tension, which is equivalent to finding the Weibull parameter of the statistical distribution of fibers strength. This fibers strength distribution might induce a progressive damage for tensile testing of the UD ply along fiber direction similar to the one presented in Fig. E.8. However, the method of testing UD composites in order to determine the corresponding $d_{i j}$ parameter has no applicability in the case of transverse matrix cracking by tensile or shear loading, corresponding to $d_{22}$ and $d_{12}$ parameters. This is because progressive matrix cracking for a single ply UD composite is not possible. The UD composite has to be embedded into a laminate, in which case damage evolution becomes dependent on laminate configuration. As a parenthesis, a complicated experimental method of transverse punch test is presented in 
Xiao and Gama $[125,126,127]$ in order to determine other 3D damage parameters required for simulation of impact damage.

More than the aspects briefly discussed so far, some other points to be in the user's attention with regard to the progressive damage implementation of transverse matrix cracking in LS-Dyna would be the following:

- crack density is not a damage variable, for applications where this information is of interest;

- damage onset is base on strength FC, which does not allow for considering the influence of laminate configuration on onset;

- the CDM type softening law can not consider the influence of laminate configuration on damage evolution;

- the influence of progressive damage on CTE of the UD damaging ply can not be evaluated. 


\section{References}

[1] J. Varna, R. Joffe, and R. Talreja. A synergistic damage-mechanics analysis of transverse cracking in $\left[ \pm \theta / 90_{4}\right]$ s laminates. Composites Science and Technology, 61(5):657$665,2001$.

[2] J. Varna, R. Joffe, N. V. Akshantala, and R. Talreja. Damage in composite laminates with off-axis plies. Composites Science and Technology, 59(14):2139-2147, 1999.

[3] S. E. Groves, C. E. Harris, A. L. Highsmith, D. H. Allen, and R. G. Norvell. Experimental and analytical treatment of matrix cracking in cross-ply laminates. Experimental Mechanics, 27(1):73-79, 1987.

[4] D. H. Allen, C. E. Harris, and S. E. Groves. A thermomechanical constitutive theory for elastic composites with distributed damage - II. Application to matrix cracking in laminated composites. International Journal of Solids and Structures, 23(9):1319-38, 1987.

[5] S. Li, S. R. Reid, and P. D. Soden. Modelling the damage due to transverse matrix cracking in fiber-reinforced laminates. In Proc. 2nd Int. Conf. on Nonlinear Mechanics (ICNP-2), pages 320-323. Peking University Press, 1993.

[6] S. Li, S. R. Reid, and P. D. Soden. A continuum damage model for transverse matrix cracking in laminated fibre-reinforced composites. Philosophical Transactions of the Royal Society London, Series A (Mathematical, Physical and Engineering Sciences), 356(1746):2379-412, 10/15 1998.

[7] J. A. Nairn and S. Hu. Damage Mechanics of Composite Materials, volume 9 of Composite Materials Series, chapter Matrix Microcracking, pages 187-244. Elsevier, 1994.

[8] S. Liu and J. A. Nairn. The formation and propagation of matrix microcracks in crossply laminates during static loading. Journal of Reinforced Plastics and Composites, 11(2):158-78, 021992.

[9] J. A. Nairn. Polymer Matrix Composites, volume 2 of Comprehensive Composite Materials, chapter Matrix Microcracking in Composites, pages 403-432. Elsevier Science, 2000. 
[10] J. A. Nairn. The strain energy release rate of composite microcracking: a variational approach. Journal of Composite Materials, 23(11):1106-29, 111989.

[11] J. A. Nairn, S. Hu, S. Liu, and J. S. Bark. The initiation, propagation, and effect of matrix microcracks in cross-ply and related laminates. In Proc. of the 1st NASA Advanced Comp. Tech. Conf., pages 497-512, Oct. 29 - Nov. 1, 19901990.

[12] J. A. Nairn. Microcracking, microcrack-induced delamination, and longitudinal splitting of advanced composite structures. Technical Report NASA Contractor Report 4472, 1992.

[13] J. A. Nairn. Applications of finite fracture mechanics for predicting fracture events in composites. In 5th Int'l Conf. on Deform. and Fract. of Comp., 18-19 March 1999.

[14] J. A. Nairn. Fracture mechanics of composites with residual stresses, imperfect interfaces, and traction-loaded cracks. In Workshop 'Recent Advances in Continuum Damage Mechanics for Composites', volume 61, pages 2159-67, UK, 20-22 Sept. 2000. Elsevier.

[15] J. A. Nairn and D. A. Mendels. On the use of planar shear-lag methods for stresstransfer analysis of multilayered composites. Mechanics of Materials, 33(6):335-362, 2001.

[16] J. A. Nairn. Finite Fracture Mechanics of Matrix Microcracking in Composites, pages 207-212. Application of Fracture Mechanics to Polymers, Adhesives and Composites. Elsevier, 2004.

[17] Y. M. Han, H. T. Hahn, and R. B. Croman. A simplified analysis of transverse ply cracking in cross-ply laminates. Composites Science and Technology, 31(3):165-177, 1988.

[18] C. T. Herakovich, J. Aboudi, S. W. Lee, and E. A. Strauss. Damage in composite laminates: Effects of transverse cracks. Mechanics of Materials, 7(2):91-107, 111988.

[19] J. Aboudi, S. W. Lee, and C. T. Herakovich. Three-dimensional analysis of laminates with cross cracks. Journal of Applied Mechanics, Transactions ASME, 55(2):389-397, 1988 .

[20] F. W. Crossman, W. J. Warren, A. S. D. Wang, and G. E. Law. Initiation and growth of transverse cracks and edge delamination in composite laminates. ii - experimental correlation. Journal of Composite Materials Suplement, 14(1):88-108, 1980.

[21] D. L. Flaggs and M. H. Kural. Experimental determination of the in situ transverse lamina strength in graphite/epoxy laminates. Journal of Composite Materials, 16:10316, 031982 .

[22] S. H. Lim and S. Li. Energy release rates for transverse cracking and delaminations induced by transverse cracks in laminated composites. Composites Part A, 36(11):14671476, 2005. 
[23] S. Li and F. Hafeez. Variation-based cracked laminate analysis revisited and fundamentally extended. International Journal of Solids and Structures, 46(20):3505-3515, 2009.

[24] J. L Rebiere and D. Gamby. A decomposition of the strain energy release rate associated with the initiation of transverse cracking, longitudinal cracking and delamination in cross-ply laminates. Composite Structures, 84(2):186-197, 2008.

[25] S. C. Tan and R. J. Nuismer. A theory for progressive matrix cracking in composite laminates. Journal of Composite Materials, 23(10):1029-47, 101989.

[26] R. J. Nuismer and S. C. Tan. Constitutive relations of a cracked composite lamina. Journal of Composite Materials, 22(4):306-21, 041988.

[27] T. Yokozeki and T. Aoki. Stress analysis of symmetric laminates with obliquely-crossed matrix cracks. Advanced Composite Materials, 13(2):121-40, 2004.

[28] T. Yokozeki and T. Aoki. Overall thermoelastic properties of symmetric laminates containing obliquely crossed matrix cracks. Composites Science and Technology, 65(1112):1647-54, 2005.

[29] D. H. Cortes and E. J. Barbero. Stiffness reduction and fracture evolution of oblique matrix cracks in composite laminates. Annals of Solids and Structural Mechanics, 1(1):29-40, 2010.

[30] E. J. Barbero and D. H. Cortes. A mechanistic model for transverse damage initiation, evolution, and stiffness reduction in laminated composites. Composites Part B, 41:124$132,2010$.

[31] A. S. D. Wang, P. C. Chou, and S. C. Lei. A stochastic model for the growth of matrix cracks in composite laminates. Journal of Composite Materials, 18(3):239-54, 051984.

[32] P. Gudmundson and S. Ostlund. First order analysis of stiffness reduction due to matrix cracking. Journal of Composite Materials, 26(7):1009-30, 1992.

[33] P. Gudmundson and S. Ostlund. Prediction of thermoelastic properties of composite laminates with matrix cracks. Composites Science and Technology, 44(2):95-105, 1992.

[34] P. Gudmundson and W. Zang. An analytic model for thermoelastic properties of composite laminates containing transverse matrix cracks. International Journal of Solids and Structures, 30(23):3211-31, 1993.

[35] E. Adolfsson and P. Gudmundson. Thermoelastic properties in combined bending and extension of thin composite laminates with transverse matrix cracks. International Journal of Solids and Structures, 34(16):2035-60, 061997.

[36] E. Adolfsson and P. Gudmundson. Matrix crack initiation and progression in composite laminates subjected to bending and extension. International Journal of Solids and Structures, 36(21):3131-3169, 1999. 
[37] W. Zang and P. Gudmundson. Damage evolution and thermoelastic properties of composite laminates. International Journal of Damage Mechanics, 2(3):290-308, 07 1993.

[38] E. Adolfsson and P. Gudmundson. Matrix crack induced stiffness reductions in $\left[\left(0_{m} / 90_{n} /+\theta_{p} /-\theta_{q}\right)_{s}\right]_{m}$ composite laminates. Composites Engineering, 5(1):107-23, 1995.

[39] P. Lundmark and J. Varna. Modeling thermo-mechanical properties of damaged laminates. In 3rd International Conference on Fracture and Damage Mechanics, FDM 2003, volume 251-252 of Advances in Fracture and Damage Mechanics, pages 381-7, Switzerland, 2-4 Sept. 2003. Trans Tech Publications.

[40] M. Kachanov. volume 30 of Advances in Applied Mechanics, chapter Elastic solids with many cracks and related problems, pages 260-445. Academic Press, Inc., 1993.

[41] T. Yokozeki, T. Aoki, and T. Ishikawa. Transverse crack propagation in the specimen width direction of CFRP laminates under static tensile loadings. Journal of Composite Materials, 36(17):2085-99, 2002.

[42] T. Yokozeki, T. Aoki, and T. Ishikawa. Consecutive matrix cracking in contiguous plies of composite laminates. International Journal of Solids and Structures, 42(9-10):2785802, 052005 .

[43] T. Yokozeki, T. Aoki, T. Ogasawara, and T. Ishikawa. Effects of layup angle and ply thickness on matrix crack interaction in contiguous plies of composite laminates. Composites Part A (Applied Science and Manufacturing), 36(9):1229-35, 2005.

[44] S. Kuriakose and R. Talreja. Variational solutions to stresses in cracked cross-ply laminates under bending. International Journal of Solids and Structures, 41(9-10):2337-47, 052004 .

[45] S. Li, S. R. Reid, and P. D. Soden. A finite strip analysis of cracked laminates. Mechanics of Materials, 18(4):289-311, 101994.

[46] S. Li and S. H Lim. Variational principles for generalized plane strain problems and their applications. Composites Part A: Applied Science and Manufacturing (Incorporating Composites and Composites Manufacturing), 36(3):353-365, 2005.

[47] J. Nairn. Some new variational mechanics results on composite microcracking. In Proceedings of the 1995 10th Internatioanal Conference on Composite Materials, pages 423-423, Aug 14 - 181995.

[48] J. A. Nairn. Exact and variational theorems for fracture mechanics of composites with residual stresses, traction-loaded cracks, and imperfect interfaces. International Journal of Fracture, 105(3):243-71, 2000. 
[49] J. A. Mayugo, P. P. Camanho, P. Maimi, and C. G. Dávila. Analytical modeling of transverse matrix cracking of $\left[ \pm \theta / 90_{n}\right]$ composite laminates under multiaxial loading. Mechanics of Advanced Materials and Structures, 17:237-45, 2010.

[50] R. Talreja. Damage Mechanics of Composite Materials, volume 9 of Composite Materials Series, chapter Damage Characterization by Internal Variables, pages 73-78. Elsevier, 1994.

[51] P. A. Smith and S. L. Ogin. Characterization and modeling of matrix cracking in a $(0 / 90)_{2 S}$ GFRP laminate loaded in flexure. Proceedings of the Royal Society of London, Series A (Mathematical, Physical and Engineering Sciences), 456(2003):2755$70,11 / 082000$.

[52] P. A. Smith and S. L. Ogin. On transverse matrix cracking in cross-ply laminates loaded in simple bending. Composites Part A: Applied Science and Manufacturing, 30(8):1003-1008, 1999.

[53] C. G. Dávila, P. P. Camanho, and C. A. Rose. Failure criteria for FRP laminates. Journal of Composite Materials, 39(4):323-345, 2005.

[54] H. T. Hahn. A mixed-mode fracture criterion for composite materials. Composites Technology Review, 5:26-29, 1983.

[55] A. Parvizi, K. W. Garrett, and J. E. Bailey. Constrained cracking in glass fiber reinforced epoxy cross-ply laminates. Journal of Materials Science, 13:195-201, 1978.

[56] E. J. Barbero. Introduction to Composite Materials Design. CRC Press, 2nd edition, 2011.

[57] J. Zhang, J. Fan, and C. Soutis. Analysis of multiple matrix cracking in $\left[ \pm \theta_{m} / 90_{n}\right] s$ composite laminates. Part II: Development of transverse ply cracks. Composites, 23(5):299-304, September 1992.

[58] D. T. G. Katerelos, J. Varna, and C. Galiotis. Energy criterion for modeling damage evolution in cross-ply composite laminates. Composites Science and Technology, 68:2318-24, 2008.

[59] S. Yalvac, L. D. Yats, and D. G. Wetters. Transverse ply cracking in toughened and untoughened Graphyte/Epoxy and Graphyte/Polycyanate cross ply laminates. Journal of Composite Materials, 25:1653-67, 121991.

[60] J. Fan and J. Zhang. In-situ damage evolution and micro/macro transition for laminated composites. Composites Science and Technology, 47:107-18, 1993.

[61] A. S. D. Wang. Fracture mechanics of sublaminate cracks in composite materials. Composites Technology Review, 6(2):45-62, 1984.

[62] K.D. Cowley and P.W.R. Beaumont. The interlaminar and intralaminar fracture toughness of carbon-fibre/polymer composites: the effect of temperature. Composites Science and Technology, 57(11):1433 - 1444, 1997. 
[63] R.W. Truss, P.J. Hine, and R.A. Duckett. Interlaminar and intralaminar fracture toughness of uniaxial continuous and discontinuous carbon fibre/epoxy composites. Composites Part A (Applied Science and Manufacturing),28A(7):627 - 36, 1997.

[64] U. Hansen and Jr. Gillespie, J.W. Dependence of intralaminar fracture toughness on direction of crack propagation in unidirectional composites. Journal of Composites Technology and Research, 20(2):89 - 99, 1998.

[65] B.F. Sorensen and T.K. Jacobsen. Large-scale bridging in composites: R-curves and bridging laws. Composites Part A (Applied Science and Manufacturing), 29A(11):1443 $-51,1998$.

[66] T. K. Jacobsen and B. F. Sorensen. Mode I intra-laminar crack growth in composites modeling of R-curves from measures bridges laws. Composites: Part A, 32:1-11, 2001.

[67] M. Iwamoto, Q.-Q. Ni, T. Fujiwara, and K. Kurashiki. Intralaminar fracture mechanism in unidirectional cfrp composites. Part I: Intralaminar toughness and AE characteristics. Engineering Fracture Mechanics, 64(6):721 - 745, 1999.

[68] S. M. Lee. Double torsion fracture toughness test for evaluating transverse cracks in composites. Journal of Materials Science Letters, 1:511-15, 1982.

[69] A.S.D. Wang, N.N. Kishore, and W.W. Feng. On mixed mode fracture in off-axis unidirectional graphite-epoxy composites. volume 1, pages 599 - 606, 1982.

[70] S.L. Donaldson. Fracture toughness testing of graphite/epoxy and graphite/PEEK composites. Composites, 16(2):103 - 12, 1985.

[71] D. R. Moore and J. C. Seferis. Intrinsic characterization of continuous fibre reinforced thermoplastic composites - I. Toughness characterization of carbon fibre/Polyether Ether Ketone (CF/PEEK) laminates. Pure ans Applied Chemistry, 63(11):1609-25, 1991.

[72] S. T. Pinho, P. Robinson, and L. Iannucci. Developing a four point bend specimen to measure the mode I intralaminar fracture toughness of unidirectional laminated composites. Composites Science and Technology, 69:1303-1309, 2009.

[73] Firehole Composites. Progressive failure of composites made easy. http://www . firehole.com/documents/mct_Progressive_Failure_Analysis_WP.pdf, 2011.

[74] Firehole Composites.

MCT multi-scale material characterization process. http://www.firehole.com/ documents/mct_multiscale_material_characterization_WP.pdf, 2011.

[75] Firehole Composites. Helius MCT, User's Guide, February 2011. Helius:MCT, Version 4.0 for ABAQUS.

[76] J. S. Mayes and Andrew C. Hansen. Multicontinuum failure analysis of composite structural laminates. Mechanics of Composite Materials and Structures, 8(4):249-262, 2001. 
[77] J. S. Mayes and A. C. Hansen. Composite laminate failure analysis using multicontinuum theory. Composites Science and Technology, 64(3-4):379-94, 2004.

[78] J. S. Mayes and A. C. Hansen. A comparison of multicontinuum theory based failure simulation with experimental results. Composites Science and Technology, 64(3-4):51727, 2004.

[79] Nelson E. E., Hansen A. C., Welsh J.S., and Mayes J. S. Recent advances in failure prediction of composite laminates utilizing multicontinuum technology. In 49th AIAA/ASME/ASCE/AHS/ASC Structures, Structural Dynamics, and Materials Conference. AIAA, April 2008.

[80] Mayes J. S. Micromechanics based failure analysis of composite structural laminates. Technical Report NSWCCD-65-TR-1999/15, Naval Surface Warfare Center, Carderock Division, West Bethesda, MD, September 1999.

[81] F. W. Crossman, W. J. Warren, A. S. D. Wang, and G. E. Law. Initiation and growth of transverse cracks and edge delamination in composite laminates. II - Experimental correlation. Journal of Composite Materials Suplement, 14(1):88-108, 1980.

[82] F. W. Crossman and A. S. D. Wang. Damage in Composite Materials, chapter The dependence of transverse cracking and delamination on ply thickness in Graphite/Epoxy laminates, pages 118-139. ASTM International, 1982.

[83] R. Joffe and J. Varna. Analytical modeling of stiffness reduction in symmetric and balanced laminates due to cracks in 90 layers. Composites Science and Technology, 59(11):1641-1652, 1999.

[84] O. Rubenis, E. Sparnins, J. Andersons, and R. Joffe. The effect of crack spacing distribution on stiffness reduction of cross-ply laminates. Applied Composite Materials, $14(2): 59-66,2007$.

[85] J. Tong, F. J. Guild, S. L. Ogin, and P. A. Smith. On matrix crack growth in quasiisotropic laminates. I. Experimental investigation. Composites Science and Technology, 57:1527-35, 1997.

[86] D. T. G. Katerelos, M. Kashtalyan, C. Soutis, and C. Galiotis. Matrix cracking in polymeric composites laminates: Modeling and experiments. Composites Science and Technology, 68:2310-17, 2008.

[87] L.N. McCartney. Physically based damage models for laminated composites. Proceedings of the Institution of Mechanical Engineers, Part L (Journal of Materials: Design and Applications), 217(L3):163 - 99, 2003.

[88] L.N. McCartney. Energy-based prediction of progressive ply cracking and strength of general symmetric laminates using an homogenisation method. volume 36, pages 119 $-128,2005$. 
[89] L. N. McCartney. Energy-based prediction of failure in general symmetric laminates. Engineering Fracture Mechanics, 72(6 SPEC. ISS.):909 - 930, 2005.

[90] H. Tada, P. C. Paris, and G. R. Irwin. Stress Analysis of Cracks Handbook. ASME Press, New York, NY, 3rd edition, 2000.

[91] J.P. Benthem and W.T. Koiter. Asymptotic approximations to crack problems, volume 1 of Mechanics of Fracture, pages 131-78. 1973.

[92] J. R. Reeder. An evaluation of mixed-mode delamination failure criteria. Technical report, NASA, 1992. NASA-TM-104210, NAS 1.15:104210.

[93] J. R. Reeder. Composite Materials: Testing and Design, volume 11, ASTM STP 1206, chapter A biliniar failure criterion for mixed-mode delamination, pages 303-322. American Society for Testing and Materials, Philadelphia, PA, 1993.

[94] M. L. Benzeggagh and M. Kenane. Measurement of mixed-mode delamination fracture toughness of unidirectional glass-epoxy composites with mixed-mode bending aparatus. Composites Science and Tecjnology, 56:439-49, 1996.

[95] P. P. Camanho and C. G. Dávila. Mixed-mode decohesion finite elements for the simulation of delamination in composite materials. Technical report, NASA Langley Research Center, June 2002. NASA/TM-2002-211737.

[96] R. Rikards, F. G Buchholz, H. Wang, A. K. Bledzki, A. Korjakin, and H. A Richard. Investigation of mixed mode I/II interlaminar fracture toughness of laminated composites by using a CTS type specimen. Engineering Fracture Mechanics, 61(3-4):325-42, 1998.

[97] Z. Hashin. Failure criteria for unidirectional fiber composites. Transactions of the ASME.Journal of Applied Mechanics, 47(2):329-34, 061980.

[98] Knops M. Analysis of Failure in Fiber Polymer Laminates: The Theory of Alfred Puck. Springer, 2008.

[99] E. J. Barbero, G. Sgambitterra, A. Adumitroaie, and X. Martinez. A discrete constitutive model for transverse and shear damage of symmetric laminates with arbitrary stacking sequence. Composite Structures, 93(2):1021-30, 012011.

[100] G. Sgambitterra, A. Adumitroaie, E. J. Barbero, and A. Tessler. A robust threenode shell element for laminated composites with matrix damage. Composites Part B: Engineering, 42(1):41-50, 2011.

[101] J. Zhang, J. Fan, and C. Soutis. Analysis of multiple matrix cracking in $\left[ \pm \theta_{m} / 90_{n}\right] s$ composite laminates. Part I: In-plane stiffness properties. Composites, 23(5):291-298, September 1992. 
[102] X. Su, F. Abdi, and R.Y. Kim. Prediction of micro-crack densities in IM7/977-2 polymer composite laminates under mechanical loading at room and cryogenic temperatures. In 46rd AIAA Structures, Structural Dynamics, and Materials Conference. AIAA, 2005.

[103] Alpha STAR Corporation. http://www.ascgenoa.com/main/, 2011.

[104] X. Su, F. Abdi, and J. A. Lavoie. Prediction of micro-crack densities in cryogenic IM7/977-2 propellant tanks. In 45rd AIAA Structures, Structural Dynamics, and Materials Conference. AIAA, 2006.

[105] Alpha STAR Corporation. Micro crack density prediction of continuous fiber reinforced polymeric composites. http://www.ascgenoa.com/main/newsletter/7/index.jsp, 2007.

[106] Simulia. Abaqus Analysis User's Manual, Version 6.10.

[107] Simulia. Abaqus Theory Manual, Version 6.10.

[108] A. Hillerborg, M. Modeer, and P.E. Petersson. Analysis of crack formation and crack growth in concrete by means of fracture mechanics and finite elements. Cemet and Concrete Research, 6:773-82, 1976.

[109] A. Hillerborg. Fictitious crack model and its use in numerical analyses. Advances in Colloid and Interface Science, pages 667-679, 1979.

[110] A. Hillerborg. Numerical methods to simulate softening and fracture of concrete, pages 141-170. Martinus Nijhoff Publ, Dordrecht, Neth, 1985.

[111] A. Hillerborg. Theoretical basis of a method to determine the fracture energy $g_{f}$ of concrete. Materials and Structures/Materiaux et Constructions, (106):291-296, 1985.

[112] A. Hillerborg. Application of the fictitious crack model to different types of materials. International Journal of Fracture, 51(2):95-102, 1991.

[113] C. G. Dávila, P. P. Camanho, and M. F. De Moura. Mixed-mode decohesion elements for analyses of progressive delamination. In 42nd AIAA/ASME/ASCE/AHS/ASC Structures, Structural Dynamics, and Materials Conference, April 16 - 19, 2001, volume 3, pages 2277-88, Seattle, WA, United states, 2001. AIAA.

[114] A. Matzenmiller, J. Lubliner, and R. L. Taylor. A constitutive model for anisotropic damage in fiber-composites. Mechanics of Materials, 20(2):125-52, 041995.

[115] J. Oliver. A consistent characteristic length for smeared cracking models. International Journal for Numerical Methods in Engineering, 28(2):461-74, 021989.

[116] E. Nelson, D. Kenik, and A. Hansen. Multicontinuum failure analysis of composites. In ICCM-17, 17th International Conference on Composite Materials. The British Composites Society, July 2009. 
[117] Firehole Composites. Helius MCT, User's Guide, February 2011. Helius:MCT, Version 4.0 for ANSYS.

[118] J. Stack, R. Fertig, and Biskner A. Modelind damage tolerance in composite structures: Selecting material degradation parameters. In SAMPE 2011, May 2011.

[119] M. Knops and C. Bogle. Gradual failure in fibre/polymer laminates. Composites Science and Technology, 66(5):616-625, 2006.

[120] Firehole Composites. Minimizing mesh sensitivity. http://www.firehole.com/ documents/HeliusMCT $\% 20$ Energy $\backslash \% 20$ Based $\ \% 20$ Degradation.pdf.

[121] Livermore Software Technology Corporation. LS-DYNA Theory Manual, March 2006.

[122] Livermore Software Technology Corporation. LS-DYNA Keyword User's Manual, version 971 edition, May 2007.

[123] Center for Composite Materials, University of Delaware. User's Manual for LSDYNA MAT162 Unidirectional and Plain Weave Composite Progressive Failure Models, November 2005.

[124] Chian-Fong Yen. Ballistic impact modeling of composite materials. In 7th International LS-DYNA Users Conference. Livermore Software Technology Corporation, May 2002.

[125] University of Delaware Center for Composite Materials. Mat162 - composite dmg msc. http://www. ccm. udel.edu/Tech/MAT162.

[126] J. R. Xiao, B. A. Gama, and J. W. Gillespie Jr. Progressive damage and delamination in plain weave S-2 Glass/SC-15 composites under quasi-static punch-shear loading. Composite Structures, 78(2):182-196, 2007.

[127] B. A. Gama, T. A. Bogetti, and J. W. Gillespie. Progressive damage modeling of plainweave composites using LS-Dyna composite damage model MAT162. In 7th European LS-DYNA Conference. DYNAmore GmbH, May 2009. 